\title{
OPTIMIZING THE MECHANICAL CHARACTERISTICS OF BAMBOO TO IMPROVE THE FLEXURAL BEHAVIOR FOR BIOCOMPOSITE STRUCTURAL APPLICATION
}

\author{
A Thesis Presented to \\ the Faculty of California Polytechnic State University, \\ San Luis Obispo
}

In Partial Fulfillment

of the Requirements for the Degree of

Master of Science in Aerospace Engineering

By

Jay M. Lopez

November 2012 
(c) 2012

Jay M. Lopez

ALL RIGHTS RESERVED 
TITLE:

AUTHOR:

DATE SUBMITTED:

COMMITTEE MEMBER:

COMMITTEE MEMBER:

COMMITTEE MEMBER:

COMMITTEE CHAIR:
Optimizing the Mechanical Characteristics of Bamboo to Improve the Flexural Behavior for Biocomposite Structural Application

Jay M. Lopez

November 2012

\section{COMMITTEE MEMBERSHIP}

Faysal Kolkailah, Ph.D, P.E.

Eltahry Elghandour, Ph.D

Bing Qu, Ph.D, P.E.

Susan Opava, Ph.D 


\begin{abstract}
Optimizing the Mechanical Characteristics of Bamboo to Improve the Flexural Behavior for Biocomposite Structural Application
\end{abstract}

Jay M. Lopez

Global awareness and preservation have spurred increasing interest in utilizing environmentally friendly materials for high-performance structural applications. Biocomposites pose an appealing solution to this issue and are characterized by their sustainable lifecycles, biodegradable qualities, light weight, remarkable strength, and exceptional stiffness. Many of these structural qualities are found in applications that exhibit flexural loading conditions, and this study focuses on improving the bending performance of engineered biocomposite structures. The current application of biocomposites is increasing rapidly, so this expanding research explores other natural constituent materials for biocomposite structures under flexural loading.

The renewable material investigated in this study was experimentally and numerically validated by optimizing the mechanical characteristics of bamboo fibers in biocomposite structures under flexural loading conditions through various thermal and organic chemical treatment methods. Therefore, bending performance of a biocomposite truss and I-beam are analyzed to demonstrate the benefits of utilizing optimally treated bamboos in their design.

To accomplish this goal, the first task consisted of treating bamboos by thermal and chemical means to determine the resulting effects on the compressive and tensile mechanical properties through experimental testing. Results indicated a significant improvement in strength, stiffness, and weight reduction. An extensive analysis determined the optimal treatment method that was utilized for flexural loading conditions.

The second task entailed studying the flexural behavior of the optimally treated bamboo in two geometric configurations, a hollow cylinder and veneer strip, to determine the resultant properties for the truss and I-beam structure. The effect of node location on flexural performance was also studied to establish design guidelines for the applied structures. Bending tests indicated that node location affects the strength and stiffness of the hollow cylindrical configuration but has minimal effects on the veneer strip. Observations discovered by this study were employed into the designs of the applied structures that yielded excellent mechanical performance through flexural testing.

The final task required conducting a finite element analysis in Abaqus/CAE on the performance of each structural application to validate experimental results. A conclusive analysis revealed good agreement between the numerical method and experimental result. 


\section{Acknowledgements}

I would first like to show my immense gratitude to my thesis advisers, Dr. Faysal Kolkailah and Dr. Eltahry Elghandour, for the opportunity to pursue my academic career on the graduate student level. Their interest in a proposal developed by the Maersk Co. inevitably drew my attention toward completing research in biocomposite structures. These gentlemen have encouraged me in all the right ways to reach for excellence in all matters regarding the work performed throughout the course of this research. The patience of these men and their unwavering support toward their students is truly humbling. My appreciation goes out to Dr. Kolkailah for all the times he has helped me proceed through my scholastic endeavors. I also send my warmest gratitude to Dr. Elghandour for always guiding me in the right direction when I needed him most and for being my rock-solid support through and through. His insight in this research and all things non-academic was immensely helpful; I certainly credit him for all his efforts in assisting me in any way he could. It is truly an honor and pleasure to have worked with Dr. Kolkailah and Dr. Elghandour on this research. Their support, generosity, and uplifting kindness will never be forgotten.

I also thank my thesis committee members, Dr. Susan Opava and Dr. Bing Qu, for taking the time to acknowledge the culmination of this work and providing me with the necessary advice to improve this thesis to its best potential. Many thanks are also due to the Cal Poly Aerospace Engineering department and all its staff for their assistance in obtaining my degree. A special thank you is also in order for Becky Powell and Debbie Hart for all their efforts and guidance throughout the closing days of my graduate career.

My appreciation also reaches far out to my friend and colleague, Cameron Chan, for his company and insight in venturing into the workings of this research with me. I certainly could not have finished this work as gracefully as I did without all his support through the seemingly never-ending hours in the lab. I would like also to thank Miles Murphy for assisting me throughout much of the l-beam assembly. His contagious enthusiasm was always a pleasure to work with in the lab.

Gratitude is also given to Cody Thompson for his assistance in machining test equipment parts for me and for his advice/company through the working days. I also thank Ray Ward for letting me test the bamboo truss with the Riehle testing apparatus in the Architecture Department's lab at Cal Poly SLO.

An honorable mention also goes out to my friends and peers in the lab that are also in the aerospace graduate program: Dennis Boettcher, Kyle Johnson, Albert Liu, Alma Melendez, Kodi Rider, Gabriel Sanchez, and Vanessa Wood. Their company was always refreshing. I give a special thanks to Albert, Etuate Varea, and Kodi for keeping Cameron and me entertained with laughs throughout those moments we were working.

Lastly and most of all, I hold a deep gratitude to my entire family for their overwhelming support throughout my entire academic career. I thank my mom, dad, and sisters for always believing in me and the person l'd like to be; I am forever indebted to them for their relentless support and patience. I also send my appreciation to all my aunts, uncles, cousins, and friends from home that also believed in me throughout my college days and always welcomed me with open arms during those short trips back home.

I dedicate this work and any of its success to all of the above. 


\section{Table of Contents}

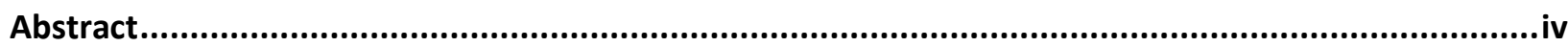

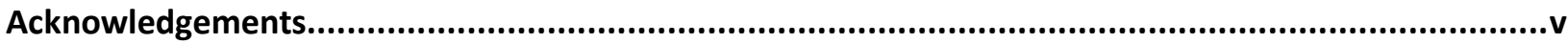

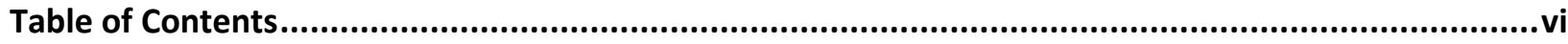

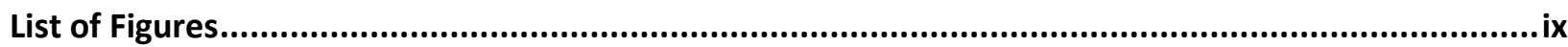

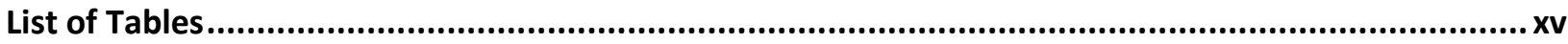

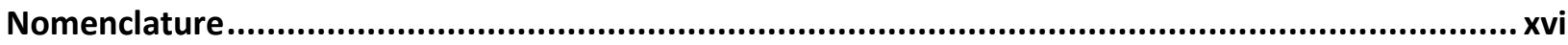

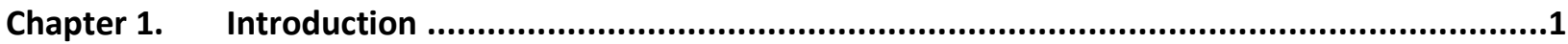

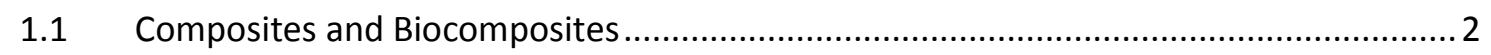

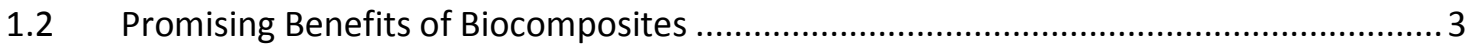

1.3 Practical Implementation of Biocomposite Structures .............................................. 6

1.4 A New Biocomposite Fiber Constituent: Bamboo …..................................................... 8

1.4.1 Understanding the Plant Bambusoideae .......................................................... 8

1.4.2 Cultivating Bamboo and Harvesting Operations................................................. 13

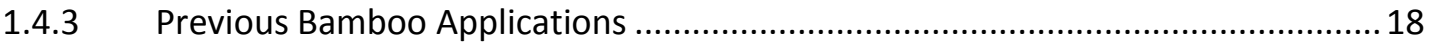

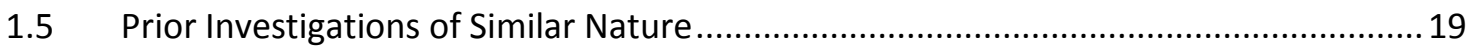

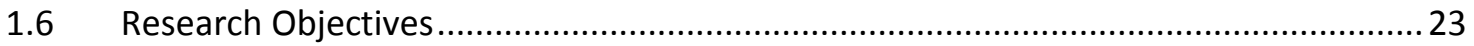

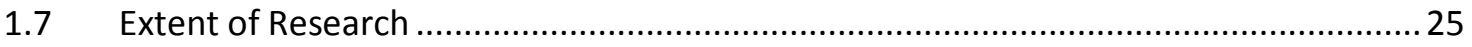

Chapter 2. Inquiring Bamboo Treatment Methods..................................................................27

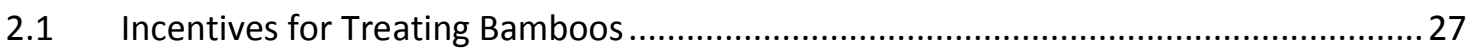

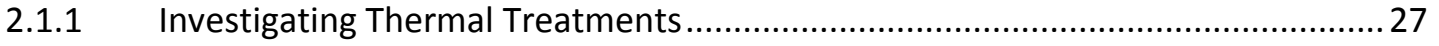

2.1.2 Investigating Organic Chemical Treatments .................................................... 28

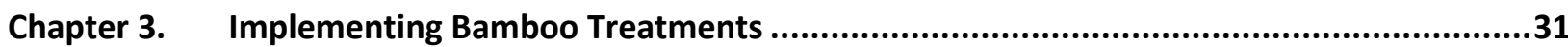

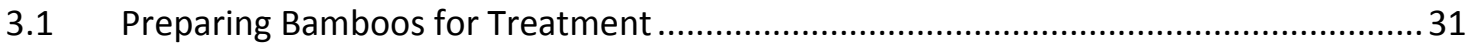

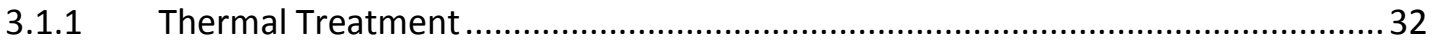

3.1.2 Natural Chemical Treatment............................................................................. 41

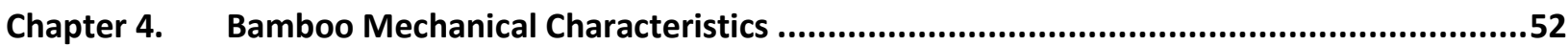

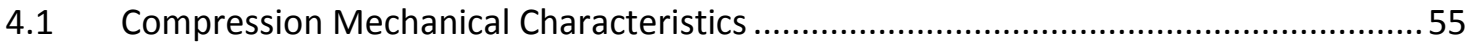

4.1.1 Preparing Compression Specimens................................................................... 56

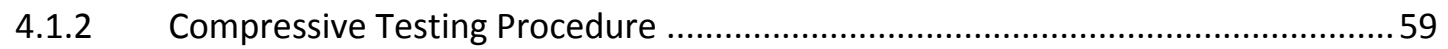

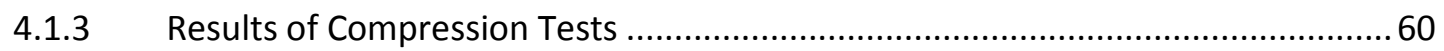




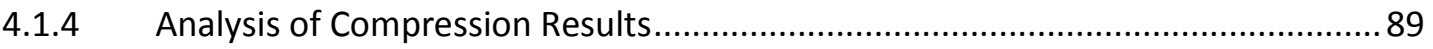

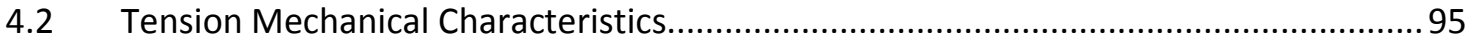

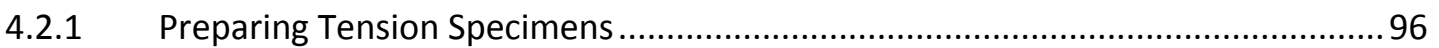

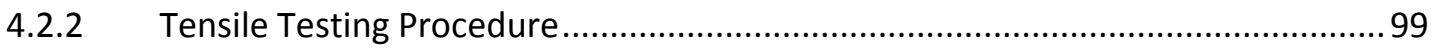

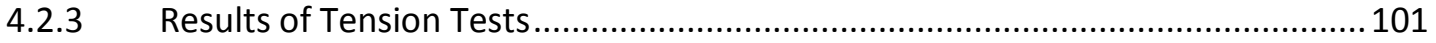

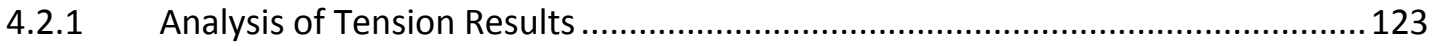

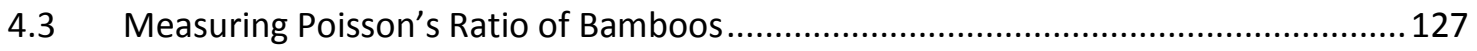

4.3.1 Preparing Poisson's Ratio Specimens …...................................................... 128

4.3.2 Poisson's Ratio Testing Procedure …............................................................. 131

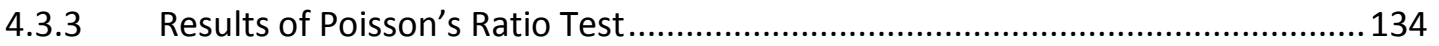

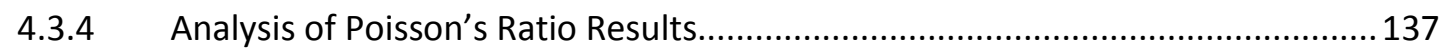

Chapter 5. Optimum Bamboo Treatment Method .....................................................................139

5.1 Optimization Analysis for Ideal Bamboo Treatment................................................139

5.2 Comparative Mechanical Performance of Bamboo to Conventional Materials.......... 146

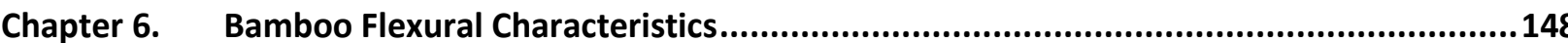

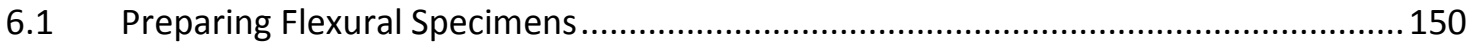

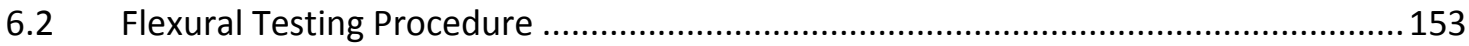

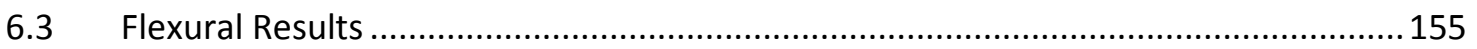

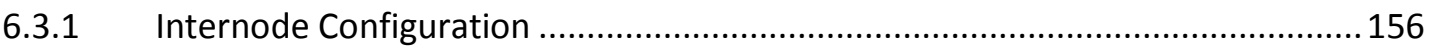

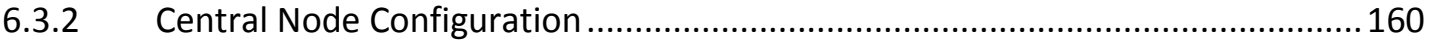

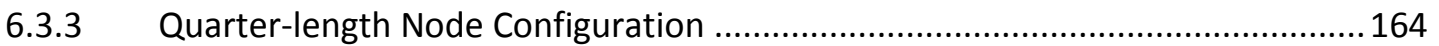

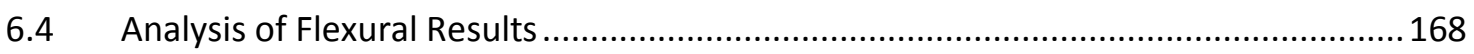

Chapter 7. Biocomposite Structural Application......................................................................177

7.1 Bamboo Member Composite Truss Application ..................................................... 178

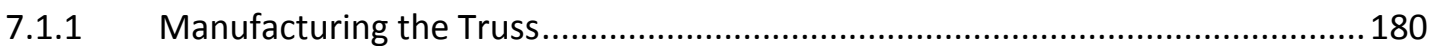

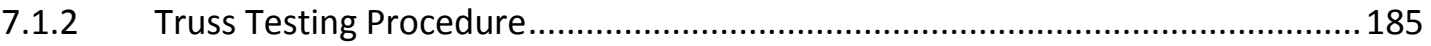

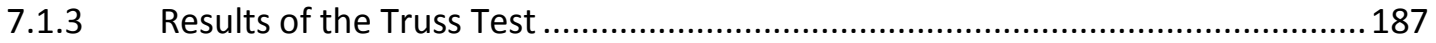

7.1.4 Finite Element Method to Validate Truss Performance Results from Experiment 192

7.1.5 Conclusion of Truss Performance and Future Notes ........................................204

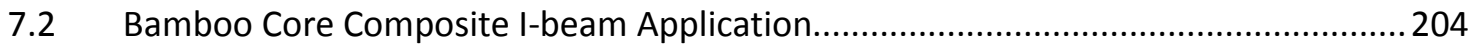

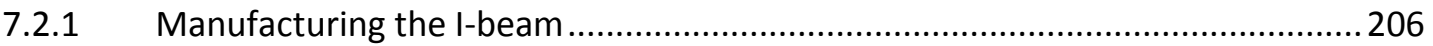




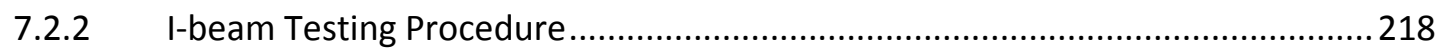

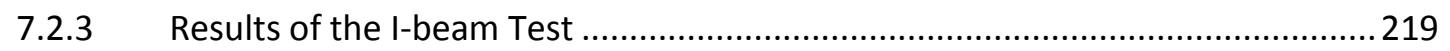

7.2.4 Finite Element Method to Validate I-beam Performance Results from Experiment 224

7.2.5 Conclusion of I-beam Performance and Future Notes .......................................235

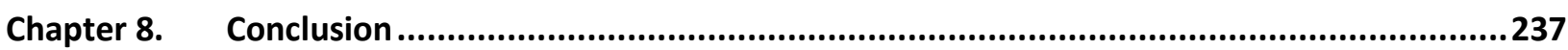

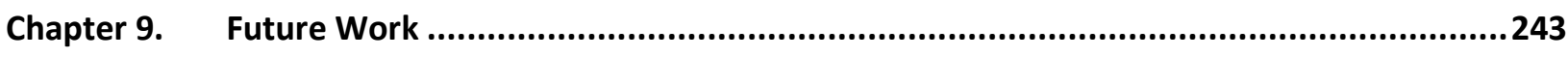

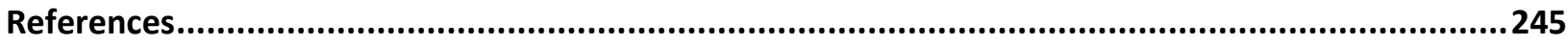

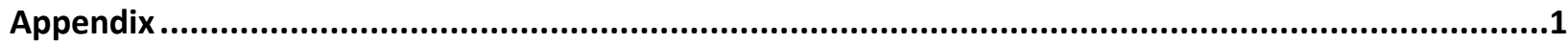

A1. Validation of ISO standard for Node Effect on Compressive Properties ........................ 1

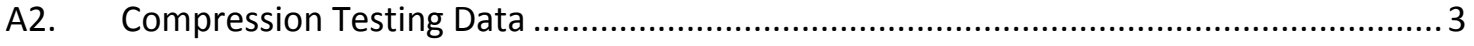

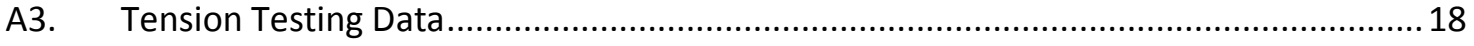

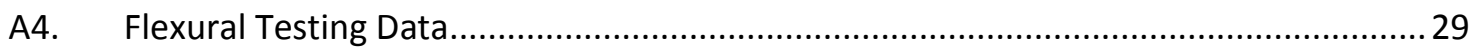

A5. Manufacturing Hemp Mold Joints for Bamboo Truss Application ............................... 35

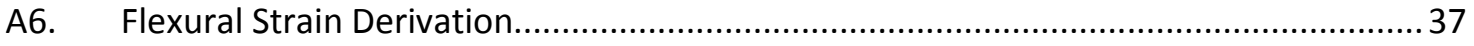

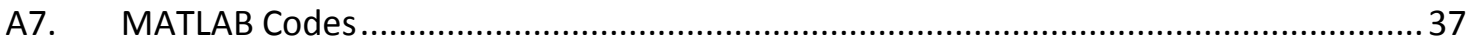




\section{List of Figures}

Figure 1-1. Constituents of a composite material [47] ..........................................................................2

Figure 1-2. Biocomposite samples made of germ, corn stover, and soybean hulls [24]...............................3

Figure 1-3. Potential lifecycle of biocomposite structures.......................................................................5

Figure 1-4. Kestrel is the first all-hemp biocomposite car body [9] ........................................................... 7

Figure 1-5. Mercedes-Benz A-Class and F-22 Raptor both utilize biocomposites in parts of their structure

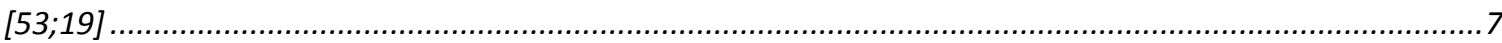

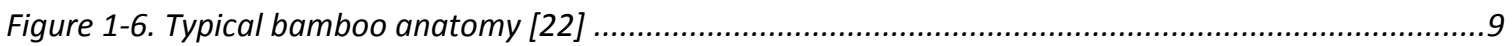

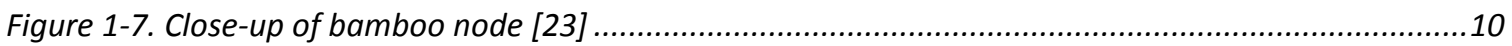

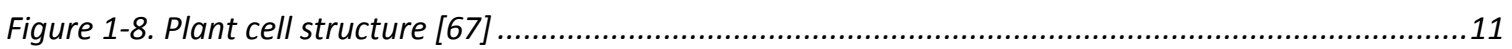

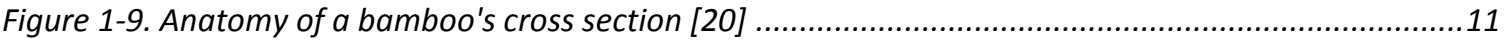

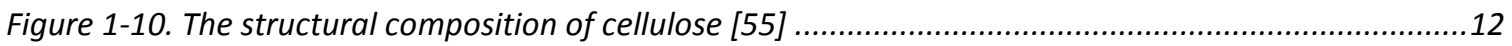

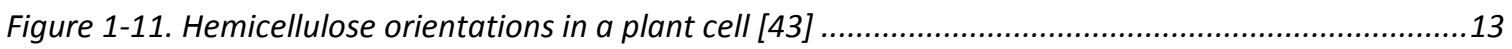

Figure 1-12. Climate zones in the United States [13] ...........................................................................15

Figure 1-13. Carefully nurtured bamboo poles personally selected from Jungle Supply Co. .......................18

Figure 1-14. Laminated bamboo surfboards and example of cross section [71] ......................................19

Figure 1-15. Water absorption data taken by FCBA [45] ...................................................................21

Figure 1-16. Schott's findings of weight loss versus treatment temperature [72] ....................................22

Figure 1-17. Schott's discovery of thermal treatment's effects on elastic modulus of bamboos [72] ..........22

Figure 1-18. Schott's data on thermal treatments' effects on strength and ultimate strain [72] ................23

Figure 2-1. Proposed natural chemical treatments salt (left), lime (center), and oil (right) [64; 26; 28] ......29

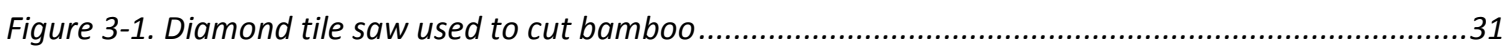

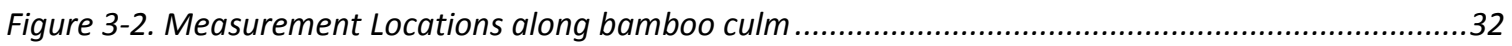

Figure 3-3. Cal Poly SLO's autoclave in Aerospace Structures/Composites laboratory .................................33

Figure 3-4. Sample cure cycle utilized: (1) heating stage (2) dwell stage (3) cooling stage (4) end stage ....34

Figure 3-5. Analysis of physical changes for all thermal treatments.....................................................40

Figure 3-6. Water salinity in different bodies of water [39] ...............................................................42

Figure 3-7. Instant Ocean Sea Salt mix [57] and soaking bamboos in the salt solution .............................43

Figure 3-8. Hydrated lime mix utilized in lime treatment [26] ...........................................................46

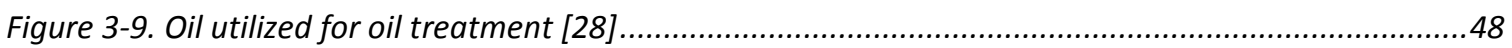

Figure 3-10. Analysis of physical changes for all organic chemical treatments .....................................50

Figure 4-1. Instron 8801 machine to perform all tests .....................................................................55

Figure 4-2. Manufacturing drawing for compression specimen with exemplary dimensions ......................57

Figure 4-3. Cutting compressions specimens to length. sanding them accordingly, and leveling them .......58

Figure 4-4. Compression test fixtures with fiberglass buffers (left), subsequent leveling (center), and

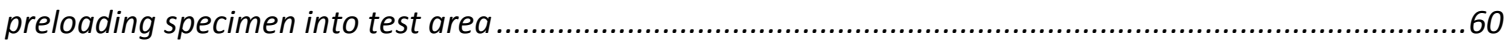

Figure 4-5. Untreated bamboo compressive stress-strain curves ........................................................62

Figure 4-6. Exaggerated view of imperfect end faces on compression specimen ......................................63

Figure 4-7. Common compressive failure types: longitudinal splitting (left) and bearing stress deformation

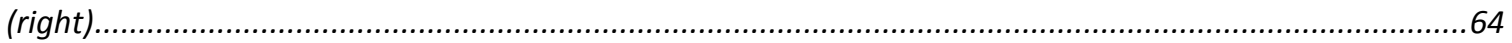

Figure 4-8. Tested untreated compression specimens ...............................................................64

Figure 4-9. Common compressive failure types for $150^{\circ} \mathrm{F}$ at $5^{\circ} \mathrm{F} / \mathrm{min}$. treatment ...................................66

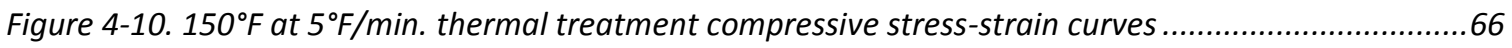

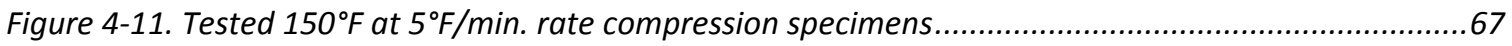


Figure $4-12.180^{\circ} \mathrm{F}$ at $5^{\circ} \mathrm{F} / \mathrm{min}$. thermal treatment compressive stress-strain curves ...............................6

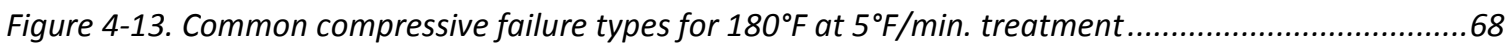

Figure 4-14. Portion of tested $180^{\circ} \mathrm{F}$ at $5^{\circ} \mathrm{F} / \mathrm{min}$. rate compression specimens ......................................69

Figure $4-15.180^{\circ} \mathrm{F}$ at $1^{\circ} \mathrm{F} / \mathrm{min}$. thermal treatment compressive stress-strain curves ...............................70

Figure 4-16. Common compressive failure types for $180^{\circ} \mathrm{F}$ at $1^{\circ} \mathrm{F} / \mathrm{min}$. treatment ..................................70

Figure 4-17. Portion of tested $180^{\circ} \mathrm{F}$ at $1^{\circ} \mathrm{F} / \mathrm{min}$. rate compression specimens ......................................71

Figure 4-18. $200^{\circ} \mathrm{F}$ at $5^{\circ} \mathrm{F} / \mathrm{min}$. thermal treatment compressive stress-strain curves ...............................72

Figure 4-19. Common compressive failure types for $200^{\circ} \mathrm{F}$ at $5^{\circ} \mathrm{F} / \mathrm{min}$. treatment .................................72

Figure 4-20. Portion of tested $200^{\circ} \mathrm{F}$ at $5^{\circ} \mathrm{F} / \mathrm{min}$. rate compression specimens ......................................73

Figure 4-21. $200^{\circ} \mathrm{F}$ at $1^{\circ} \mathrm{F} / \mathrm{min}$. thermal treatment compressive stress-strain curves ...............................74

Figure 4-22. Common compressive failure types for $200^{\circ} \mathrm{F}$ at $1^{\circ} \mathrm{F} / \mathrm{min}$. treatment..................................75

Figure 4-23. Tested $200^{\circ} \mathrm{F}$ at $1^{\circ} \mathrm{F} / \mathrm{min}$. rate compression specimens....................................................75

Figure 4-24. $220^{\circ} \mathrm{F}$ at $1^{\circ} \mathrm{F} / \mathrm{min}$. thermal treatment compressive stress-strain curves ................................77

Figure 4-25. Common compressive failure types for $220^{\circ} \mathrm{F}$ at $1^{\circ} \mathrm{F} / \mathrm{min}$. treatment ................................77

Figure 4-26. Tested $220^{\circ} \mathrm{F}$ at $1^{\circ} \mathrm{F} /$ min. rate compression specimens..................................................78

Figure 4-27. 3\% salt treatment compressive stress-strain curves ......................................................79

Figure 4-28. Common compressive failure types for $3 \%$ salt treatment ...............................................80

Figure 4-29. Portion of tested 3\% salt compression specimens.........................................................8

Figure 4-30. 6\% salt treatment compressive stress-strain curves .....................................................81

Figure 4-31. Common compressive failure types for $6 \%$ salt treatment ...............................................82

Figure 4-32. Portion of tested 6\% salt compression specimens............................................................82

Figure 4-33. 9\% salt treatment compressive stress-strain curves ......................................................83

Figure 4-34. Common compressive failure types for $9 \%$ salt treatment ..............................................84

Figure 4-35. Portion of tested $9 \%$ salt compression specimens..........................................................8.

Figure 4-36. Lime treatment compressive stress-strain curves .........................................................85

Figure 4-37. Common compressive failure types for lime treatment .................................................86

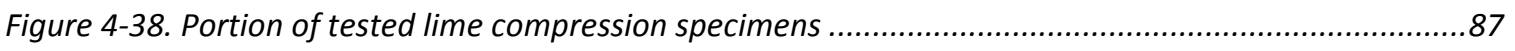

Figure 4-39. Oil treatment compressive stress-strain curves ............................................................87

Figure 4-40. Common compressive failure types for oil treatment ...................................................8

Figure 4-41. Portion of tested oil compression specimens .................................................................89

Figure 4-42. Summary of treatment effects on compressive specific strength and specific modulus..........90

Figure 4-43. Summary of treatment effects on compressive strain, stress, and modulus..........................91

Figure 4-44. Preparing tensile specimens begins by cutting culm into rectangular strips (left), sanding them

to the appropriate dimensions (center), and the final specimen geometry (right) .................................97

Figure 4-45. Cutting compressions specimens to length. sanding them accordingly, and leveling them .....97

Figure 4-46. Cutting rectangular strips from culm ...................................................................97

Figure 4-47. Manufacturing drawing for tension specimen ...................................................................98

Figure 4-48. Aligning tensile specimen within grip jaws (left) and correct setup in the grip jaws (right) ...100

Figure 4-49. Untreated bamboo tensile stress-strain curves...............................................................102

Figure 4-50. Common tensile failure types: four main failures (left), and enhanced view of fiber

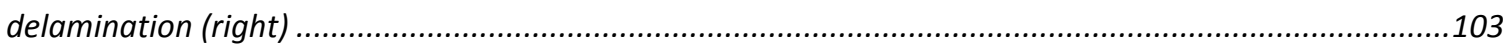

Figure 4-51. Most common tensile failure types characterized as splitting ...........................................104

Figure 4-52. Portion of tested untreated tension specimens................................................................105

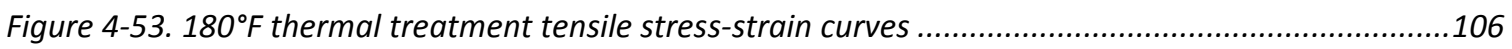

Figure 4-54. Common tensile failure types for $180^{\circ} \mathrm{F}$ thermal treatment ...............................................107

Figure 4-55. Portion of tested $180^{\circ} \mathrm{F}$ with $1^{\circ} \mathrm{F} / \mathrm{min}$. rate tension specimens .........................................107 
Figure 4-56. $200^{\circ} \mathrm{F}$ thermal treatment tensile stress-strain curves ....................................................108

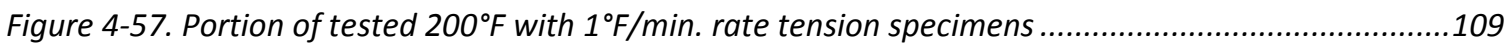

Figure 4-58. Common tensile failure types for $200^{\circ} \mathrm{F}$ thermal treatment ............................................109

Figure 4-59. $220^{\circ} \mathrm{F}$ thermal treatment tensile stress-strain curves ....................................................110

Figure 4-60. Portion of tested $220^{\circ} \mathrm{F}$ with $1^{\circ} \mathrm{F} / \mathrm{min}$. rate tension specimens ........................................111

Figure 4-61. Common tensile failure types for $220^{\circ} \mathrm{F}$ thermal treatment ..........................................111

Figure 4-62. 3\% salt treatment tensile stress-strain curves............................................................113

Figure 4-63. Common tensile failure types for 3\% salt treatment....................................................114

Figure 4-64. Portion of tested 3\% salt tension specimens ............................................................114

Figure 4-65. 6\% salt treatment tensile stress-strain curves.............................................................115

Figure 4-66. Portion of tested 6\% salt tension specimens ...............................................................116

Figure 4-67. Common tensile failure types for $6 \%$ salt treatment......................................................116

Figure 4-68. 9\% salt treatment tensile stress-strain curves.............................................................117

Figure 4-69. Common tensile failure types for 9\% salt treatment....................................................118

Figure 4-70. Portion of tested 9\% salt tension specimens .............................................................118

Figure 4-71. Lime treatment tensile stress-strain curves..............................................................119

Figure 4-72. Common tensile failure types for lime treatment ........................................................120

Figure 4-73. Portion of tested lime tension specimens..............................................................120

Figure 4-74. Oil treatment tensile stress-strain curves............................................................... 121

Figure 4-75. Common tensile failure types for oil treatment ...........................................................122

Figure 4-76. Portion of tested oil tension specimens........................................................................122

Figure 4-77. Summary of treatment effects on tensile specific strength and specific modulus .................123

Figure 4-78. Summary of treatment effects on tensile strain, stress, and modulus ................................124

Figure 4-79. Correct attachment of strain gage ...............................................................................128

Figure 4-80. Proper soldering and wiring of strain gage (top left), intermediate connection of wiring to box with alligator clips (top right), and final Wheatstone bridge connection to strain indicator box (bottom) 129 Figure 4-81. Proper attachment of strain gages and wiring ...............................................................130

Figure 4-82. Loading Poisson's ratio specimen into testing area .........................................................132

Figure 4-83. Configuring strings and tape to specimen (left) and overall test set-up for Poisson's ratio specimen loaded transverse to fibers .......................................................................................... 133

Figure 4-84. Exemplary plot utilized to determine the Poisson's ratio under longitudinal loading.............136

Figure 4-85. Exemplary plot utilized to determine the Poisson's ratio under transverse loading ...............136

Figure 5-1. Graphical representation of optimization analysis for the ideal treatment to improve the mechanical properties of bamboo................................................................................................144

Figure 6-1. Cutting bamboo poles to have the desired node locations for cylindrical configuration (left) and rectangular configuration (right) 151 Figure 6-2. Final products of flexural rectangular specimens: internode (left), mid-node (center), and quarter-node (left)..... 152

Figure 6-3. Four-point bending test fixtures (top), preloading cylindrical configuration into load area (center), and preloading rectangular configuration into load area (bottom)..... 154

Figure 6-4. Deflection of internode bamboo samples for both configurations ......................................157

Figure 6-5. Internode bamboo flexural stress-strain curves for cylindrical group ...................................158

Figure 6-6. Internode bamboo flexural stress-strain curves for rectangular group ...................................158

Figure 6-7. Common internode bending failure types: two fracture types for cylindrical group (left) and four observable fracture types for rectangular group (right) ..................................................................159

Figure 6-8. Deflection of mid-node bamboo samples for the rectangular configuration ...........................161 
Figure 6-9. Mid-node bamboo flexural stress-strain curves for cylindrical group

Figure 6-10. Mid-node bamboo flexural stress-strain curves for rectangular group.

Figure 6-11. Common mid-node bending failure types for rectangular groups: three observable fracture types (left) and the most common failure (right)

Figure 6-12. Common mid-node bending failure types for cylindrical groups seen as slit fracture (top) and

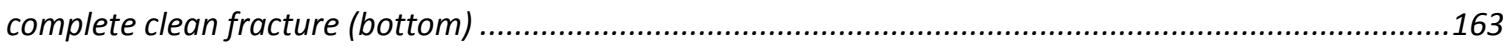

Figure 6-13. Deflection of quarter-node bamboo samples for the rectangular configuration ...................164

Figure 6-14. Quarter-node bamboo flexural stress-strain curves for cylindrical group............................165

Figure 6-15. Common quarter-node bending failure types for cylindrical group shown as a very thin

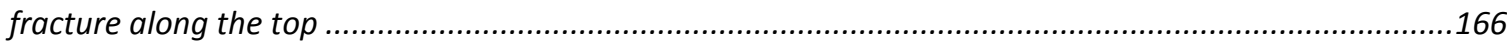

Figure 6-16. Quarter-node bamboo flexural stress-strain curves for rectangular group ..........................166

Figure 6-17. Common quarter-node bending failure types: two observable fracture types for the rectangular group (left) and enhanced view of typical failure (right)

Figure 6-18. Summary of node location effects on the flexural specific strength and specific modulus for the cylinder configuration. 168

Figure 6-19. Summary of node location effects on the flexural specific strength and specific modulus for the rectangular configuration 170 Figure 6-20. Summary of node location effects on the flexural properties for the cylinder configuration..172 Figure 6-21. Summary of node location effects on the flexural properties for the rectangular configuration

Figure 7-1. Potential applications for bamboo biocomposite truss $[48 ; 79 ; 66 ; 15]$.............................179

Figure 7-2. Manufacturing drawing for truss structural application ..................................................180

Figure 7-3. Eco-friendly resin system: Ecopxy resin and hardener ...................................................181

Figure 7-4. Initial phases of manufacturing: taping mitered truss members together (left), sanded biodegradable putty (center), and final view of joints created with Ecopoxy putty (right) .......................181

Figure 7-5. Lay-up diagram of vacuum-bagging process ...................................................................182

Figure 7-6. Curing truss joints in vacuum bag (left) and truss taken out of vacuum bag after 12-hour cure

(right).......

Figure 7-7. Sanding down excess resin and fabric (top) and resulting joint after sanding (bottom) ..........184

Figure 7-8. Raw condition of joints after cure (left) and close-up view of joint prior to sanding (right) .....184

Figure 7-9. Final product of bamboo truss with hemp joints..................................................................185

Figure 7-10. Riehle testing machine to perform compressive load ........................................................186

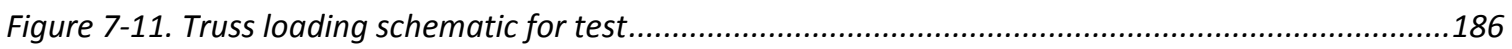

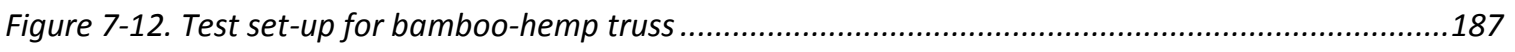

Figure 7-13. Load-deflection curve of bamboo-hemp truss test..........................................................188

Figure 7-14. Instants up until failure: (1. left) pre-failure state where top hemp joint begins to split (2. center) bamboo fiber delamination imitates in left diagonal member (3. right) top hemp joint splits further

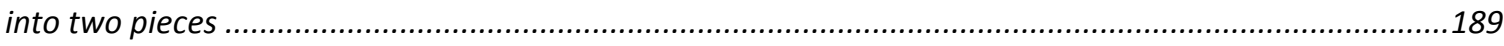

Figure 7-15. Deformation of bottom joints during the test .............................................................190

Figure 7-16. Critical joint at the conclusion of the test.......................................................................191

Figure 7-17. Deformation of the truss after the test from the front (left) and back (right) ......................191

Figure 7-18. Model based on truss dimensions and created in SolidWorks CAD software ........................193

Figure 7-19. Load and boundary conditions imposed on truss model ....................................................195

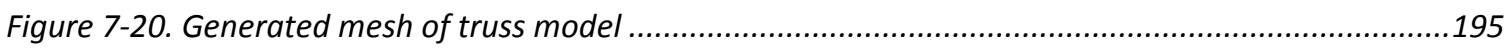

Figure 7-21. Visually enhanced views of critical areas in mesh model ...................................................196

Figure 7-22. Deformation models of truss: deformation scale 5:1 (left) and deformation scale 1:1 (right)197 
Figure 7-23. Enhanced views of deformation at critical areas with deformation scale of 1:1

Figure 7-24. Overall stress field from numerical analysis of bamboo truss.

Figure 7-25. Enhanced views of stress field in critical areas: bottom joints of truss (left) and top joint (right)

Figure 7-26. Enhanced views of stress field in left joint (top) and right joint (bottom) ...........................200

Figure 7-27. Deflection field of bamboo truss model ......................................................................201

Figure 7-28. Mesh models from the convergence study: 996 elements (top left), 3693 elements (top right), 8301 elements (bottom left), and 206904 (bottom right) ..............................................................203

Figure 7-29. Mesh convergence study of bamboo truss...............................................................203

Figure 7-30. Potential applications for bamboo biocomposite I-beams [50; 15; 56; 65] ........................205

Figure 7-31. Manufacturing drawing for I-beam structural application .............................................207

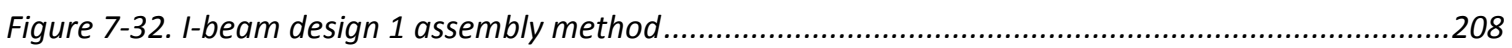

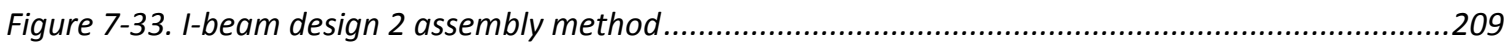

Figure 7-34. I-beam design 3 assembly method ............................................................................210

Figure 7-35. Cutting bamboo pole into strips for the web (top left), sanding strips to the correct thickness (top right), resulting product after sanding (lower left), and taping the strips together for ease in the final

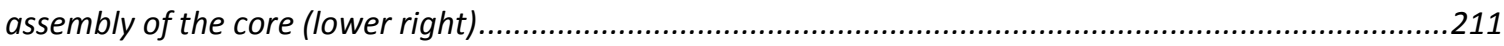

Figure 7-36. Cutting the hemp fabric to the appropriate dimensions for the plies ................................212

Figure 7-37. Set-up of adhering strip, U-channel, and flange plies prior to wet lay-up with molds...........212

Figure 7-38. Preparing the web core of the I-beam............................................................................213

Figure 7-39. Assembling the web of the I-beam ............................................................................214

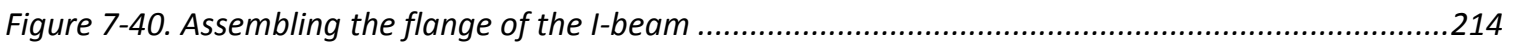

Figure 7-41. Clamping the molds together to create the I-beam ......................................................215

Figure 7-42. I-beam after taking off the molds (left) and marking the beam for cutting (right)...............216

Figure 7-43. Removing the molds from the I-beam ......................................................................216

Figure 7-44. Cutting the beam to the appropriate width (left) and sanding the flanges for final detailing 216

Figure 7-45. Final product of I-beams........................................................................................217

Figure 7-46. Free-body diagram of the I-beam under the loading case ..............................................218

Figure 7-47. Test set-up for bamboo-hemp I-beam...................................................................219

Figure 7-48. Load-deflection curves of I-beam designs ................................................................220

Figure 7-49. Instants up until failure for design 1: (1. left) pre-failure state with minor deflection (2. right) conclusion of test reveals little plastic deformation in the central region of the I-beam.....

Figure 7-50. Instants up until failure for design 2: (1. upper left) pre-failure state without crack initiation (2. upper right) minor ply delamination from the core begins (3. lower left) crack propagation (4. lower right) final failure state.

Figure 7-51. Instants up until failure for design 3: (1. upper left) pre-failure state without crack initiation (2. upper right) ply delamination near load points (3. lower left) bamboo ply delamination from web and crack propagation on one side (4. lower right) bamboo ply delamination and crack propagation on other side 222 Figure 7-52. Abaqus/CAE model based on I-beam design 2 .................................................................225

Figure 7-53. Loads and boundary conditions imposed on I-beam model..............................................227

Figure 7-54. Visually enhanced views of critical area in the meshed I-beam model ...............................228

Figure 7-55. Generated mesh of I-beam model.....................................................................................228

Figure 7-56. Deformation models of I-beam: deformation scale 5:1 (top) and deformation scale 1:1

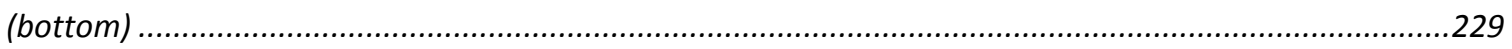

Figure 7-57. Enhanced view of I-beam deformation at critical area with deformation scale of 1:1 ...........230 Figure 7-58. Overall stress field from numerical analysis of bamboo-reinforced biocomposite I-beam .....231 
Figure 7-59. Enhanced views of the stress field in the region near boundary condition (right) ................231

Figure 7-60. Vertical deflection field of bamboo-reinforced biocomposite I-beam..................................232

Figure 7-61. Mesh models from the I-beam convergence study: 132 elements (top left), 588 elements (top

right), 1577 elements (bottom left), and 49920 (bottom right) .........................................................234

Figure 7-62. Mesh convergence study of bamboo-reinforced biocomposite I-beam ...............................235 


\section{List of Tables}

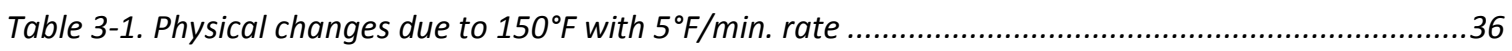

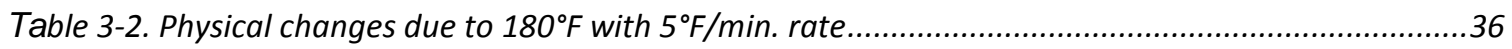

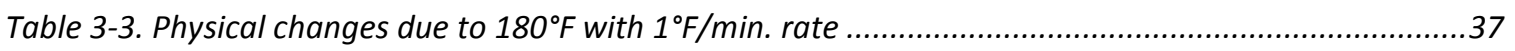

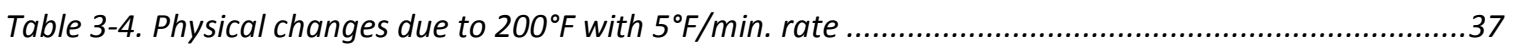

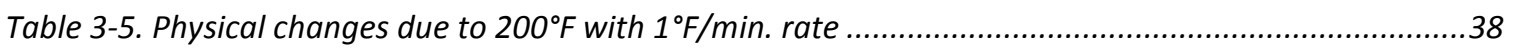

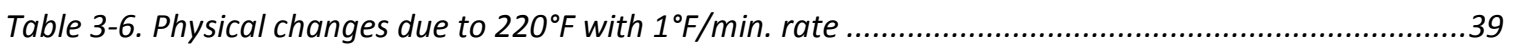

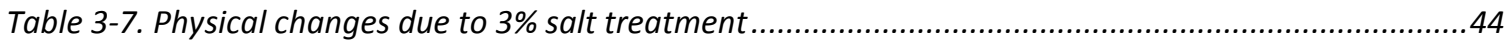

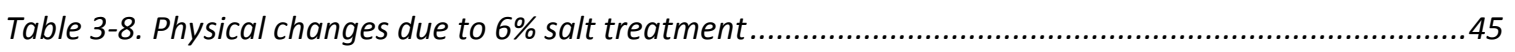

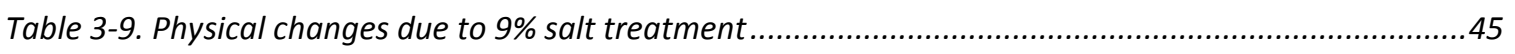

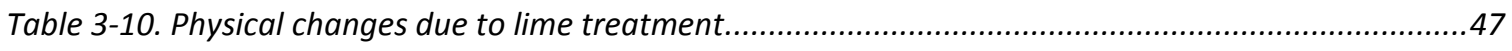

Table 3-11. Physical changes due to oil treatment................................................................................49

Table 4-1. Average percent changes of compression properties relative to the untreated green bamboo..92

Table 4-2. Average percent changes of tension properties relative to the untreated green bamboo .........125

Table 4-3. Summary of Poisson's ratio for all treatments .......................................................................137

Table 5-1. Results of the optimization analysis for the ideal treatment...................................................143

Table 5-2. Comparison of bamboo to conventional structural material ..................................................146

Table 7-1. Material properties of bamboo members in truss................................................................194

Table 7-2. Comparison of experimental and numerical results for Truss ...............................................202

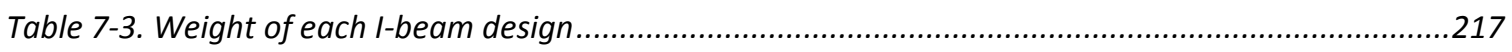

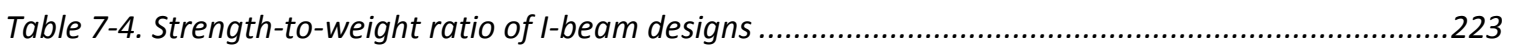

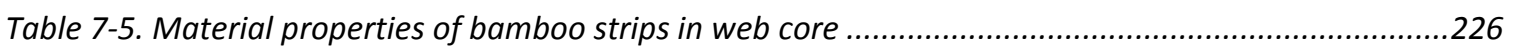

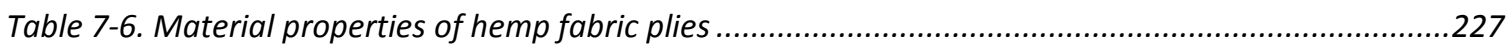

Table 7-7. Comparison of experimental and numerical results of $I$-beam ...............................................23 


\section{Nomenclature}

\begin{tabular}{|c|c|c|}
\hline$A$ & $=$ & Area \\
\hline$a$ & $=$ & distance between load and support \\
\hline$b$ & $=$ & $y$-intercept of equation \\
\hline$D$ & $=$ & diameter \\
\hline$E$ & $=$ & elastic modulus \\
\hline$e$ & $=$ & total sum error \\
\hline$F$ & $=$ & Force \\
\hline$G$ & $=$ & shear modulus \\
\hline$h$ & $=$ & height \\
\hline$L$ & $=$ & support span \\
\hline l & $=$ & length \\
\hline$m$ & $=$ & mass \\
\hline$N$ & $=$ & normalized value relative to maximum trade variable \\
\hline$n$ & $=$ & normalized value relative to untreated bamboo \\
\hline$P$ & $=$ & set percentage value \\
\hline$p$ & $=$ & product of weight factor and normalized value \\
\hline Q & $=$ & geometry factor \\
\hline$S, s$ & $=$ & error \\
\hline$T$ & $=$ & total sum \\
\hline$t$ & $=$ & thickness \\
\hline$u$ & $=$ & weight factor \\
\hline$V$ & $=$ & volume \\
\hline$w$ & $=$ & width \\
\hline$x$ & $=$ & mechanical property \\
\hline$z$ & $=$ & section modulus of beam \\
\hline 8 & $=$ & shrinkage percentage in cross-sectional area \\
\hline$\xi$ & $=$ & specific modulus \\
\hline$\varsigma$ & $=$ & specific strength \\
\hline$\Theta$ & $=$ & moisture content loss \\
\hline$\rho$ & $=$ & density \\
\hline$\sigma$ & $=$ & stress, standard deviation \\
\hline$\varepsilon$ & $=$ & strain \\
\hline$\Delta$ & $=$ & displacement \\
\hline $\mathrm{P}$ & $=$ & percentage change \\
\hline $\mathrm{E}$ & $=$ & percentage change error \\
\hline$v$ & $=$ & Poisson's ratio \\
\hline$\delta$ & $=$ & deflection \\
\hline
\end{tabular}




\section{Subscripts}

$\begin{array}{lll}1,2,3 & = & \text { principal, transverse, and out-of-plane directions } \\ \text { avg } & = & \text { average } \\ c & = & \text { compression } \\ f & = & \text { flexural } \\ g & = & \text { untreated bamboo } \\ h & = & \text { hollow cylinder beam } \\ i, j & = & \text { index } \\ l & = & \text { longitudinal } \\ m a x & = & \text { maximum } \\ o & = & \text { original } \\ r & = & \text { rectangular beam } \\ t & = & \text { tension } \\ t v & = & \text { transverse } \\ u l t & = & \text { ultimate } \\ y & = & \text { yield }\end{array}$




\section{Chapter 1. Introduction}

Biocomposites comprise an expanding field of study in today's globally aware society where current resources continue to dwindle at an alarming rate. More engineers and scientists are seeking innovative ways to interchange conventional environmentally unfriendly materials for biodegradable ones in their products' designs while still improving or maintaining their same mechanical performance characteristics for structural application. The issue at hand is that many structural products composed of natural materials do not currently outperform structures made of conventional materials. With the proper efforts and research, however, biocomposite structures have the potential to offer comparable performance. In order to accomplish this, research must expand into other materials that can satisfy this goal.

Bamboo is one remarkable example of a strong and easily renewable product that also aids in reducing the carbon footprint compared to conventional material manufacturing methods. In spite of bamboo's potential, this promising resource has not been fully exploited for its structural properties. Current uses restrict the material to mostly aesthetic and recreational purposes. This study will investigate its promise in other fields that require more strength and stiffness.

The body of this introduction describes the definition of biocomposites and their growing importance. The benefits of utilizing biocomposites over conventional materials for structural purposes are then discussed. Bamboo is sparingly used in the United States for structural applications-much less in biocomposites. Therefore, bamboo as a potential fiber reinforcement constituent in biocomposites is primarily reviewed. Previous research on bamboo is then studied to initiate a starting point for this study. The main 
objectives of this research are then outlined. Lastly, a brief summary is provided to describe the magnitude of this research in its entirety.

\subsection{Composites and Biocomposites}

Composites and biocomposites are two entities from the same origin. Technically speaking, a composite material is a combination of two or more constituents with different mechanical properties that form a useful third material [47]. Figure 1-1 illustrates how the combination of these two constituents forms a new material. Usually the resulting product has the strengths of both the constituent materials without inheriting their respective weaknesses. For example, fibrous materials demonstrate compressive tensile strength along their axis but cannot support compressive loading; a stiff resin material, however, performs well under compression but does not fare well under tension. The combination of these two constituents then yields a material that is strong in both tension and compression.

Therefore, one of the main advantages of composites is

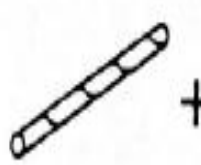

fibre

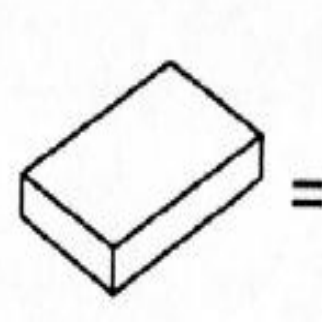

resin

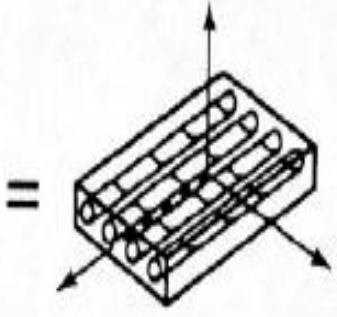

composite material

Figure 1-1. Constituents of a composite material [47]

customized for a particular loading condition. The composite definition suits the combination of these two materials, since the different mechanical properties of the two components result in a new material altogether. The fibrous constituent of carbon-fiber composite materials is often made of carbon, glass, or aramid. The resin constituent is usually a polymer-an epoxy, vinylester/thermosetting plastic, or phenol formaldehyde [49]. These constituents make up the majority of conventional composite materials. 
Similarly, biocomposites are composed of natural fibers and non-toxic resin matrices. Natural fibers may come in the form of plant cellulose while the resin is usually a vegetable or animal-based derivative. Specific plant fibers of potential use include hemp,

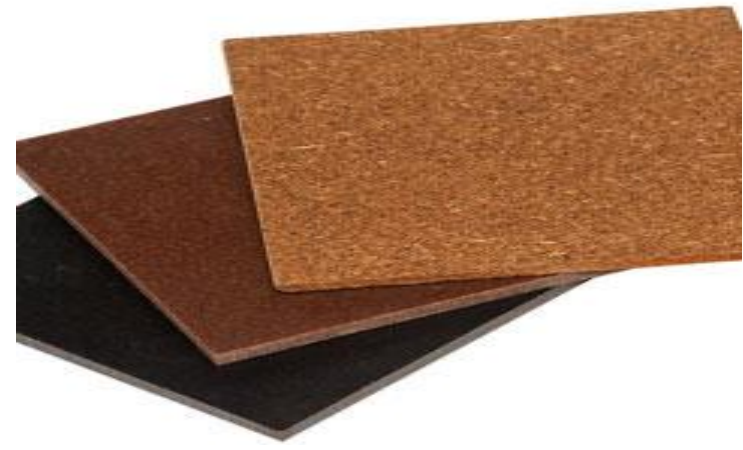

Figure 1-2. Biocomposite samples made of germ, corn stover, and soybean hulls [24] wood, flax, sisal, and jute. An example of natural resin for composite application is soybean oil. Therefore, biocomposites are simply composites that utilize all natural constituents instead of the typical carbon/fiberglass-epoxy variation.

Figure 1-2 shows an example of biocomposite plates formed from natural biodegradable materials. A biocomposite replacement for current composite materials may now be a more viable option in our current globally-aware society.

\subsection{Promising Benefits of Biocomposites}

Although the vast use of composite materials in industry demonstrates a positive effect on their product's performance, the creation and disposal of this material lays a burden on the environment. It has been shown that manufacturing these composite materials releases a substantial amount of greenhouse gases into the atmosphere. In fact, carbonfiber composites along with aluminum and magnesium have recently been reported to produce up to 20 times more $\mathrm{CO}_{2}$ emissions than the steel industry-which is estimated to be two metric tons for every metric ton of steel [12;42]. Aside from increasing greenhouse gases into the warming atmosphere, the disposal of carbon-fiber composites is a dangerous issue at the end-of-life stage of the product. International policies prohibit dumping carbon-fiber composite materials into landfills and incinerating it also releases toxic byproducts into the air [63]. Recycling practices are also employed 
but is costly and requires significant amounts of energy. As a result of these shortcomings, alternative composite constituents must be investigated to comply with the global need for sustainability.

Biocomposites, on the other hand, pose a solution to this environmental problem. Unlike the toxic chemicals found in carbon-fiber composite materials or the polluting effects of processing metals, biocomposites utilize natural fibers and non-hazardous resin matrices that vastly reduce its carbon footprint in relation to conventional materials. Unsustainable composite materials and their subsequent effect on the environment will inevitably drive biocomposites into the forefront of practical applications. In other words, environmentally-friendly scientific endeavors and engineering design methodologies are shifting toward innovative levels that incorporate greener materials into industry products. This environmental revolution is only beginning to turn away from the destructive practices of manufacturing unsustainable products.

The first shots have already been heard around the world: global awareness policies are aiming their sights at contemporary manufacturing practices and materials. The Biomass Research and Development Act, instated in 2000, requests improved recycling and waste prevention practices and pleads for a significant use of more natural products in the United States by the year 2010 [25]. In the current political atmosphere of this research, new environmental policies are being pushed by the appropriate authorities. For instance, the Recovery Act will provide funding for programs and projects that will protect the environment. Also, the national standards on greenhouse gas emissions and fuel economy will tighten its hold on current environmental policies [10]. Soon there will be more initiatives to pursue biocomposites due to government policies. It is appropriate to begin taking the correct steps forward to address these legal and moral plans for a better world of tomorrow. 
Since biocomposites utilize renewable resources, the life expectancy of these materials is estimated to be continuous throughout their lifecycle. Figure 1-3 illustrates an archetypal lifecycle of a biocomposite product. First, the necessary natural resources are harvested at the appropriate time in the season. Then the raw materials are transported to the factory where innovative chemistry and engineering are assimilated to prepare a product. The product is manufactured and the final result is used by

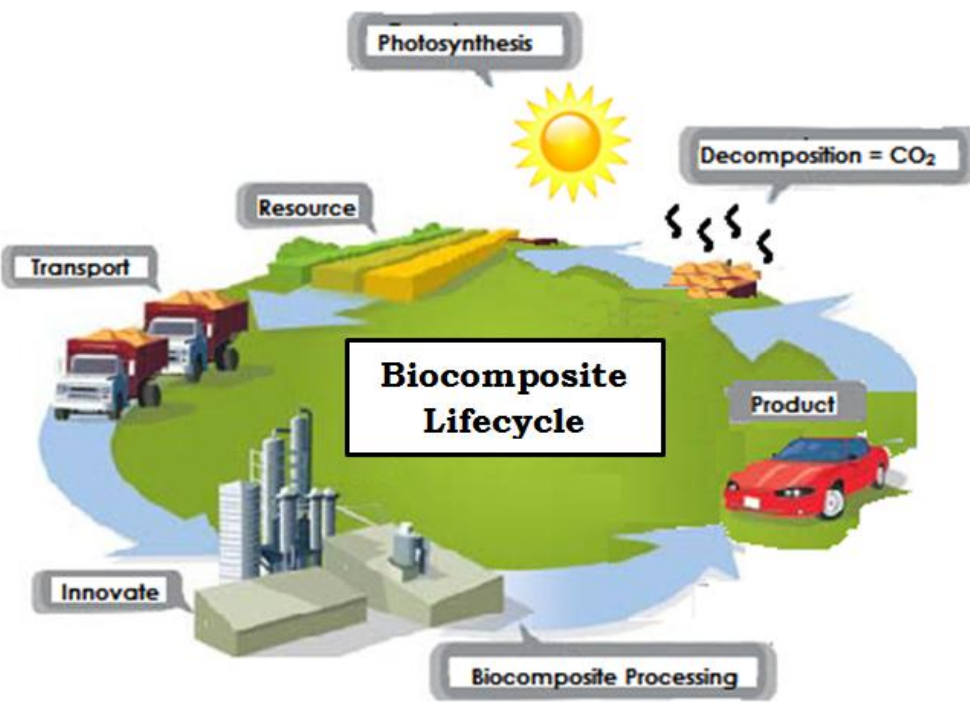

product is conducted

Figure 1-3. Potential lifecycle of biocomposite structures

back at the raw resource site; this can only be made possible by the biodegradable property of the constituents. The decomposing compost, once a biocomposite structure, is subsequently used back at the resource site to aid sustainable growth. For plantbased biocomposites, decomposition yields carbon dioxide that is combined with water and sunlight to initiate photosynthesis in the natural resource for further cultivation. Thus, a complete cycle that utilizes a renewable resource without detrimental environmental effects is possible.

In addition to positively affecting the global ecosystem, biocomposites boast economic benefits as well. Sources from research centers indicate that natural composite materials may be four to five times less expensive than fiberglass composites [8]. Sources such as 
these state that material costs for fiberglass are roughly $\$ 0.99 / 1 \mathrm{~b}_{\mathrm{m}}$ while biocomposite materials are approximately $\$ 0.25 / \mathrm{lb}_{\mathrm{m}}$. Biocomposites also reduce manufacturing costs when compared to metal processes. For example, the price of a car is largely attributed to the stamping and joining of metal components to form the frame [9]. The equipment required to accomplish this task is expensive-especially for automotive structures with several components. The typical manufacturing of biocomposites allows for more uniform structural transitions between parts: meaning fewer components. With this advantage also comes less tooling costs and manufacturing complexity required to assemble the structure.

As promising as biocomposites are in the environmental and economic aspect of the issue, they are historically known to perform not as well as synthetic-fiber composites and metals in certain high-performance applications. However, new methods and materials are being increasingly investigated for potential improvements. Hemp fibers have demonstrated successful integration into structural applications but are known to have less strength than their carbon-fiber cousins. Nonetheless, these natural fibers still have relatively high tensile strength so their use is common in many products.

\subsection{Practical Implementation of Biocomposite Structures}

Successful application of biocomposites in various structures is a proven technology although the utilization of natural fibers does not currently surpass the performance of carbon-fiber. Composites offer lighter, stronger, and more impact absorbent properties than many metal structural systems. They also resist rust and can offer cheaper means of assembly by avoiding expensive tooling. 
One impressive example of biocomposites in application is the Kestrel biocomposite electric car by Canadian automotive company Motive [9]. The innovative car is shown in

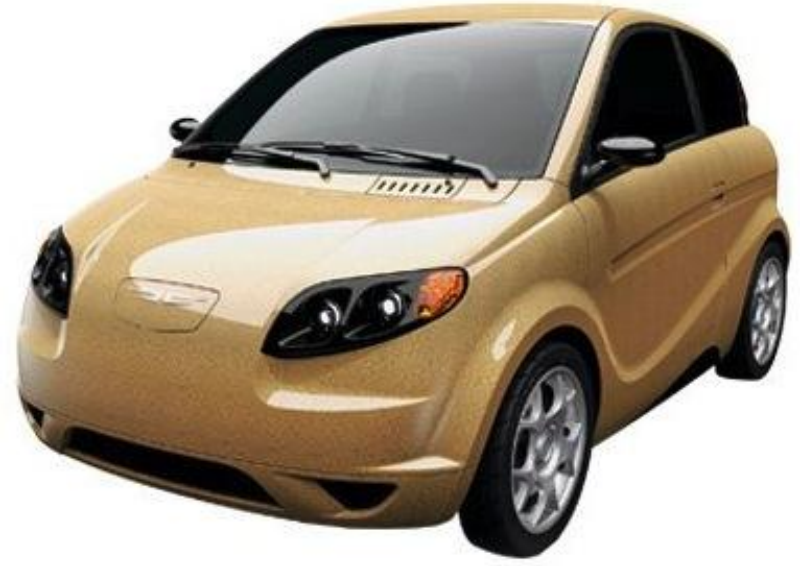

Figure 1-4. Kestrel is the first all-hemp biocomposite car body [9]
Figure 1-4. This electric car serves as a primary model for an engineered solution to human problems while still taming the issue of global pollution. Completely made of hemp fiber biocomposites, the 2000- $-\mathrm{b}_{\mathrm{m}} \quad$ Kestrel minimizes assembly costs and runs on

electric power. The damage-arresting nature of the biocomposite panels are said to create impact resistance by Motive's president.

Other remarkable examples of biocomposite application are found in the Mercedes-Benz A-Class supermini car and the F-22 Raptor fighter aircraft. External parts of the Mercedes-Benz A-Class car are composed of Abaca banana composites. Other natural composites are also known to be used internally for certain panels. The use of
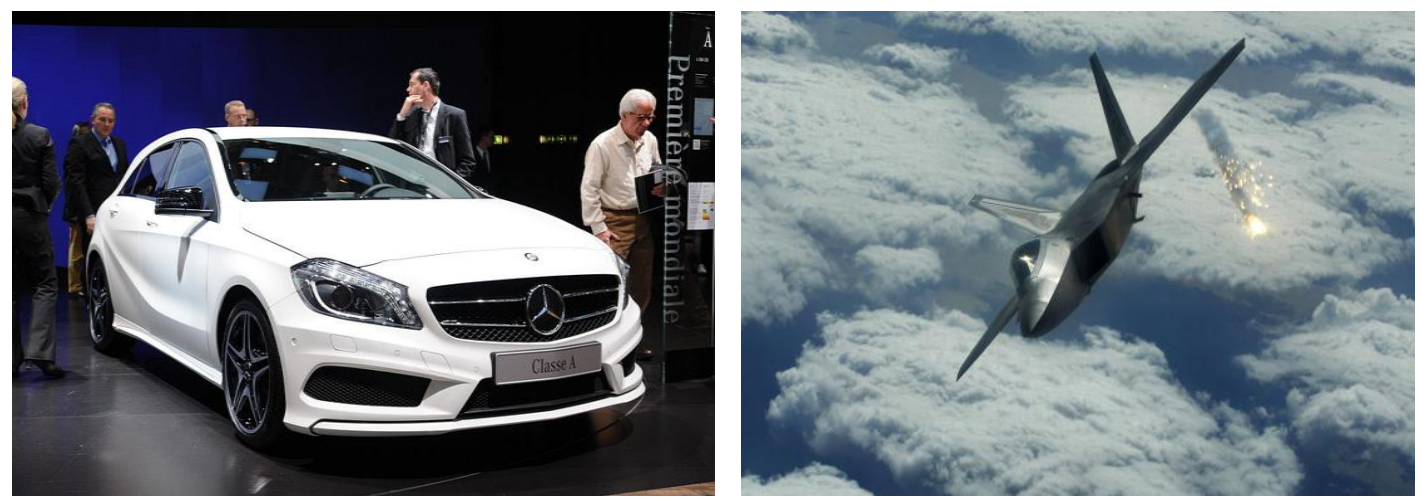

Figure 1-5. Mercedes-Benz A-Class and F-22 Raptor both utilize biocomposites in parts of their structure $[53 ; 19]$ 
biocomposites in the interior and exterior of the Mercedes-Benz A-Class car demonstrates the strength of these materials for structural purposes. Even more evident of the strength possibilities of biocomposites is its use in the F-22 Raptor. Biocomposites are said to be utilized in certain classified parts of the aircraft's structural frame [8]. As seen from these inspiring applications, biocomposites readily display proven feasibilities with undeniable success structurally and ecologically. However, these models only cast the first glimpse of the potential for biocomposite materials-there is still unknown territory in exploring other natural fibers.

\subsection{A New Biocomposite Fiber Constituent: Bamboo}

A premium natural fiber has emerged in the rapidly advancing field of biocomposites: bamboo. The list of strong fibers is short in the field of biocomposites, so a comprehensive search for another fibrous material is studied in bamboo. Incredibly strong and light, bamboo fits perfectly in the natural composite realm of new natural fibers to study for structural engineering. The unique biological composition of bamboo makes it one of the most promising materials for structures. In addition, the abundant renewable resource has one of the fastest lifecycles in the plant kingdom [36]. A summary of bamboo and its cultivation practices are discussed. In addition, previous applications in the construction industry are also discussed to research the bamboo's effectiveness in practice.

\subsubsection{Understanding the Plant Bambusoideae}

Bamboo is a group of perennial evergreens in the true grass family Poaceae under the subfamily Bambusoideae and tribe name Bambuseae [35]. Contrary to popular belief, bamboo is not a type of wood-it is a giant grass. Experts claim over 1,000 bamboo species of varying heights and diameters exist all across the world. Bamboos also vary 
in colors ranging from bright yellows with spotted greens to shimmering blacks. The variety suitably named Giant Bamboo can grow up to lengths of $65-\mathrm{ft}$ and can have a diameter of 4-in. under the appropriate growing conditions. An explanation on the physical and chemical composition of bamboo is further discussed.

The physical characteristics of bamboo can be summarized as a stiff and smooth stem composed of hollow interludes that usually grow in size as the bamboo reaches greater

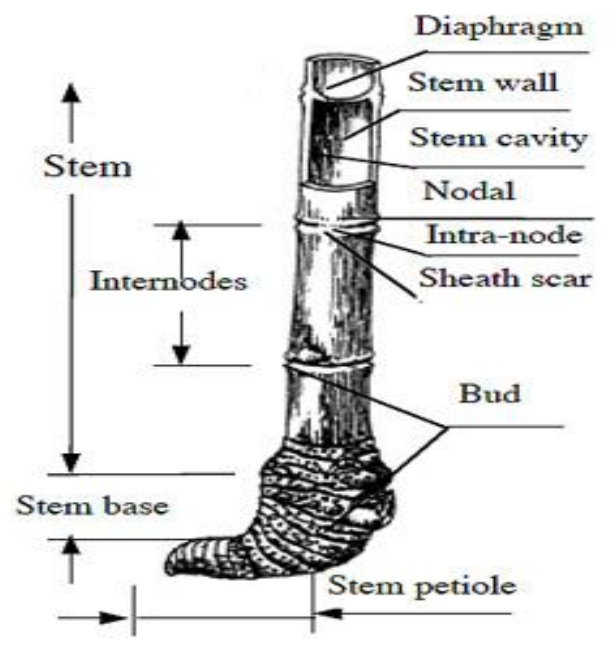

Figure 1-6. Typical bamboo anatomy [22] heights. Bamboo poles grow with a slight taper in their diameter that becomes more prominent in older individuals. These organisms grow from a rhizome system which is a term for the base of the stem underground that sends out roots and shoots in several directions. Smaller leafy branches often sprout at sparing intervals along the pole's length. Figure 1-6 illustrates the general anatomy of a bamboo pole [botany].

The stem, or culm, comprises the bamboo pole from the rhizome up while the dimension labeled as the stem base is the rhizome itself. Similarly, the stem petiole is simply the extension of the rhizome root as it grows underground through the soil. This rhizome also sprouts additional bamboo poles as the root continues to expand its intermingling shoots throughout the soil.

As seen from the figure, the culm is divided into internodes. The internodes are often smaller at the base of the pole during its younger stages of development. The internodes grow larger as the pole matures and reaches a maximum length once the stem 
advances to a certain age. Past this age, the internodes continue to grow to that maximum length. In spite of the size of each internode, the internode is always hollow as illustrated by the stem cavity label in the figure. The stem wall is simply the solid vertical structure encasing the stem cavity.

Each internode is separated by a dense section called nodes or nodals-as shown in Figure 1-6 and Figure 1-7. The outer surface of each node is irregular in the sense that each one is identical but not an exact duplicate. It can be seen from the figures that the node is composed of two segments: a lower thick lip and an upper thin one. As seen from Figure 1-7, the top of each internode diminishes into a thin lip on the outer surface of the culm wall at the location of each adjacent internode. The bottom of each internode, on the other hand, has a protruding characteristic that slightly differs from the other lip. These are the main features that all nodes share along the length of the pole.

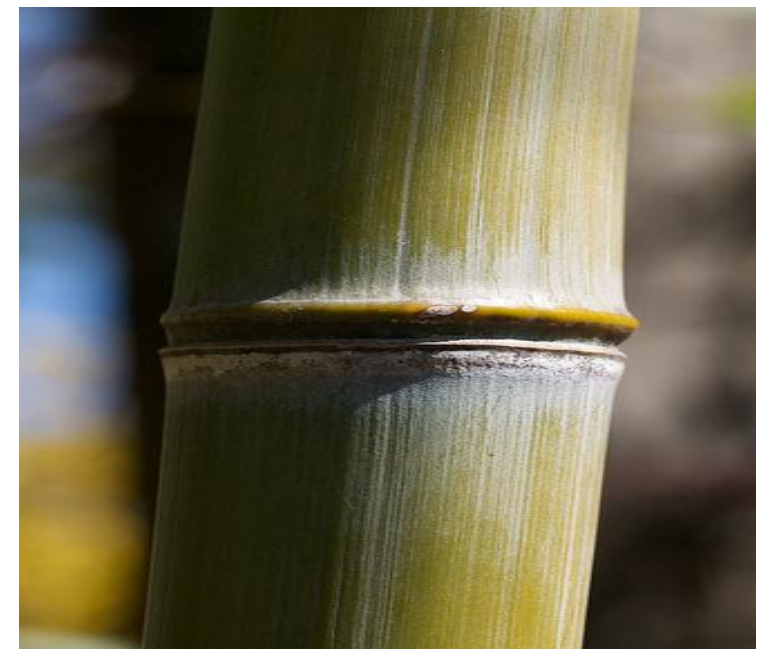

Figure 1-7. Close-up of bamboo node [23]

On the microscopic level, the biological composition of bamboo is essentially two types of fibers and a void-filling matrix. The inner cross section of the stem is hollow and houses several vascular bundles in the axial direction of the bamboo pole [71]. Vascular 


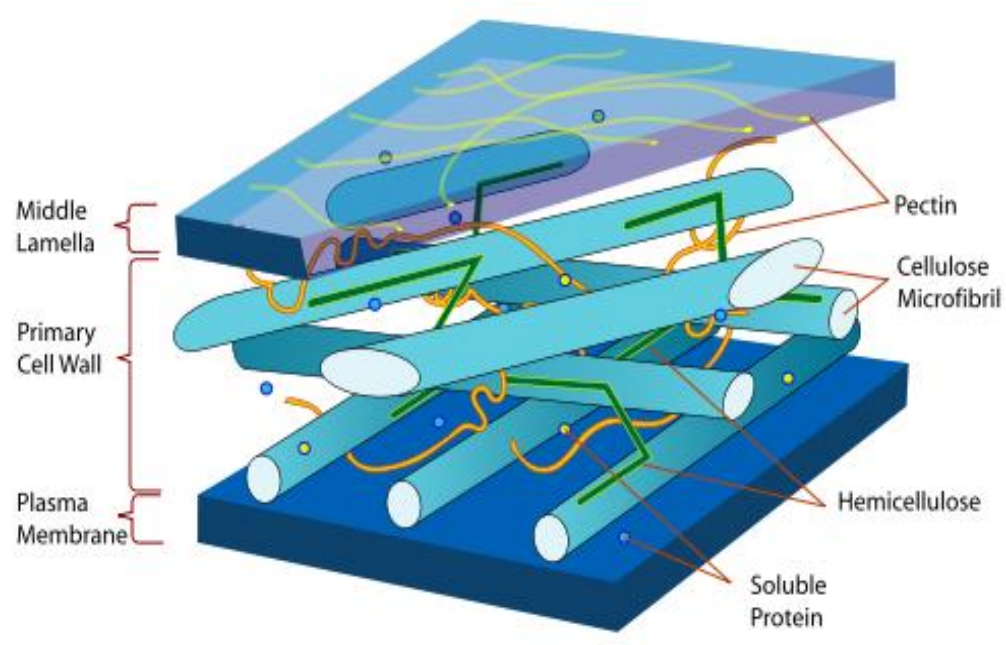

Figure 1-8. Plant cell structure [67]

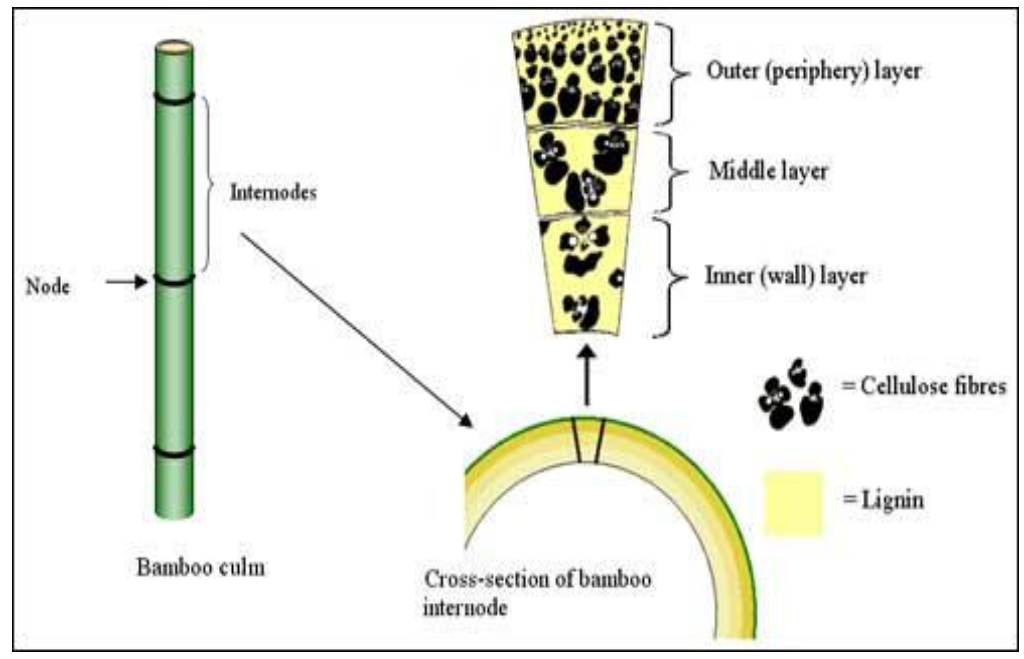

Figure 1-9. Anatomy of a bamboo's cross section [20] bundles are the transport systems in vascular plants that distribute water and nutrients throughout the entire plant body. Similarly, the main cellulose fibers run along this central axis of the pole to begin and terminate at the node locations. The volume fraction of fibers increases linearly with height although the diameter and thickness decrease [17]. Also, a bamboo has fibers that run radially throughout each internode in a similar fashion to wood. This fiber orientation scheme makes both plant organisms have an anisotropic nature due to the planes of symmetry in the radial and axial directions. However, unlike wood, bamboo has internal cavities separated by the nodes which make the material ultimately orthotropic. At these nodes, the fibers are oriented in the transverse direction to the longitudinal axis. This combination of transverse fibers and longitudinal fibers distinguishes bamboo as having different material properties in many planes. This unique material characteristic actually resembles that of multi-directional composites. 
The resemblance is also apparent in the varying fiber density along the thickness of the internode. Figure 1-9 provides a visual enhancement of a typical bamboo's cross section as well as an illustration with labels for clarity. As seen from the figure, the bamboo cross section is composed of three regions. The number of fibers increases in the positive radial direction toward the outer surface of the culm.

These fibers and the surrounding matrix are the main components of the bamboo and known as cellulose, hemicellulose, lignin, and water. Approximately $50-70 \%$ of the cross section is cellulose, $20-30 \%$ hemicellulose, and $20-30 \%$ lignin [33]. Figure 1-8 and Figure 1-9 are provided to illustrate these components in a classic plant cell structure.

Cellulose and its individual microfibrils comprise the main fibers of the bamboo structure. It is an organic compound, specifically a polysaccharide, consisting of a linear chain of several hundred to over ten thousand bonded glucose units [33]. Its main function is to be the primary structural component in the cell wall of plants, and varies in each plant species. Figure 1-10 shows the microstructure of cellulose and its microfibrils. In bamboos, the majority of its strength is attributed to

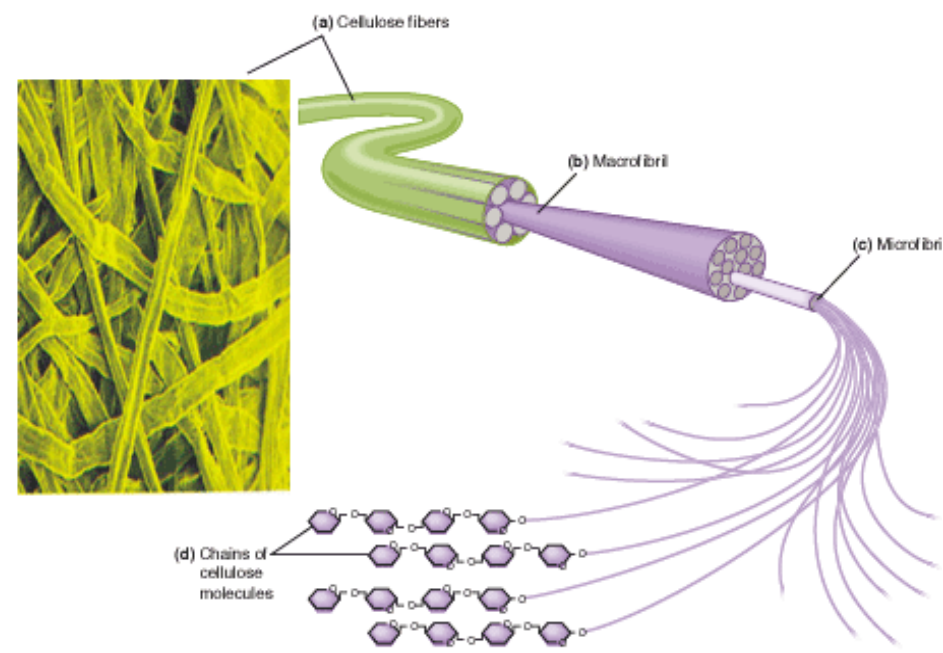
this cellulose content and its Figure 1-10. The structural composition of cellulose [55] strong covalent bonding. Although the mentioned figure illustrates an exaggerated crisscrossing of the cellulose microfibrils, these components are predominantly aligned in the longitudinal axis of the bamboo pole. The nodes, however, do not have this 
microscopic configuration due to their transverse orientation to the pole and are incredibly strong.

Hemicellulose, on the other hand, is a heteropolymer present along with cellulose in the plant cell. Unlike the strong crystalline structure of cellulose, hemicellulose is a smaller amorphous microstructure that randomly clusters around its bigger relative. This plant cell constituent bears little strength and behaves more like a matrix substance to assist holding the cellulose in place. Figure 1-11 provides another visual of hemicellulose in a plant cell structural diagram.

Another critical microstructure in bamboo is lignin; it is a fundamental and complex chemical compound in a plant's cell wall. This substance fills the spaces in the cell wall

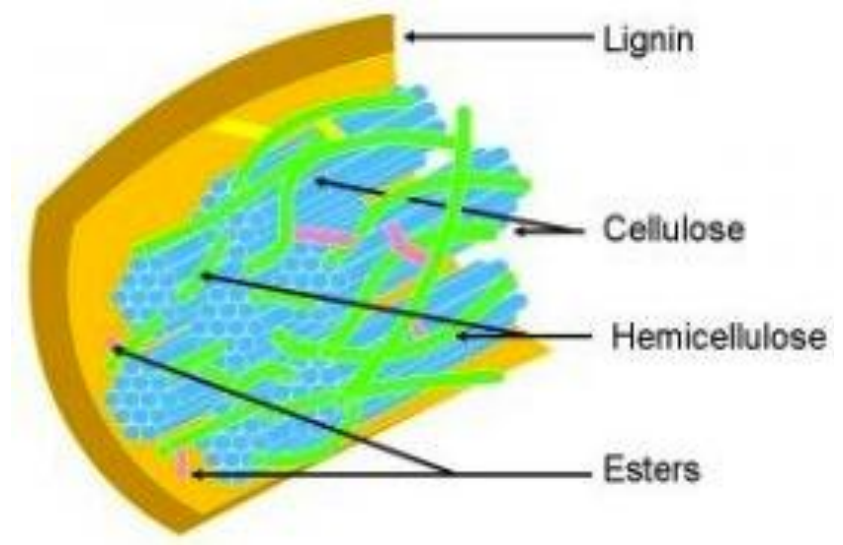

Figure 1-11. Hemicellulose orientations in a plant cell [43] and supports cellulose and hemicellulose for rigidity. The previous figures illustrate the role lignin plays in the biological makeup of bamboo. Similar to a composite lay-up, this matrix material primarily holds the other constituents together and is strong under compression loading. From

a structural standpoint, the innermost section of the bamboo wall contains the most lignin-to-fiber ratio and, therefore, the weakest part of the culm as a whole.

\subsubsection{Cultivating Bamboo and Harvesting Operations}

Bamboo's unique microstructural composition makes it an exciting constituent for a biocomposite in structural application, but the proper care and harvest of this raw 
material must be conducted for successful integration into engineered products. Bamboo has simple growing conditions and harvesting procedures that must be followed to ensure an optimum raw material.

\subsubsection{Suitable Environment Topography}

One incredible advantage of developing bamboo is that it can grow nearly anywhere in the world with the appropriate factors. The perceived factors affecting growth include water consumption, surrounding climate, sunlight exposure, soil quality, and wind presence [5]. Bamboo prolifically grows in places such as southern Asia, Central America, and North America while being relatively inexpensive to sustain. Addressing the mentioned factors in any of these geographical regions can dictate the final quality of the cultivated bamboo.

Adequate water is a key element for bamboo to grow to its optimum potential. Bamboos need adequate water to maintain moisture equilibrium within its cells. Being a hygroscopic material, bamboo gains and loses moisture depending on the water conditions of its environment, so it is important to supply the bamboo with the necessary moisture it needs. Bamboos house water in their cells in two forms: free and bound water. Free water is the type of water temporarily moving through the transport system of the plant and is interchanged regularly with the moisture levels of the surrounding air to obtain equilibrium. This water is absorbed through the leaves of the bamboo and is actively working at all times. Bound water is the water embedded in the cellulose microstructures of the plant. This water accumulates in the cells immediately from the onset of infancy and remains there for proper cell function. Under normal conditions, this water remains in the cells and directly affects the quality of the plant during growth. 
In terms of the climate, bamboos prefer tropical and warm temperate climates although it is possible to grow them in adverse conditions. Examples of unfavorable climates include deserts and cold mountain regions. Although these are adverse regions for bamboos to optimally mature, they are known to grow in the sub-Saharan deserts of Africa and cold mountains of the Himalayas [3]. The ideal locations in the United States include the western and southern regions of the country as seen in Figure 1-12. In California, successful bamboo nurseries exist in the south and north.

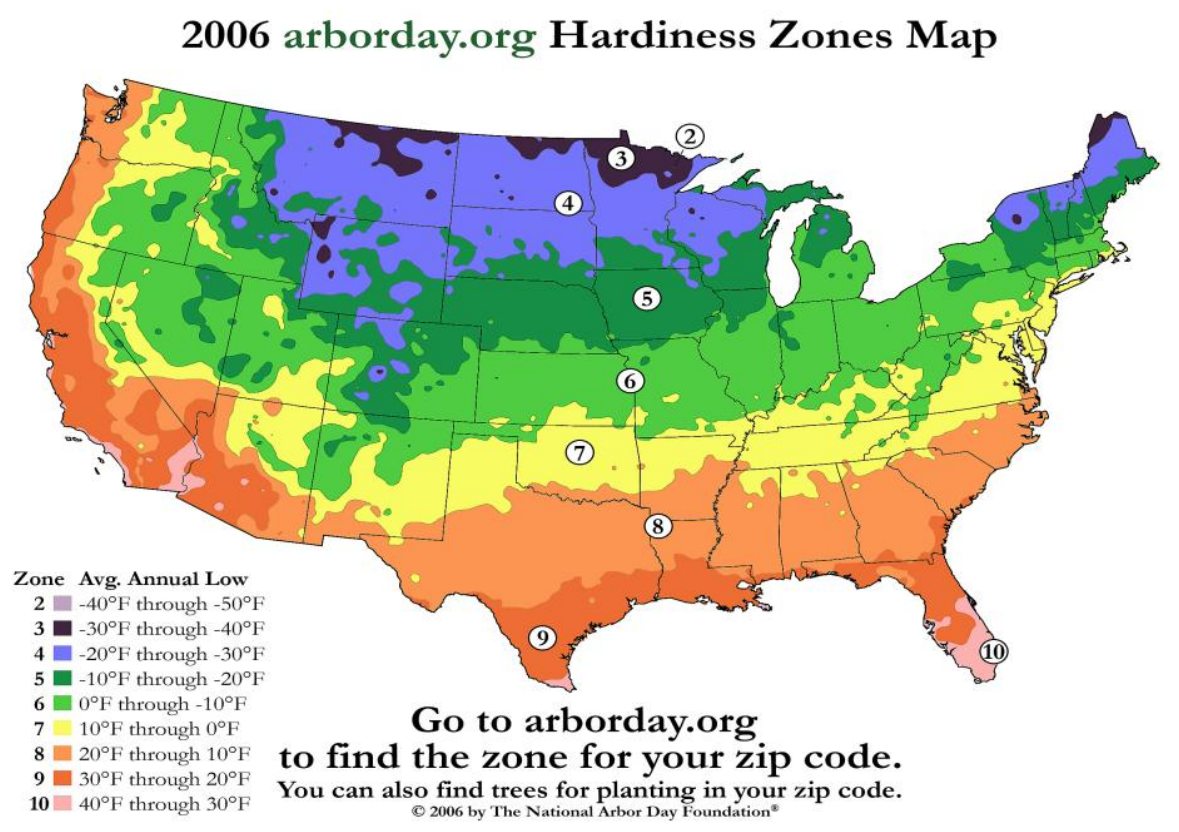

Figure 1-12. Climate zones in the United States [13]

Bamboos will often flourish in direct sunlight-especially for certain species. Some species actually prefer some shade during the hottest times of the day while others easily welcome it. During winter months when frost and direct sunlight affect the bamboo, the depletion of water from the plant is accelerated. This effect can result in premature aging and must be avoided. The ideal solution is to first select an appropriate region before rooting a plant in the ground. 
Bamboos also grow in a wide variety of soil conditions. Nearly all bamboos will grow incredibly well in either loam or marly soil [5]. These types of soil are composed of $40 \%$ sand, $40 \%$ silt, and $20 \%$ clay; it is characteristic of having high amounts of nutrients and provides a great amount of drainage. When the roots are set in the soil, they can also reclaim the land so that the soil quality improves by leaching heavy metals and holding the soil together to draw water closer to the surface [61]. Generally, bamboos prefer moderately to slightly acidic soil. Rocky and impermeable soils can slow the growth of the bamboo and can lead to pooling and rhizome rotting. However, this issue can be worked around by providing a proper drainage system. Also, a layer of mulch is recommended to shield the base of the plant during the harsh times of the season. One composed of dried leaves and compost is ideal.

An area with mild winds is ideal for optimum growth. Excessively strong winds may uproot bamboo rhizomes during early stages of growth. Once the bamboo plant has matured, however, the strong tensile strength of the bamboo culm is meant to resist high wind forces. Gardens with surrounding hedges or trees make excellent wind barriers for vulnerable young bamboos.

\subsubsection{Growth Behavior and Harvesting Processes}

The continuous harvesting cycles of bamboos is largely due to its incredibly fast growing behavior. Bamboos are the fastest growing plant in the world: certain species can grow up to 40-in. /day. Under the right conditions, bamboos can grow at a quick rate of 2in./hr. while conservative estimates measure a daily growth of 24-in./day [54]. One can literally watch the bamboo pole grow with the passing minutes. If grown properly, the bamboo plant can mature after only three to six years-a rate faster than many woods. Particular species can fully mature within just six months! Once mature, the bamboos can be simply harvested by cutting the pole at its base. Cutting bamboo poles can be 
accomplished with simple tools such as a machete. It is important to harvest the bamboos once they mature to this age to optimize its mechanical properties and maximize raw material yields. The easily renewable characteristic of the bamboo provides a significant benefit to continually harvesting this plant. Unlike hardwood trees when cut down, a new pole will simply spring from the ground and grow as fast as the harvested one within a few years [61]. Furthermore, it can be harvested every single year for the life of the plant as long as the number of cultivated poles does not exceed the number of new poles sprouted during the harvest season.

Ample growth occurs during the wet season, so it is advised to harvest the bamboo shoots at the completion of the dry season. At the end of the dry season, the sap is at its minimum level and the possibility of damaging the shoot is reduced [22]. Collecting the bamboos at dawn or dusk when the sap levels are at their daily minimum also eases harvesting. During the peak hours of sunlight, photosynthesis is operating at its maximum efficiency to promote sap quantities, which and encumbers harvesting. Therefore, dawn or dusk harvest times can yield better raw materials with minimum damage to the bamboo.

\subsubsection{Information of Bamboo Utilized in this Research}

All the bamboo raw materials obtained for this study are ensured to be from the same background to minimize inconsistencies in the results amassed in this work. It is vital that all bamboo poles are from the same geographical region so that their mechanical properties are comparable. Confirming the factors outlined previously will lend confidence that the chemical composition, and therefore, mechanical properties of the bamboo samples are similar throughout the experiments executed in this study. 
The species of bamboo utilized in this research is Phyllostachys aureosulcata, commonly known as Yellow Groove bamboo, from the Jungle Supply Company in Newcastle, California. This species of bamboo is legal to grow in the state of California as deemed by the California Invasive Plant Council [27]. A total of 34 bamboo poles

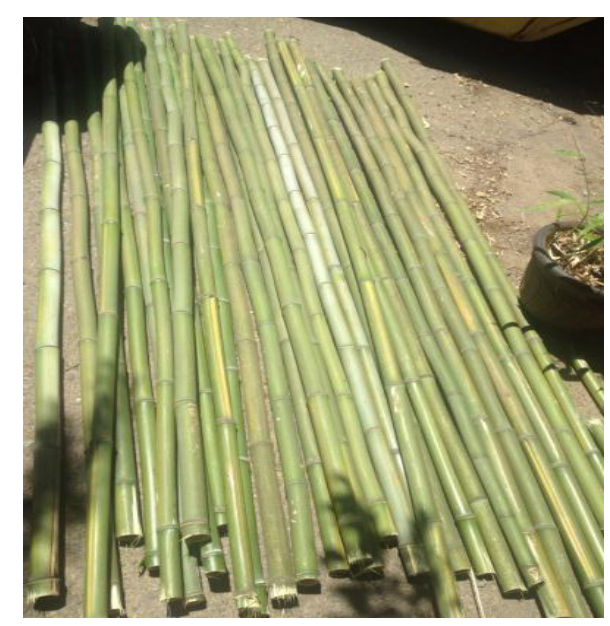

Figure 1-13. Carefully nurtured bamboo poles personally selected from Jungle Supply Co. spanning 9 -ft were obtained from this supplier. Jungle Supply Company is a prosperous fouracre bamboo nursery in northern California with an abundance of bamboo species. According to Figure $1-12$, this region of California is classified as zone 9 where the average annual temperature lows range from $30^{\circ} \mathrm{F}$ to $20^{\circ} \mathrm{F}$ [13]. This region is one of the hotter locations in the United States, so the climate is of a suitable nature for growing bamboos. The bamboos here are meticulously

cared for as seen in Figure 1-13, so it is accepted that the water consumption, sunlight exposure, and soil quality of the bamboos were adequate throughout their developing life. As mentioned earlier, the ideal age for maturity is three to six years, so only fouryear-old bamboo poles were selected from the nursery. Also, the poles were all selected personally to be within the same geometric diameter for consistency; all the poles fell within a 1.4-in. to 2.0-in. diameter range.

\subsubsection{Previous Bamboo Applications}

Past bamboo applications include a diverse range of products from cutlery handles and computer casings to bicycles and in architecture to name a select few [71]. The varied list continues to over 1,500 documented uses. The utilization of bamboo in structurally sound products is very appealing from a biocomposite engineering standpoint. A 
particularly interesting application is found in laminated bamboo composite surfboards by Bamboo Surfboards based in Hawaii; a figure is provided of their bamboo surfboards by the respective company [71]. Young's work on bamboo surfboards illuminates a path for other venues to employ bamboo. Similarly, bamboo's application in the construction industry also leads to promising hopes for other structural purposes involving bamboo. The potential for bamboo is only limited by the research poured into improving its mechanical characteristics and determining economic manufacturing methods to apply the raw material into practical structures. If these two obstacles are overcome, then bamboo biocomposites pose a serious threat to replacing plastic-reinforced composites and conventional metal materials in the struggle for a greener world.
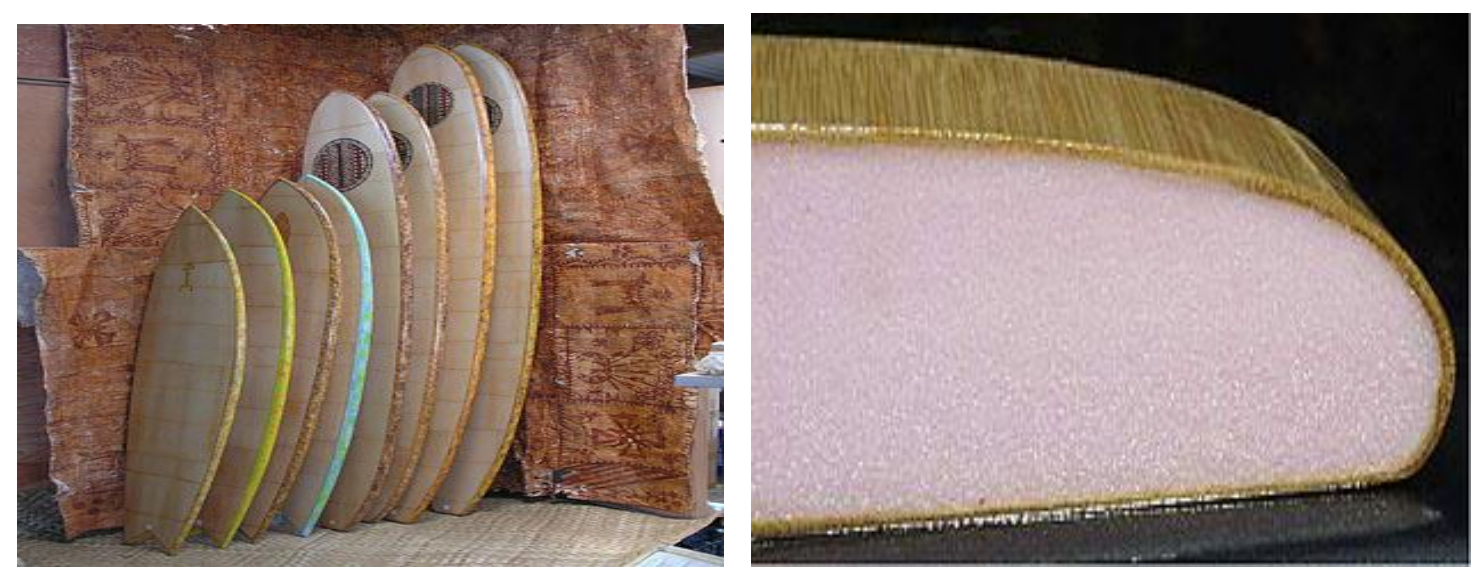

Figure 1-14. Laminated bamboo surfboards and example of cross section [71]

\subsection{Prior Investigations of Similar Nature}

As mentioned earlier, utilizing biocomposites for structural services is not a new idea. Therefore, a handful of references from previous works are recruited to form a foundation for this research. This collection is comprised of research focusing on the bamboo's mechanical properties and its various treatment/curing methods. 
Research dating back to the early 1980s provides an investigation into the potential of bamboo in building structures by placing emphasis on its mechanical properties. This research, completed in the form of a thesis by a Dutch Master's student named Janssen, is the first among the four primary references to give insight on the mechanical behavior of bamboos. Janssen goes on to explore the mechanical properties of bamboos for practical building applications in tropical climates. He performs tests on a class of bamboo called Bambusa Blumeana aged at three years, and his work encompasses compression, bending, and shear tests [46]. Unfortunately, Janssen was not able to determine a way to test the bamboo in tension, so a comprehensive tensile test is not available from his research.

Chalet and Bamboo, a contemporary Thailand-based company specializing in the manufacturing of bamboo products, gives insight on the various treatments of bamboos. Treating bamboos is known by this company to improve its quality by preserving them and protecting the bamboos from the environment after harvest. The company divides treatment methods into two distinct categories: traditional non-chemical and synthetic chemical processes [31]. Chemical processes are defined as eco-toxic substances that pose a hazard to working personnel or the environment. This resource serves primarily as a means to generate curing method ideas. Only a handful of the non-chemical treatment methods are borrowed from this source; the synthetic chemical treatments are eradicated from the potential list.

On the same level as Chalet and Bamboo, a team of students from the FCBA (French Technological Institute for Wood, Paper, and Furniture) explores various treatment methods to actually improve the mechanical properties of bamboo. Their work focused on the durability of bamboos under the persistent hazards of the elements. Experiments conducted include an investigation on the geometric swelling of bamboo soaked in 
water. Figure 1-15, taken from their experiment, bares an illustration of this effect. Geometric changes to bamboo under varying moisture levels is important knowledge if a structure is to be built with bamboos; even minor shifts at the joints of a structure, as the result of member expansion/compression due to moisture accumulation, poses a dangerous threat to its life. Another experiment explores the effects of temperature fluctuations in the bending strength and elastic modulus of bamboos. If unseen temperature changes occur to a bamboo structure, it is vital to understand the corresponding modifications in mechanical behavior. Their findings show a fairly consistent trend in the mechanical properties with the temperature effects investigated. Elements borrowed from this research now include the effects of treatments on the mechanical and geometric properties of bamboo.

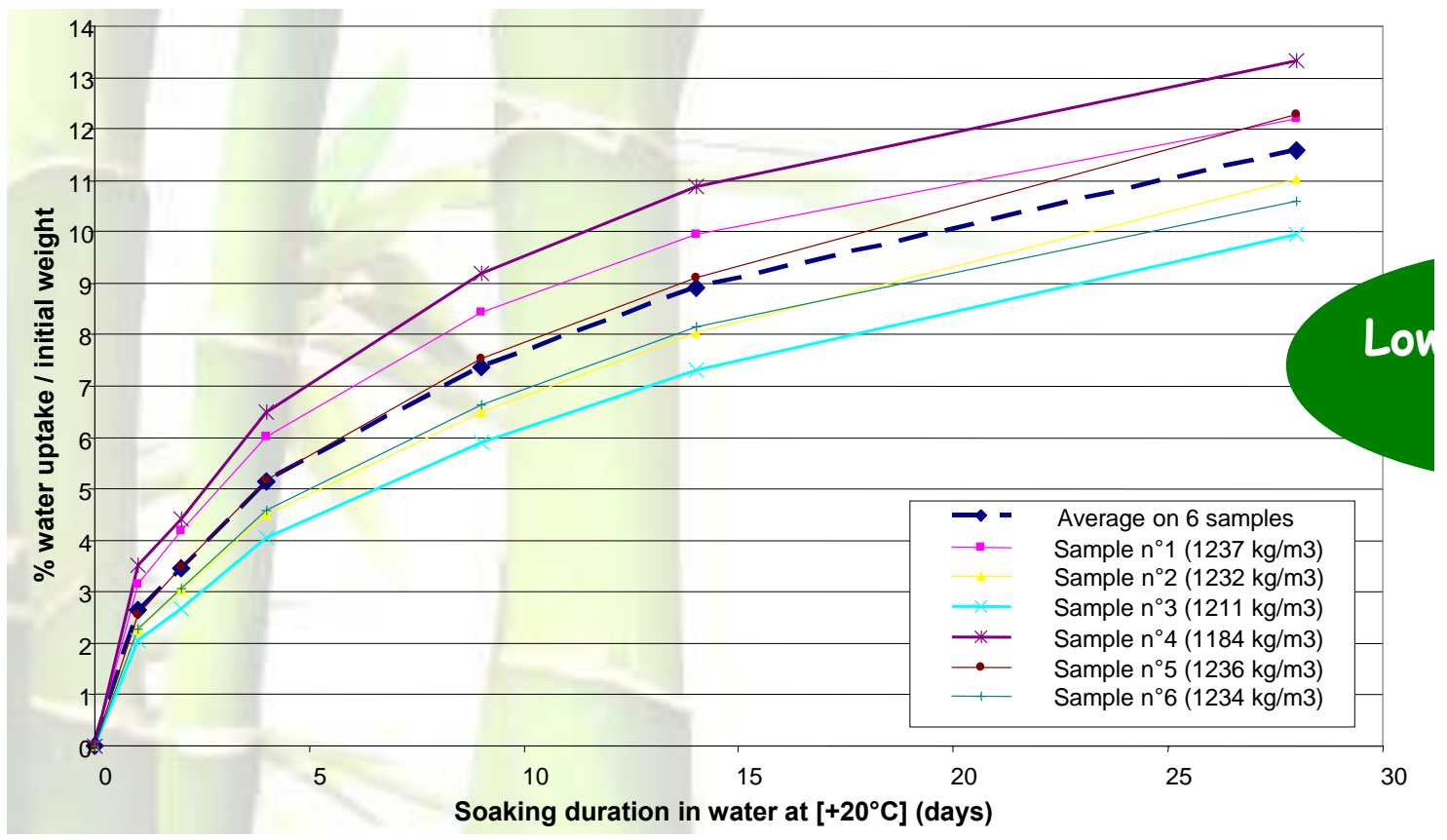

Figure 1-15. Water absorption data taken by FCBA [45] 


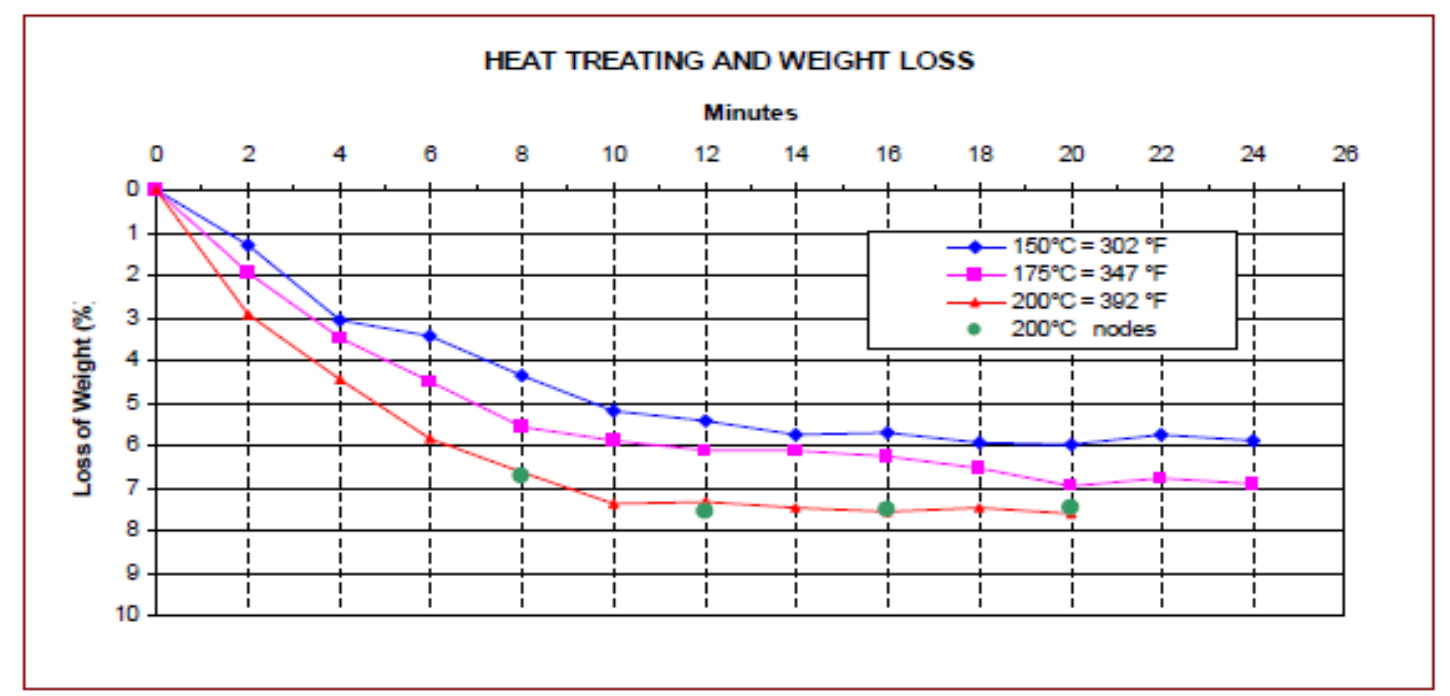

Figure 1-16. Schott's findings of weight loss versus treatment temperature [72]

The final item of research studied is Bamboo in the Lab by Wolfram Schott; his work aimed to improve the bending properties of bamboos through an assortment of heat treatments. Schott's findings are directed toward bamboo fishing pole applications. In Scott's research, heat treatments performed in a conventional house oven for up to 25 minutes served as the basis for his findings. Treatments ranging from $300^{\circ} \mathrm{F}$ to $400^{\circ} \mathrm{F}$

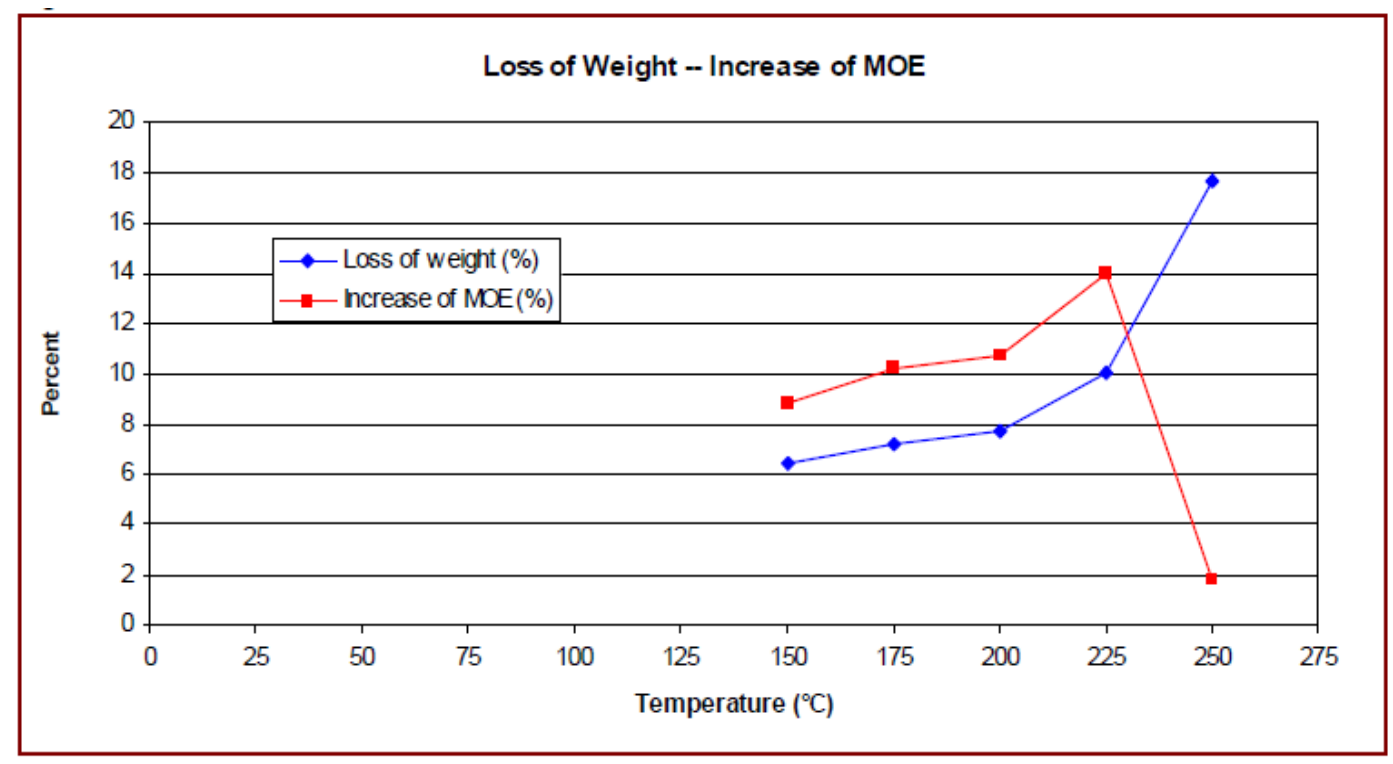

Figure 1-17. Schott's discovery of thermal treatment's effects on elastic modulus of bamboos [72] 
were conducted on bamboo cross sections to determine an optimum curing temperature. Three figures are provided to illustrate some of Schott's experimental results. Schott discovered that weight loss in the bamboo bears a direct relationship to the strength of the material. According to his findings, the optimum curing temperature is about $360^{\circ} \mathrm{F}$ for a duration of over 15 minutes. Two important facts to address are that Schott conducted these treatments on parallel plane triangular shapes measuring $6-\mathrm{mm}$ in height and 2-in. in length while only baking the specimens for relatively short times. It is understood from traditional wood preserving techniques that lower and longer heat treatments provide the best improvements in mechanical properties [37].
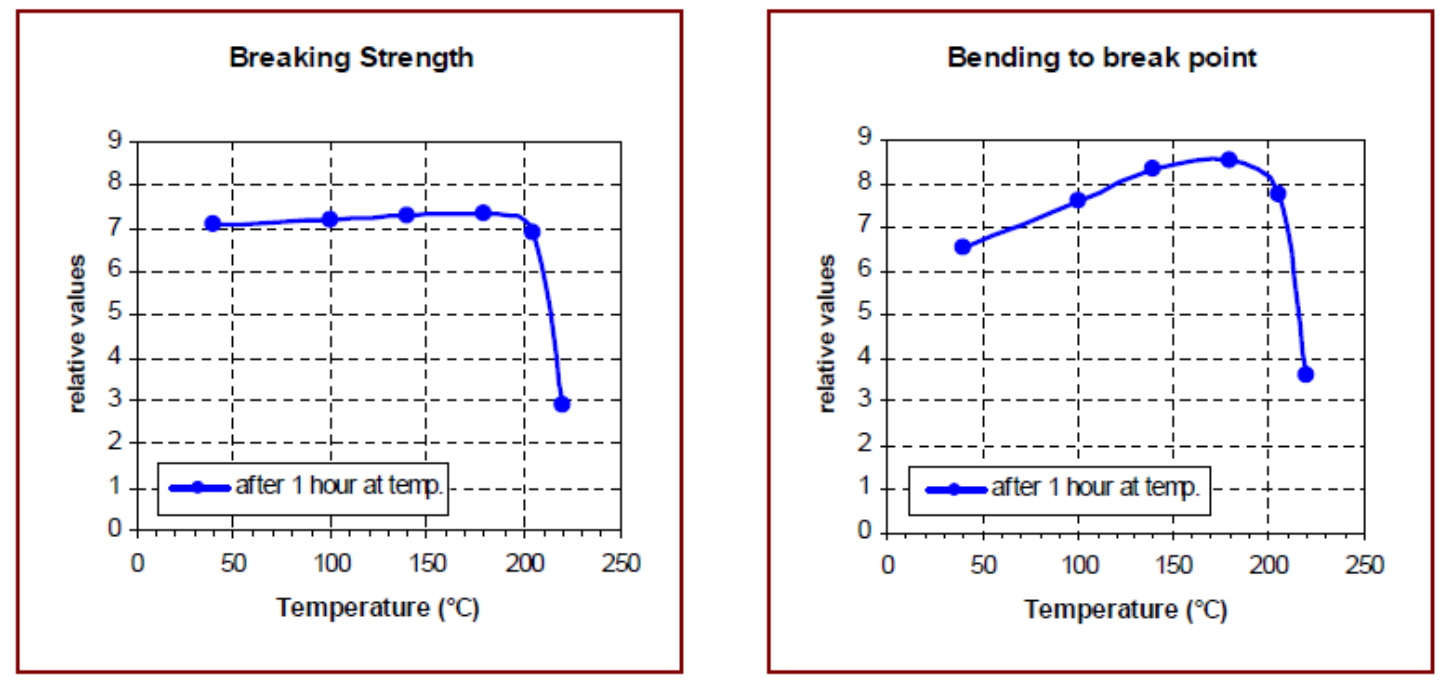

Figure 1-18. Schott's data on thermal treatments' effects on strength and ultimate strain [72]

\subsection{Research Objectives}

The purpose of this report draws inspiration from previous research and current environmental awareness policies to consider new biocomposites constituents for practical structural applications under flexural loading-specifically focusing on optimizing bamboo. A desire always exists in our rapidly changing world to create structures that are lighter, more durable, mechanically stronger, and-now-more 
environmentally friendly. The push for a greener world where more products are created from renewable resources is an eminent one. With the exception of being environmentally hazardous, modern composites successfully address these issues. As mentioned previously, engineers and scientists are drawing away from using conventional polymer-resin composites. These materials do not decompose and negatively impact the environment.

Therefore, the ultimate goal of this research is to optimize the mechanical characteristics of bamboo for biocomposite structural applications that exhibit flexural behavior. Secondary objectives of this study are as follows:

1. obtain the mechanical characteristics of non-optimized bamboos through compression and tension tests

2. explore and potentially validate varying treatment methods that alter the biological composition of the bamboo for improved performance during application

3. administer compression and tension tests on the treated bamboos to observe any changes in the mechanical properties

4. conduct an optimization analysis that maximizes the compressive and tensile characteristics for flexural loading conditions

5. perform four-point bending tests on optimally treated bamboos for two geometric configurations (hollow cylinder and veneer strip) to determine the resultant flexural properties and analyze the effect of node location in relation to applied bending loads

6. establish engineering guidelines for each flexural geometric configuration that may be implemented in biocomposite structural designs utilizing bamboos as a constituent 
7. integrate the optimum bamboo into two separate biocomposite structures, a truss and I-beam, to evaluate the feasibility and benefits of such applications under simulated loading conditions in a mechanical test of practical importance

8. develop a numerical methodology with Abaqus/CAE finite element software to validate the structures' performance from the test

9. Evaluate the performance of the structures' designs based on the results of the tested structures in conjunction with the analysis from the numerical scheme to propose improvements for future designs

Requirements of the study include using entirely "green materials" for the applications so that all constituents are biodegradable for disposal. Also, all testing experiments are performed under the guidelines of ISO standard 22157 when appropriate [44]. For the Ibeam application, the regulations of the 2012 SAMPE bridge contest are considered to manufacture and test the structure [63].

Responsibility for validity of this research is attributed to the author and any appropriate individuals that assisted in this mass of work.

\subsection{Extent of Research}

The following provides a glance at the body of work completed in this study. Chapter 2 discusses the purpose of selecting a treatment to optimize the mechanical properties of bamboos for structural application. Information on the reason for exploring these treatments is supplied in this section, and the types of treatments discussed fall into two categories: thermal and chemical. Chapter 3 chronicles all of the treatments investigated in this research and their preparation methods. Each treatment's recipe is explained, and all the curing cycles are discussed in detail. Chapter 4 dives into the resulting mechanical properties of all the treatments-specifically the compressive, tensile, and 
Poisson's ratio characteristics. Specimen preparation methods, testing procedures, results, and a comparative analysis of the data are described in detail. Chapter 5 then consists of an analysis to determine the optimal treatment based on the compressive and tensile properties of the bamboo. The analysis methodology is outlined and a comparative study to conventional eco-unfriendly materials is discussed. Chapter 6 investigates the flexural characteristics of the optimally-treated bamboo for two geometric configurations-naturally hollow cylinder and prepared veneer strip-with three different node locations. An analysis is accompanied in this section to establish guidelines for utilizing this bamboo in structures exhibiting flexural behavior. Chapter 7 describes the use of the optimum bamboo in two biocomposite structural applications, a truss and I-beam, to demonstrate the benefits of bamboo-reinforced biocomposites in practical structures. Manufacturing methods, testing procedures, results, finite element analyses for experimental validation, and conclusive notes on improving future design iterations are described in detail. Chapter 8 provides closing statements on the body of this research, and section 10 includes additional considerations that may be addressed in future work. 


\section{Chapter 2. Inquiring Bamboo Treatment Methods}

In order to obtain an optimally treated bamboo for structural application, a range of curing/treatment methods are investigated. A total of seven different types of treatment methods at varying curing temperatures and chemical additives are selected to optimize the mechanical properties of this specific bamboo species. The reasoning behind each selection is discussed for the thermal and chemical treatments presented in this study.

\subsection{Incentives for Treating Bamboos}

Bamboo must be cured and treated in order to protect the material from biological agents and to improve its mechanical properties from the raw green material. Without proper treatment, fungi and insects can consume the material if significant starch is readily available in the meat of the bamboo. Another incentive to treat bamboo is to increase the stiffness and strength of the material. In its unprepared green form, bamboo demonstrates mediocre mechanical advantages over common structural materials [46]. Also, bamboo has an average lifespan of over 15 years if correctly prepared after harvest [31]. This estimate can drop considerably under non-ideal conditions, so care is taken in determining a suitable treatment method.

\subsubsection{Investigating Thermal Treatments}

As mentioned previously, bamboos can be treated to improve its mechanical properties; thermal treatment eradicates the water in the material to increase stiffness and strength. It is mentioned in an earlier section that water exists in bamboo in two forms: bound and free water. One may recall that bound water is like the permanent resident of the cells while free water is the temporary tenant moving freely in and out of the plant cells. When considering strength and stiffness in bamboos, one must acknowledge the fiber 
saturation point. Shaku Design, a specialist in bamboo flute design, defines the fiber saturation point as the point where the free water is removed from the bamboo and only the bound water remains. They also use the excellent analogy that bamboo is like a sponge [11]. If a water-soaked sponge is squeezed with firm pressure to dispense all the "free water", then the residual moisture that remains and keeps the sponge damp is the "bound water". Only question is how to "squeeze" the water out of the bamboo. The answer lies in evaporating the water through heat treatments. At this saturation point, the majority of the bamboo's volume is composed of fibers and is measured to have a lower moisture content percentage. Moisture content is a measure of the remaining water in a dried organic sample. As water is removed from the green material, the mechanical properties of the bamboo improve due to less interference between the molecular bonding of the fiber cell walls [60].

\subsubsection{Investigating Organic Chemical Treatments}

Prior to thermal treatment in some cases, however, a series of organic chemical treatments are implemented to aid in the bamboo's optimization plan outlined previously. Employing these treatments will first deter other organisms from feeding on the bamboo; secondly, they will shield the material from the elements of the environment to aid in lengthening service life during structural application. In addition, these treatments are hypothesized to improve the microscopic imperfections of the sample since untreated bamboos have microscopic voids that are filled with free water [70]. Introducing a substance to fill these voids can reduce the number of imperfections in the bamboo to recover its optimum mechanical properties. First, a look into what substances can accomplish this feat is required for discussion.

Typically, chemical treatment systems for bamboos fall into two categories: synthetic and organic. Synthetic chemical treatments include soaking in a boric acid solution or 
imbuing the poles with pressurized chromated copper arsenate [31]. These types of treatment are discarded in an effort to avoid polluting toxic substances and promote the use of all-natural materials. Instead, natural chemical treatments are studied. It is known that certain people from rural coastal regions have traditionally soaked bamboo in the sea and also in lime solutions for construction applications [31; 7]. It is also documented that soaking wood in salt water assists in preserving and elongating its service life [1]. It is then hypothesized that the same effect can be applied to bamboos due to their close biological nature to wood. For this additional reason, the salt offers other potential benefits. Oil is also theorized to improve mechanical properties of the bamboo by filling voids and providing a barrier to moisture in the environment that facilitates premature fracture in the bamboo [11]. All of these treatments are natural chemical methods to extend or improve the service life of bamboos during application. The extent as to how the mechanical properties are affected by these treatments is unknown by practitioners.
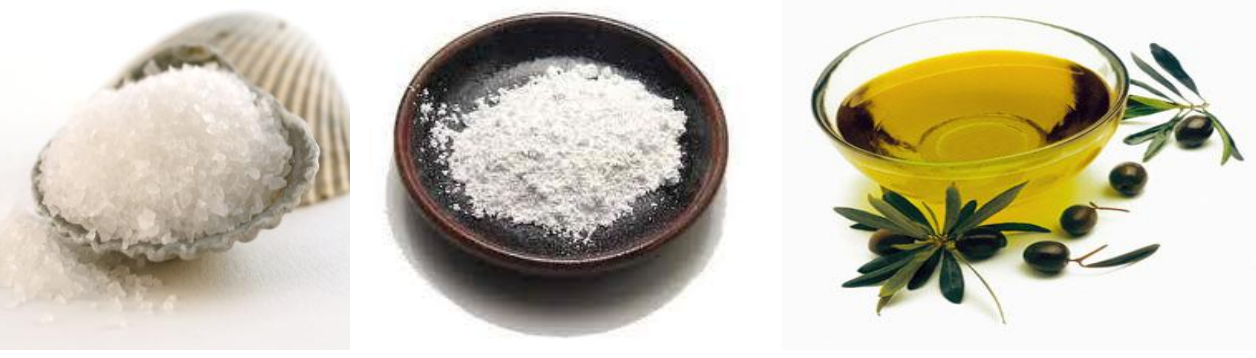

Figure 2-1. Proposed natural chemical treatments salt (left), lime (center), and oil (right) [64; 26; 28]

As a result, three types of natural chemical treatments are implemented to investigate their effects on the strength and stiffness properties of the bamboo. These chemical treatments include: varying levels of a salt solution, a lime solution, and an oil bath. Also, a series of heat treatments ranging from $150^{\circ} \mathrm{F}$ to $220^{\circ} \mathrm{F}$ are additionally executed to strengthen the bamboo and infuse the chemical additives into the bamboo's molecular anatomy. It is hypothesized that these chemical treatments will promote the leaching of 
starches, protect the bamboo from the environment, and fill the voids in the material. The thermal treatment will evaporate moisture and cement the supplements in the molecular anatomy of the bamboo. Furthermore, this theory leads to the conclusion that strength in bamboo is also dependent on specific gravity. Schott states, "[as] specific gravity increases, strength properties increase because internal stresses are distributed among more molecular material." In other words, stresses can be transferred with more ease among the bamboo's internal microstructures due to the bridged gaps built by the chemical additives and thermal treatment. The bamboo can, therefore, be optimized by applying only the chemical treatments that do not negatively affect the mechanical properties and also protect the material from the environment during application. 


\section{Chapter 3. Implementing Bamboo Treatments}

To begin this research on the optimization of bamboo, all the treatment methods must first be prepared for subsequent testing. Then a set of guidelines is followed to fabricate the specimens for successive chemical/heat treatment. Once the bamboo samples are treated, then an analysis is conducted on any moisture content and geometric changes. This is necessary so that a prediction can be made on the amount of volume shrinkage caused by the treatment and proper preparation can be considered accordingly before application. As mentioned before, shrinkage can pose a problem when making a structure since these geometric changes can prematurely fail it. This process will also provide a preview on the improvement in mechanical properties since the moisture content is a good indicator of the bamboo's strength.

\subsection{Preparing Bamboos for Treatment}

To prepare the bamboo specimens for each treatment method, a procedure is established so that all the curing treatments are not affected by the manner in which the specimen is prepared. First, initial measurements are made for each green bamboo pole. The weight and outer diameter are recorded to determine the taper of the culm. Each bamboo pole's internode is then marked for easy identification after treatment. The nodes are then removed-exposing the inner cavity-in order to

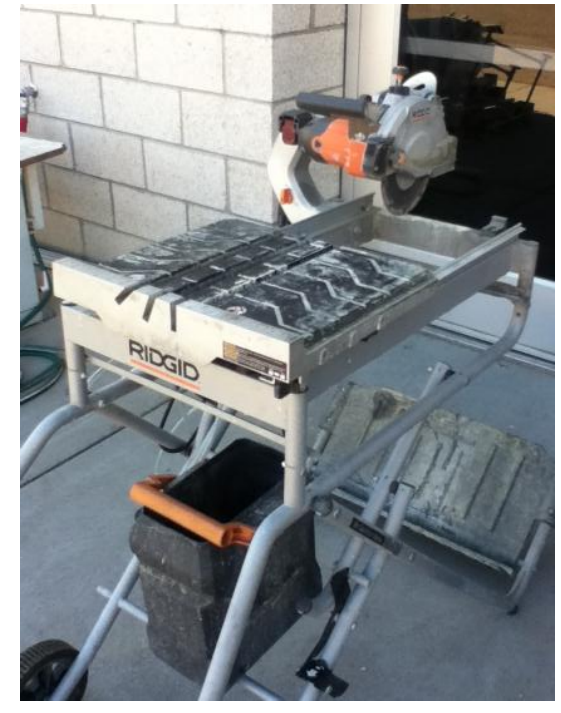

Figure 3-1. Diamond tile saw used to cut bamboo allow even treatment throughout the entire section of the culm. The internodes of the bamboo are cut using a diamond tile saw by Rigid, as shown in Figure 3-1. After the 
internodes are cut, each one is measured in accordance with ISO/TR 22157 to determine the volumetric shrinkage and moisture content of the sample after the treatment. To maintain consistency in recording each geometric measurement after treatment, each bamboo

internode is marked in 12

different locations to

precisely re-measure the

thickness and outer

diameter. Figure 3-2

provides an illustration of

the measurement

locations. The red stars

designated on the culm

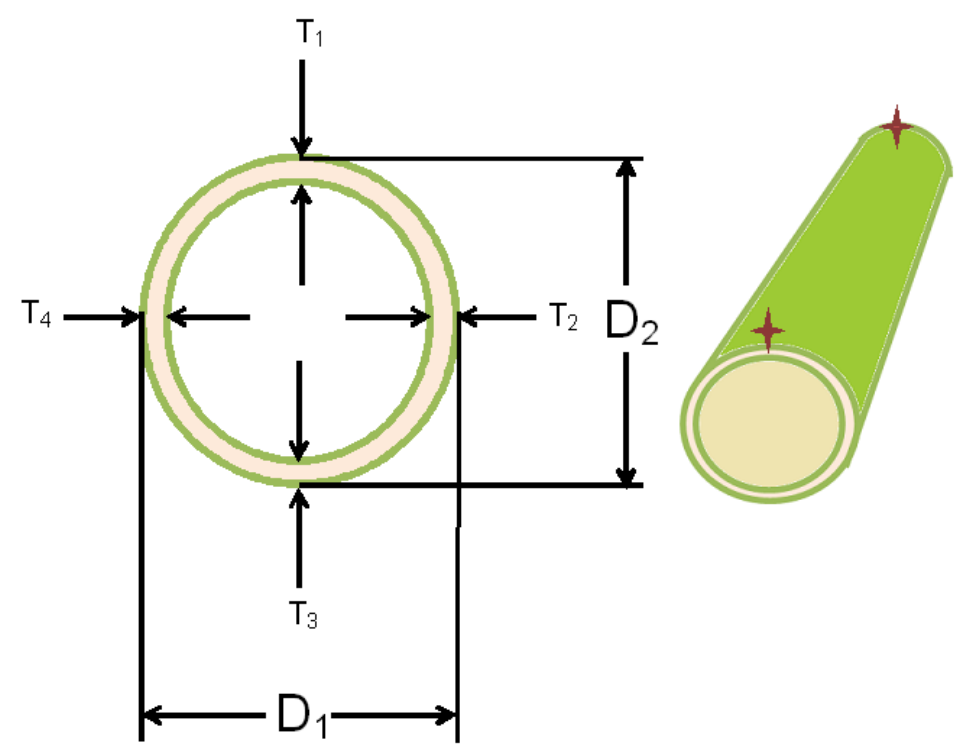

indicate where the

Figure 3-2. Measurement Locations along bamboo culm

thickness and diameter measurements are taken at both ends of the culm. The second benefit of using such extensive measuring procedures is to get an accurate mean for the dimensions; natural materials such as bamboos never yield uniform geometric measurements. Now at this point, the samples are ready to begin treatment.

\subsubsection{Thermal Treatment}

The bamboo samples are subjected to a series of thermal treatments to determine the ideal temperature for all the natural chemical treatments. Heat treatments of $150^{\circ} \mathrm{F}$, $180^{\circ} \mathrm{F}, 200^{\circ} \mathrm{F}$, and $220^{\circ} \mathrm{F}$ at two different heating rates are investigated. The relatively short intervals between the temperatures are to identify a value where the mechanical properties are damaged due to thermal degradation. The heating rates are $1^{\circ} \mathrm{F} / \mathrm{min}$. and $5^{\circ} \mathrm{F} / \mathrm{min}$. during the initial ramp-up phase of the heat treatment; these low ramp-up rates 
are chosen to prevent thermal shock on the bamboo during heat treatment. Two different heating rates are selected because the effect of initial heat rates on the bamboo's mechanical properties is difficult to assess at this point in the research. Preliminary tests using a conventional house oven showed that crack initiation occurred with ramp-up rates of $10^{\circ} \mathrm{F} / \mathrm{min}$. Efforts to avoid this event were taken into account early on in

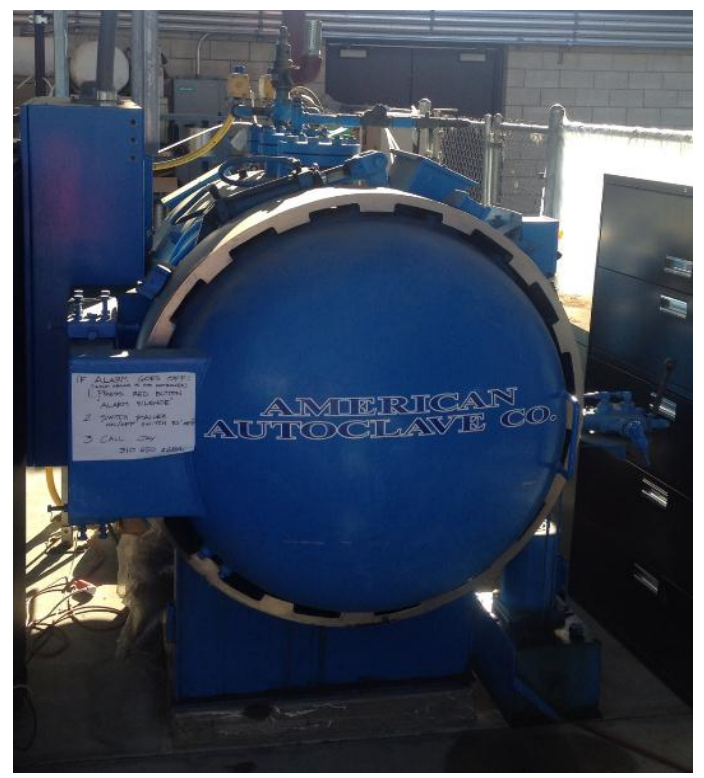

Figure 3-3. Cal Poly SLO's autoclave in Aerospace Structures/Composites laboratory the study. The combination of temperatures and rates all sum to seven different heat treatments. All heat treatments done in this research are performed in an autoclave by the American Autoclave Co. at the Cal Poly Structures/Composites Laboratory. Figure 3-3 depicts the autoclave from the aerospace department at Cal Poly SLO. It is important to note that temperatures above $220^{\circ} \mathrm{F}$ are not explored due to the mechanical limitations of the autoclave at the time. Nonetheless, this device is the only machine in the Aerospace Department capable of conducting a heat treatment that resembles the curing cycles required in this research. Its inner chamber measurements include a diameter of 2 -ft. and a length of 4 -ft. It also comes equipped with chamber-pressure capabilities and a blowing fan to circulate the air to the desired temperature. Although the autoclave is capable of applying pressure during the curing cycle, this feature was excluded for each thermal treatment to prevent any damage to the bamboos. Therefore, the initial ramp-up and cool-down stages are the major reasons for utilizing the autoclave. 


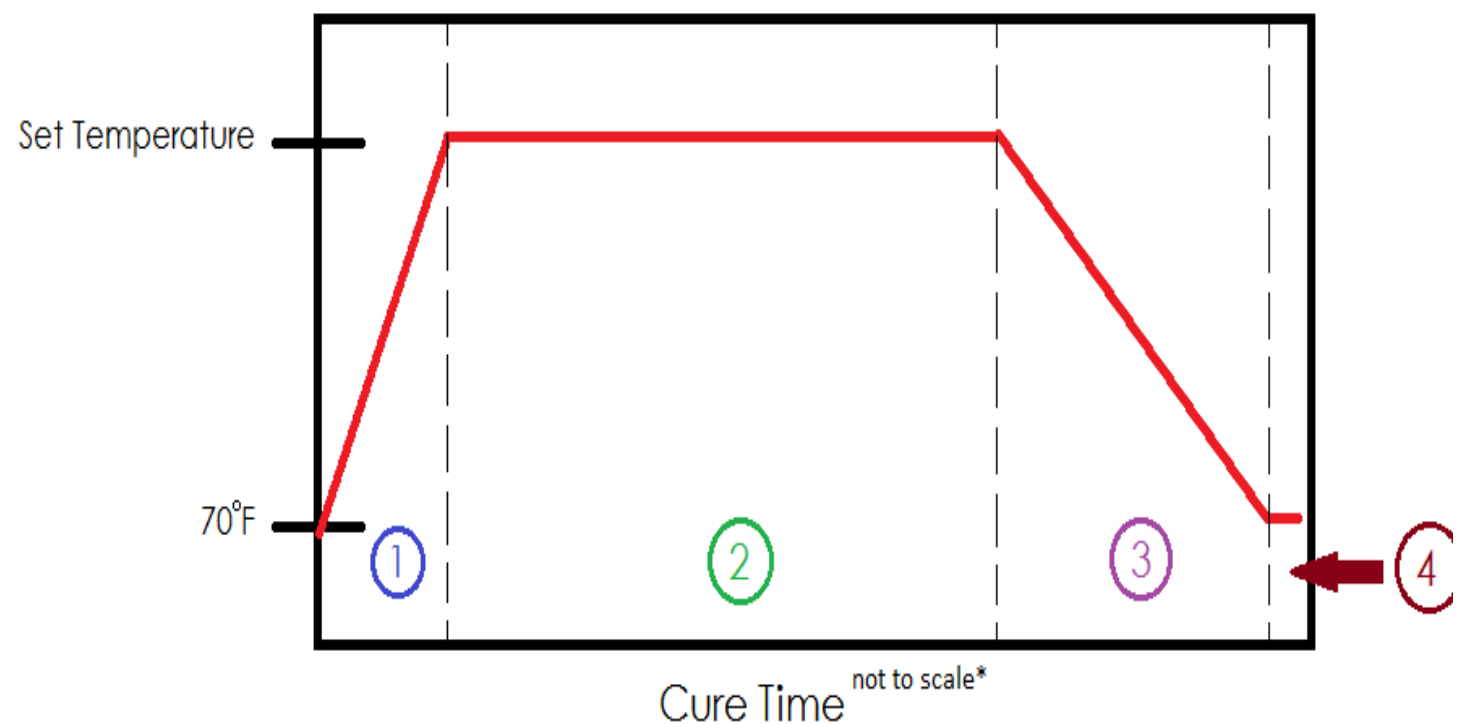

Figure 3-4. Sample cure cycle utilized: (1) heating stage (2) dwell stage (3) cooling stage (4) end stage

The four-stage curing cycles created with the autoclave's software program for all the heat treatments follow a base template. This template is constructed as follows: first, the curing cycle begins at ambient temperature of approximately $70^{\circ} \mathrm{F}$ and ramps up at the set heat rate towards the prescribed temperature, subsequently the bamboo samples sit in a dwell period for 4 hours, the curing cycle nears its end with a ramp-down cooling phase of $0.2^{\circ} \mathrm{F} / \mathrm{min}$. back to $70^{\circ} \mathrm{F}$, lastly the bamboo waits $1-\mathrm{min}$. in the final stage before being pulled out of the autoclave. Figure 3-4 illustrates a generic cure cycle for clarity. The $0.2^{\circ} \mathrm{F} / \mathrm{min}$. cooling phase is selected so as to minimize thermal shock in the bamboo caused by the transition between the dwell temperature and the end temperature. It is important to note that all cure cycles did not make use of any additional chamber pressure-a common practice for curing composites. On a similar note, the four-hour dwell time is chosen due to precautions taken to not damage the machine by running it for extensive periods of time at the mentioned temperatures. Lastly, it is worth noting that great obstacles were posed to the author of this study in using the autoclave. Performing the treatments with the mentioned parameters was only made possible due 
to extensively learning the inner workings of the autoclave and diagnosing the issues with a bit of practicality.

After each curing method was complete, the moisture content loss and the volumetric shrinkage are measured for each sample internode. The moisture content of the bamboo is taken in accordance with ISO $22157-1$ and is defined as,

$$
\Theta=\frac{m_{0}-m}{m_{0}} \cdot 100
$$

Where $\Theta$ is the moisture content loss, $m_{0}$ is the mass of the original internode, and $m$ is the mass of the internode after the heat treatment. In addition, the shrinkage percentage of the bamboo is defined as,

$$
\mathcal{Z}=\frac{\left[D_{o_{a v g}}^{2}-\left(D_{o_{a v g}}-2 t_{o_{a v g}}\right)^{2}\right]-\left[D_{\text {avg }}^{2}-\left(D_{\text {avg }}-2 t_{\text {avg }}\right)^{2}\right]}{D_{\text {avg }}^{2}-\left(D_{\text {avg }}-2 t_{\text {avg }}\right)^{2}} \cdot 100
$$

Where $\partial$ is the shrinkage in percent of the cross-sectional area, $D_{o \text { avg }}$ and $t_{o \text { avg }}$ are the average outer diameter and thickness of the original internode, and $D_{\text {avg }}$ and $t_{\text {avg }}$ are the dimensions corresponding to post-treatment. It is noted that the length is not incorporated in the equation above because the shrinkage in this direction is negligible. Consequently, the volumetric shrinkage can be approximated by analyzing the crosssectional shrinkage. After being armed with these variables, the treatments may be administered and analyzed.

\subsubsection{1 $150^{\circ} \mathrm{F}$ with $5^{\circ} \mathrm{F} / \mathrm{min}$. Initial Ramp Rate}

The first heat treatment consists of heating the bamboo from room temperature to $150^{\circ} \mathrm{F}$ at a rate of $5^{\circ} \mathrm{F} / \mathrm{min}$. during the initial ramp-up phase. A total of four different internodes were cured using this method of treatment, Table 3-1 shows the moisture content and 
shrinkage of each internode. The average moisture content and shrinkage that resulted from this curing method is $18.1 \%$ and $5.56 \%$, respectively.

Table 3-1. Physical changes due to $150^{\circ} \mathrm{F}$ with $5^{\circ} \mathrm{F} / \mathrm{min}$. rate

\begin{tabular}{|c|c|c|c|c|c|c|}
\hline Specimen & $\begin{array}{c}\text { Weight } \\
\text { Before } \\
\text { (g) }\end{array}$ & $\begin{array}{l}\text { Weight After } \\
\text { Treatment } \\
\text { (g) }\end{array}$ & $\begin{array}{c}\text { Moisture } \\
\text { Content } \\
\text { Loss }\end{array}$ & 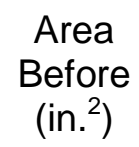 & $\begin{array}{c}\text { Area After } \\
\text { (in. }^{2} \text { ) }\end{array}$ & Shrinkage \\
\hline 1 & 0.281 & 0.229 & $18.5 \%$ & 0.976 & 0.916 & $6.14 \%$ \\
\hline 2 & 0.274 & 0.223 & $18.6 \%$ & 1.01 & 0.957 & $5.69 \%$ \\
\hline 3 & 0.253 & 0.207 & $17.9 \%$ & 1.03 & 0.974 & $5.22 \%$ \\
\hline 4 & 0.203 & 0.168 & $17.5 \%$ & 1.01 & 0.953 & $5.18 \%$ \\
\hline \multirow{4}{*}{\begin{tabular}{||c|} 
Average \\
$\begin{array}{l}\text { Standard } \\
\text { Deviation }\end{array}$ \\
Maximum \\
Minimum \\
\end{tabular}} & & & $18.1 \%$ & & & $5.56 \%$ \\
\hline & & & $0.5 \%$ & & & $0.45 \%$ \\
\hline & & & $18.6 \%$ & & & $6.14 \%$ \\
\hline & & & $17.5 \%$ & & & $5.18 \%$ \\
\hline
\end{tabular}

\subsubsection{2 $180^{\circ} \mathrm{F}$ with $5^{\circ} \mathrm{F} / \mathrm{min}$. and $1^{\circ} \mathrm{F} / \mathrm{min}$. Initial Ramp Rate}

The second thermal treatment set consisted of heating the bamboo to a temperature of $180^{\circ} \mathrm{F}$ with $5^{\circ} \mathrm{F} / \mathrm{min}$. and $1^{\circ} \mathrm{F} / \mathrm{min}$. rates. As mentioned before, the effect of heating the bamboo at different rates needs to be investigated to find an optimum treatment.

Table 3-2. Physical changes due to $180^{\circ} \mathrm{F}$ with $5^{\circ} \mathrm{F} / \mathrm{min}$. rate

\begin{tabular}{|c|c|c|c|c|c|c|}
\hline Specimen & $\begin{array}{l}\text { Weight } \\
\text { Before } \\
\text { (g) }\end{array}$ & $\begin{array}{c}\text { Weight } \\
\text { After } \\
\text { Treatment } \\
\text { (a) }\end{array}$ & $\begin{array}{c}\text { Moisture } \\
\text { Content } \\
\text { Loss }\end{array}$ & $\begin{array}{c}\text { Area } \\
\text { Before } \\
{\text { (in. }{ }^{2} \text { ) }}^{\text {a }}\end{array}$ & $\begin{array}{c}\text { Area After } \\
\text { (in. }{ }^{2} \text { ) }\end{array}$ & Shrinkage \\
\hline 1 & 0.308 & 0.222 & $27.90 \%$ & 0.931 & 0.844 & $9.31 \%$ \\
\hline 2 & 0.272 & 0.198 & $27.14 \%$ & 0.979 & 0.888 & $9.22 \%$ \\
\hline 3 & 0.286 & 0.208 & $27.12 \%$ & 0.971 & 0.887 & $8.68 \%$ \\
\hline \multirow{4}{*}{\begin{tabular}{|c|} 
Average \\
$\begin{array}{l}\text { Standard } \\
\text { Deviation }\end{array}$ \\
Maximum \\
Minimum \\
\end{tabular}} & & & $27.39 \%$ & & & $9.07 \%$ \\
\hline & & & $0.44 \%$ & & & $0.34 \%$ \\
\hline & & & $27.90 \%$ & & & $9.31 \%$ \\
\hline & & & $27.12 \%$ & & & $8.68 \%$ \\
\hline
\end{tabular}

Three total specimens were cured using the higher rate and four different specimens were cured using the lower rate. The results of the moisture content and shrinkage measurements for each rate can be seen in Table 3-2 and 
Table 3-3. The average moisture content loss for the $5^{\circ} \mathrm{F} / \mathrm{min}$. and $1^{\circ} \mathrm{F} / \mathrm{min}$. rates are $27.39 \%$ and $26.15 \%$, respectively. Similarly, the average shrinkage for the $5^{\circ} \mathrm{F} / \mathrm{min}$. and $1^{\circ} \mathrm{F} / \mathrm{min}$. rates are $9.07 \%$ and $7.70 \%$, respectively. The results for the $180^{\circ} \mathrm{F}$ treatments were very similar to each other in moisture content loss and shrinkage.

Table 3-3. Physical changes due to $180^{\circ} \mathrm{F}$ with $1^{\circ} \mathrm{F} / \mathrm{min}$. rate

\begin{tabular}{|c|c|c|c|c|c|c|}
\hline Specimen & $\begin{array}{c}\text { Weight } \\
\text { Before } \\
(\mathrm{g})\end{array}$ & $\begin{array}{c}\text { Weight After } \\
\text { Treatment } \\
(\mathrm{g})\end{array}$ & $\begin{array}{c}\text { Moisture } \\
\text { Content } \\
\text { Loss }\end{array}$ & $\begin{array}{c}\text { Area } \\
\text { Before } \\
\left(\text { in. }{ }^{2}\right)\end{array}$ & $\begin{array}{c}\text { Area After } \\
\left(\text { in. }{ }^{2}\right)\end{array}$ & Shrinkage \\
\hline 1 & 0.288 & 0.214 & $25.54 \%$ & 0.907 & 0.840 & $7.45 \%$ \\
\hline 2 & 0.169 & 0.120 & $28.72 \%$ & 0.845 & 0.769 & $9.01 \%$ \\
\hline 3 & 0.267 & 0.197 & $26.07 \%$ & 0.880 & 0.816 & $7.31 \%$ \\
\hline 4 & 0.194 & 0.147 & $24.26 \%$ & 0.910 & 0.846 & $7.04 \%$ \\
\hline Average & & & $26.15 \%$ & & & $7.70 \%$ \\
\hline $\begin{array}{l}\text { Standard } \\
\text { Deviation }\end{array}$ & & & $1.88 \%$ & & & $0.89 \%$ \\
\hline Maximum & & $28.72 \%$ & & & $9.01 \%$ \\
\hline Minimum & & & $25.54 \%$ & & & $7.04 \%$ \\
\hline
\end{tabular}

\subsubsection{3 $200^{\circ} \mathrm{F}$ with $5^{\circ} \mathrm{F} / \mathrm{min}$. and $1^{\circ} \mathrm{F} / \mathrm{min}$. Initial Ramp Rate}

Table 3-4. Physical changes due to $200^{\circ} \mathrm{F}$ with $5^{\circ} \mathrm{F} / \mathrm{min}$. rate

\begin{tabular}{|c|c|c|c|c|c|c|}
\hline Specimen & $\begin{array}{c}\text { Weight } \\
\text { Before } \\
(\mathrm{g})\end{array}$ & $\begin{array}{c}\text { Weight After } \\
\text { Treatment } \\
(\mathrm{g})\end{array}$ & $\begin{array}{c}\text { Moisture } \\
\text { Content Loss }\end{array}$ & $\begin{array}{c}\text { Area } \\
\text { Before } \\
\text { in. }^{2} \text { ) }\end{array}$ & $\begin{array}{c}\text { Area } \\
\text { After } \\
\left.\text { (in. }^{2}\right)\end{array}$ & Shrinkage \\
\hline 1 & 0.194 & 0.135 & $30.23 \%$ & 0.924 & 0.867 & $6.18 \%$ \\
\hline 2 & 0.318 & 0.235 & $26.28 \%$ & 1.11 & 1.04 & $5.65 \%$ \\
\hline 3 & 0.365 & 0.258 & $29.28 \%$ & 0.988 & 0.920 & $6.87 \%$ \\
\hline 4 & 0.378 & 0.273 & $27.62 \%$ & 1.04 & 0.964 & $7.53 \%$ \\
\hline Average & & $28.35 \%$ & & & $6.56 \%$ \\
\hline $\begin{array}{c}\text { Standard } \\
\text { Deviation }\end{array}$ & & $1.60 \%$ & & & $0.82 \%$ \\
\hline Maximum & & & $30.23 \%$ & & & $7.53 \%$ \\
\hline Minimum & & & $26.28 \%$ & & & $5.65 \%$ \\
\hline
\end{tabular}

Similar to the second set, the third thermal treatment set consisted of heating the bamboo to a temperature of $200^{\circ} \mathrm{F}$ with $5^{\circ} \mathrm{F} / \mathrm{min}$. and $1^{\circ} \mathrm{F} / \mathrm{min}$. rates. Four sample internodes are cured under this test and the resulting moisture content loss and shrinkage are recorded in the following tables. The average moisture content loss for the 
$5^{\circ} \mathrm{F} / \mathrm{min}$. and $1^{\circ} \mathrm{F} / \mathrm{min}$. rates are $28.35 \%$ and $27.18 \%$, respectively. Similarly, the average shrinkage for the $5^{\circ} \mathrm{F} / \mathrm{min}$. and $1^{\circ} \mathrm{F} / \mathrm{min}$. rates are $6.56 \%$ and $10.72 \%$, respectively.

Table 3-5. Physical changes due to $200^{\circ} \mathrm{F}$ with $1^{\circ} \mathrm{F} / \mathrm{min}$. rate

\begin{tabular}{|c|c|c|c|c|c|c|}
\hline Specimen & $\begin{array}{l}\text { Weight } \\
\text { Before } \\
\text { (g) }\end{array}$ & $\begin{array}{l}\text { Weight After } \\
\text { Treatment } \\
\text { (g) }\end{array}$ & $\begin{array}{c}\text { Moisture } \\
\text { Content } \\
\text { Loss }\end{array}$ & $\begin{array}{c}\text { Area } \\
\text { Before } \\
\text { (in. }{ }^{2} \text { ) }\end{array}$ & $\begin{array}{c}\text { Area After } \\
\text { (in. }{ }^{2} \text { ) }\end{array}$ & Shrinkage \\
\hline 1 & 0.357 & 0.258 & $27.62 \%$ & 1.02 & 0.926 & $9.09 \%$ \\
\hline 2 & 0.477 & 0.353 & $26.06 \%$ & 1.02 & 0.888 & $12.84 \%$ \\
\hline 3 & 0.491 & 0.354 & $27.85 \%$ & 1.05 & 0.939 & $10.51 \%$ \\
\hline \multirow{4}{*}{\begin{tabular}{|c|} 
Average \\
Standard \\
Deviation \\
Maximum \\
Minimum \\
\end{tabular}} & & & $27.18 \%$ & & & $10.72 \%$ \\
\hline & & & $0.97 \%$ & & & $1.89 \%$ \\
\hline & & & $27.85 \%$ & & & $12.84 \%$ \\
\hline & & & $26.06 \%$ & & & $9.09 \%$ \\
\hline
\end{tabular}

\subsubsection{4 $220^{\circ} \mathrm{F}$ with $1^{\circ} \mathrm{F} / \mathrm{min}$. Initial Ramp Rate}

The final curing treatment conducted has a dwell temperature of $220^{\circ} \mathrm{F}$ at a rate of $1^{\circ} \mathrm{F} / \mathrm{min}$. The reasoning behind omitting the $5^{\circ} \mathrm{F} / \mathrm{min}$. rate for this set is due to a mechanical error that occurred during this curing cycle. Certain technical issues with the autoclave's blowing fan at this temperature resulted in an emergency shutdown before the cycle could finish. Before irreparable damage could take place, the curing cycle with $5^{\circ} \mathrm{F} / \mathrm{min}$. rate was canceled for some other time since these samples could no longer be treated again without tainting the data. In order to economize the stock of bamboo, the $5^{\circ} \mathrm{F} /$ min sample group was omitted. Once the autoclave was ready, the treatment process continued again with the $1^{\circ} \mathrm{F} / \mathrm{min}$. rate. Four different specimens were treated at this temperature. The moisture content loss and shrinkage values are displayed in Table 3-6. The average moisture content for this curing method is $26.42 \%$, and the average shrinkage is $9.57 \%$. 
Table 3-6. Physical changes due to $220^{\circ} \mathrm{F}$ with $1^{\circ} \mathrm{F} / \mathrm{min}$. rate

\begin{tabular}{|c|c|c|c|c|c|c|}
\hline Specimen & $\begin{array}{c}\text { Weight } \\
\text { Before } \\
(\mathrm{g})\end{array}$ & $\begin{array}{c}\text { Weight After } \\
\text { Treatment } \\
(\mathrm{g})\end{array}$ & $\begin{array}{c}\text { Moisture } \\
\text { Content } \\
\text { Loss }\end{array}$ & $\begin{array}{c}\text { Area } \\
\text { Before } \\
\left.\text { (in. }{ }^{2}\right)\end{array}$ & $\begin{array}{c}\text { Area After } \\
\left.\text { (in. }{ }^{2}\right)\end{array}$ & Shrinkage \\
\hline 1 & 0.48752 & 0.35728 & $26.71 \%$ & 0.906 & 0.814 & $10.20 \%$ \\
\hline 2 & 0.47828 & 0.35332 & $26.13 \%$ & 0.967 & 0.880 & $8.99 \%$ \\
\hline 3 & 0.49456 & 0.36696 & $25.80 \%$ & 0.970 & 0.878 & $9.54 \%$ \\
\hline 4 & 0.30844 & 0.22506 & $27.03 \%$ & 0.726 & 0.657 & $9.52 \%$ \\
\hline Average & & $26.42 \%$ & & & $9.57 \%$ \\
\hline $\begin{array}{c}\text { Standard } \\
\text { Deviation }\end{array}$ & & $0.56 \%$ & & & $0.31 \%$ \\
\hline Maximum & & $27.03 \%$ & & & $10.20 \%$ \\
\hline Minimum & & & $25.80 \%$ & & & $8.99 \%$ \\
\hline
\end{tabular}

\subsubsection{Analysis of Thermal Treatments}

As a reminder, the purpose of this analysis is to give insight on the optimum thermal treatment to combine with the chemical one. Figure 3-5 summarizes the mean moisture content loss and shrinkage values of all the thermally treated samples for discussion. All the cure temperatures above $150^{\circ} \mathrm{F}$ certainly indicate a higher moisture content loss and shrinkage value. A second observation is that using either rate does not significantly affect the results of moisture loss and shrinkage-differences of a few percent are observed. Therefore, the data seem to say that using the $5^{\circ} \mathrm{F} / \mathrm{min}$. rate for treatment would yield similar moisture loss with quicker treatment times. Additionally, by merely looking at the average raw numbers, one can postulate that the optimum thermal treatment is $200^{\circ} \mathrm{F}$ with a $5^{\circ} \mathrm{F} / \mathrm{min}$. rate. This treatment offers the highest nominal moisture evaporation while providing the smallest amount of shrinkage next to the $150^{\circ} \mathrm{F}$ treatment. Upon closer analysis, however, an obvious optimum heat treatment becomes obscure. Error bars on the plot illustrate that the $200^{\circ} \mathrm{F}$ heat treatments have high deviations; this reason alone cannot allow this treatment to be the optimum one at this point in the research. It appears that none of the treatments above $180^{\circ} \mathrm{F}$ offer an overwhelming decrease in moisture content or shrinkage over the others. In light of this, additional observations can be drawn from the sample geometries. First, the $180^{\circ} \mathrm{F}$ heat 
treatments yield the lowest deviations but also have a sample size of three or four. Second, specimen 1 from the $200^{\circ} \mathrm{F}$ treatment group at $5^{\circ} \mathrm{F} / \mathrm{min}$. is considerably lighter than the rest and consequently has a higher moisture content loss that may have skewed the results. With this change, the average moisture content loss for that group would drop to $27.7 \%$ with a deviation of $1.50 \%$ - equalizing it among the rest.

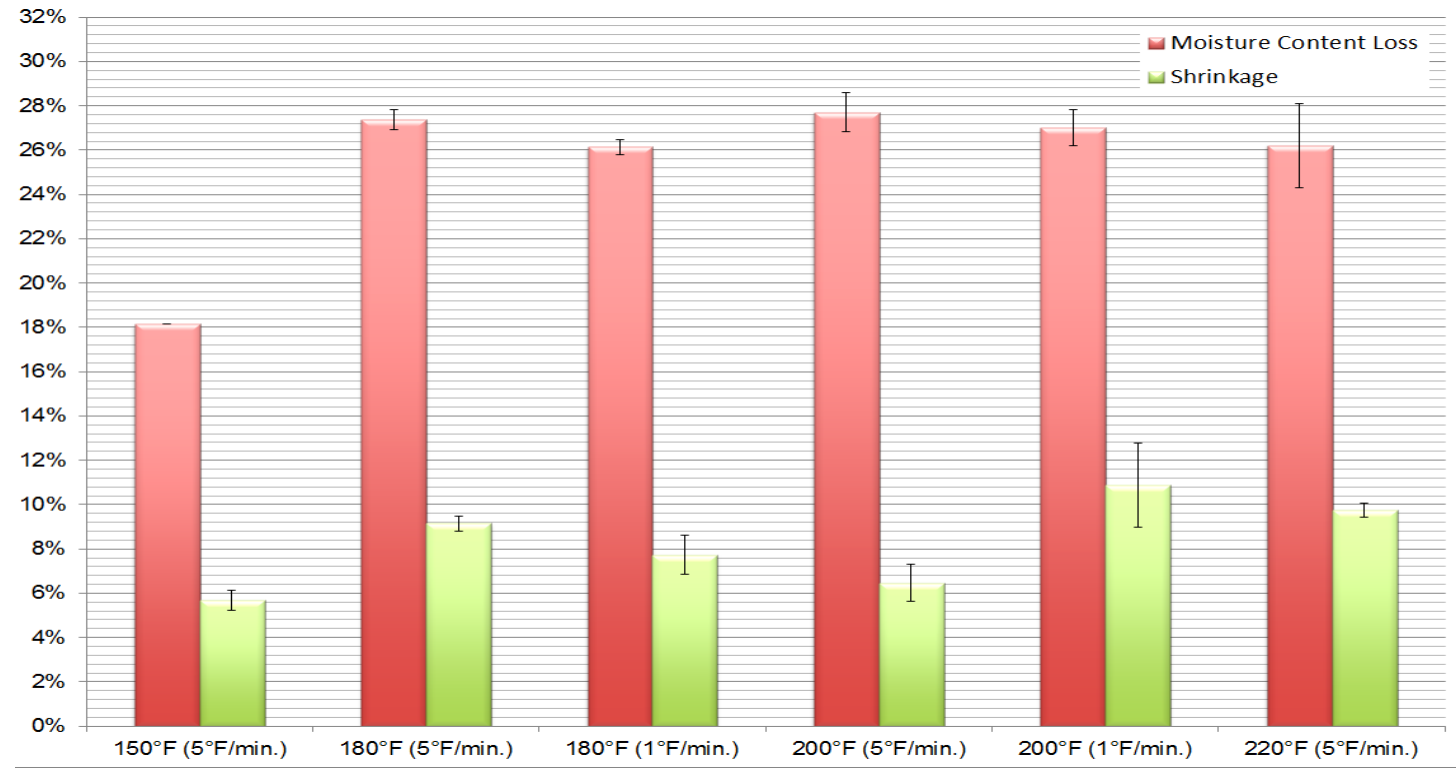

Figure 3-5. Analysis of physical changes for all thermal treatments

In summary, the results of the thermal treatment indicate moisture content losses in the range of $18 \%$ to $28 \%$ with shrinkage values of $6 \%$ to $10 \%$. The shrinkage observed suggests that structures built with bamboo poles must expect area reductions of up to $10 \%$ after curing at temperatures up to $220^{\circ} \mathrm{F}$. Therefore, the design of a structure must account for this change when selecting bamboos to strengthen through thermal treatment. On another note, the $220^{\circ} \mathrm{F}$ treatment would intuitively yield the highest moisture content loss which leads to better mechanical properties in the bamboo. For this reason, the $220^{\circ} \mathrm{F}$ treatment at $1^{\circ} \mathrm{F} / \mathrm{min}$. is favored when proceeding with the following treatments. Committing to this decision, however, should not cause alarm. Curing bamboo at $220^{\circ} \mathrm{F}$ should theoretically show similar mechanical properties to a 
$200^{\circ} \mathrm{F}$ or $180^{\circ} \mathrm{F}$ treatment-since they yield similar moisture loss. Consequently, it is hypothesized at this point from the plot that four-hour treatments with temperatures above $180^{\circ} \mathrm{F}$ do not show significant advantages in moisture loss for bamboo. Therefore, for future reference, improving the mechanical properties of bamboo by reducing its moisture content with a four-hour $180^{\circ} \mathrm{F}$ heat treatment at $5^{\circ} \mathrm{F} / \mathrm{min}$. should suffice. The lower temperature and faster cure time should save time, energy, and economic resources.

\subsubsection{Natural Chemical Treatment}

Now that some insight has been gained from the preliminary thermal treatments, the natural chemical treatments can be pursued to begin investigating their effect on the mechanical properties of bamboo. Again, chemical treatments are carried out on bamboo groups as an attempt to fill the microscopic voids in the cellular structure. They also are known to prolong material life and to seal off the raw material from biological attacks. A total of three salt-water solutions are first studied: $3 \%, 6 \%$, and $9 \%$. Second, a $5 \%$ slaked lime-water solution is prepared to chemically treat the bamboo. Finally, an oil bath is implemented to examine potential benefits after curing. All of these chemical treatments are followed by thermal curing in the autoclave to solidify the natural additives into the bamboo's anatomical structure, so the $220^{\circ} \mathrm{F}$ heat treatment at $1^{\circ} \mathrm{F} / \mathrm{min}$. is employed to cure the sample and its additive. Once again, the moisture content loss and shrinkage is analyzed to provide an estimate of which treatments would provide the optimal mechanical properties.

\subsubsection{Salt-Water Solution Treatment}

A set of salt-water solutions is the first chemical treatment investigated in this portion of the research. As previously mentioned, the purpose of the salt treatment is to fill the microscopic voids in the bamboo and observe any effects on the strength of the 
surrounding matter while extending the service life of the material. Salt and water are mixed together creating a compound that easily penetrates the bamboo samples. Difficulty would arise in simply placing bamboo samples into a volume of salt because chemical penetration through the culm may not occur. Consequently, water is deemed as a better vehicle to transport the salt through the inner structure.

The basis for selecting a $3 \%$ salt solution is to simulate the salinity of seawater that natives have traditionally used. On average, the salinity of the world's oceans is about thirty-five parts per thousand-or 3.5\% [58]. For example, this means that $35-\mathrm{g}$ of salt dispensed in a $1000-\mathrm{g}$ volume of water yields a salinity of $3.5 \%$. Figure 3-6 is shown to illustrate the varying salinity in bodies of water across the globe. This process also brought up an interesting question of whether higher salinity levels could further

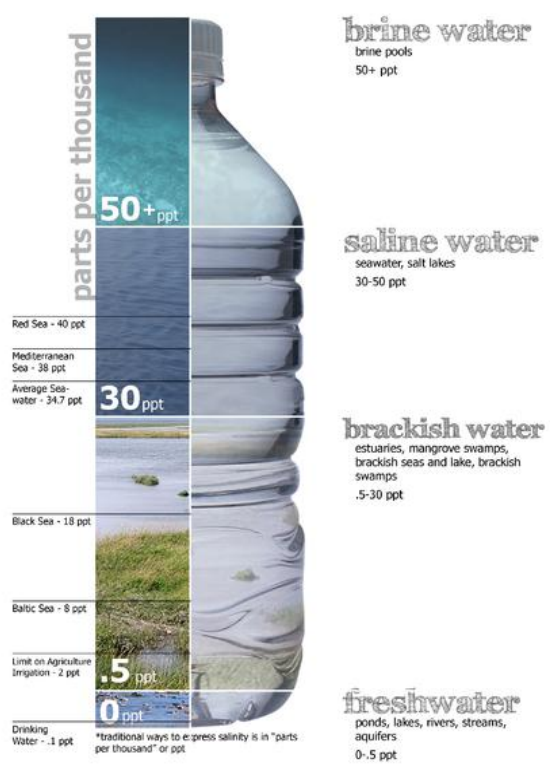

Figure 3-6. Water salinity in different bodies of water [39]

improve the mechanical properties of bamboo, so the solution is increased by increments of $3 \%$ for two other salt treatments. A study is then carried out by using varying salt solutions that are twice or three times the ocean's average salinity to determine how much additive is necessary to fill the bamboo's voids, thereby improving mechanical properties.

In order to conduct this chemical treatment, only a few necessary items are required. Instant Ocean Sea Salt Mix is obtained to simulate the ocean's seawater as much as possible-this particular brand is recognized as one of the best at simulating natural seawater. A mass of $0.62-\mathrm{kg}$ of this salt is dissolved into $20.58-\mathrm{kg}$ of water to create the 
$3 \%$ salt solution in a plastic bin. A $3 \%$ salt solution is created instead of a $3.5 \%$ solution because the hydrometer used to check the salinity of the solution did not go up past $10 \%$; to keep the $3.5 \%$ salt solution meant having to make the other two $7 \%$ and $10.5 \%$. Such a difference is considered negligible for the study. Once mixed, bamboo samples taken from different poles are then placed into the salt solution and left uncovered in a well-ventilated area for a period of five days. Figure 3-7 shows a photograph of the sea salt mix used and some of the specimens soaking in the salt solution. The process is then repeated for the other salt treatments with the appropriate salt-to-water ratios.
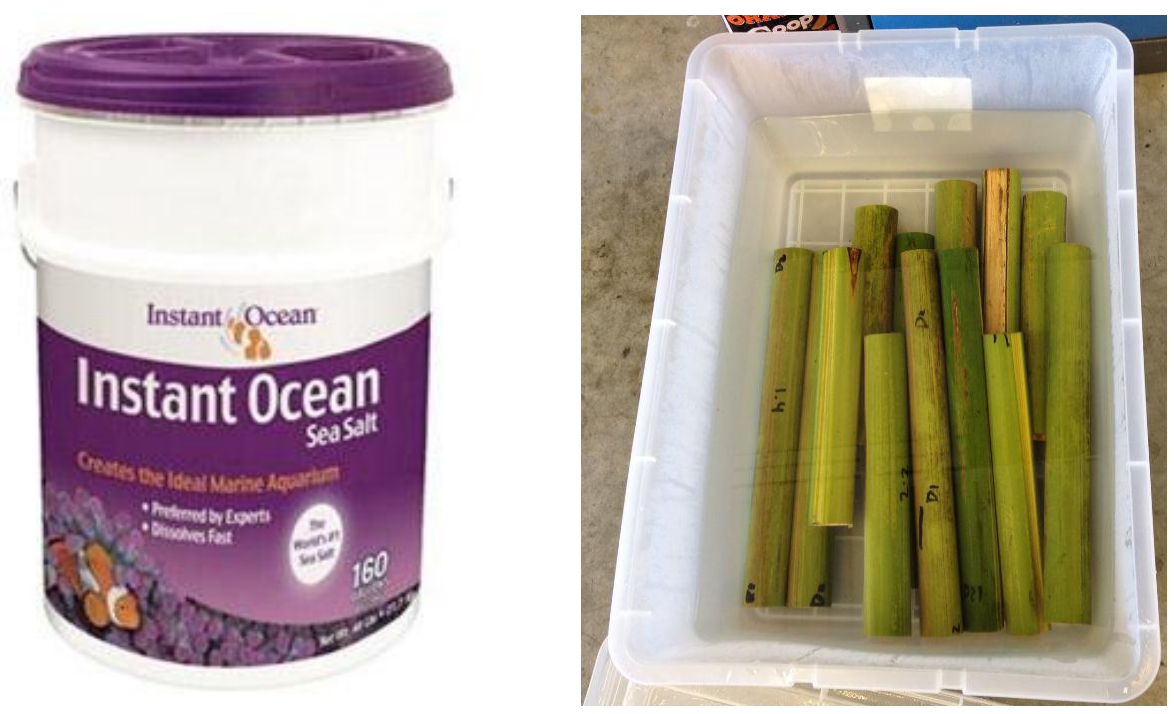

Figure 3-7. Instant Ocean Sea Salt mix [57] and soaking bamboos in the salt solution

A total of 11 bamboo samples are used for the $3 \%$ and $9 \%$ solution while 6 are used for the $6 \%$ salt solution. About a week for chemical treating was simply the time frame chosen to ensure that the saltwater saturated the bamboos completely. Also, the compound solution was mixed several times a day to safeguard against the issue of salt settling to the bottom of the bin. It is very likely that mixing was unnecessary since the solution appeared almost clear, but the extra precaution was taken as it did not involve much effort. 
Table 3-7. Physical changes due to $3 \%$ salt treatment

\begin{tabular}{|c|c|c|c|c|c|c|}
\hline Specimen & $\begin{array}{c}\text { Weight } \\
\text { Before } \\
(\mathrm{g})\end{array}$ & $\begin{array}{c}\text { Weight } \\
\text { After } \\
\text { Treatment } \\
\text { (g) }\end{array}$ & $\begin{array}{c}\text { Moisture } \\
\text { Content } \\
\text { Loss }\end{array}$ & 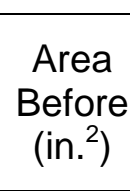 & $\begin{array}{l}\text { Area } \\
\text { After } \\
\left(\text { in. }{ }^{2} \text { ) }\right.\end{array}$ & $\begin{array}{c}\text { Cross } \\
\text { section } \\
\text { Shrinkage }\end{array}$ \\
\hline 1 & 0.328 & 0.196 & $40.27 \%$ & 0.594 & 0.504 & $15.15 \%$ \\
\hline 2 & 0.359 & 0.225 & $37.18 \%$ & 0.681 & 0.563 & $17.37 \%$ \\
\hline 3 & 0.284 & 0.177 & $37.77 \%$ & 0.589 & 0.515 & $12.58 \%$ \\
\hline 4 & 0.304 & 0.191 & $37.34 \%$ & 0.653 & 0.580 & $11.24 \%$ \\
\hline 5 & 0.318 & 0.199 & $37.48 \%$ & 0.580 & 0.610 & $10.42 \%$ \\
\hline 6 & 0.320 & 0.207 & $35.44 \%$ & 0.752 & 0.692 & $8.02 \%$ \\
\hline 7 & 0.364 & 0.225 & $38.26 \%$ & 0.684 & 0.593 & $13.38 \%$ \\
\hline 8 & 0.378 & 0.238 & $37.21 \%$ & 0.720 & 0.594 & $17.53 \%$ \\
\hline 9 & 0.392 & 0.246 & $37.26 \%$ & 0.719 & 0.617 & $14.26 \%$ \\
\hline 10 & 0.362 & 0.230 & $36.37 \%$ & 1.030 & 0.903 & $12.07 \%$ \\
\hline 11 & 0.379 & 0.239 & $36.82 \%$ & 0.947 & 0.841 & $11.20 \%$ \\
\hline \multirow{4}{*}{$\begin{array}{l}\text { Average } \\
\text { Standard } \\
\text { Deviation } \\
\text { Maximum } \\
\text { Minimum } \\
\end{array}$} & & & $37.62 \%$ & & & $14.57 \%$ \\
\hline & & & $1.20 \%$ & & & $2.91 \%$ \\
\hline & & & $40.27 \%$ & & & $17.53 \%$ \\
\hline & & & $35.44 \%$ & & & $8.02 \%$ \\
\hline
\end{tabular}

After sitting in the salt solution for five days, the bamboo samples are taken out of their bin and prepared for thermal treatment. Each bamboo internode is lightly dried off with paper towels to remove any water that would cause excess dripping. Once lightly dried, the bamboo group is placed in the autoclave and ready for thermal treatment at $220^{\circ} \mathrm{F}$ with a $1^{\circ} \mathrm{F} / \mathrm{min}$. ramp-up rate. When placed into the autoclave, the samples were stacked cross-wise on top of a pair of carbon-fiber elevators to provide adequate ventilation throughout the curing cycle. It is important to note that each of these chemical treatments was done sequentially so as to maintain consistency in the total soak time. Once cured from the thermal treatment, the samples are removed from the autoclave and inspected visually.

Examination of all three groups reveals a glossy golden brown hue to the surface of the bamboo. Again, the moisture content loss and shrinkage are measured after the thermal treatment is complete. Three tables for each chemical treatment are provided to 
Table 3-8. Physical changes due to $6 \%$ salt treatment

\begin{tabular}{|c|c|c|c|c|c|c|}
\hline Specimen & $\begin{array}{c}\text { Weight } \\
\text { Before } \\
\text { (g) }\end{array}$ & $\begin{array}{c}\text { Weight } \\
\text { After } \\
\text { Treatment } \\
\text { (g) }\end{array}$ & $\begin{array}{c}\text { Moisture } \\
\text { Content } \\
\text { Loss }\end{array}$ & $\begin{array}{c}\text { Area } \\
\text { Before } \\
\text { (in. }{ }^{2} \text { ) }\end{array}$ & $\begin{array}{l}\text { Area } \\
\text { After } \\
\left(\text { in. }{ }^{2} \text { ) }\right.\end{array}$ & $\begin{array}{c}\text { Cross } \\
\text { section } \\
\text { Shrinkage }\end{array}$ \\
\hline 1 & 0.315 & 0.192 & $39.05 \%$ & 0.567 & 0.497 & $12.29 \%$ \\
\hline 2 & 0.347 & 0.212 & $38.91 \%$ & 0.682 & 0.550 & $19.38 \%$ \\
\hline 3 & 0.393 & 0.241 & $38.63 \%$ & 0.830 & 0.643 & $15.74 \%$ \\
\hline 4 & 0.297 & 0.198 & $33.56 \%$ & 0.787 & 0.750 & $9.68 \%$ \\
\hline 5 & 0.333 & 0.221 & $33.60 \%$ & 0.860 & 0.786 & $8.61 \%$ \\
\hline 6 & 0.386 & 0.249 & $35.46 \%$ & 1.010 & 0.877 & $12.81 \%$ \\
\hline Average & & & $35.99 \%$ & & & $13.14 \%$ \\
\hline $\begin{array}{l}\text { Standard } \\
\text { Deviation }\end{array}$ & & & $2.64 \%$ & & & $3.98 \%$ \\
\hline Maximum & & & $39.05 \%$ & & & $19.38 \%$ \\
\hline Minimum & & & $33.56 \%$ & & & $8.61 \%$ \\
\hline
\end{tabular}

Table 3-9. Physical changes due to $9 \%$ salt treatment

\begin{tabular}{|c|c|c|c|c|c|c|}
\hline Specimen & $\begin{array}{l}\text { Weight } \\
\text { Before } \\
\text { (g) }\end{array}$ & $\begin{array}{c}\text { Weight After } \\
\text { Treatment } \\
\text { (g) }\end{array}$ & $\begin{array}{c}\text { Moisture } \\
\text { Content } \\
\text { Loss }\end{array}$ & $\begin{array}{c}\text { Area } \\
\text { Before } \\
\left(\text { in. }{ }^{2}\right)\end{array}$ & $\begin{array}{l}\text { Area } \\
\text { After } \\
\left(\text { in. }^{2}\right)\end{array}$ & $\begin{array}{c}\text { Cross } \\
\text { section } \\
\text { Shrinkage }\end{array}$ \\
\hline 1 & 0.342 & 0.239 & $29.99 \%$ & 0.665 & 0.559 & $15.94 \%$ \\
\hline 2 & 0.387 & 0.271 & $30.03 \%$ & 0.781 & 0.695 & $10.96 \%$ \\
\hline 3 & 0.298 & 0.205 & $31.31 \%$ & 0.669 & 0.623 & $6.94 \%$ \\
\hline 4 & 0.386 & 0.285 & $26.22 \%$ & 0.812 & 0.675 & $16.90 \%$ \\
\hline 5 & 0.400 & 0.294 & $26.40 \%$ & 0.859 & 0.753 & $12.35 \%$ \\
\hline 6 & 0.422 & 0.312 & $26.17 \%$ & 0.897 & 0.801 & $9.71 \%$ \\
\hline 7 & 0.442 & 0.328 & $25.87 \%$ & 0.974 & 0.904 & $7.21 \%$ \\
\hline 8 & 0.424 & 0.312 & $26.35 \%$ & 0.984 & 0.892 & $9.36 \%$ \\
\hline 9 & 0.450 & 0.311 & $30.89 \%$ & 0.869 & 0.791 & $8.92 \%$ \\
\hline 10 & 0.433 & 0.299 & $31.00 \%$ & 0.826 & 0.724 & $12.25 \%$ \\
\hline 11 & 0.349 & 0.241 & $30.90 \%$ & 0.909 & 0.804 & $12.02 \%$ \\
\hline Average & & & $28.65 \%$ & & & $10.96 \%$ \\
\hline $\begin{array}{l}\text { Standard } \\
\text { Deviation }\end{array}$ & & & $2.38 \%$ & & & $3.21 \%$ \\
\hline Maximum & & & $31.0 \%$ & & & $15.94 \%$ \\
\hline Minimum & & & $26.17 \%$ & & & $6.94 \%$ \\
\hline
\end{tabular}

depict the resulting moisture content loss and shrinkage. It is vital to note that shrinkage along the length of the culm is measured to be negligible, so this variable is omitted from the results. The average moisture content losses for the $3 \%, 6 \%$, and $9 \%$ treatments 
are: $37.62 \%, 35.99 \%$, and $28.65 \%$, respectively. The mean shrinkage of each treatment is $14.57 \%, 13.14 \%$, and $10.96 \%$, respectively.

\subsubsection{Lime-Water Solution Treatment}

The second chemical treatment studied is a $5 \%$ hydrated lime-water solution. Similar to the salt solution, hydrated lime and water are mixed into a compound that the bamboo samples can absorb. Hydrated lime is an environmentally friendly compound that is normally in a white powder form and its typical uses include being a protector and sanitizer of materials. Hydrated lime absorbs carbon dioxide from the air to convert back to limestone which provides a protective layer over the material's surface [73]. It also

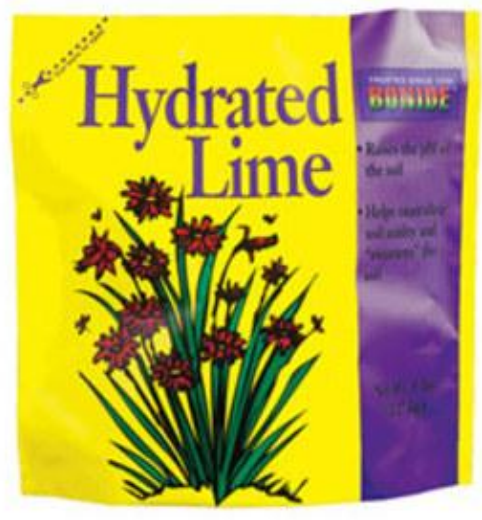

Figure 3-8. Hydrated lime mix utilized in lime treatment [26] sanitizes surfaces by insulating the material from fungi, bacteria, and insects; this insulating property also protects against moisture absorption from the air and resists high temperature fluctuations. A study is then conducted to see if a hydrated lime solution treatment affects the mechanical properties of bamboo by filling microscopic voids with the protective and insulating properties of the chemical compound.

Preparation of the chemical treatment is simple and requires minimal ingredients. The solution consists of Bonide Hydrated Lime mix and water. To begin, 1.54-kg of hydrated lime is dispensed into $30.85-\mathrm{kg}$ of water in a bin and mixed thoroughly. This mixture provides a $5 \%$ lime-water compound solution; the reasoning for using this ratio is because initial observations showed that the water was fairly saturated with this amount of hydrated lime. Therefore, it was deemed adequate to keep the solution at this mixture 
ratio. Sufficient mixing produced a white milky consistency at which mixing is stoppedthis reaction is characteristic of the calcium hydroxide solute. A period of approximately five minutes is long enough for the remaining calcium hydroxide particles to settle towards the bottom of the bin. Once settled, a board with adequate height is placed into the bin so its plane rests above the milky layer at the bottom of the solution. The bamboo samples are then placed on the board for five days and allowed to sit, similar to the salt solution procedure.

Table 3-10. Physical changes due to lime treatment

\begin{tabular}{|c|c|c|c|c|c|c|}
\hline Specimen & $\begin{array}{c}\text { Weight } \\
\text { Before } \\
\text { (g) }\end{array}$ & $\begin{array}{c}\text { Weight } \\
\text { After } \\
\text { Treatment } \\
\text { (g) }\end{array}$ & $\begin{array}{c}\text { Moisture } \\
\text { Content } \\
\text { Loss }\end{array}$ & $\begin{array}{c}\text { Area } \\
\text { Before } \\
{\text { (in. }{ }^{2} \text { ) }}^{\text {a }}\end{array}$ & $\begin{array}{l}\text { Area } \\
\text { After } \\
\text { (in. }{ }^{2} \text { ) }\end{array}$ & Shrinkage \\
\hline 1 & 0.334 & 0.222 & $33.47 \%$ & 0.848 & 0.739 & $12.88 \%$ \\
\hline 2 & 0.342 & 0.227 & $33.72 \%$ & 0.851 & 0.744 & $12.62 \%$ \\
\hline 3 & 0.346 & 0.233 & $32.70 \%$ & 0.882 & 0.777 & $11.97 \%$ \\
\hline 4 & 0.306 & 0.201 & $34.39 \%$ & 0.916 & 0.764 & $16.6 \%$ \\
\hline 5 & 0.345 & 0.230 & $33.42 \%$ & 0.814 & 0.659 & $18.99 \%$ \\
\hline 6 & 0.340 & 0.227 & $33.29 \%$ & 0.842 & 0.693 & $17.67 \%$ \\
\hline 7 & 0.316 & 0.211 & $33.15 \%$ & 0.849 & 0.676 & $20.41 \%$ \\
\hline 8 & 0.214 & 0.140 & $34.7 \%$ & 0.846 & 0.684 & $19.36 \%$ \\
\hline 9 & 0.198 & 0.129 & $34.81 \%$ & 0.864 & 0.724 & $16.24 \%$ \\
\hline 10 & 0.176 & 0.117 & $33.33 \%$ & 1.030 & 0.907 & $12.07 \%$ \\
\hline 11 & 0.194 & 0.279 & $32.73 \%$ & 0.882 & 0.759 & $13.94 \%$ \\
\hline 12 & 0.374 & 0.250 & $33.14 \%$ & 0.873 & 0.735 & $15.72 \%$ \\
\hline 13 & 0.290 & 0.197 & $32.17 \%$ & 0.858 & 0.746 & $13.02 \%$ \\
\hline Average & & & $33.45 \%$ & & & $15.76 \%$ \\
\hline $\begin{array}{l}\text { Standard } \\
\text { Deviation }\end{array}$ & & & $0.78 \%$ & & & $2.97 \%$ \\
\hline Maximum & & & $34.81 \%$ & & & $20.41 \%$ \\
\hline Minimum & & & $32.17 \%$ & & & $11.97 \%$ \\
\hline
\end{tabular}

After the chemical treatment is complete, the samples are removed from the solution and prepared for thermal treatment. Again, the internode samples are wiped with paper towels to remove excess water and then carefully stacked on the elevators inside the autoclave to begin thermal treatment for the same $220^{\circ} \mathrm{F}$ curing cycle. Once thermal 
treatment is complete, the bamboos are inspected visually; this solution yields a slightly dull surface color compared to the salt-treated variety. The moisture content loss and shrinkage are measured after the thermal treatment is complete. A table is provided to outline the results of the hydrated lime treatment. The average moisture content loss and shrinkage are $33.45 \%$ and $15.76 \%$, respectively.

\subsubsection{Oil Treatment}

The final curing treatment investigated is an oil treatment. As a reminder, the purpose of the oil treatment is to observe its effect on the bamboo's mechanical properties by filling internal microscopic voids and providing a barrier to resist premature fracture caused by environmental elements. Preparation of the treatment simply requires pouring $3-\mathrm{L}$ of Kirkland Canola Oil into a bin. Bamboo samples are then placed at the bottom of the bin and allowed to soak for five

days. After soaking in the treatment for five days, the

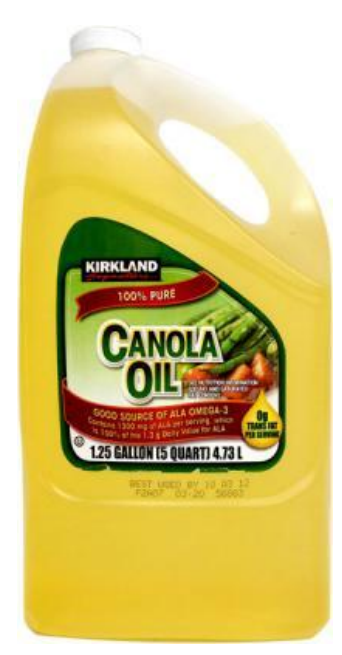

Figure 3-9. Oil utilized for oil treatment [28] internode samples are removed and wiped with towels to reduce the excess oil. Also, extensive precautions must be taken so that flammable materials are nowhere near the samples when placing them in the autoclave. Once the thermal treatment was complete, the bamboo internodes are removed from the autoclave and observed for visual changes. Upon inspection, the oil-treated bamboos displayed a darker shade of golden brown than in those treated with salt. Lastly, the table shown displays the values of moisture content loss and shrinkage for the treatment. These average values are respectively calculated to be $30.49 \%$ and $8.19 \%$. 
Table 3-11. Physical changes due to oil treatment

\begin{tabular}{|c|c|c|c|c|c|c|}
\hline Specimen & $\begin{array}{l}\text { Weight } \\
\text { Before } \\
\text { (g) }\end{array}$ & $\begin{array}{l}\text { Weight After } \\
\text { Treatment } \\
\text { (g) }\end{array}$ & $\begin{array}{c}\text { Moisture } \\
\text { Content } \\
\text { Loss }\end{array}$ & $\begin{array}{l}\text { Area } \\
\text { Before } \\
\text { (in. }^{2} \text { ) }\end{array}$ & $\begin{array}{l}\text { Area } \\
\text { After } \\
\left(\text { in. }{ }^{2}\right)\end{array}$ & Shrinkage \\
\hline 1 & 0.39116 & 0.27060 & $29.13 \%$ & 0.785 & 0.743 & $5.32 \%$ \\
\hline 2 & 0.32692 & 0.22792 & $30.28 \%$ & 0.743 & 0.654 & $12.01 \%$ \\
\hline 3 & 0.29788 & 0.20460 & $31.31 \%$ & 0.669 & 0.623 & $6.95 \%$ \\
\hline 4 & 0.28380 & 0.19800 & $30.23 \%$ & 0.636 & 0.584 & $8.06 \%$ \\
\hline 5 & 0.27104 & 0.19008 & $29.87 \%$ & 0.610 & 0.552 & $9.48 \%$ \\
\hline 6 & 0.25476 & 0.17600 & $30.92 \%$ & 0.576 & 0.531 & $7.84 \%$ \\
\hline 7 & 0.23760 & 0.16368 & $31.11 \%$ & 0.542 & 0.528 & $2.59 \%$ \\
\hline 8 & 0.30932 & 0.22220 & $28.17 \%$ & 0.720 & 0.642 & $10.81 \%$ \\
\hline 9 & 0.28116 & 0.20328 & $27.70 \%$ & 0.667 & 0.621 & $6.90 \%$ \\
\hline 10 & 0.26576 & 0.19096 & $28.15 \%$ & 0.647 & 0.604 & $6.75 \%$ \\
\hline 11 & 0.25740 & 0.18128 & $29.57 \%$ & 0.608 & 0.551 & $9.33 \%$ \\
\hline Average & & & $30.49 \%$ & & & $8.19 \%$ \\
\hline $\begin{array}{l}\text { Standard } \\
\text { Deviation }\end{array}$ & & & $1.26 \%$ & & & $2.60 \%$ \\
\hline Maximum & & & $31.31 \%$ & & & $12.01 \%$ \\
\hline Minimum & & & $27.70 \%$ & & & $2.59 \%$ \\
\hline
\end{tabular}

\subsubsection{Analysis of Natural Chemical Treatments}

A figure summarizing the mean moisture content loss and shrinkage values of all the chemically treated samples is presented for analysis. As a reminder, the purpose of this analysis is to determine predicted shrinkages for each curing temperature and to predict an optimum chemical treatment.

A decline in moisture content loss among the salt treatments is evident from the plot. The cause for the trend is simply due to the increasing amount of salt in the solution preventing greater moisture evaporation due to the added mass. The figure also indicates that the $3 \%$ and $6 \%$ salt treatment may yield the highest moisture content loss and, therefore, strength increase. However, the relatively high standard deviation does not allow a clear distinction between $3 \%$ and $6 \%$ salt treatments. The remaining treatments do indicate a clear difference between their moisture losses: lime yields higher losses than oil. Compared to oil, the lime treatment illustrates a higher average 
moisture loss of about 3\%. The higher moisture loss in lime is attributed to the oil's ability to shield the inner moisture from the heat treatment. On the other hand, the greater moisture loss seen in the salt treatments is due to the heat treatments having more of an adverse effect on the moisture loss. Another important point to realize is that including these natural chemicals into the overall treatment process increases the amount of moisture loss by roughly $5 \%$.

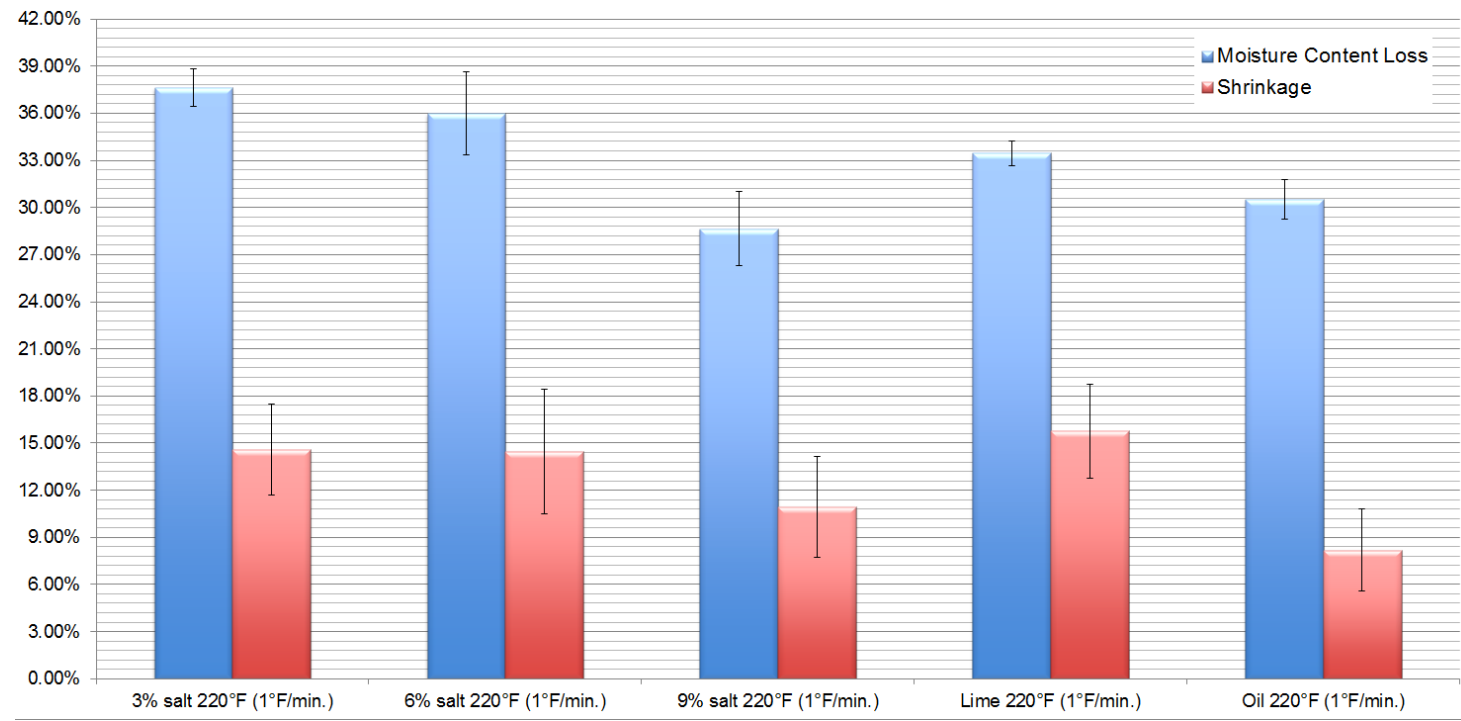

Figure 3-10. Analysis of physical changes for all organic chemical treatments

In terms of shrinkage, the lime indicates the highest value among the treatments. However, the deviation for all the samples is relatively high and make it difficult to clearly state that one chemical treatment offers less shrinkage than another. It can only be noted that the oil provides the least amount of shrinkage among the treatments-in addition to providing the least amount of moisture loss. At least it can be said that shrinkage percentages are expected to be greater than $10 \%$ for the majority of the chemical treatments, which is slightly higher than the group with only thermal treatment. 
By merely looking at the numbers, it appears the $3 \%$ or $6 \%$ salt treatment is likely to yield the best mechanical properties due to its greater moisture content loss. Testing each sample for its mechanical properties will ultimately provide the truth about the treatments' effectiveness. Also, shrinkage at this temperature is relatively the same to the $220^{\circ} \mathrm{F}$ thermal treatment without organic chemical enhancement. Therefore, the same design principle of accounting for a $10 \%$ reduction in area is advised when curing bamboos for structural application. 


\section{Chapter 4. Bamboo Mechanical Characteristics}

Understanding the mechanical characteristics of this particular species of bamboo is vital to gain insight on the material's potential behavior during application. This research aims to investigate the resulting mechanical properties of bamboo under the thermal and chemical treatments outlined previously. It is important to establish a precise database for these characteristics if plans are implemented to investigate bamboos as a serious building material. Aside from obtaining the stresses and strains under various loading conditions, other classic material properties are investigated. These material properties include the: elastic modulus, yielding conditions, and ultimate strength. Additionally, two other variables-specific modulus and specific strength-are incorporated into the previous set of material characteristics to construct a base for comparison among all the specimens. It is impossible to produce two specimens that behave exactly the same under loading due to the inconsistencies between bamboo poles. Tests on several specimens proved that they may all behave similarly but no two perform the same-

much like all things in nature. As a result, the specific modulus and specific strength are included to properly compare the data at an unbiased level across all treatments. A final tabulation summarizing all the bamboo's mechanical properties is provided at the end of this section.

The first of the main characteristics studied is the elastic modulus. An important variable in the study of structural mechanics, the modulus measures the stiffness of a material under linear-elastic deformation. In classic structural mechanics, the elastic modulus is defined as,

$$
E=\frac{d \sigma}{d \varepsilon}
$$


Where $E$ is the elastic modulus, $\sigma$ is the stress, and $\varepsilon$ is the strain from the stress-strain curve. The elastic modulus in this research is calculated by fitting a line to the linear elastic region of the stress-strain curve. The slope of this fitted line then becomes the elastic modulus. All curve-fits to calculate the modulus yielded an average $R^{2}$ value of at least 0.999 . The equation for this fitted line is,

$$
\sigma(\varepsilon)=E \varepsilon+b
$$

Where $\sigma$ is stress as a function of strain, $\varepsilon$ is strain, $E$ is the slope (elastic modulus), and $b$ is the $y$-intercept of the line. A MATLAB code that performs these tasks is created to determine the modulus and is provided in the appendix.

Second, the specific modulus is of vital importance to properly compare the stiffness between specimens. The specific modulus is simply the stiffness of a material per unit mass density. This variable is a quantifiable measurement that allows fair comparison between materials of varying sizes and mass. It is defined as,

$$
\xi=\frac{E}{\rho}
$$

Where $\xi$ is the specific modulus, $E$ is the elastic modulus, and $\rho$ is the density of the specimen material.

Yield conditions—-that is, yield stress and strain-are determined by the threshold-slope method. In essence, this method takes the yield point to be the location where the slope of the stress-strain curve falls below some set percentage of the modulus in the linearelastic region. The slope-threshold method is flexible in that it can determine a yield point for any specified fraction of the modulus. For example, if the proportionality limit is desired, a percentage value of $100 \%$ will determine the stress at which the slope falls below the linear portion of the curve. In contrast, a $10 \%$ set percentage value can 
calculate a yield point that occurs when the slope nears an inflection point on the curve; in other words, the point on the stress-strain curve where it begins to level off before plastic deformation. Mathematically speaking, the yield point is defined as,

$$
\left(\sigma_{y}, \varepsilon_{y}\right)=\lim _{\varepsilon \rightarrow 0} \frac{d \sigma}{d \varepsilon} \leq P
$$

Where $\sigma_{y}$ and $\varepsilon_{y}$ are defined as the yield stress and strain point on the stress-strain curve, $P$ is the set percentage value, and $\frac{d \sigma}{d \varepsilon}$ is the first derivative of the curve. For all tests conducted in this section, a value for $P$ of $90 \%$ was selected to determine a yield point slightly above the proportionality limit. A MATLAB code created to perform this function is also supplied in the appendix.

The strength of the material is simply determined as the maximum stress on the stressstrain curve. Therefore, the maximum stress is

$$
\sigma_{u l t}=\max \left(\sigma_{i}\right)
$$

Where $\sigma_{u l t}$ is the ultimate stress, and $\sigma_{i}$ is the indexed stress at a point along the stressstrain curve. A MATLAB code is also created to determine the strength of each specimen and is located in the appendix as another reference. The final mechanical characteristic of interest is the specific strength, simply defined as,

$$
\varsigma=\frac{\sigma_{u l t}}{\rho}
$$

Where $S$ is specific strength, $\sigma_{u l t}$ is ultimate stress, and $\rho$ is density. As mentioned before, the specific strength of the material is an important mechanical characteristic to properly analyze the strengths of each geometrically varying specimen.

The two methods utilized to investigate these mechanical properties included tests under compression and tension. All testing experiments performed in this research are done on 
the Instron 8801 machine at the Aero Structures/Composites lab as seen in Figure 4-1. Manufacturing procedures are also supplied for all testing experiments done in this report. It is quickly realized that the specimen's manufacturing procedure for each respective test is distinct. Specimens for these tests are fabricated accordingly with a tight tolerance outlined to the original testing plans. Introducing more variability in the experiments would only generate disastrously inconclusive data; consequently, these testing specimens are the product of diligent fabrication. All manufacturing processes performed at this level are carried out by the author, and great care is taken to conduct standardized testing in all possible cases.

Unlike most material testing procedures, standardized tests for bamboo are scarce. Only a handful of standards are known to exist to the current knowledge of the author. Intuition and insightful structural experience from advisors was relied on in the gray areas not specified in the

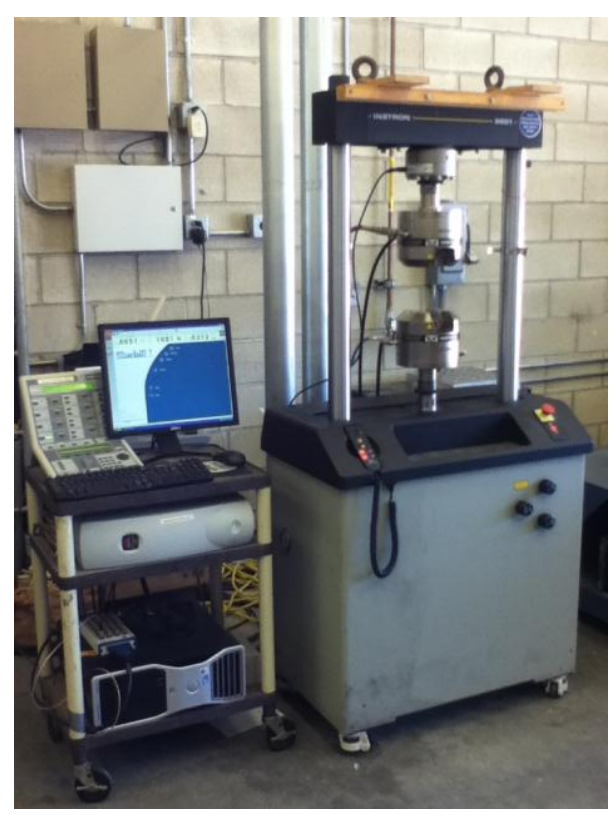

Figure 4-1. Instron 8801 machine to perform all tests

testing standards. The standard employed is ISO standard 22157 as provided by the ISO-also known as the International Organization for Standardization [44]. The full name of the standard selected to properly investigate the mechanical characteristics is Bamboo-Determination of physical and mechanical properties.

\subsection{Compression Mechanical Characteristics}

As outlined in the compression section of the ISO standard, each bamboo specimen was tested using internodes only. The standard specifies, "There is no significant difference 
between test results on compression on nodes and internodes." However, validation of this statement is recognized with additional testing, and the results confirming the claim are found in the appendix. The standard additionally requires each specimen to have a length equal to its diameter. The end planes of the specimen must also be flat, with a deviation less than $0.2-\mathrm{mm}$, and at perfectly right angles to the length along the fiber direction. The standard also requires a minimum of 12 samples to determine the mechanical characteristics of the bamboos. Furthermore, a loading rate of $0.024-\mathrm{in} . / \mathrm{min}$. is utilized during the compression test. Last, a proper method must be devised during compression testing in order to prevent any severe friction or slipping effects.

These aforementioned instructions constitute the only guidelines for the compression test. Specifically, the issue of what the tolerance is for the equal diameter-length guideline is not addressed. Consequently, that tolerance is left to the author to be 0.1 -in.

\subsubsection{Preparing Compression Specimens}

Each compression specimen was carefully prepared at the Cal Poly SLO Aerospace Structures/Composites Laboratory according to the geometric dimensions specified in the standard. A bamboo internode is first selected and carefully inspected for any defects along its length. Defects in this sense are defined as cracks or irregular cavities on the outer surface of the culm. These features would produce inconclusive data, so they are either cut off if the internode can be salvaged or simply discarded from the experiment. After inspection, the internode's maximum and minimum diameters are measured at the ends-resulting in four diameter measurements. (Bamboo culms usually grow thinner in diameter as the pole reaches higher heights, so measurement of the center of the internode is not necessary). These measurements are then averaged to determine the overall average diameter of the internode. The overall average diameter is subsequently used to mark sections along the internode's length. Figure 4-2 illustrates 
exemplary dimensions of a compression specimen; the average diameter of the culm ultimately determines the height of the specimen.

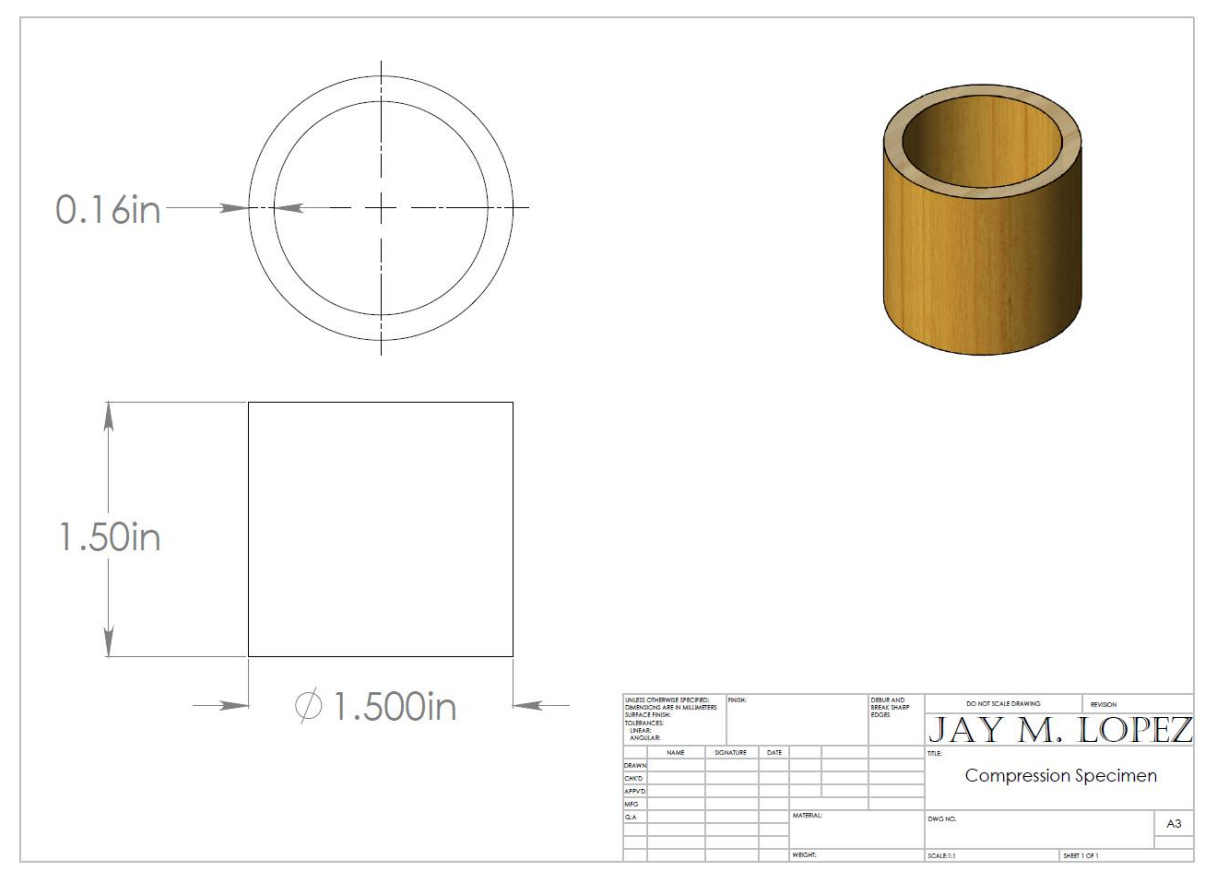

Figure 4-2. Manufacturing drawing for compression specimen with exemplary dimensions

Once marked, the internode is cut with a diamond-tile saw at the marked locations as shown in Figure 4-3. These cuts are then inspected to be perpendicular to their lengths and fiber direction of the culm. After the internode is cut into several specimens of roughly equal length and diameter, the end planes of each specimen are meticulously sanded with a belt sander to make them perpendicular to its lengths as seen in the figure. A leveler is employed to validate that the end planes are perpendicular. A final inspection of each specimen is conducted and any imperfections caused by the belt sander are sanded by hand with fine grade sand paper. The procedure is then repeated for additional bamboo internodes. 

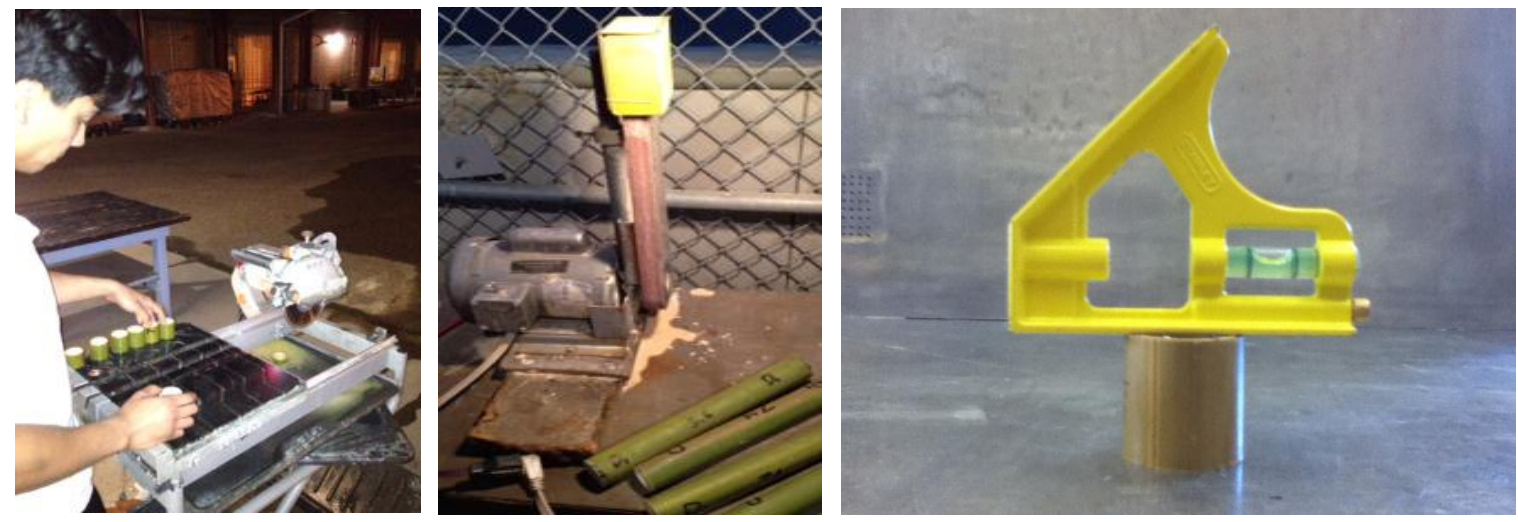

Figure 4-3. Cutting compressions specimens to length. sanding them accordingly, and leveling them

Once all the specimens are prepared, tables of the dimensions prior to testing are investigated to help account for any anomalies that might later be found in the plots. The average height, outer diameter, and wall thickness of each sample was measured with an electronic caliper to \pm 0.001 -in. accuracy. The mean of four height measurements is taken as the average height; likewise, the mean of four outer diameter measurements is taken as the average diameter. On the other hand, the mean of eight thickness measurements-four from each end-face-is determined to be the average thickness. The weight of each specimen was measured on an electronic scale to within \pm 0.0005 $\mathrm{lb}_{\mathrm{m}}$. The average area of each specimen was calculated using the equation,

$$
A_{c}=\frac{\pi}{4}\left(D_{\text {avg }}^{2}-\left(D_{\text {avg }}-2 t_{\text {avg }}\right)^{2}\right)
$$

Where $A_{c}$ is the average cross-sectional area of the compression specimen, $D_{\text {avg }}$ is the average outer diameter, and $t_{\text {avg }}$ is the average wall thickness. Subsequently, the average volume of each specimen is calculated by,

$$
V_{c}=A_{c} h_{a v g}
$$

Where $V$ is the corresponding average volume and $h_{\text {avg }}$ is the average height. Lastly, the density of each specimen is determined using the following equation: 


$$
\rho_{c}=\frac{m}{V_{c}}
$$

Where $\rho$ is density, $m$ is the mass of the specimen, and $V_{c}$ is the volume of the entire sample. Testing could then ensue after defining these physical properties.

\subsubsection{Compressive Testing Procedure}

As mentioned previously, all compression testing is performed with the Instron 8801 testing machine in the Aero Structures/Composites lab. All data for the tests are acquired with a data acquisition computer, and the Instron is controlled with its own Bluehill 2 software. The compression testing procedure begins by configuring special compression fixtures to the Instron machine. A lack of instruction from the ISO standard exists for how to address the bearing stress created between the contact points created from the bamboo top face and the compression plate from the testing apparatus. Preliminary testing demonstrated that adding a buffer in the form of a composite fiberglass sheet between the bamboo specimen and the compression plate produced less bearing stress on the specimen. A leveler is then used once again to ensure the plates are parallel to the horizontal plane.

Once the specimen group is ready, the compression specimen is loaded into the testing area of the Instron machine with the appropriate fixtures in place. Figure 4-4 illustrates the setup of the test. The ISO standard dictates the specimen must be set in the center of the compression plates and pre-loaded only to create contact between the specimen end plane and compression plate. All compression specimens were pre-loaded to within $10-\mathrm{lb}_{\mathrm{f}}$ to ensure contact between the specimen and plate, as shown in the figure. $A$ bamboo compression program, specially created with the Instron Bluehill 2 controller software, is loaded onto the computer and the compression rate is defined to be $0.024-$ in./min. as recommended by the standard. This testing procedure measures the 
compressive shortening and load applied to the specimen from the crossheads of the Instron. Once the specimen is pre-loaded correctly, the test initiates and concludes at failure when a $40 \%$ drop in load is observed. The sample is then removed, the testing area cleaned, and the procedure is repeated for the remaining specimens.
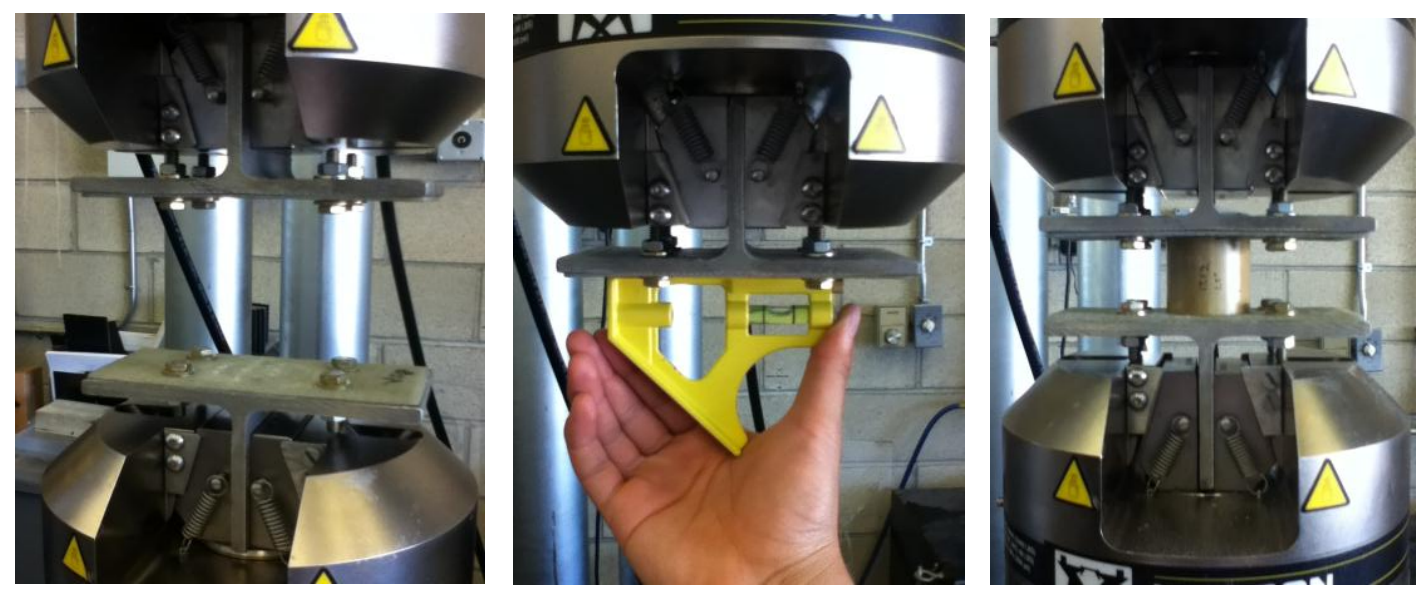

Figure 4-4. Compression test fixtures with fiberglass buffers (left), subsequent leveling (center), and preloading specimen into test area

\subsubsection{Results of Compression Tests}

The compressive material properties of this bamboo for various thermal and chemical treatments are investigated to help determine the optimum treatment combination. First, green bamboo without treatment is studied to form a baseline for the mentioned treatments. Thermal treatments of $150^{\circ} \mathrm{F}$ through $220^{\circ} \mathrm{F}$ at two initial ramp-up cure rates are investigated. Also, chemical treatments studied include the varying salt solutions, lime solution, and oil treatment. The outcomes of the compression tests are gathered into the seven mentioned categories to illustrate the mechanical properties of the bamboo. One of the major difficulties associated with this research is the variability among all the samples due to nature's original manufacturing, so a large sample size is strived for in all testing. Tables displaying all the specimens' geometries are first provided in the appendix to illustrate any variations that may be pertinent to the tests. A 
plot of the raw data from the test, i.e. the compressive force-shortening plot, is also supplied in the appendix. Aside from the aforementioned compression mechanical characteristics of importance, each treatment group includes its stress-strain plot. In addition to this plot, an array of photographs is shown to display the types of failure for each treatment group under compression. Finally, a table summarizing all the specimens' mechanical characteristics for each treatment group is provided in the appendix.

The proper analytic equations are required to study the mechanical behavior of bamboos under compression. The following describes the manner in which the mechanical properties are calculated. Before any mechanical properties can be calculated, it is vital to establish the definition of compressive stress and strain in this study. Compressive stress is defined as,

$$
\sigma_{c}=\frac{F_{c}}{A_{c}}
$$

Where $F_{c}$ is the compressive force and $A_{c}$ is the cross-sectional area computed from the average dimensions of the specimen. Additionally, compressive strain is defined as,

$$
\varepsilon_{c}=\frac{\Delta}{h_{a v g}}
$$

Where $\varepsilon_{C}$ is the compressive strain, $\Delta$ is the shortening displacement that compresses the specimen, and $h_{a v g}$ is the average height of the specimen. Once these central variables are identified, the proper equations for the main mechanical properties can be employed. 


\subsubsection{Untreated Bamboo Compression Results}

The green bamboo group serves as the baseline case from which to compare the results of the different treatments. In this way, any improvements in its mechanical properties can be noted and optimized for structural application.

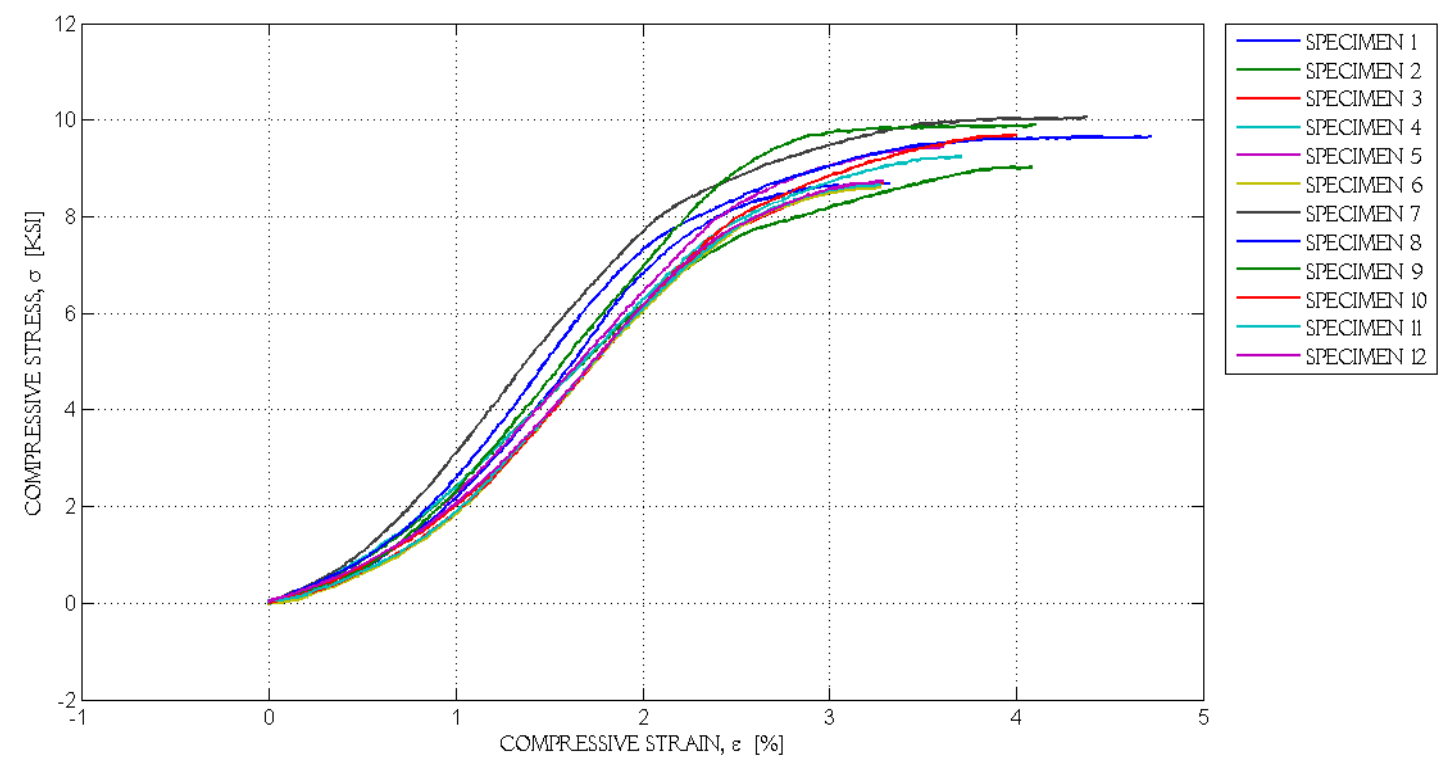

Figure 4-5. Untreated bamboo compressive stress-strain curves

Figure 4-5 displays the stress-strain curve of the green bamboo group. These curves cluster uniformly and lend confidence that the group yields reliable data for a natural material. The variation seen among the specimens is due to their natural biological differences. One thing to notice about the plot is the nonlinear portion of the curve at the very beginning of the test. This feature is also apparent in the force-shortening curve. A plausible reason for this may be from sanding the end faces of the specimen during manufacturing. Since contact between the compression plate and specimen top-end face was established by pre-loading the specimen to within a few pounds, extremely small imperfections at the end faces may have created a circumference of unleveled height measurements. Figure 4-6 shows a diagram with exaggerated imperfections of 
the end faces to illustrate the stress concentrations created at contact. Compressions at these locations cause the observed high strain-small stress region at the beginning of the curve. It is incredibly difficult to correct these inaccuracies during specimen fabrication although great care was taken to ensure the end planes were level before pre-loading the specimen.

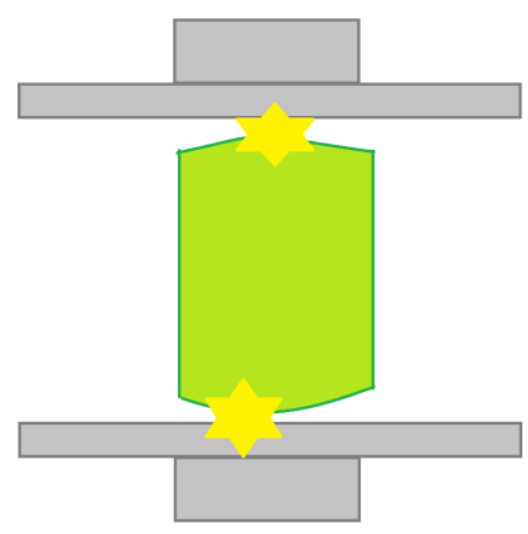

Figure 4-6. Exaggerated view of imperfect end faces on compression specimen

The linear elastic and nonlinear plastic regions of the curves are clearly evident from the plot. Immediately following the small section at the beginning of the curve is the linear elastic regionwhich compromises about half of the stress-strain curve. All the curves visually display a similar slope in this portion of the curve. The end of the elastic region appears to be at around $7-\mathrm{ksi}$; a corresponding strain of $2.0 \%$ is evident. As noted from the plot, the variation picks up again when entering the nonlinear portion of the curve. Several unseen factors attributed to the natural character of the bamboo may explain the variation in the plastic region of the stress-strain curve. However, for designs based on yielding criteria, one would steer clear from this portion of the curve, so less emphasis is placed on this area of the plot under those considerations.

Two failure types are apparent from the green bamboo compression test. Photographs of the failure types are shown in Figure 4-7. One of the failures observed is a complete fracture running all the way down the height of the specimen. This fracture is sometimes accompanied by others but is usually concentrated on one side of the sample's circumference-very much like the shown figure. Such an effect may be the final blatant clue that the end faces were not truly perpendicular to the height-possibly off by a 
minute degree. The other failure mode detected was due to bearing stress, as one can see that the bamboo material compresses within itself. This caused large stresses on the end planes causing the bamboo material to fail. Lastly, these failure modes are also apparent in the remaining compression specimens for every treatment.
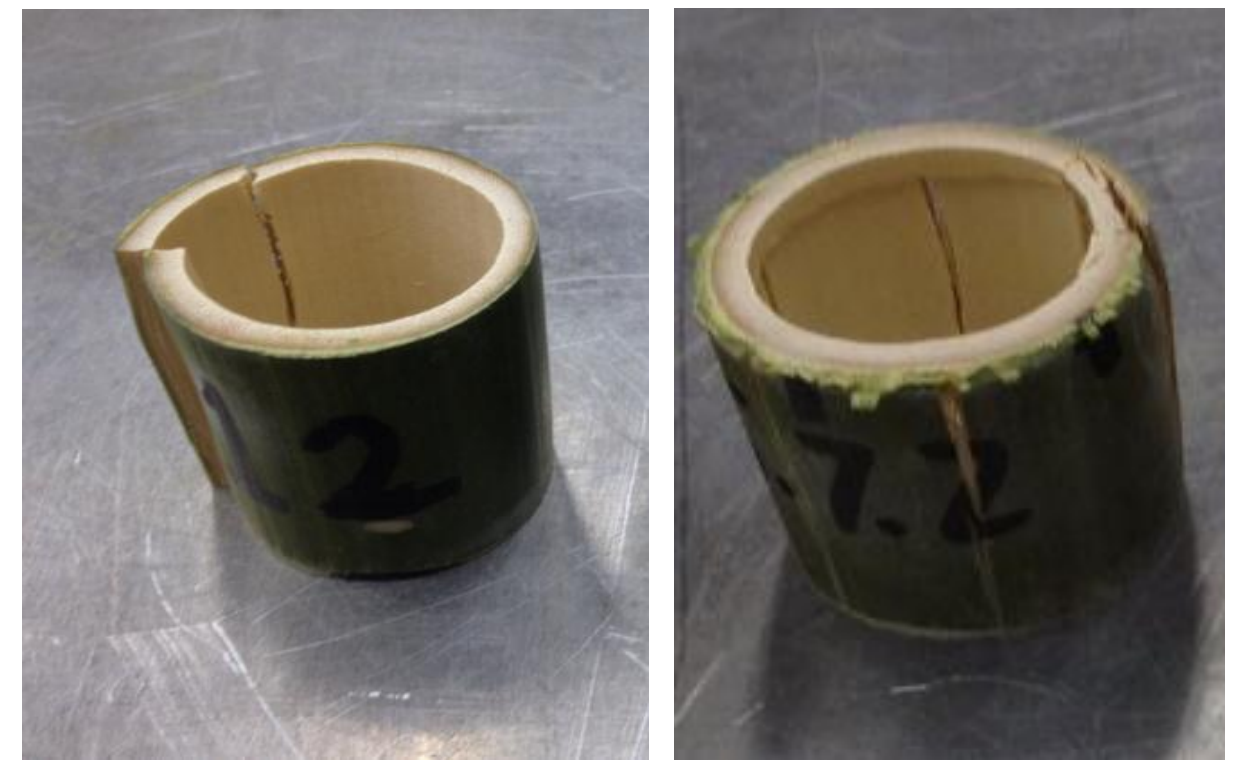

Figure 4-7. Common compressive failure types: longitudinal splitting (left) and bearing stress deformation (right)

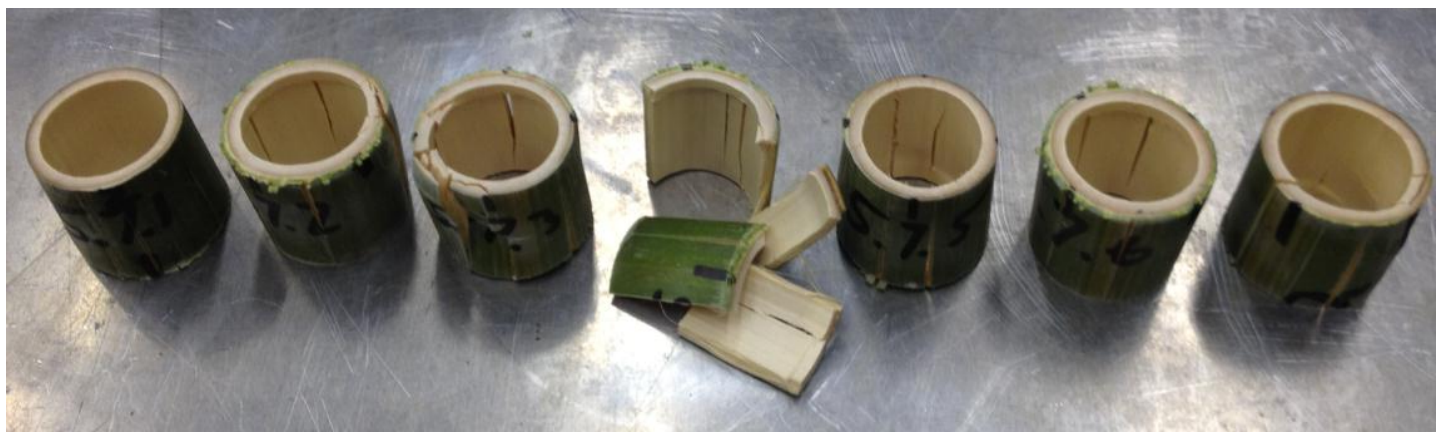

Figure 4-8. Tested untreated compression specimens

In summary of the untreated bamboo compression test, a table outlining the seven main mechanical characteristics is shown in the appendix. For green bamboo of this variety, a specific strength of 223-kips-in./lb $\mathrm{b}_{\mathrm{m}}$ and ultimate stress of $9.34-\mathrm{ksi}$ is observed. Also, a 
compressive elastic modulus of $442-\mathrm{ksi}$ and specific modulus of $10543-\mathrm{kips}-\mathrm{in} . / \mathrm{lb}_{\mathrm{m}}$ is measured. Statistical analysis of this table exemplifies consistent data considering the natural characteristic of the material. Most of the specimens show a deviation from the average of about $8 \%$; the modulus, yield and ultimate strain were the two properties to have a deviation of $10 \%$.

\subsubsection{Thermal Treatment Compression Results}

The first aim in this portion of the research is to determine the optimum heat treatment to cure the bamboo for increased compressive mechanical performance. As mentioned previously, it is known from farmers of foreign lands that smoking or roasting the bamboo over an open fire can improve bamboo performance. After all, these farmers used these methods to construct entire houses and provide scaffolding for other building projects in their communities [7]. A scientific approach, however, as to how the mechanical performance is affected remains obscure. The first set of investigative treatments to improve the mechanical characteristics of bamboo is the heat-treated group. This group is comprised of heat treatments ranging from $150^{\circ} \mathrm{F}, 180^{\circ} \mathrm{F}, 200^{\circ} \mathrm{F}$, and $220^{\circ} \mathrm{F}$ - with varying heat rates. As a result, the treatment temperatures previously mentioned are investigated from a standpoint of the bamboo's mechanical characteristics.

\subsubsection{1 $150^{\circ} \mathrm{F}$ at $5^{\circ} \mathrm{F} / \mathrm{min}$ Thermal Treatment Results}

First among the heat treatment group is the specimens treated with $150^{\circ} \mathrm{F}$ at a $5^{\circ} \mathrm{F} / \mathrm{min}$. rate. Figure 4-10 displays the resulting stress-strain curve for the group. The initial nonlinear portion is again evident in the curve and the linear portion appears to be consistent among all specimens. The end of the linear portion of the curve occurs around a compressive strain and stress of $2.08 \%$ and $6.50-\mathrm{ksi}$, respectively. In addition, 
the plastic region of the curves also appear to follow a similar slope, although not as closely as the linear region, for most of the samples.

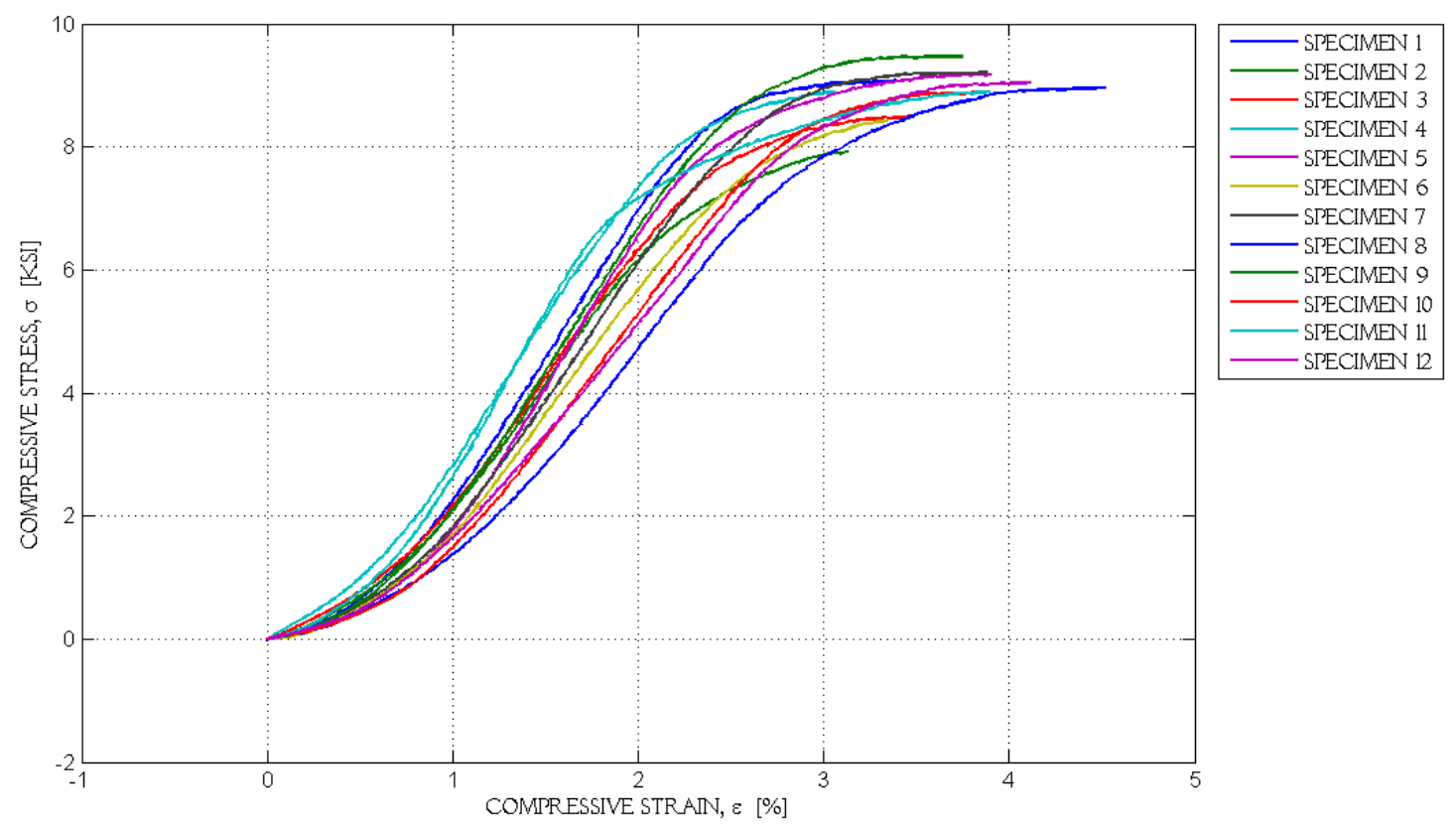

Figure $4-10.150^{\circ} \mathrm{F}$ at $5^{\circ} \mathrm{F} / \mathrm{min}$. thermal treatment compressive stress-strain curves

In terms of the failure types observed from the test, many of the specimens exhibit clean fractures running the entire height. Figure 4-9 shows this notable failure. Very few of the specimens show any
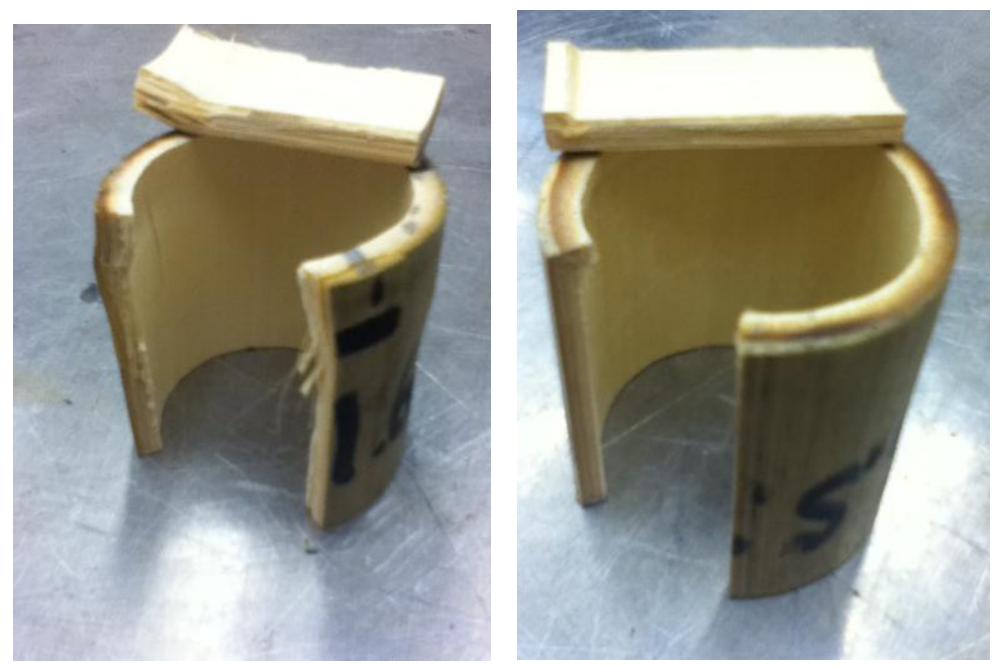
specimens show any

Figure 4-9. Common compressive failure types for $150^{\circ} \mathrm{F}$ at $5^{\circ} \mathrm{F} / \mathrm{min}$. treatment failures due to bearing stress. Most specimens show a single clean fracture from the top to bottom faces of the specimens. In some cases, fractions of the culm wall separate 
from the rest of the specimen. It can be seen that the major failure is due to material failure and often in a segment that breaks away from the rest of the culm.

In summary of the bamboo treated at $150^{\circ} \mathrm{F}$, a table is shown in the appendix displaying the four main mechanical properties. All of the equations mentioned previously are used to determine these properties. At $150^{\circ} \mathrm{F}$, an average specific strength of $239-\mathrm{kips}-\mathrm{in} . / \mathrm{lb}_{\mathrm{m}}$ and ultimate stress of $8.87-\mathrm{ksi}$ is observed. Also, a compressive elastic modulus of 446 ksi and specific modulus of $12033-\mathrm{kips}-\mathrm{in} . / \mathrm{lb}_{\mathrm{m}}$ is expected from this cure. In this group, the modulus and strains deviate around $10 \%$ from the average.

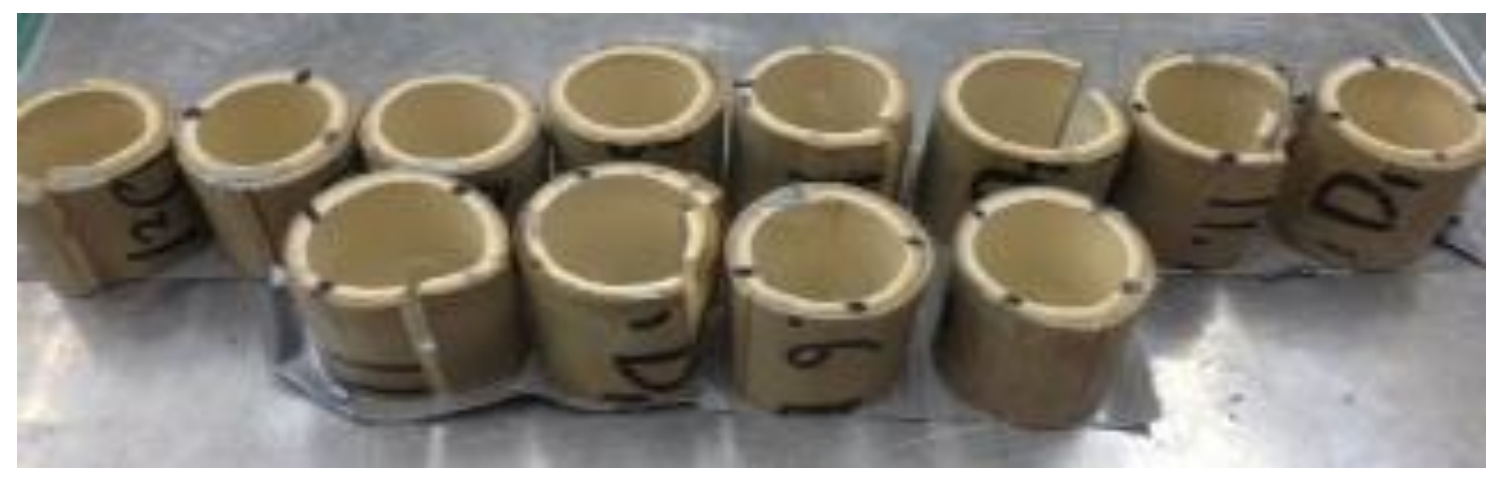

Figure 4-11. Tested $150^{\circ} \mathrm{F}$ at $5^{\circ} \mathrm{F} / \mathrm{min}$. rate compression specimens

\subsubsection{2 $180^{\circ} \mathrm{F}$ at $5^{\circ} \mathrm{F} / \mathrm{min}$ Thermal Treatment Results}

Secondly tested among the heat treatment group is the $180^{\circ} \mathrm{F}$ at $5^{\circ} \mathrm{F} / \mathrm{min}$. set. Figure 4-12 displays the resulting stress-strain curve for the group. The initial nonlinear portion is again evident in the curve and the linear portion appears to be consistent among all specimens. The end of the linear portion of the curve occurs around a compressive strain less than $2.10 \%$ while the compressive stress is about $7.76-\mathrm{ksi}$, respectively. The linear elastic region of most all samples follows a fairly similar path-with the exception of one specimen in cyan coloring. Looking at the samples' geometry leads to a conclusion that perhaps the reason for this anomaly is rooted in some irregularity not 
related to the specimen's geometry. Namely, it is conceivable that a variation in the biological composition of this specimen exists, or an error like slipping in the grips may have occurred during testing. The latter, however, is rather unlikely. The plastic region of these curves also appear to follow a similar slope for most of the samples-although not as closely as the linear region.

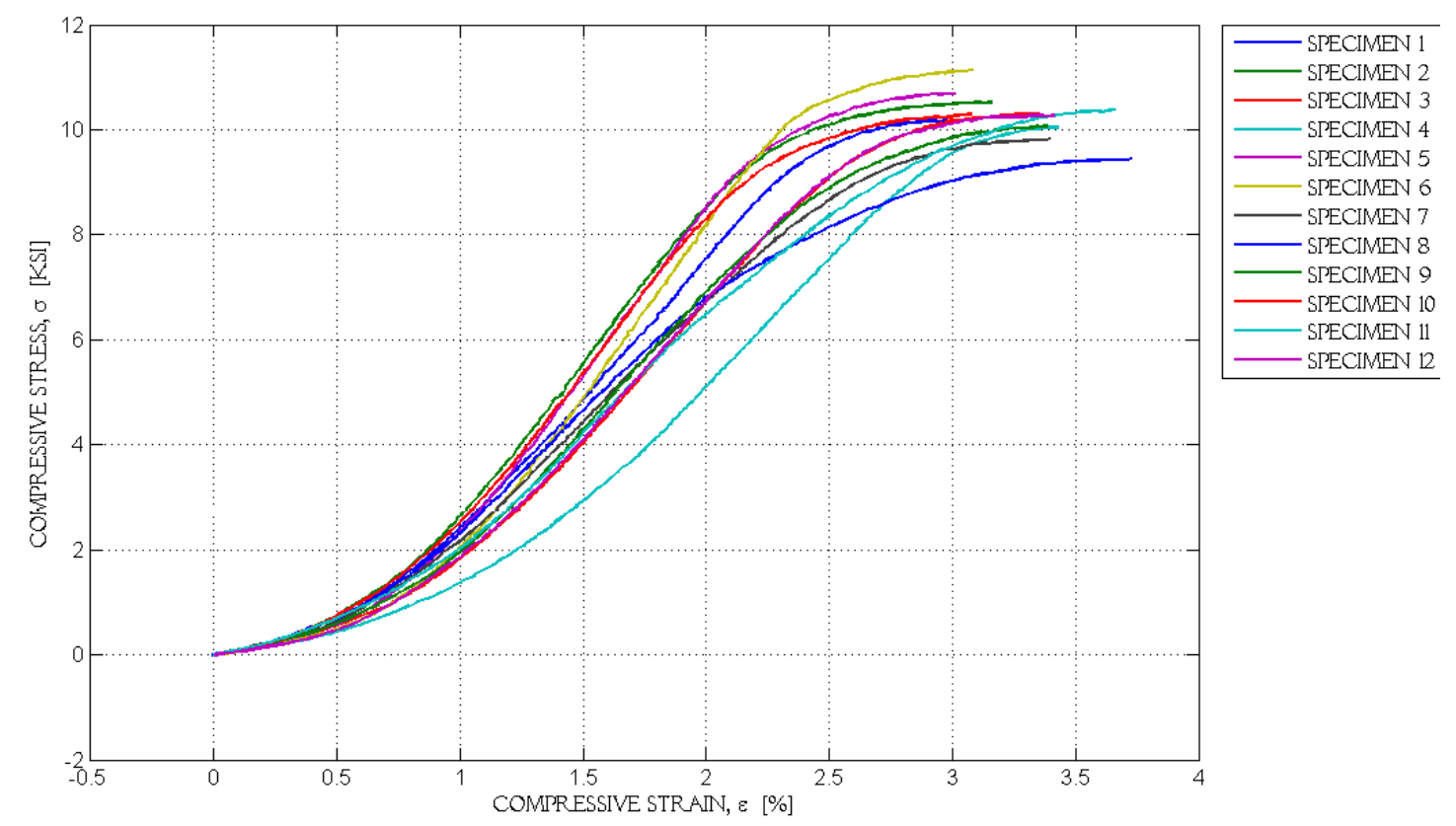

Figure 4-12. $180^{\circ} \mathrm{F}$ at $5^{\circ} \mathrm{F} / \mathrm{min}$. thermal treatment compressive stress-strain curves

The failure types seen from this treatment depict either splitting or local buckling. Local buckling in this sense is defined as the outer layers of the culm separating from the inner portion of the wall to
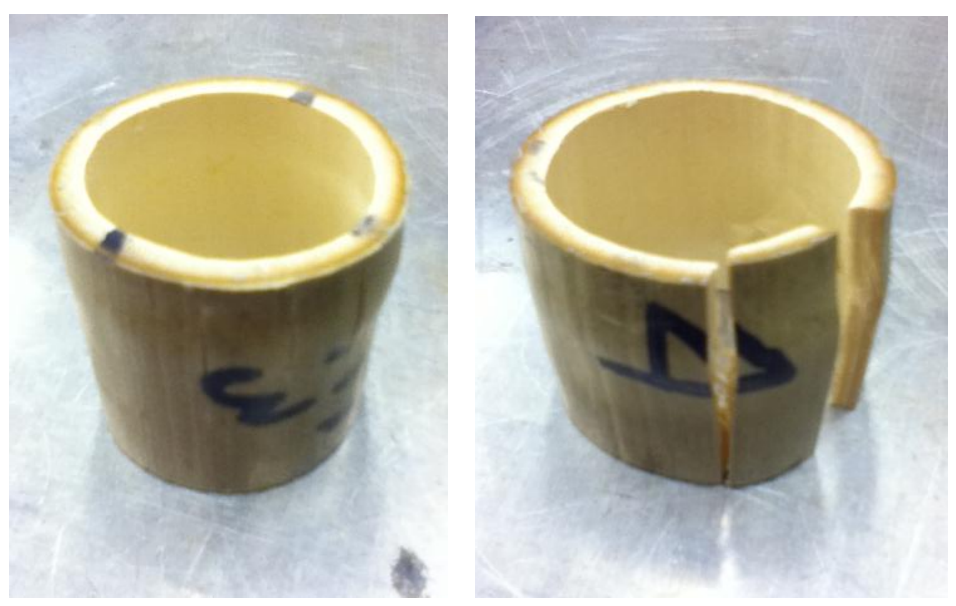

Figure 4-13. Common compressive failure types for $180^{\circ} \mathrm{F}$ at $5^{\circ} \mathrm{F} / \mathrm{min}$. 
protrude from the longitudinal axis in a buckled fashion. As seen from the figure below, the location of the buckling region ranges from the center to a location near the face. Again, a portion of the specimen often breaks away from the major part of the culm.

In summary of the bamboo treated at $180^{\circ} \mathrm{F}$, a table is shown in the appendix displaying the four main mechanical properties. All of the equations mentioned previously are used to determine these properties. In this group, the average specific strength is 286-kips-in./lb $\mathrm{b}_{\mathrm{m}}$ and the ultimate stress is 10.3-ksi. Also, the compressive modulus is $544-\mathrm{ksi}$ and specific modulus is 15135 kips-in./lb $\mathrm{b}_{\mathrm{m}}$. The modulus deviates the most from the average at approximately $14 \%$ while the remaining properties vary

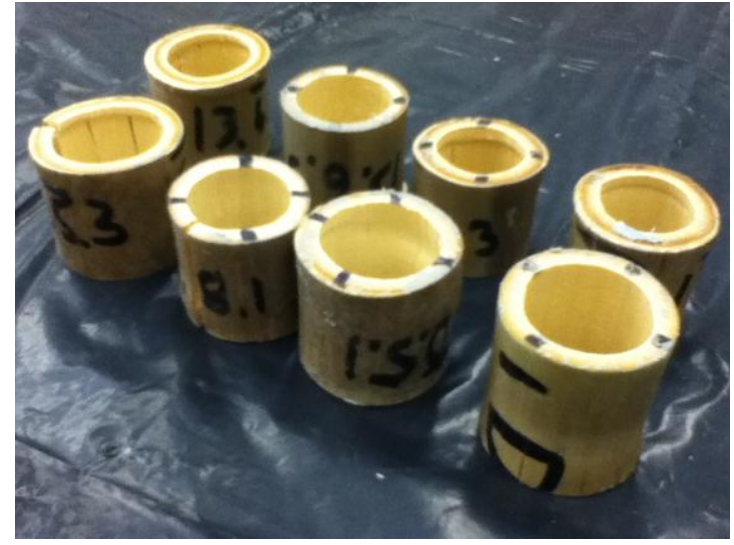

Figure 4-14. Portion of tested $180^{\circ} \mathrm{F}$ at $5^{\circ} \mathrm{F} / \mathrm{min}$. rate compression specimens by about $5 \%$.

\subsubsection{3 $180^{\circ} \mathrm{F}$ at $1^{\circ} \mathrm{F} / \mathrm{min}$ Thermal Treatment Results}

Third among the heat treatment group is the $180^{\circ} \mathrm{F}$ at $1^{\circ} \mathrm{F} / \mathrm{min}$ set. Figure $4-15$ displays the resulting stress-strain curve for the group heat treated at $180^{\circ} \mathrm{F}$ with a $1^{\circ} \mathrm{F} / \mathrm{min}$ initial rate. As seen from the figure, the trend of this plot is more consistent than the raw data supplied in the appendix. Analyzing the physical data yields slightly higher deviations across the different dimensions. The density of this sample group has a deviation relative to the average to be only $4 \%$; the area also shares the same deviation for this treatment set. The remaining dimensions fall within a $4 \%$ to $9 \%$ deviation range relative to the average. In spite of this, the true measure of precision will be the resulting mechanical properties obtained from the test. The initial nonlinear portion is again evident in the curve and the linear portion appears to be consistent among all 
specimens. The end of the linear portion of the curve occurs around a compressive strain less than $2.31 \%$ while the compressive stress is about $7.62-\mathrm{ksi}$, respectively.

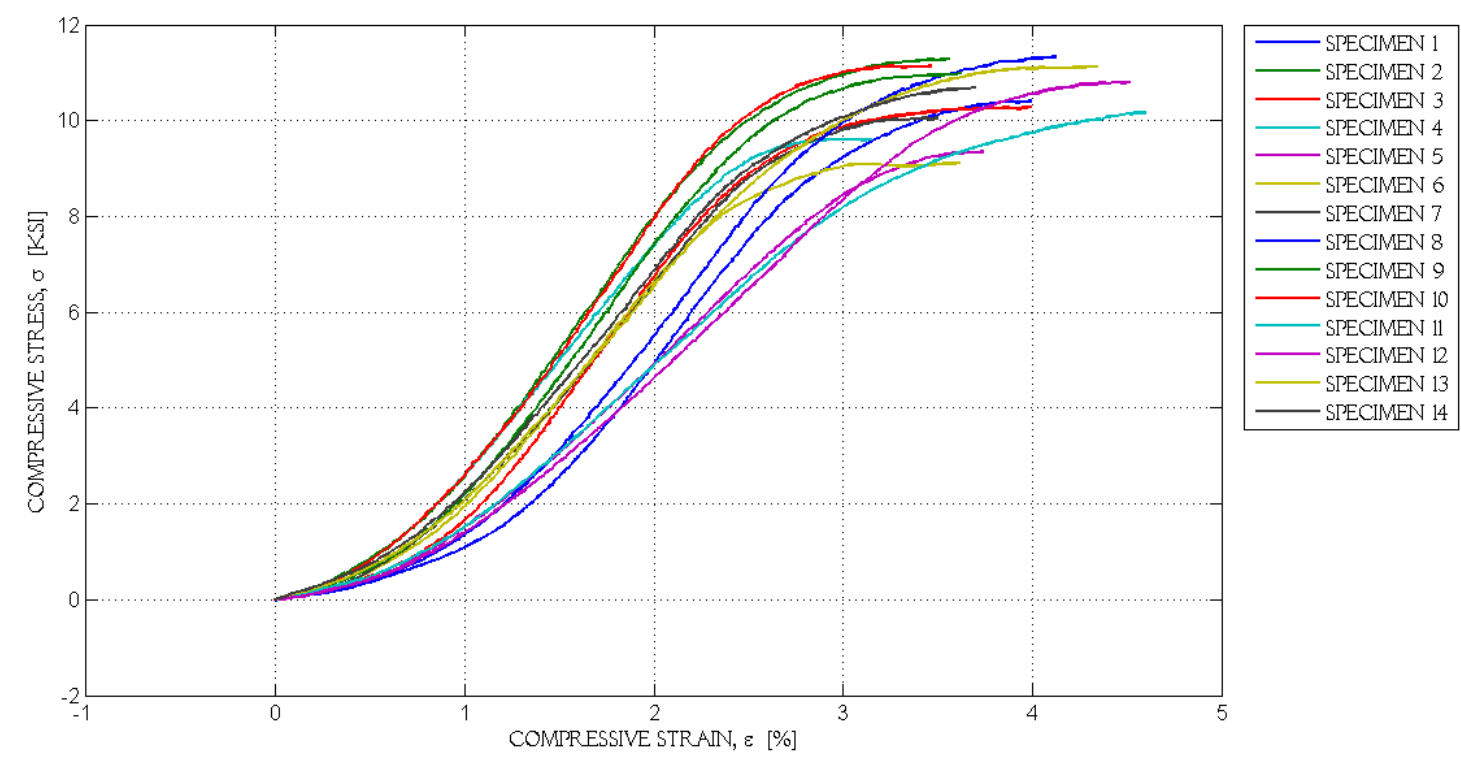

Figure $4-15.180^{\circ} \mathrm{F}$ at $1^{\circ} \mathrm{F} / \mathrm{min}$. thermal treatment compressive stress-strain curves

The linear elastic region of most all samples follows a similar slope; the plastic region, however, varies considerably. One point to bear in mind for this group is that the stressstrain curves remained fairly consistent for the most part although the manufacturing deviation increased to $7 \%$.

The two prominent failure types observed in this test are shown in Figure 4-16. Splitting and bearing stress failures are observed. The majority of the specimens, however, exhibit minor crack propagations. Cracks that
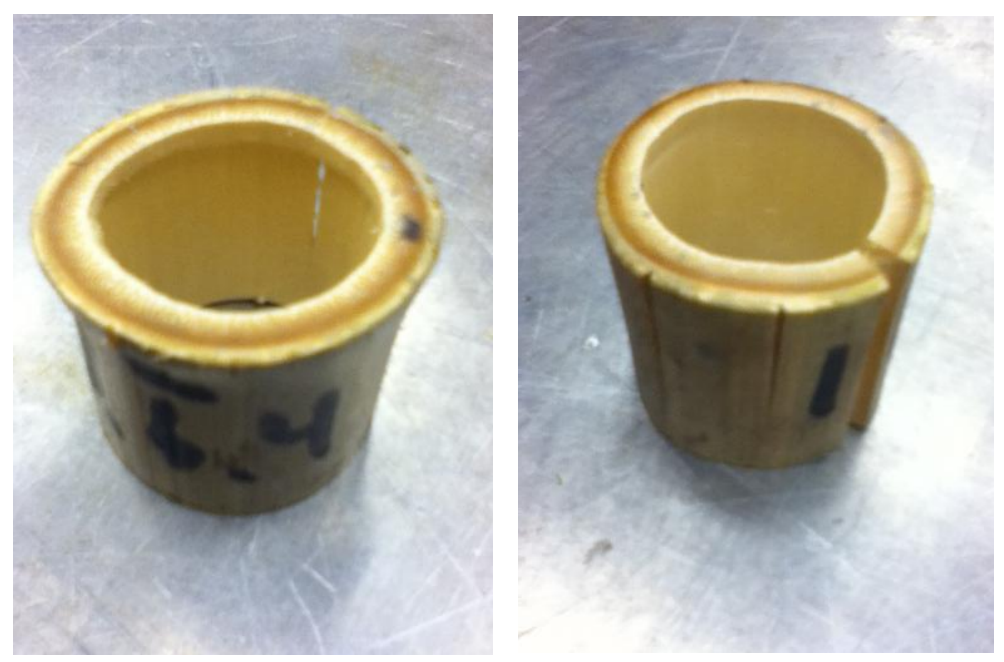

Figure 4-16. Common compressive failure types for $180^{\circ} \mathrm{F}$ at $1^{\circ} \mathrm{F} / \mathrm{min}$. treatment 
do form as a result of the compression test are really small and may be classified as slit fractures. Failure types illustrated with a split demonstrate a fracture running the entire height of the specimen with a minor opening.

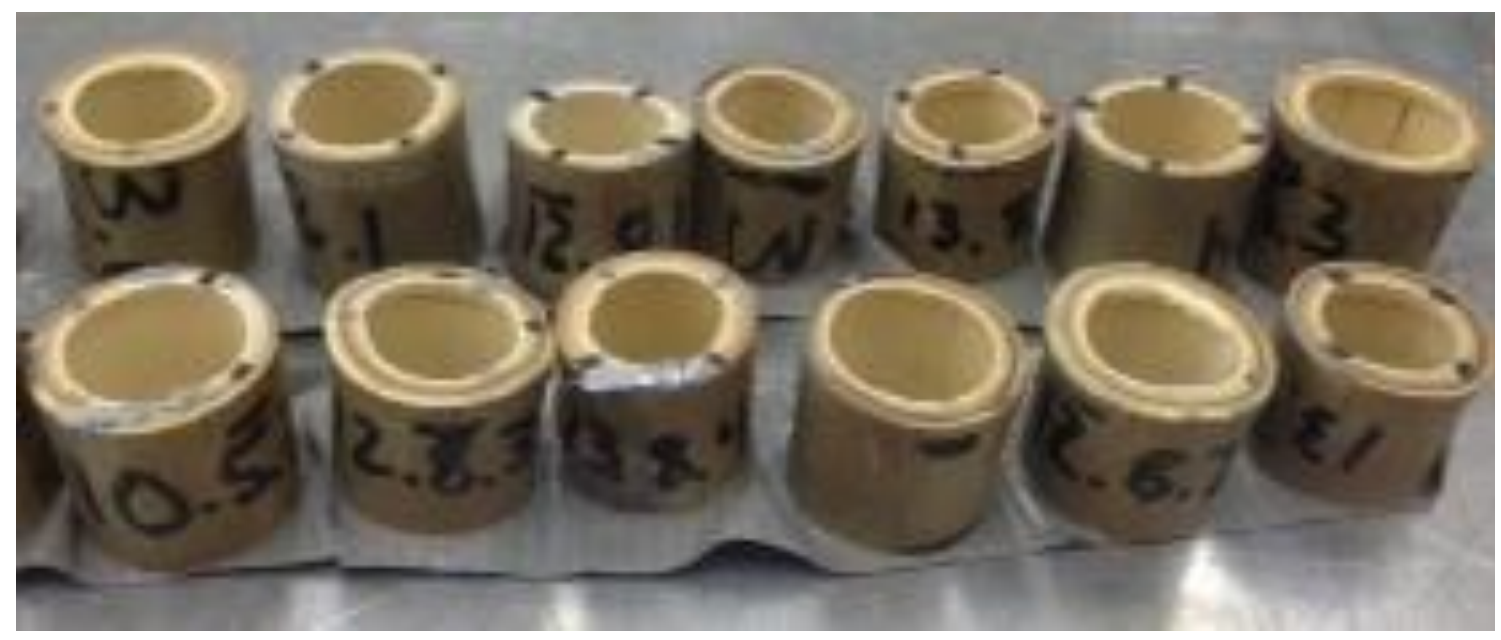

Figure 4-17. Portion of tested $180^{\circ} \mathrm{F}$ at $1^{\circ} \mathrm{F} / \mathrm{min}$. rate compression specimens

In summary of the bamboo treated at $180^{\circ} \mathrm{F}$ with a $1^{\circ} \mathrm{F} / \mathrm{min}$ rate, a table is shown in the appendix displaying the main mechanical properties. In this group, the average specific strength is 303-kips-in./lb $\mathrm{b}_{\mathrm{m}}$ and the ultimate stress is $10.5-\mathrm{ksi}$. In addition, the compressive modulus is $480-\mathrm{ksi}$ and the specific modulus is $13894-\mathrm{kips}-\mathrm{in} . / \mathrm{lb}_{\mathrm{m}}$. From this test group, the modulus also deviates from the average approximately $14 \%$ while the remaining properties vary by about $12 \%$. Although the deviations are slightly higher than normal, the data are still representative of the mechanical characteristics of this treatment.

\subsubsection{4 $200^{\circ} \mathrm{F}$ at $5^{\circ} \mathrm{F} / \mathrm{min}$ Thermal Treatment Results}

The following group among the heat treated bamboos is the $200^{\circ} \mathrm{F}$ at $5^{\circ} \mathrm{F} / \mathrm{min}$. set. Figure 4-18 displays the resulting stress-strain curve for the group heat treated at $200^{\circ} \mathrm{F}$ at $5^{\circ} \mathrm{F} / \mathrm{min}$. The high sample size is due to a second group being added. Relatively high deviation in the first group warranted a second round of specimens. 


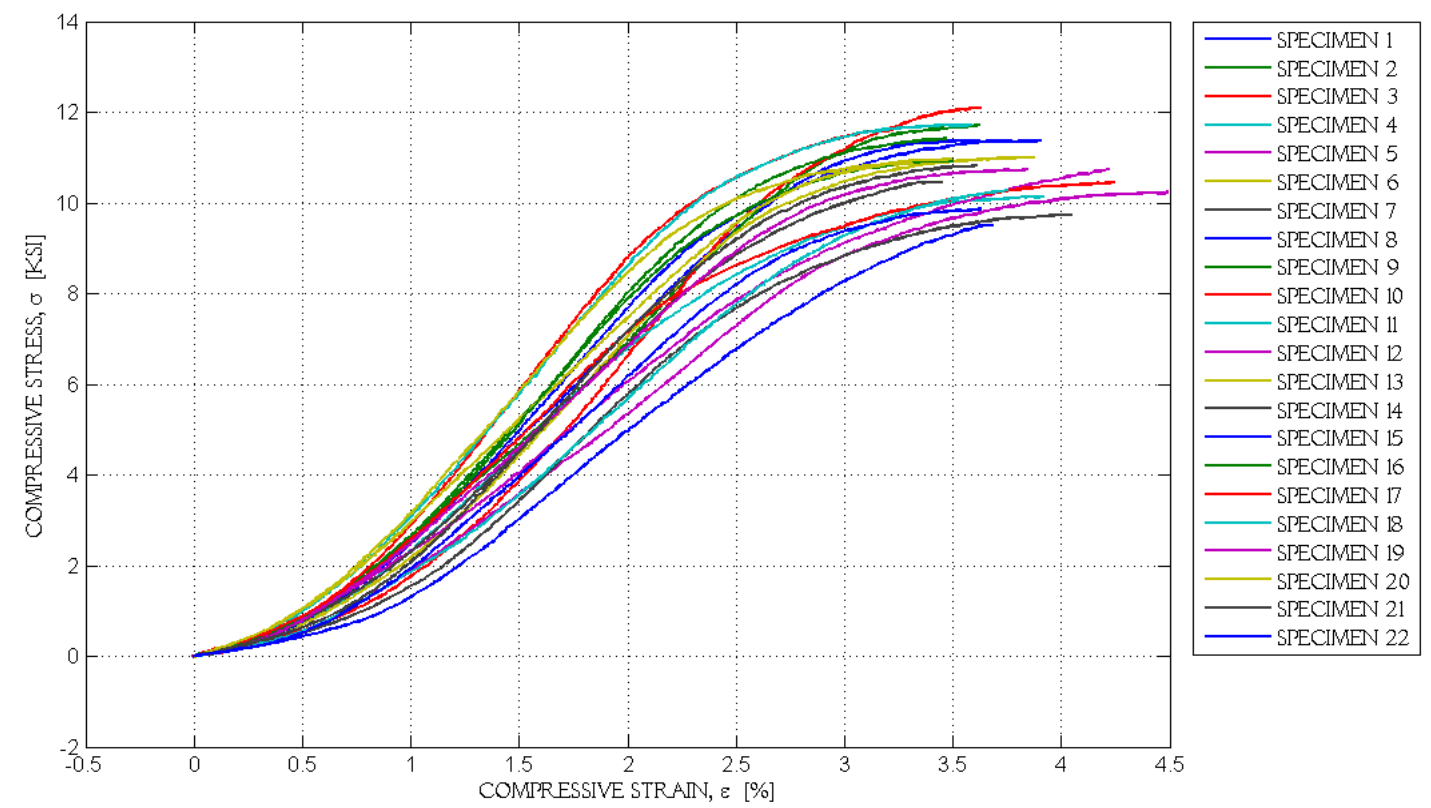

Figure $4-18.200^{\circ} \mathrm{F}$ at $5^{\circ} \mathrm{F} / \mathrm{min}$. thermal treatment compressive stress-strain curves

As seen from the figure, the trend of this plot is more consistent than the previous one.

The initial nonlinear portion is again evident in the curve and the linear portion seems fairly consistent among all specimens. The end of the linear portion of the curve occurs around a compressive strain of $2.27 \%$ while the compressive stress is about $8.16-\mathrm{ksi}$, respectively. In this specimen group, the linear elastic portion of the curve varies notably in slope. On a positive note, however, all the specimens follow a stress-strain path in much the same way; even the nonlinear plastic region of the curve appears consistent.
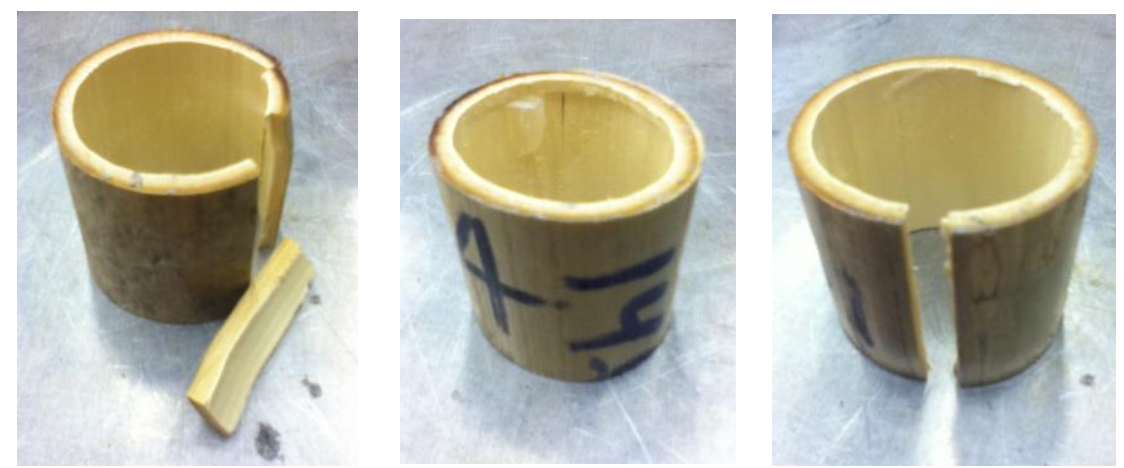

Figure 4-19. Common compressive failure types for $200^{\circ} \mathrm{F}$ at $5^{\circ} \mathrm{F} / \mathrm{min}$. treatment 
Failure types observed from this treatment indicate splitting, complete separation, and bearing stress. The majority of the specimens treated in this manner demonstrate splitting failure. As usual, the splitting failure observed runs the length of the specimen from end to end. Culm separation is the following most common failure type. As seen from the figures shown, about $20 \%$ of the culm separates from the rest of the specimen. The majority of the specimens demonstrating bearing stress exhibit minor deformation. As a result, it is noted that this type of treatment reduces the probability of complete culm separation-fiber splitting is more common.

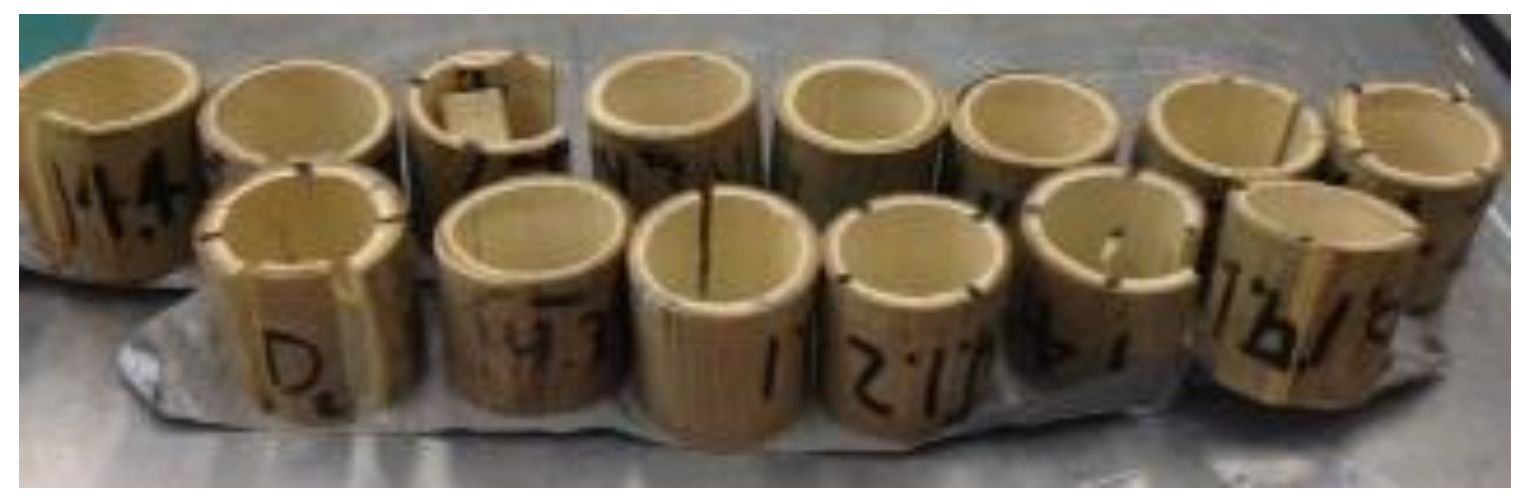

Figure 4-20. Portion of tested $200^{\circ} \mathrm{F}$ at $5^{\circ} \mathrm{F} / \mathrm{min}$. rate compression specimens

In summary of the bamboo treated at $200^{\circ} \mathrm{F}$ with a $5^{\circ} \mathrm{F} / \mathrm{min}$. rate, a table is shown in the appendix displaying the main mechanical properties. In this group, an average specific strength of 336-kips-in. / $/ \mathrm{b}_{\mathrm{m}}$ and ultimate stress of 10.8-ksi is observed. The compressive modulus is $487-\mathrm{ksi}$ and the specific modulus of $15130-\mathrm{kips}-\mathrm{in} . / \mathrm{lb} \mathrm{m}$ as measured from the data. In this test group, the modulus also deviates from the average approximately $14 \%$ while the remaining properties vary by about $7 \%$. A safe assumption may be made at this point concerning the precision of the elastic modulus under compression: it carries the highest standard deviation among all variables. This statement is not necessarily a terrible one since the modulus deviates by a difference of $9 \%$ compared to the other properties - pretty comforting for a material that is not man-made. 


\subsubsection{5 $200^{\circ} \mathrm{F}$ at $1^{\circ} \mathrm{F} / \mathrm{min}$ Thermal Treatment Results}

Next among the thermally treated group is the bamboo specimens treated at $200^{\circ} \mathrm{F}$ with $1^{\circ} \mathrm{F} / \mathrm{min}$. Figure 4-21 displays the resulting stress-strain curve for the group heat treated

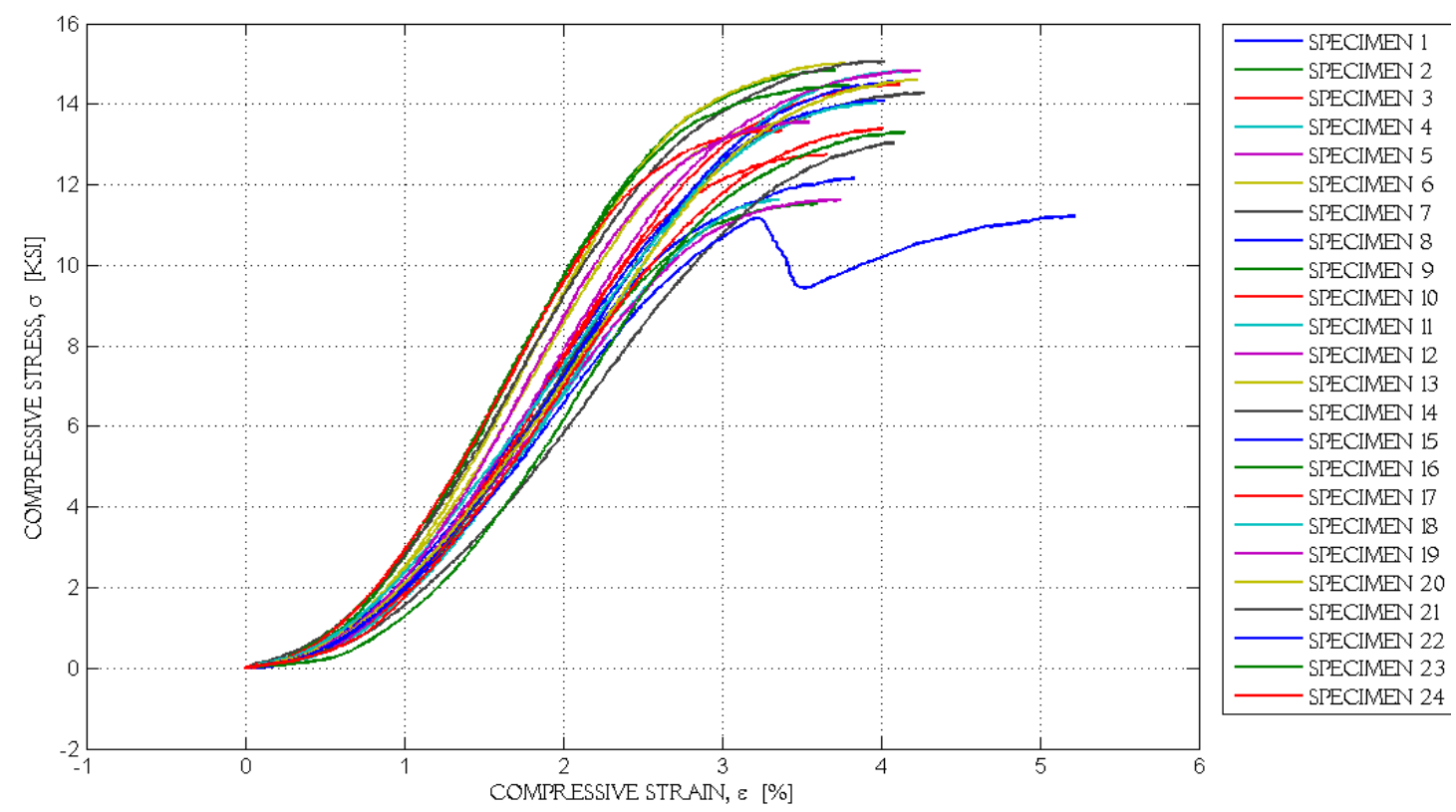

Figure $4-21.200^{\circ} \mathrm{F}$ at $1^{\circ} \mathrm{F} / \mathrm{min}$. thermal treatment compressive stress-strain curves

at $200^{\circ} \mathrm{F}$ with a $1^{\circ} \mathrm{F} / \mathrm{min}$. rate. Confidence in the data is established with the consistent curves shown. The initial nonlinear portion is again evident in the curve and the linear portion appears to be fairly consistent among all specimens. The end of the linear portion of the curve occurs around a compressive strain of $2.43 \%$ while the compressive stress is about $9.55-\mathrm{ksi}$. The linear elastic region of most all samples follows a similar slope while the plastic region varies in about half the specimens. The specimens that reach a higher ultimate stress demonstrate a fairly consistent stress-strain curve in the plastic region. 

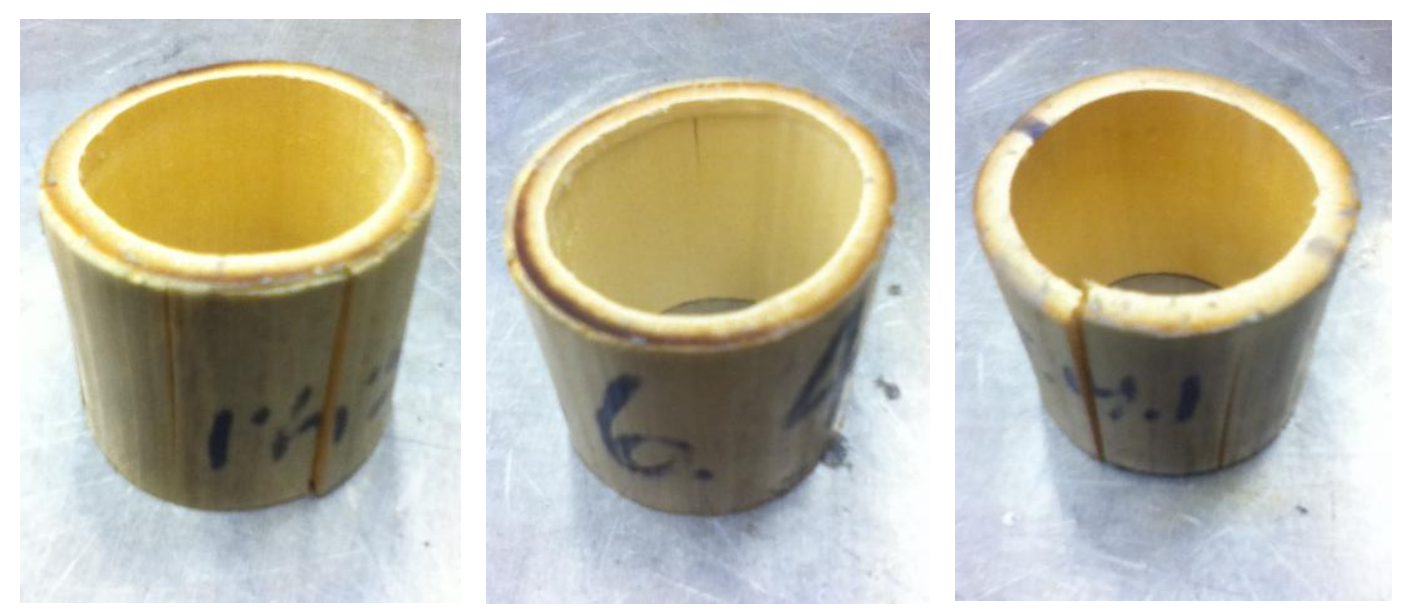

Figure 4-22. Common compressive failure types for $200^{\circ} \mathrm{F}$ at $1^{\circ} \mathrm{F} / \mathrm{min}$. treatment

Minor bearing stress failures and splitting are observed from curing bamboos with this thermal treatment. Splitting among these specimens show little crack opening compared to the previous treatment specimens. The majority of these specimens indicate this type of failure. Consequently, culm separation is much less prominent with this treatment as seen in the shown figures. Bearing stress is also rare in the results of this compression test since less than $10 \%$ of the total sample size exhibit this characteristic.

In summary of the bamboo treated at $200^{\circ} \mathrm{F}$ with a $1^{\circ} \mathrm{F} / \mathrm{min}$. rate, a table is shown in the appendix displaying the main mechanical properties. In this group, the average specific

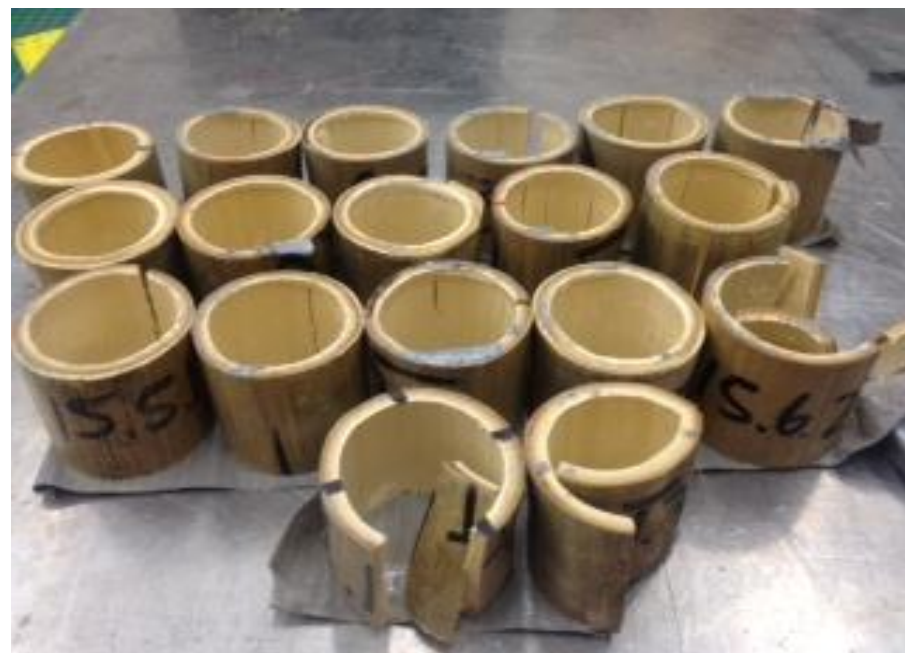

Figure 4-23. Tested $200^{\circ} \mathrm{F}$ at $1^{\circ} \mathrm{F} / \mathrm{min}$. rate compression specimens strength is $373 \mathrm{kips}-\mathrm{in} . / \mathrm{lb}$ and the ultimate stress is $13.6-\mathrm{ksi}$. The compressive modulus is 623-ksi and the specific modulus is 17136-kips-in./lb $\mathrm{b}_{\mathrm{m}}$. In this test group, the yield 
stress deviates the most at $24 \%$ while the rest are at $8 \%$. The deviations in this group are higher than the previous ones. It is probable that the specimen geometries are the cause for blame in this case. The weight of the specimen varies considerably in relation to the samples in the test group.

\subsubsection{6 $220^{\circ} \mathrm{F}$ at $1^{\circ} \mathrm{F} / \mathrm{min}$ Thermal Treatment Results}

The final group among the heat treatment set is the $220^{\circ} \mathrm{F}$ at $1^{\circ} \mathrm{F} / \mathrm{min}$ rate. Figure $4-24$ displays the resulting stress-strain curve for the bamboo group heat treated at $220^{\circ} \mathrm{F}$ with a $1^{\circ} \mathrm{F} / \mathrm{min}$. rate. The large sample size seen is due to variation in the first sample set tested. Initial testing data demonstrated a relatively high deviation among the samples, so it was imperative to include another set of specimens. After testing it was discovered that the deviations for the majority of the mechanical characteristics dropped substantially. The density deviation among all of the specimens is less than $9 \%$. Similarly, the area deviation relative to the average is approximately $8 \%$. The deviation in the remaining physical properties is also low; they all have deviations less than $5 \%$ relative to the average. Confidence in the data is established with fairly consistent curves added to the figure. The initial nonlinear portion is again evident in the curve and the linear portion appears to be largely inconsistent among the specimens. The end of the linear portion of the curve occurs around a compressive strain of $3.63 \%$ and compressive stress of about $12.7-\mathrm{ksi}$. The linear elastic region of most all samples follows a similar slope while the plastic region varies in about half the specimens. The specimens that reach a higher ultimate stress demonstrate a fairly consistent stressstrain curve in the plastic region. 


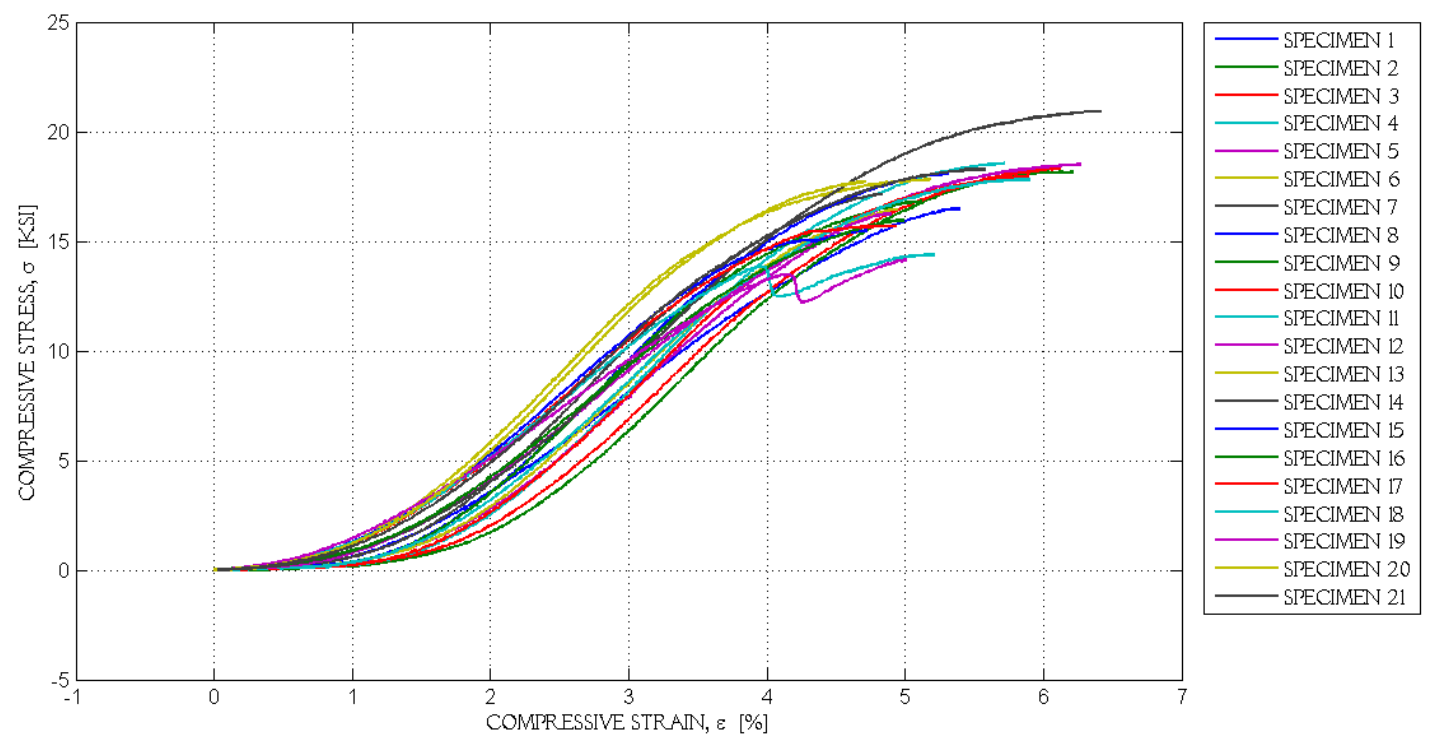

Figure $4-24.220^{\circ} \mathrm{F}$ at $1^{\circ} \mathrm{F} / \mathrm{min}$. thermal treatment compressive stress-strain curves

The results of the compression test performed on bamboos cured with this thermal treatment indicate less crack opening than the previous thermal treatments. Culm separation and fiber splitting are the two main observations from compressed bamboo treated with this thermal treatment. Figure 4-25 shows exaggerated examples of the two features observed in this test. Figure $4-26$ displays the specimens tested under this type of thermal treatment and shows the small crack opening for the majority of the
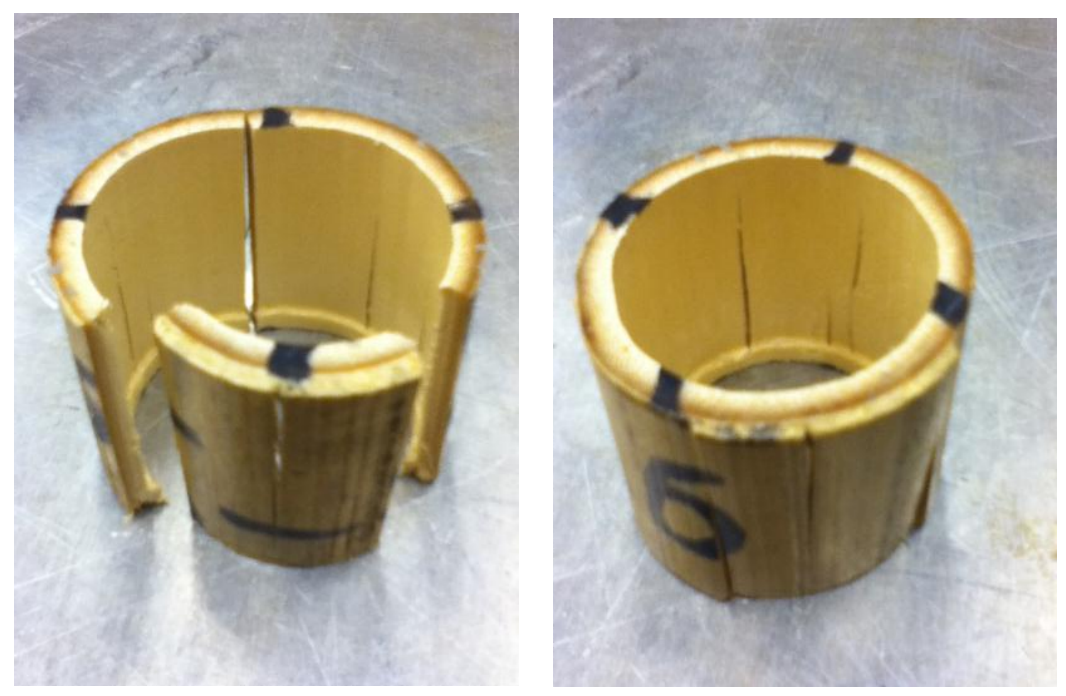
specimens.

Figure 4-25. Common compressive failure types for $220^{\circ} \mathrm{F}$ at $1^{\circ} \mathrm{F} / \mathrm{min}$. treatment 
In summary of the bamboo treated at $220^{\circ} \mathrm{F}$ with a $1^{\circ} \mathrm{F} / \mathrm{min}$. rate, a table is shown in the appendix displaying all the main mechanical properties. In this group, the average specific strength is $489-\mathrm{kips}-\mathrm{in} . / \mathrm{lb}_{\mathrm{m}}$ and ultimate stress is 17.1-ksi. A compressive modulus of $579-k s i$ and specific compressive modulus of $16572-k i p s-i n . / / b_{m}$ is observed. From this test group, the ultimate strain deviates the most from the average at $11 \%$ while the rest are at $9 \%$. The deviations in this group are slightly higher than the previous ones but not high enough to trigger warnings. These deviations are, in actuality, expected of this bio-material.

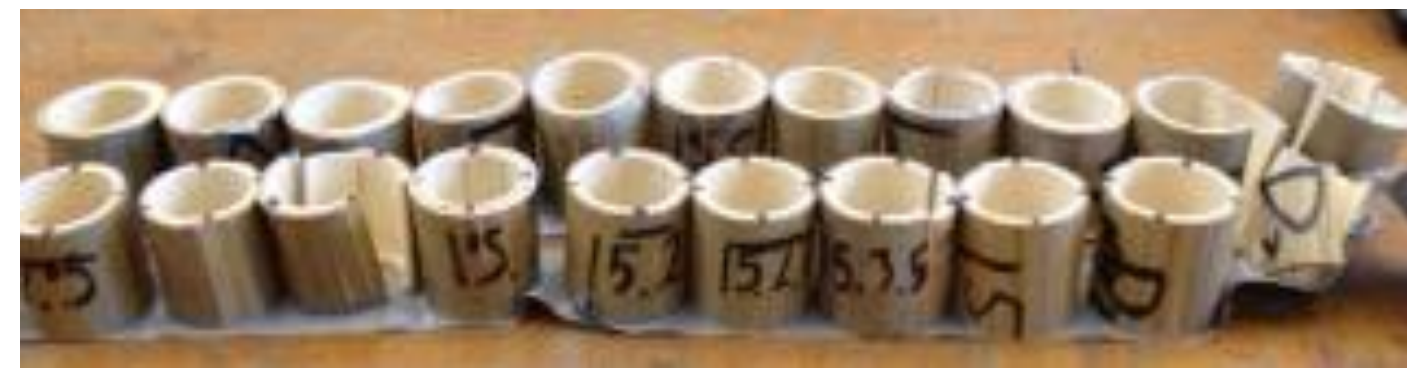

Figure 4-26. Tested $220^{\circ} \mathrm{F}$ at $1^{\circ} \mathrm{F} / \mathrm{min}$. rate compression specimens

\subsubsection{Thermal Treatment with Organic Chemical Additives Compression Results}

The second aim of this portion of the research is to determine the effect of organic chemical treatment on the bamboo's compressive material properties. Sources from foreign lands indicate that these chemical treatments may improve performance and provide lasting protection to the material, so the validity of these claims is explored [7]. It is imperative to discover any side effects of these treatments so as to raise awareness of the consequences of trying to chemically protect and improve the performance of bamboo. The scientific approach is utilized to investigate the magnitude of mechanical performance improvement in the bamboo. This last set of investigative treatments to improve the mechanical characteristics of bamboo is the chemically treated group followed by a heat treatment. As mentioned earlier, these groups consist of salt, lime, 
and oil recipes. In order to economize the reserve of bamboo available for this research, it was decided to conduct the chemical treatments only at $220^{\circ} \mathrm{F}$ with the $1^{\circ} \mathrm{F} / \mathrm{min}$. rate. At this temperature, the bamboo would surely be cured to reach its best mechanical properties although the $180^{\circ} \mathrm{F}$ treatment is also adequate for obtaining similar results. As mentioned, the goal in this portion of the research is to investigate the effects of these natural chemical treatments; exploring varying thermal treatments alongside the chemical ones is left for future work.

\subsection{3\% Salt}

First among the chemically treated groups is the bamboo saturated in the $3 \%$ salt solution. Figure 4-27 displays the resulting stress-strain curve for the group treated with $3 \%$ salt. By examining the figure, it can be seen that there is a slight "dip" found in one of the specimens colored in blue. Analyzing the physical properties of the test group indicates lack of a difference that would cause this effect in the mechanical response of the specimen. Therefore, biological causes are likely to blame. The end of the linear portion of the curve occurs around a compressive strain of $3.70 \%$

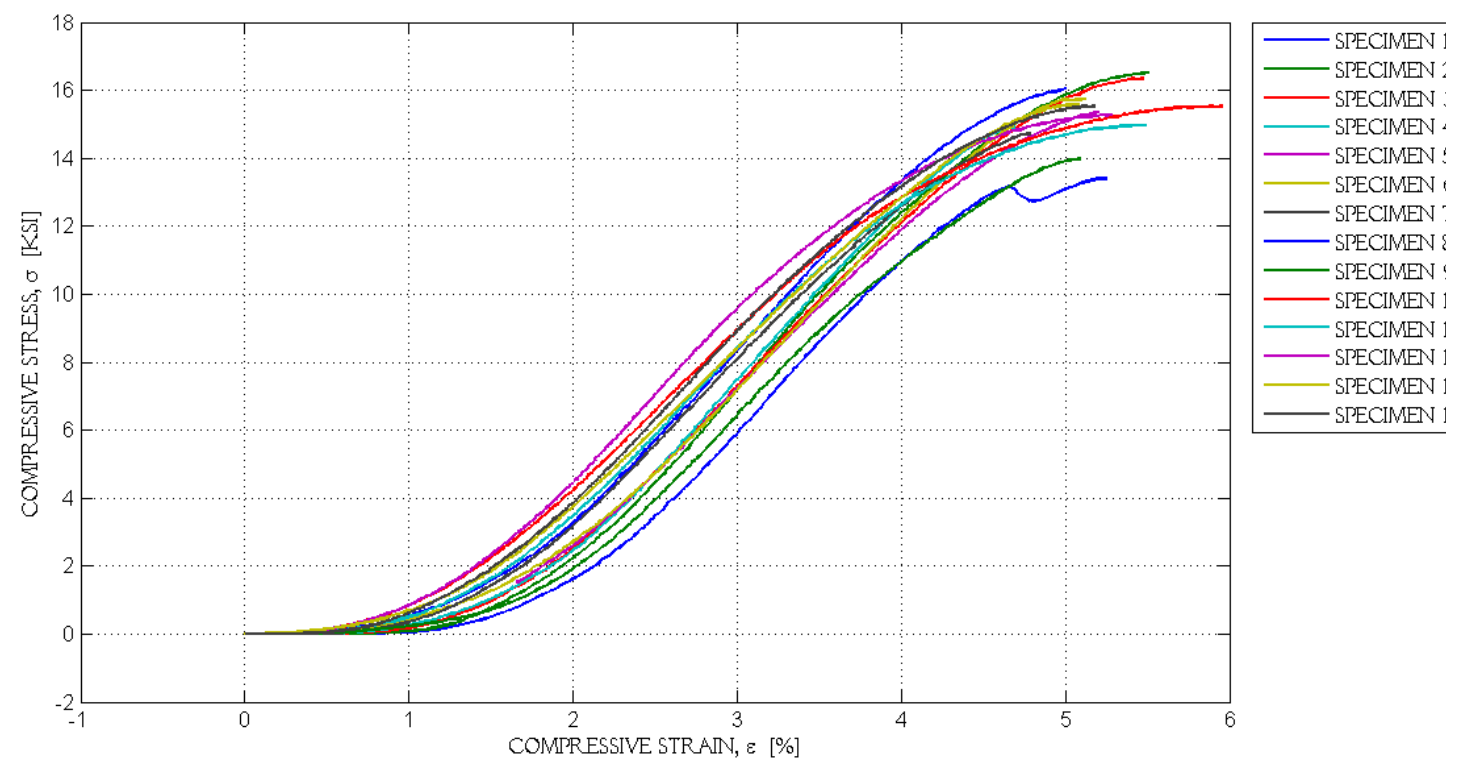

Figure 4-27. 3\% salt treatment compressive stress-strain curves 
and compressive stress

around 10.6-ksi. The linear

elastic region of most

samples follows a similar

slope while the plastic region

is unpredictable.

Inspection of the $3 \%$ salt treated specimens indicates
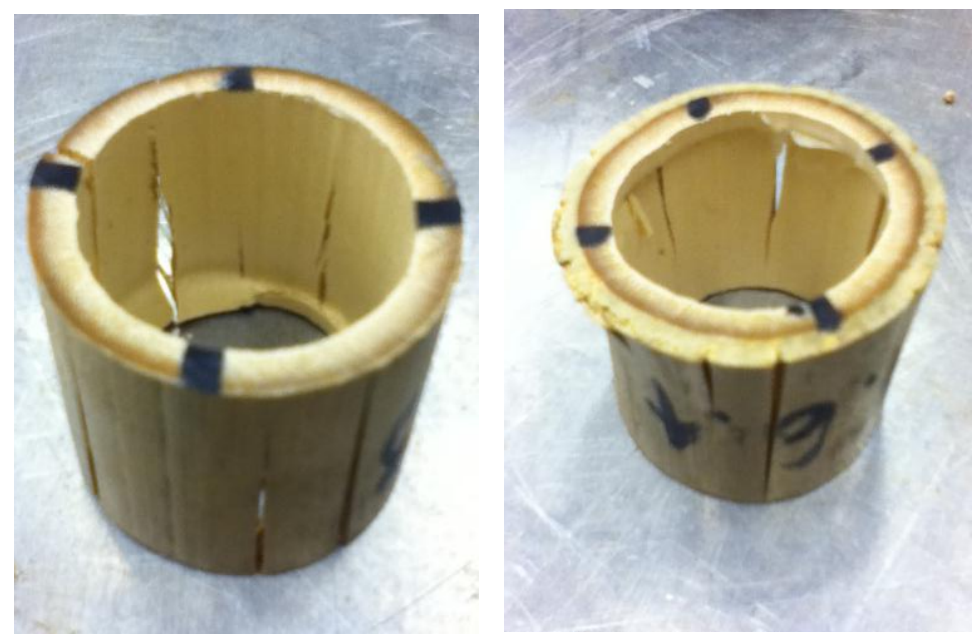

Figure 4-28. Common compressive failure types for $3 \%$ salt treatment slightly larger crack openings at the end of the test. Bearing stress and splitting is also evident from the specimens. As seen in Figure 4-28, bearing stress in the specimens is a prominent feature. Splitting in most of the specimens is also evident, but an interesting fact to notice is that the fractures do not propagate entirely through the specimen from end to end. Also, numerous fractures are seen around the entire circumference of the specimen to illustrate a symmetric fracture

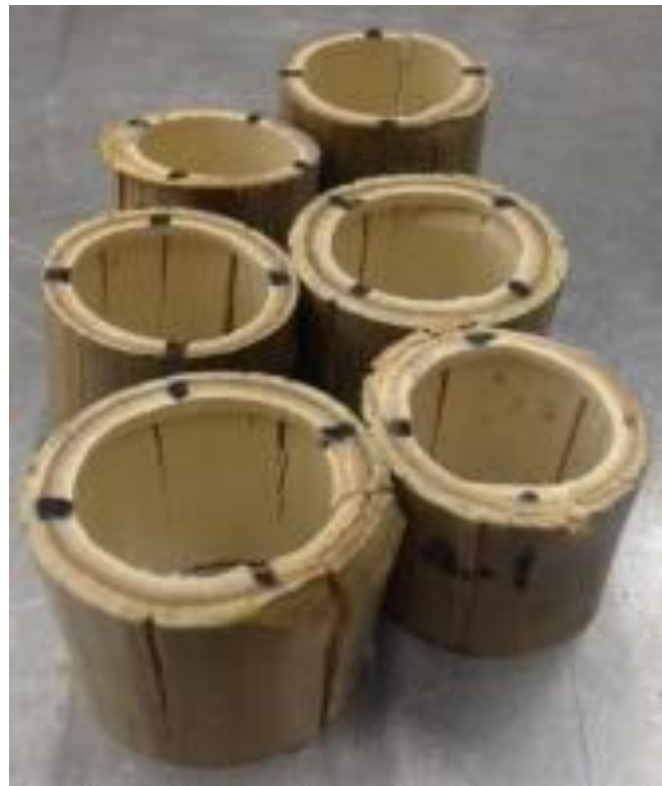

Figure 4-29. Portion of tested $3 \%$ salt compression specimens geometry.

In summary of the bamboo treated with $3 \%$ salt, a table is shown in the appendix displaying the main mechanical properties. In this group, the average specific strength is 490-kips-in.//b $\mathrm{b}_{\mathrm{m}}$ and ultimate stress is $15.3-\mathrm{ksi}$. The compressive modulus is $514-\mathrm{ksi}$ and specific compressive modulus is 16770 -kips-in./lb $\mathrm{b}_{\mathrm{m}}$. Oddly enough, the ultimate stress deviates the most from the average at $7 \%$ while the rest is $6 \%$. One important lesson to 
gain from this group is that a high variation in geometry still yields comparatively precise data.

\subsection{6\% Salt}

The second group among the chemically treated sets is the bamboos soaked in the $6 \%$ salt solution. Figure 4-30 displays the resulting stress-strain curve for the group treated with $6 \%$ salt. The data appear to be consistent for all specimens except one. One specimen is shown to have a considerably lower ultimate stress than the rest.

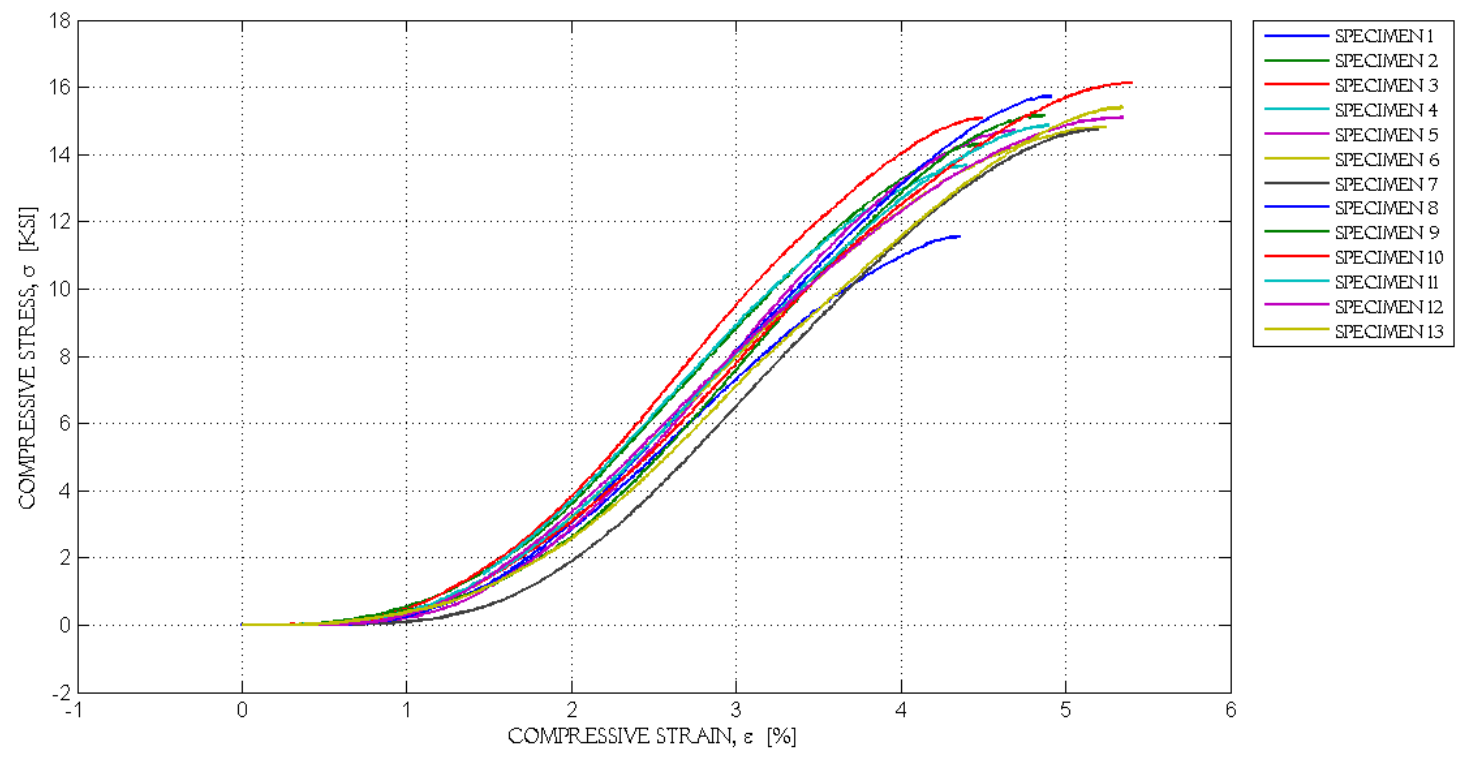

Figure 4-30. 6\% salt treatment compressive stress-strain curves

The initial nonlinear portion is again evident in the curve and the linear portion is consistent among all specimens. The end of the linear portion of the curve occurs around a compressive strain of $3.51 \%$ and the corresponding compressive stress is 10.5-ksi. The linear elastic region of almost all samples follows a similar slope while the plastic region is unpredictable as the sample continues to strain.

Similarly to the $3 \%$ salt treatment, this group yields splitting, bearing stress, and complete culm separation. It is also observed that the fractures along the specimen span the entire circumference of the culm. One case deomonstrates culm separation across 
the entire specimen that yields many pieces. Other cases show crack openings that are relatively small and do not span the whole thickness of the specimen wall. Similar to the previous treatment, the crack opening for bamboos treated with $6 \%$ salt is more prominent.
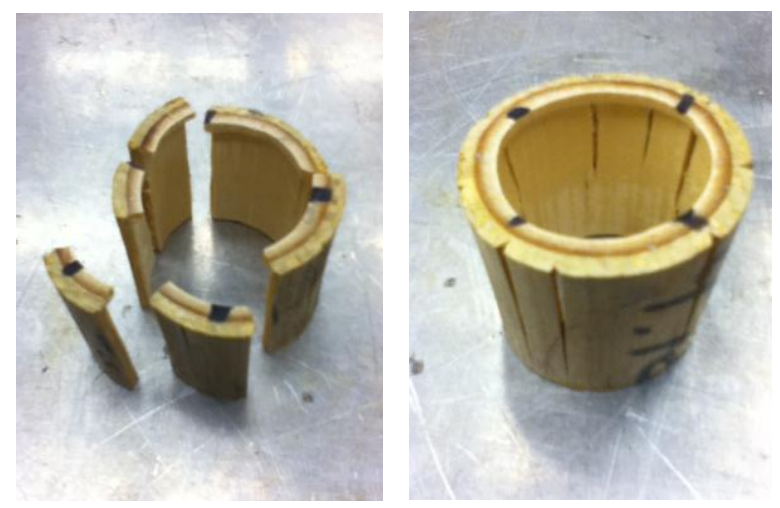

Figure 4-31. Common compressive failure types for $6 \%$ salt treatment

In summary of the bamboo treated with $6 \%$ salt, a table is shown in the appendix displaying the main mechanical properties. In this group, the average specific strength is 472-kips-in./lb $\mathrm{b}_{\mathrm{m}}$ and the ultimate stress is $14.7-\mathrm{ksi}$. The compressive modulus is $518-\mathrm{ksi}$ and the specific modulus is $16658-k i p s-i n . / / b_{m}$. In this set, the yield stress deviates the most from the average at $12 \%$ while the rest are at $6 \%$.

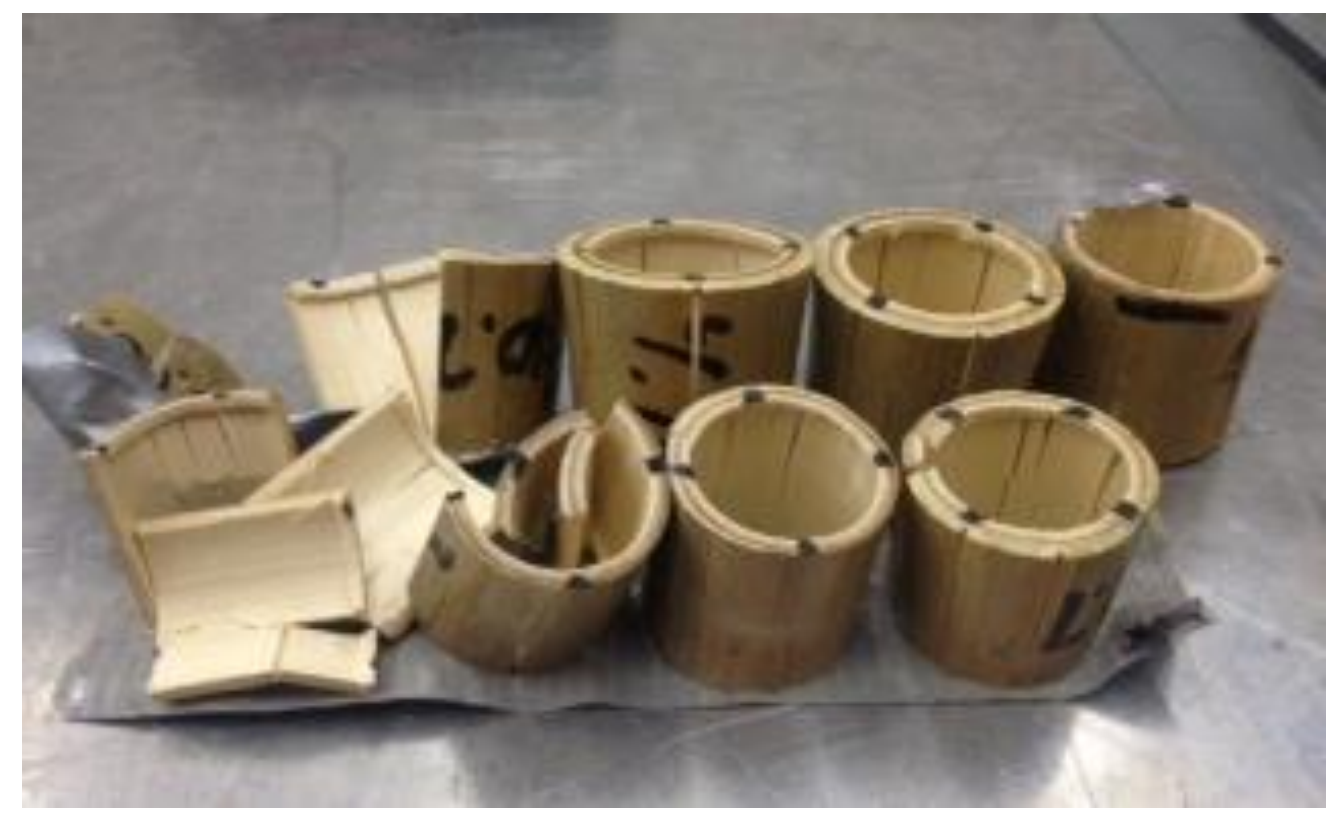

Figure 4-32. Portion of tested $6 \%$ salt compression specimens 


\subsection{9\% Salt}

The last group among the salt-treated sets is the bamboos soaked in the $9 \%$ salt solution. Figure 4-33 displays the resulting stress-strain curve for the group treated with $9 \%$ salt. The data appear to be consistent for all of the specimens. In fact, the deviations in this sample set are some of the lowest among all the treatment data.

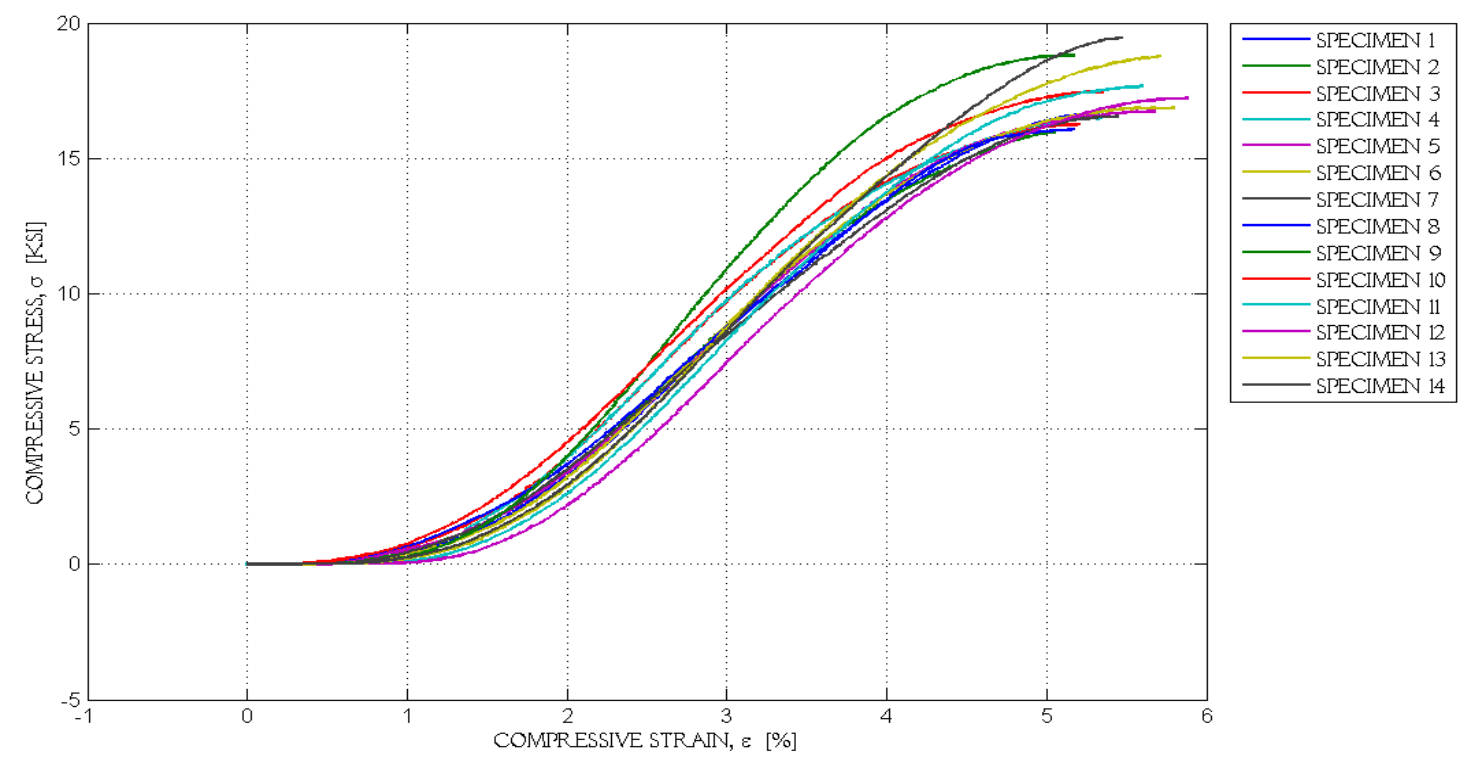

Figure 4-33. 9\% salt treatment compressive stress-strain curves

Looking at the physical dimensions of the $9 \%$ salt treatment shows the highest deviation relative to the average to be only $8 \%$. This deviation is attributed to the average density of the group. Most of the physical dimensions are within $3 \%$ to $5 \%$ relative to each other. This consistency is inherently due to the great care taken in preparing the specimens. As mentioned before, it is extremely difficult to maintain consistent geometries across all of the specimens since their dimensions are at the will of the culm's diameter-an inconsistent variable along the length of the pole. Nonetheless, a thorough study on the mechanical properties of this sample set will demonstrate the care inveseted in manufacturing these specimens. 
The initial nonlinear portion is again evident in the curve and the linear portion is consistent among all samples. The end of the linear portion of the curve occurs around a compressive strain slightly above $3.40 \%$ while the compressive stress occurs around 11.2-ksi. The linear elastic region of almost all samples follows a similar slope while the plastic region is unpredictable as the sample continues to strain.

The dominant failure types from this treated bamboo indicate mostly culm
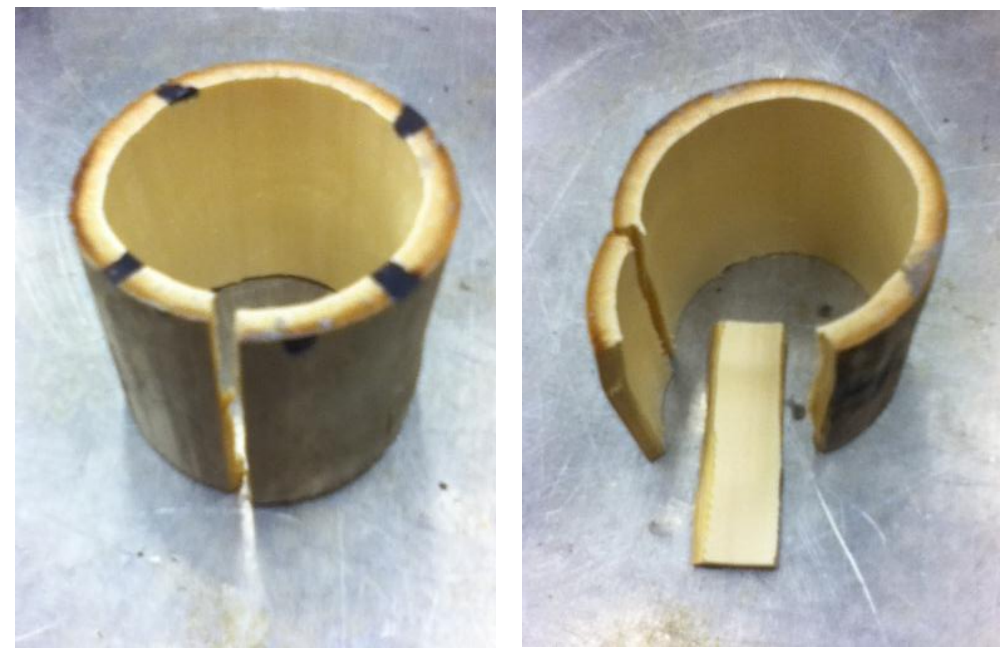

Figure 4-34. Common compressive failure types for $9 \%$ salt treatment separation and fiber splitting. Complete fracture from end to end with a large crack opening is also evident in a number of samples in this test. Minor crack propagation is also observed, but the majority of the bamboo samples treated with $9 \%$ salt demonstrates culm separation. It appears that adding salt to the bamboos may promote the likelihood of culm separation or fiber splitting in the wall.

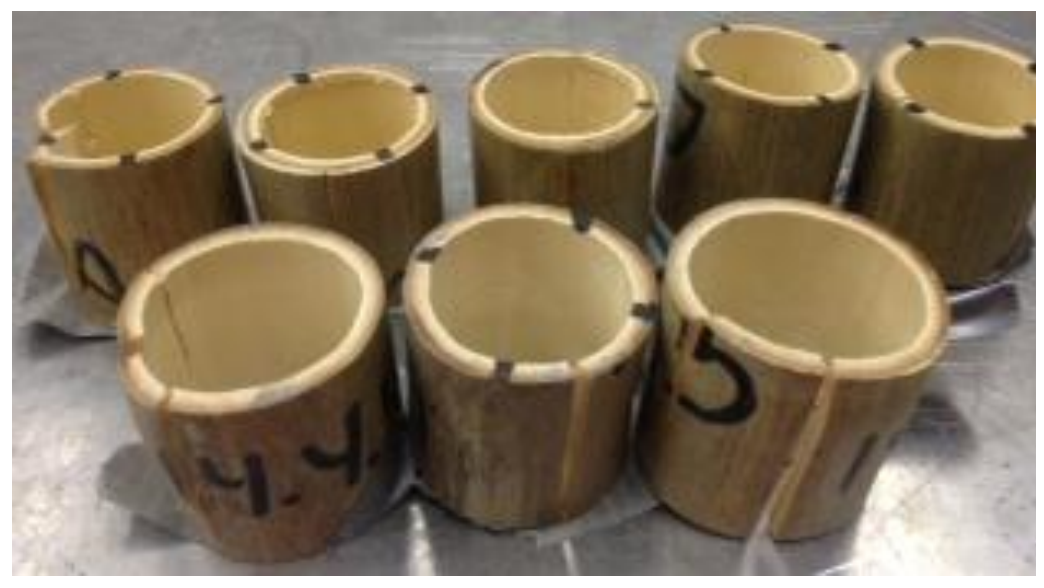

Figure 4-35. Portion of tested $9 \%$ salt compression specimens 
In summary of the bamboo treated with $9 \%$ salt, a table is shown in the appendix displaying the main mechanical properties. As seen from the table, the mechanical properties are all consistent relative to the average. The care taken in preparing these specimens is evident in the low standard deviations of the compressive material properties of this treatment set. In this group, the average specific strength is $472-k i p s-$ in.// $\mathrm{b}_{\mathrm{m}}$ and ultimate stress is $17.2-\mathrm{ksi}$. A compressive modulus of $585-\mathrm{ksi}$ and specific compressive modulus of $16025-\mathrm{kips}-\mathrm{in} . / \mathrm{b}_{\mathrm{m}}$ is observed. In this set, the yield stress and modulus deviate the most from the average at $8 \%$ while the rest are at $5 \%$.

\subsection{Lime}

Another chemically treated set used is the bamboo soaked in a $5 \%$ lime-water solution. Figure 4-36 displays the resulting stress-strain curve for the group treated with lime. The slopes of the curves in the linear elastic region begin to differ as they near their

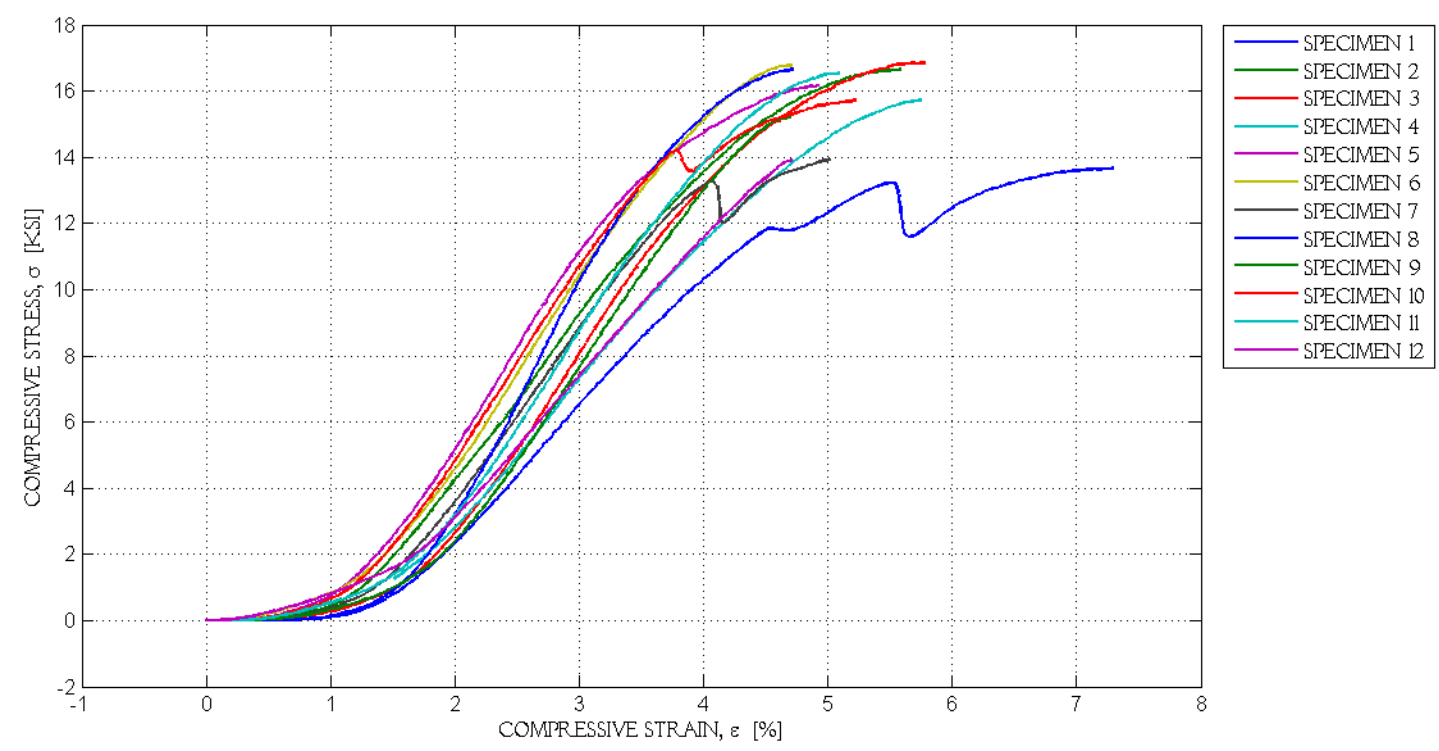

Figure 4-36. Lime treatment compressive stress-strain curves

proportional limit. The initial nonlinear portion is again evident. The end of the linear portion of the curve occurs around a compressive strain of $3.38 \%$ and stress of $10.7-\mathrm{ksi}$. The nonlinear portions of the curves are highly erratic and difficult to surmise in a 
qualitative expression. Some specimens show a behavior that peaks and quickly drop while others depict a more stable reaction after entering the ultimate stress.

Observing the lime specimens after the test demonstrates that fiber splitting and bearing stress are the primary responses to the treatment. Crack openings for these specimens are also large and dominate the majority of the fracture behavior for this treatment. As seen from Figure 4-37, the crack displacement for one particular specimen is significantly large. Many of the specimens also display dramatic forms of deformation, so utilizing lime to treat the bamboos results in a substantial deformation characteristic.
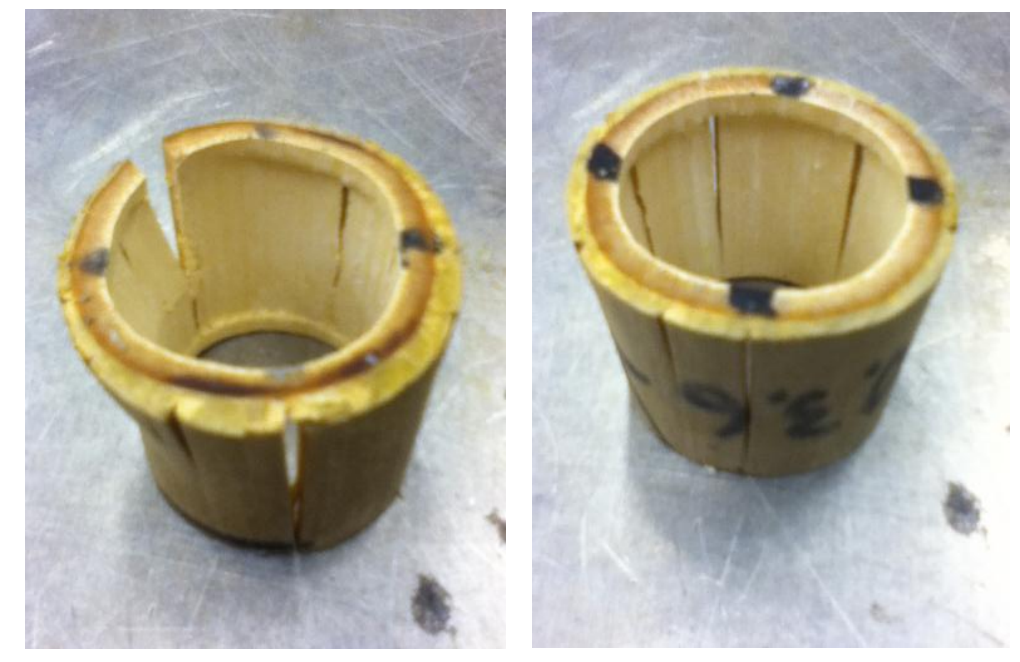

Figure 4-37. Common compressive failure types for lime treatment

In summary of the bamboo treated with lime, a table is shown in the appendix displaying the seven main mechanical properties. In this group, the average specific strength is 439-kips-in.//l $\mathrm{b}_{\mathrm{m}}$ and ultimate stress is $15.6-\mathrm{ksi}$. The compressive modulus is 556-ksi and the specific modulus is $15687-k i p s-i n . / / b_{m}$. In this set, the modulus and ultimate strain deviate the most from the average at $16 \%$ and $15 \%$, respectively. The yield stress has a deviation of $4 \%$ while the remaining variables have a deviation around $9 \%$. Biological imperfections may be the root of this deviation. 


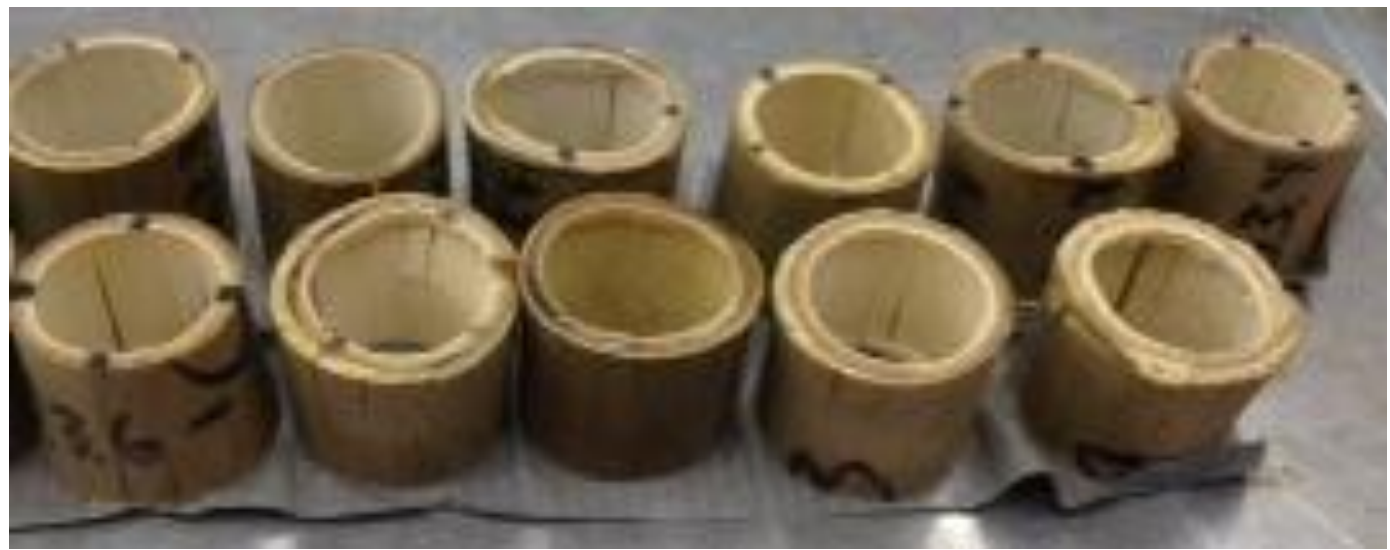

Figure 4-38. Portion of tested lime compression specimens

\subsection{Oil}

Another chemically treated set is the bamboo soaked in oil. Figure 4-39 displays the resulting stress-strain curve for the group treated with oil. The slopes of the curves all appear to match well with the exception of the specimen colored in green. Investigating the physical properties of the treatment group yields an anomaly that should raise a red flag. The density of this specimen is found to be the maximum of the group. Intuitively,

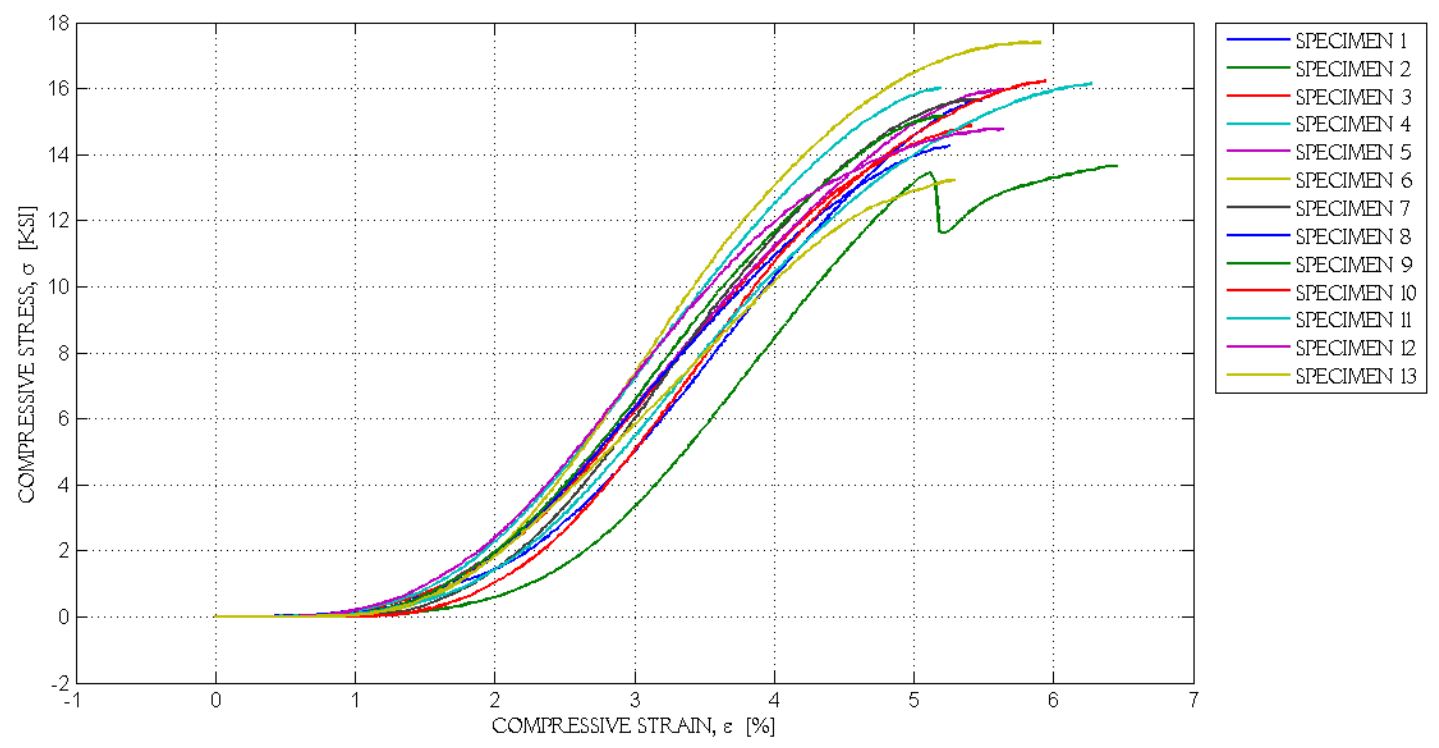

Figure 4-39. Oil treatment compressive stress-strain curves

this sample should have performed quite well among the data set. In all likelihood, the specimen may have incurred an unexpected mechanical response caused by a 
particularly dense area near the middle section of its height. Consequently, bearing stress predominantly occurred near the ultimate load of the test and slightly skewed the data. As seen from the figure, a large "dip" in stress occurs before increasing back up to the ultimate load. It was also observed during the test that the specimen's top face failed before overall fracture occurred, leading to the conclusion that the biological composition in the middle portion of the specimen is to blame.
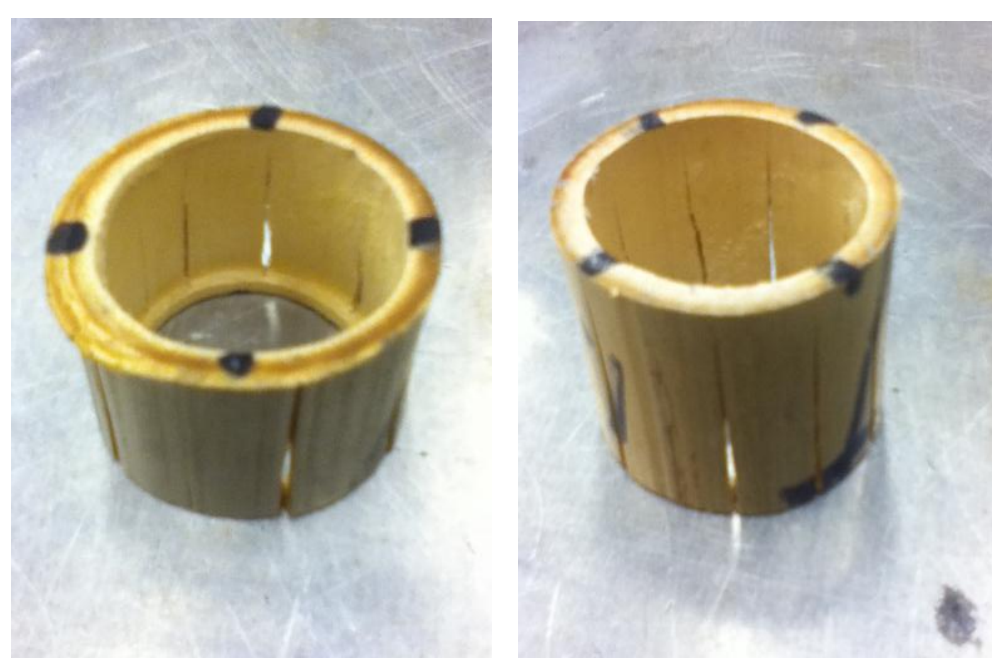

Figure 4-40. Common compressive failure types for oil treatment

The initial nonlinear portion is again evident. The end of the linear portion of the curve occurs around a compressive strain of $3.39 \%$ and the corresponding compressive stress is $10.7-\mathrm{ksi}$. The nonlinear portions of the curves-for the most part-characterize a gradual level-off region. One specimen colored in green demonstrates a sudden drop before increasing to the ultimate limit.

Bamboos treated with oil display splitting failure as well as bearing stress failure types. It is also interesting to note that fractures do not run the entire height of the samples. Crack openings appear to be great in some samples while quite small in others. On another note, bearing stress failure is a major failure type observed in this group. More than half of the specimens show signs of bearing stress at the conclusion of the test.

In summary of the bamboos treated with oil, a table is shown displaying the four main mechanical properties. In this group, the average specific strength is $439-k i p s-i n . / / b_{m}$ and 
the ultimate stress is $15.6-\mathrm{ksi}$. The compressive modulus is 556-ksi and the specific compressive modulus is $15687-k i p s-i n . / / b_{m}$. In this set, the yield stress deviates the most from the average at $9 \%$ while the rest are at $7 \%$. This final group shows a low deviation compared to the previous compression specimens and suggests high precision of the data. A deviation of about $6 \%$ is evident across all the mechanical properties in this sample group.

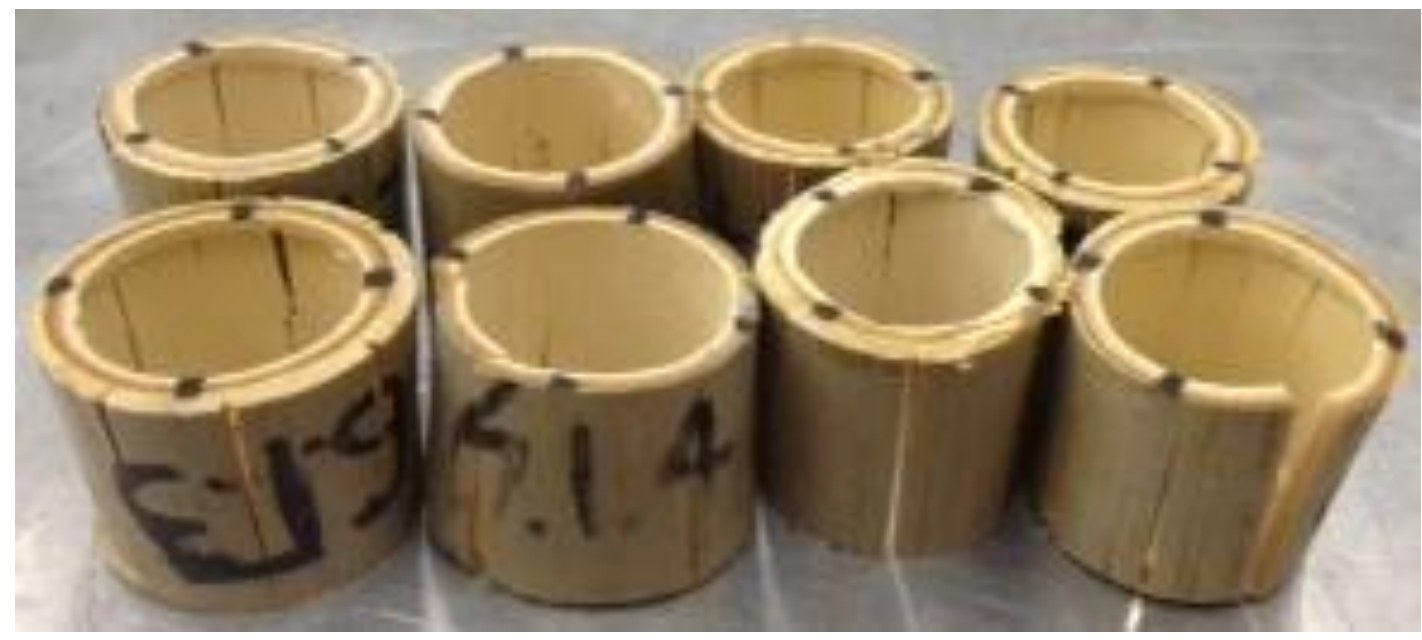

Figure 4-41. Portion of tested oil compression specimens

\subsubsection{Analysis of Compression Results}

A final analysis for the bamboos under compression is conducted, and two plots summarizing all the mechanical properties are shown. In addition, a separate table shows the percentage changes for all of the mechanical properties relative to the green bamboo group. It is worth mentioning that all errors bars generated in these plots are determined using the standard deviation of each sample group. In particular, the percent changes (P) and associated errors (E) for each value are calculated by conventional data calculation methods. In other words, the standard deviations of each characteristic are factored into the overall percent error by the square of the sum of errors for every mechanical property. It is vital to perform this analysis on the compressive mechanical 
characteristics in order to understand the effects of the treatments over the whole spectrum of the bamboo's behavior under compression.

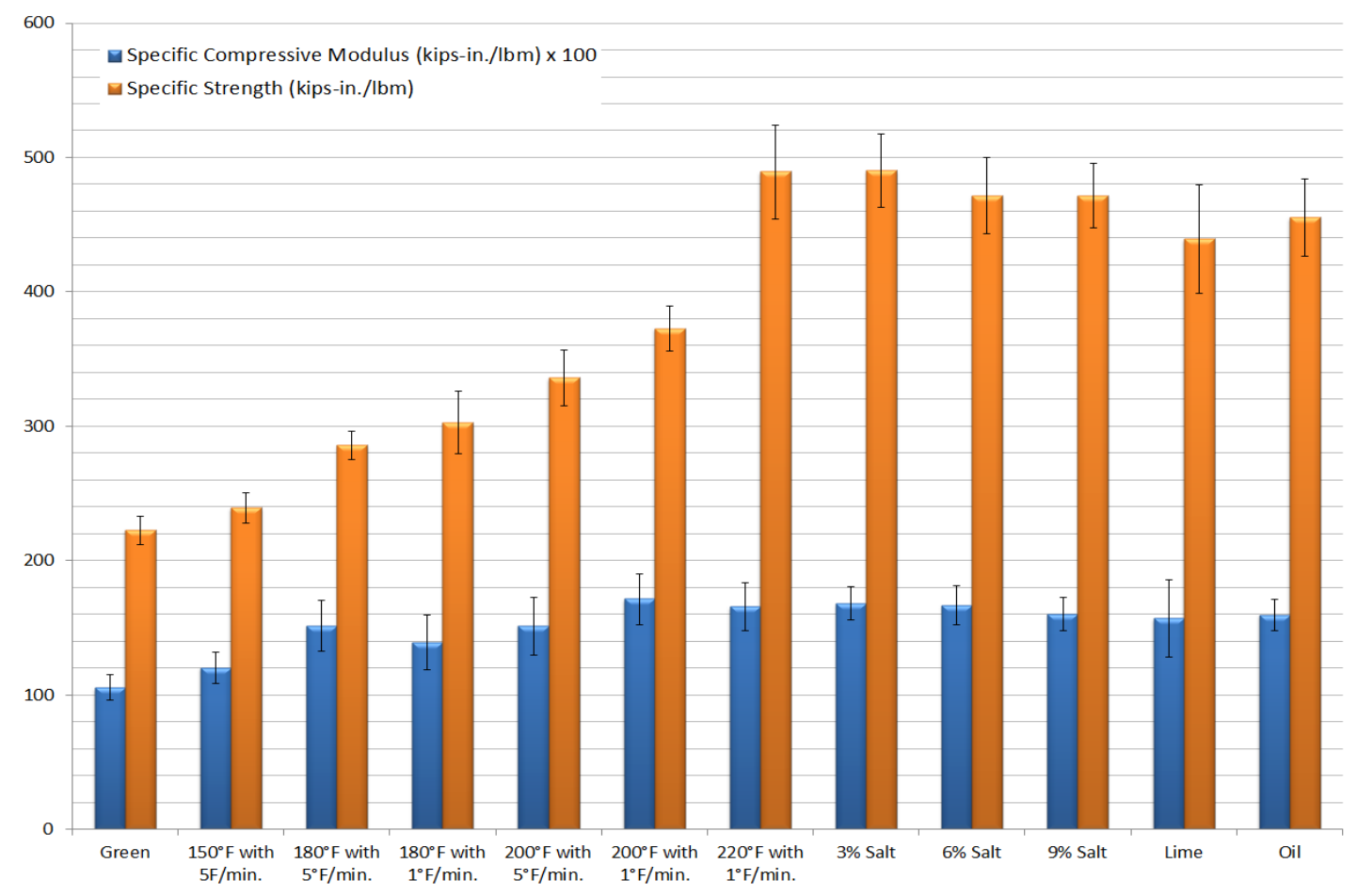

Figure 4-42. Summary of treatment effects on compressive specific strength and specific modulus

Analysis of the critical variable of this study, the compressive specific strength, shows a trend that $220^{\circ} \mathrm{F}$ heat or chemical treatments can improve green bamboo's compressive specific strength by an average of $111 \%$ relative to untreated bamboo. Increasing the cure temperature up to $220^{\circ} \mathrm{F}$ shows a substantial increase in compressive specific strength to approximately $120 \%$. It is possible that curing the bamboo at higher temperatures may yield greater strength improvements, but another higher-temperature autoclave must be employed to validate this assumption. Referring to the appropriate figures in the table, it seems that running the thermal treatments at the lower initial rampup

rate 


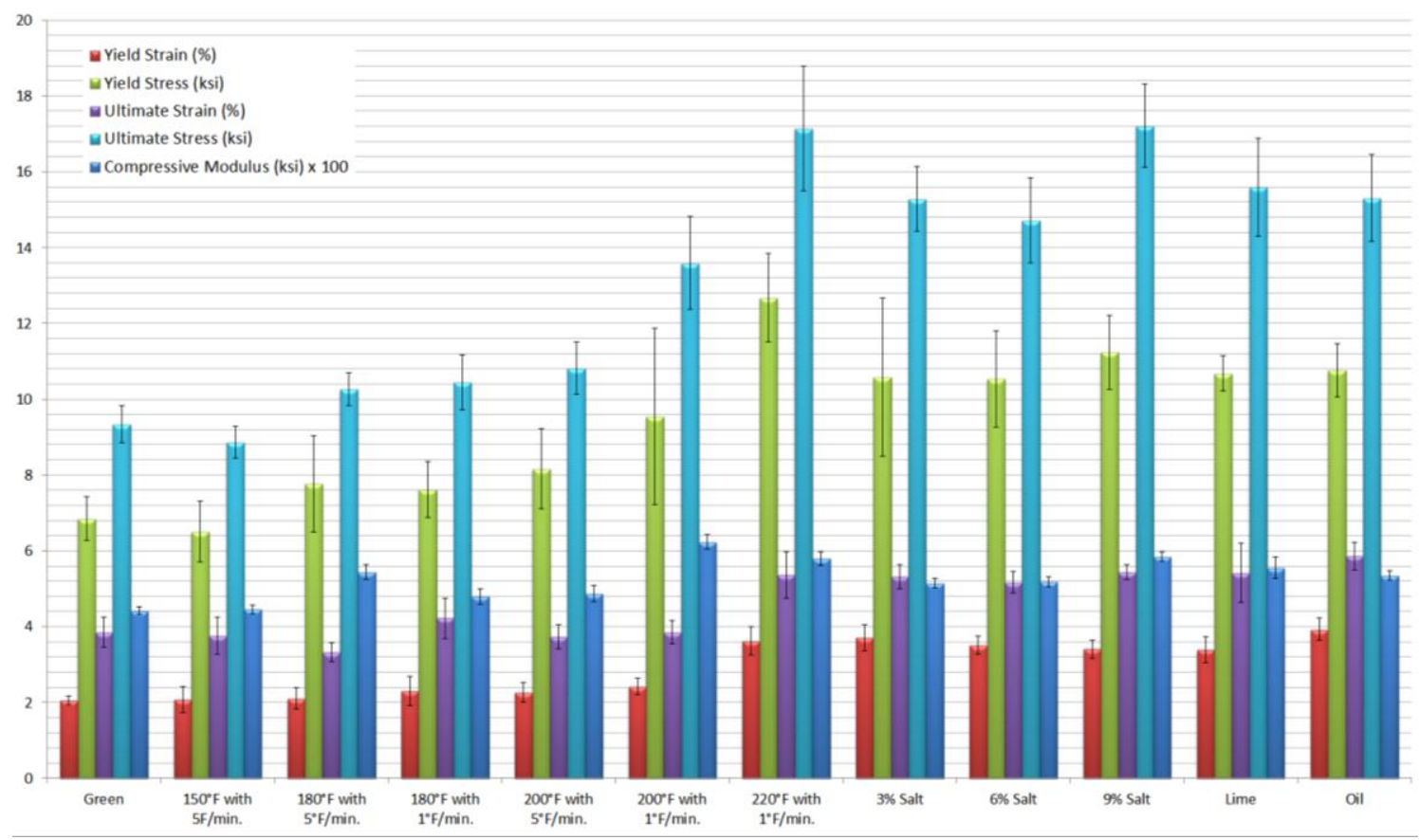

Figure 4-43. Summary of treatment effects on compressive strain, stress, and modulus

produces specimens with improved specific strengths by about $8 \%$ or $16 \%$. However, the respective deviation for each test cannot confirm this difference with absolute certainty. The $3 \%$ salt solution shows an improvement of $120 \%$ while the $6 \%$ and $9 \%$ demonstrate an increase of about $112 \%$. Furthermore, the lime and oil samples show less outstanding improvement at $97 \%$ and $105 \%$, respectively. If taking into account the deviation of each test with a chemical treatment, it is quite difficult to argue that one is better than the other. All of the salt treatments fall within the same range generated by deviation limits. In light of this, it is concluded that treating bamboos with these natural chemicals-particularly the salt treatments-for their protective properties against the environment will not diminish their compressive strength. In fact, thermally treating bamboos at $220^{\circ} \mathrm{F}$ with/without the treatments can significantly improve the load-carrying capacity under compression.

In terms of the specific compressive modulus, there is a clear trend that chemical treatments improve the stiffness of untreated bamboo by over $50 \%$. The groups with a 
heat treatment of $180^{\circ} \mathrm{F}$ increased by over $30 \%$, and the groups at $200^{\circ} \mathrm{F}$ increased by over $40 \%$. Also, treatments above $200^{\circ} \mathrm{F}$ at $1^{\circ} \mathrm{F} / \mathrm{min}$. show improvements of about $60 \%$. Consequently, it is understood that green bamboo's stiffness may be increased by curing it at higher temperatures-an intuitive observation now affirmed with testing. As seen from the table, the stiffness improvements increase almost linearly with increasing curing temperatures. However, the data indicates that compressive stiffness improvement past $200^{\circ} \mathrm{F}$ is unlikely since a plateau is observed to extend to the $220^{\circ} \mathrm{F}$

Table 4-1. Average percent changes of compression properties relative to the untreated green bamboo

\begin{tabular}{|c|c|c|c|c|c|c|c|c|c|c|c|c|c|c|}
\hline Treatment & \multicolumn{2}{|c|}{$\begin{array}{c}\text { Modulus } \\
(\%)\end{array}$} & \multicolumn{2}{|c|}{$\begin{array}{c}\text { Specific } \\
\text { Modulus } \\
(\%)\end{array}$} & \multicolumn{2}{|c|}{$\begin{array}{c}\text { Yield } \\
\text { Strain (\%) }\end{array}$} & \multicolumn{2}{|c|}{$\begin{array}{c}\text { Yield } \\
\text { Stress } \\
(\%)\end{array}$} & \multicolumn{2}{|c|}{$\begin{array}{c}\text { Ultimate } \\
\text { Strain } \\
(\%)\end{array}$} & \multicolumn{2}{|c|}{$\begin{array}{c}\text { Ultimate } \\
\text { Stress } \\
(\%)\end{array}$} & \multicolumn{2}{|c|}{$\begin{array}{c}\text { Specific } \\
\text { Strength } \\
(\%)\end{array}$} \\
\hline \multirow{2}{*}{ Green } & \multicolumn{2}{|c|}{--} & \multicolumn{2}{|c|}{--} & \multicolumn{2}{|c|}{--} & \multicolumn{2}{|c|}{--} & \multicolumn{2}{|c|}{--} & \multicolumn{2}{|c|}{--} & \multicolumn{2}{|c|}{--} \\
\hline & $\mathrm{P}$ & $\mathrm{E}$ & $\mathrm{P}$ & $\mathrm{E}$ & $\mathrm{P}$ & $\mathrm{E}$ & $\mathrm{P}$ & $\mathrm{E}$ & $\mathrm{P}$ & $\mathrm{E}$ & $\mathrm{P}$ & $\mathrm{E}$ & $\mathrm{P}$ & $\mathrm{E}$ \\
\hline $\begin{array}{c}150^{\circ} \mathrm{F} \\
\text { at } \\
5^{\circ} \mathrm{F} / \mathrm{min} .\end{array}$ & 1 & 0 & 14 & \pm 2 & 2 & 0 & -5 & \pm 1 & -2 & 0 & -5 & 0 & 7 & 0 \\
\hline $\begin{array}{c}180^{\circ} \mathrm{F} \\
\text { at } \\
5^{\circ} \mathrm{F} / \mathrm{min} .\end{array}$ & 23 & \pm 4 & 44 & \pm 7 & 3 & 0 & 13 & \pm 2 & -14 & \pm 2 & 10 & \pm 1 & 28 & \pm 2 \\
\hline $\begin{array}{c}180^{\circ} \mathrm{F} \\
\text { at } \\
1^{\circ} \mathrm{F} / \mathrm{min} .\end{array}$ & 9 & \pm 1 & 32 & \pm 5 & 12 & \pm 2 & 11 & \pm 1 & 10 & \pm 2 & 12 & \pm 1 & 36 & \pm 3 \\
\hline $\begin{array}{c}200^{\circ} \mathrm{F} \\
\text { at } \\
5^{\circ} \mathrm{F} / \mathrm{min} .\end{array}$ & 10 & \pm 2 & 44 & \pm 7 & 11 & \pm 1 & 19 & \pm 3 & -3 & 0 & 16 & \pm 1 & 51 & \pm 4 \\
\hline $\begin{array}{c}200^{\circ} \mathrm{F} \\
\text { at } \\
1^{\circ} \mathrm{F} / \mathrm{min} .\end{array}$ & 41 & \pm 6 & 63 & \pm 9 & 19 & \pm 2 & 39 & \pm 10 & 0 & 0 & 46 & \pm 5 & 67 & \pm 4 \\
\hline $\begin{array}{c}220^{\circ} \mathrm{F} \\
\text { at } \\
1^{\circ} \mathrm{F} / \mathrm{min} .\end{array}$ & 31 & \pm 4 & 57 & \pm 8 & 77 & \pm 9 & 85 & \pm 11 & 39 & \pm 6 & 84 & \pm 9 & 120 & \pm 10 \\
\hline $3 \%$ Salt & 16 & \pm 2 & 59 & \pm 7 & 81 & \pm 8 & 54 & \pm 12 & 38 & \pm 5 & 64 & \pm 5 & 120 & \pm 9 \\
\hline $6 \%$ Salt & 17 & \pm 2 & 58 & \pm 7 & 71 & \pm 6 & 54 & \pm 8 & 34 & \pm 4 & 58 & \pm 5 & 112 & \pm 9 \\
\hline $9 \%$ Salt & 32 & \pm 4 & 52 & \pm 6 & 66 & \pm 6 & 64 & \pm 8 & 41 & \pm 5 & 84 & \pm 7 & 112 & \pm 8 \\
\hline Lime & 26 & \pm 5 & 49 & \pm 10 & 65 & \pm 8 & 56 & \pm 5 & 41 & \pm 7 & 67 & \pm 7 & 97 & \pm 10 \\
\hline Oil & 21 & \pm 3 & 51 & \pm 6 & 92 & \pm 8 & 57 & \pm 6 & 52 & \pm 6 & 64 & \pm 6 & 105 & \pm 8 \\
\hline
\end{tabular}


treatment. Higher thermal treatment temperatures would have to be investigated to confirm with certainty. Also, the plot suggests the two heat rates do not significantly yield a difference in the stiffness improvement of the bamboo. This observation is an important note when trying to economize energy and time. By analyzing the salt treatments, it is apparent that adding small percentile increases in the salt solution does not significantly affect the stiffness of the bamboo. Perhaps increasing the salt in the solution may increase the stiffness further but that is left for future work. Also, the lime and oil treatments demonstrate minor differences in stiffness improvement compared to green bamboos. Consequently, it is concluded that curing temperatures above $200^{\circ} \mathrm{F}$ and natural chemical treatments do not negatively affect the compressive stiffness of bamboo; therefore, these treatments can be performed on this natural material for their protective properties without a stiffness penalty.

Unlike the specific modulus, the compressive modulus shows a bit more variation in the data. Similarly, all the treatments indicate an improvement relative to the untreated bamboo. However, an anomaly exists at $220^{\circ} \mathrm{F}$ where the compressive modulus suddenly drops to a $30 \%$ increase. In other words, the bamboo does not gain as much stiffness when cured at a temperature of $220^{\circ} \mathrm{F}$. It is very likely, however, that this difference is simply due to the modulus of the specimen-i.e. normalizing this figure by density is required. By analyzing the modulus for the chemically treated groups, the assumption is further cemented by the lower percentage increases relative to the control case. Increasing the amount of salt increases the stiffness by a range of $16 \%$ to $30 \%-$ another intuitive observation confirmed with testing. The $9 \%$ salt increased stiffness the most at $32 \%$ among the salt treatments while the oil and lime fall in between the $6 \%$ and $9 \%$ salt solutions. One interesting observation is that all-except the $9 \%$ salt grouphave lower moduli than the cure temperature at $200^{\circ} \mathrm{F}$. 
Furthermore, examining the stresses of each group also partially validates the initial hypothesis that increasing the salt solution would increase strength. The $9 \%$ salt solution clearly shows a higher yield and ultimate stress over the other two treatments, but an anomaly occurs in the $6 \%$ salt treatment. The $3 \%$ salt solution has a $4 \%$ higher ultimate stress than the $6 \%$ solution. However, the deviation in both samples suggests that the values are within the same range. Another observation to note is that the $9 \%$ salt solution yields a lower specific strength although the $9 \%$ salt solution performed better in terms of ultimate stress.

Similar to the compressive modulus, heat or chemical treatments increase the yield strain of green untreated bamboo. Also, a less obvious pattern to the yield strain does not manifest itself easily from the data. However, one apparent point is that all strains are quite low-very characteristic of brittle materials. The strain of about $5 \%$ is much lower than metals; the brittleness of this material is more closely aligned with carbon fiber. Closely examining the data from this group reveals the majority of the chemically treated specimens to have considerably higher strains. Only the first two specimens exhibit normal strains at yield. The $200^{\circ} \mathrm{F}$ group shows a near absence of change in yield strain, but the group heat treated at $220^{\circ} \mathrm{F}$ demonstrates a strain increase of nearly $80 \%$. The salt treatments do not clarify the situation as their yield strains fluctuate when increasing the salt content. What can be said, nonetheless, is that oil-soaked bamboo has the highest yield strain increase at $92 \%$ among all the groups. It is unlikely that the variability in yield strain is due to testing errors because every single specimen loaded into the testing apparatus followed the same procedure. Perhaps biological variation in the composition of the bamboos after treatment significantly affects this factor.

The ultimate strains of the chemically treated test groups show an increase relative to the untreated bamboo although the simple thermal treatments indicate similar 
performance. The highest increase in strain is seen in the oil group with a value of $52 \%$. The bamboos thermally treated at $180^{\circ} \mathrm{F}$ with a $1^{\circ} \mathrm{F} / \mathrm{min}$. rate demonstrate an ultimate strain approximately $10 \%$ higher than those untreated. Oddly, the $200^{\circ} \mathrm{F}$ group show nearly no change in strain while the $220^{\circ} \mathrm{F}$ has an increase of $39 \%$. Any causes for this are likely explained by the specimens' individual biological imperfections.

\subsection{Tension Mechanical Characteristics}

As outlined in the tension section of ISO standard 22157, the tensile mechanical characteristics are determined with a set of guidelines. These guidelines have regulations for preparing, testing, and analyzing the material properties of bamboos under tension. Many who desire to determine the tensile properties of bamboo understand that performing any tensile test on a whole culm proves to be extremely difficult. Initially, the idea was explored to plug both ends of a culm with a wooden dowel so the Instron grips would not crush the specimen. However, this method proved to be much more time-consuming and a different solution was sought. The next idea was to cut a culm into strips around the circumference and test rectangular-shaped specimens. This method proved to be more practical but a different issue arose during testing: failure often occurred near the grips. Such a failure is classified as an inappropriate failure type, so two solutions are introduced to solve the problem. First, the grip pressure on the Instron is dropped below 100-psi, and secondly each specimen is prepared in a dogbone shape to promote failure near the center of the specimen. The dog-bone shape for tensile testing is also recommended by the standard, so this type of geometry is selected for the specimens. The standard also requires a minimum of 12 samples to determine the mechanical characteristics of bamboo. Furthermore, a loading rate of $0.024-\mathrm{in} . / \mathrm{min}$ is used during the tension test. Lastly, a proper method must be devised during tensile 
testing in order to prevent any slipping effects. The serrated jaws of the Instron grips are selected to provide contact between the specimen and the testing apparatus.

In spite of these guidelines, the standard contains a few issues. This standard strongly advises the tensile specimens to be manufactured in a dog-bone-like shape with dimensions much greater than those permissible with this variety of bamboo. The standard assumes working with bamboo culms much larger than Phyllostachys aureosulcata bamboo. As a result, the specimens are prepared with the smaller dimensions described in the subsequent section. Also, tolerances on the dimensions are not specified so all them are determined to be fabricated with a 0.01 -in tolerance.

\subsubsection{Preparing Tension Specimens}

Each tensile specimen is also carefully prepared at the Aero Structures/Composites lab according to the geometric shape advised in the standard. A bamboo internode is first selected and carefully inspected for any defects along its length. Again, defects in this sense are defined as cracks or irregular cavities on the outer surface of the culm. Consequently, these features are either removed or the bamboo culm is discarded for a different one. After inspection, the culm is cut in half along the longitudinal direction parallel to the fibers using a tile saw. It is important to cut slowly as this procedure places considerable stress on the culm. This will produce two pieces, and then each piece is also cut in half to produce a total of four sections. Each piece is then cut in half one last time to yield a total of eight strips. Once cut, the long strips are transversely cut to a length of six inches in the fiber direction. Figure 4-44 depicts the long strips being cut in half, and Figure 4-46 illustrates how the culm is cut into strips. 

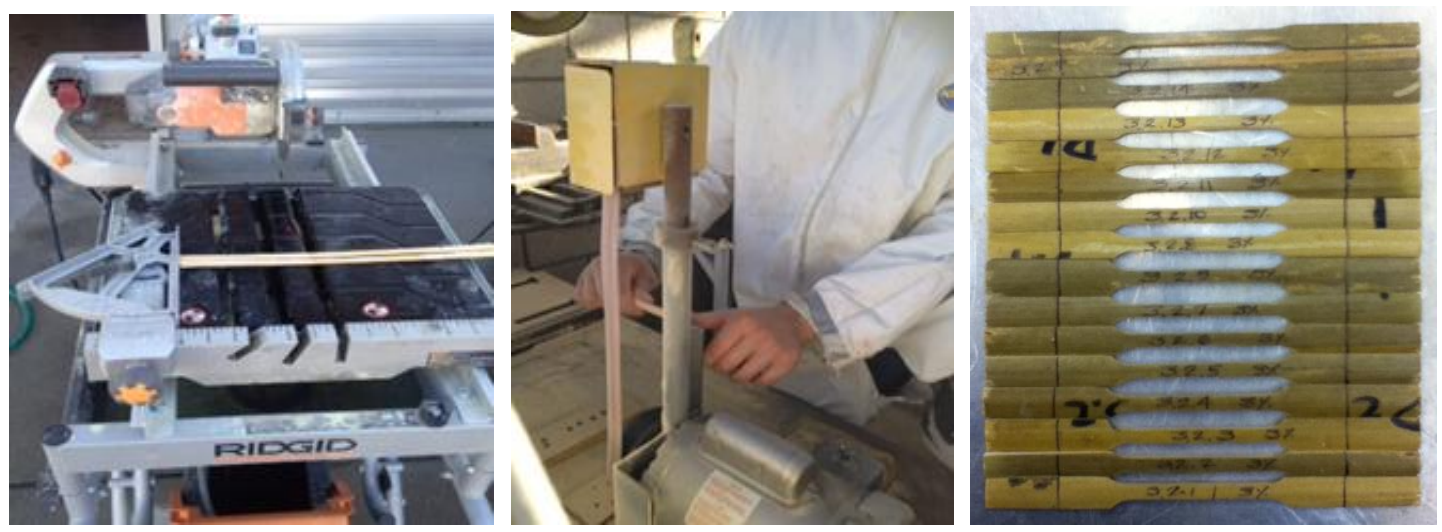

Figure 4-44. Preparing tensile specimens begins by cutting culm into rectangular strips (left), sanding them to the appropriate dimensions (center), and the final specimen geometry (right)

At this point, the dog-bone shape can be fabricated for each bamboo section. Figure 4-47 is provided to illustrate the general dimensions of the specimen. All dimensions are in inches and have a tolerance of 0.01 -in. First, all specimens are sanded with a belt sander on the inner side of the wall to achieve a uniform thickness that is relatively the

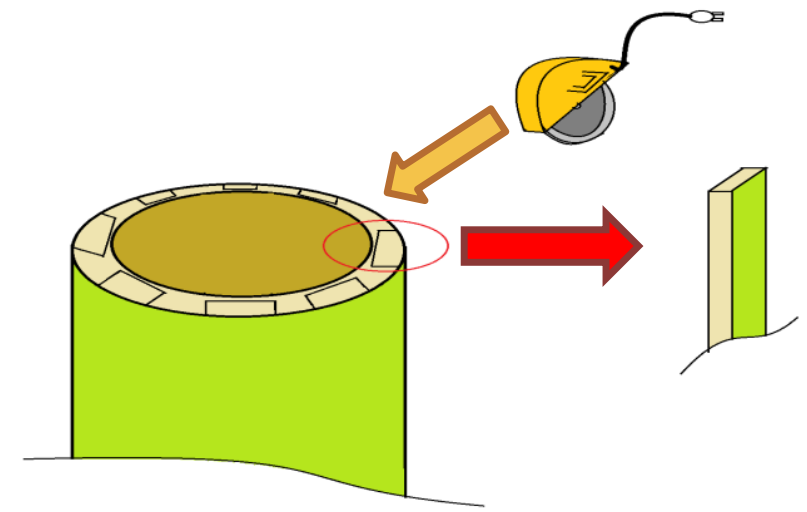

Figure 4-46. Cutting rectangular strips from culm same among all the specimens. It is critical not to sand the outer side of the wall where the strongest fibers are located. In this way, both the inner and outer layers of the bamboo are mechanically tested. In addition to the inner side, the edges of each rectangular strip are sanded to make them straight. The subsequent step requires each strip to be marked on the inner side at the appropriate locations to create the gauge length area. In order to achieve the desired shape in the gauge length area, the strips of bamboo are sanded with a belt sander. Similarly, the transition area between the gauge length and tab section of the specimen is created by using a Dremel tool with the correct diameter. In this manner the gauge 
length should measure 2.00-in. x 0.20-in. x 0.12-in. Figure 4-44 illustrates the final product of an exemplary group of tensile specimens. A note is taken in Figure 4-44 that a line is drawn 1-in. offset toward the center from both edges. This is to provide a guiding area for the grips jaws to clamp on the tabs of the specimen.

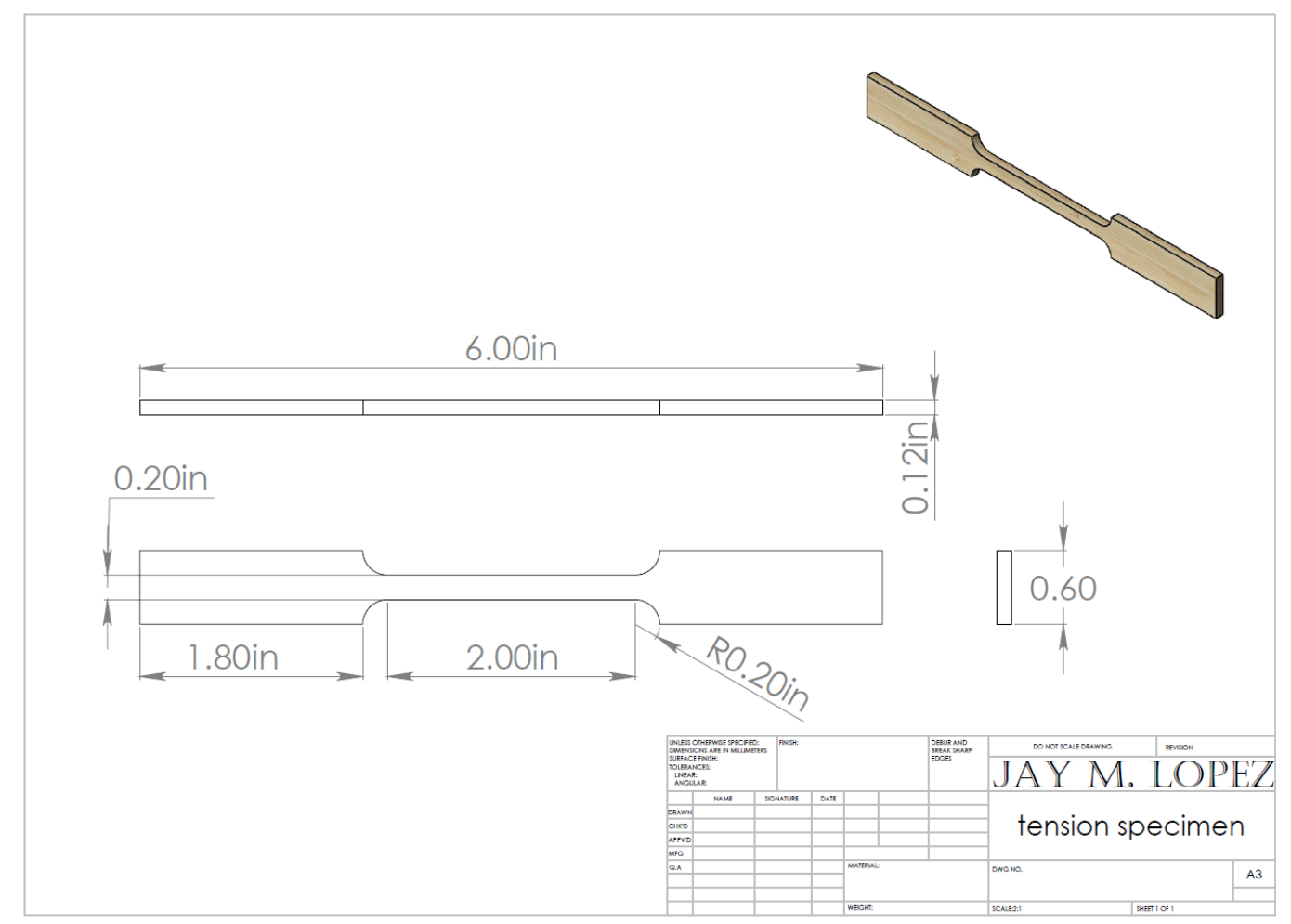

Figure 4-47. Manufacturing drawing for tension specimen

The average area of each tensile specimen is calculated using the equation,

$$
A_{t}=w_{\text {avg }} \cdot t_{\text {avg }}
$$

Where $A_{t}$ is the average cross-sectional area, $w$ is average width of the gauge length, and $t_{\text {avg }}$ is the average thickness of the gauge length. Subsequently, the average volume of each specimen is calculated by,

$$
V_{t}=2 Q t_{\text {avg }}+A_{t} l
$$


Where $V_{t}$ is the corresponding average volume of the tensile specimen, $Q$ is a factor determined by the geometry of the specimen's tabs, $t_{\text {avg }}$ is the average thickness, $A_{t}$ is the average cross-sectional area of the gauge length, and / is the length of the gauge region. The factor $Q$ is calculated by determining the area of the specimen's tab and is measured to be 1.1294 for the vast majority of the samples. Lastly, the density of each specimen is determined with the following equation:

$$
\rho_{t}=\frac{m}{V_{t}}
$$

Where $\rho$ is density, $m$ is the mass of the specimen, and $V_{t}$ is the volume of the entire specimen. Testing can then proceed once these physical parameters are defined.

\subsubsection{Tensile Testing Procedure}

All tensile testing is also conducted using the Instron 8801 machine. Again, the Bluehill 2 software is used to regulate the testing device and the data acquisition computer obtains all testing data. First, the correct tension program created in the Bluehill 2 software is uploaded to the computer and a rate of $0.024-\mathrm{in} . / \mathrm{min}$. is set. This testing procedure measures the tensile extension and load applied to the specimen from the crossheads of the Instron. Then the tensile testing procedure is initiated by calibrating the correct grip pressure to about 100-psi-the lowest setting. The tensile section of the standard does not provide a value on the ideal pressure, but it is known from preliminary testing that pressure values above 100-psi can cause large compressive stresses on the specimen and yield premature fracture and subsequent failure. These grip jaws also provide a contact shear force to adequately place a tension force on the specimen, so it is important not to impart too much transverse stress.

Once the grip pressure is calibrated, the tensile specimen is loaded into the jaw area. The common usage of metal tabs on the grip areas of the specimen is disregarded after 
preliminary tests showed that adding them causes stress concentrations when the grips closed. The relatively soft inner part of the bamboo wall becomes crushed by this effect and premature failure occurred. As a result, only the specimen itself is required to complete the test. It is also important to maintain a gauge length that is parallel to the pulling direction of the jaws; otherwise, off-axis mechanical measurements of the bamboo fibers are tested. Figure 4-48 is shown to demonstrate the correct manner for loading the specimen into the test area.
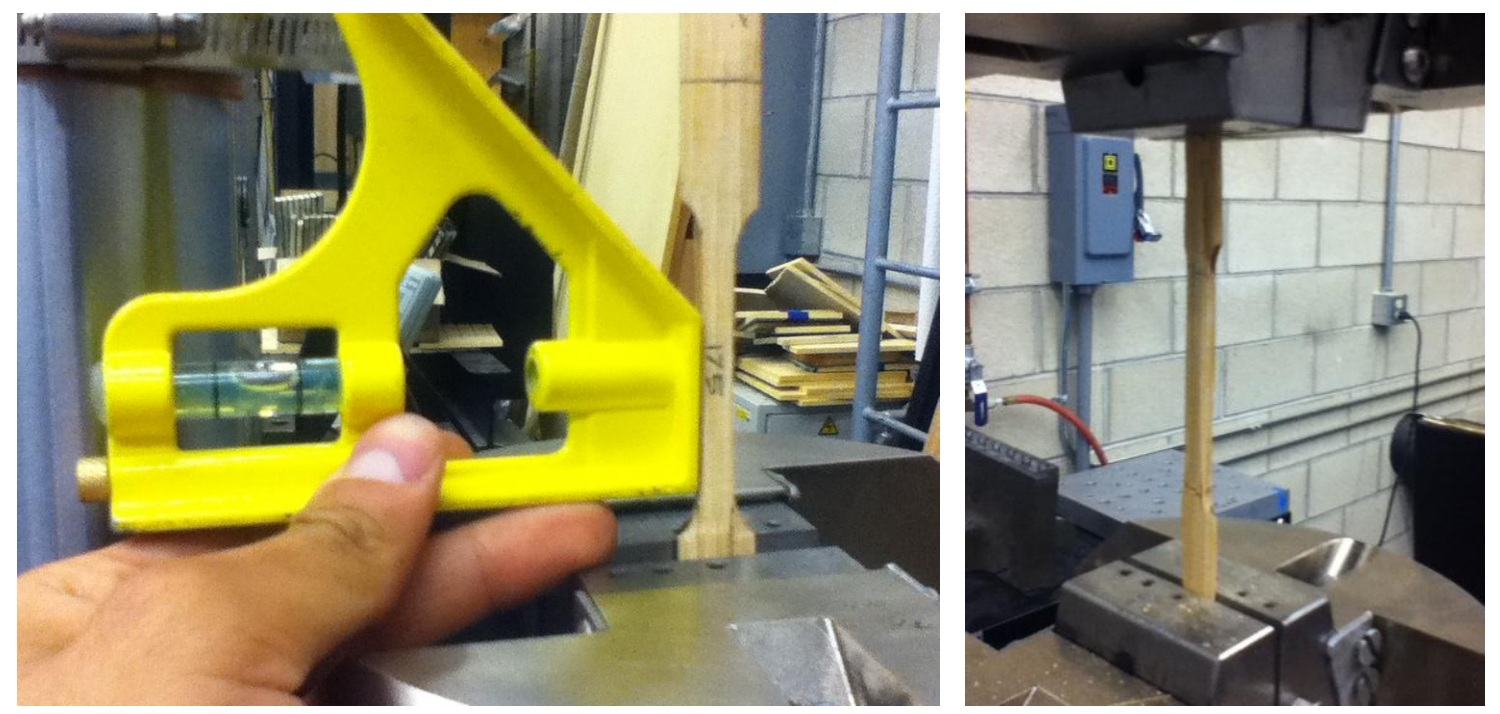

Figure 4-48. Aligning tensile specimen within grip jaws (left) and correct setup in the grip jaws (right)

The jaws are then closed when the correct alignment is achieved for the gauge length. This procedure often places a compressive force on the specimen that will cause it to buckle out of its vertical plane. When this occurs, the detail extensometer controller is utilized to pull the jaws apart and create a tensile force. The tensile force is added until the specimen ceases to buckle and a preload tensile force of less than $10-\mathrm{Ib}_{\mathrm{f}}$ is observed. At this point, the test may begin and the specimen is monitored for the duration of the test. Once the test ends, the specimen is removed from the jaw area, and the area is cleaned of debris for the following test. 


\subsubsection{Results of Tension Tests}

The tensile material properties of this bamboo for various thermal and chemical treatments are investigated to help determine the optimum treatment combination. First, green bamboo without treatment is studied to form a baseline for the mentioned treatments. Thermal treatments of $180^{\circ} \mathrm{F}$ through $220^{\circ} \mathrm{F}$ at a $1^{\circ} \mathrm{F} / \mathrm{min}$. initial ramp-up cure rate are investigated next. Lastly, chemical treatments studied include the varying salt solutions, lime solution, and oil treatment. The results of the tension tests are gathered into a table with the seven mechanical properties to illustrate the tensile properties of the bamboos. Once again a large sample size is favored in these tests to reduce the variability associated with the natural material. Tables displaying all the specimens' geometries are first studied to illustrate any variations that may be pertinent to the tests. In addition to the mechanical properties of interest, a plot of the tensile stress-strain curves are provided. Also, a discussion of the failure modes is supplied with visualizations for clarity. Lastly, a table summarizing all of the specimens' tensile characteristics for each treatment group is provided in this section. It is important to note that the $150^{\circ} \mathrm{F}$ treatment and all the treatments cured at $5^{\circ} \mathrm{F} / \mathrm{min}$. are omitted from the tension study due to the expected low performance discovered in the compression analysis and the dwindling supply of bamboo at this point in the research.

The proper analytic equations are defined to study the mechanical behavior of bamboo under tension. The following discussion describes the manner in which the mechanical properties are calculated. Before any mechanical properties can be calculated, it is vital to establish the definition of tensile stress and strain in this study. Tensile stress is defined as, 


$$
\sigma_{t}=\frac{F_{t}}{A_{t}}
$$

Where $\sigma_{t}$ is the tensile stress, $F_{t}$ is the tensile force and $A_{t}$ is the cross-sectional area computed from the average dimensions of the gauge length region in the specimen. Additionally, tensile strain is defined as,

$$
\varepsilon_{t}=\frac{\Delta}{l}
$$

Where $\varepsilon_{t}$ is the tensile strain, $\Delta$ is the extension of the specimen, and $I$ is the gauge length of the specimen. The main mechanical properties can then be employed to characterize the tensile behavior of the tests, so reference is given to the equations established in the Bamboo Mechanical Characteristics section.

\subsubsection{Untreated Bamboo Tension Results}

The first group tested under tension is the untreated bamboo sample set. This green bamboo group serves as the baseline case from which to compare the results of the different treatments. In this way, any improvements in its mechanical properties can be noted and optimized for structural application.

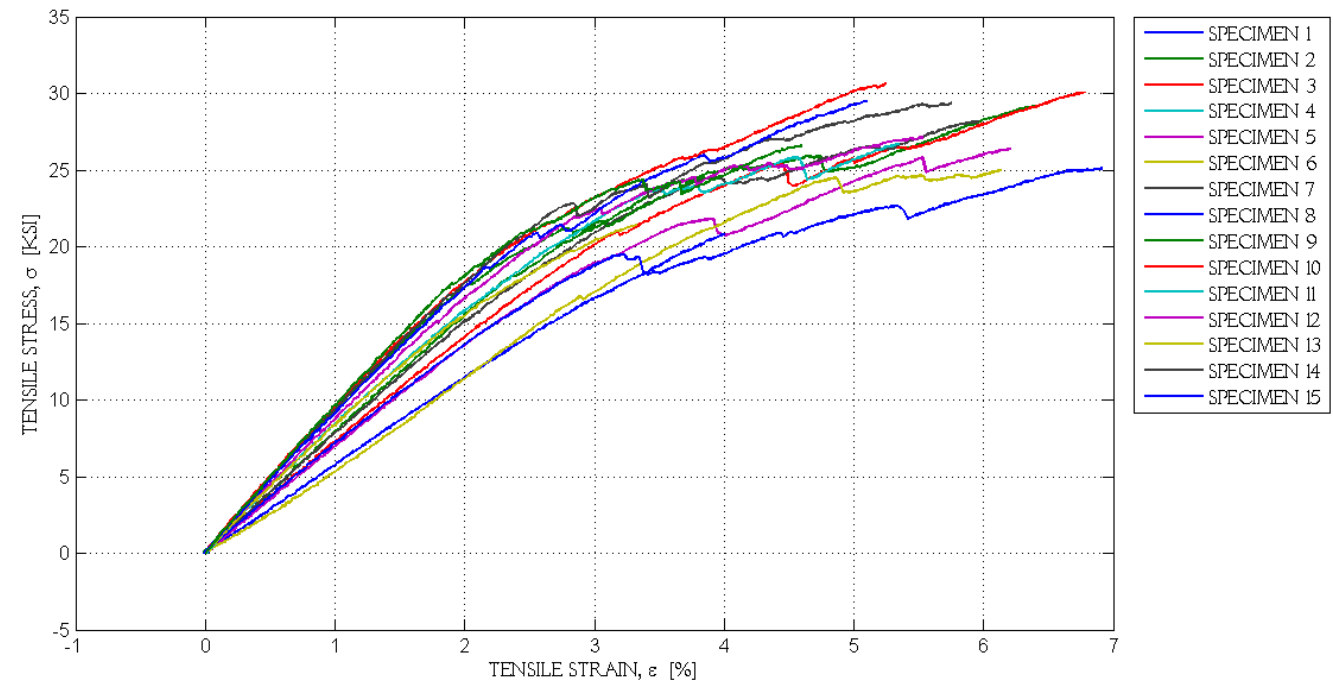

Figure 4-49. Untreated bamboo tensile stress-strain curves 
Figure 4-49 displays the stress-strain curve of the green bamboo group. By analyzing the raw data supplied in the appendix, one can see that these curves show less variation. Once again, the variation seen among the specimens is due to natural factors of the bamboos.

Unlike the compression tests, the tensile test shows a linear elastic region from the very beginning of the test and demonstrates a largely brittle curve. The linear elastic and nonlinear regions of the curves are clearly evident in the plot. Unlike ductile materials, such as metals, that display a nonlinear portion in the elastic zone, brittle materials do not explicitly show a yield point or prolonged plastic region. Many of the curves visually display varying slopes in the linear portion of the curve. The end of the elastic region appears to be around $13.1-\mathrm{ksi}$; a corresponding strain of $1.71 \%$ is also evident. As noted from the plot, the variation picks up again when entering the nonlinear portion of the curve. Several unseen factors attributed to the natural character of the bamboo may explain the variation in the plastic region of the stress-strain curve. Lastly, all tensile tests reveal this type of stress-strain curve.
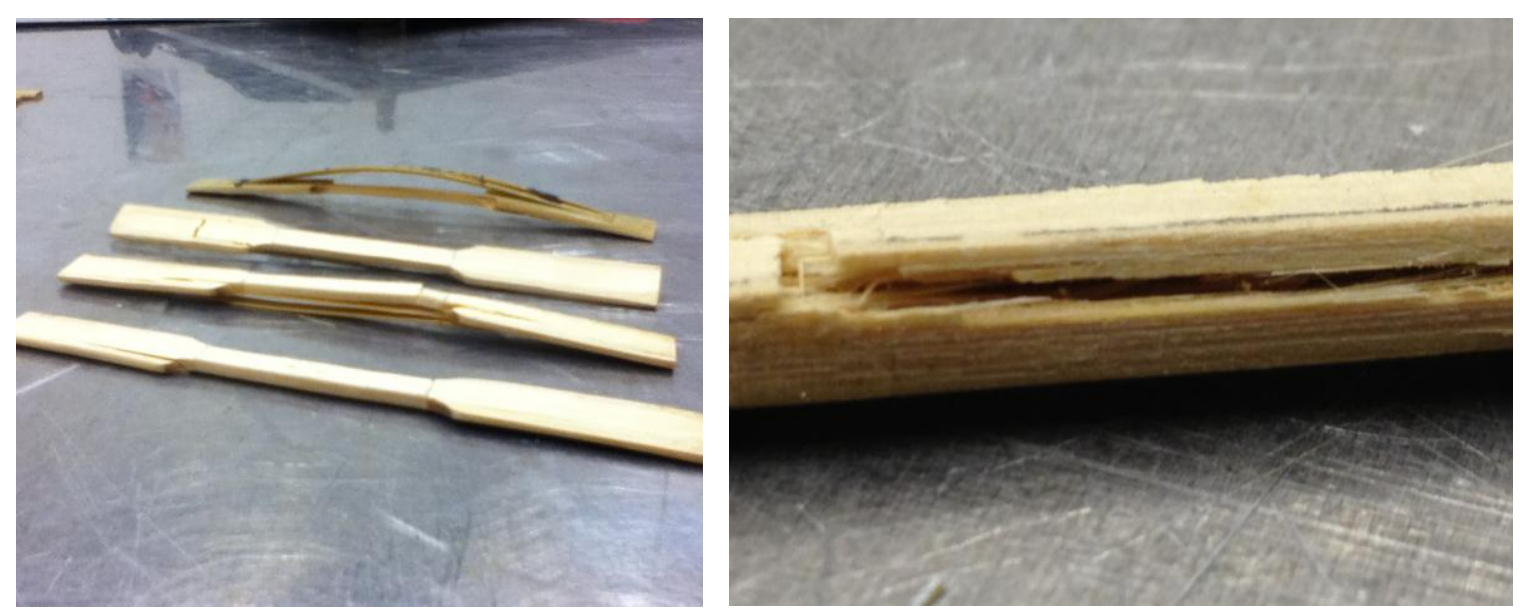

Figure 4-50. Common tensile failure types: four main failures (left), and enhanced view of fiber delamination (right) 
Bamboo material is characterized as having a few failure types under tensile forces. A few notable failure types are observed in the tension test. Figure 4-50 is shown to provide a visualization of the common failures. The stress-strain curve often displays brief "dips" on the way up to the ultimate stress; each of these dips is a segment of fibers failing under tension. Often times the localized failure events are only heard by audible cracks in the specimen. Eventually, the outer layers of the bamboo culm fails and is noticed as a sort of fiber-shredding in the longitudinal direction. The common failure type often shows the specimen split in half or a region of splitting that protrudes from the
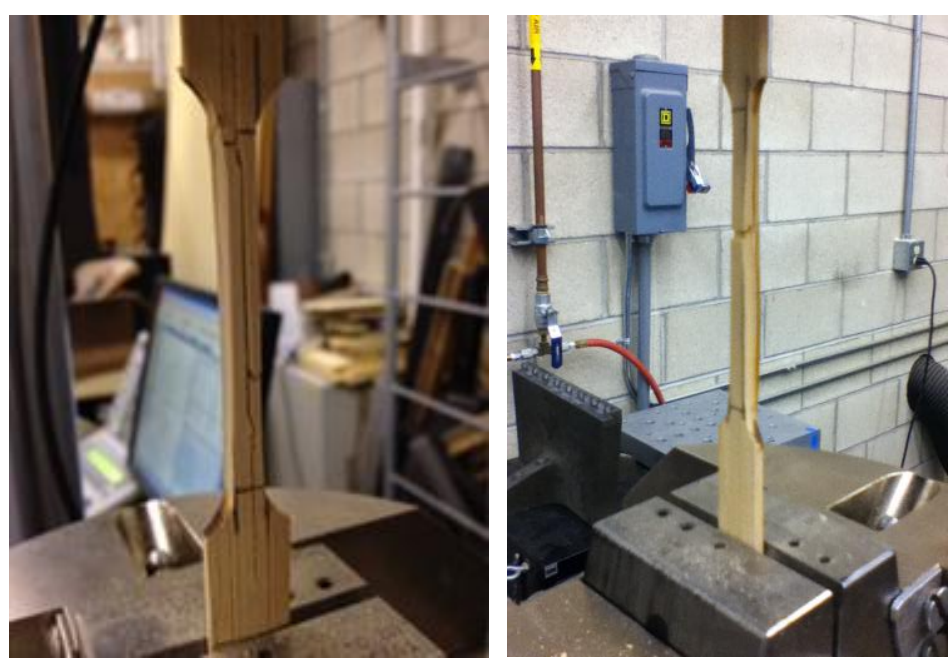

Figure 4-51. Most common tensile failure types characterized as splitting specimen in a "buckling" manner. An example of this characteristic is shown in

Figure 4-50. It is also apparent from the failures that bamboo exhibits some of the same characteristics of bi-directional fiberreinforced composites. In a biological sense this observation corresponds with the molecular make-up of bamboo. As discussed previously, bamboo is composed of the main cellulose fibers and transverse-running hemicellulose fibers. The dominant cellulose fibers carry much of the stress but often do not fail during loading. On the other hand, the hemicellulose fibers are often the elements of the bamboo that fail first. Since many of these fibers sit off-axis to the force direction, failure is much more likely to happen. These fibers characterize the shear-like fractures observed in the photograph provided. Unlike unidirectional composites that can demonstrate fiber failures all within one region, bamboo exhibits fractures that travel in the transverse direction as well as the longitudinal direction. It is 
important to note that the splitting occurrence in the tab areas are due to the grip force of the jaws. Remedying this issue was very difficult since the grip force could not be lowered any further. Lastly, these failure types are also common in the remaining tensile specimens.

In summary of the green bamboo tensile test, a table outlining the seven main mechanical characteristics is shown in the appendix. For green bamboo of this variety, a specific strength of $\quad 796-k i p s-i n . / / b_{m}$ and ultimate stress of $26.0-\mathrm{ksi}$ is observed. The measured

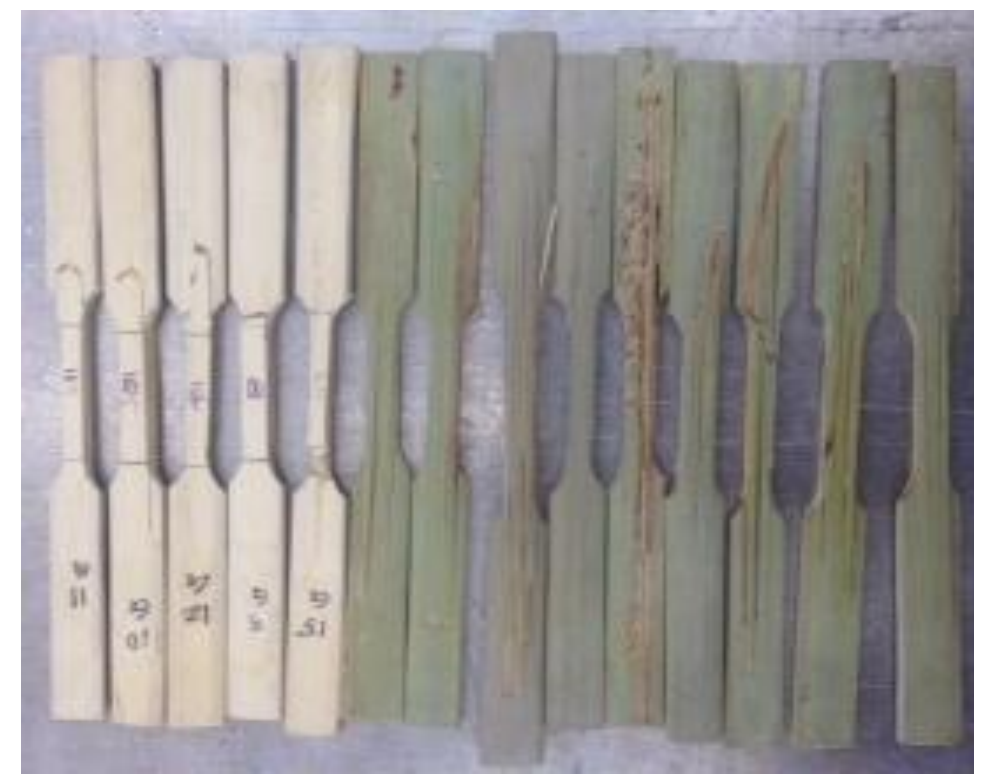

Figure 4-52. Portion of tested untreated tension specimens tensile elastic modulus is $773.2-\mathrm{ksi}$ and the specific modulus is $23690-\mathrm{kips}-\mathrm{in} . / \mathrm{lb}_{\mathrm{m}}$. Statistical analysis of this table exemplifies consistent data considering the natural element of the material. Most of the specimens show a deviation from the average of less than $15 \%$; the strain values demonstrate a deviation of $20 \%$.

\subsubsection{Thermal Treatment Tension Results}

Similar to the compression test, the first goal of thermal treatment is to investigate the potential benefits in improving the tensile mechanical properties of bamboo. The first set of treatments studied in the tension test is the heat-treated group. For the same reasons mentioned in the compression section, these heat treatments are investigated to determine the quantifiable amount of improvement these treatments can yield. This group is comprised of heat treatments ranging from $180^{\circ} \mathrm{F}, 200^{\circ} \mathrm{F}$, and $220^{\circ} \mathrm{F}$-with a 
$1^{\circ} \mathrm{F} / \mathrm{min}$. ramp-up rate. Once again, the $150^{\circ} \mathrm{F}$ heat treatment is excluded from the study since the compressive results indicate this thermal treatment to be the least beneficial in improving the mechanical properties of bamboos. Also, the lower ramp-up rate is selected in an effort to prevent any trace of material degradation-although potentially unnecessary as suggested by the compression analysis.

\subsubsection{1 $180^{\circ} \mathrm{F}$ Thermal Treatment}

The first thermal treatment bamboo studied is the $180^{\circ} \mathrm{F}$ thermal treatment. Figure $4-53$ displays the resulting stress-strain curve for this group. The yield stresses of this group average approximately to $27.8-\mathrm{ksi}$. The corresponding average strain is measured to be 2.39\%. The curves of most of the samples illustrate some variation. Looking at the samples' geometry table leads to a conclusion that perhaps the reason for this anomaly is rooted in some irregularity not related to the specimen's geometry. All of the geometric deviations are below $11 \%$ of the calculated averages-not an uncommon deviation range. Again, it is likely that biological causes are to blame for the observation.

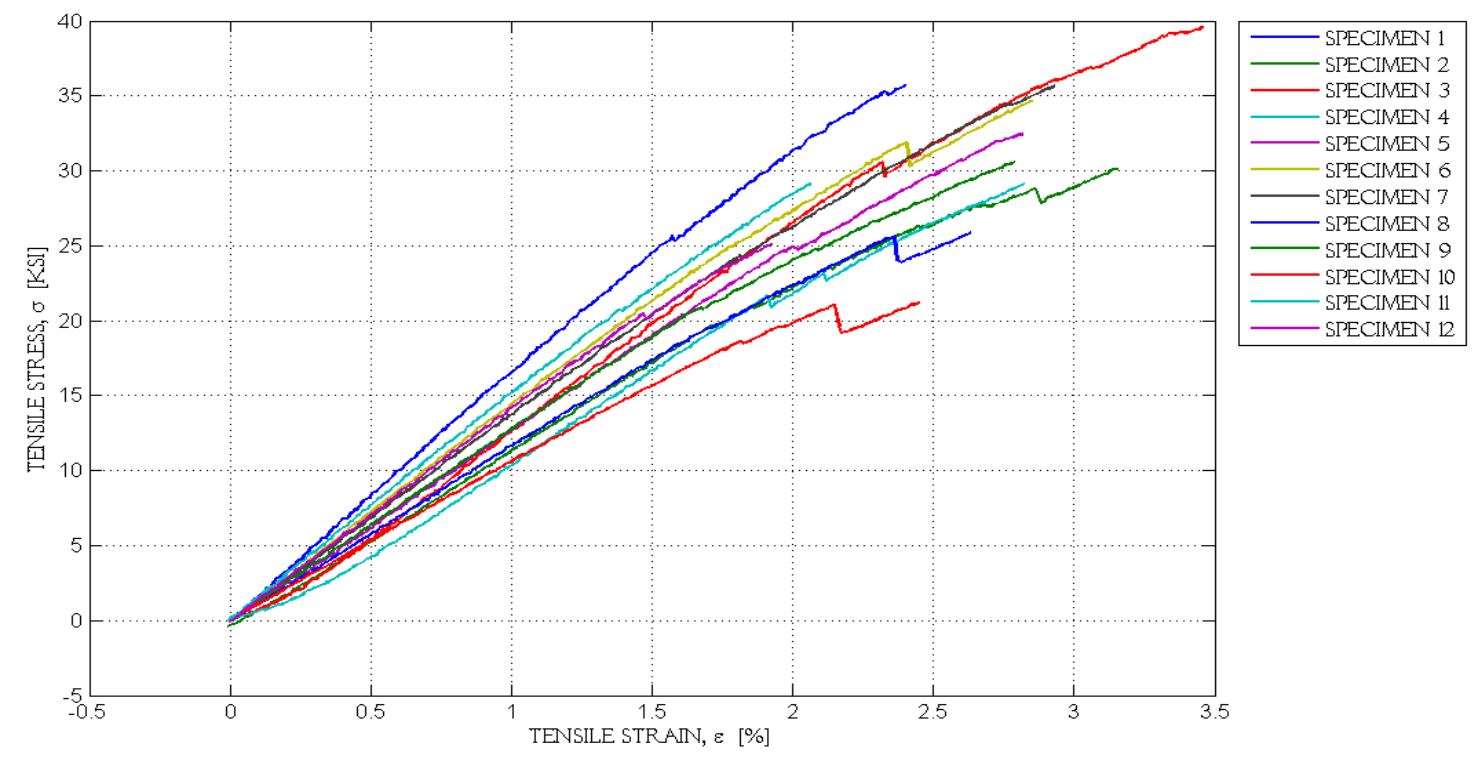

Figure $4-53.180^{\circ} \mathrm{F}$ thermal treatment tensile stress-strain curves 


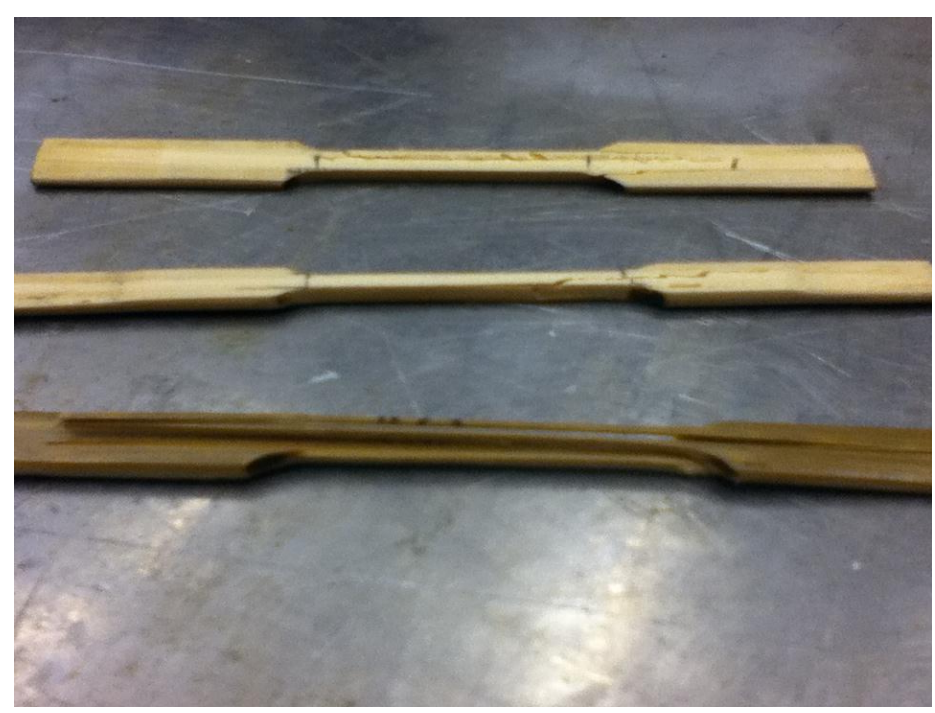

Figure 4-54. Common tensile failure types for $180^{\circ} \mathrm{F}$ thermal treatment
The conclusion of the tensile tests for this thermally treated bamboo group indicates fiber splitting in the longitudinal direction of the fibers. It seems that certain sections of the fibrous wall can withstand greater levels of tensile stress. A few of the specimens show straight

fractures along the fiber direction while others exhibit portions of the fracture that are initially straight and then suddenly change directions in the crack propagation. Furthermore, other samples show fiber splitting on the outer wall. These failure types indicate the separation of the outer fibers from the inner wall of the culm where substantial strength losses are known to exist. Separation of the fibers in this way is attributed to breakage of the bond between the lignin matrix and hemicellulose fibers.

In summary of the bamboo treated at $180^{\circ} \mathrm{F}$, a table is shown displaying the seven main tensile mechanical properties. All of the equations mentioned previously are used to determine these properties. In this group, the average specific strength is

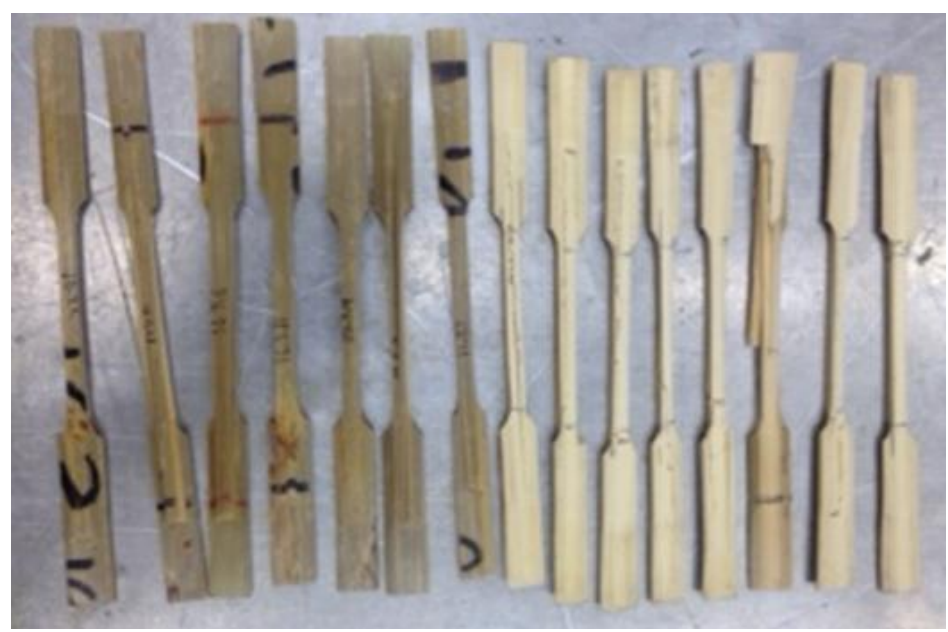

Figure 4-55. Portion of tested $180^{\circ} \mathrm{F}$ with $1^{\circ} \mathrm{F} / \mathrm{min}$. rate tension specimens 
$1335-\mathrm{kips}-\mathrm{in} . / \mathrm{l} \mathrm{b}_{\mathrm{m}}$, and the ultimate stress is $31.2-\mathrm{ksi}$. The tensile modulus is measured to be $1350-\mathrm{ksi}$ and the specific tensile modulus is $58253-\mathrm{kips}-\mathrm{in} . / \mathrm{lb} \mathrm{m}$. The yield strain deviates the most from the average at approximately $20 \%$ while the remaining properties vary by about $13 \%$.

\subsubsection{2 $200^{\circ} \mathrm{F}$ Thermal Treatment}

The second heat-treated bamboo group is the thermal treatment at $200^{\circ} \mathrm{F}$. Figure $4-56$ shows the stress-strain curve of the $200^{\circ} \mathrm{F}$ thermal treatment. As precedent from the force-extension curve supplied in the appendix, the variation is also apparent in this figure. The end of the linear portion of the stress strain curves occurs approximately at 30.5-ksi and $2.51 \%$ strain. Possible explanations for the deviation may lie in the varying thickness of the specimens or the natural groove on the outer wall that gives this species of bamboo its name. It is important to address the fact that excluding this groove can give an inaccurate representation of this bamboo species. Therefore, little can be done to avoid this issue.

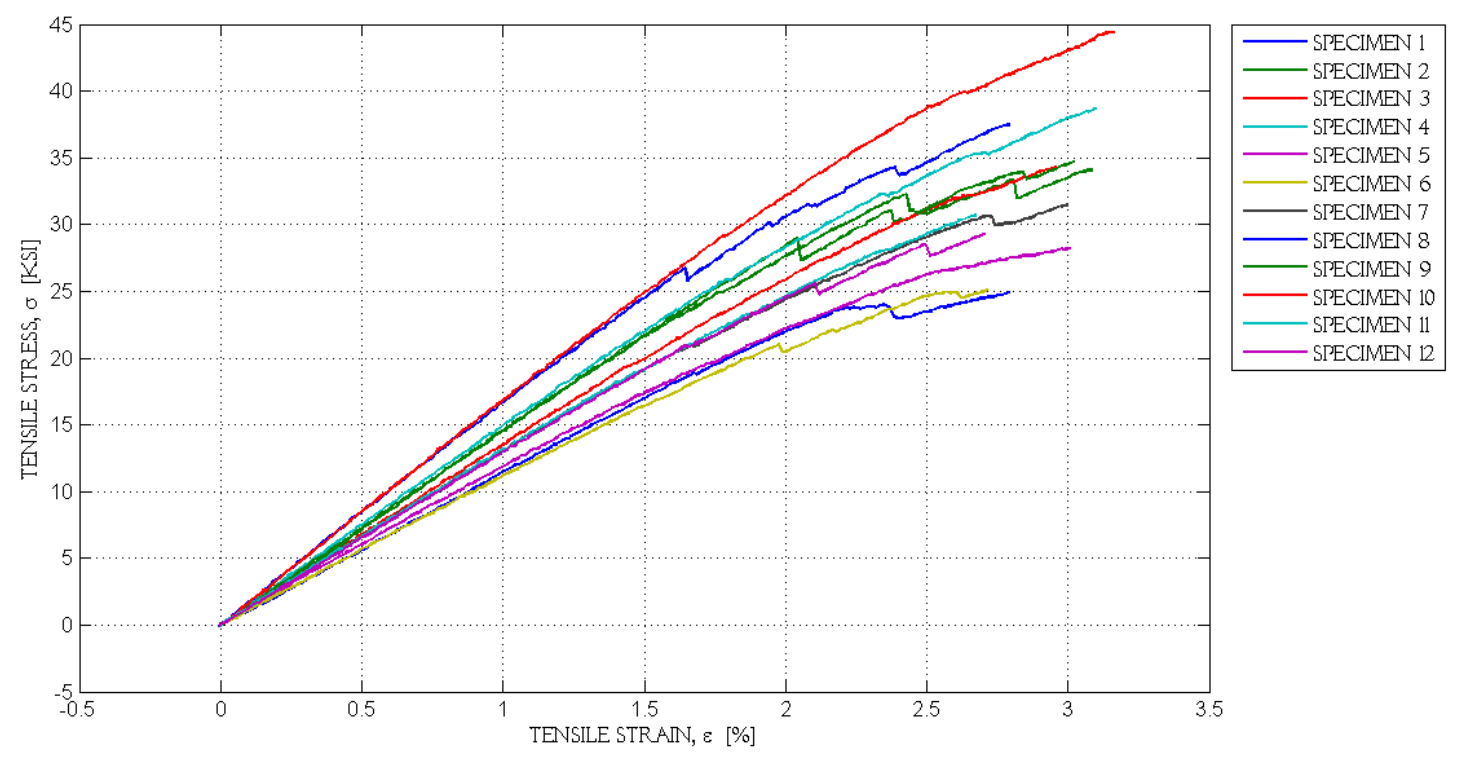

Figure $4-56.200^{\circ} \mathrm{F}$ thermal treatment tensile stress-strain curves 
Failure types observed for bamboos thermally treated at $200^{\circ} \mathrm{F}$ indicate mostly fiber splitting. A few exceptions demonstrate little fracture propagation, and one instance shows a complete separation between the fibers as seen in Figure 4-58. The majority of the specimens, however, failed in a similar

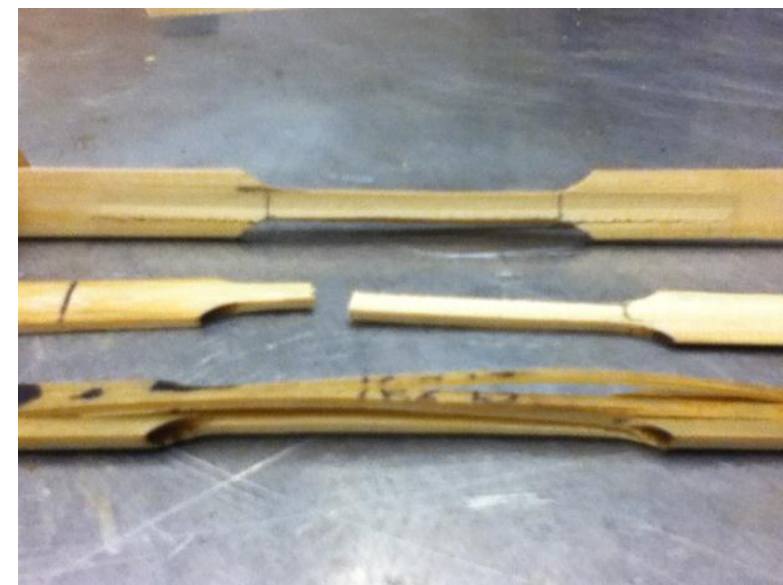

Figure 4-58. Common tensile failure types for $200^{\circ} \mathrm{F}$ thermal treatment

fashion to the bottom-most specimen in Figure 4-58. Evidently this type of thermal treatment promotes little fiber splitting in the bamboo. Very few fractures are also observed from the samples in this test.

In summary of the bamboo treated at $200^{\circ} \mathrm{F}$, a table is shown in the appendix displaying the main mechanical properties. In this group, an average specific strength of $1333.7-\mathrm{kips}-\mathrm{in} / \mathrm{l} \mathrm{b}_{\mathrm{m}}$ and ultimate stress of $32.8-\mathrm{ksi}$ is measured. A tensile modulus of 1390 $\mathrm{ksi}$, and specific modulus of 56733-kipsin. $/ \mathrm{lb}_{\mathrm{m}}$ is also calculated. In this test group, the yield strain also deviates from the average by approximately $21 \%$

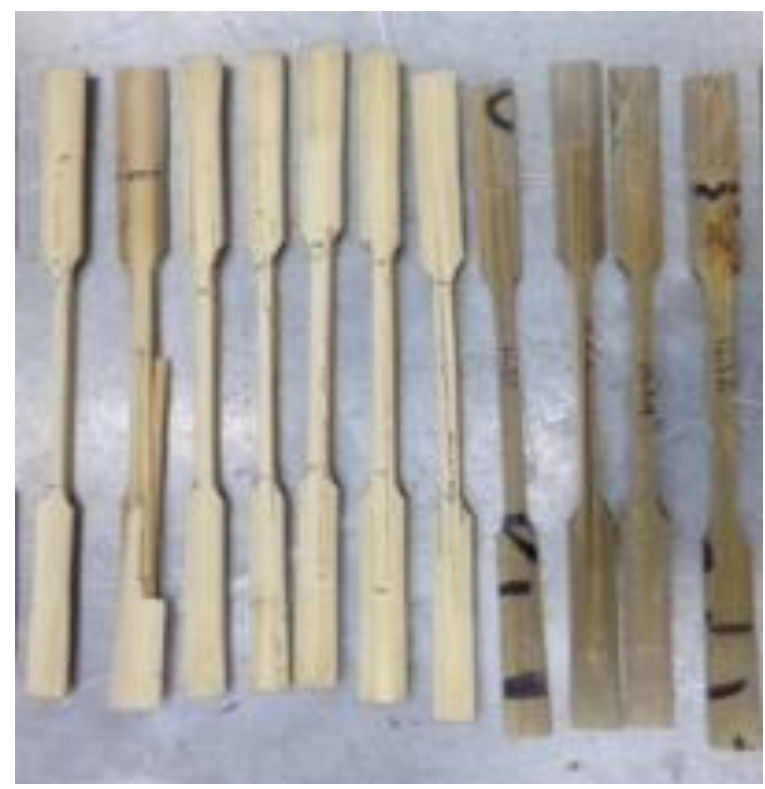

Figure 4-57. Portion of tested $200^{\circ} \mathrm{F}$ with $1^{\circ} \mathrm{F} / \mathrm{min}$. rate tension specimens

while the remaining properties vary by about $15 \%$. At this point, a trend is starting to develop of the yield strain producing the highest deviation. The physical explanation 
behind the analysis is found in some of the specimens not demonstrating a yield point at all. A few specimens demonstrate this outcome: specimens three, four, five, and ten.

\subsubsection{3 $220^{\circ} \mathrm{F}$ Thermal Treatment}

The final thermal treatment conducted is the heat treatment at $220^{\circ} \mathrm{F}$. The importance of this heat treatment warranted several specimens to adequately capture the correct tensile properties of this bamboo group. Initial testing data demonstrated a relatively high deviation among the samples, so it was imperative to include another set of specimens. After testing it was discovered that the deviations for the majority of the mechanical characteristics dropped by more than half. Figure 4-59 displays the resulting stressstrain curve for the group heat treated at $220^{\circ} \mathrm{F}$. Confidence in the data is again

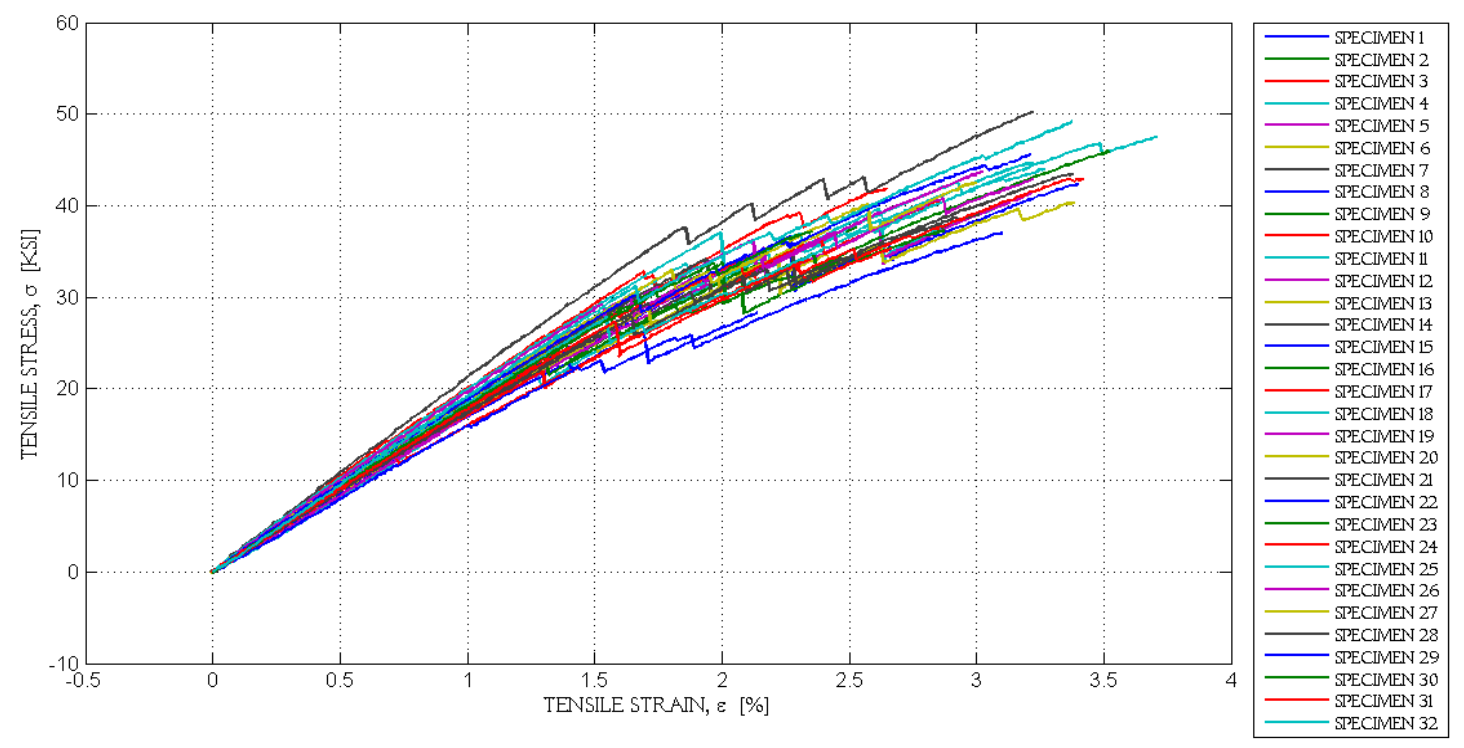

Figure 4-59. $220^{\circ} \mathrm{F}$ thermal treatment tensile stress-strain curves

established with the consistent curves shown. The yield stress of the group varies mostly around $29.7-\mathrm{ksi}$ and the yield strain is calculate to be approximately $1.73 \%$. It is determined that some of the variation attributed to this group is due to geometric inconsistencies and natural biological factors. Reasoning behind this conclusion is found in the table of physical properties housed in the appendix of this work. As seen from the 
figure, a few specimens display a "saw-tooth" behavior in the stress-strain curve. Sudden minor drops in stress are observed and are explained by the individual fiber failures within the specimen. An explanation of this phenomenon is briefly discussed earlier in the Untreated Bamboo Tension Results subsection. This characteristic is highly variable among the specimens and is due to biological imperfections in each sample.

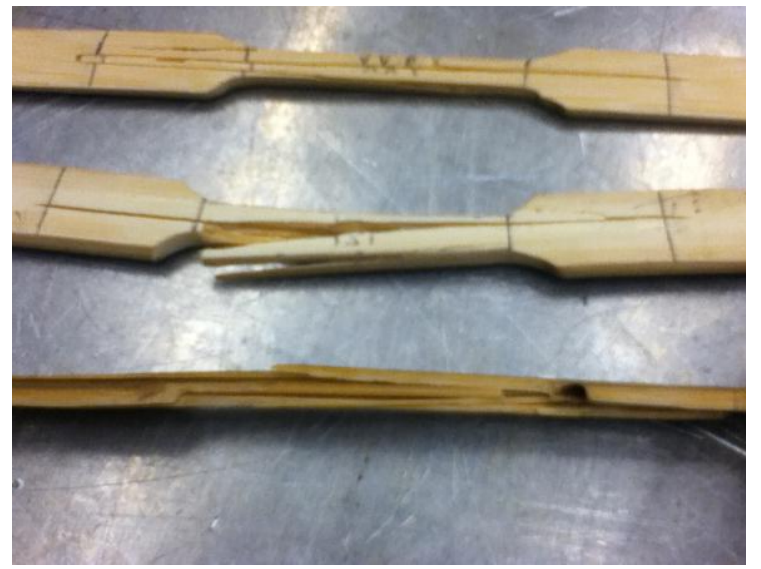

Figure 4-61. Common tensile failure types for $220^{\circ} \mathrm{F}$ thermal treatment

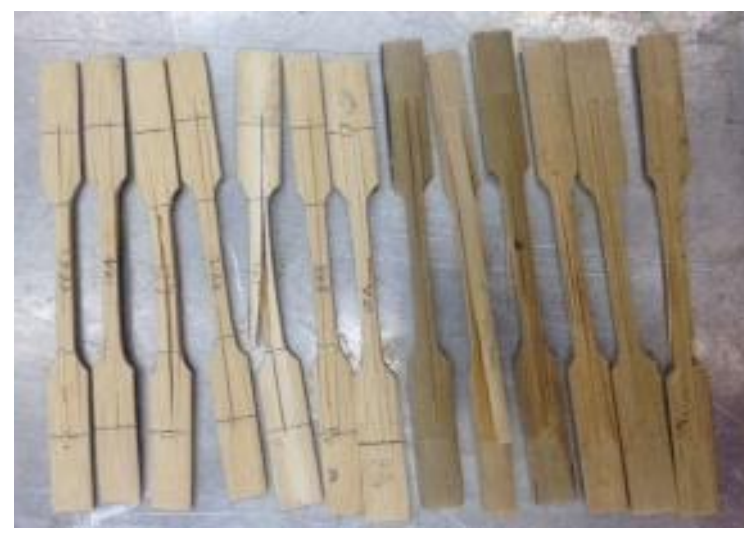

Figure 4-60. Portion of tested $220^{\circ} \mathrm{F}$ with $1^{\circ} \mathrm{F} / \mathrm{min}$. rate tension specimens
Unlike the previous treatments tested, straight longitudinal fractures and directionally-changing fiber splitting dominate the failure types observed in this sample group. Segments of the samples are shown to crack in certain areas to demonstrate a lot of fractures along the length of the specimen. Also, it appears the deformation for this type of treatment is significantly different from the previous bamboo treatments. The damage in the samples is self-evident in the figures.

In summary of the bamboo treated at $220^{\circ} \mathrm{F}$, a table is shown displaying the main mechanical properties. In this group, the average specific strength is 1393-kips-in.// $\mathrm{b}_{\mathrm{m}}$ and the ultimate stress is 40.2 ksi. The elastic modulus is $1866-k s i$ and the specific tensile modulus is 64597-kipsin./lb $b_{m}$. From this test group, the yield strain deviates the most from the average at $19 \%$ while the rest are at $13 \%$. The deviations in this group are lower than the others in some 
respective points. Surely the size of the sample set plays a large part in reducing the deviation in this sample group.

\subsubsection{Thermal Treatment with Organic Chemical Additives Tension Results}

In the same manner as the compression test, the aim of this section of the research is to determine the validity of the natural chemical treatments and their effects on the bamboo's tensile material properties. The final set of treatments investigated to improve the mechanical characteristics of bamboo is the thermally treated group with organic chemical additives. The line-up of chemical treatments is composed of the salt, lime, and oil additives mentioned previously. Also, as a reminder, all of these chemical treatments are cured at $220^{\circ} \mathrm{F} / \mathrm{min}$. with a $1^{\circ} \mathrm{F} / \mathrm{min}$. rate for the same reason of rationing the bamboo supply. These treatments are known to protect and preserve this material, but the resultant effects on the mechanical properties have not been studied. Again, the protective properties of these treatments are investigated through the scientific method to search for any effects on the tensile mechanical properties of bamboos.

\subsection{3\% Salt}

First among the chemically treated groups is the bamboo saturated in the $3 \%$ salt solution. Again, initial testing conclusions demonstrated high deviations for this sample group, so additional specimens are tested. The sample size for this group nearly doubled and consequently brought down the deviation across the tensile properties. Figure 4-62 displays the resulting stress-strain curves for the group treated with $3 \%$ salt. The consistency of the data is evident in the stress-strain plot; even the outlier of the raw data-as supplied in the appendix-conforms to the other samples. The yield stress is approximately $32.2-\mathrm{ksi}$; the corresponding strain is determined to be $2.20 \%$. As seen from the figure, many of specimens exhibit the familiar "dips" along its stress-strain 
curve. This phenomenon is, again, due to individual fiber failures in the interior of the specimen.

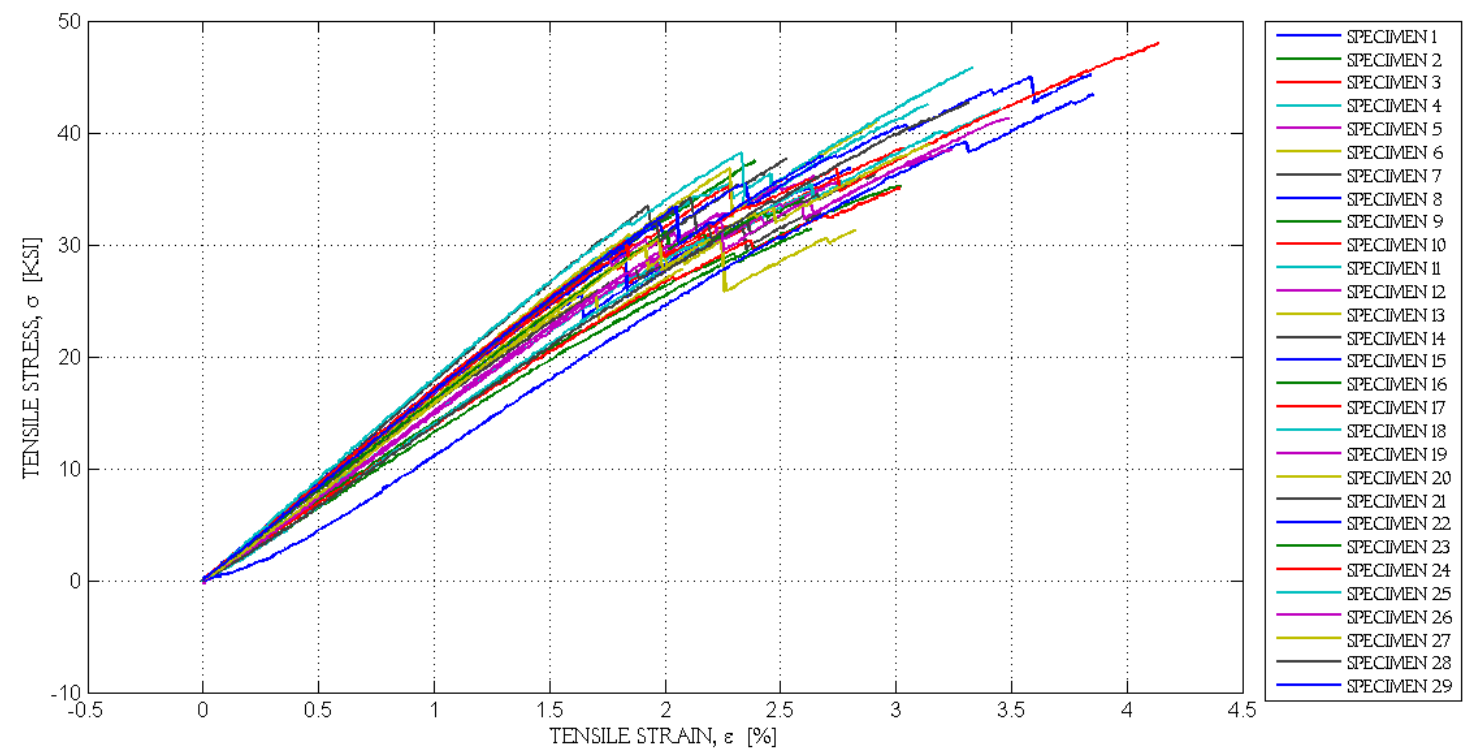

Figure 4-62. 3\% salt treatment tensile stress-strain curves

Directional splitting, straight longitudinal fractures, and fiber splitting are evident from the bamboos treated with 3\% salt. The dominant failure behavior observed is fiber splitting and longitudinal fracture. Longitudinal fracture failure types mostly exhibit a single fracture running the length of the middle portion of the specimen. The majority of the specimens indicate this type of failure as seen in Figure 4-63. Again, the deformation of the specimen indicates much more drastic behavior than the previous thermal treatments.

In summary of the bamboo treated with $3 \%$ salt, a table is shown in the appendix displaying the main mechanical properties. In this group, the average specific strength is 1467-kips-in.//l $\mathrm{b}_{\mathrm{m}}$ and the ultimate stress is $36.5-\mathrm{ksi}$. The tensile modulus is 1613-ksi and the specific modulus is $63117-k i p s-i n . / / b_{m}$. In this set, the yield strain and specific strength deviate the most from the average at $17 \%$ while the rest are at approximately 
$10 \%$. It is interesting to note that the yield strain has consistently driven the deviation to its highest among the other properties. This trend is likely due to the tiny geometric variation in the transition region of the tensile specimen. A few of the specimens had varying transition region geometries during

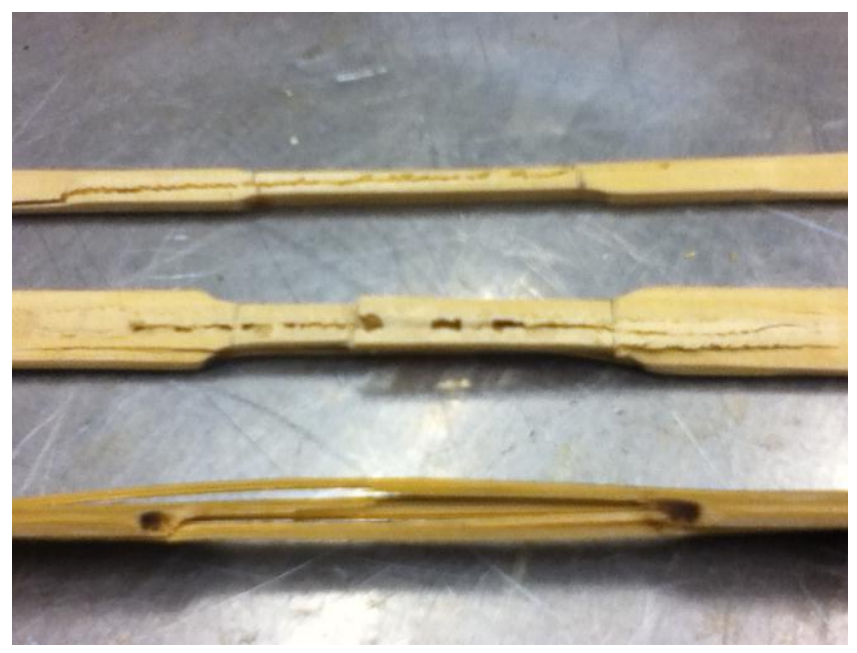

Figure 4-63. Common tensile failure types for $3 \%$ salt treatment preparation and these ultimately manifested differences in the test as seen from the curves. A number of the specimen tests displayed initial fracture propagation in this region before fracture in the gauge area.

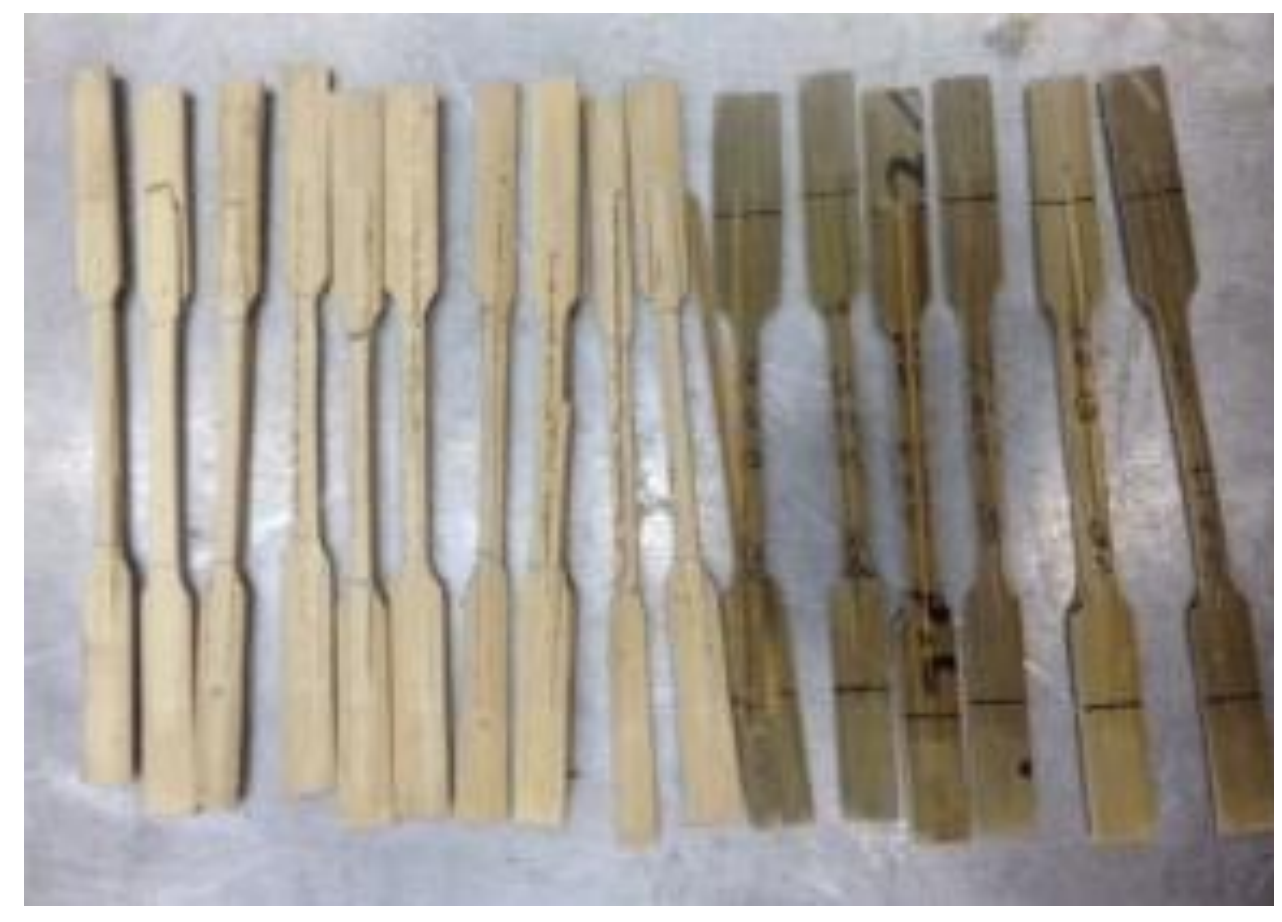

Figure 4-64. Portion of tested $3 \%$ salt tension specimens 


\subsection{6\% Salt}

The second group among the chemically treated sets is the bamboo soaked in the $6 \%$ salt solution. Figure 4-65 displays the resulting stress-strain curves for the group treated with $6 \%$ salt. The data appears to be really consistent for the majority of the specimens. Many of the specimens represent very brittle behavior as their yield point lies very close to the ultimate stress on the stress-strain curve. A few of the specimens exhibit the dipping effect referenced earlier. Of course, this dipping behavior is very characteristic of the tensile nature of the bamboo and is confirmed again under this type of treatment. In either case, the curves demonstrate fairly consistent data. The yield stress is determined to be $26.4-\mathrm{ksi}$ and the corresponding strain is $2.08 \%$.

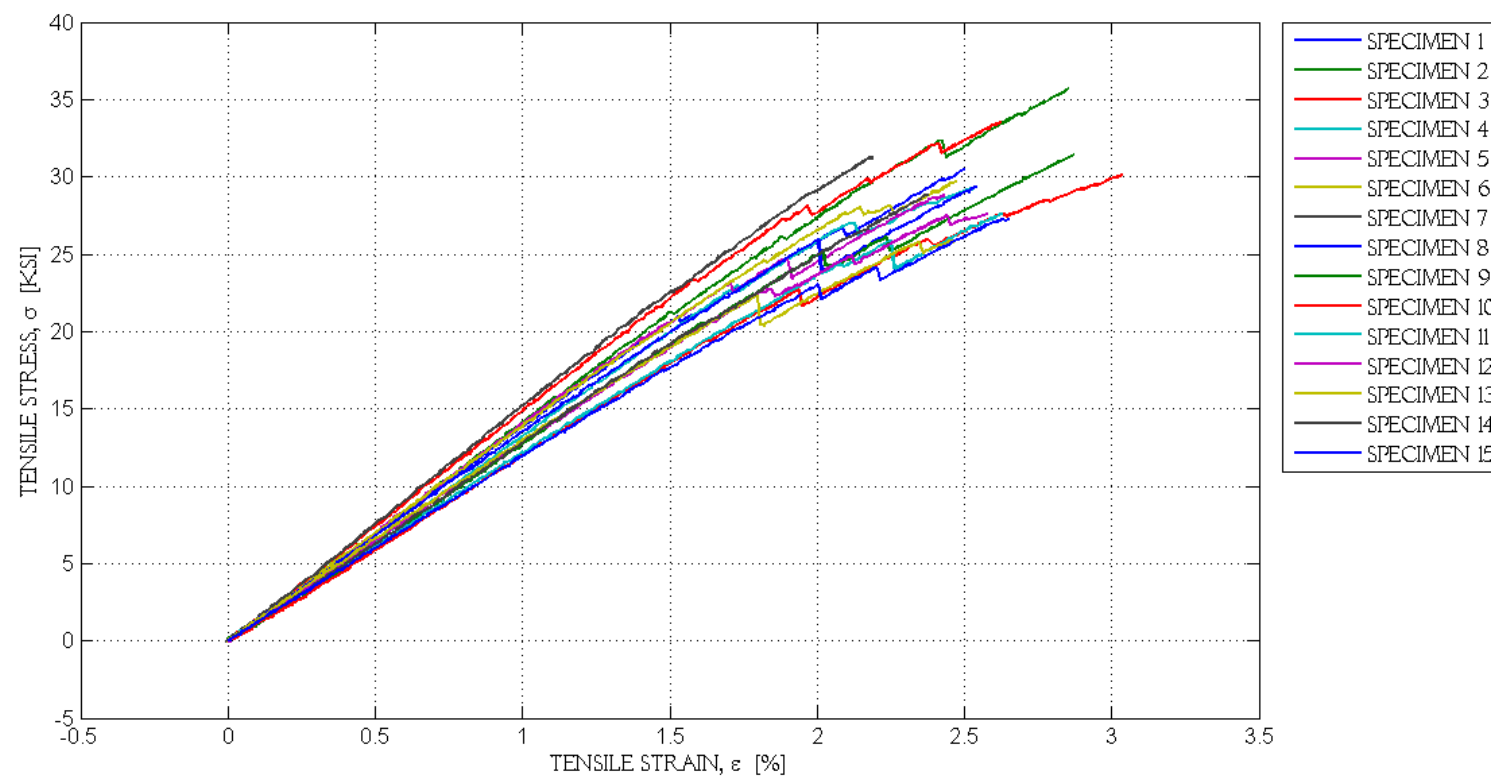

Figure 4-65. 6\% salt treatment tensile stress-strain curves

Bamboos treated with $6 \%$ salt indicate fiber splitting, complete fiber separation, and longitudinal fracture. Longitudinal fracture and fiber splitting are the most common failures among the three types observed. Complete fiber separation in this group is quite rare and all the specimens demonstrate only part of the specimen thickness to separate 
in the gauge area. Another notable fact is that mostly single fractures are found near the center of the gauge region on the specimen.

In summary of the bamboo treated with $6 \%$ salt, a table is shown displaying the main mechanical properties for the tensile test. In this group, the average specific strength is 1286 -kips-in.//b $/ \mathrm{b}_{\mathrm{m}}$ and the ultimate stress is 29.8-ksi. The tensile modulus is $1355-\mathrm{ksi}$ and the specific tensile modulus is 58543-kipsin. $/ \mathrm{lb}_{\mathrm{m}}$. Again, the yield strain deviates the most from the average at $11 \%$ while the rest are at $8 \%$. The $6 \%$ salt treatment group

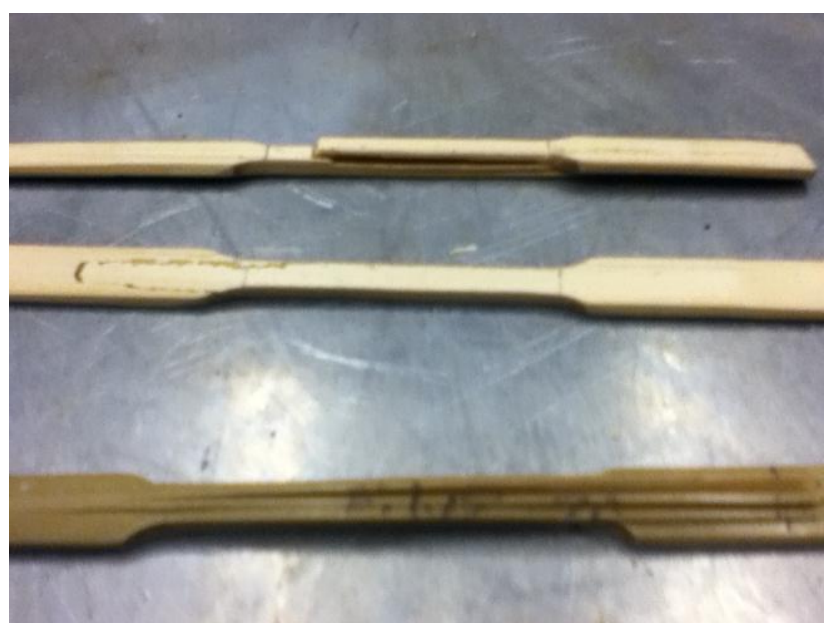

Figure 4-67. Common tensile failure types for $6 \%$ salt treatment

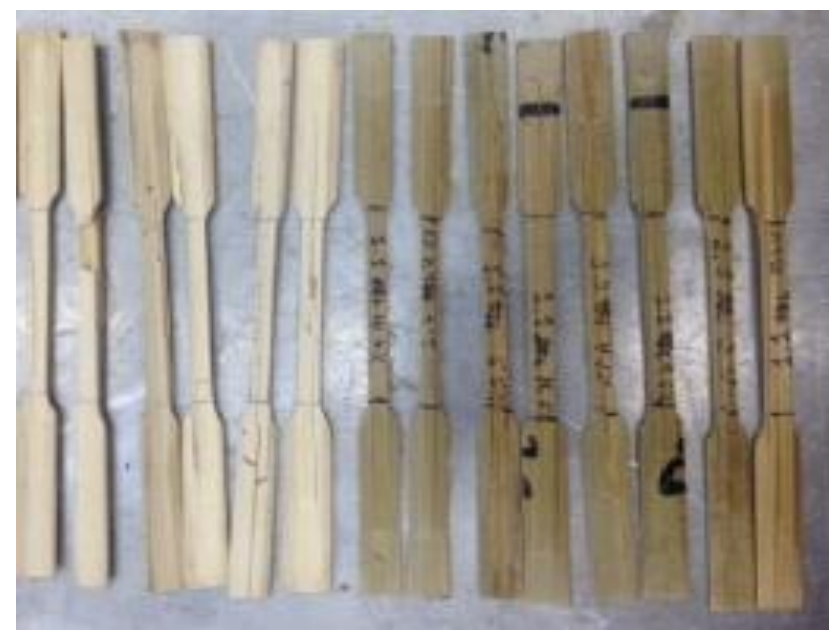

Figure 4-66. Portion of tested $6 \%$ salt tension specimens demonstrates fairly consistent data.

\subsection{9\% Salt}

The last group among the salt-treated sets is the bamboo soaked in the $9 \%$ salt solution. Figure 4-68 displays the resulting stress-strain curve for the group treated with $9 \%$ salt. The average yield stress is determined to be 25.8-ksi and corresponding strain to be $2.29 \%$. Oddly enough, the variation increases with this stress-strain figure in relation to the load-deflection plot located in the appendix of this work. It can be noted from the geometry table provided in the appendix that a few specimens show a considerable 
difference in their cross sectional area. One specimen in particular displays a prominent dipping effect, and this can be explained simply by the same specimen having a lower cross-sectional area. It is also possible that an unexpected occurrence during testing is the reason for the discrepancy, but it is highly unlikely.

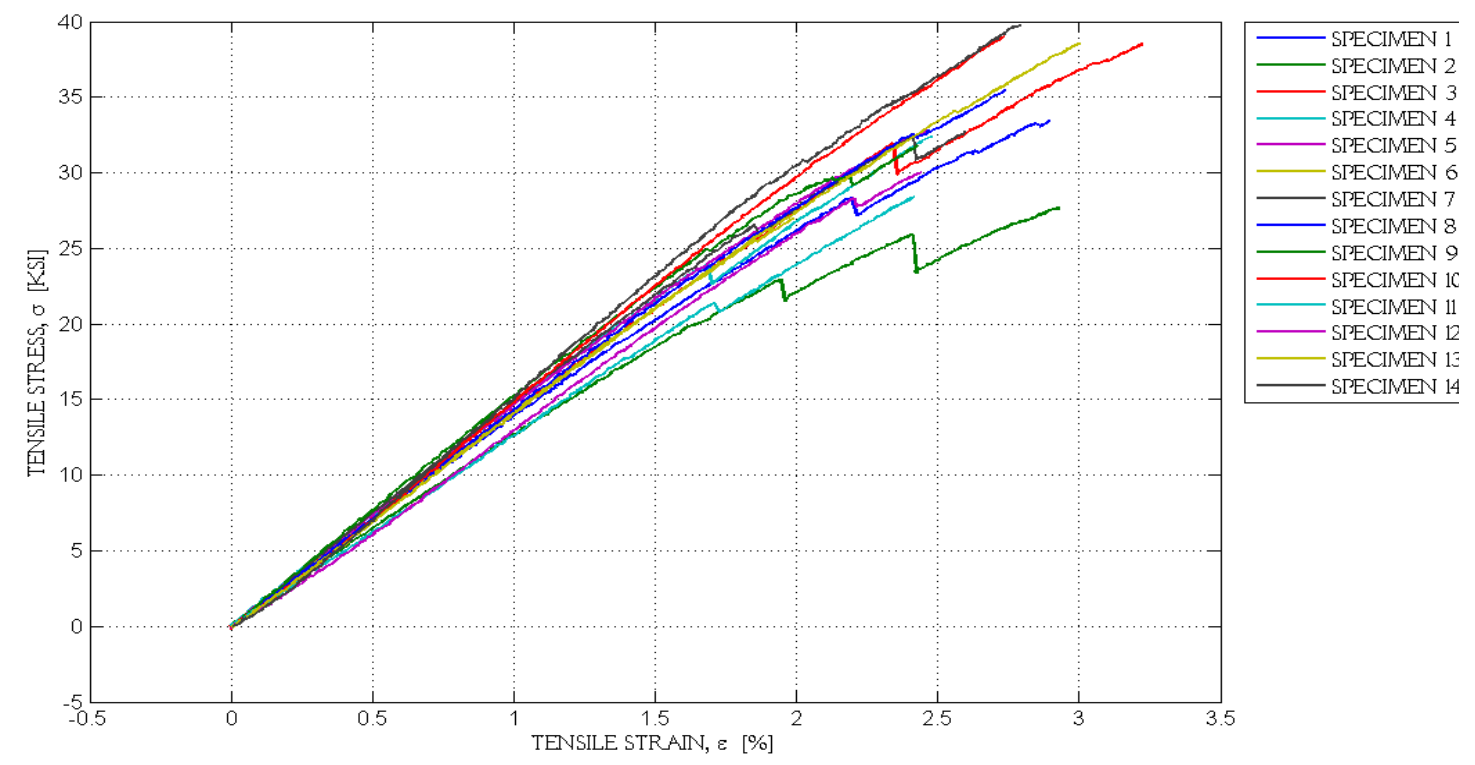

Figure 4-68. 9\% salt treatment tensile stress-strain curves

In a similar fashion to the $6 \%$ salt solution, the three failure types observed in the bamboos treated with this $9 \%$ salt include fiber splitting, culm separation, and directional fracture. The majority of the specimens exhibit fiber splitting as seen in Figure 4-69. One specimen, on the other hand, demonstrates culm separation. However, the specimen does not completely separate into two pieces as the fracture only propagates through half of its thickness. Only one occurrence of this failure behavior is observed after inspecting the specimens following the test. As mentioned previously, the majority of the specimens indicate splitting failure in the fibers. The extent of the fracture for most of the samples exhibits a single jagged propagation path and not a clean or straight crack. 
In summary of the bamboo treated with

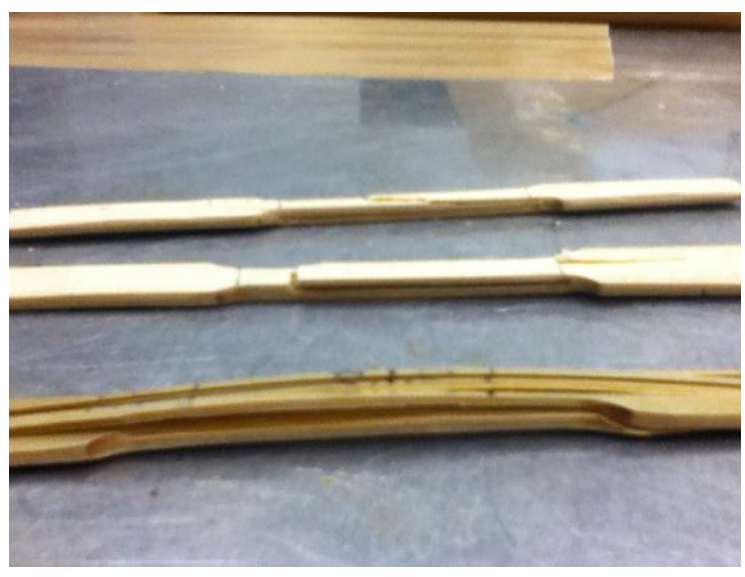

Figure 4-69. Common tensile failure types for $9 \%$ salt treatment

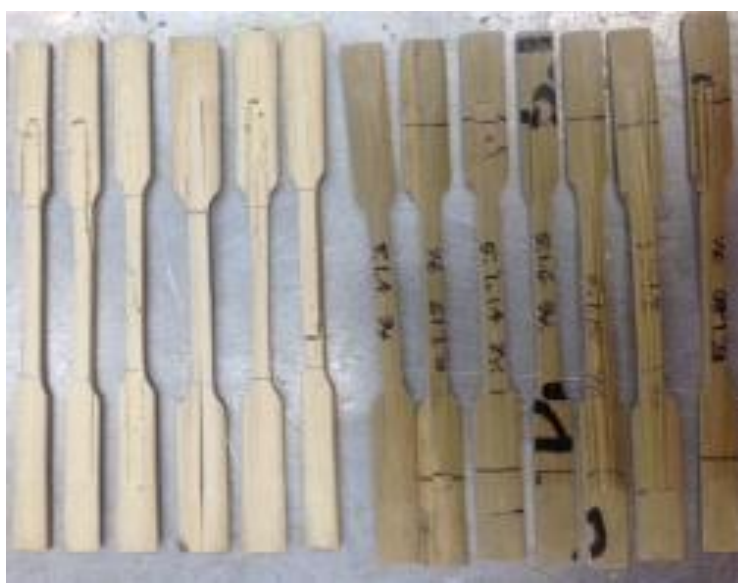

Figure 4-70. Portion of tested $9 \%$ salt tension specimens
$9 \%$ salt, a table is shown in the appendix displaying the seven main mechanical properties. In this group, the average specific strength is $1296-\mathrm{kips}-\mathrm{in} . / \mathrm{lb} \mathrm{m}$ and ultimate stress is $33.8-\mathrm{ksi}$, tensile modulus is $1458.0-\mathrm{ksi}$, and specific modulus is $56636-k i p s-i n . / / \mathrm{b}_{\mathrm{m}}$. As expected, the deviation is high for the yield strain at a value of $14 \%$. The specific strength also has a deviation of $14 \%$. In addition, the ultimate stress has the next highest deviation at about $12 \%$. However, the deviation for the specific tensile modulus is low at $9 \%$. Overall, the data are still usable and lends great insight on the tensile properties of bamboo treated with a $9 \%$ salt solution.

\subsection{Lime}

Another chemically treated set is the bamboo soaked in the $5 \%$ lime solution. Figure 4-71 displays the resulting stress-strain curve for the group treated with lime. Although difficult to see from this figure, the mean yield stress and mean yield strain of the group is $29.9-\mathrm{ksi}$ and $1.83 \%$, respectively. Once again, the sample size of this group is large on the account of high deviations among the tensile mechanical properties for the original test data. The sample size was consequently doubled to lower the deviation. The figure 
also demonstrates fairly consistent data for the majority of the sample size. Adding the specimens greatly improved the reliability of the test as seen from the consistency found in the plot. As seen in the figure, a few of the specimens exhibit the "dipping" effect common in this material behavior. However, it can also be noted that the dip is fairly large compared to the previous tests. This indicates that major fiber failures occurred in these specimens. A large drop could only be attributed to the main longitudinal fibers. Therefore, it is possible that the lime treatment affects the main fibers of the bamboo in a way unseen before. Only an investigation into the tensile mechanical properties of interest can conclusively say what the effect may be.
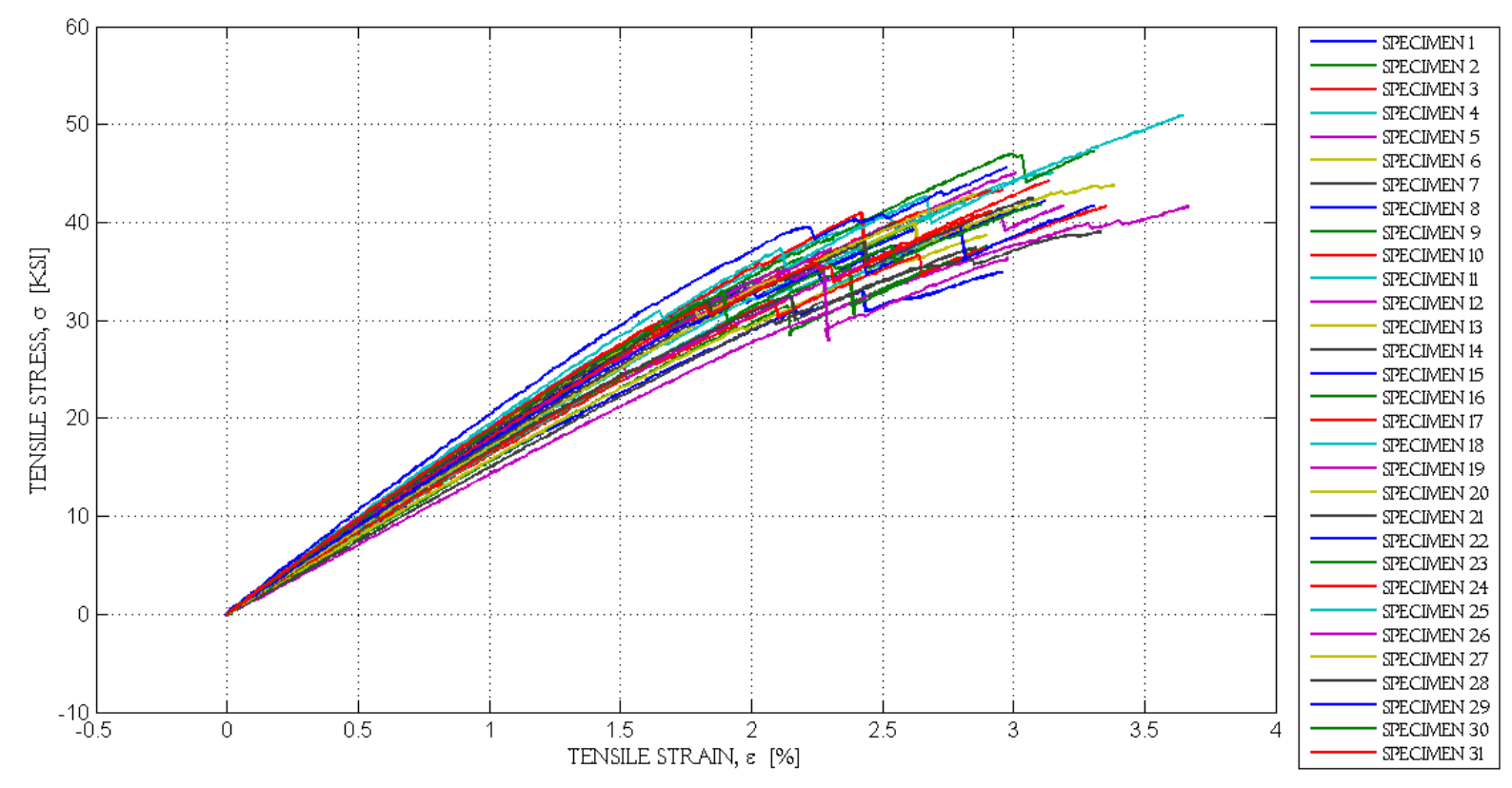

Figure 4-71. Lime treatment tensile stress-strain curves

Lime treatment on the bamboo demonstrates longitudinal fracture, complete fiber separation, and fiber splitting along the outer wall of the culm after tensile testing. It seems that fiber splitting is the more prominent failure type observed as seen from the figures provided. The least common failure type is shown to be the one specimen with complete separation-the specimen essentially parted into two segments. It is noted that 
the deformation at the conclusion of testing these samples is not as severe as most of the previously treated specimens.

In summary of the bamboo treated with lime, a table is shown in the appendix displaying the seven main mechanical properties of the tensile test. In this group, the average specific strength is 1159 kips-in./lb $b_{m}$ and the ultimate stress is $41.2-\mathrm{ksi}$. The tensile modulus is 1783-ksi and the specific tensile

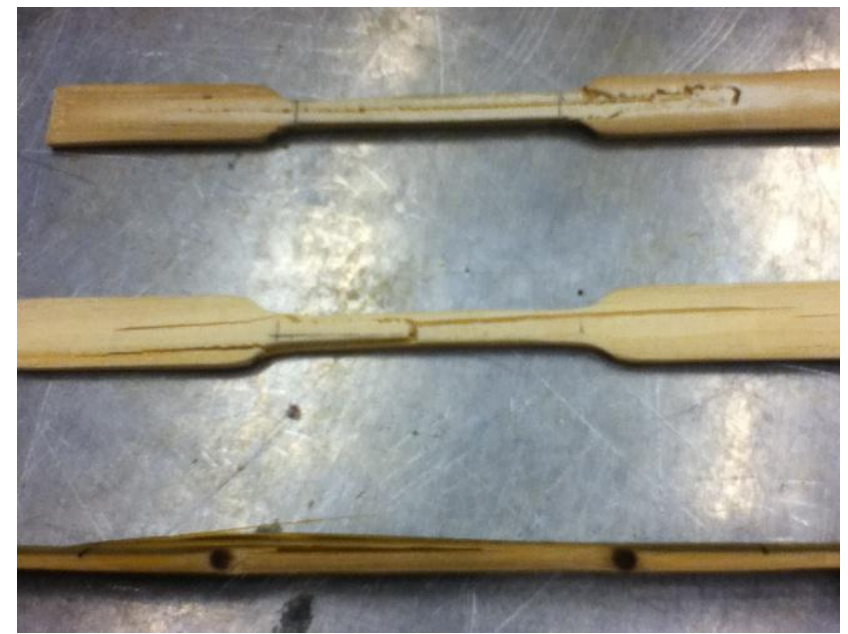

Figure 4-72. Common tensile failure types for lime treatment modulus is 63446-kips-in./lb $\mathrm{b}_{\mathrm{m}}$. In this set, the ultimate strain deviates the most at approximately $16 \%$ from the respective average. The yield stress data contains the next highest deviation at $12 \%$. The remaining specimens demonstrate low deviations with respect to the average. The tensile modulus has a deviation of $8 \%$. The yield strain has a deviation of $10 \%-$ a value considerably low in comparison to the previous tests. Similarly, the ultimate stress is determined to have a deviation from the average at roughly $12 \%$. The specific tensile modulus is determined to have a deviation of $10 \%$, and the specific strength demonstrates a deviation from the average at approximately $9 \%$. Considering the variation in some

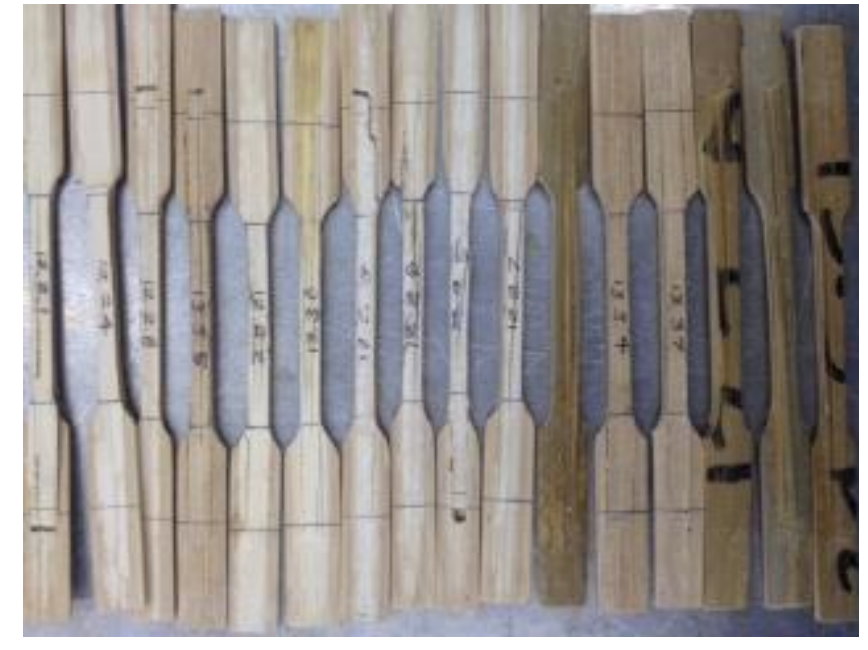

Figure 4-73. Portion of tested lime tension specimens 
of the other tensile tests, this variation is quite low overall. Lastly, it is important to note that the tensile material properties of interest, the specific modulus and specific strength, are low for the summary of these treatments.

\subsection{Oil}

The final natural chemical treatment investigated is the group of bamboo samples soaked in oil. Figure 4-74 displays the resulting stress-strain curve for the group treated with oil. The yield stress for the group is determined to be approximately $28.7-\mathrm{ksi}$. The ultimate strain for most of the specimens is determined to be $2.08 \%$. The slopes of the curves all appear to match well for most of the specimens. There is some deviation, as seen from the figure, but it must be kept in mind that the material in question is naturally made. Obtaining data more consistent than this is incredibly difficult. Again, the amplified "dipping" effect is apparent in the data. It appears that oil treatment of bamboos can affect the mechanical response of the material near the ultimate stress. Once more, the results of the tensile mechanical properties will ultimately determine the effects of this treatment on the bamboo.

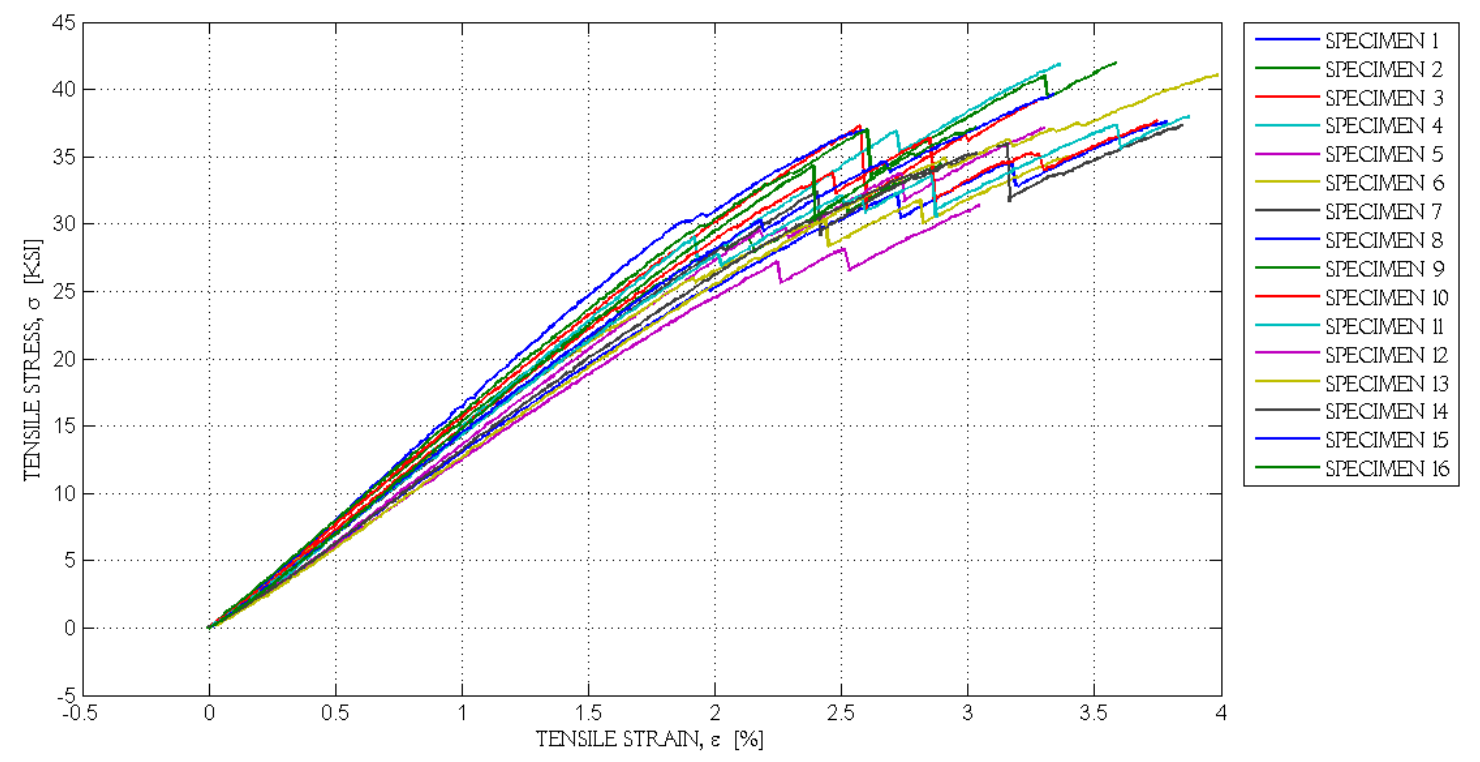

Figure 4-74. Oil treatment tensile stress-strain curves 


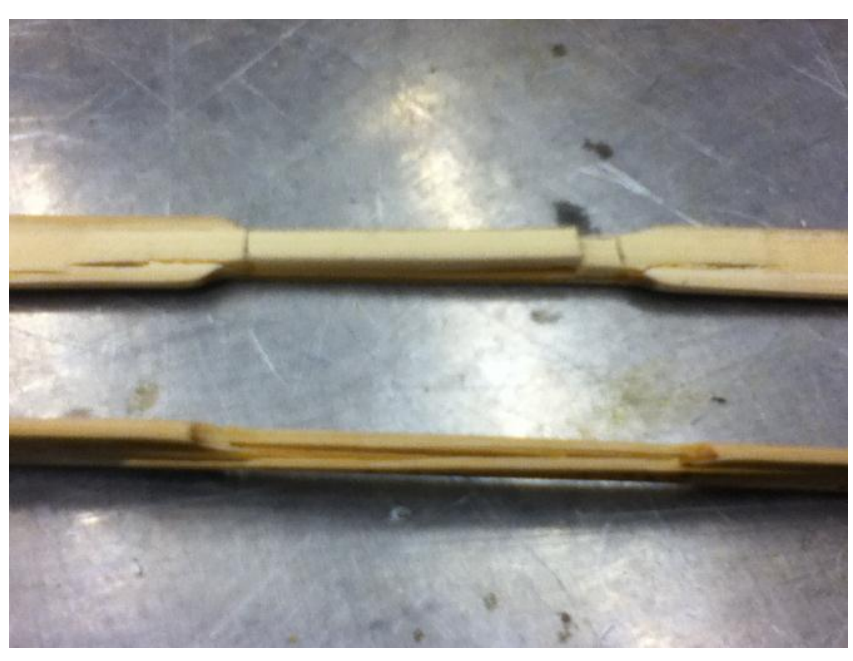

Figure 4-75. Common tensile failure types for oil treatment
Fiber splitting and directional splitting are the prominent failure behaviors shown by bamboo treated with oil and tested under tension. Again, fiber splitting is the most prominent behavior seen from this test. One instance shows nearly complete separation of the specimen; the separation only reaches half-way through the thickness of the sample. In any case, fiber splitting is seen more evidently without severe deformation on the specimen at the end of the test. Another specimen illustrates directional splitting where the fracture lies approximately $45^{\circ}$ to the vertical in the central region of the gauge area.

In summary of the bamboo treated with oil, a table is shown in the appendix displaying the main tensile mechanical properties. In this group, the average specific strength is 1116-kips-in./l $\mathrm{b}_{\mathrm{m}}$ and the ultimate stress is $37.5-\mathrm{ksi}$. The tensile modulus is determined

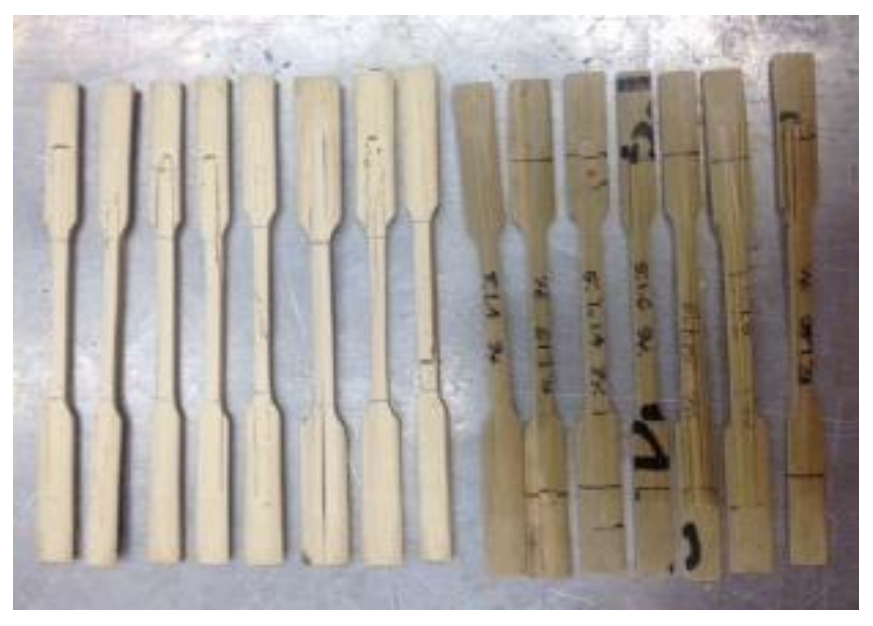

Figure 4-76. Portion of tested oil tension specimens to be 1486-ksi while the specific tensile modulus is 53391-kipsin./lb $b_{m}$. Again, the yield strain has the highest deviation at approximately $15 \%$. In this set, the yield stress deviates from the average at $9 \%$. The remaining properties have a deviation of only $7 \%$. For the majority of these data, 
a precise characteristic is observed and reliability is assured in the material properties obtained for this treatment group.

\subsubsection{Analysis of Tension Results}

For the final analysis of bamboo under tension, two figures summarizing all of the mechanical properties are shown. In addition, a separate table shows the percentage changes for all of the mechanical properties relative to the untreated bamboo group. Again, all the errors bars generated in the plots are determined using the standard

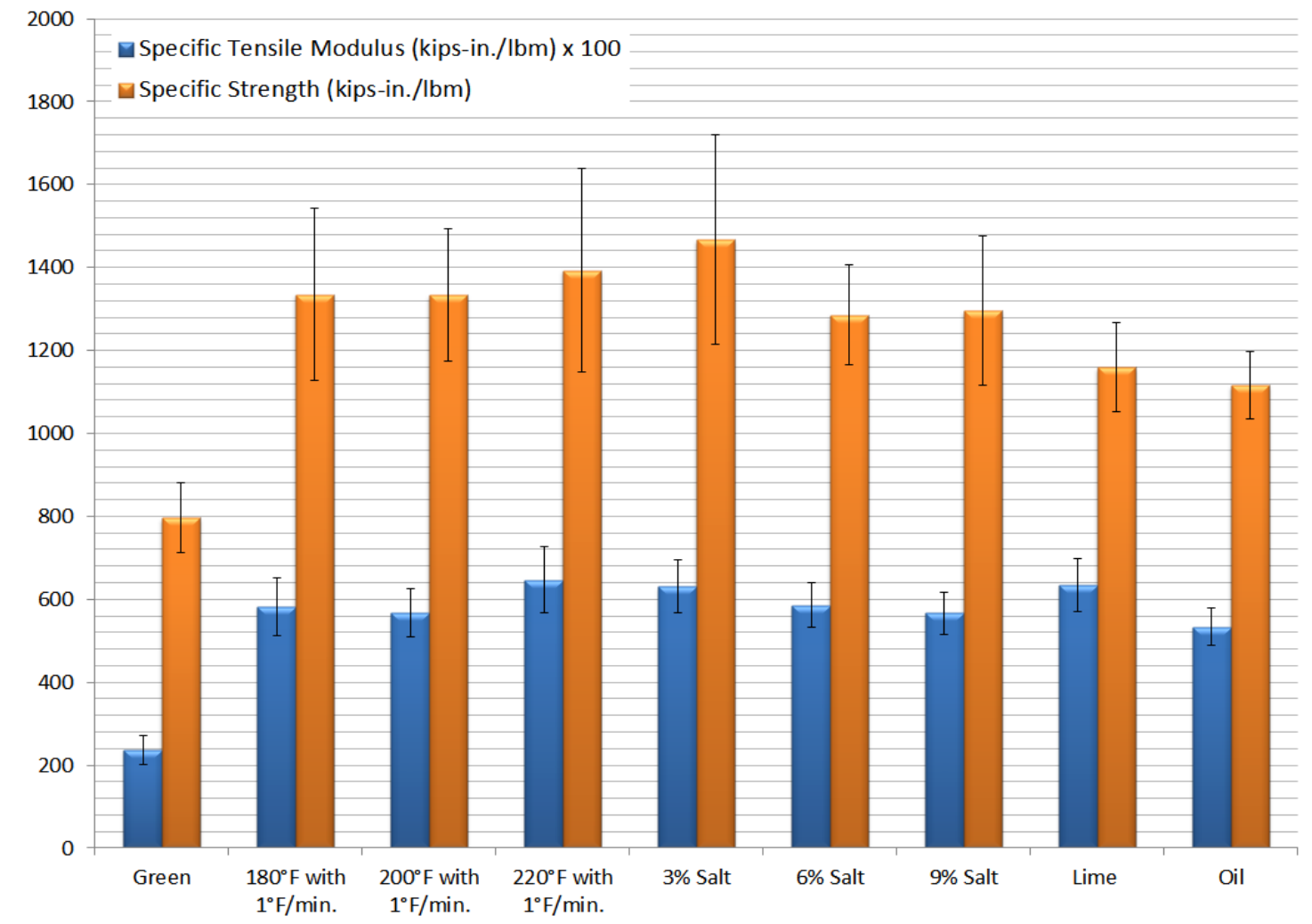

Figure 4-77. Summary of treatment effects on tensile specific strength and specific modulus

deviation of each sample group. In terms of the percent changes relative to the untreated bamboo, all the errors calculated are determined by the same method of the square of the sum of errors over each mechanical property. It is vital to perform this analysis on the tensile mechanical characteristics in order to understand the effects of the treatments over the whole spectrum of the bamboo's behavior under tension. 


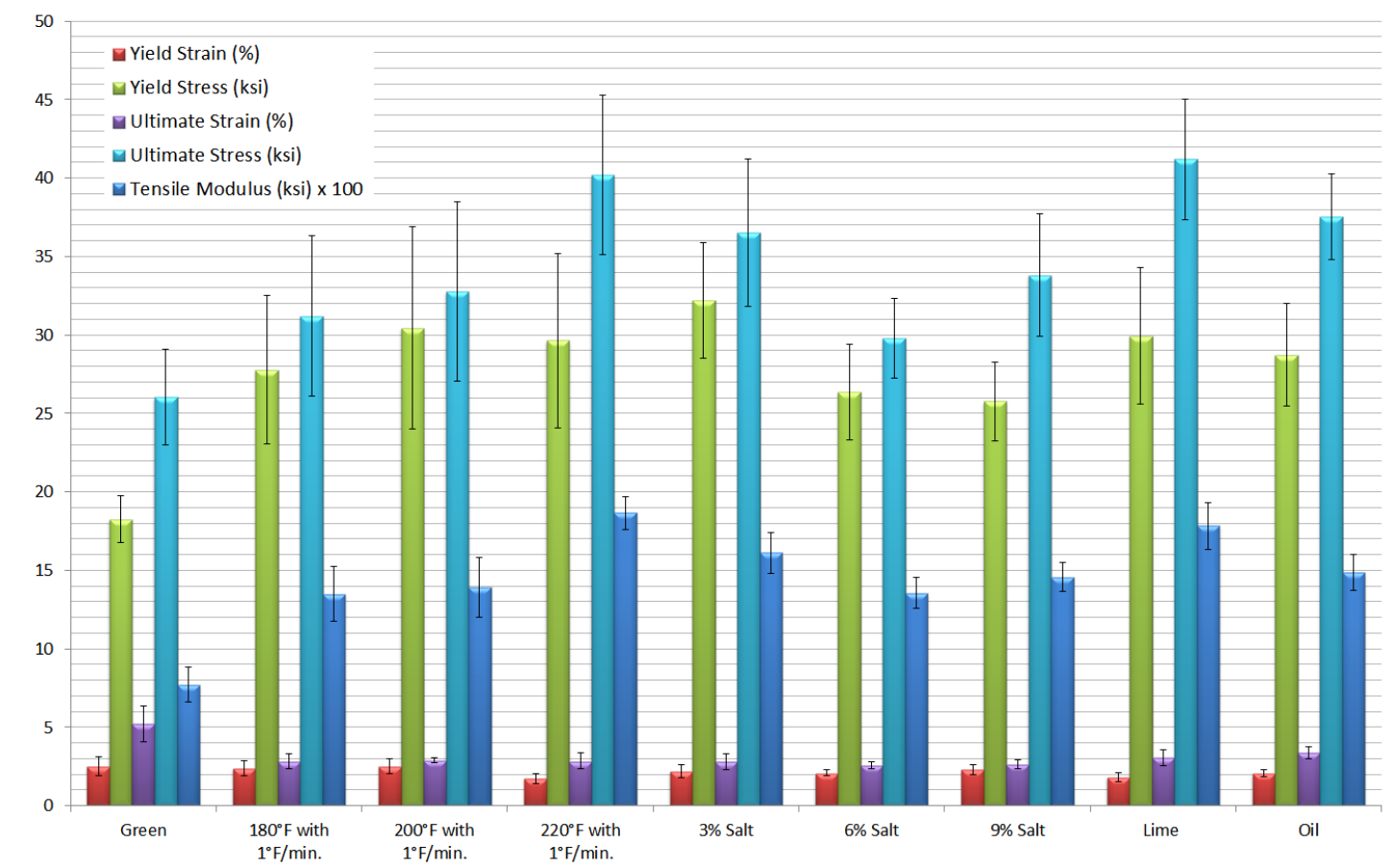

Figure 4-78. Summary of treatment effects on tensile strain, stress, and modulus

The vital mechanical characteristic of this study, the specific strength, indicates that all of the treatments investigated can improve this characteristic by an average of $60 \%$. As seen in the table, an increase in specific strength of $40 \%$ to $80 \%$ can be expected by utilizing these treatments. Among the treatments without chemical additives, the $220^{\circ} \mathrm{F}$ thermal treatment has a slight advantage over the others in terms of the average, but the deviation quickly renders it level with the other two thermal treatments. As hypothesized in the Investigating Thermal Treatments section, mechanical improvement in treatments over $180^{\circ} \mathrm{F}$ is marginally better. In terms of the thermal treatments, an optimum treatment is indiscernible-which contrasts the analysis from the compressive test. In another surprising divergence, the chemical treatments with higher salt concentrations appear to negatively affect the tensile strength of the bamboo. It is possible that the chemical additions to the bamboo on the microscopic level may ultimately degrade the quality of the fibers under tensile forces. The salt treatments show the least amount of damage to the strength compared to the lime and oil treatments. Specific strength 
performance also appears to be indistinguishable between the oil and lime chemical treatments. In addition, the table indicates that the $3 \%$ salt treatment and the $220^{\circ} \mathrm{F}$ thermal treatment provide the most strength increase relative to untreated bamboo. $\mathrm{A}$ clear advantage of using one over the other is not readily apparent. Therefore, it is concluded that the best improvements to the tensile strength of bamboo can be accomplished with the $220^{\circ} \mathrm{F}$ thermal treatment or the $3 \%$ salt treatment without sacrificing the longevity-increasing property of the salt.

Table 4-2. Average percent changes of tension properties relative to the untreated green bamboo

\begin{tabular}{|c|c|c|c|c|c|c|c|c|c|c|c|c|c|c|}
\hline Treatment & \multicolumn{2}{|c|}{$\begin{array}{l}\text { Modulus } \\
(\%)\end{array}$} & \multicolumn{2}{|c|}{$\begin{array}{c}\text { Specific } \\
\text { Modulus } \\
(\%)\end{array}$} & \multicolumn{2}{|c|}{$\begin{array}{c}\text { Yield } \\
\text { Strain (\%) }\end{array}$} & \multicolumn{2}{|c|}{$\begin{array}{c}\text { Yield } \\
\text { Stress } \\
(\%)\end{array}$} & \multicolumn{2}{|c|}{$\begin{array}{c}\text { Ultimate } \\
\text { Strain } \\
(\%)\end{array}$} & \multicolumn{2}{|c|}{$\begin{array}{c}\text { Ultimate } \\
\text { Stress } \\
(\%)\end{array}$} & \multicolumn{2}{|c|}{$\begin{array}{c}\text { Specific } \\
\text { Strength } \\
(\%)\end{array}$} \\
\hline \multirow{2}{*}{ Green } & \multicolumn{2}{|c|}{--} & \multicolumn{2}{|c|}{--} & \multicolumn{2}{|c|}{--} & \multicolumn{2}{|c|}{--} & \multicolumn{2}{|c|}{--} & \multicolumn{2}{|c|}{--} & \multicolumn{2}{|c|}{-- } \\
\hline & $\mathrm{P}$ & $\mathrm{E}$ & $\mathrm{P}$ & $\mathrm{E}$ & $\mathrm{P}$ & $\mathrm{E}$ & $\mathrm{P}$ & $\mathrm{E}$ & $\mathrm{P}$ & $\mathrm{E}$ & $\mathrm{P}$ & $\mathrm{E}$ & $\mathrm{P}$ & E \\
\hline $180^{\circ} \mathrm{F}$ & 75 & \pm 14 & 146 & \pm 28 & -5 & \pm 2 & 52 & \pm 10 & -46 & \pm 13 & 20 & \pm 9 & 68 & \pm 4 \\
\hline $200^{\circ} \mathrm{F}$ & 80 & \pm 16 & 139 & \pm 25 & -1 & \pm 0 & 67 & \pm 15 & -44 & \pm 10 & 26 & \pm 9 & 68 & \pm 4 \\
\hline $220^{\circ} \mathrm{F}$ & 141 & \pm 22 & 173 & \pm 33 & -31 & \pm 10 & 62 & \pm 13 & -46 & \pm 13 & 54 & \pm 8 & 75 & \pm 11 \\
\hline $3 \%$ Salt & 109 & \pm 18 & 166 & \pm 30 & -13 & \pm 4 & 76 & \pm 11 & -46 & \pm 13 & 40 & \pm 8 & 84 & \pm 8 \\
\hline $6 \%$ Salt & 75 & \pm 12 & 147 & \pm 26 & -18 & \pm 4 & 44 & \pm 6 & -50 & \pm 12 & 14 & \pm 7 & 61 & \pm 2 \\
\hline $9 \%$ Salt & 89 & \pm 14 & 139 & \pm 24 & -9 & \pm 3 & 41 & \pm 5 & -49 & \pm 12 & 30 & \pm 8 & 63 & \pm 5 \\
\hline Lime & 131 & \pm 22 & 168 & \pm 30 & -28 & \pm 8 & 64 & \pm 11 & -41 & \pm 11 & 58 & \pm 6 & 46 & \pm 8 \\
\hline Oil & 92 & \pm 15 & 125 & \pm 21 & -18 & \pm 5 & 57 & \pm 8 & -35 & \pm 9 & 44 & \pm 5 & 40 & \pm 6 \\
\hline
\end{tabular}

On a better note, the specific modulus is substantially improved across all treatments by an average of $150 \%$ relative to untreated bamboos. As seen in the table, these treatments can improve the stiffness of the material by $125 \%$ to $170 \%$. Nearly all of the treatments provide similar stiffness improvement. A similar trend is also observed from 
the analysis of the compression test results. As a result, it is concluded that curing temperatures above $200^{\circ} \mathrm{F}$ with a chemical treatment of $3 \%$ salt or lime solution does not negatively affect the tensile stiffness of bamboo; therefore, these treatments can be performed on this natural material for their protective properties without a stiffness penalty.

In analyzing the tensile modulus, a clear trend can be seen that all the thermal and chemical treatments improve the stiffness of green bamboo-especially the $220^{\circ} \mathrm{F}$ curing cycle and the lime treatment. In particular, the $220^{\circ} \mathrm{F}$ thermal and lime treatments provide the most stiffness increase by over $100 \%$ in relation to the green bamboo. The $220^{\circ} \mathrm{F}$ thermal treatment has a clear advantage over the other two thermal treatments. Similarly, the $3 \%$ salt solution has a clear benefit over the other two salt treatments. From the data, the $220^{\circ} \mathrm{F}$ treatment offers a slight advantage over the $3 \%$ salt treatment while the lime and $3 \%$ salt treatment show similar stiffness increases. It is possible that these treatments filled the voids more adequately over the other chemicals to add stiffness to the cellulose fibers for tensile loading.

In addition, the best ultimate stress improvement over the green bamboo is found in a few of the treatments but the yielding stress seems to improve across the board. The $220^{\circ} \mathrm{F}$ curing cycle, lime, and oil treatments offer the best increases in terms of the ultimate stress. Among those three, the lime shows the highest average but is not a clear winner due to the deviation. The remaining treatments seem to offer less significant increases in strength. In terms of the yield stress, a clear winner is also indistinguishable among the salt treatments. It is shown that the $3 \%$ salt treatment offers a higher improvement than the $9 \%$ salt treatment. It appears the hypothesis of increasing the salt content further from that of seawater is unfruitful in producing better tensile mechanical properties under yielding. All of the salt treatments demonstrate similar results; perhaps 
the $3 \%$ incremental increase is not sufficient. Increasing the salt content further is left for future work. All in all, confidence is attained in utilizing these treatments to increase the load-bearing capabilities of this material under tension.

In contrast to the compressive tests, heat or chemical treatments do not significantly affect the yield strain of green bamboo like it does for the ultimate strain. Among the simple thermal treatments, only the $220^{\circ} \mathrm{F}$ treatment significantly lowered the yield strain relative to the green bamboo. Similarly, the lime and oil treatments clearly outperform the other two salt treatments. On the other hand, the ultimate strains for all the treatments decrease by about $50 \%$ relative to the green bamboo. This is important for engineering applications where deflections need to be considerably lowered. Aside from these two observations, a clear improvement among the treatments is seen from the results.

\subsection{Measuring Poisson's Ratio of Bamboos}

In order to capture all of the mechanical properties of bamboo, the Poisson's ratio is also measured for the untreated bamboo group and all of the treated ones. Additionally, determining these values also demonstrates the effect the treatments have on the Poisson's ratio. This material property is simply the negative ratio of transverse to axial strain under some applied load. In other words, it measures the amount a material contracts—or expands, in a few rare cases_in the perpendicular direction of an applied tensile force. The Poisson's ratio in the 1-2 direction is measured for all of the specimens, and the 2-1 direction is measured for only one of the treatments. The sparse section in the ISO standard for measuring Poisson's ratio is utilized as a guideline for all of the specimen groups. 


\subsubsection{Preparing Poisson's Ratio Specimens}

The manufacturing of these specimens follows a similar procedure as the tensile test groups. As outlined in the standard, a strain gage is utilized to measure the strain in the transverse direction of the load. However, the dog-bone shape recommended in the standard is disregarded so that the strain gage can fit within the specimen. All of these specimens are manufactured without the dog-bone shape and are kept simply rectangular. The approximate dimensions for all of the specimens are 9.0-in. $x$

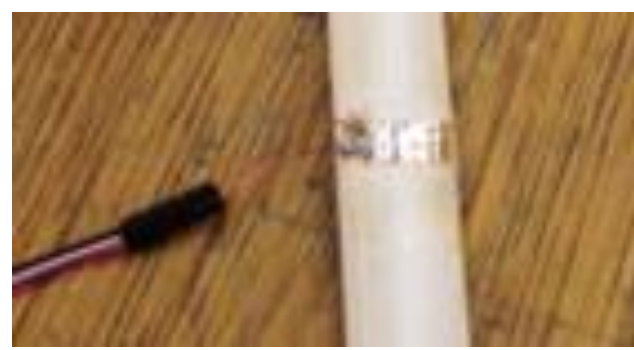

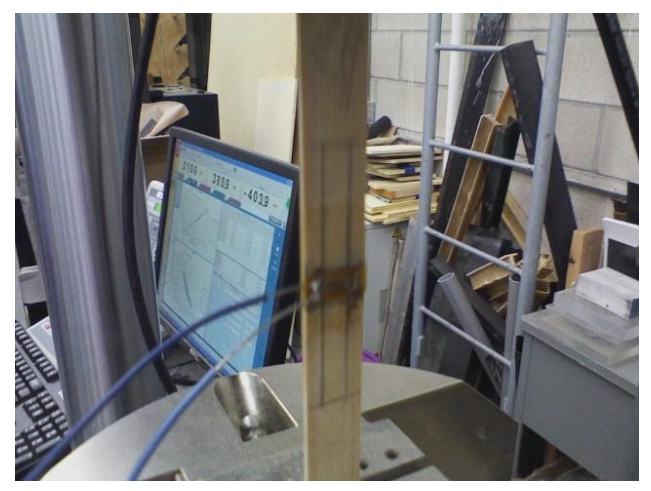

Figure 4-79. Correct attachment of strain gage 0.99-in. x 0.09-in. Manufacturing the specimens begins in the same fashion as the tensile samples where the bamboos are inspected and cut around the culm into rectangular strips. Reference is given back to Figure 4-46 on how to extract the rectangular strips from the culm. Each strip is then sanded on the inner side and the edges to the mentioned dimensions.

At this point in the manufacturing process, the specimens can be fitted with a strain gage. The directions provided by the Student Strain Gage Application Manual from Vishay are followed [68]. First, the area where the strain gage will be applied is cleaned with acetone and a base cleaner as supplied by Vishay Micro measurements. It is important to use a swab to clean the area and only use strokes in one direction; swabbing back and forth is not recommended so as not to spread any debris. After cautiously cleaning the area, a strain gage of the appropriate size is placed face-down on the area. The majority of the specimens utilized the $13-250 \mathrm{BG}-120$ class of strain 


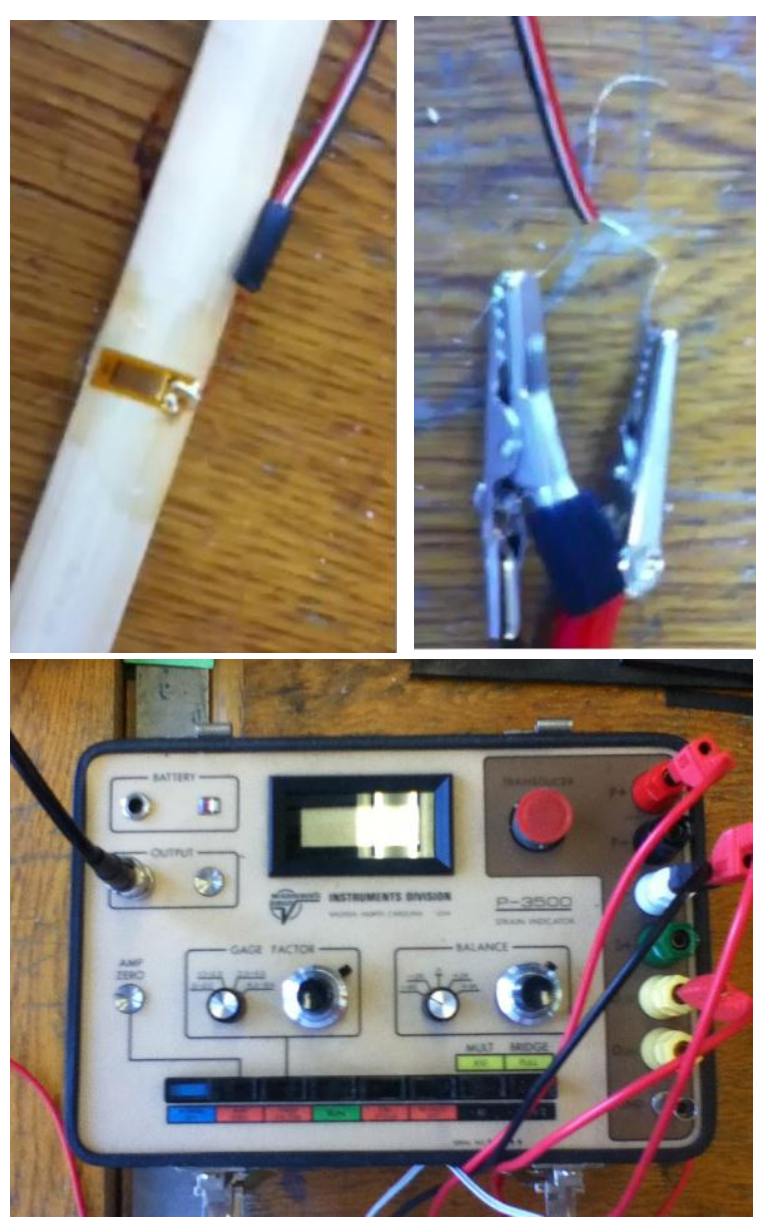

Figure 4-80. Proper soldering and wiring of strain gage (top left), intermediate connection of wiring to box with alligator clips (top right), and final Wheatstone bridge connection to strain indicator box (bottom)

gage by Vishay Micro-Measurements. It is critical to place the strain gage perpendicular to the fiber direction for accurate measurements of the Poisson's ratio. Scotch tape about an inch in length is then applied over the strain gage and made sure to stick well on one of the edges. The tape is peeled back so the strain gage is now attached to the tape while a catalyst is applied. One minute is then waited so the catalyst can dry before applying the adhesive. A drop of M-bond 200 adhesive is applied to the cleaned area of the specimen. The tape is then reapplied quickly over the adhesive so that the catalyst-coated strain gage is in contact with the part and adhesive. A sweep of the finger is smeared over the tape to remove any trapped air bubbles and improve proper mixing of the catalyst and adhesive. Firm pressure is placed over the tape for a total of three minutes for the adhesive to dry. Then the tape is removed and the strain gage is inspected for proper alignment and adhesion to the specimen. Once properly attached to the specimen, electrical wires are soldered to the strain gage at the two output tabs. Figure 4-80 is provided for an exemplary specimen fitted with a strain gage and the proper connections attached. 
Once the strain gage is attached to the specimen, the wiring is connected to the data acquisition system to measure the appropriate strain. The strain gage is then connected to a Wheatstone bridge strain indicator box. Then additional wires are attached and connected to a National Instruments BNC-2111 External Connector Box, which is also connected to a data acquisition PC running the Bluehill 2 software. By connecting the appropriate wires to the data acquisition system, the strain gage measurement can be recorded by the Versa Channel feature in the testing software of Bluehill 2.

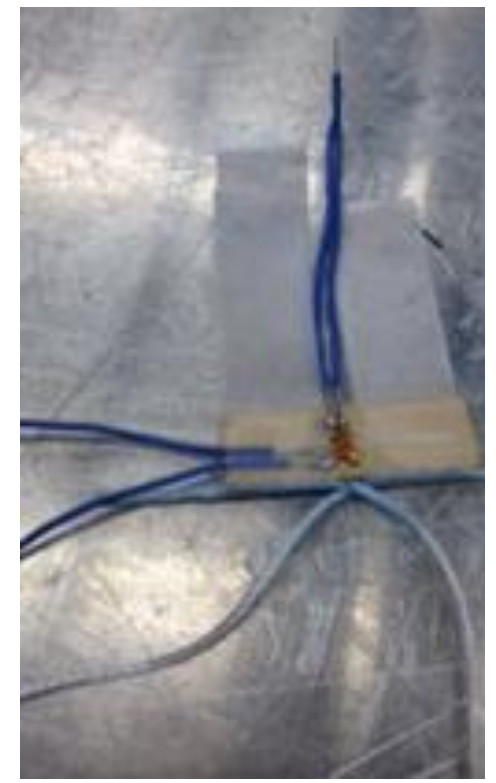

Figure 4-81. Proper attachment of strain gages and wiring

Figure 4-80 illustrates how the proper connections and the correct specimen set-up appear. Next, the strain box indicator is calibrated accordingly. The proper gage factor is set on the indicator box and the initial reading is adjusted to zero. Once calibrated on the strain indicator, the Bluehill 2 software is calibrated to be synchronized with the strain gage and the indicator box. Further detailed instructions on how to use the versa channel option and calibrate the strain gage is supplied in the thesis by Amini [18].

The manufacturing procedure differs slightly for the samples loaded in the transverse direction of the fibers. Similar to the green bamboo, no references exist-to the knowledge of the author-as to the Poisson's ratio for bamboo loaded perpendicular to the fiber direction. Consequently, a method had to be devised for this particular experiment. Since the diameter of the culm makes it exceptionally difficult to provide flat surfaces for the jaws of the Instron machine to attach to the specimen, this machine is quickly ruled out as a potential testing apparatus. Instead, it is decided to tape a small specimen to the edge of a table and at its center for loading. Only one specimen is 
prepared due to the enormous amount of work required to perform this test. The procedure initiates by cutting a culm out of the specified bamboo pole. Very much like the tension specimens, the culm is split radially into several sections to produce a nearly flat specimen in terms of its thickness. Unlike the tension specimens, however, a larger width is required to allow proper attachments of two strain gages. It is necessary to attach two strain gages in order to measure the strain in the transverse and longitudinal direction. Consequently, the necessary precautions are taken to ensure that the widest rectangular strip is extracted from the culm. Since the width of the specimen must accommodate two strain gages in the same area, a much smaller strain gage is utilized. Consequently the EA-06-062AK-120 class of strain gage by Vishay MicroMeasurements is implemented in this test. The specimen is then prepared in the same aforementioned manner to apply the strain gages oriented in the direction transverse and longitudinal to the fibers; the direction of the applied load and the additional strain gage are the contrasting factors to the previous tests. The transverse strain gage is placed offset from the center toward the top edge, and the longitudinal strain gage is positioned near the center toward the bottom edge. Again, the proper wiring is attached and soldered to the strain gages once they are positioned on the specimen. Figure 4-81 depicts the proper attachment of the strain gages and wiring for this sample. In this manner, it is assumed that the general strain in the region containing the gages is appropriate for determining the Poisson's ratio for this loading condition.

\subsubsection{Poisson's Ratio Testing Procedure}

Since the ISO standard does not give detailed instructions on how to measure the Poisson's ratio of the bamboo, a testing procedure from the tensile test and ASTM E13204 are considered for calculating the normal Poisson's ratio [14]. Maintaining the same procedure as the tensile test, the grip jaws are first calibrated to within 100-psi jaw 


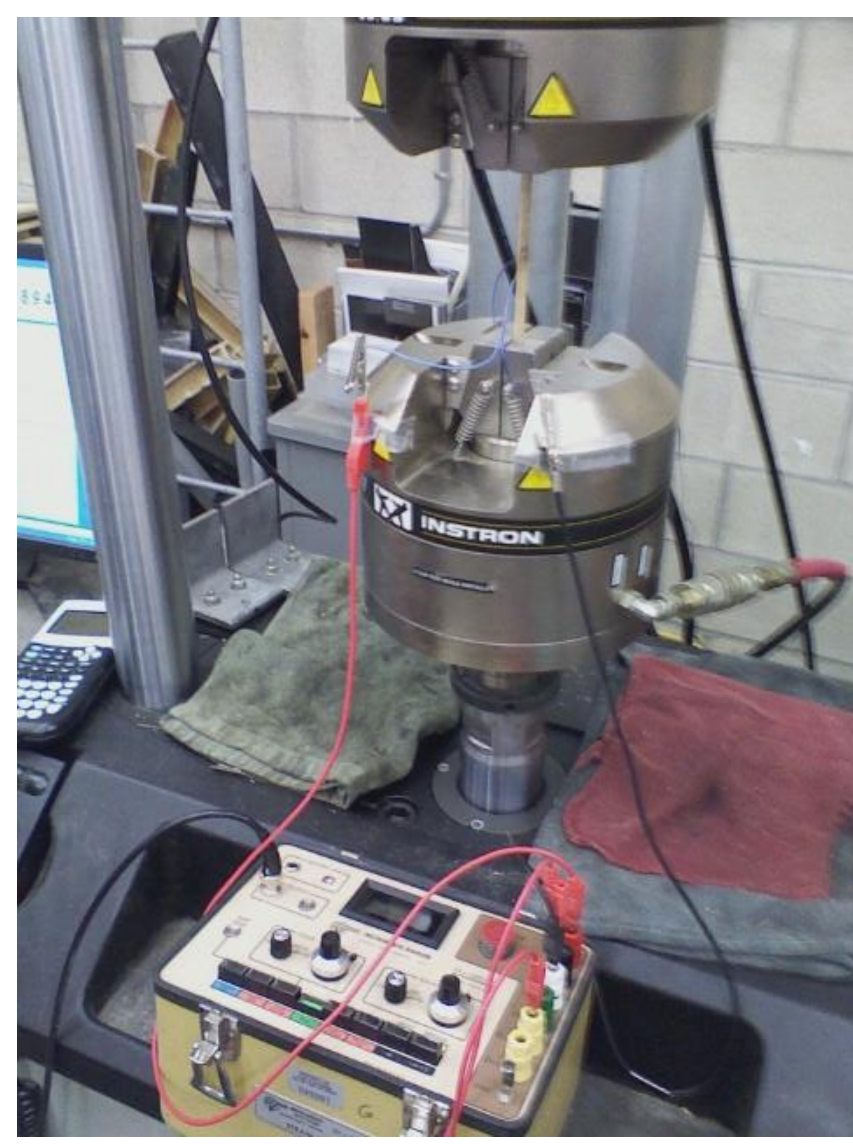

Figure 4-82. Loading Poisson's ratio specimen into testing area pressure. This testing procedure measures the tensile extension and load applied to the specimen from the crossheads of the Instron machine. The correct loading rate is then selected in the Bluehill 2 software. Next, the specimen is loaded into the grip jaws and aligned so the fibers are parallel to the applied force. Any necessary adjustments are completed to preload the specimen under $10-\mathrm{lb}_{\mathrm{f}}$. Figure 4-82 depicts the test set up for the normal Poisson's ratio test. Once preloaded, the test is started and continues until a $500-1 b_{f}$ load is reached. Loading until failure would have been unnecessary since the Poisson's ratio is only measured for the linear-elastic region of the stress-strain plot. At the end of the test, the specimen is removed and another specimen is set up into the loading area. A total of 10 specimens are tested for each group.

In contrast, the Poisson's ratio test with transverse loading requires an entirely different testing procedure without the use of the Instron machine. Once the strain gages are on the coupon, the specimen is taped at its flanks up to the table and left to hang. In order to do this, a two-step procedure must be followed. First, a piece of tape is wrapped around the width of the specimen so it can be sealed at the top to form a closed loop. This process is done twice: a loop of tape at both sides of the strain gages. Before 
closing the loop at the top, two long pieces of string are placed horizontally directly below the strain gages near the bottom edge. The reason for using the two segments of string is to evenly distribute the load across the bottom edge of the specimen. It must also be noted that the pieces of string are oriented so that they met at the center for adequate weight attachment. Figure 4-83 shows how the tape and strings are set up for
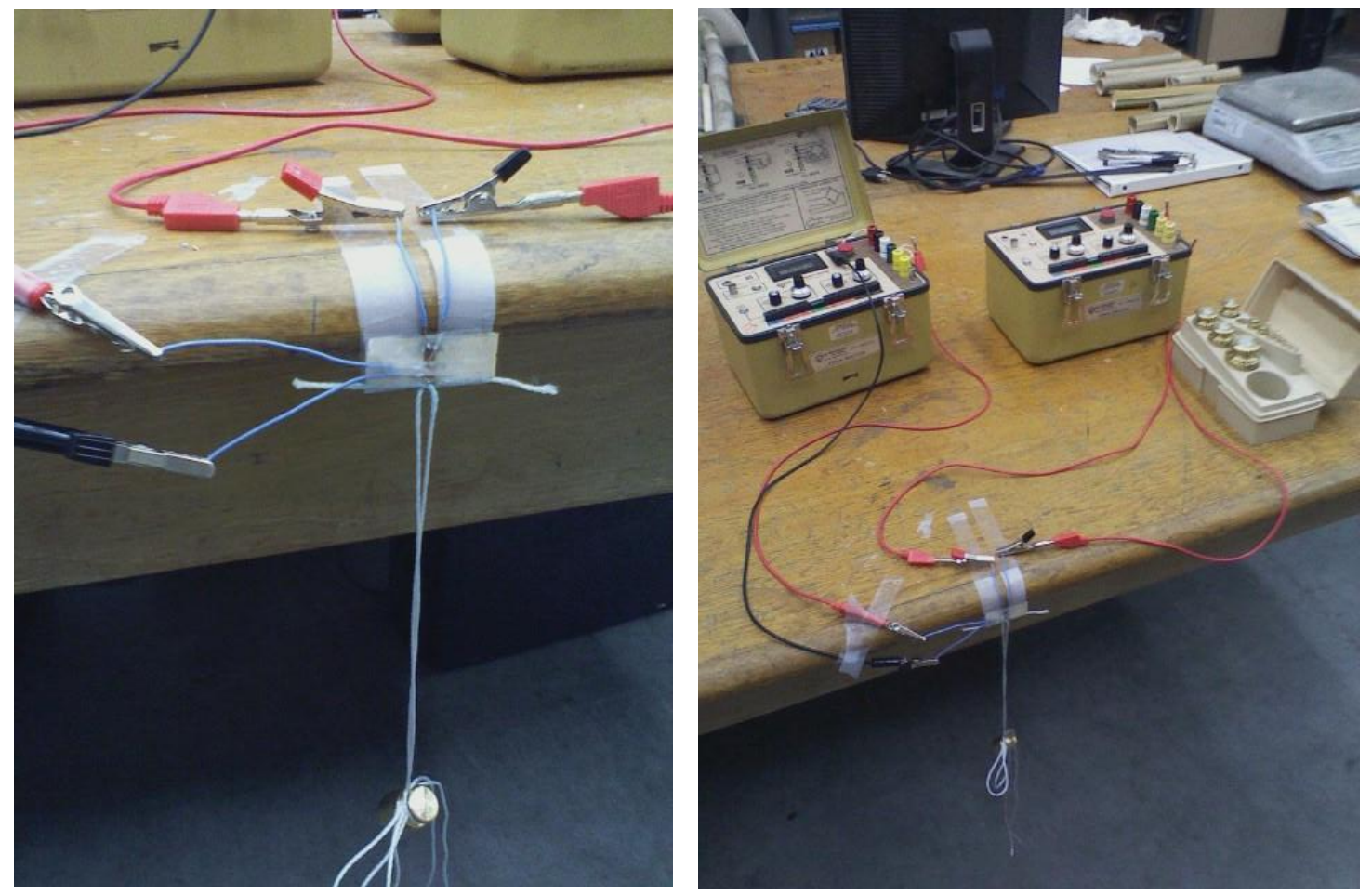

Figure 4-83. Configuring strings and tape to specimen (left) and overall test set-up for Poisson's ratio specimen loaded transverse to fibers

proper testing. The necessary connections are then attached from the specimen's strain gages and to the two strain indicator boxes. Again, the utilization of two strain indicator boxes is necessary in order to capture the strain in the longitudinal and transverse directions. Originally, the Instron machine measured the longitudinal strain but obviously that convenience no longer was possible for this testing procedure. The test begins by tying small weights to the string and letting them hang while recording the value on the strain indicator box. It is important to record the value measured when the reading on the strain indicator box reaches a steady value-otherwise inaccurate data will be recorded. 
A total of six weights, and therefore six strains, make up the data for this test. Figure 4-83 illustrates the experimental set-up for this test.

\subsubsection{Results of Poisson's Ratio Test}

The results of the Poisson's ratio tests are summarized for the treatments of interest in this research. Eight different groups are measured for the Poisson's ratio, and the testing procedure follows the same one as the tensile test. The samples included the groups of: untreated bamboo, no additive with $220^{\circ} \mathrm{F}$ heat treatment, $3 \%$ salt, $6 \%$ salt, $9 \%$ salt, lime, oil, and the $3 \%$ salt loaded in the transverse direction of the fibers. It should also be noted that all treatments are cured at $220^{\circ} \mathrm{F}$. The subsequent section, Optimum Bamboo Treatment Method, will explain this further. As mentioned earlier, this last specimen group of $3 \%$ salt is to determine the Poisson's ratio in the $2-1$ direction. The reason for selecting the $3 \%$ salt-treated bamboo for the transverse loading condition is also explained in the subsequent discussion. A table summarizing all of the data is also provided at the closing of this section.

First, the basic variables are defined to calculate the Poisson's ratio. The longitudinal strain is defined as,

$$
\varepsilon_{l}=\frac{\Delta_{l}}{l}
$$

Where $\Delta_{l}$ is the extension of the specimen in the longitudinal direction of the fibers and I is the average length of the specimen. Similarly, the transverse strain is defined as,

$$
\varepsilon_{t v}=\frac{\Delta_{t v}}{w}
$$

Where $\Delta_{t v}$ is the displacement of the specimen in the transverse direction of the fibers, and $w$ is the average width of the specimen. 
Once the variables are defined, a plot of strain versus force is made from the test results. Two curves—both longitudinal strain and transverse strain-are plotted against the applied load. These two lines are then used to calculate the Poisson's ratio in the primary and secondary directions. Therefore, Poisson's ratio is defined as,

$$
v_{12}=\frac{\frac{d \varepsilon_{t v}}{d F}}{\frac{d \varepsilon_{l}}{d F}}
$$

Where $v_{12}$ is Poisson's ratio, $F$ is the applied tensile load, $\varepsilon_{t v}$ is the transverse strain, and $\varepsilon_{l}$ is the longitudinal strain relative to the fibers. Similarly, the shear modulus is also determined to completely define this set of mechanical properties. For bamboo that is predominantly a unidirectional material with varying moduli in the primary and secondary direction of the fibers, the following definition is adequate to determine the shear modulus. The shear modulus is defined by Huber's equation as

$$
G_{12}=\frac{\sqrt{E_{1} E_{2}}}{2\left(1+\sqrt{v_{12} v_{21}}\right)}
$$

Where $G_{12}$ is the shear modulus, $E$ is the elastic modulus, and $v_{12}$ and $v_{21}$ is the previously determined Poisson's ratio [74]. The shear modulus is not particularly measured for performance evaluation but for finite element purposes in a subsequent section. In order to calculate these properties, a MATLAB code is created and supplied in the appendix similarly to the one used to calculate the modulus of a test specimen. Figure 4-84 and Figure 4-85. show examples of the plot used to calculate the Poisson's ratio. Due to the similar nature of the plots, only one example is shown in Figure 4-84 to demonstrate how the Poisson's ratio is determined. The remaining plots are supplied in the appendix. It is critical that the appropriate definitions for transverse and longitudinal are defined when utilizing the equation above for the Poisson's ratio. Transverse is 
always referenced as the direction perpendicular to the load, and longitudinal is defined as the direction parallel to the applied load.

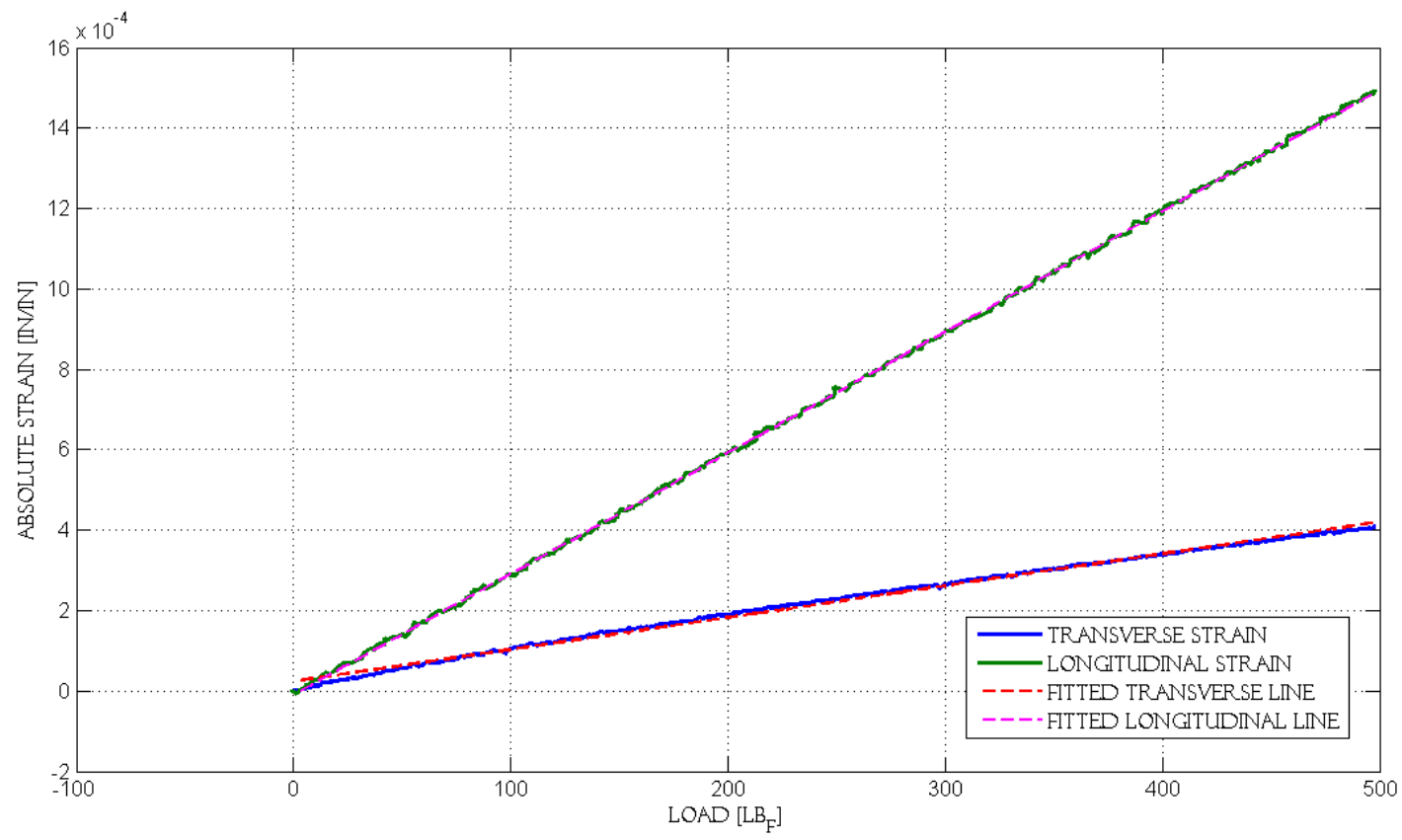

Figure 4-84. Exemplary plot utilized to determine the Poisson's ratio under longitudinal loading

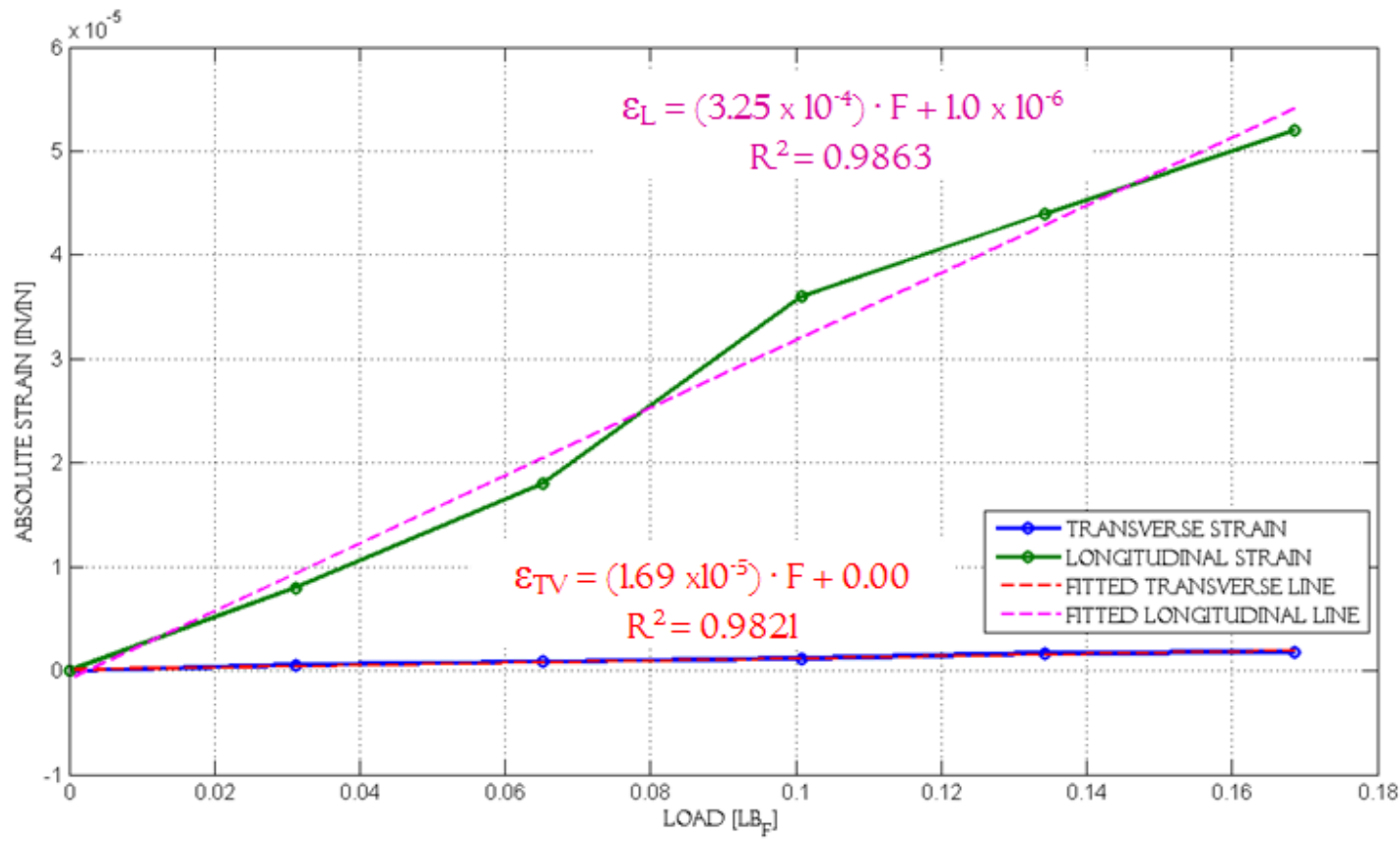

Figure 4-85. Exemplary plot utilized to determine the Poisson's ratio under transverse loading 


\subsubsection{Analysis of Poisson's Ratio Results}

The measured Poisson's ratio for each of the curing treatments were all correlative to one another as the Poisson's ratio ranged from 0.260 to 0.311 for specimens loaded in the longitudinal direction. The Poisson's ratio for the specimen loaded transversely to the

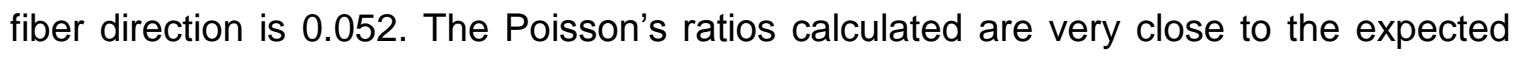
Poisson's ratio referred to by Janssen and Laemlaksakul—their experiments yield values ranging from 0.31 to $0.35[46]$.

Table 4-3. Summary of Poisson's ratio for all treatments

\begin{tabular}{|c|c|c|}
\hline Treatment & Poisson's Ratio $(-)$ & Standard Deviation \\
\hline Untreated & 0.481 & \pm 0.014 \\
\hline $220^{\circ} \mathrm{F}$ & 0.300 & \pm 0.006 \\
\hline Lime & 0.311 & \pm 0.024 \\
\hline Oil & 0.298 & \pm 0.026 \\
\hline $3 \%$ Salt & 0.260 & \pm 0.021 \\
\hline $6 \%$ Salt & 0.301 & \pm 0.020 \\
\hline 9\% Salt & 0.300 & \pm 0.026 \\
\hline 3\% Salt & 0.052 & - \\
\hline (Transverse Loading) & & \\
\hline
\end{tabular}

Table 4-3 displays a summary of the Poisson's ratios determined in this study. First, it is observed that the Poisson's ratio across all treatments yield the same value. Second, the majority of the groups have a Poisson's ratio within the range established by previous works. An exception, however, may be seen in the green bamboo group. The ratio determined from this experiment is higher than the rest of the sample groups. As of now, there are no references available for the Poisson's ratio of untreated green bamboo. Both references discovered for this mechanical property only investigated the value for construction-grade bamboo-implying the bamboo is a few years old and already air-dried for several months. Green bamboo is much more flexible than treated 
bamboo, so intuitively, an increase in both longitudinal/transversal strains is expected. This is seen in the strain-load curve provided in the appendix; the strains are higher than the other treated groups. Thus, strong confidence stands behind the measured value obtained. If any errors could have possibly occurred during the test, it would be found in the grip jaws. Perhaps the moisture from the green bamboo may have produced slippage at the contact surfaces of the grip jaws and specimens.

The value for the Poisson's ratio under transverse loading is the lowest among all the groups as expected. As mentioned earlier, bamboo is a naturally unidirectional material that has fibers running in the axial direction. During a transverse load applied to the fibers, the only substance carrying most of the stress is the lignin and hemicellulose matrix. The matrix is not expected to withstand the majority of a tensile load-much like conventional composite structures. As a result, lower transversal-to-longitudinal strains are noted from the strain-load curve provided. In summary, it is concluded that implementing any of these treatments will not significantly affect the Poisson's ratio of the bamboos. 


\section{Chapter 5. Optimum Bamboo Treatment Method}

An optimization analysis is performed in order to determine the ideal treatment method for improving the mechanical characteristics of bamboo, and the result is compared to conventional structural materials. The material properties in compression and tension are two behaviors present in flexural mechanics. By optimizing the bamboo in terms of compression and tension, a corresponding improvement in the bending behavior of this material is expected. Determination of the optimum treatment can also be utilized during the manufacturing process of this material in structural applications. Utilizing such a treatment will improve the performance of the structure in relation to using untreated bamboos. This analysis is conducted in the form of a quantitative trade study involving all of the treatments investigated. First, objectives and requirements are established for the trade study. Second, trade variables of interest are identified. Third, a weighting factor for each trade variable is assigned according to its importance relative to the others. Last, the analysis is conducted, and an optimum treatment is concluded. Once a conclusion is reached, a comparison is performed of the optimized bamboo to conventional structural materials. Understanding the role bamboo can play in the search for new biocomposite constituents is important in order to consider utilizing this material over conventional ones.

\subsection{Optimization Analysis for Ideal Bamboo Treatment}

The objectives and requirements of this trade study can be summarized into one statement: determine the bamboo treatment with the optimum compressive and tensile properties for use in structural application. The compressive and tensile properties are utilized in this analysis because they provide the fundamental elements for characterizing the mechanical nature of this material. By selecting the ideal treatment 
under these parameters, a general notion may be obtained for the performance of the material under flexural loading. It is of no surprise that bending materials exhibit compression and tension regions about the neutral axis. On the grand order of this research, this analysis will assist in optimizing bamboo for use in more structural applications to replace conventional eco-destructive materials.

Next, four trade variables are selected to summarize the mechanical properties of interest. The specific strength and modulus in tension and compression are deemed to be the ideal variables. Specific strength in this analysis indicates two items of information: the resultant strength and weight acquired from the treatment. The specific strength essentially defines the strength of the bamboo in relation to its mass contained in a volumetric unit. Additionally, this variable fairly compares the strength performances across all of the treatments; comparing the ultimate stress of each group is inadequate since this property does not capture the vital characteristic of mass in the system. On another note, the specific modulus supplies insight on the resultant stiffness of the treatment per unit mass in a contained volume of the material. Many ideal structural systems minimize the amount of deflection caused by nominal loading conditions during operation. Minimizing displacements and optimizing the stiffness of the system for design conditions is therefore represented by the specific modulus.

Selecting weighting factors for each trade variable is the next vital step in the analysis. Factors of $20 \%$ are chosen for each variable in order to determine the optimum treatment in a completely objective and unbiased manner. In this sense, a general improvement of the mechanical properties is studied. Maintaining equivalent weighting factors is also appropriate in order to optimize the flexural performance of the bamboo. As mentioned before, it is well known that bending behavior is equally composed of compression and tension elements in the material. 
The simple algorithm for conducting the trade study is as follows. It is also important to note that the standard deviation of each trade variable is incorporated in this analysis and is factored into the algorithm in the same manner of the square of the sum of errors. First, all the mechanical properties of interest for the treatments are normalized by the untreated bamboo properties. Equation (23) indicates how to conduct this operation.

$$
n_{i, j}=\frac{x_{i, j}}{x_{g, j}} \pm s_{i, j}
$$

In the equation above, $n$ is the normalized value of the mechanical property respective to the untreated bamboo, $x$ is the value of the trade variable, the subscript $i$ denotes the treatment in question, the subscript $j$ denotes any of the four trade variables, the subscript $g$ references the untreated bamboo, and $s$ is the error associated with performing this quotient. The error of the equation above is defined as

$$
s_{i, j}=\sqrt{\left(\frac{\sigma_{g, j}}{x_{g, j}}\right)^{2}+\left(\frac{\sigma_{i, j}}{x_{i, j}}\right)^{2}}
$$

Where $s$ is the error and $\sigma$ is the standard deviation of the mechanical property given in the variable $x$. Then all of the respective variables are normalized again relative to the highest performing variable. Equation (25) defines this operation at this step of the algorithm.

$$
N_{i, j}=\frac{n_{i, j}}{n_{\max , j}} \pm S_{i, j}
$$

The equation above defines $N$ as the normalized value relative to the maximum of the trade variables. The subscript max denotes the treatment with the highest performing variable, and $S$ is another error associated with performing this operation. This particular error is defined as 


$$
S_{i, j}=\sqrt{\left(\frac{s_{\max , j}}{n_{\max , j}}\right)^{2}+\left(\frac{s_{i, j}}{n_{i, j}}\right)^{2}}
$$

Where $S$ is the error of normalizing the trade variables relative to the highest performing treatment. It is critical to note that $s_{m a x, j}$ denotes the previously calculated error associated with the highest performing treatment-not the maximum error for the trade variable among the treatments. Once normalized, all the resulting variables are multiplied by the respective weighting factor of each trade variable. Equation (27), shown below, outlines this step as

$$
p_{i, j}=u_{j} \cdot N_{i, j}
$$

Where $p$ is the product of these two variables and $u$ is the corresponding weight factor of each trade variable. Then each product calculated in the previous step is summed for every treatment. Equation (28) defines how to determine the final score for each treatment as

$$
T_{i}=\sum_{j=1}^{4} N_{i, j} \pm e_{i, j}
$$

Where $T$ is the total sum score of the treatment, and $e$ is the total sum error for the score. This final error is defined as

$$
e_{i, j}=\sqrt{\sum_{j=1}^{4}\left(u_{j} \cdot S_{i, j}\right)^{2}}
$$

Where $e$ is the sum of the errors for this analysis, and $u$ is the weight factor associated with the respective trade variable. Finally, the highest scoring treatment is then selected as the optimum. It is important to take into account the error associated with each treatment score in order to effectively determine the optimum treatment. It is deemed 
necessary to associate the errors with this trade study due to the variability in the measured mechanical properties. If the tests yielded lower standard deviation values, then the analysis would be performed without the error shown.

Table 5-1. Results of the optimization analysis for the ideal treatment

\begin{tabular}{|c|c|c|c|c|c|c|c|c|c|c|}
\hline \multirow{3}{*}{$\begin{array}{c}\text { Weight } \rightarrow \\
\begin{array}{c}\text { Treatment } \\
\downarrow\end{array}\end{array}$} & \multicolumn{2}{|c|}{$\begin{array}{c}\text { Specific } \\
\text { Compressive } \\
\text { Modulus }\end{array}$} & \multicolumn{2}{|c|}{$\begin{array}{l}\text { Specific } \\
\text { Tensile } \\
\text { Modulus }\end{array}$} & \multicolumn{2}{|c|}{$\begin{array}{c}\text { Specific } \\
\text { Compressive } \\
\text { Strength }\end{array}$} & \multicolumn{2}{|c|}{$\begin{array}{l}\text { Specific Tensile } \\
\text { Strength }\end{array}$} & \multirow{3}{*}{$\begin{array}{l}\text { Total } \\
\text { Score }\end{array}$} & \multirow{3}{*}{ Error } \\
\hline & \multicolumn{2}{|c|}{$25 \%$} & \multicolumn{2}{|c|}{$25 \%$} & \multicolumn{2}{|c|}{$25 \%$} & \multicolumn{2}{|c|}{$25 \%$} & & \\
\hline & Value & Score & Value & Score & Value & Score & Value & Score & & \\
\hline $\begin{array}{c}180^{\circ} \mathrm{F} \\
\text { with } \\
1^{\circ} \mathrm{F} / \mathrm{min} .\end{array}$ & 138.936 & 12.7 & 582.526 & 21.1 & 302.602 & 7.5 & 1334.626 & 20.1 & 61.4 & 8.5 \\
\hline $\begin{array}{c}200^{\circ} \mathrm{F} \\
\text { with } \\
1^{\circ} \mathrm{F} / \mathrm{min} .\end{array}$ & 171.357 & 25.0 & 567.331 & 20.2 & 372.602 & 14.0 & 1333.696 & 20.0 & 79.2 & 9.1 \\
\hline $\begin{array}{c}220^{\circ} \mathrm{F} \\
\text { with } \\
1^{\circ} \mathrm{F} / \mathrm{min} \text {. }\end{array}$ & 165.720 & 22.9 & 645.967 & 25.0 & 489.425 & 24.9 & 1392.772 & 22.2 & 95.0 & 10.8 \\
\hline $3 \%$ Salt & 167.937 & 23.7 & 631.171 & 24.1 & 490.210 & 25.0 & 1466.878 & 25.0 & 97.8 & 10.8 \\
\hline $6 \%$ Salt & 166.582 & 23.2 & 585.431 & 21.3 & 471.648 & 23.3 & 1285.881 & 18.3 & 86.0 & 8.7 \\
\hline $9 \%$ Salt & 160.246 & 20.8 & 566.359 & 20.1 & 471.578 & 23.3 & 1295.988 & 18.6 & 82.8 & 8.5 \\
\hline Lime & 156.872 & 19.5 & 634.461 & 24.3 & 439.314 & 20.2 & 1159.370 & 13.5 & 77.6 & 9.1 \\
\hline Oil & 159.369 & 20.5 & 533.910 & 18.2 & 455.323 & 21.7 & 1115.550 & 11.9 & 72.2 & 7.0 \\
\hline $\begin{array}{c}150^{\circ} \mathrm{F} \\
\text { with } \\
5^{\circ} \mathrm{F} / \mathrm{min} \text {. }\end{array}$ & 120.329 & 5.6 & -- & -- & 239.267 & 1.6 & -- & -- & 7.2 & 1.1 \\
\hline $\begin{array}{c}180^{\circ} \mathrm{F} \\
\text { with } \\
5^{\circ} \mathrm{F} / \mathrm{min} .\end{array}$ & 151.346 & 5.6 & -- & -- & 285.596 & 5.9 & -- & -- & 23.3 & 3.7 \\
\hline $\begin{array}{c}200^{\circ} \mathrm{F} \\
\text { with } \\
5^{\circ} \mathrm{F} / \mathrm{min} .\end{array}$ & 151.300 & 17.4 & -- & -- & 335.762 & 10.6 & -- & -- & 28.0 & 4.0 \\
\hline
\end{tabular}

The conclusion of the analysis deems the $3 \%$ salt solution cured at $220^{\circ} \mathrm{F}$ with a $1^{\circ} \mathrm{F} / \mathrm{min}$. initial ramp rate to be the optimum treatment. Table 5-1 and 
Figure 5-1 supply a comprehensive representation of the results from the optimization analysis. As seen from previous analyses, all the treatments investigated provide a substantial improvement over the untreated bamboo. For this analysis, an Initial inspection warrants the $3 \%$ salt treatment to be the optimum, but it can also be observed

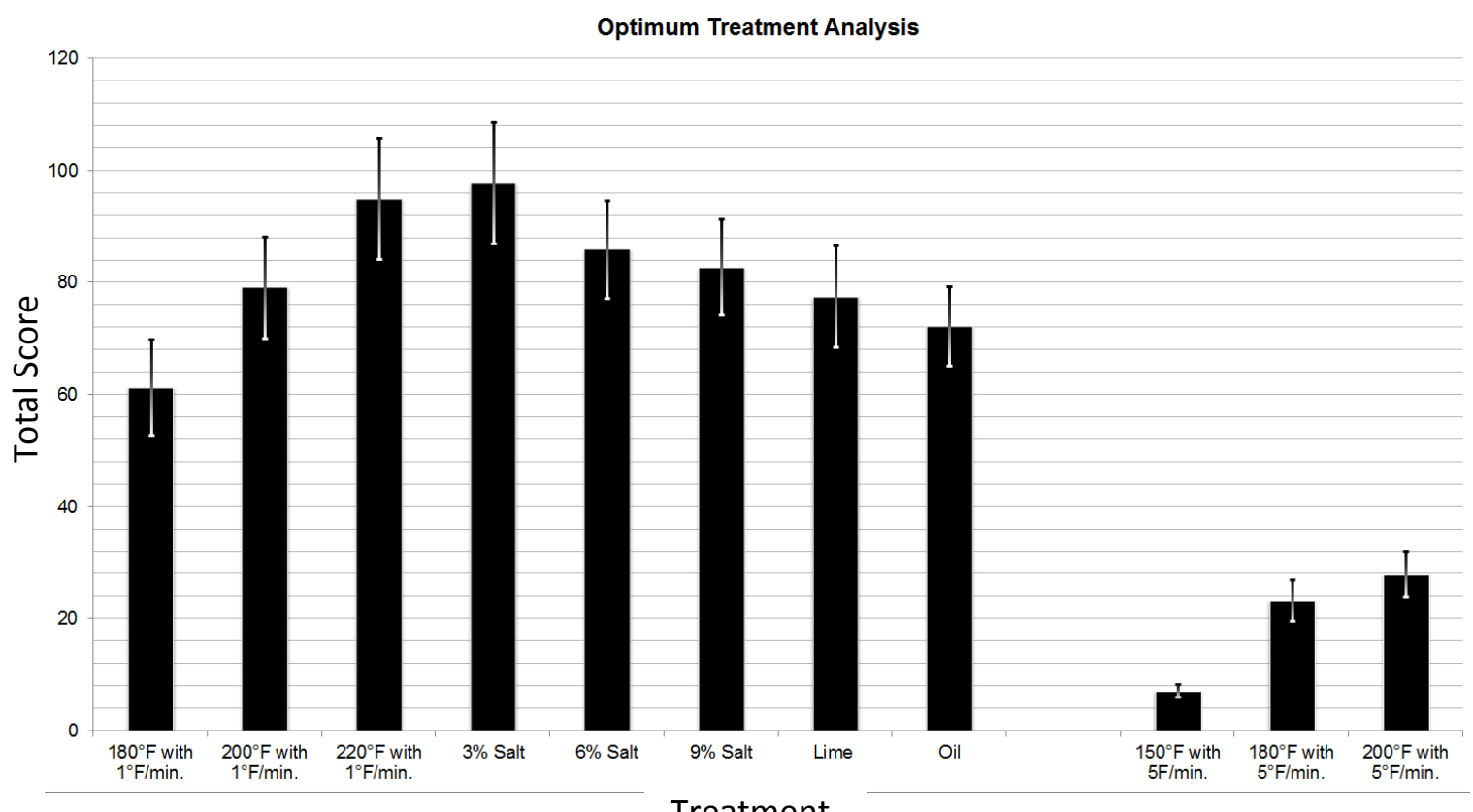

Figure 5-1. Graphical representation of optimization analysis for the ideal treatment to improve the mechanical properties of bamboo

that the $220^{\circ} \mathrm{F}$ treatment fairs well in the study also. It is apparent that these two treatments optimally improve the mechanical properties of the bamboo. However, the analysis also suggests that the $6 \%$ and $9 \%$ salt treatments are additional contenders for ideal treatments. The $200^{\circ} \mathrm{F}$ also takes notice but its proximity to the two best treatments is not as significant as the salt treatments. Finally, it is said with confidence that the highest thermal treatment and lowest salt treatment are the only options considered. The likelihood of the remaining treatments performing better than the considered options is low since their maximum possible score considering the error does not reach the total score of these two treatments. Selecting either treatment would consequently yield similar performance, but ultimately the $3 \%$ salt treatment is nominated over the $220^{\circ} \mathrm{F}$ as 
the optimum treatment for two reasons. First, this treatment scored the highest in terms of the raw total score of the analysis. More importantly, it is noted that the $3 \%$ salt treatment provides additional benefits by preserving the bamboo to have longer operational life. These grounds motivate the selection of the $3 \%$ salt treatment. A valid statement is now imposed in saying that the treatments with the $5^{\circ} \mathrm{F} / \mathrm{min}$. rate do not score as high due to insufficient data from the tension experiments. However, even if these treatments are awarded the maximum points for those tensile trade variables, the final outcome of the trade study would not have changed.

In addition, a brief weight factor sensitivity analysis is also conducted to investigate how varying the importance of each trade variable affects the outcome of the study. The details of the study are supplied in the appendix. By placing more emphasis on the compressive material characteristics, the $3 \%$ salt treatment still scores the highest. The remaining salt treatments also score higher and enter deeper into the range of the $3 \%$ salt treatment. Similarly, if the tensile trade variables are given more importance, the $3 \%$ salt treatment scores the highest again. Therefore, it is concluded that favoring either loading condition does not considerably affect the outcome of the study. Similarly, placing more emphasis on the strength trade variables also results in the $3 \%$ salt treatment scoring the highest. The only other treatment to perform within the range of the lowest salt treatment is the $200^{\circ} \mathrm{F}$. In contrast, more importance on the modulus trade variables yields an obscure win for the $3 \%$ salt treatment. Intuitively, this outcome makes sense since the modulus improvement for these treatments are similar. In summary, changing these variables in the mentioned ways always yields the $3 \%$ salt treatment scoring the highest; the win becomes more apparent if the strength variables are favored while emphasizing the compression variables complicates the win for the $3 \%$ salt treatment. 


\subsection{Comparative Mechanical Performance of Bamboo to Conventional}

\section{Materials}

With the optimal treatment now determined, it is imperative to compare its material properties to conventional materials to validate its use in application. Many of the conventional materials utilized in industry yield destructive practices on the environment whether it is through the manufacturing process or in the disposal phase of the structure's lifecycle. These environmentally unfriendly materials are 4130 steel, two aircraft grade aluminums, titanium, and carbon fiber-epoxy composites. Specific strengths of each material are compared to that of this bamboo. Table 5-2 outlines the comparative results of all the materials.

Table 5-2. Comparison of bamboo to conventional structural material

\begin{tabular}{|c|c|}
\hline Material & Tensile Specific Strength $\left(\frac{\text { kips-in. }}{\mathrm{Ib}_{\mathrm{m}}}\right)$ \\
\hline AISI 4130 Steel (Normalized at $\left.1600^{\circ} \mathrm{F}\right)^{[4]}$ & 342 \\
\hline Aluminum 2024-T3 ${ }^{[4]}$ & 700 \\
\hline $\begin{array}{l}\text { Phyllostachys aureosulcata Bamboo } \\
\text { (Untreated) }\end{array}$ & 796 \\
\hline Aluminum $7075-\mathrm{T}^{[4]}$ & 814 \\
\hline Titanium Ti-6Al-4V (Grade 5), Annealed ${ }^{[4]}$ & 863 \\
\hline $\begin{array}{l}\text { Phyllostachys aureosulcata Bamboo } \\
\text { (220F Thermal Treatment Only) }\end{array}$ & 1393 \\
\hline $\begin{array}{l}\text { Phyllostachys aureosulcata Bamboo } \\
\text { (3\% Salt Treatment) }\end{array}$ & 1,467 \\
\hline Carbon-Epoxy Composite ${ }^{[52]}$ & 3,145 \\
\hline
\end{tabular}

As seen from the table, bamboo shows comparable performance to many of the conventional materials used in structures. In fact, the tensile specific strength of the optimum bamboo is 4.3 times that of steel and 1.7 times that of titanium. Specific strength of carbon fiber, however, is approximately 2 times greater than this bamboo. It 
important to note that this bamboo is only one species of the many that grow in the wild. There is a possibility that greater mechanical improvement can be yielded by other species of bamboo. Although bamboo currently does not surpass the performance of conventional carbon-fiber composites, the gap between these two can close with furhter research. In any case, bamboo is shown to provide competitive performance to other structural-grade materials. Critical evaluation of these data does not imply that this bamboo is an appropriate material for all applications; it merely illustrates the possibilities of utilizing this material as a viable option in more structural system architectures. 


\section{Chapter 6. Bamboo Flexural Characteristics}

Once the optimization analysis illustrates the optimum treatment, the mechanical properties of bamboos for two geometric configurations under bending loading conditions are investigated. Many structures in engineered designs contain beams under bending loads, so it is imperative to study the flexural mechanics of the bamboo to adequately predict its behavior during operation. The bending behavior of the bamboo varies between the different geometries in biocomposites, so this study will also investigate the flexural properties of bamboos for whole bamboo culms and prepared bamboo rectangular strips. This analysis will provide insight on the flexural behavior of using the bamboo in its natural form or veneer shape for structural applications. The term "veneer" in this case is defined as a thin rectangular strip of approximately 0.1 -in. thickness cut from the bamboo's culm. Utilizing entire bamboo culms are beneficial for applications requiring strong support in main lightweight structural components. Conversely, employing bamboo veneers is valuable in shell bodies typical of composite laminate structures that also call for strength.

In addition, research into the effect of nodes in bamboo structures has not been comprehensively studied under bending. Therefore, bending tests with varying node placement along the length of the beam are conducted. It is well known that bamboo nodes beneficially assist the load-bearing characteristics of the material by absorbing much of the stress in compression-particularly if the load point is close to the node. One can also theorize that specimens with nodes are stronger under flexural stress. However, as previously alluded, a node cannot always be guaranteed in the desirable vicinity of a structural member, so it is beneficial to examine the effects of the node's placement along the internode. A test where the node is located at the same location for 
every specimen aids in yielding more precise data, and further insight can be gained by analyzing the bending characteristic of a bamboo pole when loaded at locations near a node. In addition, these configurations will also determine how much of a difference nodes make on the overall performance of bamboos. To summarize the testing analysis, a set of guidelines are developed for utilizing bamboo in structures that exhibit bending behavior during application.

The bending section of the ISO standard outlines a procedure and method to determine these bending mechanical properties. First, a four-point bending scheme is chosen over a three-point method in order to satisfy failure due to flexural stress. Applying two equally spaced forces helps ensure a bending failure. The standard also dictates that bamboo poles must be used with nodes "evenly" spaced. Friction effects are addressed by utilizing frictionless supports that only restrain the sample specimen in the vertical direction. Consequently, any restraints from friction are reduced by using a smooth roller support. A loading rate that will cause failure within 1 to 10 minutes is also advised.

However, a few issues presented themselves at the beginning of the flexural investigation-ones for which solutions were absent in the ISO standard. For instance, the standard does not differentiate between bamboo internodes and specimens with nodes. This may pose a problem for applications where only one configuration is used. Consequently, both bamboo configurations are investigated: one arrangement for just the internode, another where the node is located at the center, and an alternative at the quarter-length location. Another issue is the question of what lengths the samples should be for the test. After inspecting multiple bamboo poles remaining from the previous tests, it was decided that most internodes of this bamboo variety span about 9-in. Consequently 9-in. samples are tested in an effort to simulate an average bamboo pole's performance at the lowest level. Lastly, the loading rate selected to comply with the 
standard is 0.024 -in./min. On another note, obtaining poles for a test group with nodes exactly spaced apart at the same locations is difficult to acquire and often does not occur naturally. This issue raised a red flag for data inconsistency. As mentioned previously, a solution devised to mitigate the problem manifested itself in the form of the three specimen configurations. Finally, the same matter of maintaining a constant diameter and thickness among the samples in the "whole culm" groups persisted to be a potential issue; an improved solution besides a large test group was not employed.

\subsection{Preparing Flexural Specimens}

Like the specimens before, these flexural test samples are carefully prepared at the Cal Poly SLO Aero Structures/Composites Laboratory according to the geometric dimensions specified earlier. Bamboo poles are first gathered to accommodate the numerous long specimens required for the test. It is imperative that each different pole be selected to have similar geometric dimensions such as outer diameter and thickness. Both geometric configurations entail different manufacturing methods, so each procedure is described in this section.

Three specimen configurations warrant slightly different preparation procedures for the test with the entire culm configuration. Initially, a bamboo pole is examined for any terrible irregularities or cracks. Irregularities in this case are surmised by any culm with end-diameter differences of over $50 \%$, any really oval cross sections, and lastly by exaggerated bends along the axial direction of the pole. These irregularities are then excluded from the test group after cutting each culm at the proper locations. For the internode group that does not carry any nodes: the diamond tile saw is used to cut each culm to span 9.5-in. between each node. The test group with a node at the center of the specimen is cut 4.75 -in. from each side of the node. Lastly, the final sample collection for 
the node located at the quarter-point was cut 2.5-in. from the node on one side and 7.0in. on the other. Figure 6-1 depicts the process of cutting these specimens. The maximum and minimum diameter of each specimen is measured at both ends; likewise the thickness is measured four times at equal distances around the circumference of each end. Once cut and measured, the specimens are inspected once again for safe measure and are ready for testing.
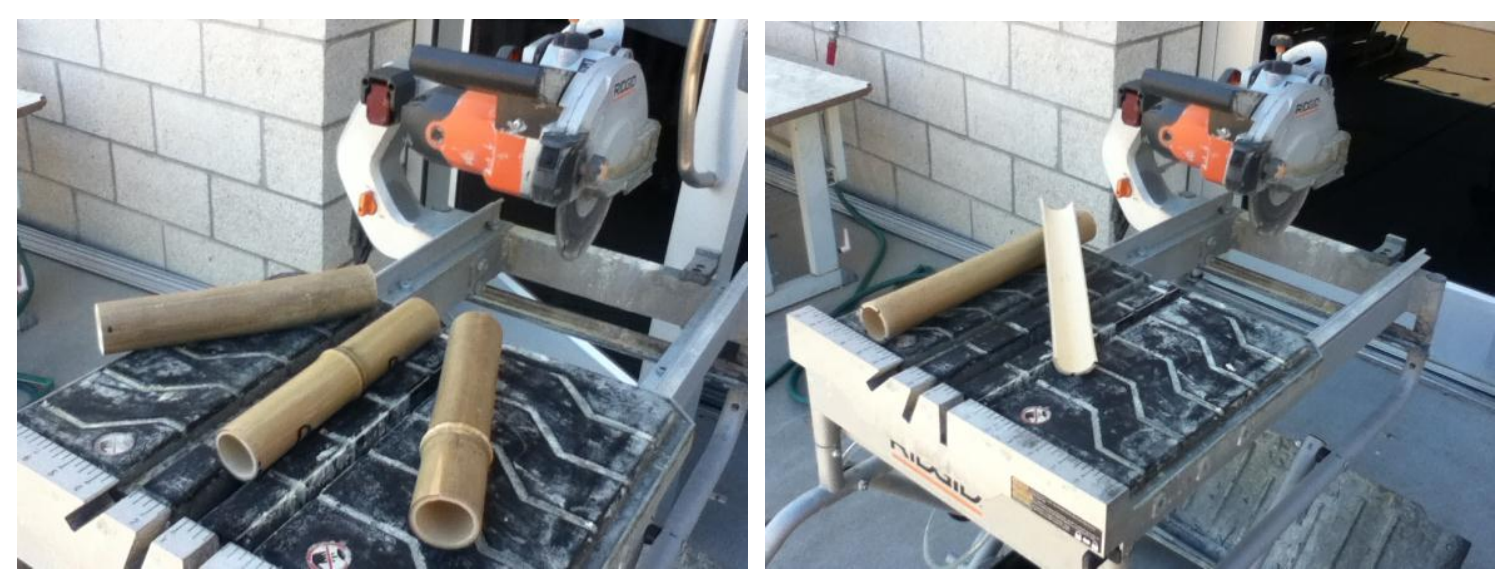

Figure 6-1. Cutting bamboo poles to have the desired node locations for cylindrical configuration (left) and rectangular configuration (right)

Although not as simple as the previous procedure, the geometric configuration involving the bamboo veneer group initially follows a similar preparation procedure. First, all the bamboo poles are inspected and cut to the proper length from each node using the same initial procedures defined previously for the whole-culm configuration. At this point the preparation similarities cease, and the procedure outlined in the Preparing Tension Specimens section is utilized to cut and sand each culm into the desired rectangular strips. Figure 6-2 demonstrates the final products for the flexural rectangular specimens. Once cut to the appropriate dimensions and sanded accordingly, the samples are inspected and marked ready to test. 

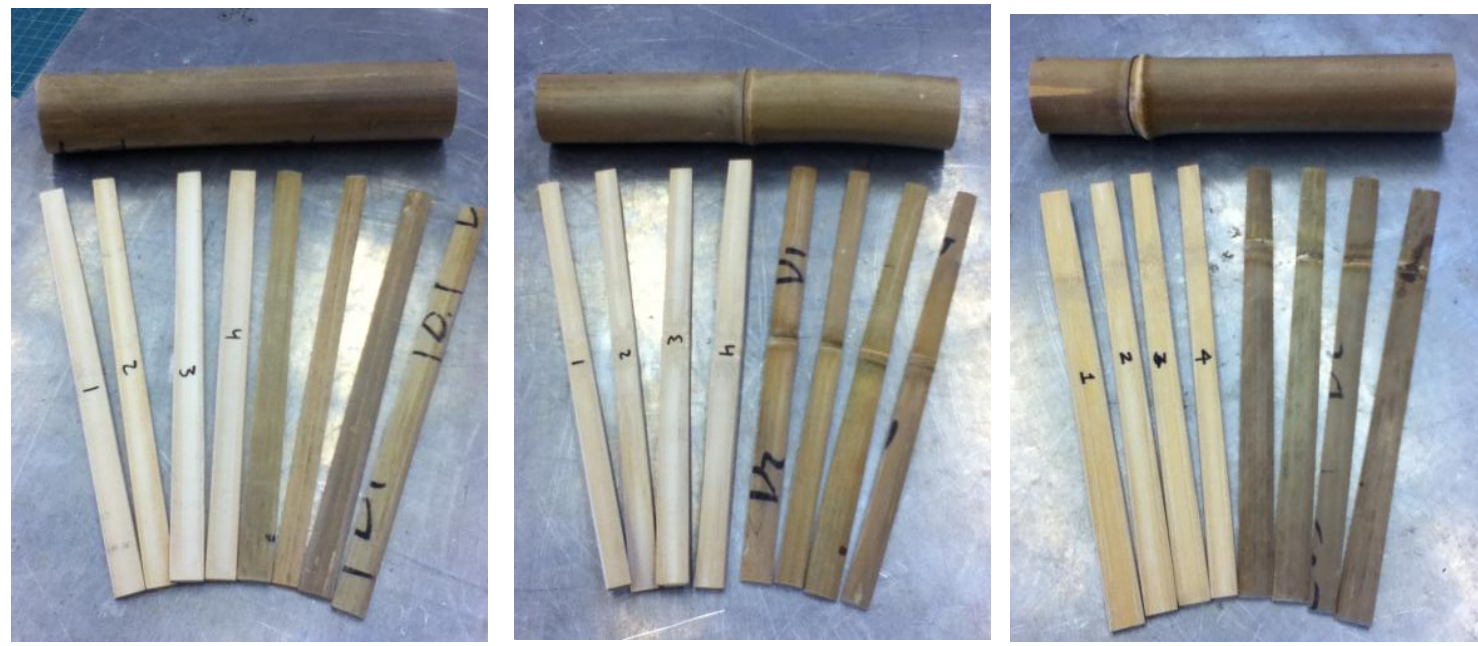

Figure 6-2. Final products of flexural rectangular specimens: internode (left), mid-node (center), and quarter-node (left)

After preparing each specimen, a table summarizing all the specimens' dimensions prior to testing is investigated to help account for any anomalies that may exist in the plots. The average outer diameter and wall thickness of each sample are measured with an electronic caliper to \pm 0.001 -in. accuracy. The mean of four outer diameter measurements is taken as the average diameter. On the other hand, the mean of eight thickness measurements-four from each end-face-is determined to be the average thickness. The mass of each specimen is measured on an electronic scale to within $\pm 0.0005-\mathrm{lb}_{\mathrm{m}}$.

The average cross-sectional area of each specimen calculated requires two different equations for each flexural configuration. For the cylindrical specimen group, Eq. (9) is utilized to determine its cross-sectional area. Similarly, Eq. (14) is used to calculate the cross-sectional area for the rectangular configuration group. All of the dimensions are measured in the same manner as explained in earlier sections. The average volume of these flexural specimens is then defined as, 


$$
V_{f}=A_{f} l_{\text {avg }}
$$

Where $V_{f}$ is the average volume of the flexural specimen under either geometric configuration, $A_{f}$ is the corresponding cross-sectional area, and $l_{\text {avg }}$ is the average length of the specimen in the axial direction to the fibers. The average cross-sectional area is defined the same as that of the tensile specimens, so reference is given to the equation located in the Preparing Tension Specimens section. Finally, the average density is subsequently defined as,

$$
\rho_{f}=\frac{m}{V_{f}}
$$

Where $\rho_{f}$ is the average density of the flexural specimen, $m$ is the measured mass, and $V_{f}$ is the previously defined volume on the whole specimen.

\subsection{Flexural Testing Procedure}

The flexural testing procedure followed for both geometric configurations is that outlined in the bending section of the ISO standard mentioned. All bending tests are conducted using the same Instron machine from the Cal Poly SLO Aero Structures/Composites Laboratory. As suggested by the standard, a four-point bend arrangement is installed on the Instron machine. Using a four-point bending scheme aids in ensuring a correct failure due to flexural stress. In other words, failure due to shear is avoided by not utilizing a three-point bending set-up. Once the correct fixtures are installed, the proper support span and length between the half-loads are adjusted on the fixtures. Unfortunately, the standard does not specify the values for these dimensions, so ones tailored for this specific experiment are devised. For all tests these two distances remain constant at 9-in. and 3-in., respectively. The average length of the internodes for all groups is slightly above 9-in., so this dimension seems like the logical choice. Consequently, the distance between both half-loads needs to be 3-in. for proper spacing 
between the center and supports. At this point the testing apparatus is ready, and a bamboo specimen is then loaded onto the roller supports. First, the roller supports are cleaned of any debris that may cause friction; the free end of the beam specimen must allow rotation at the support. Careful attention is then paid when aligning the sample on the supports: the specimen, roller supports, and load fixtures are all aligned to this same vertical plane. For the rectangular specimens, the outer side of the culm is always facing the top of the sample when placed on the supports. Figure 6-3 depicts how the specimens are loaded into the testing area. As instructed in the standard, a constant load rate of $0.024-\mathrm{in} . / \mathrm{min}$. is entered into the Instron software. The specimen is then preloaded just enough to provide surface contact between the load points and
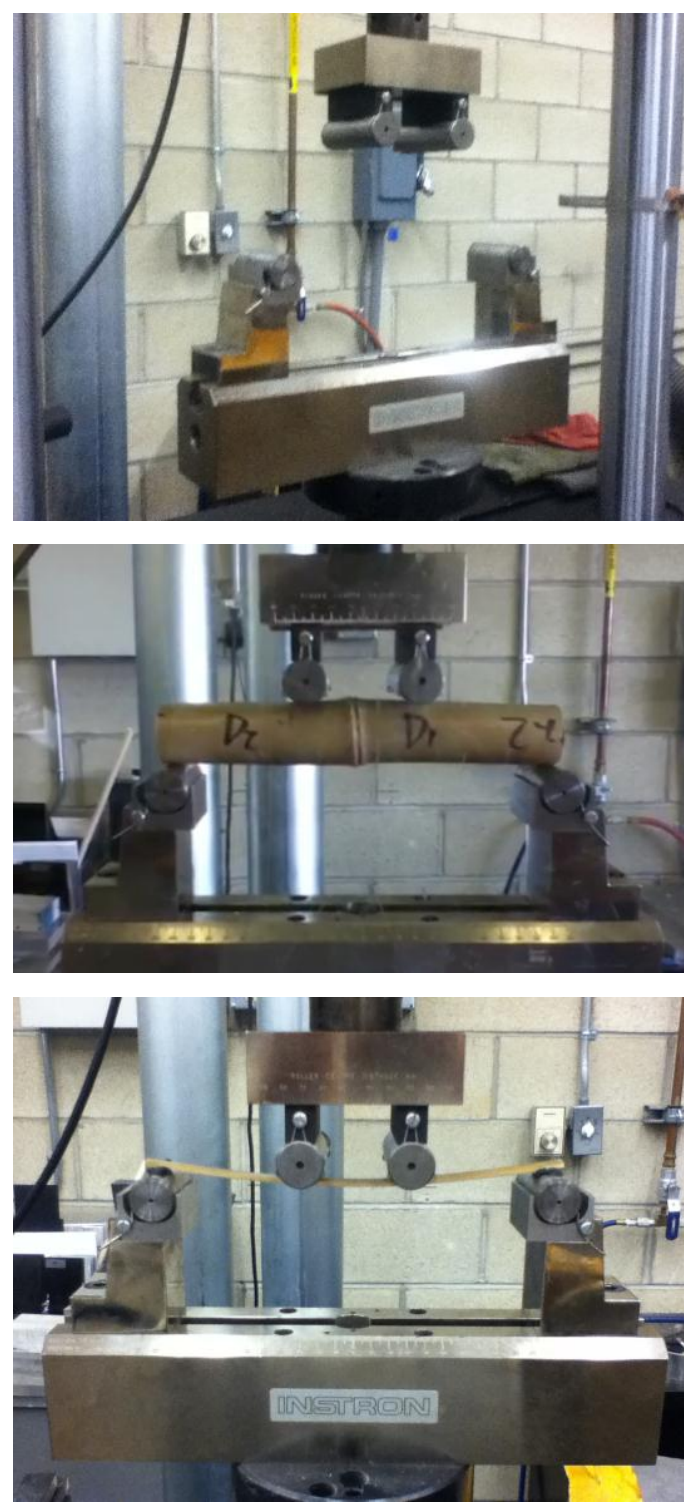

Figure 6-3. Four-point bending test fixtures (top), preloading cylindrical configuration into load area (center), and preloading rectangular configuration into load area (bottom)

the specimen body. Finally, the test initiates and ends at failure when the load measured by the load cell falls $40 \%$ from the average force determined at the high sampling rate. The specimen is then removed and the testing area is cleaned before repeating the last two steps for the remaining samples. 


\subsection{Flexural Results}

The following encompasses the results of the flexural tests for all three bamboo node locations in both geometric configurations. As with the other tests, a table showcasing the geometric dimensions of all specimens is investigated for anomalies. Again, this table is shown for reassuring purposes that all testing samples are within the same general dimensions and to pin-point any discrepancies resulting from the tests. Each bamboo configuration's load-deflection curve is illustrated as well as its stress-strain plots. The mechanical properties included in this section are the specific strength, specific modulus, yield stress/strain, and ultimate stress/strain. Also, a discussion of the failure types of each test is analyzed. Lastly, a table summarizing the flexural mechanical characteristics for each sample group is provided in the appendix for convenience.

The proper analysis tools for these tests require slightly different equations for each geometric configuration and are defined in this section. For a four-point bend on a beam with equidistant half-loads from the supports, the flexural stress is defined as,

$$
\sigma_{f}=\frac{F a}{z}
$$

Where $\sigma_{f}$ is flexural stress, $F$ is the force applied, $a$ is the distance between the nearest load and support, and $z$ is the section modulus of the beam. It is important to note that this is the maximum stress along the length of the beam. For a hollow cylindrical bamboo specimen assumed to be perfectly circular, the section modulus is computed with the equation,

$$
z_{h}=\frac{\pi}{32 D_{\text {avg }}}\left[D_{\text {avg }}^{4}-\left(D_{\text {avg }}-2 t_{\text {avg }}\right)^{4}\right]
$$


Where $z_{h}$ is the section modulus for a hollow cylinder, $D_{\text {avg }}$ is the average outer diameter of the specimen and $t_{\text {avg }}$ is the average thickness of the hollow cylinder. Similarly, the section modulus for a rectangular beam is defined as,

$$
z_{r}=\frac{w_{\text {avg }} t_{\text {avg }}^{2}}{6}
$$

Where $z_{r}$ is the section modulus for a rectangular beam, $w_{a v g}$ is the average thickness of the specimen, and $t_{\text {avg }}$ is the average thickness of the rectangular beam.

In addition, the flexural strain for both geometric configurations of the bamboo also differs. The flexural strain for the hollow cylindrical beam under this loading condition is defined as,

$$
\varepsilon_{f_{h}}=\frac{12 D_{a v g}}{3 L^{2}-4 a^{2}} \delta
$$

Where $\varepsilon_{f_{h}}$ is the flexural strain of a hollow cylinder beam, $D_{a v g}$ is the average outer diameter of the beam, $L$ is the support span, and $\delta$ is the flexural deflection (positive for deflections increasing in the vertical direction). A derivation of this strain equation is provided in the appendix. Likewise, the flexural strain for the rectangular beam with the same loading condition is defined as,

$$
\varepsilon_{f_{r}}=\frac{12 t}{3 L^{2}-4 a^{2}} \delta
$$

Now that the proper analytical equations are developed, the testing results can be analyzed appropriately.

\subsubsection{Internode Configuration}

The internode specimens are the first tested among the three sample groups and are deemed as the baseline from which to compare the other two groups. Figure 6-4 depicts 
one of the samples under a flexural load during a test as an example of the deformation of the beam.

The following figures, Figure 6-5 and Figure 6-6, display the stress-strain curve of the internode bamboo group. The average ultimate stress for the hollow cylinder group is determined to be $4.22-\mathrm{ksi}$. The rectangular group has an average ultimate stress of 20.3ksi. The ultimate strain for the cylindrical group is averaged to be $0.85 \%$ and the rectangular group is determined to be $1.43 \%$. Although the cylindrical group displays slightly high variation in the data, the rectangular group demonstrates consistent curves for most of the specimens. Variation in the cylindrical group is due to the uncontrollable
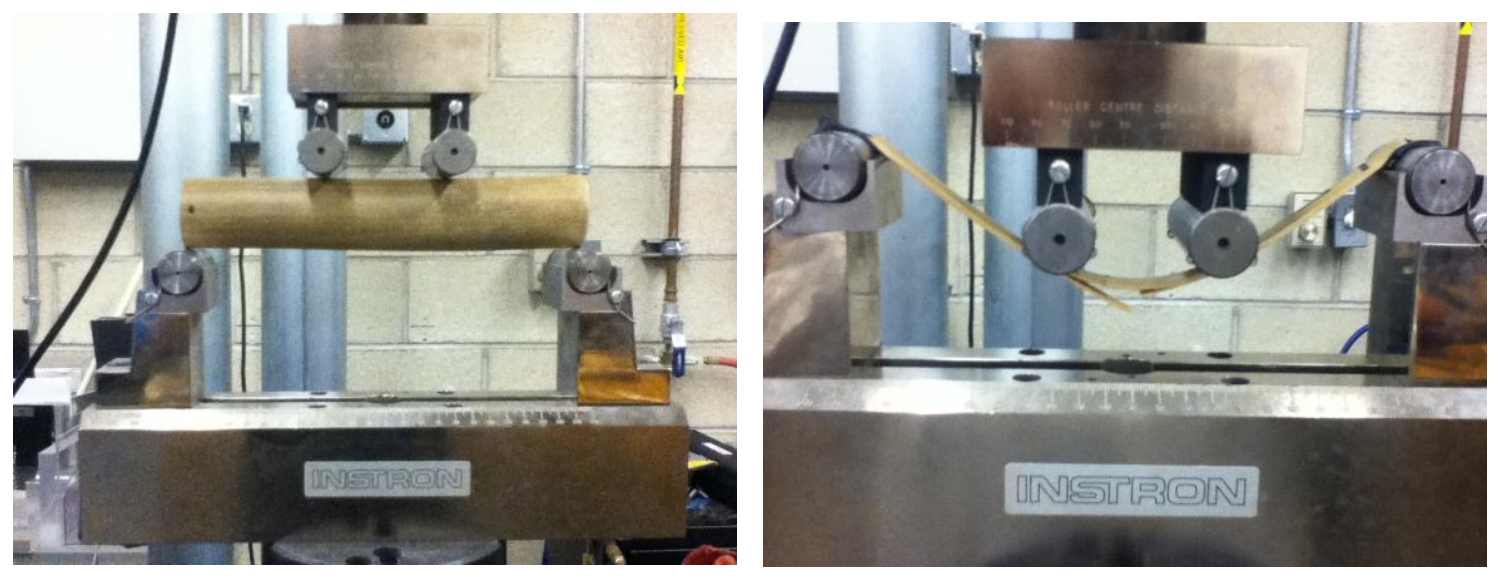

Figure 6-4. Deflection of internode bamboo samples for both configurations

thickness and diameter of the few samples. Care was taken in selecting similar bamboo internodes, but ultimately the data indicate these variables still differed substantially. It was noticed during the mounting portion of the test procedure that the specimen often times had to be rotated in order to provide adequate surface contact at both load points. As mentioned earlier, bamboo poles have a taper to them that runs the length of the pole from the base to the top. Significant stress can be placed on one side if only one load fixture makes contact with the specimen. As a result, the slight fluctuations in diameter along the length of the specimen were used to discover a position where both load fixtures made proper contact. In addition to this observation, specimens one and four are 
exceptionally oval. This compounded with the rotation of the specimen may have affected the results of the internode bending test. The rectangular group avoids this problem by having a constant thickness made during manufacturing.

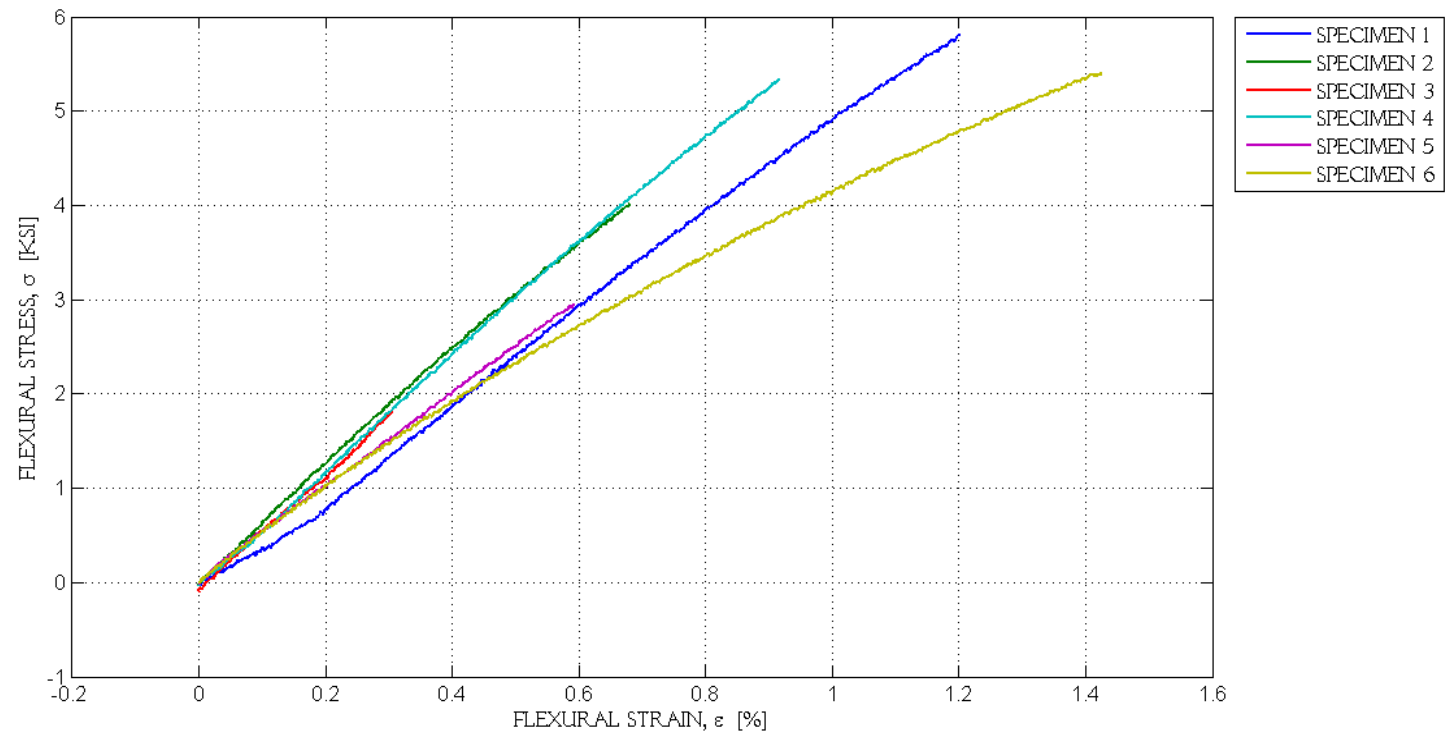

Figure 6-5. Internode bamboo flexural stress-strain curves for cylindrical group

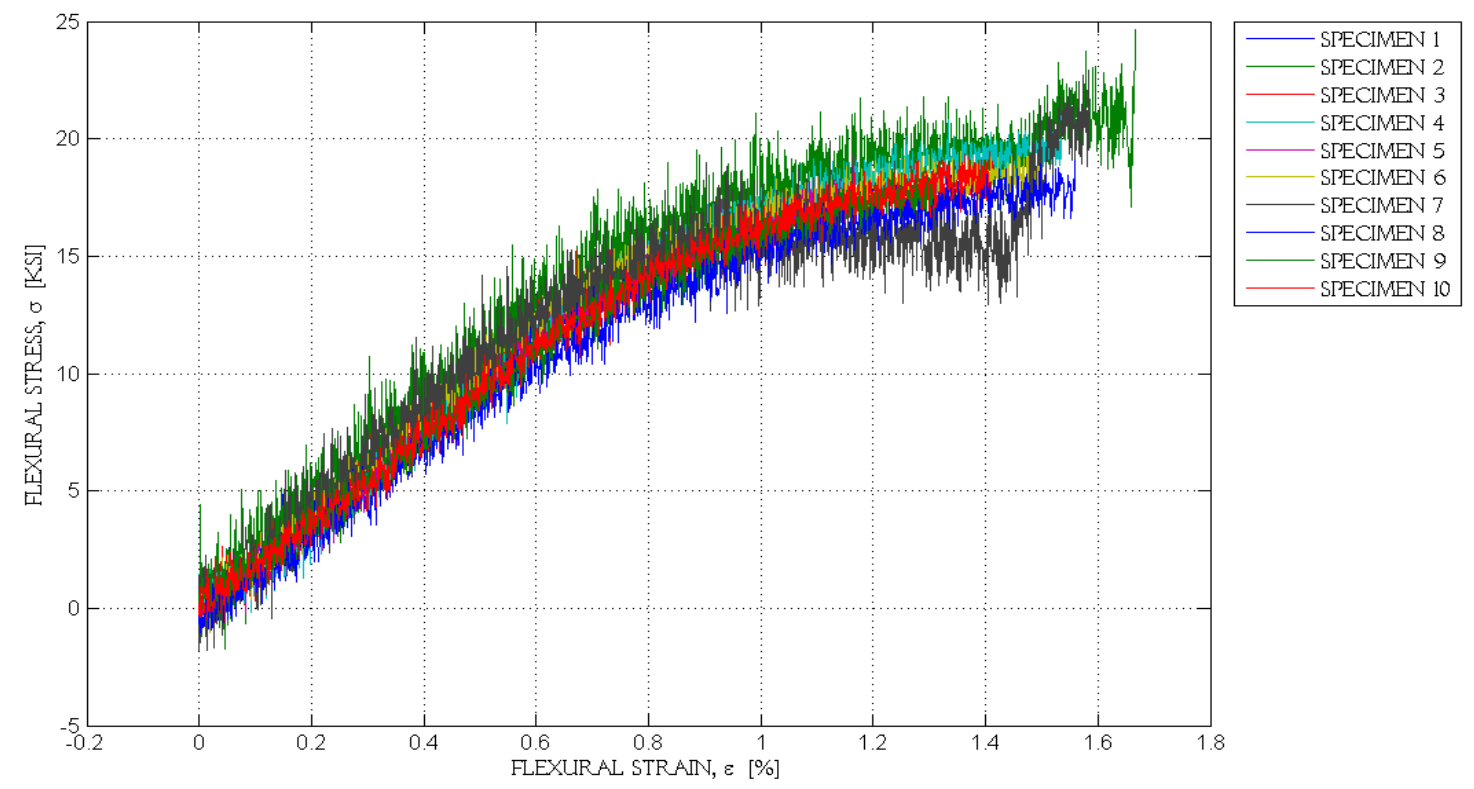

Figure 6-6. Internode bamboo flexural stress-strain curves for rectangular group 
The common failure types observed from both tests are shown in the Figure 6-7. The cylindrical group illustrates a cleaner fracture that is apparent in all the specimens. As seen from the photograph, a single straight fracture spanning the top length of the specimen is how all the specimens failed. Sometimes the fracture would be a clean one while other times a few fibers would remain clinging at certain locations. At the top of this region, the fibers being compressed transversely to their principal direction cause the fracture down the center. In contrast, the rectangular specimens display four common failure types. The order of failure frequency is shown in the figure and increases from bottom to top. All fractures occurred in the lightly colored inner side of the culm since this is the weaker portion of the cross section. As seen from the figure, the most observed failure mode is a specimen that exhibits two transverse fractures at the load points. In one instance, the specimen did not fracture at all, which is a reminder of a bamboo's flexibility.
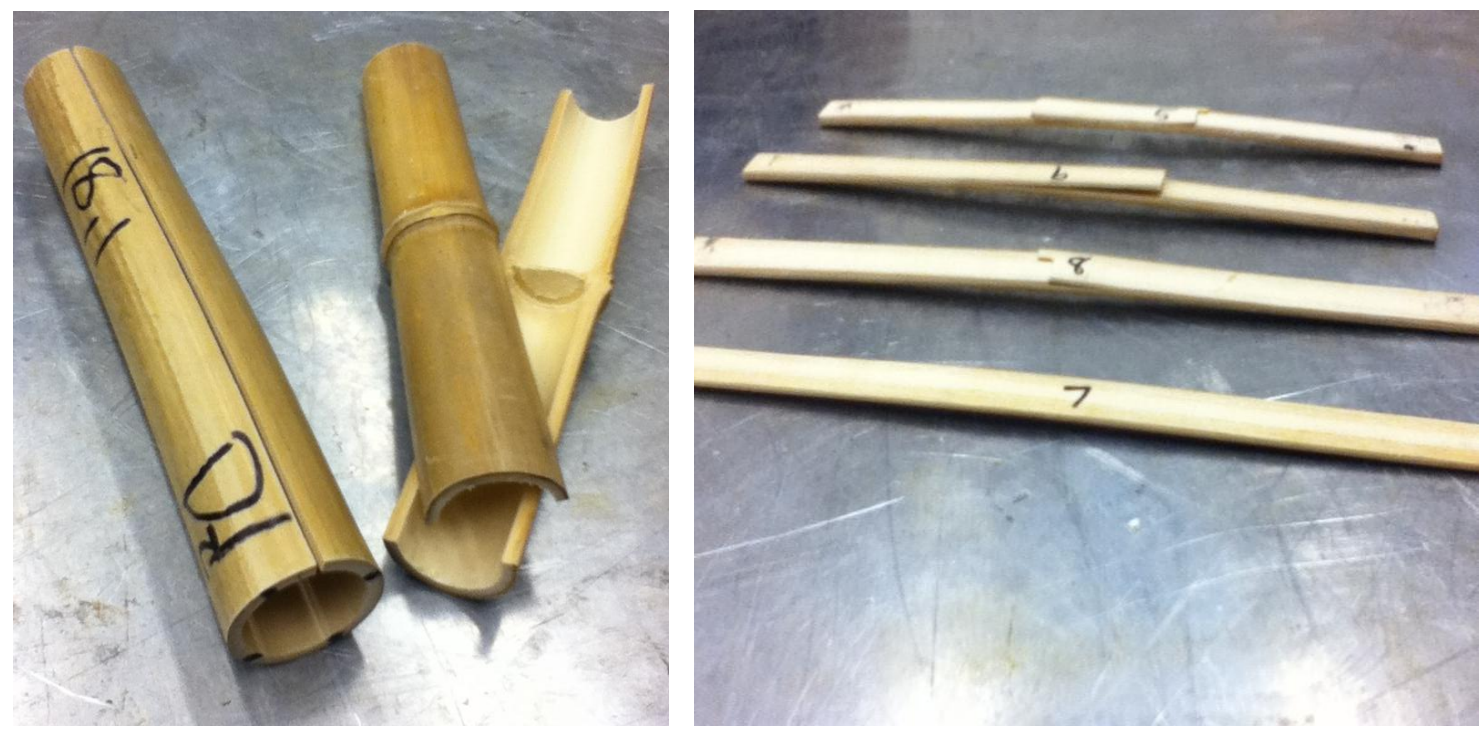

Figure 6-7. Common internode bending failure types: two fracture types for cylindrical group (left) and four observable fracture types for rectangular group (right) 
In summary of the bamboo internode flexural test, two tables outlining the main mechanical characteristics of both groups are shown. It is important to note that both of these tests cannot be compared to each other in terms of their mechanical properties; they are simply supplied together for convenience. All of the properties shown are calculated in the same manner as the previous tests. The bending modulus for the cylindrical test is averaged to be $529-\mathrm{ksi}$, the yield stress is $3.99-\mathrm{ksi}$, the ultimate stress is $4.22-\mathrm{ksi}$, the specific modulus is $19984-\mathrm{kips}-\mathrm{in} . / \mathrm{lb}_{\mathrm{m}}$, and the specific strength is $162-$ kips-in./lb $\mathrm{b}_{\mathrm{m}}$. As expected, most of the percent deviations are over $35 \%$-except for the bending modulus. The bending modulus for the rectangular specimens is averaged to be $1926-\mathrm{ksi}$, the yield stress is $14.1-\mathrm{ksi}$, the ultimate stress is greater at $20.3-\mathrm{ksi}$, the specific strength is 757-kips-in./lb $\mathrm{b}_{\mathrm{m}}$, and the specific modulus is $71862-\mathrm{kips}-\mathrm{in} . / \mathrm{lb}_{\mathrm{m}}$. All the percent deviations for this test are less than $14 \%$. The specific properties relative to their respective averages vary by $5 \%$. The bending modulus also deviates from the average by $5 \%$, but the yielding properties have a greater deviation at $14 \%$ and $9 \%$ for the strain and stress, respectively. On a slightly better note, the ultimate strain and stress have a deviation of $12 \%$ and $10 \%$, respectively. A reminder is stated in noting that these deviations are expected for a material with varying biological factors.

\subsubsection{Central Node Configuration}

The following group is where a node is located at the center of the bamboo specimen. Figure 6-8 depicts one of the rectangular samples under a flexural load during a test as an example of the deformation of the beam. A photograph of a loaded cylindrical specimen with a central node is shown previously in Figure 6-3. 

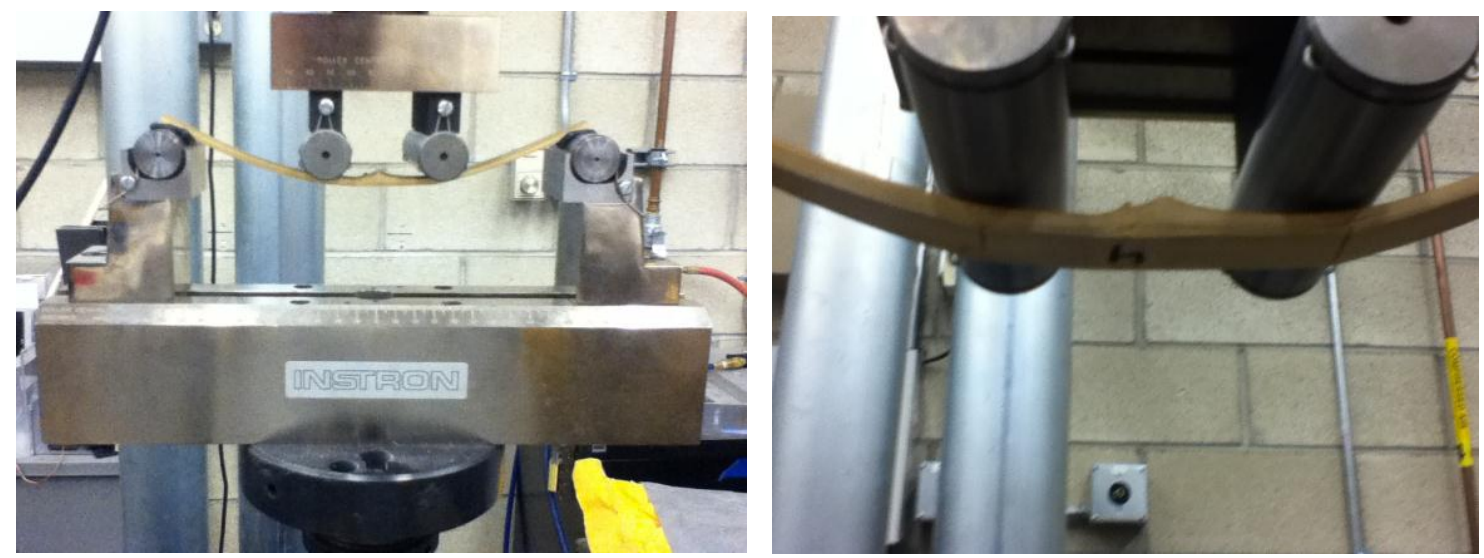

Figure 6-8. Deflection of mid-node bamboo samples for the rectangular configuration

Figure 6-9 and Figure 6-10 display the resulting stress-strain curves for the bending group with a node at the center. For the cylindrical group, two types of curves are evident from the plot: a brittle one and another that is characterized by an upper and lower yield limit. The first two specimens exhibit a normal brittle stress-strain curve and

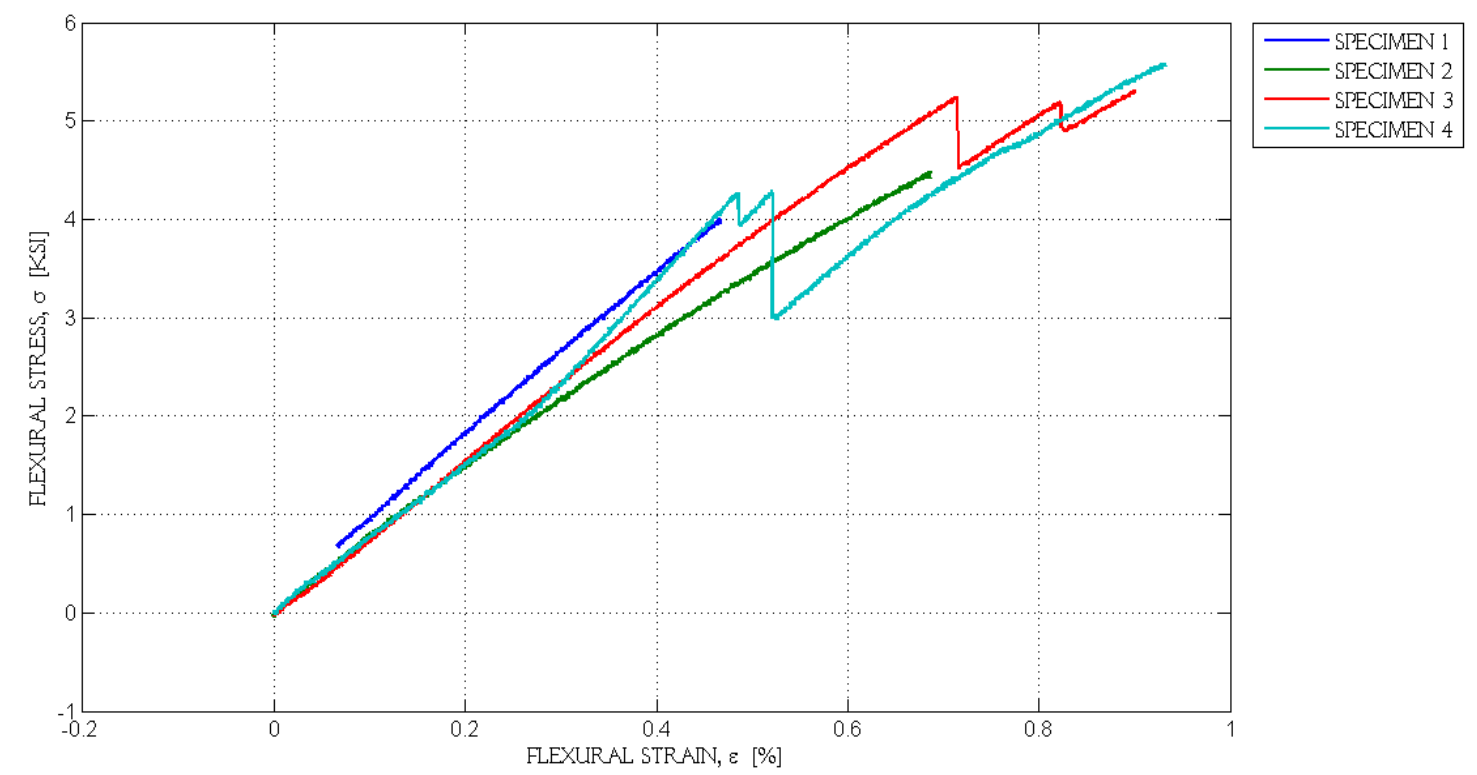

Figure 6-9. Mid-node bamboo flexural stress-strain curves for cylindrical group

are characterized by really oval diameters. The rectangular specimen group indicates much more of a yielding region nearing the plastic zone. The ultimate stress for the cylindrical specimens is averaged to $4.85-\mathrm{ksi}$ while the ultimate strain is determined to 
be $0.75 \%$. Similarly, the rectangular group shows an average ultimate stress of $19.4-\mathrm{ksi}$ and an average ultimate strain of $1.10 \%$.

The common failure types observed from both configurations are shown in Figure 6-12 and Figure 6-11; notice the different fractures in both configurations. Upon failure, thin slits develop on one side of the cylindrical specimen and closes back to a seemingly unbroken specimen after removing the load. A crack forms only on one side because of the varying diameters between both sides of the node. Consequently, the side with a slightly greater diameter fails first and a thin fracture develops. In one case, the failure load causes both the bottom and top face to fracture-resulting in two halves of the specimen. This fracture also illustrates a clean break as shown from the figure. The rectangular specimens display three common failure types. The order of failure frequency is shown in the figure and increases from top to bottom. Again, all the fractures occurred in the lightly colored inner side of the culm since this is the weaker portion of the cross section. As seen from the figure, the most observed failure type is a

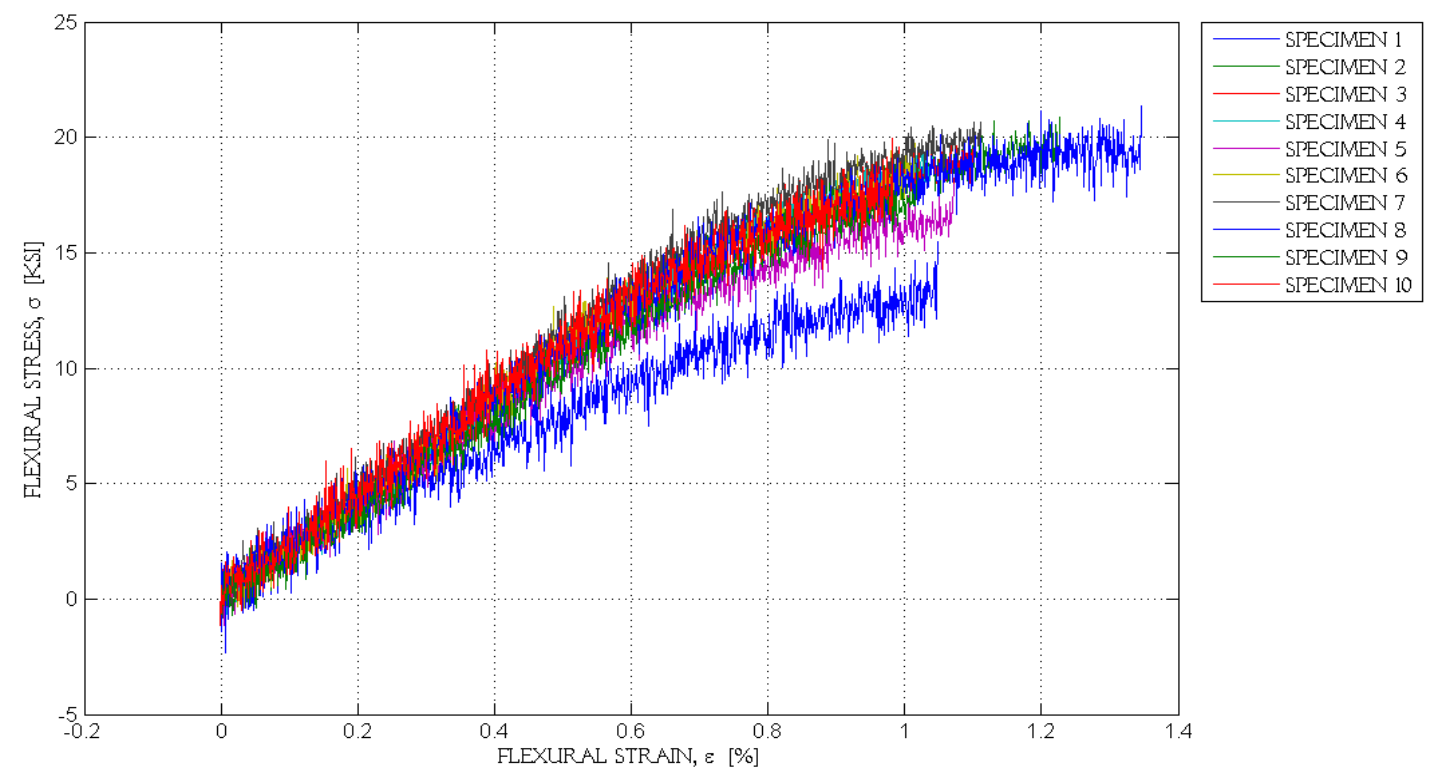

Figure 6-10. Mid-node bamboo flexural stress-strain curves for rectangular group 
specimen that exhibits two transverse fractures at the load points. The second most common failure mode occurs at the side of the central node. The differences between the last two failure modes are simply the amount of fiber delamination and the side from which the fracture propagates. Figure 6-11 illustrates a close-up view of the extent of fiber delamination on one of the specimens. As expected, the delamination region propagates from the center of the beam during flexure.

In summary of the bamboo with a node at the center, two tables are shown displaying the main mechanical properties. In the cylindrical group, the average specific strength is 137kips-in./lb $\mathrm{b}_{\mathrm{m}}$, ultimate stress is $4.85-\mathrm{ksi}$, bending modulus is 753-ksi, and specific bending modulus 21096-kips-in./l $\mathrm{b}_{\mathrm{m}}$. From this test group, strains deviate the most at over $25 \%$. The specific strength deviates the second most at $21 \%$ while the rest are less than 15\%. An interesting note: the
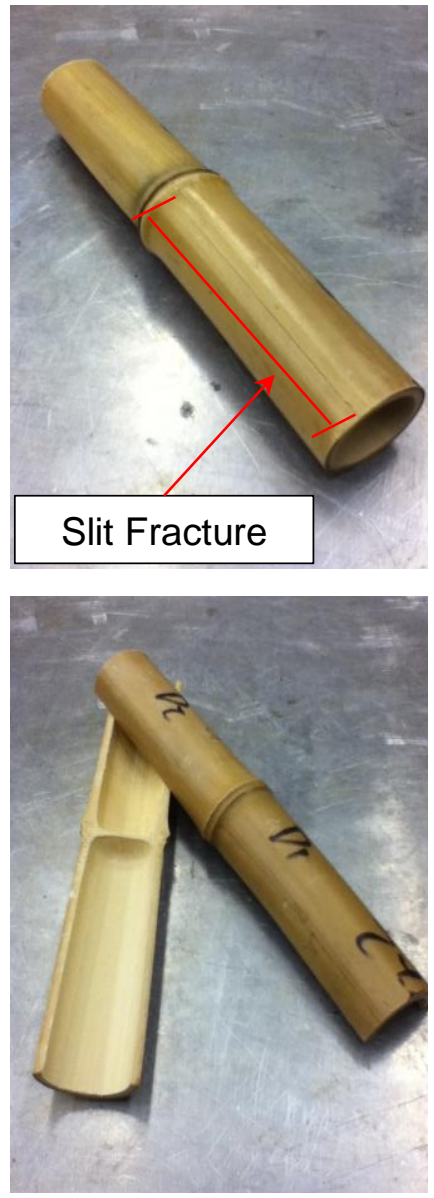

Figure 6-12. Common mid-node bending failure types for cylindrical groups seen as slit fracture (top) and complete clean fracture (bottom)
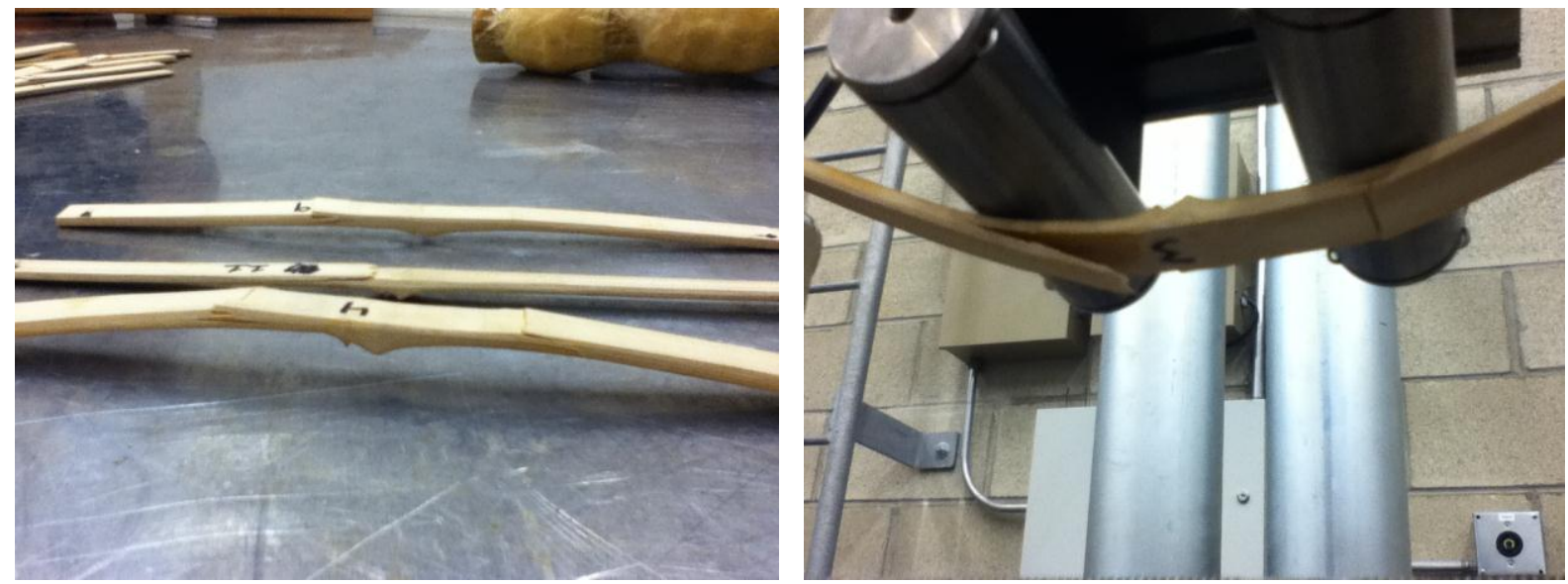

Figure 6-11. Common mid-node bending failure types for rectangular groups: three observable fracture types (left) and the most common failure (right) 
mechanical properties have less deviation than the previous group although the average geometry of the group deviates more. In the rectangular samples, the average specific strength is $687-\mathrm{kips}-\mathrm{in} . / \mathrm{b}_{\mathrm{m}}$, ultimate stress is $19.4-\mathrm{ksi}$, bending modulus is $2047-\mathrm{ksi}$, and specific bending modulus is $72221-\mathrm{kips}-\mathrm{in} . / \mathrm{lb}_{\mathrm{m}}$. From the rectangular group, most of the deviations with respect to the average are under $10 \%$. The highest deviation from the average among the properties is the yield stress. This property demonstrates a deviation of $16 \%$. The specific properties both show a deviation of $10 \%$-a fairly reasonable deviation for data reliability.

\subsubsection{Quarter-length Node Configuration}

The final bending group is where a node is located at the quarter-length of the testing specimen. Figure 6-13 depicts one of the samples under a flexural load during a test as an example of the deformation of the beam. A photograph of the cylindrical specimen is omitted since the deflection behavior looks the same as that of the previous two cylindrical configurations.
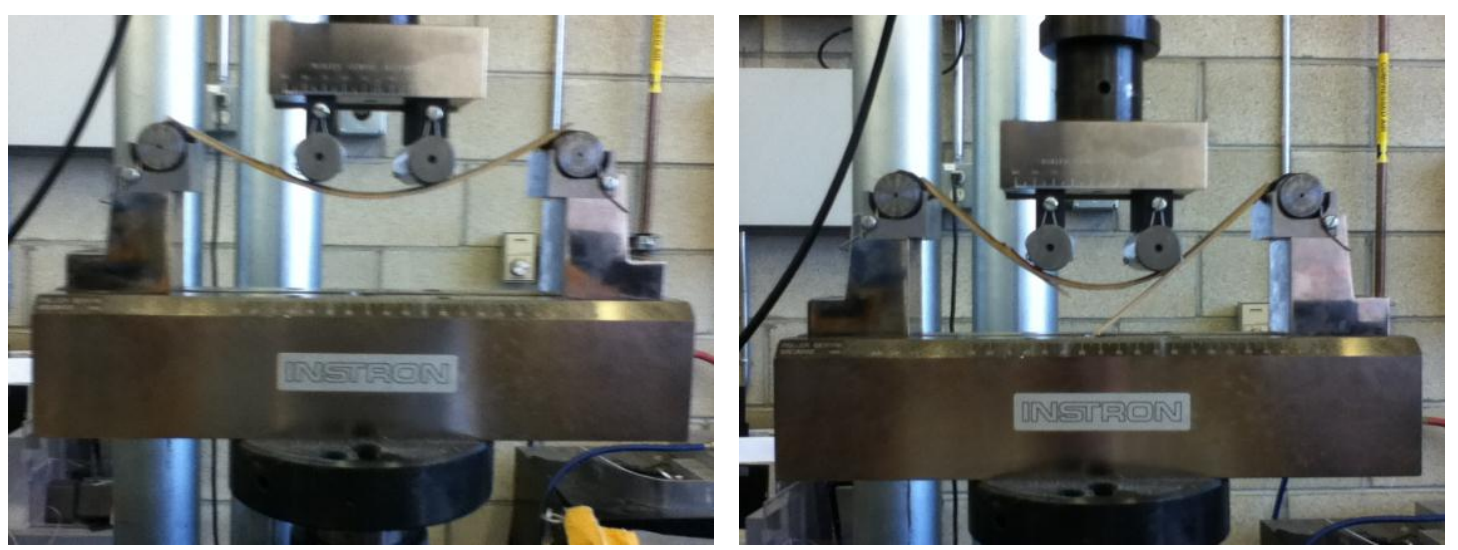

Figure 6-13. Deflection of quarter-node bamboo samples for the rectangular configuration

Figure 6-14 displays the resulting stress-strain curve for the bending group with a node at the quarter-span. The same two types of curves are present in this test. The 
rectangular specimens also show consistency in the linear region of the curve but start to deviate near the plastic zone. This effect occurs because of the individual characteristics of the node begin to dominate the stress imposed on the beam at higher loads. An effect such as this is expected from this type of test. For the cylindrical group, the average ultimate stress is $4.21-\mathrm{ksi}$ while the ultimate strain is averaged to be $1.11 \%$. A yielding characteristic near the plastic zone is much more apparent in the test for the rectangular specimens, and the average ultimate stress is $23.1-\mathrm{ksi}$. Ultimate strains for this configuration are determined to be $1.50 \%$.

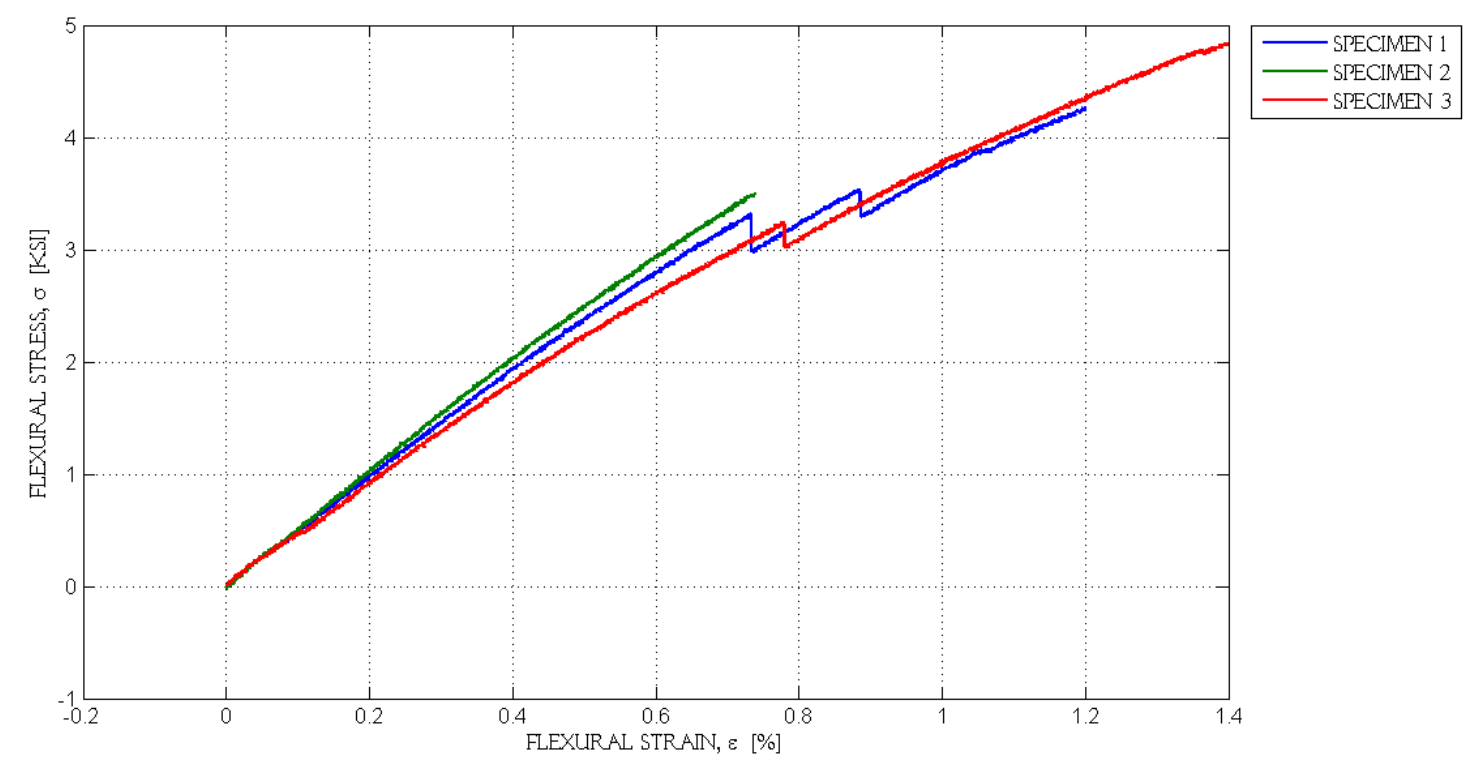

Figure 6-14. Quarter-node bamboo flexural stress-strain curves for cylindrical group

Once again, the common failure types observed are shown for the cylindrical and rectangular groups. Figure 6-15 illustrates the typical failure for the cylindrical group. Failure in the cylindrical group is very similar to the mid-node group where a thin fracture propagates from one side. Again, once the load is relieved from the specimen, the open fracture closes shut. The rectangular specimens display two common failure types. The order of failure frequency is shown in Figure 6-17 and increases from top to bottom. Again, all the fractures occurred in the lightly colored inner side of the culm since this is 


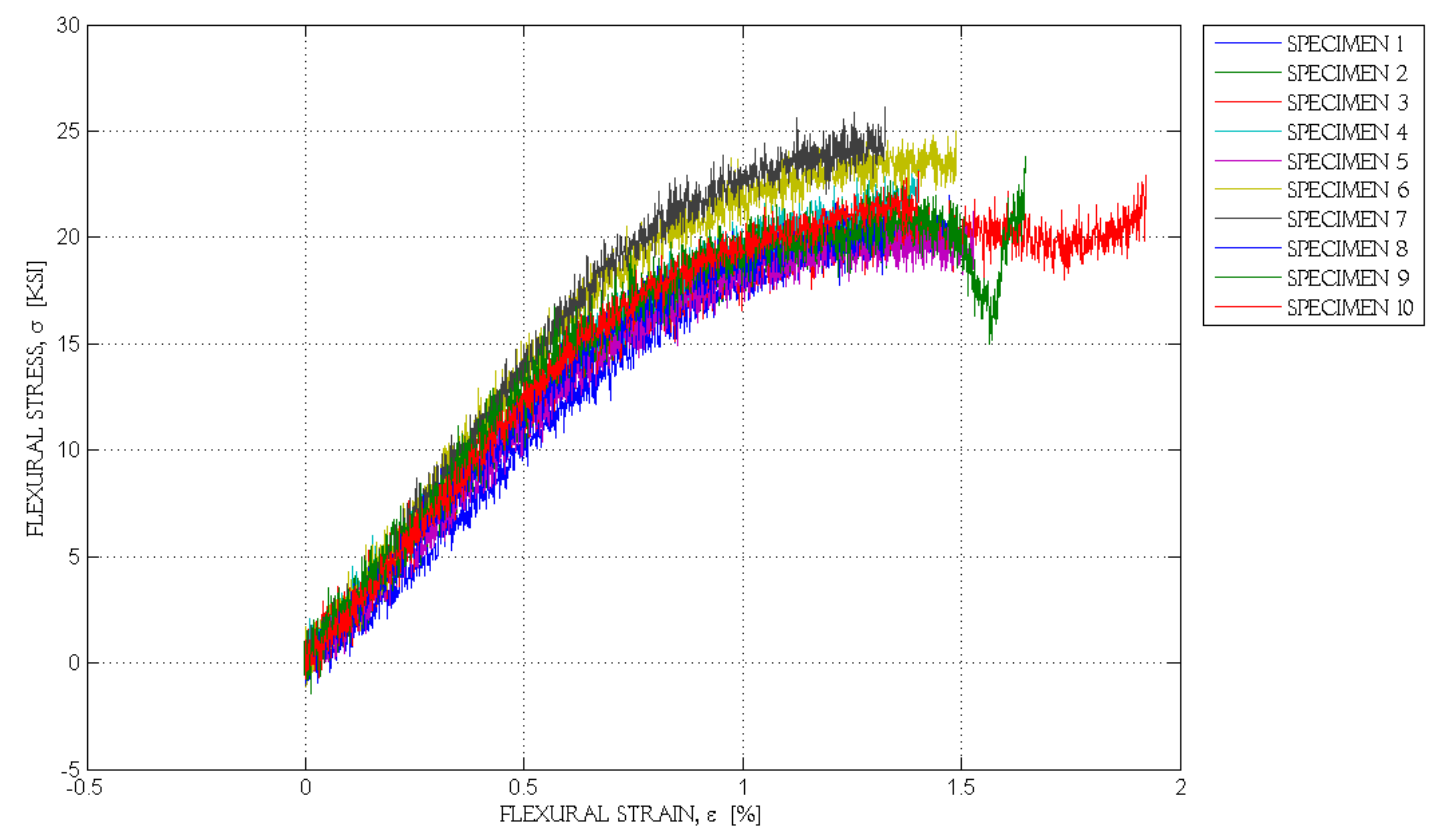

Figure 6-16. Quarter-node bamboo flexural stress-strain curves for rectangular group

the weaker portion of the cross section. As seen from the figure, the most observed failure type is a specimen that exhibits a single fracture in the center of the beam. The second most common failure type occurs off-center and is likely caused by an imbalance of the loading points. Figure 6-17 shows an example of the amount of fiber delamination characteristic of the quarter-point node specimen. Also evident in this figure is the exceptional amount of deflection withstood by this specimen configuration. Further analysis of this characteristic is investigated in the subsequent section.

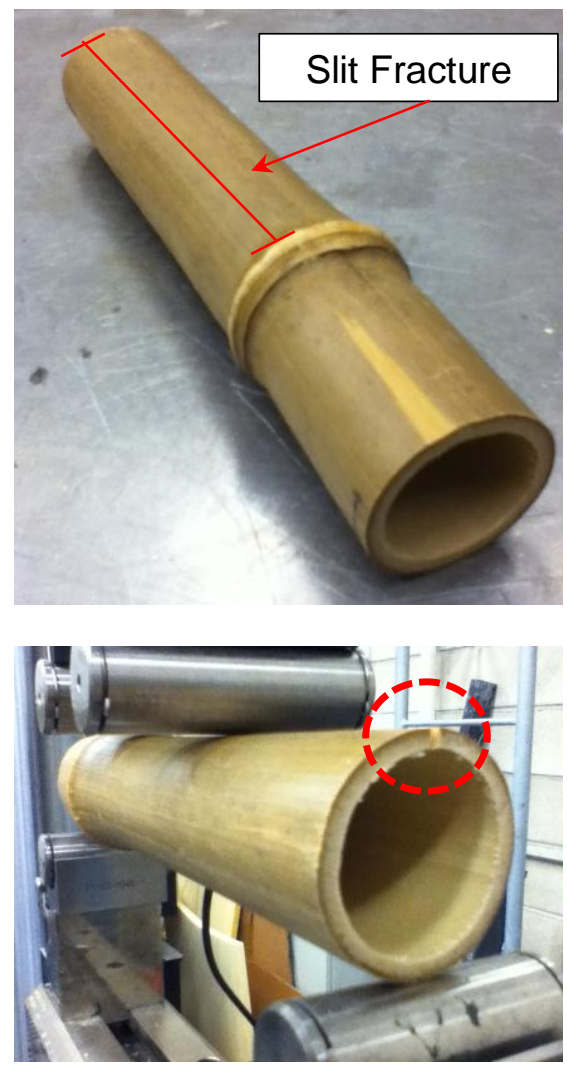

Figure 6-15. Common quarter-node bending failure types for cylindrical group shown as a very thin fracture along the top 
In summary of the bamboo with a node at the quarter-point, two tables are shown displaying the main mechanical properties of this specimen configuration. In the cylindrical group, the average specific strength is 120 -kips-in.// $\mathrm{b}_{\mathrm{m}}$, ultimate stress is 4.21 $\mathrm{ksi}$, flexural modulus is $464-\mathrm{ksi}$, and specific flexural modulus is $13207-\mathrm{kips}^{-i n}$./lb $\mathrm{b}_{\mathrm{m}}$. From this test group, strains deviate the most at over $30 \%$. The yield stress deviates from the average by $21 \%$ and the remaining properties deviate less than $16 \%$. The rectangular group demonstrates a bending modulus of 2334-ksi, a yield stress of 19.4-ksi, ultimate stress of 23.1-ksi, specific bending modulus of 71587-kips-in.//b $\mathrm{b}_{\mathrm{m}}$, and a specific strength of $709-\mathrm{kips}-\mathrm{in} . / \mathrm{l} \mathrm{b}_{\mathrm{m}}$. The greatest standard deviation for the rectangular group is $18 \%$ for the yield strain. The second highest deviation is found in the yield stress at $12 \%$. The specific modulus yields a standard deviation of $8 \%$ while the specific strength only shows a deviation of $5 \%$. The ultimate strain and stress indicate a deviation of $12 \%$ and $7 \%$, respectively. Lastly, the bending modulus shows a deviation of $10 \%$. Overall, the variability of the data is fairly low to indicate favorable reliability in the results.
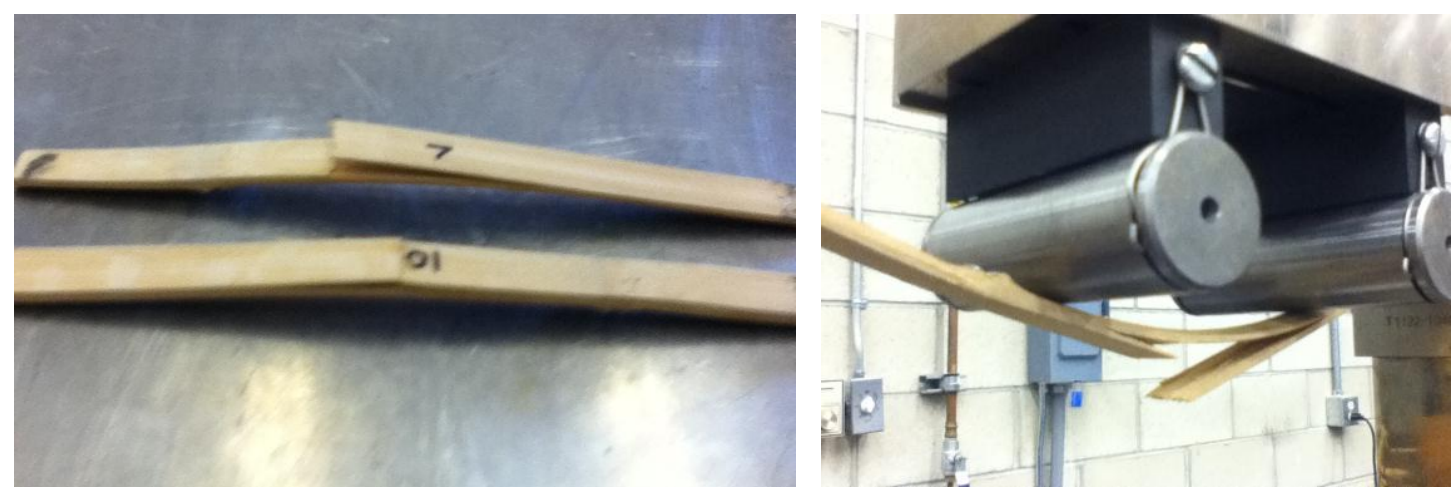

Figure 6-17. Common quarter-node bending failure types: two observable fracture types for the rectangular group (left) and enhanced view of typical failure (right) 


\subsection{Analysis of Flexural Results}

An analysis comparing all the bending specimens' results is reported in this section. A series of figures summarize all the mechanical properties of the groups. A note is taken in stating that all error bars produced in the plots are derived from the standard deviation of each respective bending property. As a reminder, this analysis entails discovering the general flexural behavior of bamboo, as well as the effects nodes have on the strength and stiffness of the material, and finally the effect of node placement on bending behavior during loading conditions. Analysis of the flexural characteristics of the specimens infers that utilizing bamboo with or without nodes will affect the flexural behavior on the naturally cylindrical form but not so much in a laminate form. It is important to bear in mind that explicit comparisons are only appropriate between the different node locations for each geometric configuration.

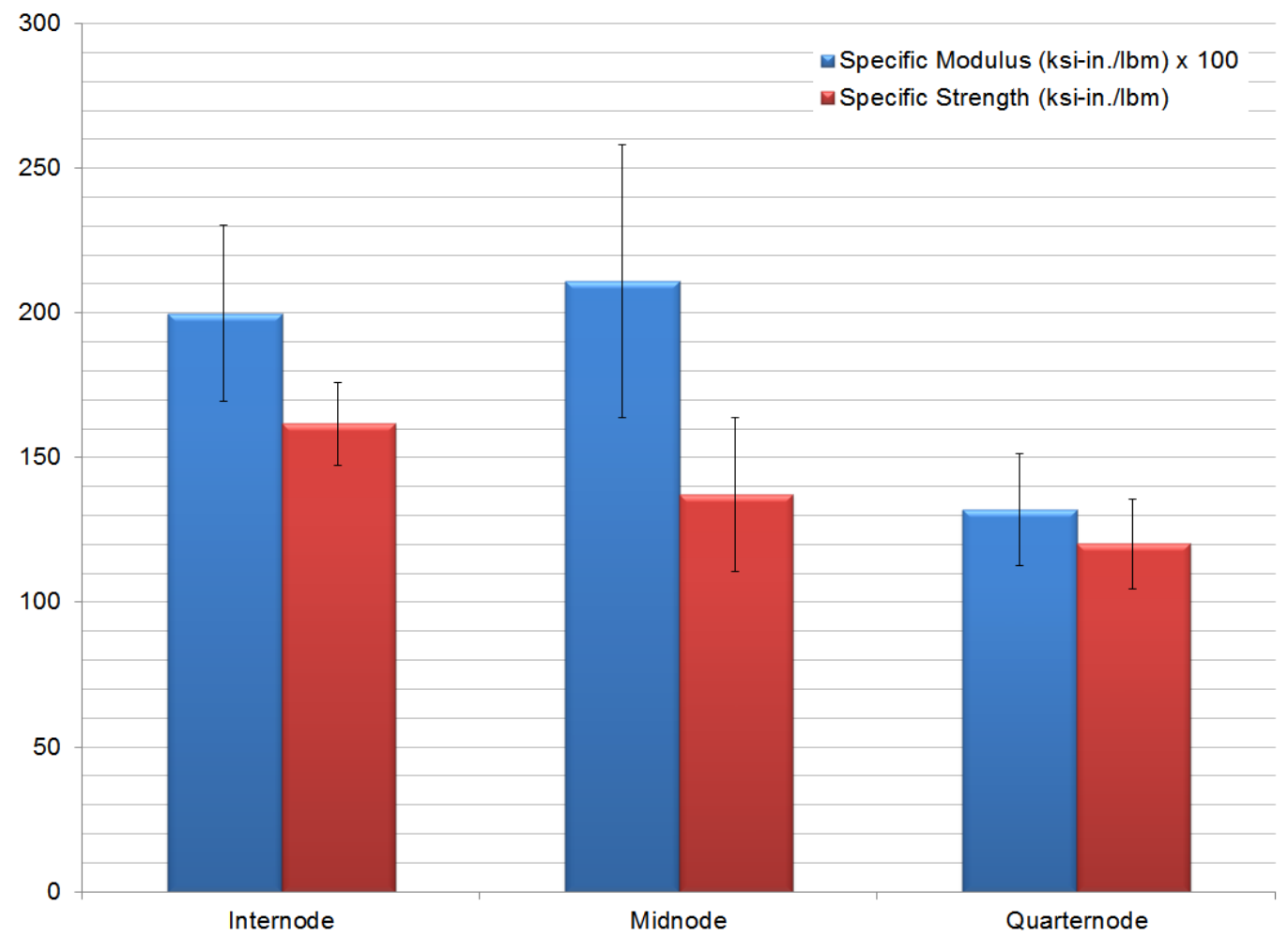

Figure 6-18. Summary of node location effects on the flexural specific strength and specific modulus for the cylinder configuration 
In terms of the specific strength property of these geometric configurations, the data indicates that the internode configuration performs slightly better than the others in a cylindrical form while less significant strength changes occur for the rectangular group. Therefore, it is recommended for designs utilizing bamboo to place nodes away from the immediate vicinity of an applied flexural load when permissible to maximize strength capabilities. First glance into the data from Figure 6-18 shows that the cylindrical configuration has more variability in the specific strength for the different node locations than the rectangular group. The specific strength for the rectangular specimen illustrates much more of a consistent rate. A note is taken in observing that the orientation of the outer wall of the bamboo relative to the applied load has a significant effect on the material strength. In fact, preliminary tests done on rectangular specimens with the outer culm facing down towards the bottom supports showed no fracture at the end of the test. This confirms the higher strength of the outer culm and suggests possibly better performance from the rectangular group if provided enough stiffness.

For both configurations, the average specific strength for the internode performed slightly better than the other two node locations. For the cylindrical group, it appears that the internode and mid-node perform relatively the same while a minor decrease in performance is observed in the quarter-node. At best, the quarter-node configuration performs at $92 \%$ of the internode. In contrast, Figure 6-20 illustrates that the rectangular group has consistent performance for each node placement. Also, the difference between the mid-node and quarter-node is more apparent in the cylindrical group while the rectangular group shows much closer values. Although the raw average values show that the internode has a higher specific strength, the standard deviation of each group suggests all of them to be within the same range. This observation leads to the conclusion that having nodes in bamboo in its natural veneer form will result in similar 
performance for their respective configurations but using it in its natural cylindrical form will only yield similar strength performance for the internode and mid-node.

Therefore, placing a concentrated load on a structure utilizing whole bamboo culms at one side of a node will result in lower performance as opposed to having a load far from a node. However, this can be mitigated by balancing the load across the length of the node. Speaking from a mechanical point of view, the similar performances between the three specimen groups suggests a structure built out of bamboo veneers would not significantly be affected in its strength capability by the location of its nodes. In terms of a veneer composite structure, this characteristic would be beneficial since the nodes would not have to be removed before extracting the outer layer from the culm. Preparation simplicity is achieved for structural applications, and comfort is found in knowing that including the nodes will not significantly affect strength performance.

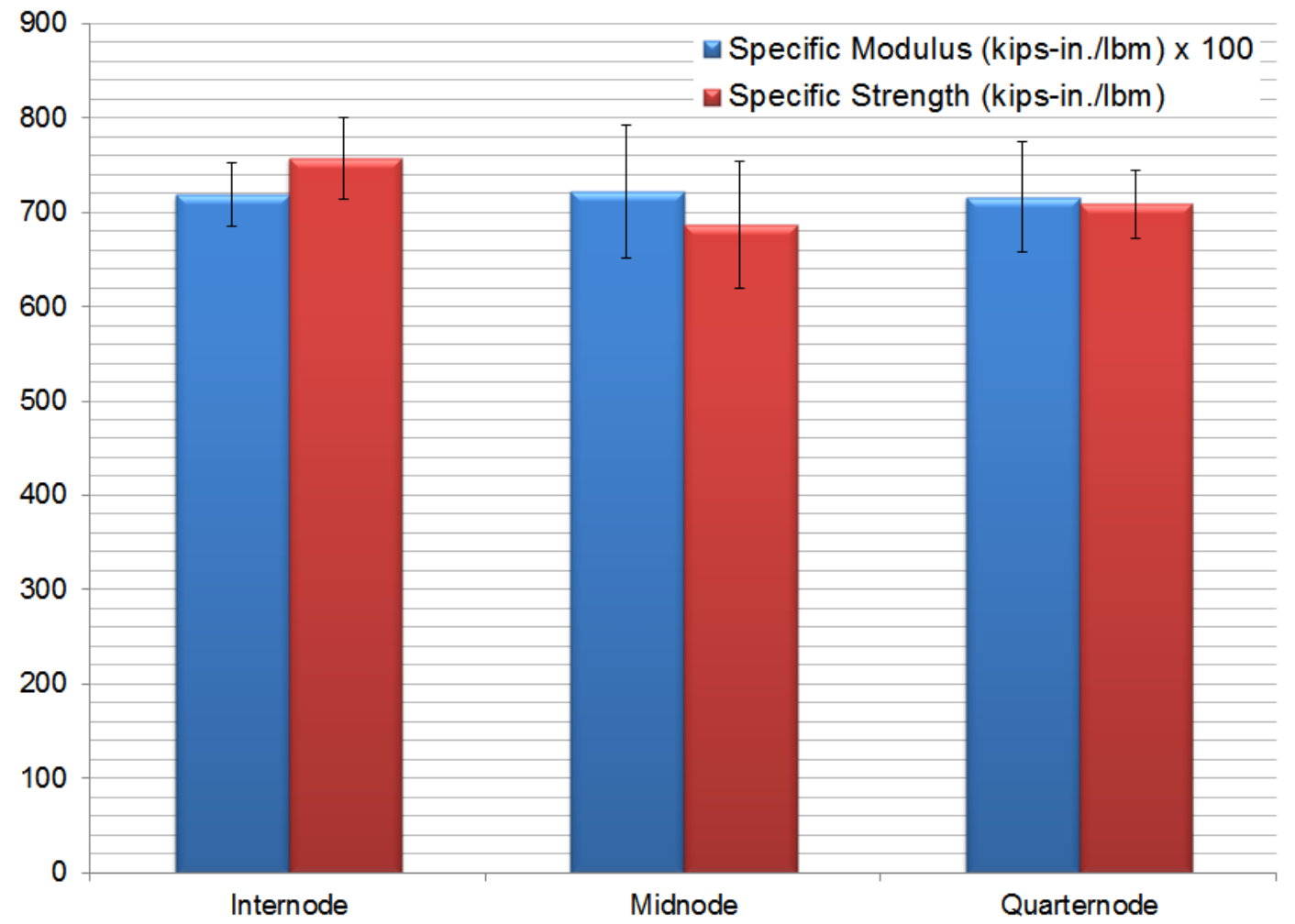

Figure 6-19. Summary of node location effects on the flexural specific strength and specific modulus for the rectangular configuration 
The specific bending modulus illustrates that node placement has more of a prominent behavior for the cylindrical configuration than for the rectangular geometry. Consequently, it is advised that designs aimed at maximizing stiffness and implementing the natural form of the bamboo have a node located directly underneath an applied flexural load or sufficiently far from it while designs utilizing the veneer strip can disregard the location of the node. First, it is noted that the veneer form illustrates much less change than the natural bamboo configuration for all node locations in terms of stiffness. As seen in Figure 6-18, the modulus is greater for the internode and mid-node. On average, the quarter-node displays $63 \%$ of the mid-node's stiffness and $66 \%$ of the internode. At best, it performs at $93 \%$ of the mid-node and $90 \%$ of the internode. The quarter-node, at worst, performs at $44 \%$ of the mid-node and $49 \%$ of the internode.

Therefore, stiffness performance is expected to degrade by about $35 \%$ for structures with nodes located on one side near the vicinity of a flexural load point. Unless the load point is directly in the center of a node during application, the structure is not expected to perform as well in terms of stiffness. It is certainly possible in some cases, however, that a node location in the general area offset from the load point would have adequate performance to a structure without the node. The likelihood of this occurring would be low, so it is advised in designing systems using whole bamboo culms to position nodes far from the point of a bending load application or directly underneath the point of application where the load can be evenly distributed across the length of the node and not just on one side. For the rectangular configuration, the location of a node has much less of an effect on the overall stiffness. It is observed from Figure 6-19 that the internode is seen to have slightly stiffer performance by approximately $7 \%$. Aside from this observation, the stiffness remains very similar despite the location of the node. Reflection on this behavior suggests again much more simplicity in systems utilizing this 
type of configuration. The practicality of utilizing bamboos in a veneer form would not significantly affect its stiffness depending on the locations of its nodes. As a result, it is recommended that utilizing bamboo veneers would be very beneficial for structural systems in terms of simplicity.

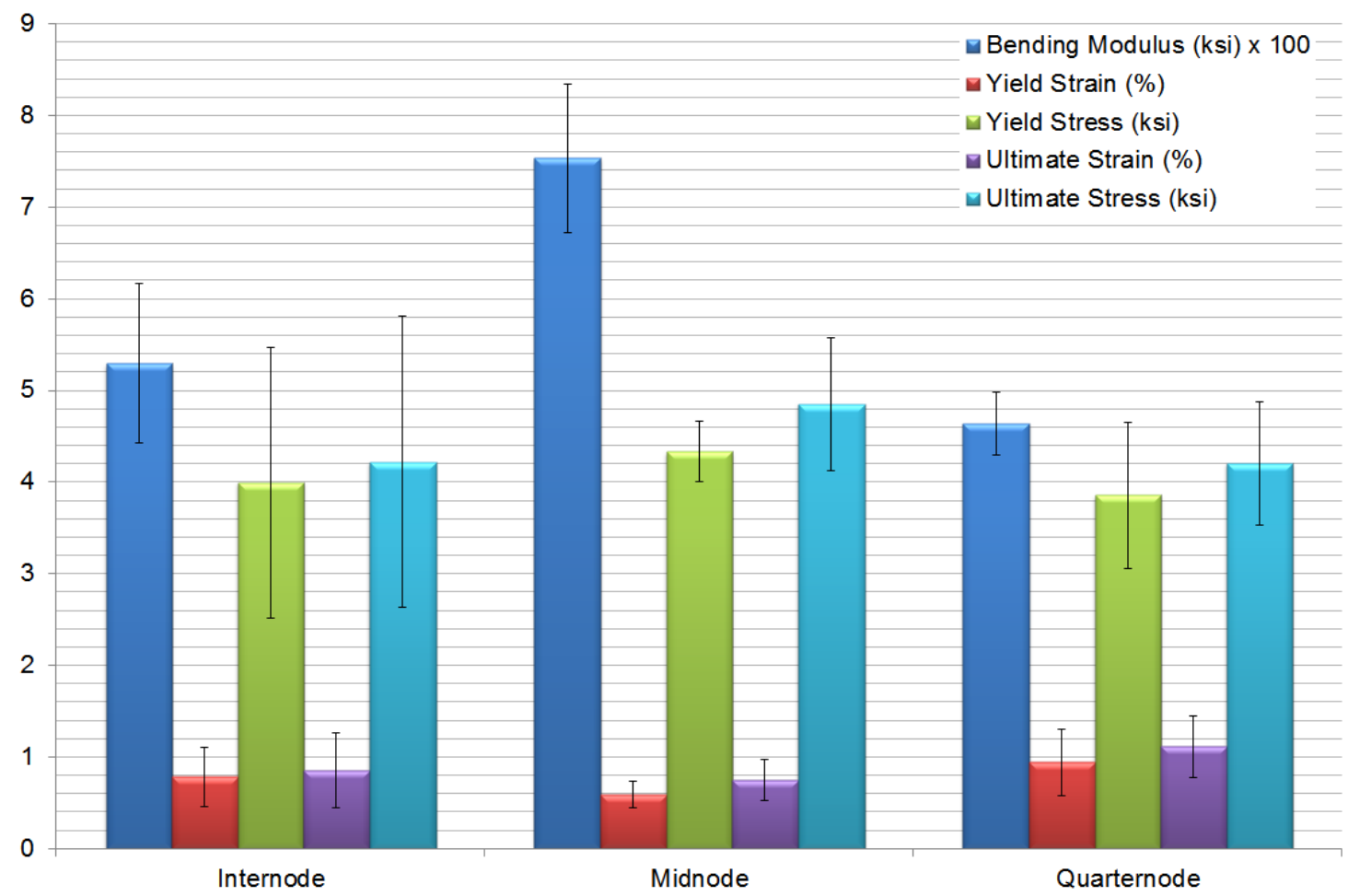

Figure 6-20. Summary of node location effects on the flexural properties for the cylinder configuration

In terms of the normal bending modulus, a different behavior is observed for the configurations. First, it is important to remember that ultimately the specific modulus is the measure for overall stiffness in the structure. In this case, one configuration may have a greater stiffness as represented by the modulus but may also incur more weight. For structural applications favoring light-weight materials, this notion is critical. For the cylindrical specimens as seen in Figure 6-20, the mid-node is stiffer than the internode by $42 \%$ and by $63 \%$ than the quarter-node. Among all three specimens for the 
rectangular group as seen in Figure 6-21, the quarter-node yields the stiffest configuration by roughly $21 \%$ over the internode and $14 \%$ over the mid-node.

In the cylindrical group, the bending modulus for the mid-node group is clearly the highest among the rest. Minor differences between the quarter-node and internode, however, indicate they may have the same stiffness. Probable reasons for the discrepancy between the internode and mid-node configurations lie in the transverse

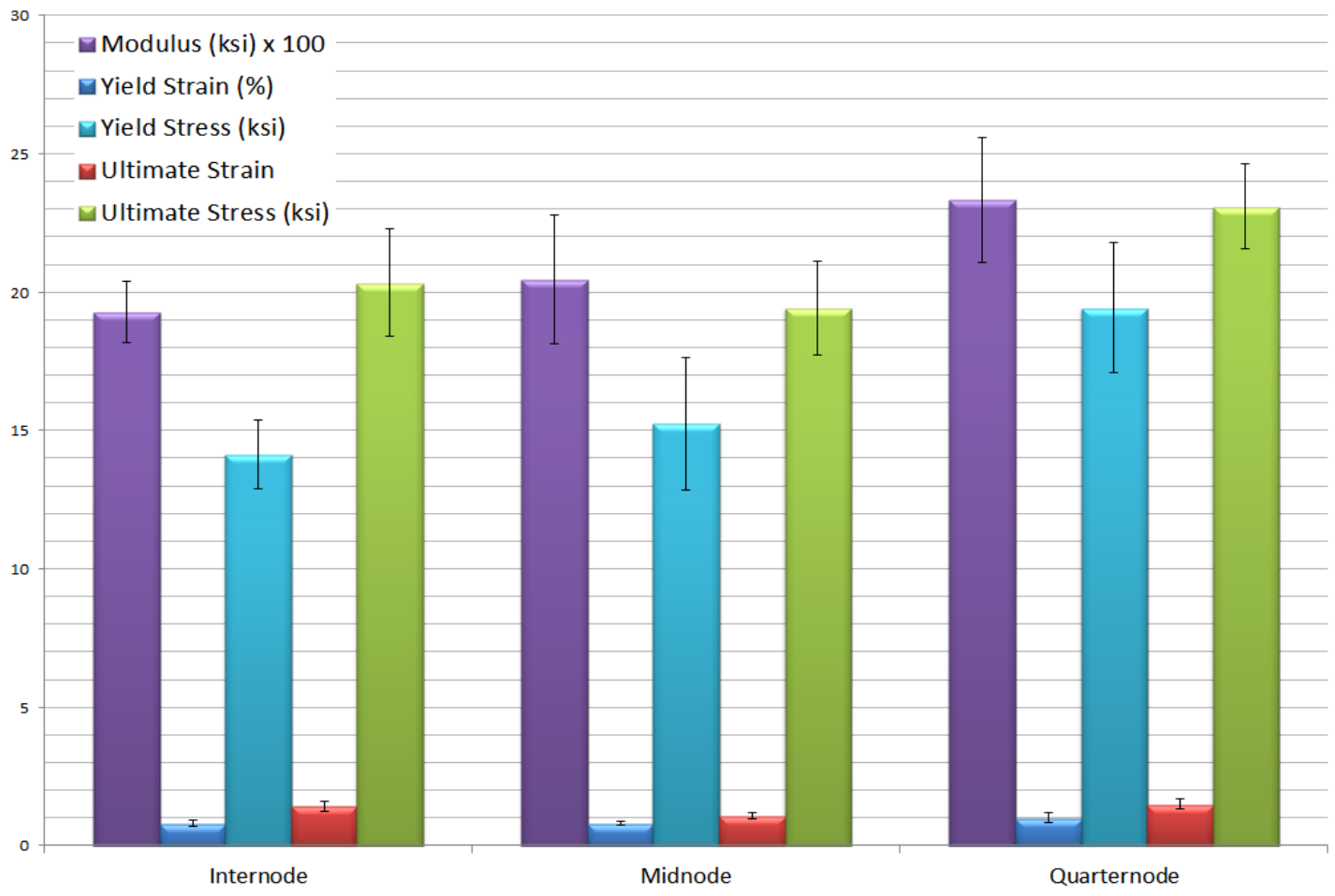

Figure 6-21. Summary of node location effects on the flexural properties for the rectangular configuration

fiber wall characteristic of the node. In fact, this diaphragm separating the two cell cavities adds stiffness to the beam for loading conditions where loads flank the node. Conversely, it is likely that the close proximity of the node on one side of the loading point in the quarter-node group depreciated the sample in achieving greater stiffness qualities. 
Perhaps placing a load only at one side of the node results in a concentrated region of stress in the transverse fibers than on the other side. Much stress is then placed on the main longitudinal fibers by the node. The added wall of the node adds considerable stiffness to the system without suffering a terrible weight penalty. In the rectangular group, the specific bending modulus obtained from the experiment illustrates a different trend. The modulus for the quarter-node is stiffer than the internode but is similar to the mid-node-a contrast to the cylindrical configuration. Again, it is likely the fibers in the transverse direction aid in resisting bending deflection. Therefore, the use of bamboo in a veneer configuration favors the presence of nodes near the loading application.

The yield and ultimate strains of all three node configurations indicate different performances in both groups. For the cylindrical group, yield and ultimate strains are all surely within the same range. This may be the direct result of the loading span directly causing deflection on the longitudinal fibers. Under this type of configuration, the node must not have a critical effect on the deflection since most of the main fibers take the majority of the stress. On the other hand, these two strains vary substantially in all of the rectangular specimens. On average, the ultimate strain is $55 \%$ higher than the yield strain for the three node arrangements.

Unlike the brittle behavior observed in the cylindrical specimens, a yielding behavior much more characteristic in a flexural member is evident in the rectangular samples. The yield strain among all three sample groups fall within the same region which is insightful. However, the mid-node displays the minimal amount of ultimate strain among the three by about $5 \%$ at worse-taking into account the deviations. On average, the ultimate strain for the mid-node is less by about $25 \%$. This important feature indicates the mid-node to be the optimum configuration to minimize flexural displacement if weight and volume are not of significant concern. In structural applications, this suggests that 
strategically placing a node in the location of a load point may significantly decrease the amount of expected deflection from a yielding perspective.

As introduced earlier, the strength and yield stress for all three configurations indicate similar performances-particularly for the cylindrical group. Average ultimate stress of the rectangular group indicates a $29 \%$ increase over the yield stress. The specific strengths mentioned previously alluded to this theme in the flexural properties of the bamboo. The ultimate and yield stress are all in the same range for the cylindrical group; the trend is clear across the three node configurations. Unlike the cylindrical configuration, however, the ultimate stress is higher than the yield stress in the rectangular internode group. Aside from this exception, the stresses are the same across the scope of the data. Once again, the true measure of strength in this study is the specific strength.

In summary, the effects of node placement reveal a few beneficial characteristics that may greatly assist a structure's flexural performance if accommodated correctly. First, the specific strength is minimally affected on the global performance of a structure by including nodes. This effectively has two benefits: manufacturing efforts can simplify by keeping the nodes and eliminating the need to reattach the longitudinal fibers with some adhesive mechanism, or respectable weight savings can be achieved while maintaining strength in the material when excluding the nodes. Secondly, significant stiffness can be added to a structure by strategically placing nodes near critical load points for applications where the whole bamboo culm is utilized. In contrast, applications using the bamboo in veneer form indicate the same stiffness regardless of node location. For a design considering the use of whole bamboo culms, this recommendation can easily increase the stiffness of a structure in areas of interest without paying a significant weight penalty. On average, a node cut-out only weighs $20 \%$ more than an internode of 
the same diameter and length. Thirdly, yielding strains can be expected to the be the same whether employing either configuration, but utilizing bamboo sections with a node near the load point can minimize the ultimate strain in laminate-like applications. Finally, the yield stress and strength of this bamboo material under any configuration would produce similar performance in their respective structural applications. Employing these guidelines will significantly aid in optimizing structures exploiting bamboos for flexural applications. 


\section{Chapter 7. Biocomposite Structural Application}

In order to implement the benefits of bamboo in flexural biocomposite structures, a bamboo member composite truss and bamboo core composite I-beam are constructed with all the established guidelines from the previous studies and tested to investigate the performance of bamboo-reinforced biocomposites. The truss application represents the benefits of utilizing bamboo in its naturally cylindrical form in biocomposite structures. Under this configuration, sectioned bamboo culms are utilized in combination with joints made of environmentally-friendly materials. CT-PT12 hemp fabric and organic resin are combined to form a practical truss structure. Conversely, the I-beam application represents the advantages of using bamboo fibers explicitly in biocomposite structures. This type of application employs split bamboo fibers also merged with CT-PT12 hemp fabric and organic resin to develop an innovative biocomposite l-beam.

Biocomposite research is further expanded by utilizing bamboos as the primary material in the structural design of these practical structures. Most conventional structural materials in industry throughout the United States do not currently exploit the use of natural resources in their designs; this research attempts to change this notion through the investigation of these structural applications. All efforts begin with a design based on current light-weight applications emphasizing structural rigidity. Subsequently, specialized manufacturing methods are employed for each respective application. The appropriate testing protocols are then implemented on the structures, and their respective performances are evaluated. Finally, validation of the test results are determined with finite element methods. 


\subsection{Bamboo Member Composite Truss Application}

The main objective of this truss study is to determine the feasibility and performance of using sectioned bamboo culms for long-spanning lightweight structures under an applied load that would normally result in great flexural stresses on a single beam; a biocomposite truss is developed with the guidelines established in the previous study to minimize this flexural stress on the beam. For loading conditions that would normally exhibit large bending in a single bamboo culm, two diagonal members and a central vertical member are added to create a truss and reduce the bending stress on the horizontal member. It is imperative to state the only requirement for this truss is using only natural materials for its entire construction. In terms of the design, the bamboo utilized for this truss are treated with the $3 \%$ salt treatment at $220^{\circ} \mathrm{F}$ with a $1^{\circ} \mathrm{F} / \mathrm{min}$. initial curing rate. Furthermore, as outlined in the Analysis of Flexural Results section, the nodes of the bamboo culms are excluded from the design in order to optimize strength and stiffness.

The selected truss design is a common element in many aircraft structures and civil architecture. Figure 7-1 is provided to depict some examples of this type of truss in application. As seen from the figure, this biocomposite truss may be utilized as part of a roof, bridge frame, aircraft wing-rib structure, or aircraft fuselage. The investigation of this truss study embodies manufacturing, testing, result presentation, and numerical validation. Briefly speaking, the manufacturing process entails cutting the bamboo poles into truss member sections, mitering each member's end to provide a good fit, and finally lashing each joint together with resin-soaked hemp fabric before curing the structure in a vacuum bagging process at room temperature. Testing the truss structure is conducted by applying a uniformly distributed load across the top joint until failure, and an

evaluation report is generated by the ultimate load observed. Experimental validation is 
sought with Abaqus/CAE finite element analysis software to simulate the maximum deflection of the structure under the same loading conditions.
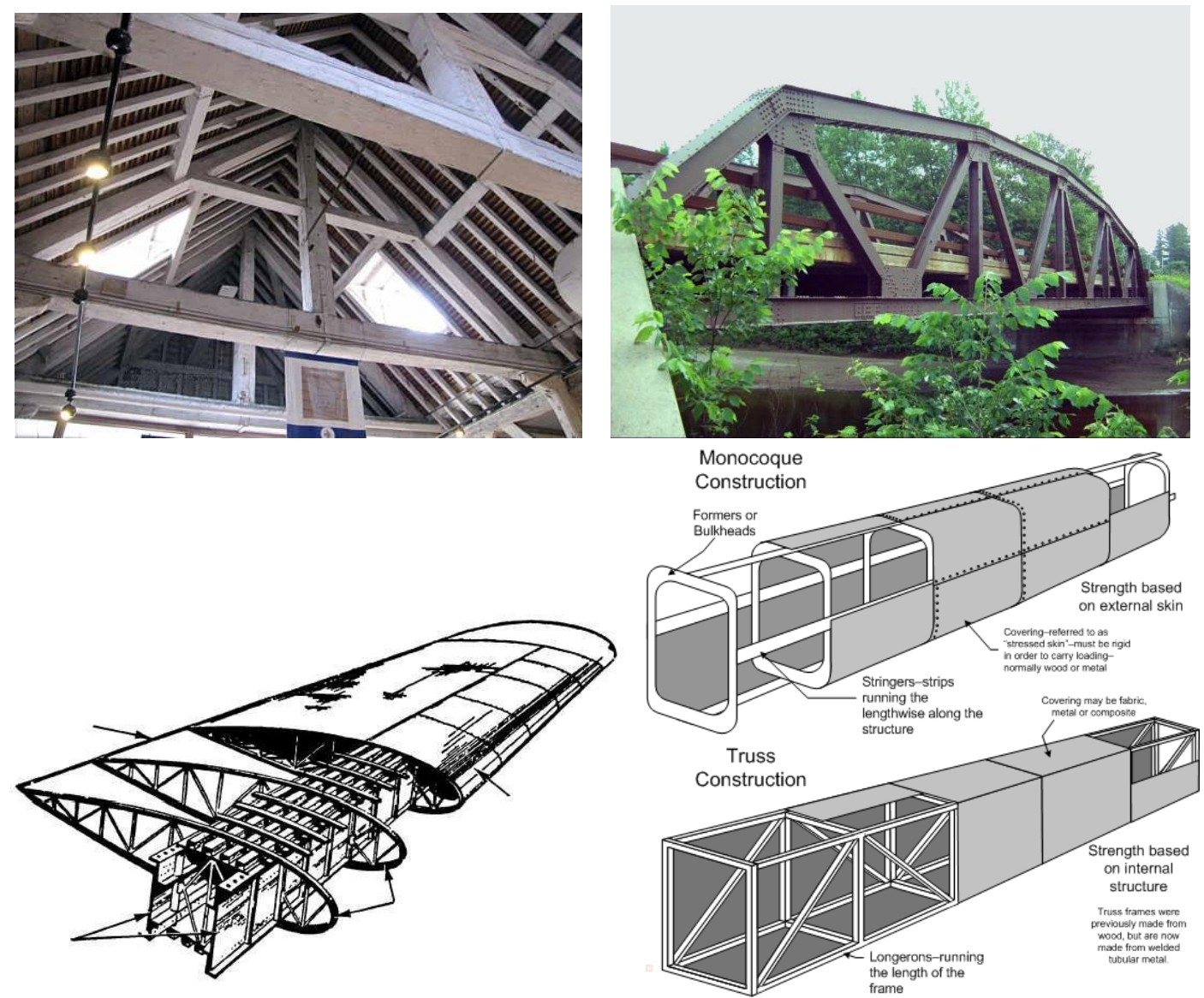

Figure 7-1. Potential applications for bamboo biocomposite truss [48; 79; 66; 15$]$

Initially, two designs with different joint fabrications were originally conceived for the truss application. As in many truss designs, the reliability of the joint must be ensured to be strong in order for the truss to perform at its true potential. The first design required the joints to be made from molded fittings of hemp fabric coated with resin-eliminating the need to miter the bamboo members. The second design incorporated a lashing technique with strips of resin-soaked hemp fabric at the joints of the truss. The idea behind the first idea was to simplify the truss manufacturing by having bamboo internodes interconnected with joint fittings that are laid-up on the bamboo truss 
assembly. Ultimately, the first design was abandoned because of the poor quality of the joint product. Perhaps, the first design may be more beneficial if improvements can be made in manufacturing the joints during the traditional composite lay-up process.

\subsubsection{Manufacturing the Truss}

The entire construction of the truss structure is done in the Cal Poly Structures/Composites Laboratory, and all the materials used in the truss fabrication are green certified. Also, it is decided to fabricate a small-scale prototype version of this application in order to evaluate the effectiveness of bamboo in the structure. A full-scale prototype is left for future work. The mentioned materials include bamboo internodes cured with the $3 \%$ salt treatment, joints made of the same bi-directional hemp fabric, and biodegradable putty from Ecopoxy. The joints are fabricated through a wet lay-up process using organic resin and hardener, and the entire truss is vacuum bagged to properly cure the part for a desirable final product.

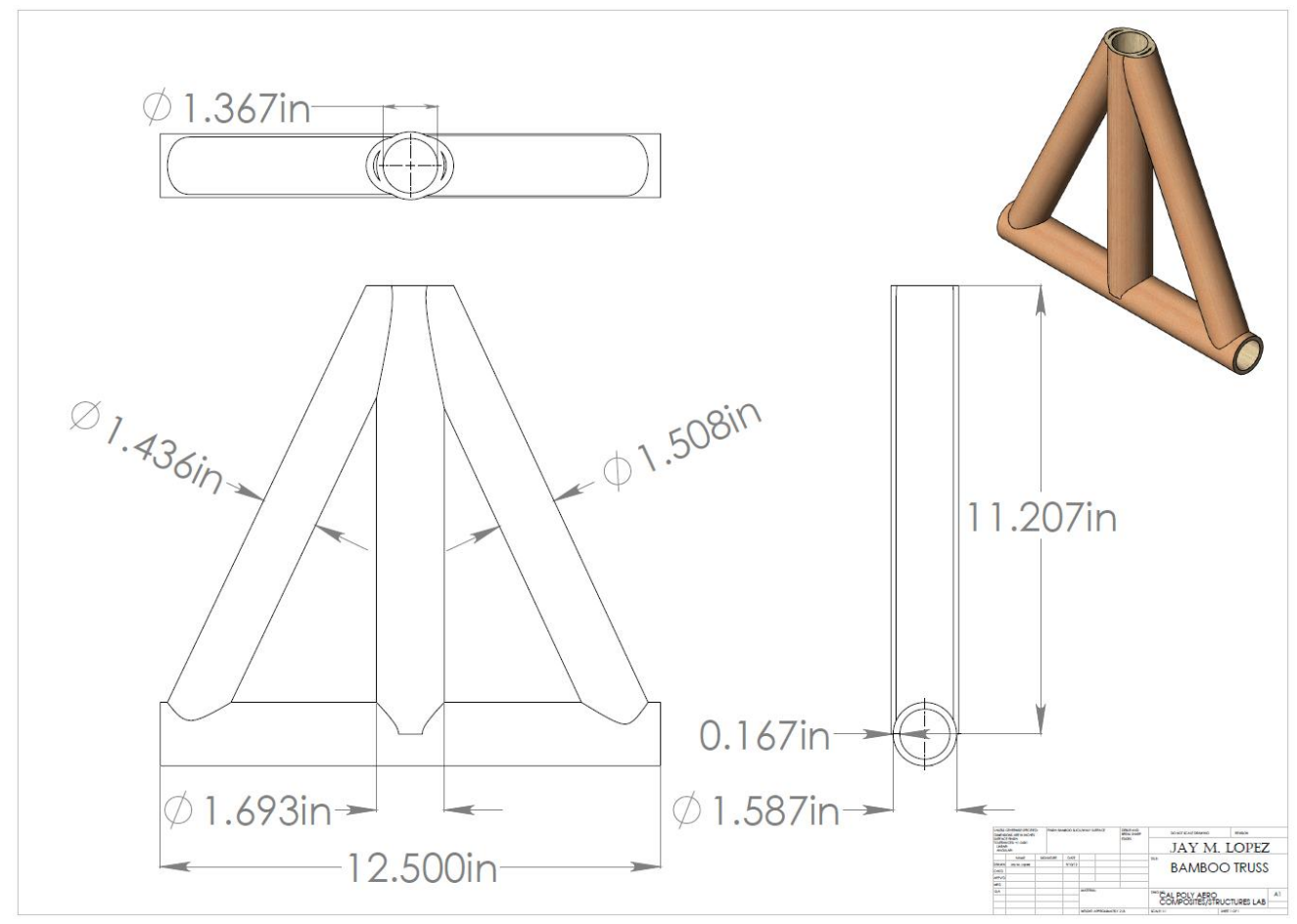

Figure 7-2. Manufacturing drawing for truss structural application 

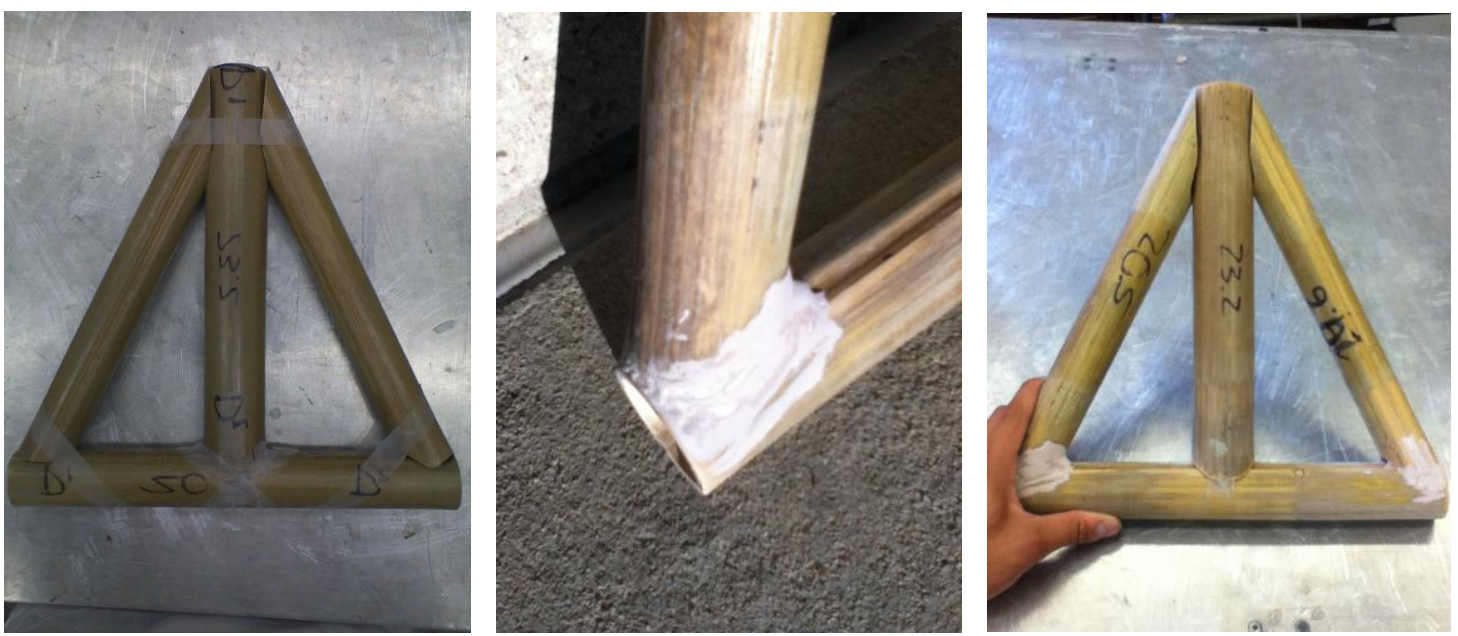

Figure 7-4. Initial phases of manufacturing: taping mitered truss members together (left), sanded biodegradable putty (center), and final view of joints created with Ecopoxy putty (right)

The design and its manufacturing technique is now discussed. A drawing created in SolidWorks is provided in Figure 7-2 to map out the design of the truss. This design is followed closely in order to fabricate the truss with the correct dimensions. First, the properly cured bamboo poles are cut to the approximate lengths of each truss member: 13.5-in. for the diagonals, 12 -in. for the vertical, and 12.5-in. for the horizontal member. Subsequently, each diagonal member is mitered at both ends while the vertical member is mitered only at its bottom end. A tile saw is used in order to cut off the majority of the material required to properly miter the bamboo members. Once the major portion is cut from the bamboo, a Dremel tool is used to fine-tune the work to ensure a custom fit to adjacent members when assembling the truss. Course sandpaper is then lightly utilized at each member's end to provide a proper adhesion surface for the remaining steps; the slick waxy surface of the

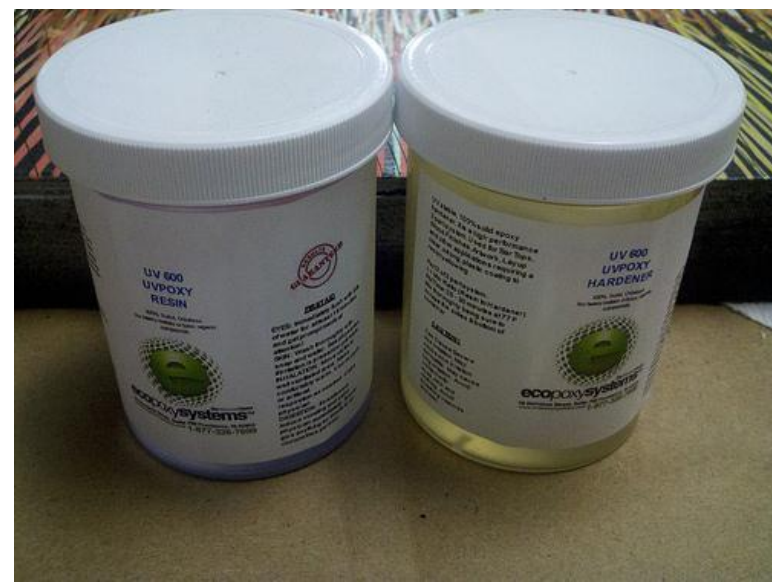

Figure 7-3. Eco-friendly resin system: Ecopxy resin and hardener 
bamboo culm prevents

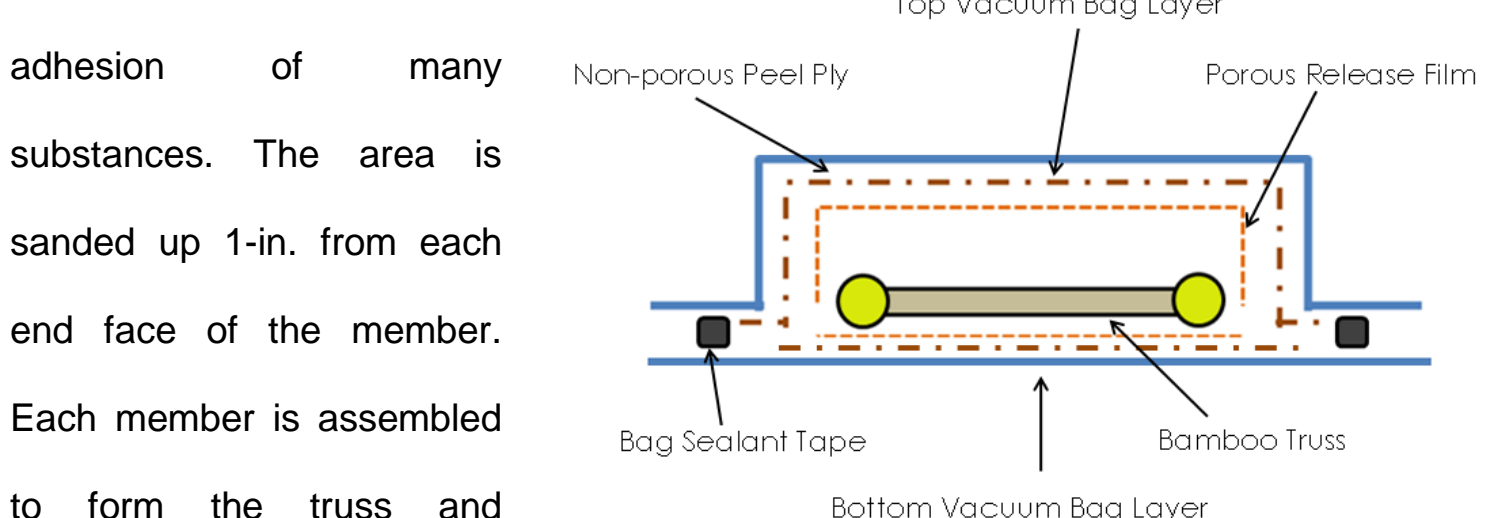
temporarily taped in place. Figure 7-5. Lay-up diagram of vacuum-bagging process

Figure 7-4 shows how the taping lay-out is done on the truss. Afterward, the tape at each bottom-side joint is removed one at a time, and a light application of Ecopoxy putty is then applied with a spreader and allowed to cure. The cure time for this putty is approximately 45 -min. for a full cure. The truss is ensured to be placed in a wellventilated area in order to expedite the cure time. After the putty solidifies into a hard joint, the excess is removed with the Dremel tool and final touches are sanded with finegrade sandpaper. Precaution is taken at this step so as not to sand vigorously at any time, or else the putty would be removed. Figure 7-4 shows the sanded finish at the respective joints.

Once the truss members are assembled into the appropriate configuration, the laminate joint lashings are prepared. First, two 1.5-in. x 5-ft. strips are cut from a roll of bidirectional hemp fabric. Two strips are initially used for each joint, so a total of 8 strips are cut from the roll of fabric. Next, the strips of hemp are coated with Ecopoxy resin ER500 and EH725 medium hardener with a 4:1 resin-hardener ratio. It is worth noting that quicker coating times can be achieved by dumping tablespoons of resin on a group of strips before using spreaders and glove-fitted hands to saturate the strips with resin. After the strips are thoroughly saturated with resin, a brisk working pace is stressed to 
lash the joint in order to avoid early curing. A standard figure-8 lashing technique is used for all the joints except the top. The top joint is lashed in a modified diagonal method by first lashing one diagonal to the center and then repeating it for the other diagonal member. It is of critical importance that the lashings are created with symmetry and with a tight wrap. Otherwise, stress concentrations can result from wrapping a joint excessively on one side or the lashing can easily undo itself. Also, it is incredibly helpful to temporarily stick pins through the joint after both strips are lashed to prevent the lashing from falling apart when moving along to the next joint.

After all the joints are lashed with the eight resin-coated hemp strips, the truss is placed on a 2-ft. $\times 2$-ft. square vacuum bag with a porous release film sheet on top of a nonporous peel ply spanning 6-in. x 6-in. at each joint location. Once the truss is placed on the vacuum bag and its release media, the pins are removed and another layer of
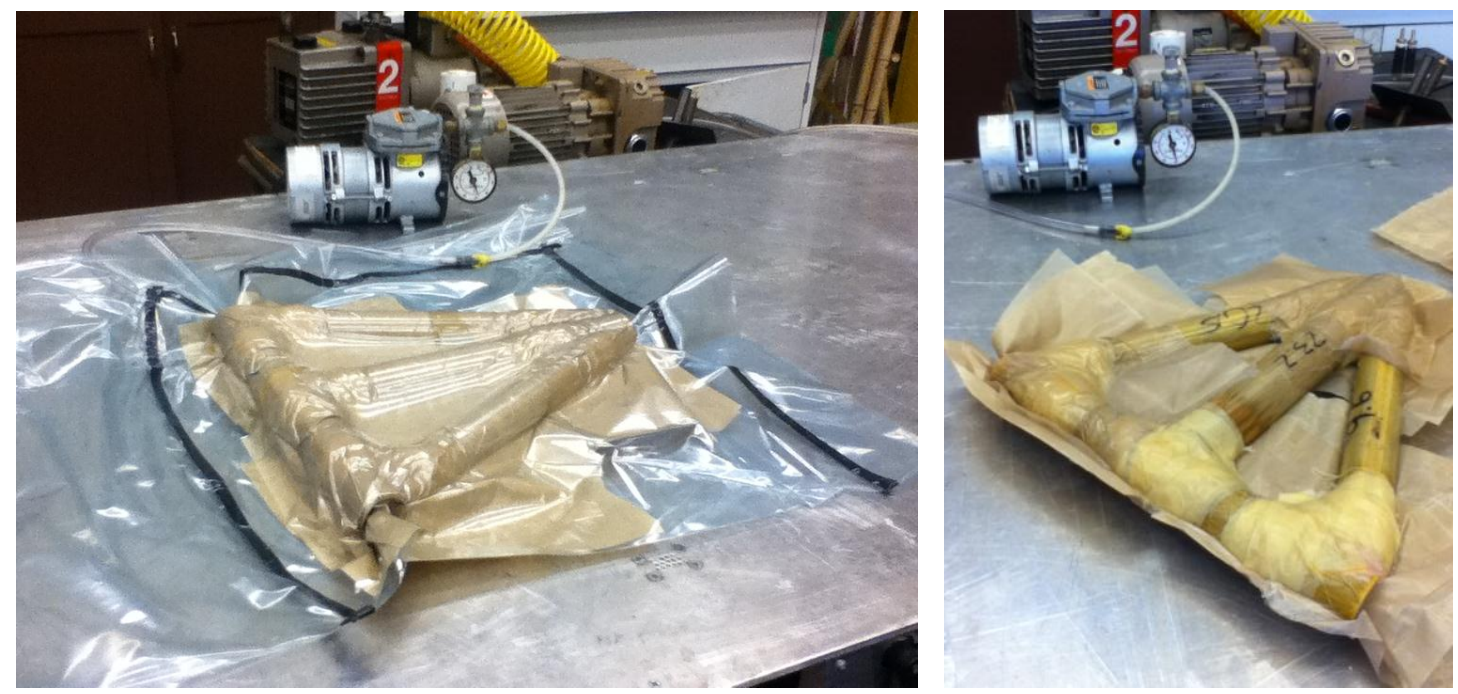

Figure 7-6. Curing truss joints in vacuum bag (left) and truss taken out of vacuum bag after 12-hour cure (right)

porous release film is placed at each joint followed by one more layer of non-porous peel ply. Next, bag sealant tape is lined around the truss in a square fashion. A vacuum pump with a hose covered in a thick layer of cloth at the end of its plastic tube is set on the corner of the tape, and another small section of tape is applied transverse to the 

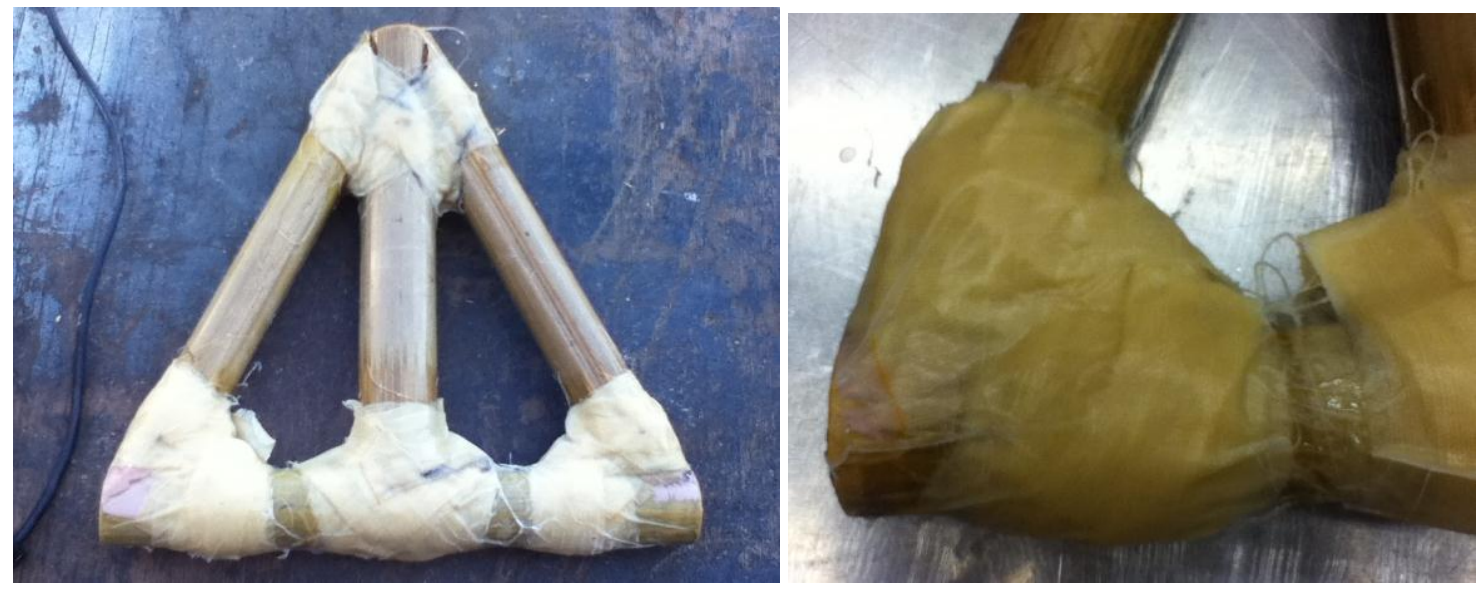

Figure 7-8. Raw condition of joints after cure (left) and close-up view of joint prior to sanding (right)

longitudinal direction of the hose. The vacuum bag is then folded over the truss and sealed with the tape. An illustration is provided in Figure $7-5$ to show the composite bagging stacking order from a side view. Lastly, the vacuum pump is turned on so that a $30-p s i$ vacuum pressure is placed on the part. Using the vacuum pump allows the joints to have a tight fit to the truss members and evenly distributes the resin along the laminate for a better quality product. Figure 7-8 portrays the truss sealed in a vacuum bag. The bagged laminate is then allowed to cure at room temperature for a total of 12 hours before being removed from the bag.

Once cured, the truss is removed from the bagging, and all the joints are lightly sanded with a Dremel and sandpaper to remove any sharp edges and excess

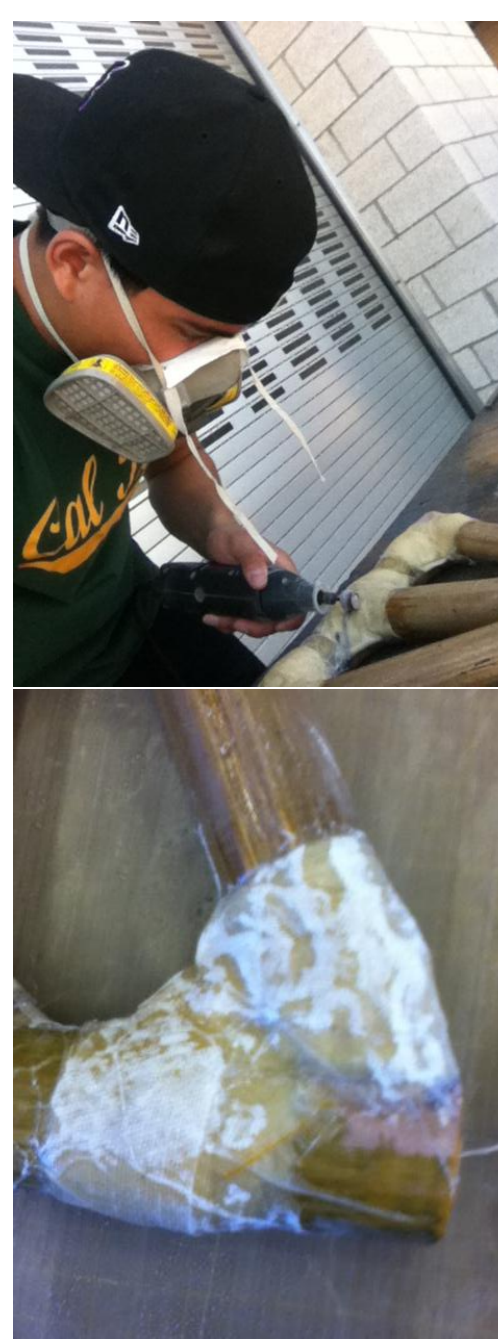

Figure 7-7. Sanding down excess resin and fabric (top) and resulting joint after sanding (bottom) 


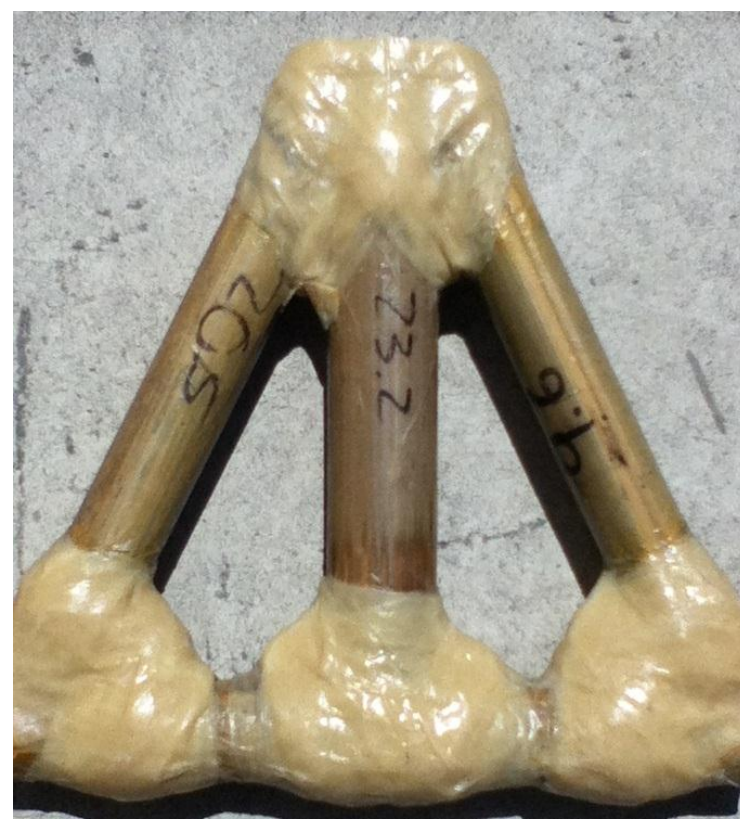

Figure 7-9. Final product of bamboo truss with hemp joints resin/fabric that did not cure flush to the joint. Careful care is taken in not using the Dremel excessively so as not to shave the strips too far down-this would result in much less strength capability in the joint. Figure 7-6 shows the truss during its 12-hour cure and also it being recently taken out of the vacuum bag. Also, Figure 7-8 and Figure 7-7 show certain unfinished joints and an example of what a sanded joint should look like.

After each joint is sanded to the appropriate degree, the entire process was redone one more time in order to ensure a strong joint. After one more cycle of joint manufacturing, the truss is weighed for specification purposes. The weight is measured to be $2.175-\mathrm{Ib}_{\mathrm{f}}$. The main goal of the truss test is to ensure a failure load due to the bamboo members and not the joints, so the extra precaution is necessary.

\subsubsection{Truss Testing Procedure}

The main objective of the truss test is to determine the failure point under a compressive load with two supports at both bottom ends. Best judgment and intuition was used in determining testing requirements and guidelines due to the lack thereof. First, a compressive load at the top central joint is designated as the load point. Second, the truss is nested into two custom supports placed on a stable flat surface. Each support is fabricated from a rectangular block with a semicircle of the same outer diameter as the horizontal member. The supports are of sufficient height to allow spacing between the 
horizontal member and the floor. An illustration of the test set-up is shown in Figure 7-11 for clarity.

The purpose of this fixture is to minimize the point load caused by placing the structure on a flat roller support. Lastly, a metal plate fixture is required to evenly apply the load

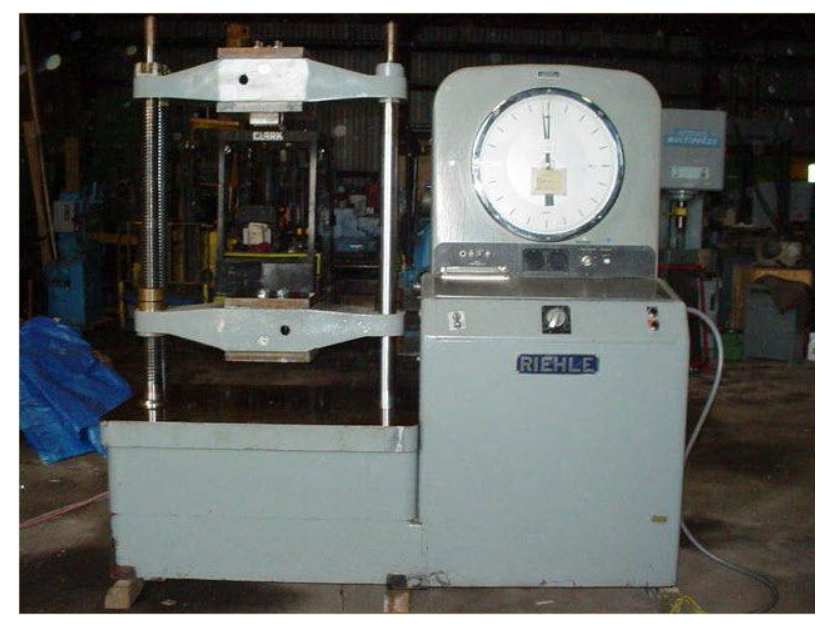

Figure 7-10. Riehle testing machine to perform compressive load

across the top joint. The testing apparatus is selected to be the Riehle testing machine from the Architectural Engineering department as it is capable of applying loads in excess of $10000-1 b_{f}$. Figure 7-10 depicts an image of the Riehle testing apparatus used for this experiment.

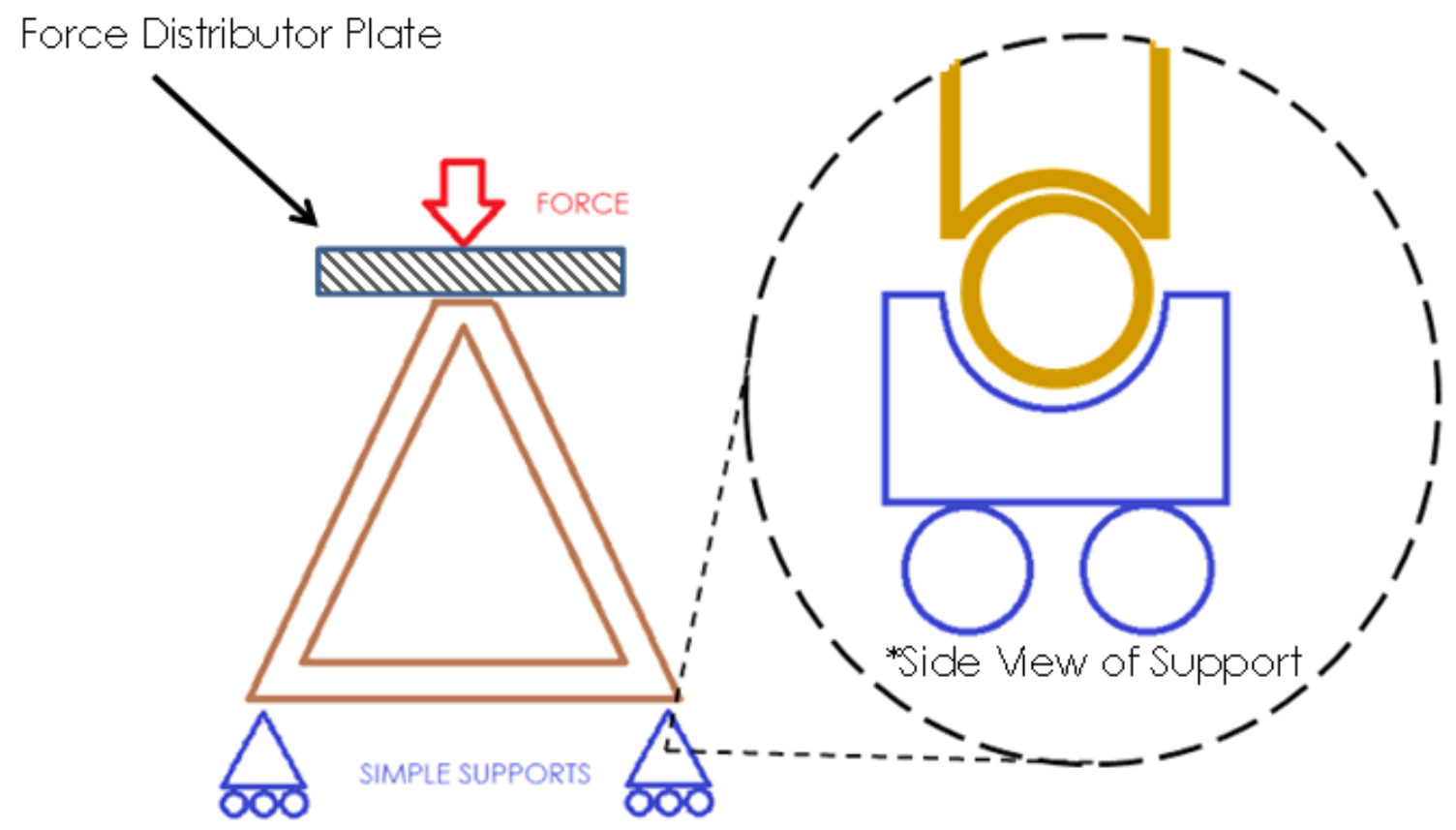

Figure 7-11. Truss loading schematic for test 
Setting up the test begins by placing the supports onto the flat surface under the loading apparatus. The truss is then set within the semicircles of the supports and made sure that it is stands vertically. It is important that the truss is placed vertically onto the supports so that all the compressive force is applied normal to the face at the top joint. A metal plate is then set on top of the joint and the crosshead is lowered so that it makes contact with the plate. Figure $7-12$ is shown to portray the truss in the correct configuration. Usually an applied force of a few pounds is sufficient to keep the structure in place so that it will not move. Subsequently, the loading rate is manually adjusted on the testing apparatus to be 0.088 in/min. Testing can begin once the preload is

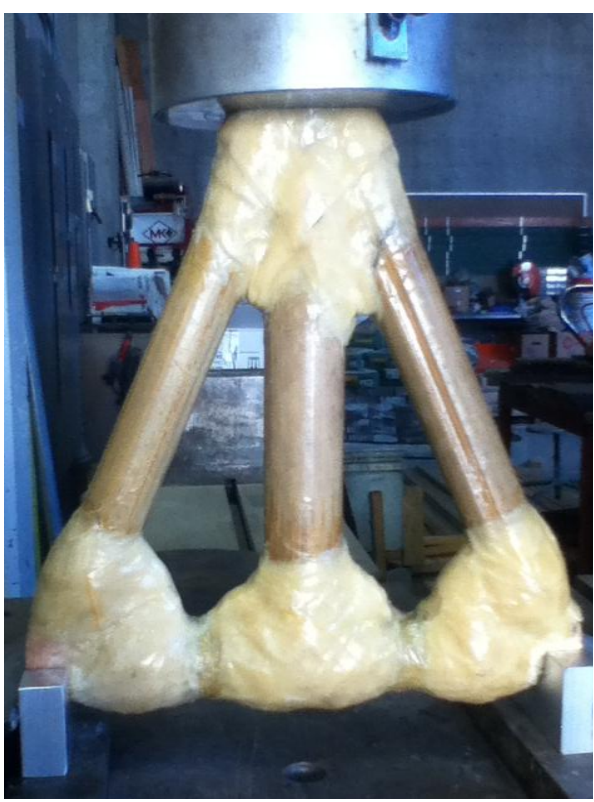

Figure 7-12. Test set-up for bamboo-hemp truss applied and the load rate is set.

\subsubsection{Results of the Truss Test}

The results of this test indicate beneficial practicality of utilizing bamboo for structures such as a truss that would exhibit flexural loading in the horizontal member. In addition, the mechanical behavior of this bamboo truss structure is determined to lend insight on its use in real applications. The testing result of the truss is shown in Figure 7-13. The load-deflection curve illustrates that the truss withstands high loads with little deflection. As seen from the plot, the maximum load endured is $15,371-\mathrm{Ib}_{\mathrm{f}}$ with a corresponding deflection of 0.46963 -in. The load curve is very similar to the compression ones seen previously in this research. A low loading gradient is observed at the beginning of the test, insinuating relatively greater displacement changes at lower loads. The linear 


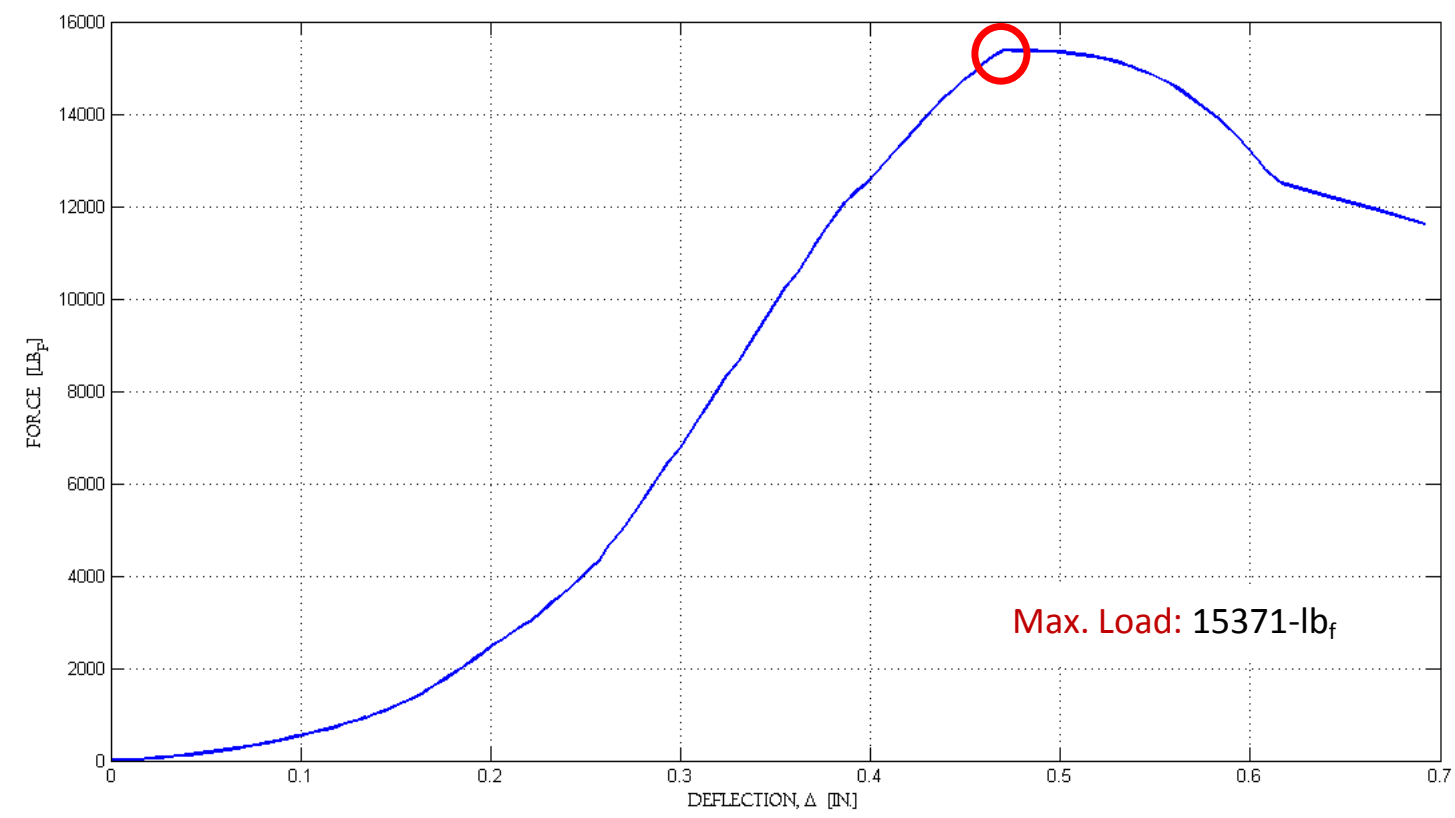

Figure 7-13. Load-deflection curve of bamboo-hemp truss test

portion of the curve passes through several thousand pounds before the slope begins to drop. Once the slope begins to drop the structure is approaching its ultimate load before failure. The ultimate load applied to the truss suggests that this material has an exemplary strength-to-weight ratio under this type of loading condition-a value of 7067 . This ratio is achieved with the use of only a few natural materials weighing about $2-\mathrm{Ib}_{\mathrm{f}}$. Furthermore, it can be predicted that a tensile force applied to the structure would yield an even greater ultimate load. Tensile tests shown previously suggest much higher allowable stresses for this type of cured bamboo. Considering the mechanical behavior of this structure, two key points are evident from the test. First, this structure is incredibly stiff and strong for a natural material. Few other plant materials exhibit such strength under compressive loads. Second, considerable changes in the design of the top joint must be implemented if greater structural strength is desired. 

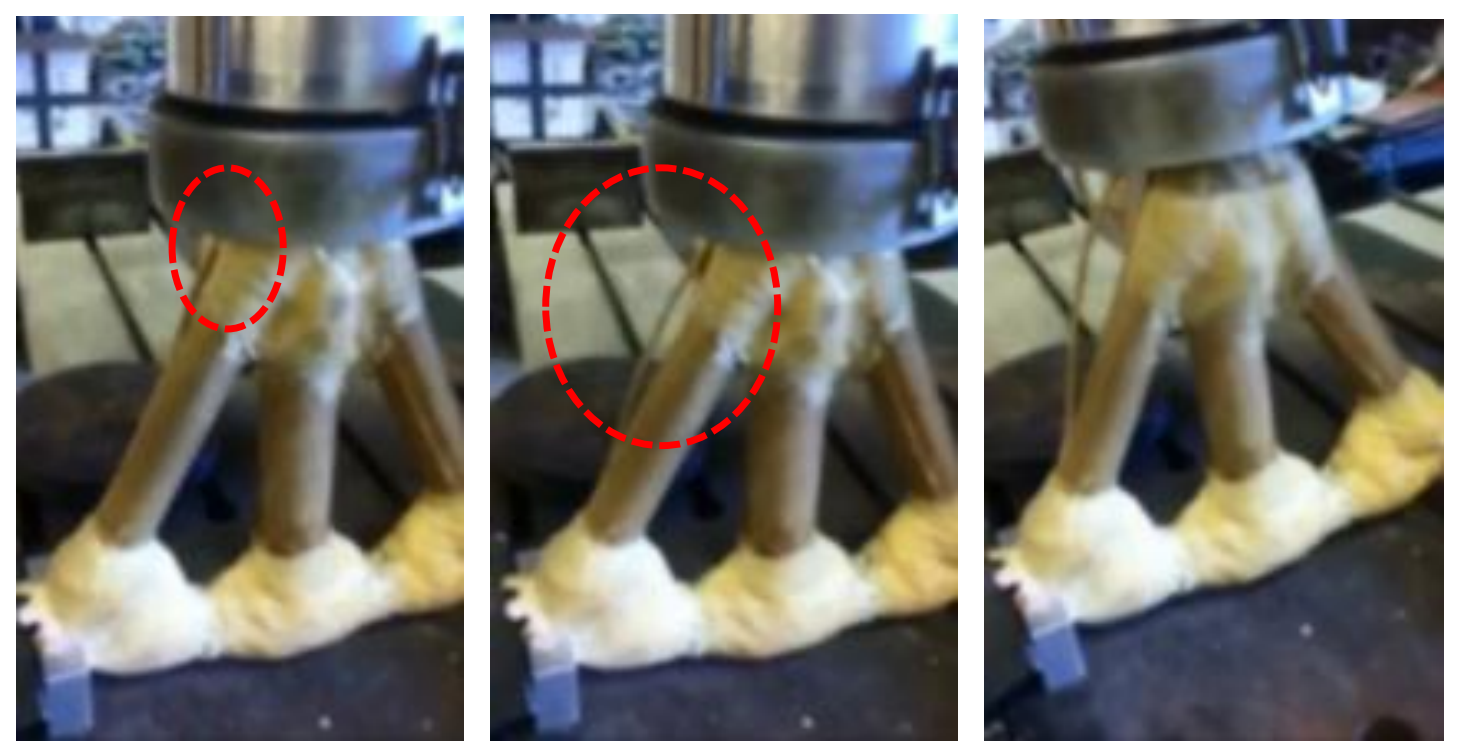

Figure 7-14. Instants up until failure: (1. left) pre-failure state where top hemp joint begins to split (2. center) bamboo fiber delamination imitates in left diagonal member (3. right) top hemp joint splits further into two pieces

To illustrate the failure of this structure, Figure $7-17$ is shown to indicate the critical failure locations. As expected, the truss failed primarily at the top center joint. The stress at the top joint experienced so much force that it caused the hemp joint to split into two pieces. The tension in the lashed joint clearly shows a clean split in the fabric. Therefore, it can be seen that a stronger hemp joint is necessary in order to improve performance. Hemp fabric delamination also occurred at the side joints, but the damage is minimal compared to the center section. Delamination initially occurred at the end where the wrapping was thinnest and slowly propagated up and along the edge of the joint. Delamination in the center joint occurs much more drastically and is shown in Figure 7-16.

It can also be seen that the center vertical member did not experience catastrophic failure. Fiber separation around the circumference of the top joint prevented large deformations that would lead to overall buckling failure. It is proposed by Chan that a longer vertical member would increase the likelihood of causing the center column to 
buckle in the traditional sense where large lateral deformation is observed. His work on the buckling behavior of this bamboo indicates that a slenderness ratio under 34.7 must be followed in order to avoid global buckling. The results of this test suggest this recommendation is followed adequately. Exploring truss dimensions greater than this is left for future work.
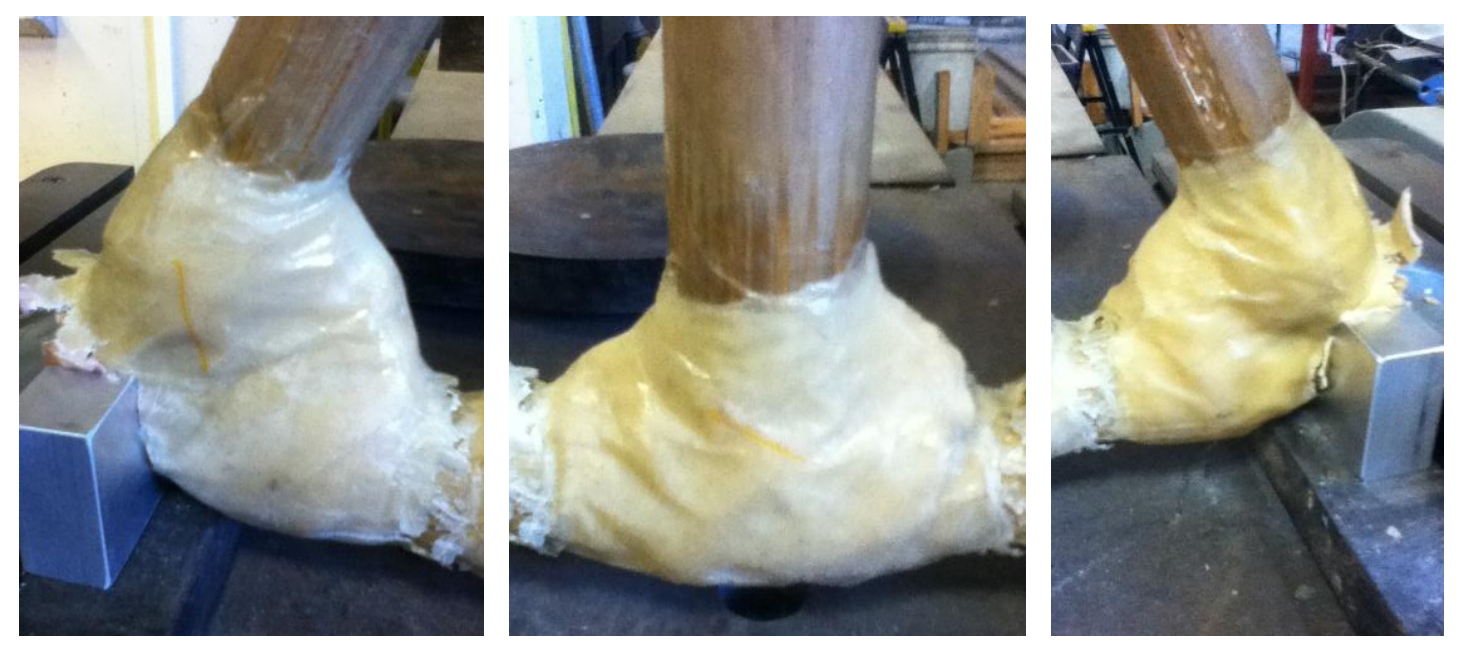

Figure 7-15. Deformation of bottom joints during the test

The horizontal member also did not demonstrate a failure as prominent as the top end of the diagonal members. It is then observed that much stress is alleviated in the central portion of the truss with diagonal members. The minor deflection and heavy deformation at the top joint is testament to this observation. Most of the deformation occurred at the extreme ends of the horizontal member where the metal supports provided much of the normal force on the joints. It is important to note that shear forces also dominated this stress region. Added stiffness can be introduced on these ends by lashing more hemp fabric around this area. Overall, the deformation in the bottom member was fairly minimal, and it seems the reinforced joints in these locations largely benefited the performance of the truss. 

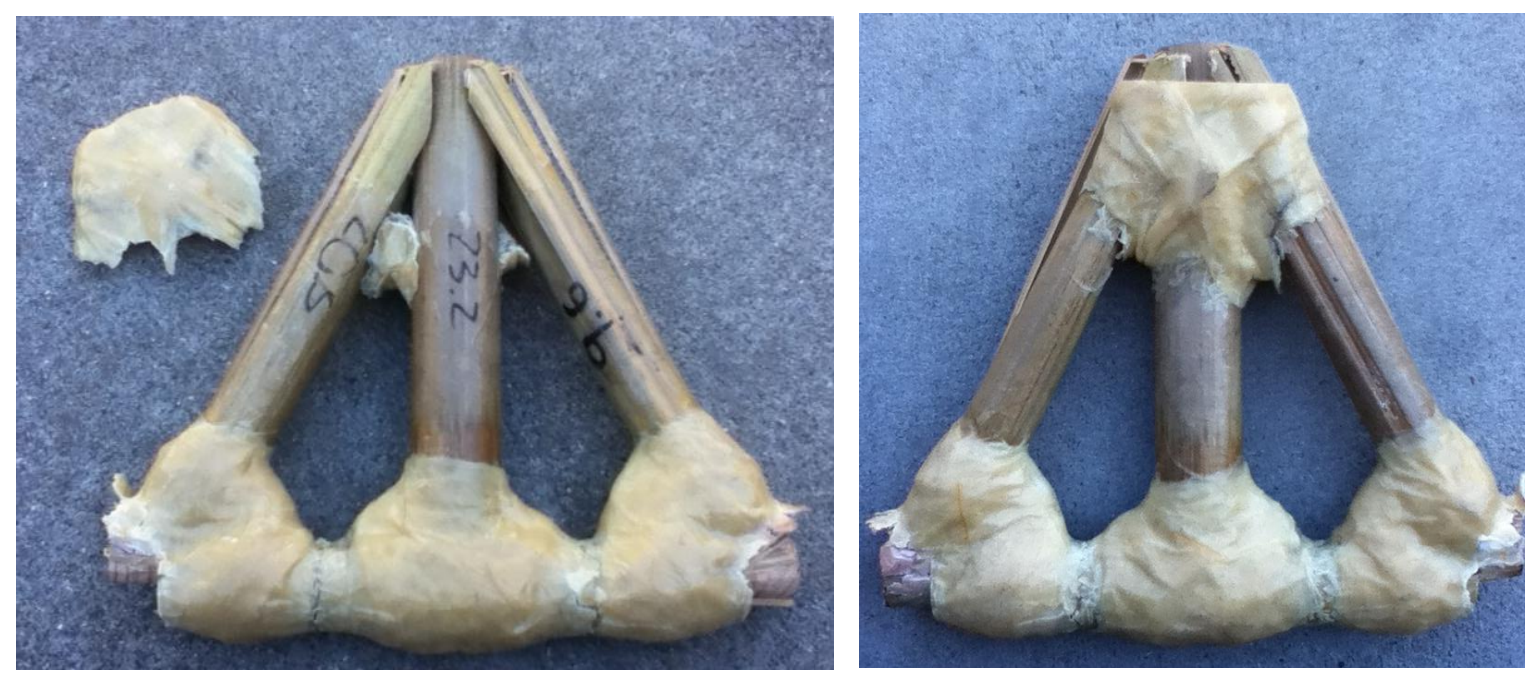

Figure 7-17. Deformation of the truss after the test from the front (left) and back (right)

Further inspection also reveals that stress in the transverse direction to the fibers accounts for the main cause of failure at the top joint. The top ends of the diagonal members demonstrate an "opening" characteristic that split the culm into an array of strips. As seen in the figure, it can be observed that the diagonal members were the weakest elements of the structure. In contrast, the center vertical and horizontal members show little-to-absent deformation. These two observations yield the conclusion that better performance can be expected from this structure if the end faces of each member are fitted with a different type of joint that distributes the stress across the face
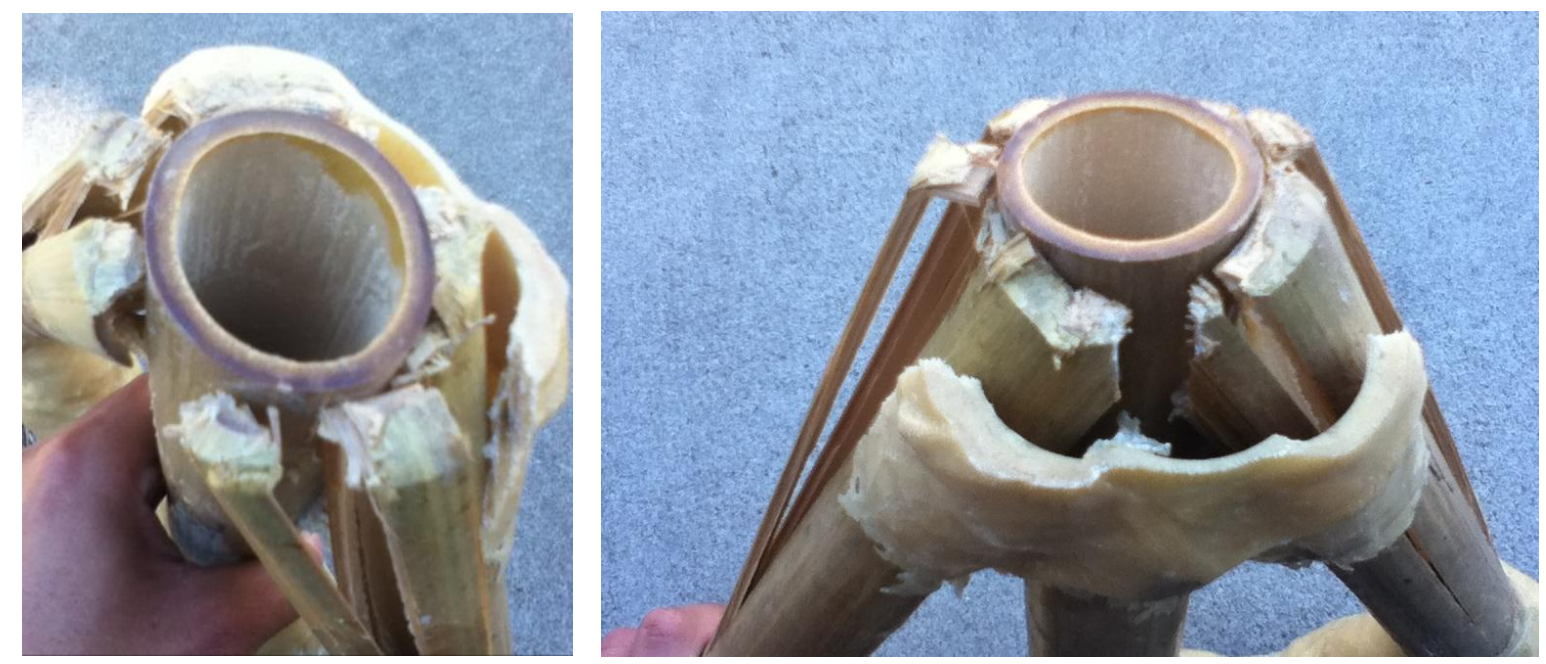

Figure 7-16. Critical joint at the conclusion of the test 
more evenly. The current configuration of the joint has the members mitered to each other at an angle that results in shear forces acting on the fibers of the bamboo members. Testing on bamboo demonstrates that the shear stress capability of this material transverse to the fiber orientation is nowhere near the tensile or compressive strength. This is why the deformation in the top portion of the diagonal members is blatantly apparent while the other vertical and horizontal members are in fairly good condition. As a result, a better joint system can drastically improve the performance of the system.

\subsubsection{Finite Element Method to Validate Truss Performance Results from Experiment}

It is of vital importance to explore a means to validate the results from the truss test numerically in order to efficiently design future structures using bamboo. Validation of the application's test results is conducted with a finite element analysis using the software Abaqus with the pre-processor CAE. The flexural behavior described by the numerical analysis will be compared to that exhibited in the actual test. Also, the finite element analysis can reveal information about unseen factors of the test such as the stresses inherent in the truss. The finite element analysis is deemed necessary in order to validate the use of numerical techniques on this truss structure. As with many preprocessors in conjunction with finite element software, the analysis procedure is followed by: identifying the model's geometry, defining the material properties, specifying the interaction between any individual parts in the model, assigning the boundary/load conditions, and finally generating the mesh to represent the model. A linear implicit solver is utilized to analyze the model under compressive loading conditions. 


\subsubsection{Numerical Analysis Methodology for Bamboo Truss}

First, the geometry of the truss model must be in accordance with the actual structure. The general dimensions are modeled in SolidWorks and imported into the Abaqus software along with the mechanical properties of the bamboos. Figure 7-18 illustrates the transition from the real-life specimen to the computer-generated model. For simplicity, the joints are absent from the model under the assumption that the truss members are perfectly joined. The basis for this assumption is why the extra measures were taken in fabricating strong joints. It is also important to note that the geometric dimensions in all the members differ slightly. Variables such as the outer diameter, thickness, and angle between the joining members are not exactly the same. These effects are due to the manufacturing method conducted during the structure's fabrication. In order to obtain accurate measurements, these inconsistencies are modeled as close as allowable. Therefore, the individual diameters, thicknesses, and lengths of each member are modeled to the same dimensions measured from the untested truss. However, the tapering effect
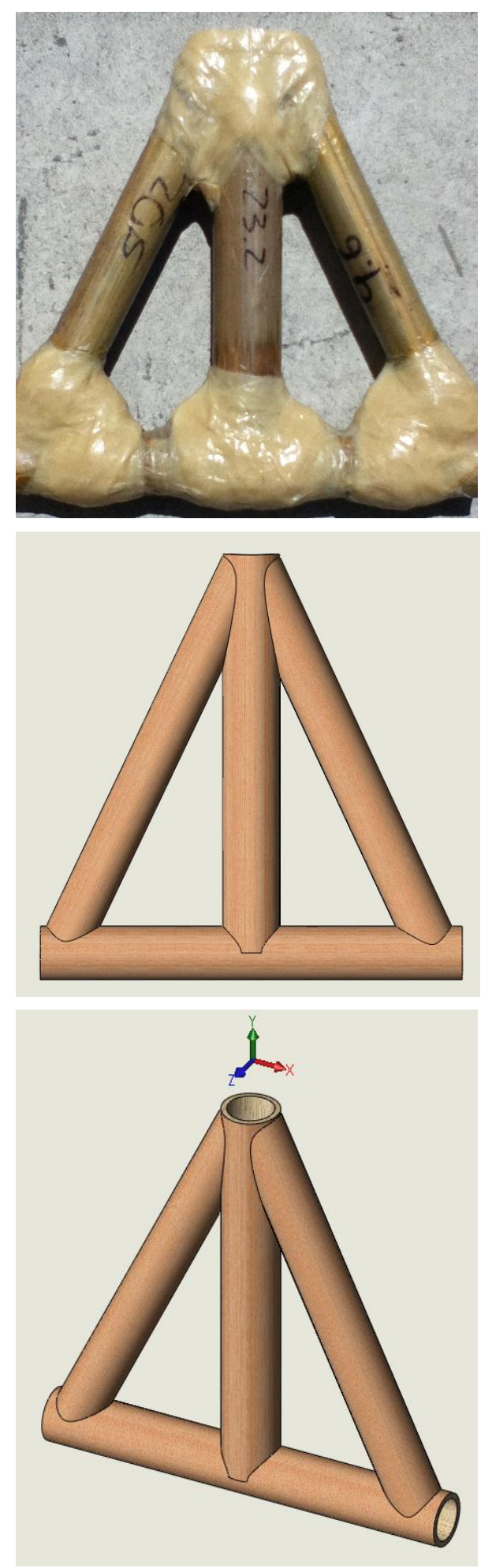

Figure 7-18. Model based on truss dimensions and created in SolidWorks CAD software 
common in the bamboo internodes is neglected, and each member is assumed to be a perfectly hollow cylindrical shape. It is worth mentioning that these assumptions do not significantly affect the final outcome of the analysis.

Table 7-1. Material properties of bamboo members in truss

\begin{tabular}{|c|c|}
\hline Material Property & Value \\
\hline$E_{11}$ & $513,536-p s i$ \\
\hline$E_{22}$ & $8,829-p s i$ \\
\hline$E_{33}$ & $8,829-p s i$ \\
\hline$v_{12}$ & 0.260 \\
\hline$v_{13}$ & 0.052 \\
\hline$v_{23}$ & 0.052 \\
\hline$G_{12}$ & $30,160-p s i$ \\
\hline$G_{13}$ & $30,160-p s i$ \\
\hline$G_{23}$ & $4,196-p s i$ \\
\hline
\end{tabular}

The model's material properties are defined as those obtained from prior experimental results of the optimum bamboo treatment. A table is provided to supply the respective material properties. As mentioned previously, the internal material property of the bamboo culm is known to be orthotropic. Although previous analyses indicate that using an isotropic material definition may greatly simplify the pre-processing procedure, the orthotropic material definition is selected in order to obtain better accuracy in the analysis. As a result, this orthotropic property is defined in the model. Since the main fibers are known to run longitudinally along the axis of the culm, the proper material orientations corresponding to the given material constants are assigned in the preprocessor. The principal direction of each truss member is assigned in the longitudinal direction. The remaining 2 and 3-axes are assumed to be symmetric, so the appropriate material constants defined in the table are assigned to the model. This assumption is rendered safe since the lignin matrix and fibers are predominantly distributed evenly across the cross section of the culm. 


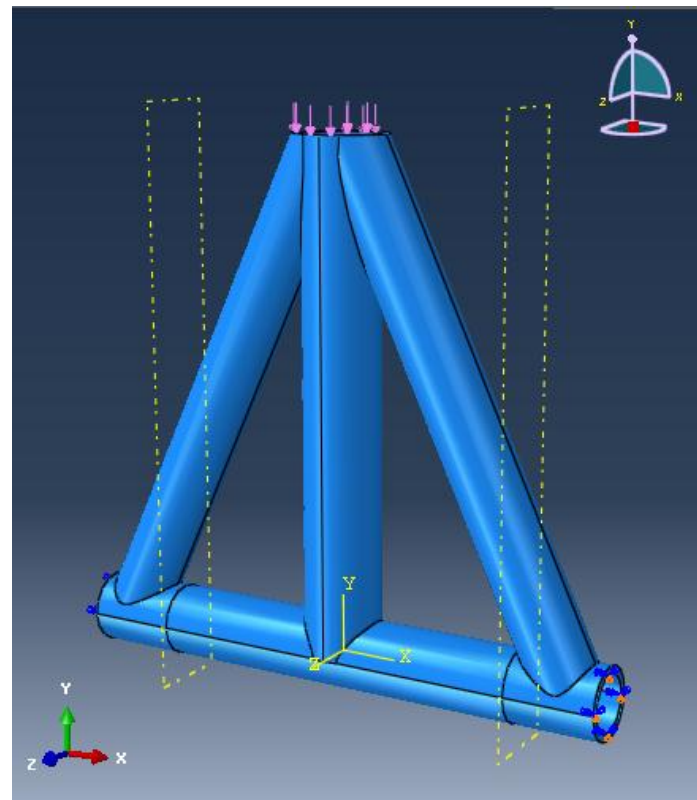

Figure 7-19. Load and boundary conditions imposed on truss model

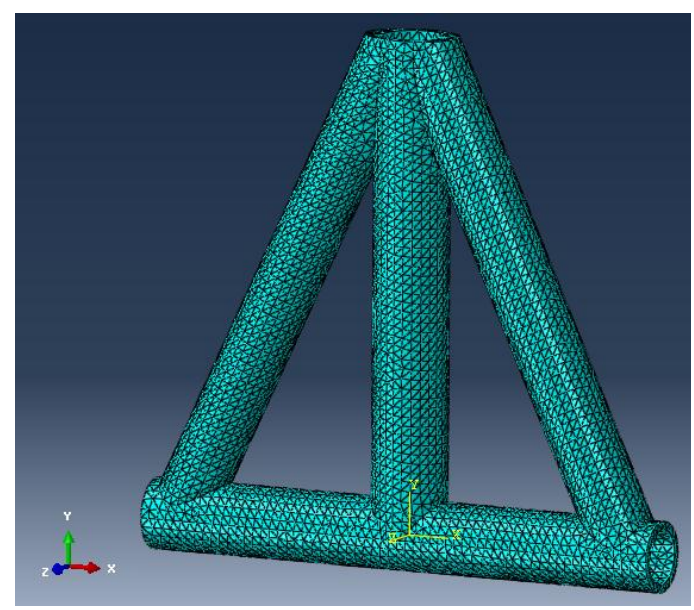

Figure 7-20. Generated mesh of truss model

Since the truss model is assumed to be composed of four perfectly joined members, it is fully defined without any interactions from other structural geometries. Next, the standard step size for this type of static, general load case is set for a maximum of 100 increments with an increment size of $5.0 \times 10^{-5}$.

Subsequently, loading and boundary conditions are applied after designating the correct assembly for the model. In order to validate the experiment in the linear-elastic region of the material, a $5000-\mathrm{Ib}_{\mathrm{f}}$ force is imposed on the model. A static $5000-\mathrm{Ib}_{\mathrm{f}}$ force is uniformly placed on the top face of the model along the negative vertical axis of the central member. Constraints are then applied to both of the bottom joints. The bottom member is partitioned longitudinally at the center of its diameter and subsequently the bottom half is fixed at its ends. Both end faces are constrained in the vertical direction and horizontal plane coming out from the model. Similarly, the end faces are constrained to have zero rotation about the $x$-axis and $z$-axis. The load and boundary conditions of the model are shown in Figure 7-19. The boundary conditions are illustrated by the blue and orange triangles, and the load is shown by the pink arrows. 
Once the boundary conditions are defined, the mesh is subsequently modeled. Figure 7-20 shows the mesh generated for the model in Abaqus. Figure 7-21 illustrates closeup views of the important regions of the model. As seen from the figure, the model is generated with tetrahedral continuum elements. This type of element is selected in the model's mesh largely by the cylindrical nature of the geometry in conjunction with the merging sections at the joints. Selecting another type of element would consequently result in high element distortion that yields inaccurate results.
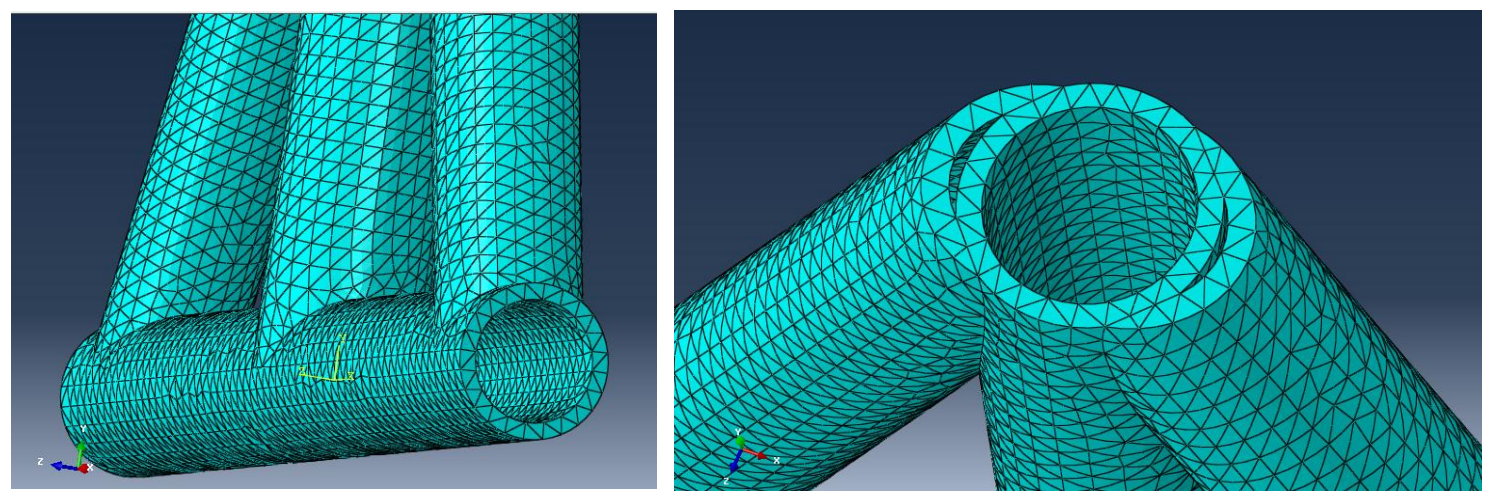

Figure 7-21. Visually enhanced views of critical areas in mesh model

\subsubsection{Comparison of Finite Element Analysis and Experimental Results for Truss}

For the finite element analysis, a series of deformation, stress, and displacement models are generated from the finite element analysis. Figure 7-22 illustrates the deformation of the model from the analysis, and the deformation scale is set to $5: 1$ for visual clarity. Overall deformations developed in the analysis comply with those observed in the experiment. Note must also be taken in the flexural behavior that the numerical results yield. The horizontal member exhibits the expected bending response observed from the experiment. Higher deformations are seen in the top and bottom of the structure. The top joint of the structure is observed to have the highest deformation since this joint is 
vertically and transversely compressed. A close-up view of this top joint deformation is provided-the deformation scale is $1: 1$. The lower central joint deforms vertically and slightly out of plane. Another close-up view of this bottom joint deformation is provided and its scale is also 1:1. Asymmetric truss members are the cause for this out-of-plane distortion since they differ in diameter and location. Also, the applied load is only about a third of the ultimate load, so its deformation is appropriately different from the plasticity effects seen in the actual structure.
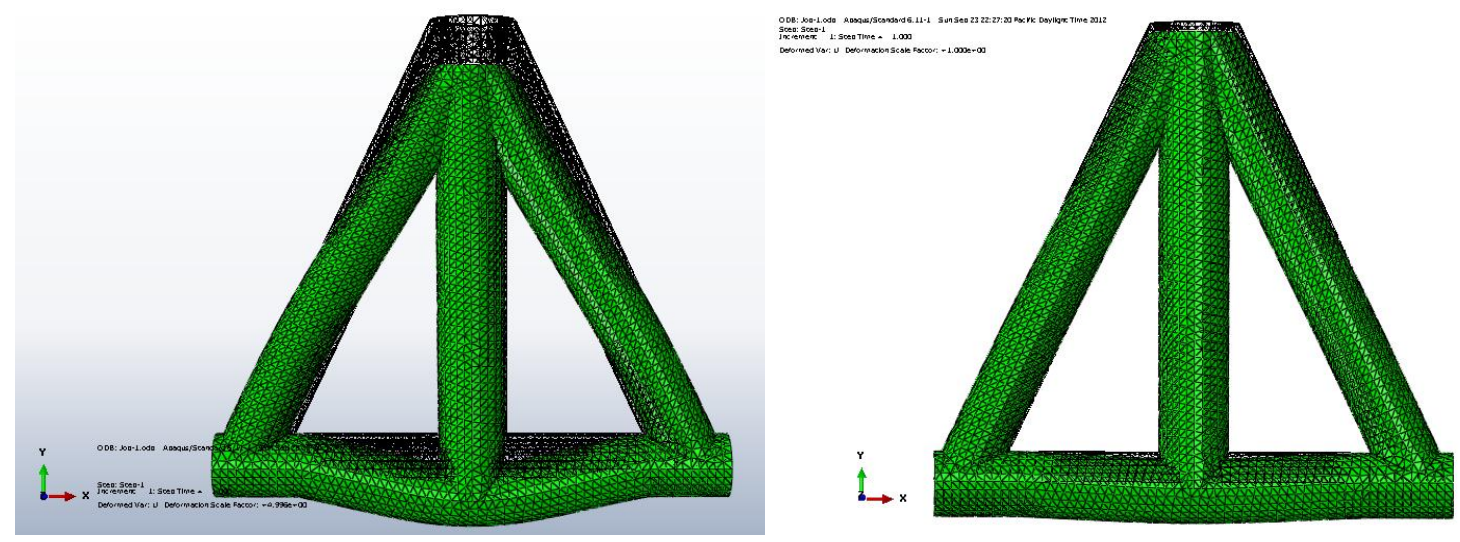

Figure 7-22. Deformation models of truss: deformation scale 5:1 (left) and deformation scale 1:1 (right)
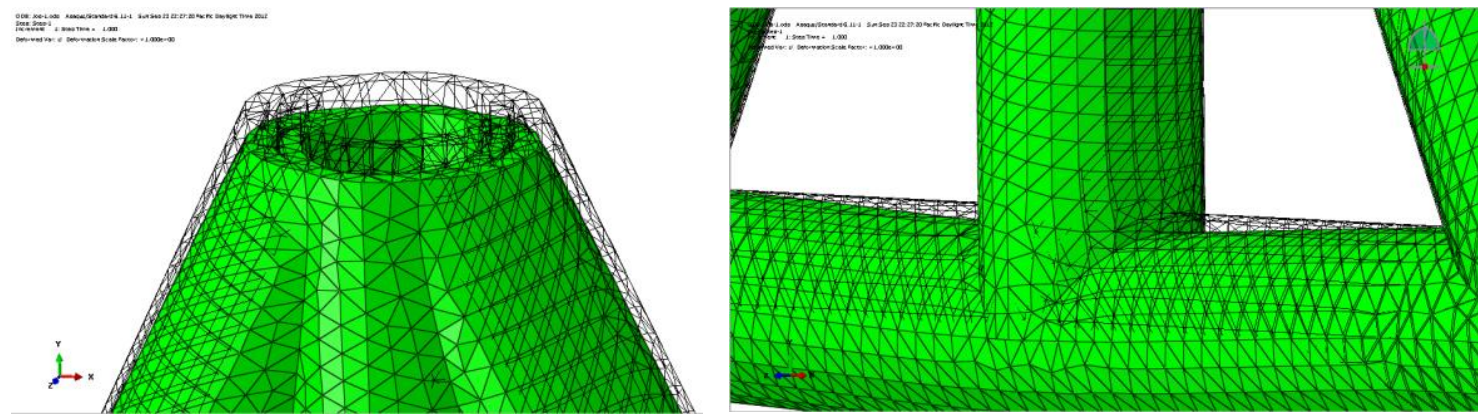

Figure 7-23. Enhanced views of deformation at critical areas with deformation scale of 1:1

Figure 7-24 shows the stress model from the analysis. The provided figures illustrate some of the estimated stresses throughout the structure of the truss under flexural loading. As seen from the Von Mises stress figure, the highest stresses are located in 
the vicinity of the joints. This figure reassures the assumption that the slight geometric differences in the members do not demonstrate a strong effect on the stress distribution in the structure. As seen from the figure, the stress field is fairly symmetrical about the mid-vertical plane and fairly low for the majority of the structure. Striking differences are mostly observed at the individual joints where the geometric deviation begins to have a more significant effect. In general, the diagonal members are observed to be the highly stressed members of the structure while the central vertical member exhibits the same stresses only near the top. This is expected, however, due to the boundary conditions imposed on the side joints.

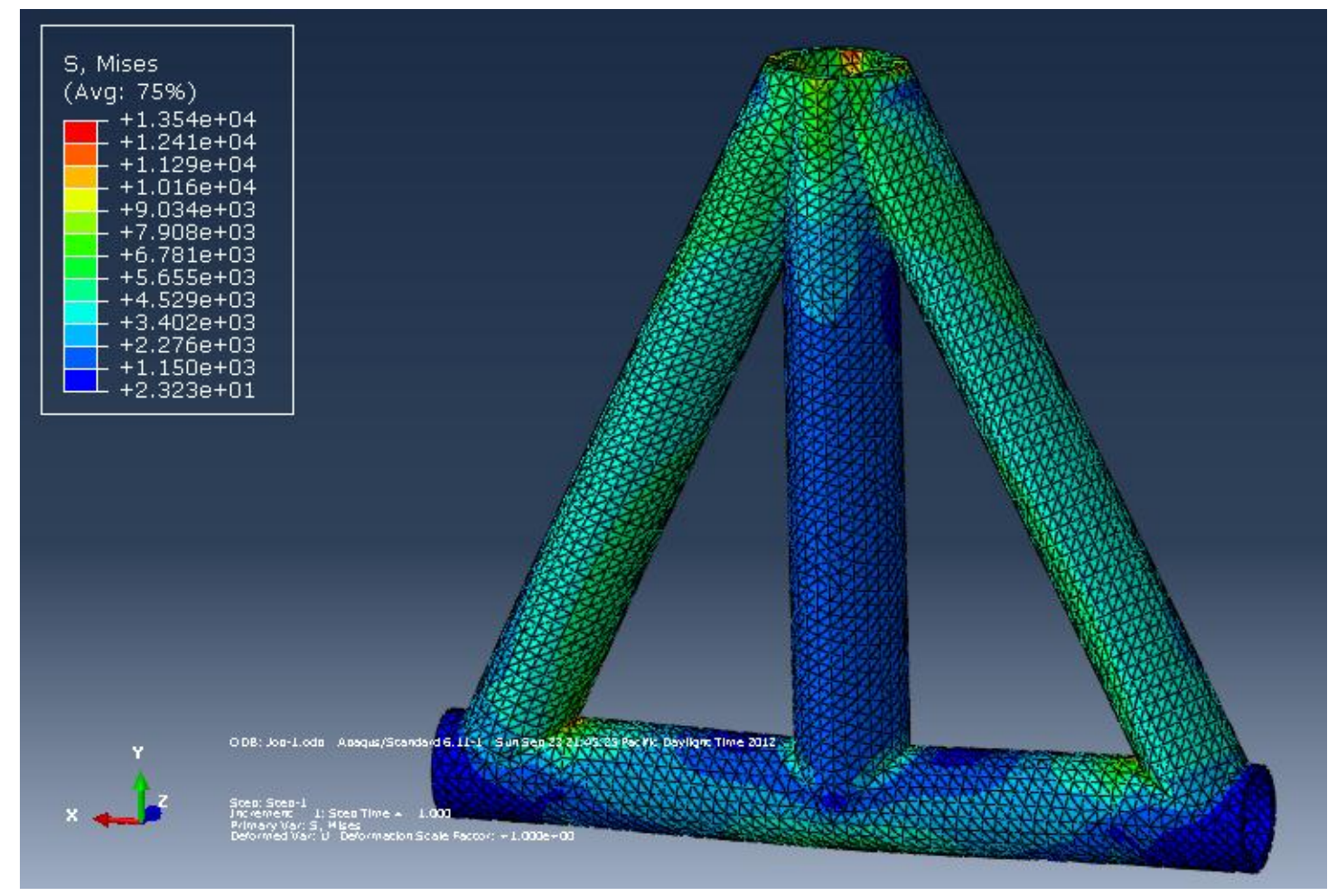

Figure 7-24. Overall stress field from numerical analysis of bamboo truss

Overall stresses obtained from the analysis also suggest the horizontal member does not experience highly stressed regions at the bottom flanks of the central member as expected. However, the center of the horizontal member does exhibit one of the higher 
stress locations in the model which is expected. By observing the top joint, a stress concentration is seen at the sides of the central member where the three bamboo internodes meet. The load applied to this top face causes the two diagonal members to deflect toward the center which causes the stress concentrations in the central internode. It can also be seen that stress concentrations occur at the inner portion of the
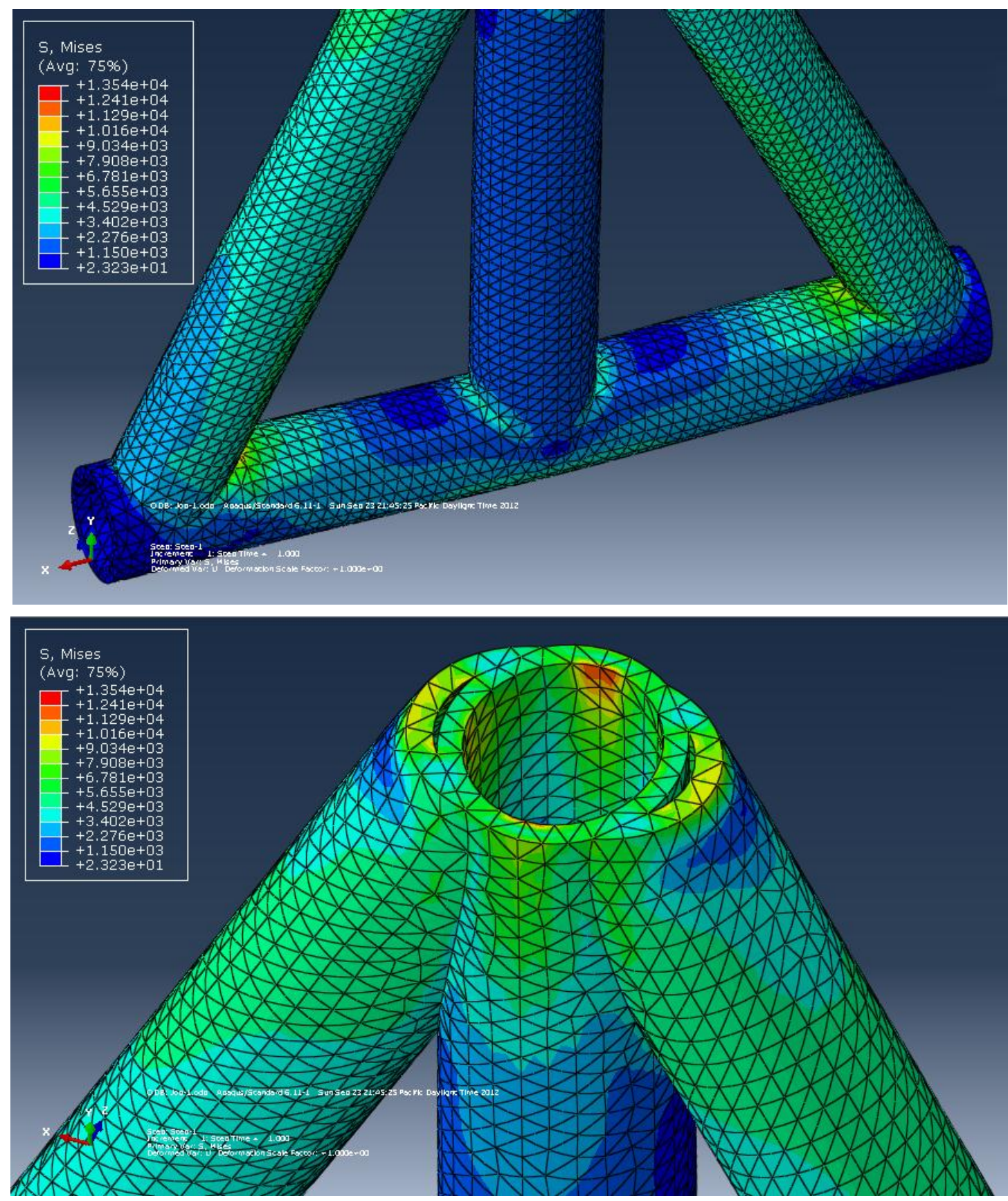

Figure 7-25. Enhanced views of stress field in critical areas: bottom joints of truss (left) and top joint (right) 
left joint. This stress concentration is attributed to the minor asymmetry inherent in the geometry.

The model illustrating the deflection caused by the compressive load in the analysis is shown in Figure 7-27. Only the deflection in the vertical is shown to comply with the results obtained from the experiment. The deflection model determined from the analysis also agrees with experimental results. It is important to observe the flexural deformations
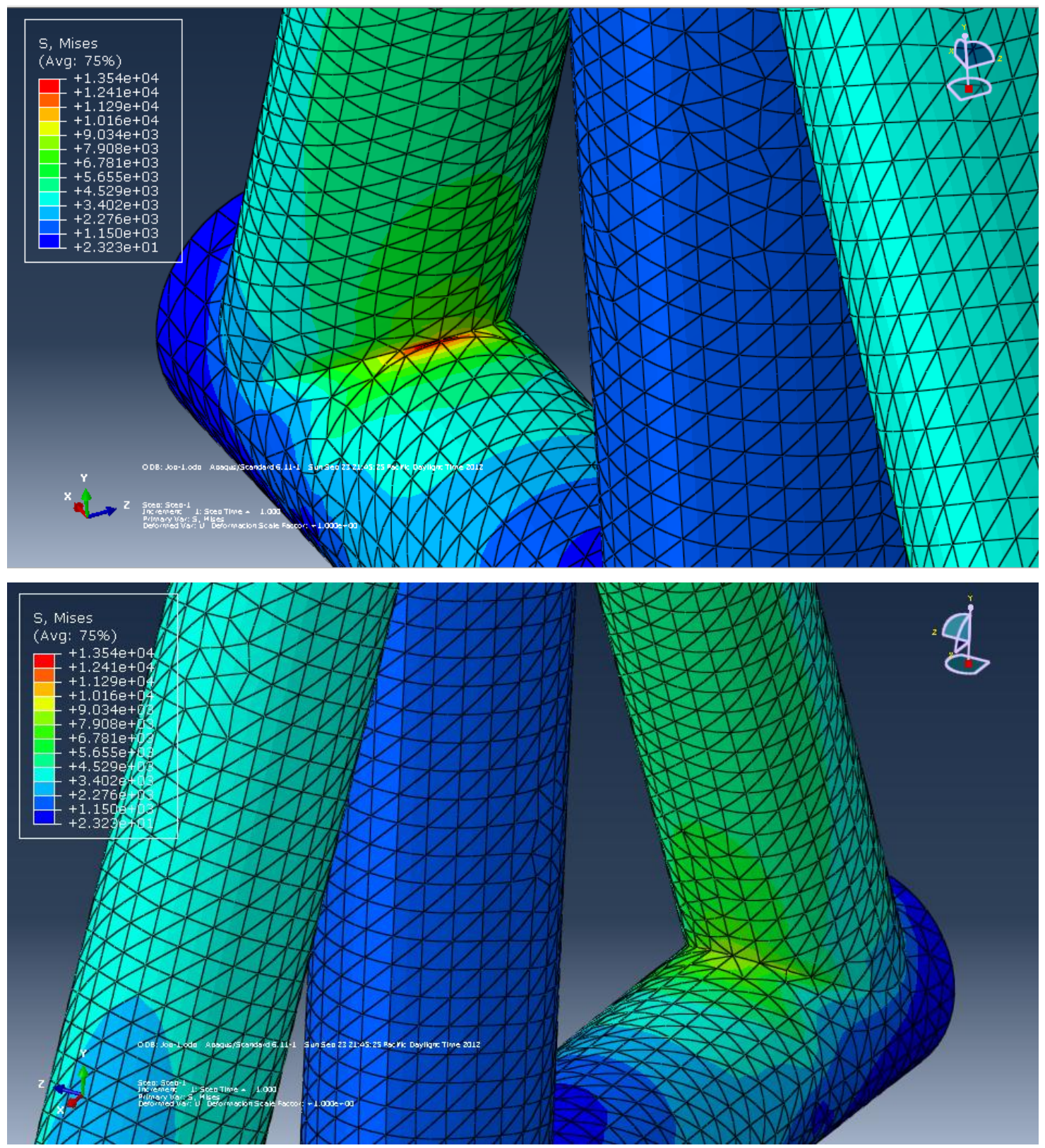

Figure 7-26. Enhanced views of stress field in left joint (top) and right joint (bottom) 
apparent at the central joint of the model. As seen from the figure, the highest deflection is observed at the top joint. Almost no deflection is observed at the bottom side joints where the boundary conditions are imposed. The figure also shows that the central member indicates the most displacement and suggests this will be the location for failure. Deformation at this location and in this manner is the primary deflection observed during the test-giving good credibility to the results of the analysis.

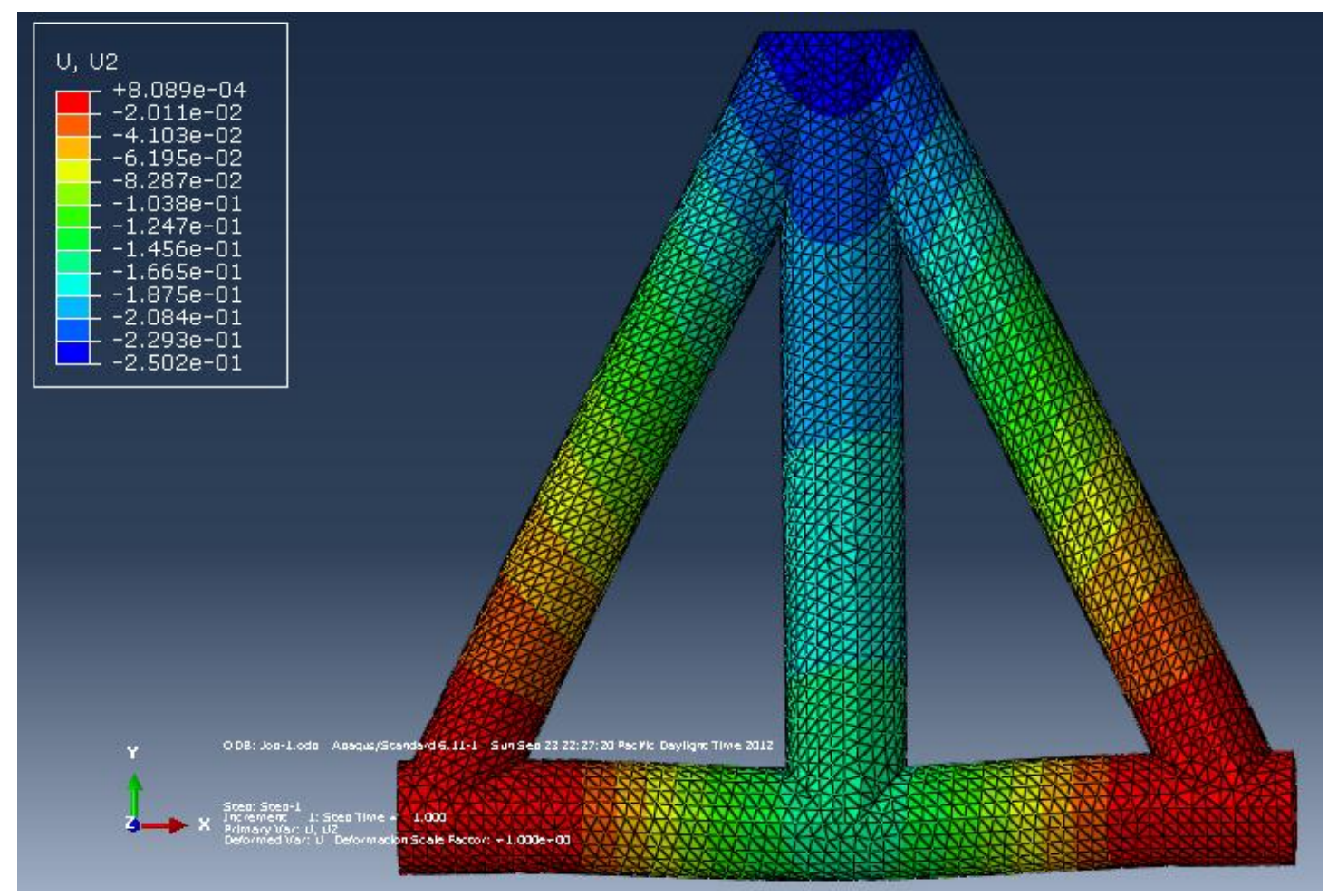

Figure 7-27. Deflection field of bamboo truss model

A table comparing the numerical and experimental results is outlined below. The maximum displacement corresponding to the vertical axis is compared to the experimental result because the Instron machine measures the relative change in displacement referenced from the starting position of the fixture prior to initializing the test. The stress obtained from the finite element method matches fairly well with the experiment-yielding a $7.90 \%$ error. The numerically obtained displacement 
demonstrates a $7.61 \%$ error from the experimental results. Once again, this analysis does not take into account any biological defects and geometric inconsistencies in the actual structure. Factors such as the tapering effect, where the model assumes a perfect hollow cylinder, may deviate from the test structure's actual geometry. The Poisson's ratio is also noted to be from a different sample group-contributing to the discrepancy between the model and the actual test. In any case, finite element analysis is still an appropriate tool for simulating the loads on this structure.

Table 7-2. Comparison of experimental and numerical results of truss

\begin{tabular}{|c|c|c|}
\hline \multirow{2}{*}{$\begin{array}{c}\text { Bamboo-Hemp Truss } \\
\text { Analysis }\end{array}$} & \multicolumn{2}{|c|}{$\begin{array}{r}\text { Static 5000- }-\mathrm{lb}_{\mathrm{f}} \text { vertical force on top central joint of } \\
\text { simply supported truss }\end{array}$} \\
\cline { 2 - 3 } & Stress (ksi) & Displacement (in.) \\
\hline \hline Experimental & $\sim 6.78$ & 0.2708 \\
\hline Numerical & 6.24 & 0.2502 \\
\hline Error (\%) & 7.90 & 7.61 \\
\hline
\end{tabular}

\subsubsection{Mesh Convergence Study for Bamboo Truss}

In order to determine an efficient manner in which to conduct this finite element analysis, a mesh convergence study is necessary for the truss. In addition, this study will provide insight on whether the analysis has reached the correct result after increasing the mesh size of the model. The procedure to conduct this analysis follows a simple algorithm. To begin, a small mesh element density is selected and the analysis is conducted again. A node from the mesh is probed in the area of interest and is logged as a data point for the convergence study. For this study, the probed element is inspected for the maximum deflection at the top joint of the model. The subsequent step requires returning to the mesh and lowering the global element size to increase model's mesh density. Again, the analysis is conducted, and the probed element is recorded as a data point for the 
convergence study. This process continues until the probed solution yields the same value within a set tolerance value of 0.001 -in. for an increased mesh densitysignifying convergence. It can be noted that this process effectively aids in selecting an optimum mesh density for an efficient analysis. Some of the mesh models from the
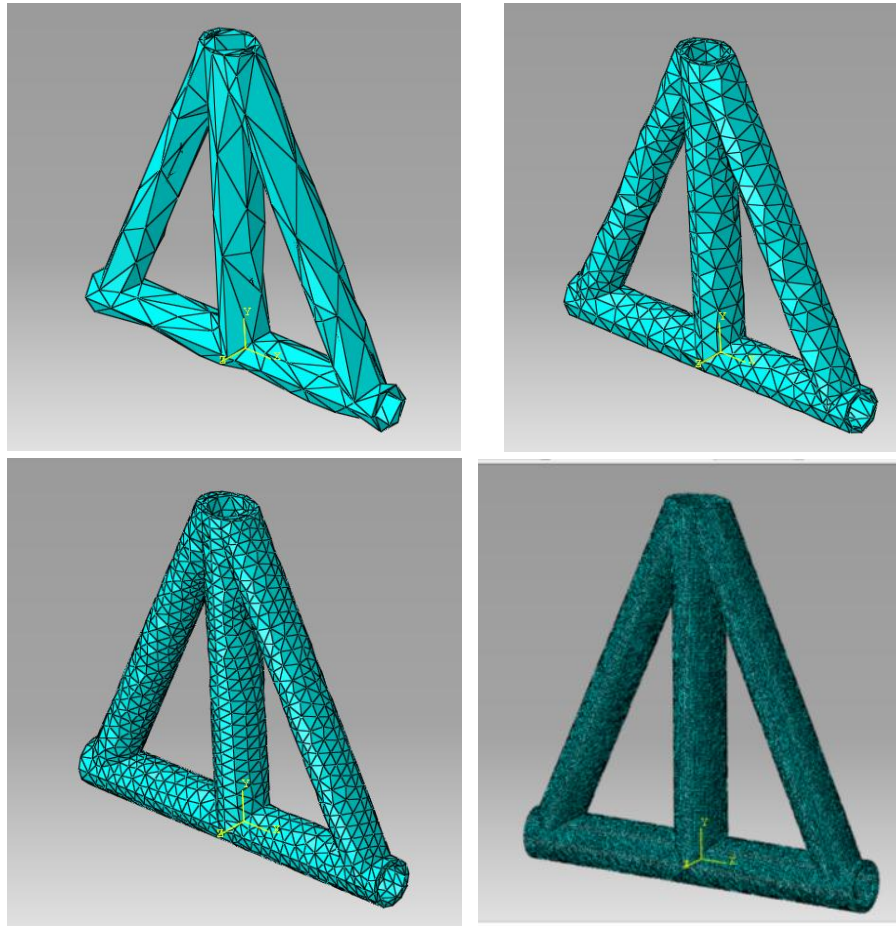

Figure 7-28. Mesh models from the convergence study: 996 elements (top left), 3693 elements (top right), 8301 elements (bottom left), and 206904 (bottom right)

analysis are shown in Figure 7-28. Figure 7-29 illustrates the results of the study and suggests 32,643 elements are adequate for reaching mesh convergence of the solution.

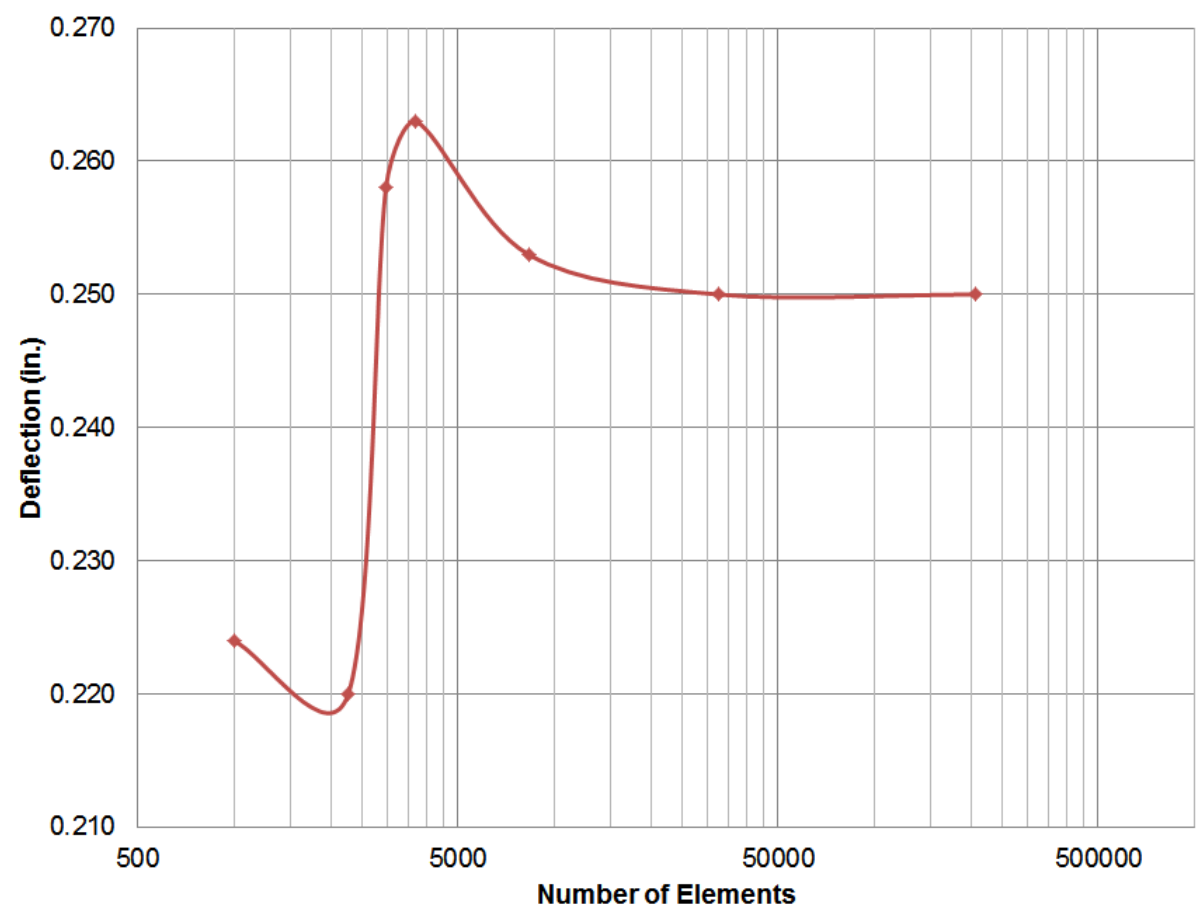

Figure 7-29. Mesh convergence study of bamboo truss 


\subsubsection{Conclusion of Truss Performance and Future Notes}

In summary of the bamboo biocomposite truss, the results of the experiment demonstrate a successful structure capable of withstanding considerable loads, and the numerical method conducted adequately predicts the behavior observed in the mechanical test. The ultimate load and strength-to-weight ratio is deemed impressive for a structure such as this. Flexural behavior of the bottom horizontal member shows minimal deformation indicating the structure properly distributed the vertical force better than initially predicted. In spite of this positive performance outcome, the design of the structure can be improved further. A few noted considerations in the design are made by inspecting the deformation of the structure at the end of the test and analyzing the numerical model. First, the top joint may be reinforced to strengthen the structure in this region. The extensive damage found in the top joint in the tested structure and the high stresses observed in this region of the simulated model testify to this suggestion. Secondly, the structure's weight can be optimized by reducing the hemp plies around the lower central joint as the stress anticipated in this area was not as high. Again, the little deformation observed in the tested structure and the lower stresses seen in this area of the simulated model provide the seed for this note. Overall, these structures give insight into the benefits of utilizing bamboo cylindrical culms in a biocomposite structure, and the mentioned notes can improve the performance of future design iterations.

\subsection{Bamboo Core Composite I-beam Application}

The main objective of the I-beam study is to develop the manufacturing process and performance characteristics for using veneer bamboo fibers as the web core for a flexural biocomposite I-beam considered by rules from the 2012 SAMPE bridge contest. This I-beam can demonstrate the benefits of utilizing bamboos as the main fiberreinforcement in a biocomposite structure exhibiting flexural behavior. Three similar 
designs are created and tested to investigate the benefits of utilizing different bamboo configurations. The first design involves utilizing a 2-ply bamboo core while the second design utilizes a 3-ply bamboo core. The third design requires using a 4-ply bamboo core with a ply of bamboo in the bottom flange and hemp twine fillers in the corners of the web. An evaluation on the performance of each design is then conducted to determine the benefits of design's unique characteristic. The manufacturing process entails fabricating the I-beam through a wet lay-up method using eco-friendly resin, bamboo strips, and hemp fabric joined with the use of molds. Figure 7-30 is provided to depict some conventional applications for a bamboo biocomposite I-beam. As seen from the figure, this biocomposite I-beam may be utilized as part of a building frame, major bridge beam, aircraft wing spar, or automotive suspension.

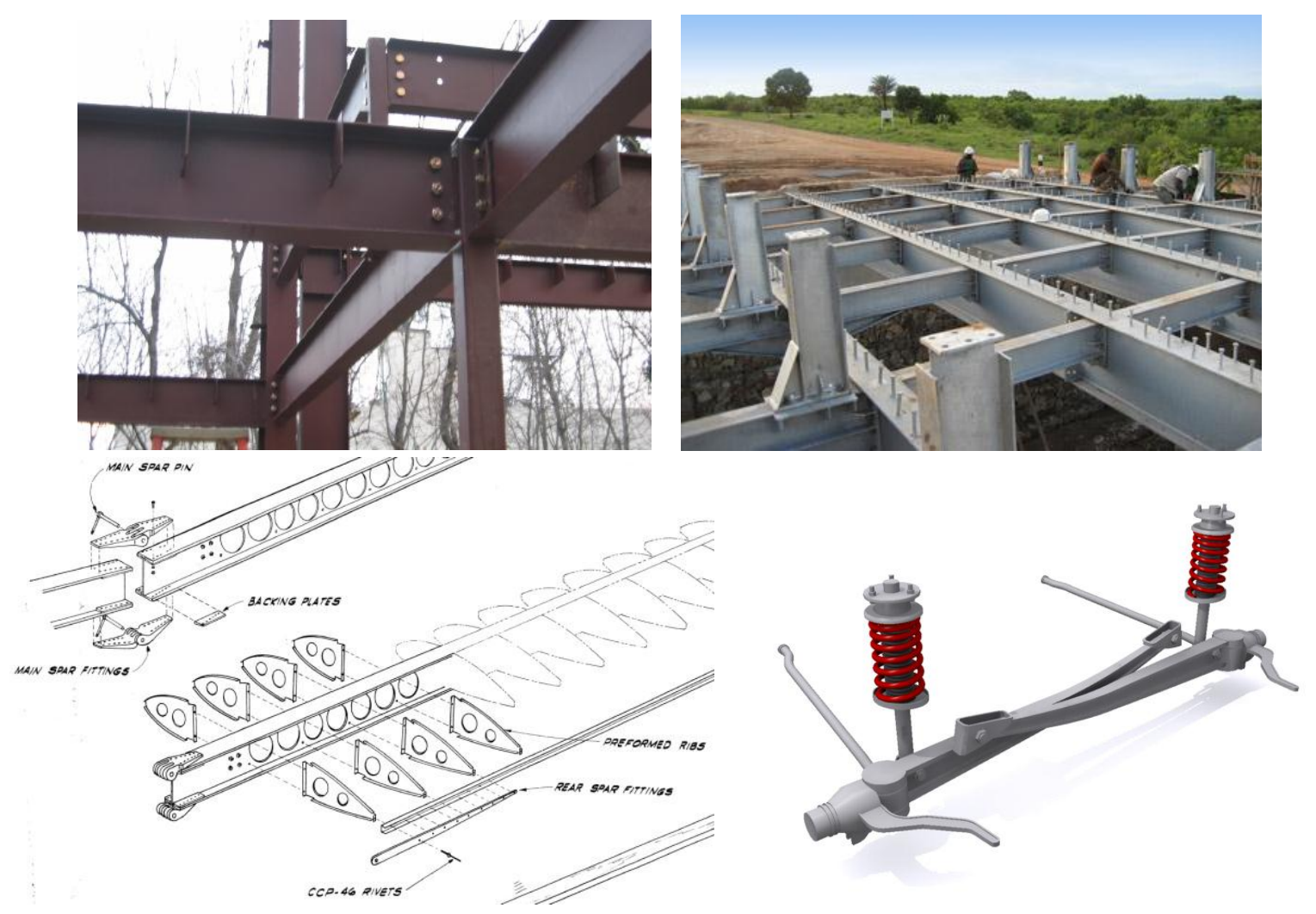

Figure 7-30. Potential applications for bamboo biocomposite I-beams $[50 ; 15 ; 56 ; 65]$ 
The following contest rules and requirements are designated for the natural fiber I-beam category of the contest [63]. The maximum load is taken to be the primary criterion for performance evaluation. The maximum load is defined as the minimum compressive ultimate load, the load at a 1 -in. deflection, or the maximum design load of $25000-1 \mathrm{~b}_{\mathrm{f}}$. The beam is loaded under a four-point bend configuration with a specialized fixture; the use of braces against the loading fixture is strictly prohibited. The maximum dimensions for the I-beam cross section is 4-in. $x$ 4-in. The I-beam is allowed to have a single web with a thickness of 0.6 -in. or less and a cross section that varies along the length of the beam. The minimum length of the beam is 23-in., and a distributed load spanning 4-in. at the center is applied midway across the length. Lastly, the maximum beam weight must be $1.32277-\mathrm{Ib}_{\mathrm{f}}$.

The investigation of this I-beam study begins with manufacturing, testing, result presentation, and numerical validation. Briefly speaking, the manufacturing process entails cutting the bamboo poles into thin strips of the appropriate length, sanding the strips to the correct thickness, joining the bamboo fibers to create the web, and merging the web with the flanges with the use of resin-soaked hemp fabric. Testing the I-beam is conducted by applying a uniformly distributed load through a plate spanning 4-in. at the midpoint until failure, and an evaluation report is generated by the strength-to-weight ratio observed. Experimental validation is sought with Abaqus/CAE finite element analysis software to simulate the maximum deflection of the structure under the same loading conditions.

\subsubsection{Manufacturing the I-beam}

The entire construction of the I-beam structure was also done in the Cal Poly Structures/Composites Laboratory, and all the materials used in the beam are all natural materials. As previously mentioned, the I-beam is manufactured through a wet lay-up 
process under considered regulations provided by the SAMPE bridge contest rules. A total of two square beams and two rectangular plates are utilized for the mold to clamp the I-beam after the lay-up process. The square beams each have cross section dimensions of 2-in., and the rectangular plates span 4-in. x 1-in. for its cross section. Both the beams and the plates span a length of 30-in. Once again, established design guidelines warrant the bamboos of the I-beam to be treated with the $3 \%$ salt solution at $220^{\circ} \mathrm{F}$ with an initial cure rate of $1^{\circ} \mathrm{F} / \mathrm{min}$. In addition, the locations of the nodes are placed minor significance in terms of placement along the length of the beam as determined in the Analysis of Flexural Results section. All the designs are made with the optimally treated bamboo and hemp fabric.

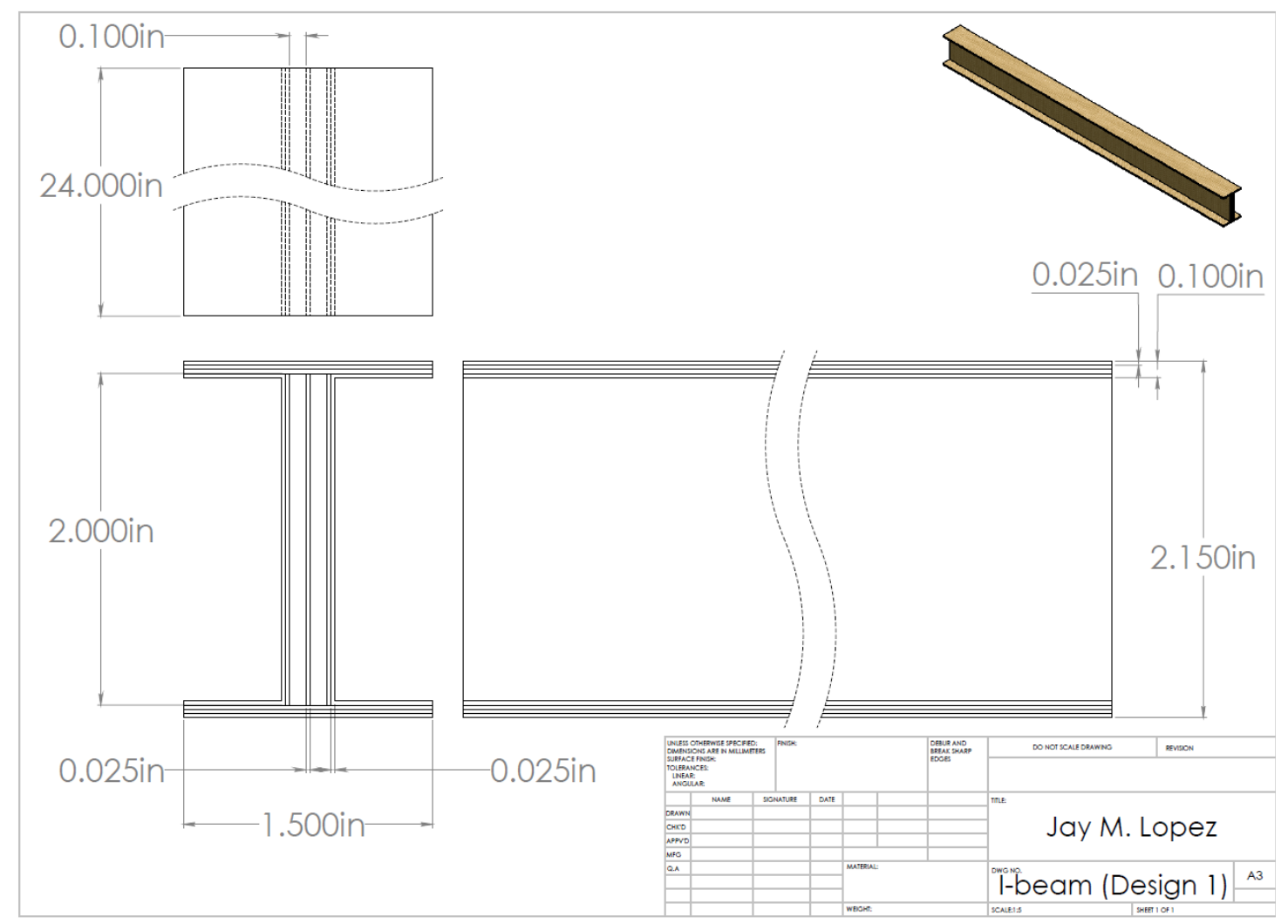

Figure 7-31. Manufacturing drawing for I-beam structural application

A total of three similar designs are chosen for the I-beam and their manufacturing techniques are now discussed. A drawing created in SolidWorks is provided in Figure 
7-2 to manufacture the I-beam to the appropriate dimensions-the major difference among all the designs is the number of bamboo/hemp fabric plies in the web. Again, the dimensions of the beam conform to the regulations imparted by SAMPE for the contest. It is important to note that the web of the beam is composed of bamboo strips oriented vertically so that their longitudinal fibers run the length of the beam and their inner/outer walls are horizontal. This is done to maximize strength and stiffness in the beam.

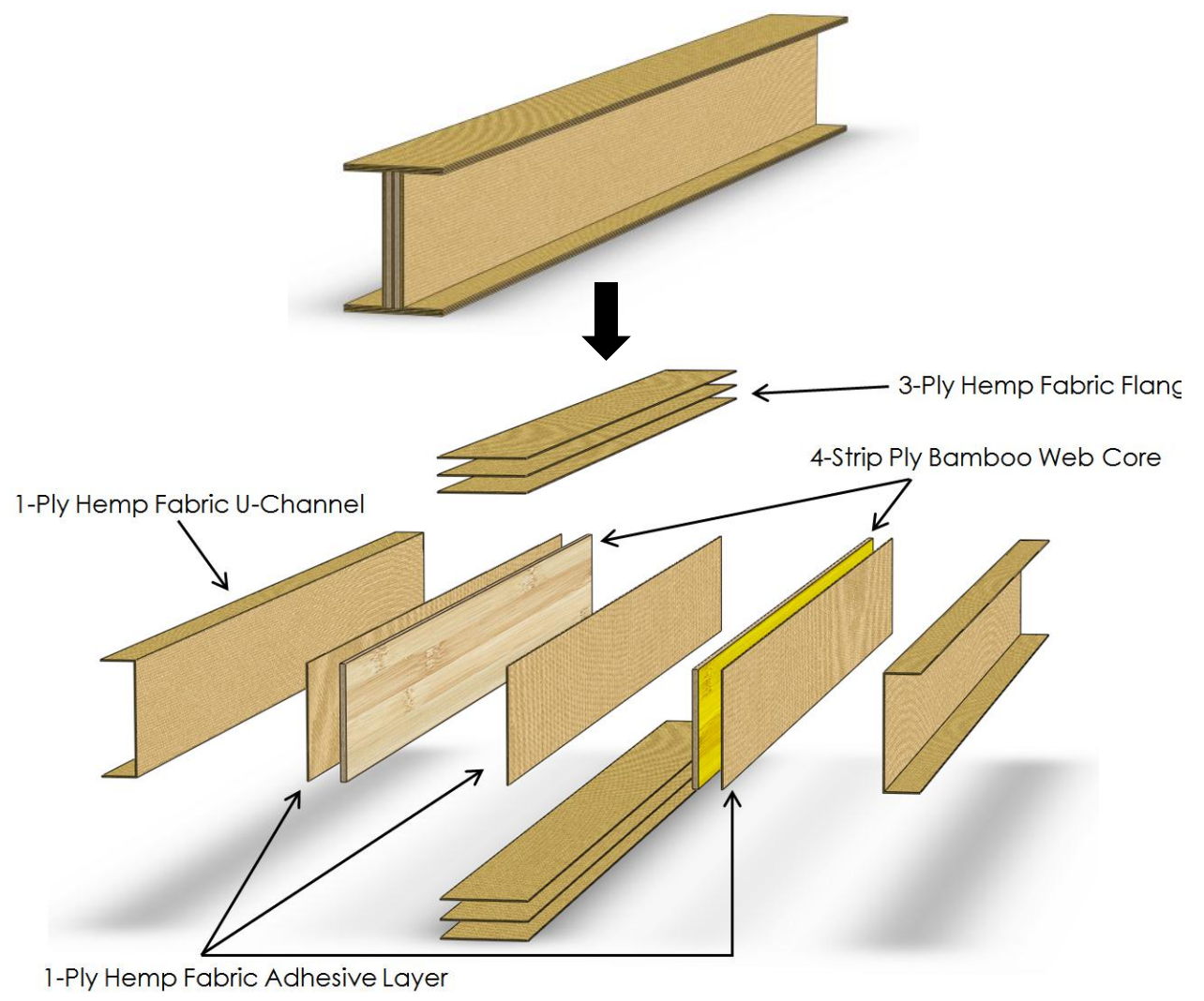

Figure 7-32. I-beam design 1 assembly method

To begin the manufacturing process, the bamboo is prepared to create the I-beam's web core. First, a correctly treated bamboo pole is cut to the approximate lengths of 26 -in. to allow the necessary tolerance for the final product. Similarly to the tensile and rectangular bending specimens, the culm is cut in halves along the longitudinal direction of the fibers until a total of eight strips are obtained. Each strip is then sanded to 0.10 -in. 
thickness across its entire length. Placing numerous strips on a flat rigid plate can expedite this part of the manufacturing process. Once sanded to the correct thickness, the strips are inspected and collected into groups of four that fit well when placed side by side. The groups of bamboo strips are then laid side by side with the inner culm facing down-making sure to minimize gaps between each strip-and taped across to keep them in place. It must be remembered that in this critical step the strips must be taped appropriately so that the strips are positioned with their inner/outer walls in the horizontal direction.

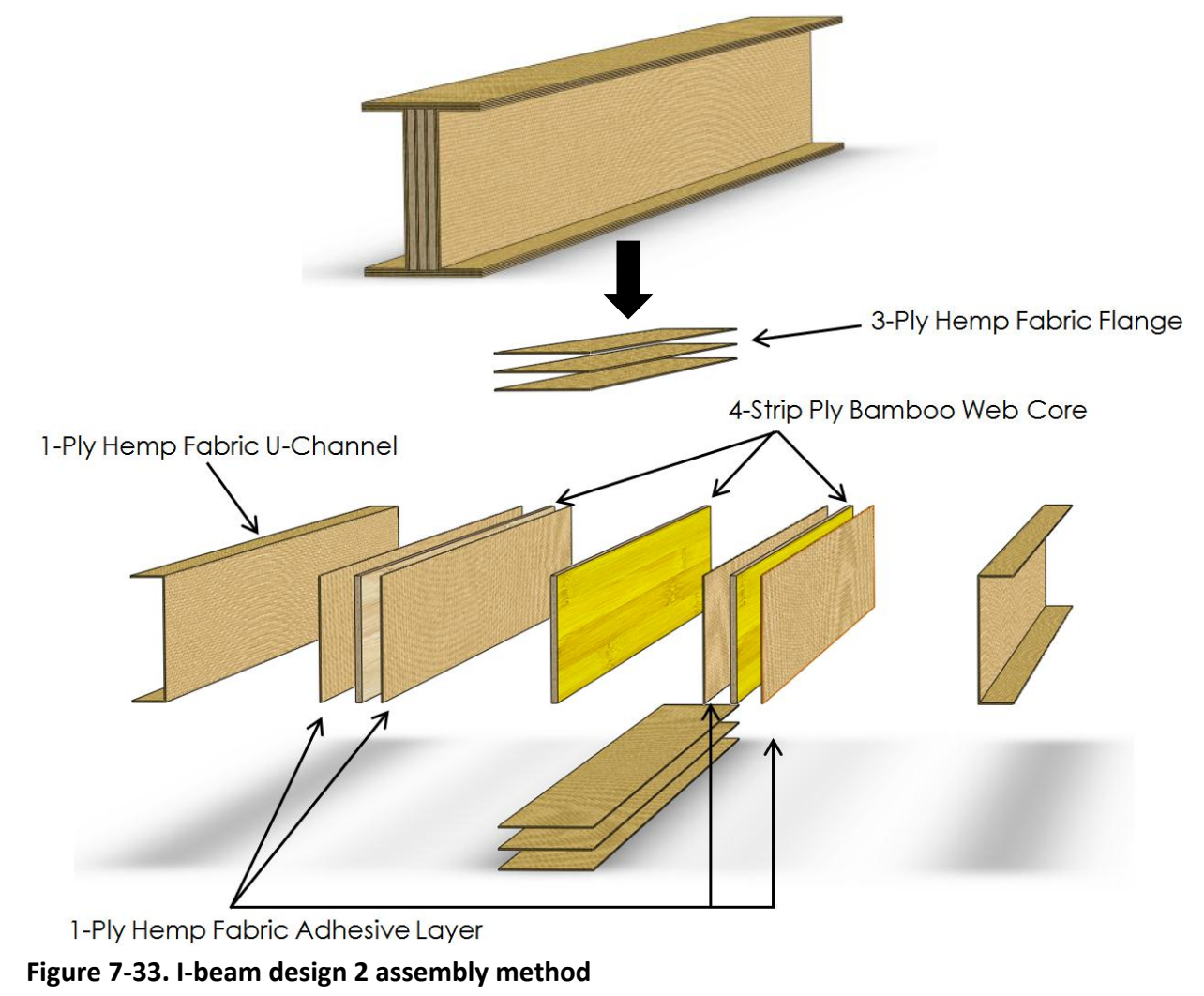

Figure 7-35 depicts this process of preparing the bamboo for the web. For the first design, the web consists of two bamboo plies. The second design utilizes three bamboo plies while the third design utilizes four plies in the web. In addition, the third design utilizes four of these bamboo strips as one ply for the bottom flange of the I-beam. When 
stacking these plies for the web, it is vital that the inner culms face each other to provide a good bonding surface for each ply. After stacking the plies, the group is sanded down until the web height is 2.0 -in. Accomplishing this task can be done with ease if bamboo plies are clamped in between two plates that are 2.0-in. high. In this manner, the web can be of uniform height across the entire length and will lessen the likelihood of creating voids in the beam during the lay-up process. In addition, the outer walls of the bamboos plies are lightly sanded with coarse sandpaper to facilitate adhesion between the core and hemp plies for the web.

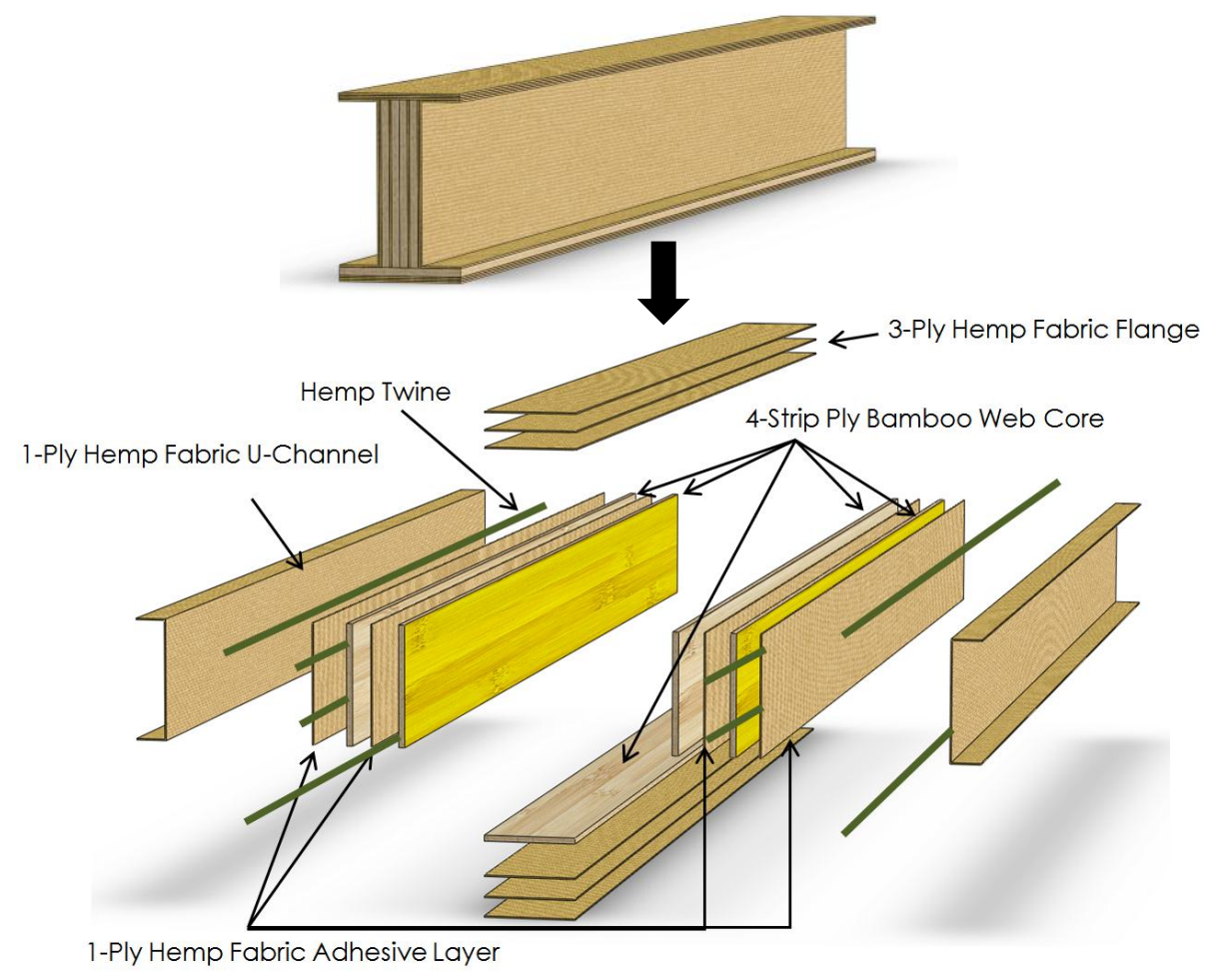

Figure 7-34. I-beam design 3 assembly method

Once the web is prepared in the correct stacking manner, the hemp fabric plies are prepared in relation to the web. First, a pair of 26-in. $\times 2$-in. rectangular sections are cut from a roll of hemp fabric. These rectangular strips serve as the main adhesive plies that are sandwiched between the plies of bamboo in the web. Cutting the fabric may be done 
with ease by using a T-square and a marker as shown in Figure 7-36. The next hemp fabric plies to be prepared are the flanges. Six rectangular sections, spanning 26 -in. $\times 3$ in., are cut for three plies on the top and bottom flange. The next plies required are the "U" channel plies that provide the adhering mechanism for the flange to the web. These plies are also cut from the roll of fabric and span 26-in. x 5-in. Two plies are required for each design. At this point, the individual components are ready for the lay-up process as shown in Figure 7-37.
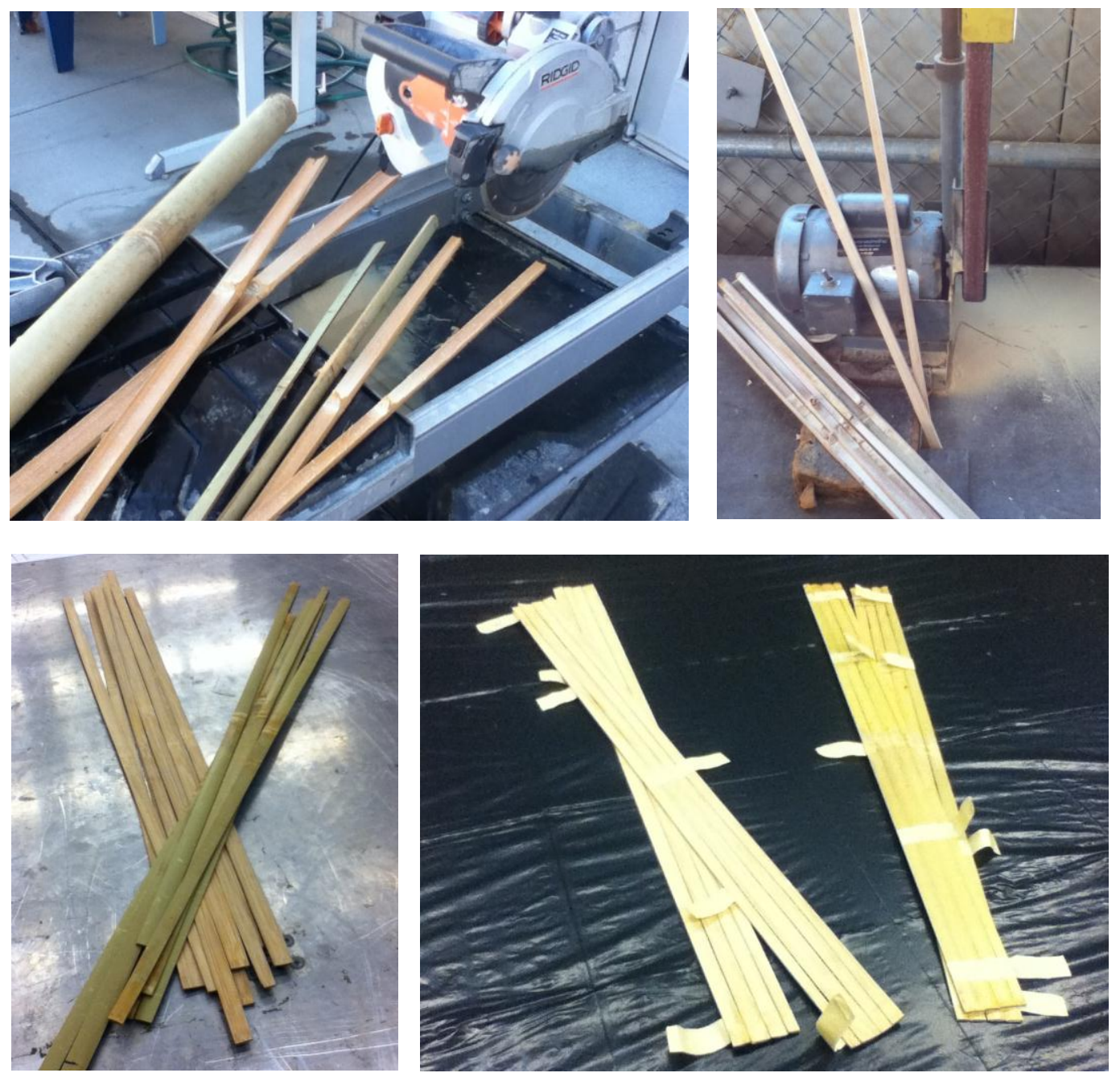

Figure 7-35. Cutting bamboo pole into strips for the web (top left), sanding strips to the correct thickness (top right), resulting product after sanding (lower left), and taping the strips together for ease in the final assembly of the core (lower right) 
Prior to the lay-up, however, the proper molds must be prepared to house the beam under a tight clamp during the curing process. First, each mold part is cleaned and scraped of debris on the faces of the molds making contact with the I-beam. Applying acetone and scraping the molds' surface with a razor is the proper way to accomplish this task. Each beam and rectangular plate is then wrapped in a vacuum bag and taped in place to prevent the beam from ruining the molds with resin. It is important that the tape be located in regions of the mold that do not make contact with the I-beam during

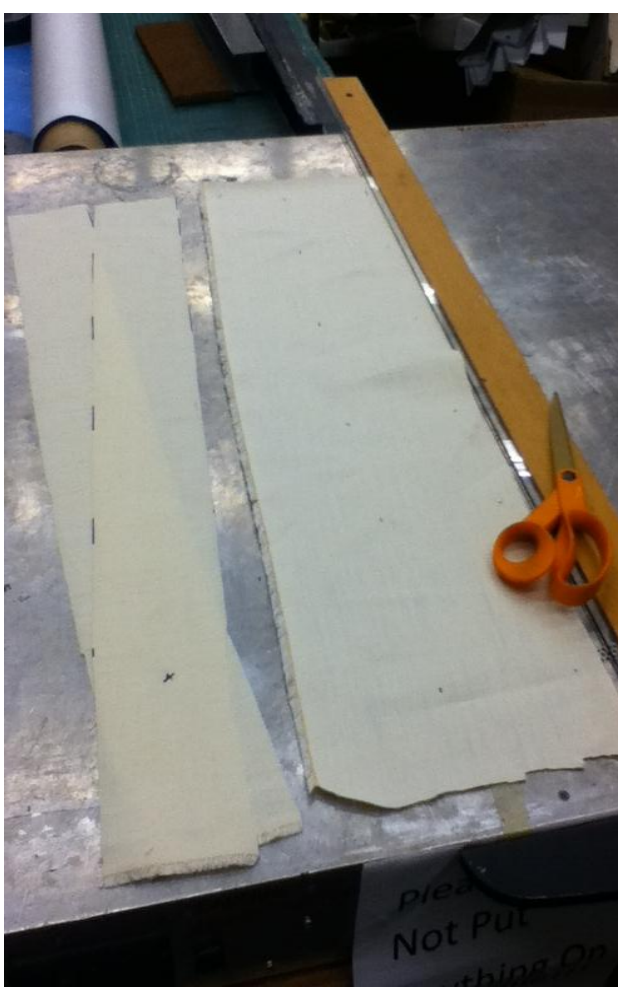

Figure 7-36. Cutting the hemp fabric to the appropriate dimensions for the plies the curing process.

The following step in the manufacturing process is laying up the I-beam with the previously prepared components of the web and flange. As mentioned previously, the resin used in this lay-up is Ecopoxy resin ER500 and the hardener is EH725. To initiate the process, the resin compound is created by mixing a 4:1 resin-to-hardener ratio. It is strongly recommended to vigorously mix the resin-hardener compound with an adequately long utensil in a disposable cup so that

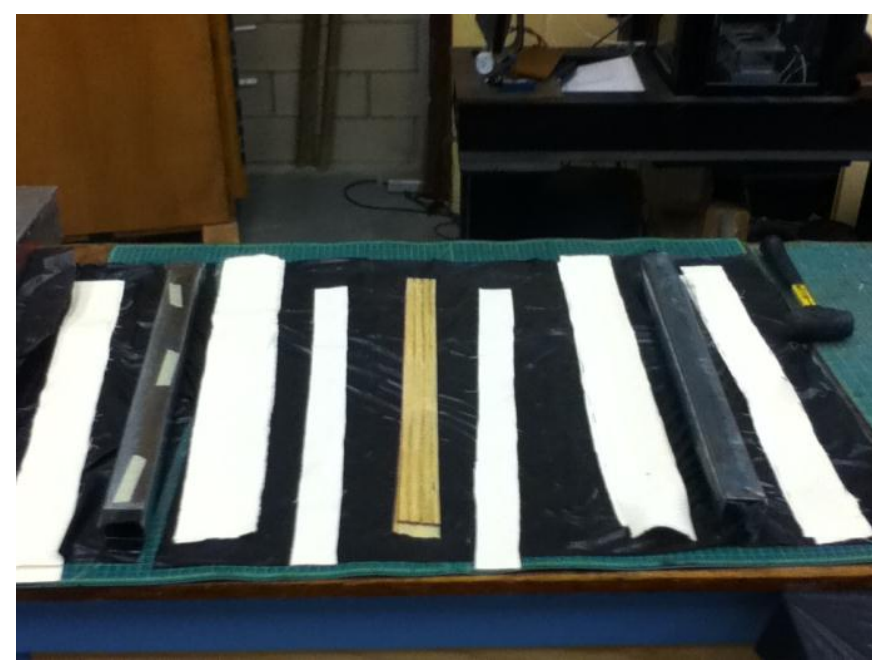

Figure 7-37. Set-up of adhering strip, U-channel, and flange plies prior to wet lay-up with molds 
proper mixing can take place. Once the resin is prepared, the hemp and bamboo plies are organized on the table in the same fashion shown in Figure 7-37.

The first component to prepare is the web of the I-beam. Resin is then poured over each component on the table and evenly distributed across the surface with a spreader until the fabric is thoroughly saturated. A good indicator of saturation is seen when the fabric becomes transparent after resin application. Working in a swift pace, the adhering layer of hemp fabric for the web of the beam is first laid in between each ply comprising of bamboos taped together. It is critical that the adhering hemp ply be laid on the inner wall surface of the bamboo ply, for the other side of the web core does not easily allow the hemp plies to adhere to the bamboos' outer wall. Then a ply of bamboo core is laid on top of the hemp fabric. This process is done once more for the second and third designs, and the two bamboo-hemp sandwiches are pressed together to create the web.
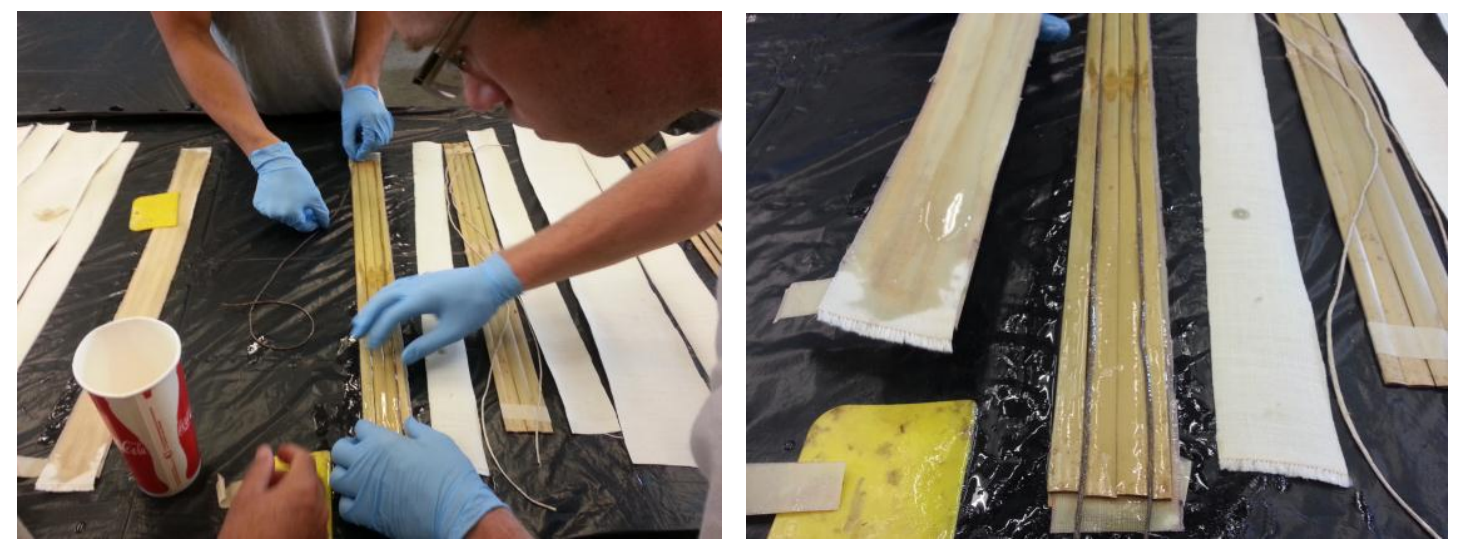

Figure 7-38. Preparing the web core of the I-beam

The following components of the I-beam to prepare are the U-channels of the web. Again, the resin is evenly coated over the appropriate hemp plies and spreaders are used to evenly saturate the plies. Once full saturation is reached for the fabrics, the plies are laid one-by-one on top of the box beam molds-paying special attention in aligning the ply across the centerline of the beam mold. After aligning the ply correctly, the 
bamboo core is placed on top of the hemp ply on the mold. The other mold, after being fitted with another hemp ply, is then positioned over the other half of the web. Special care is taken in removing air bubbles when assembling the plies and bamboo core of the web. Figure 7-39 depicts a graphic summary of this process for clarity.
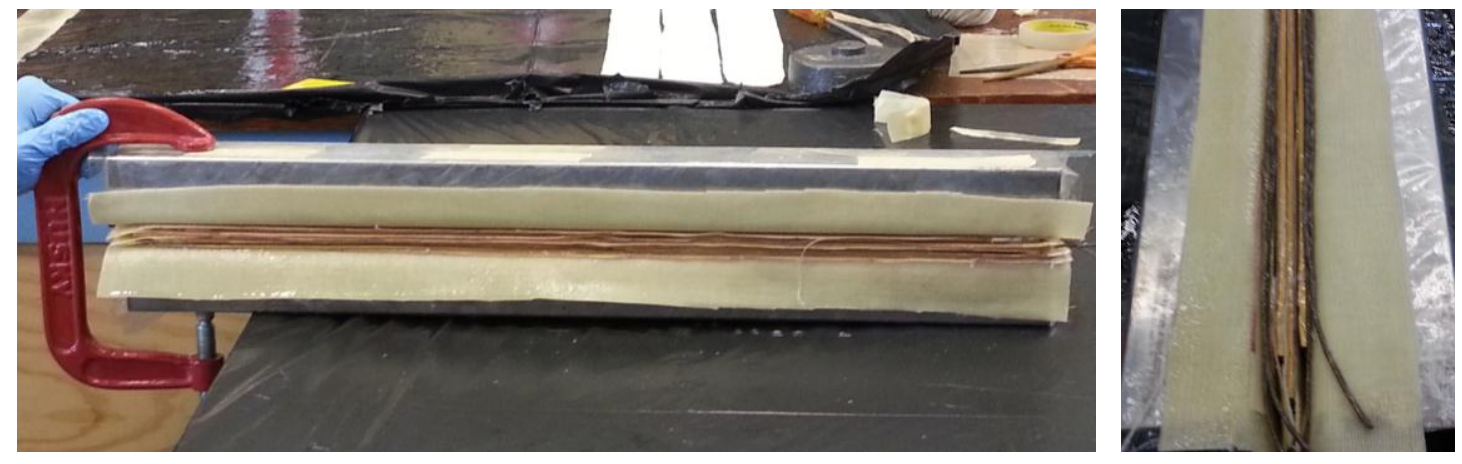

Figure 7-39. Assembling the web of the I-beam

The next step entails preparing the flanges of the I-beam. Once more, the hemp fabric is soaked in resin and prepared with a spreader until full saturation is reached. The three plies of fabric are then placed over each other while carefully orienting the plies so that the weave of each ply matches the correct direction. After stacking the plies correctly, the flange is then is positioned over the web after making the proper alignment measurements. Once positioned correctly, the rectangular beam mold is placed on top. The same procedure is done for the bottom by flipping the part and mold over and repeating the process. At this
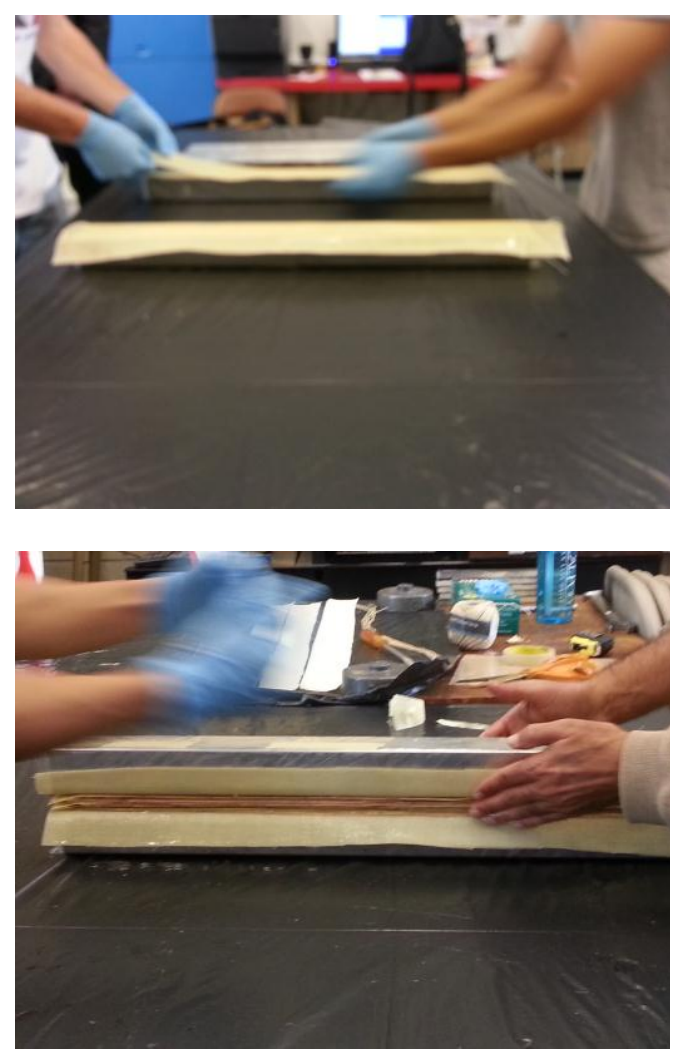

Figure 7-40. Assembling the flange of the I-beam 
point, the assembly is nearly finalized. For the third design, this process is repeated slightly different to accommodate a bamboo ply in the flange. Figure 7-40 depicts a graphic summary of this process for clarity.

The final step in manufacturing the I-beam is then clamping the molds together to provide pressure on the part for an even distribution of resin and to remove any voids inherent in the part. C-clamps are used to provide the desired pressure distribution across the entire structure. It is important to maintain symmetry when clamping the part as shown in Figure 7-41.
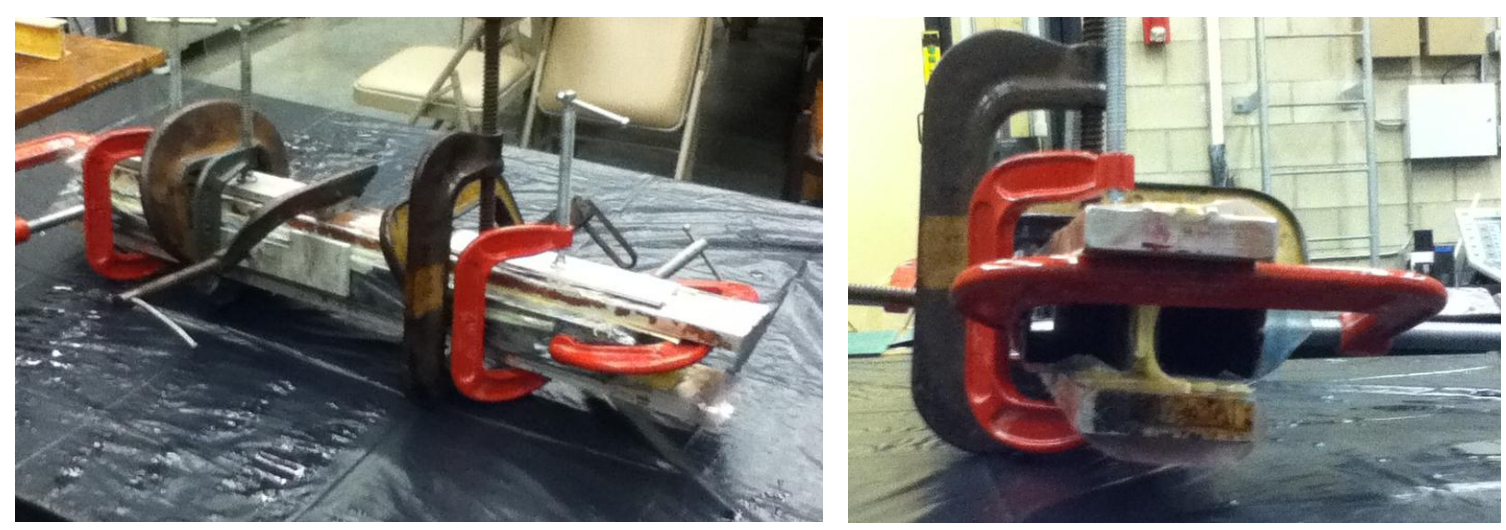

Figure 7-41. Clamping the molds together to create the I-beam

The part is then allowed to cure at room temperature for three days to ensure a fully cured I-beam. After allowing the part to cure for an adequate amount of the time, the clamps and molds are subsequently removed from the I-beam. Using a razor to chip away at the edges of the I-beam's face greatly assists in removing the molds as the curing process often makes them difficult to pry from the part. The razor can then be used to remove the vacuum bag from the molds; the molds are easily pulled from the part using a lever and the bag adhering to the I-beam can be simply pulled away from the part. Figure 7-43 depicts a graphic summary of this process for clarity. 

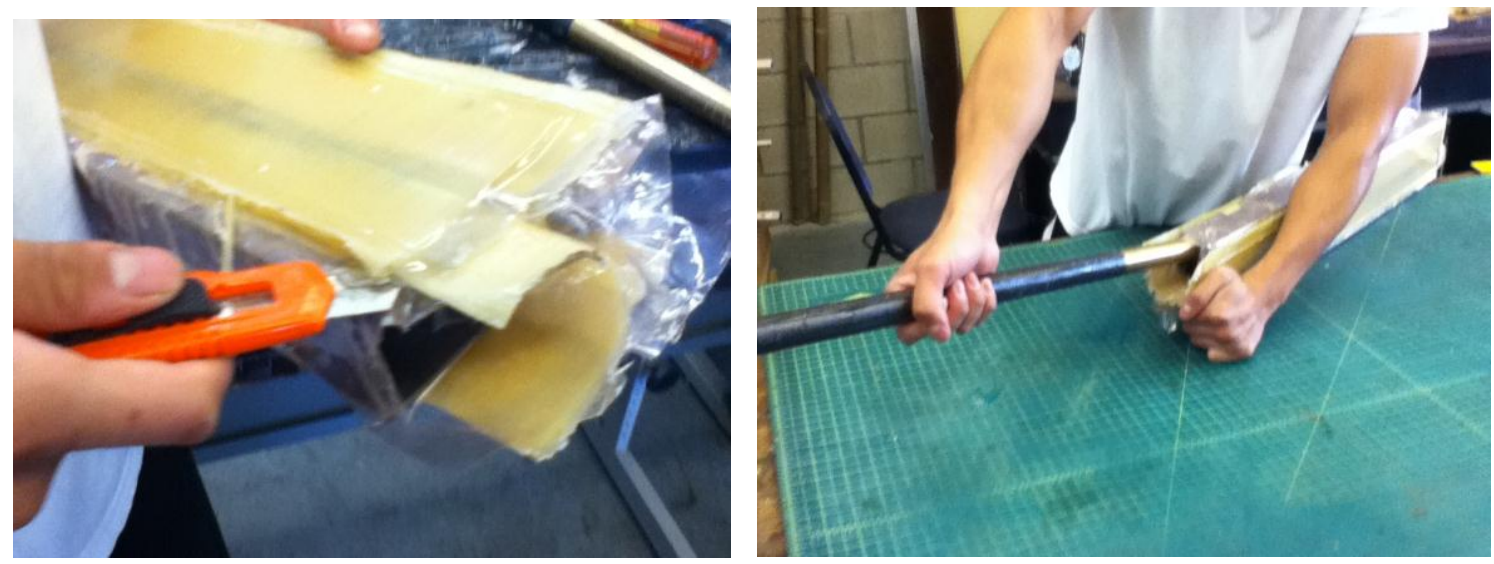

Figure 7-43. Removing the molds from the I-beam

Once the molds are removed from the I-beam, the part is cut to the appropriate flange width dimensions. This task is performed by using a tile saw to cut the excess flange material after marking the Ibeam to the correct width.
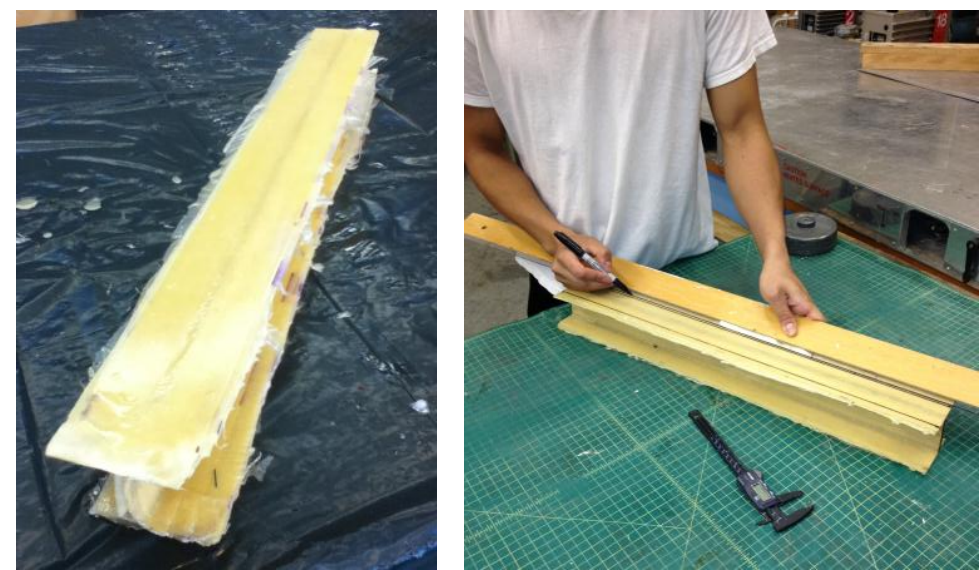

Figure 7-42. I-beam after taking off the molds (left) and marking the beam for cutting (right)

The dimensions illustrated in Figure 7-31 provides the information for correctly cutting the I-beam to size. The nonstraight edges of the beam produced after cutting is then taken care of by sanding the flanges with a belt sander. Figure 7-44 depicts a graphic
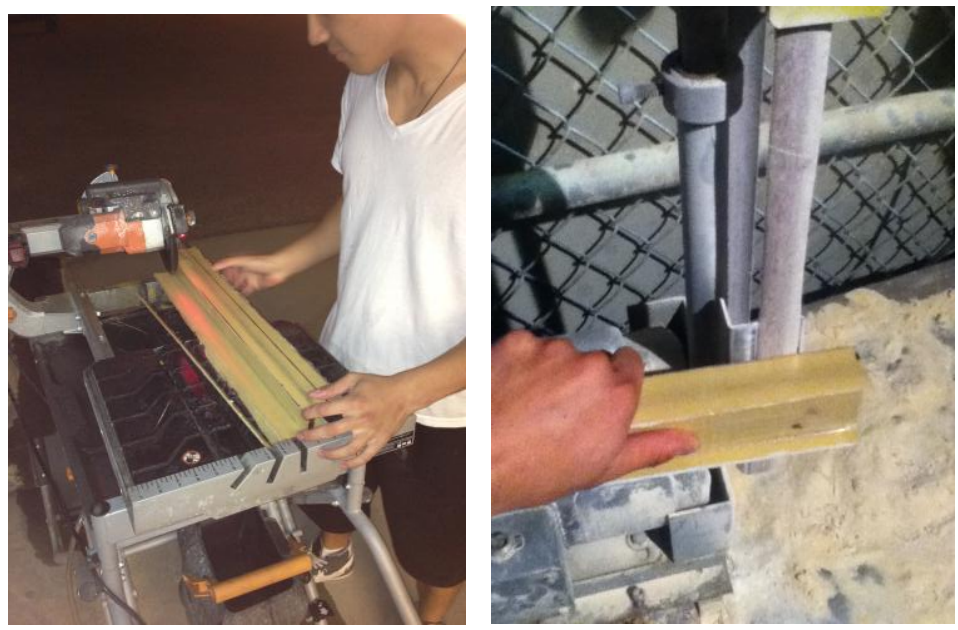

Figure 7-44. Cutting the beam to the appropriate width (left) and sanding the flanges for final detailing 
summary of this process for clarity. The final product of the I-beams as well as their close-up views are shown in Figure 7-45 and the corresponding weights are shown in Table 7-3.
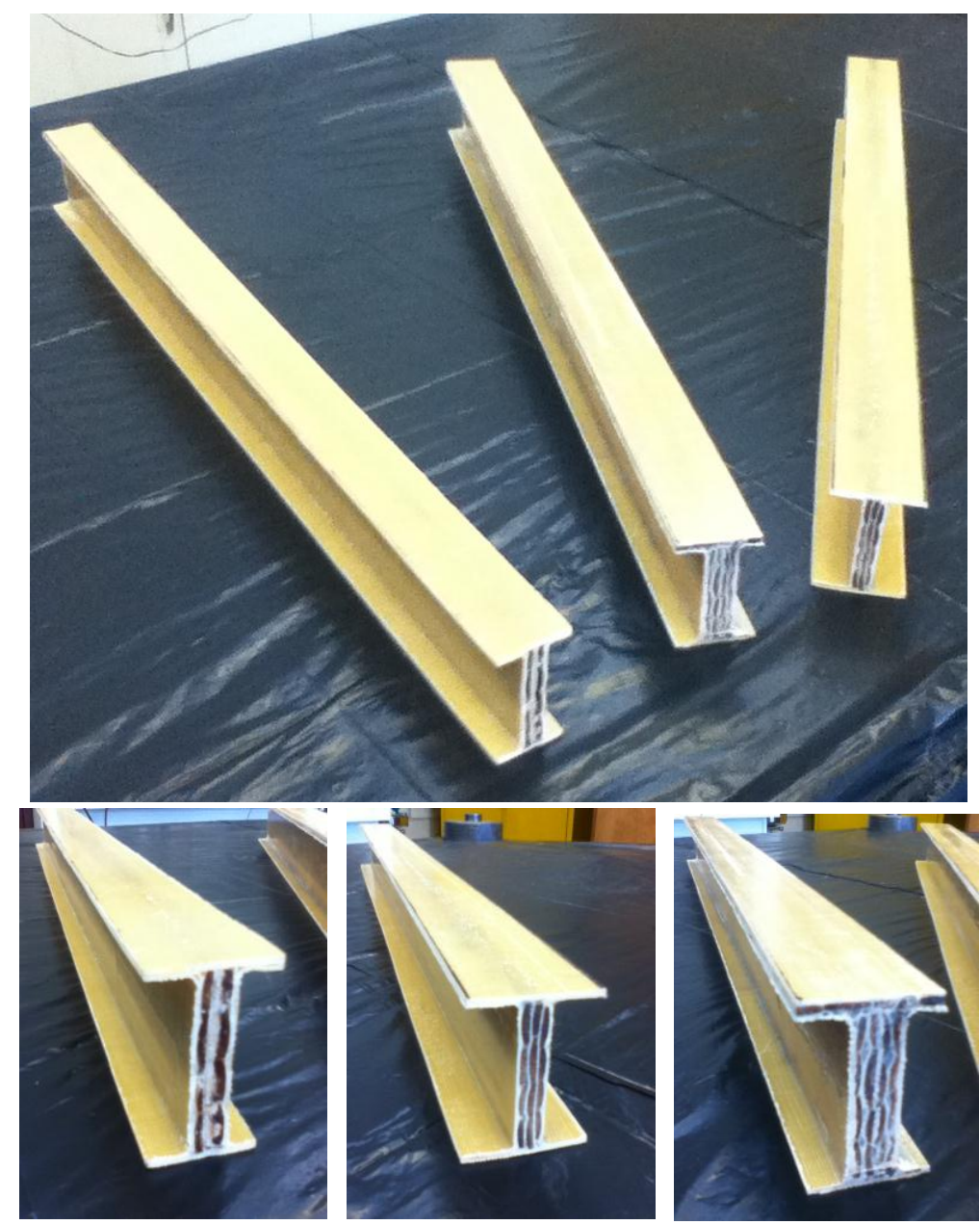

Figure 7-45. Final product of I-beams

Table 7-3. Weight of each I-beam design

\begin{tabular}{|c||c|c|}
\hline Design Number & Characteristic Trait & Weight $\left(\mathrm{lb}_{\mathrm{f}}\right)$ \\
\hline \hline 1 & 2-Ply Bamboo Web Core & 1.13053 \\
\hline 2 & 3-Ply Bamboo Web Core & 1.20990 \\
\hline 3 & $\begin{array}{c}\text { 4-Ply Bamboo Web Core, } \\
\text { Bamboo Flange, \& Hemp Twine }\end{array}$ & 1.58601 \\
\hline
\end{tabular}




\subsubsection{I-beam Testing Procedure}

The main objective of the I-beam test is to determine a failure point under a four-point compressive load with two simple vertical supports at each end. As previously discussed, the test set-up and procedure follows the considered regulations set by the contest rules. As a reminder, the maximum load is taken to be the primary criterion for performance evaluation of the test. The maximum load is defined as the minimum compressive ultimate load, the load at a 1-in. deflection, or the maximum design load of $25000-\mathrm{lb}_{\mathrm{f}}$. The beam is loaded under a four-point bend configuration with a specialized fixture for the support span required for the test; the use of braces against the loading fixture is strictly prohibited. A distributed load through a four-point bending fixture is applied midway across the length of the beam. The Instron machine is once again used to conduct the test. An illustration of the test set-up is shown in Figure 7-46 and displays the free-body diagram of the I-beam under the correct loading condition.

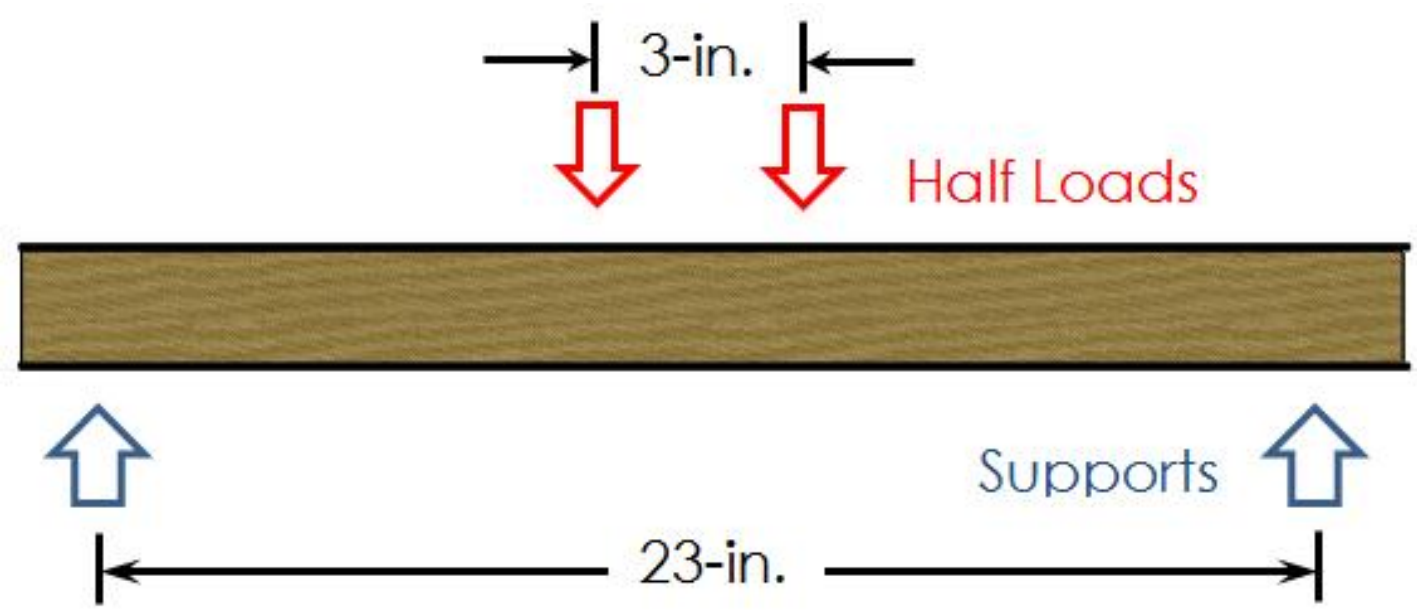

Figure 7-46. Free-body diagram of the I-beam under the loading case

Initial set-up for the test begins by installing the correct bending test fixtures. Once the fixtures are installed, the correct support span and loading span of each fixture is adjusted. The support span marked by the length in Figure 7-46 is designated to be 23- 
in. The loading span illustrated by the half loads in the figure spans three inches, so the correct length is adjusted on the top fixture. The subsequent step is placing the I-beam on the support fixtures and making sure the center axis of the beam is aligned with the

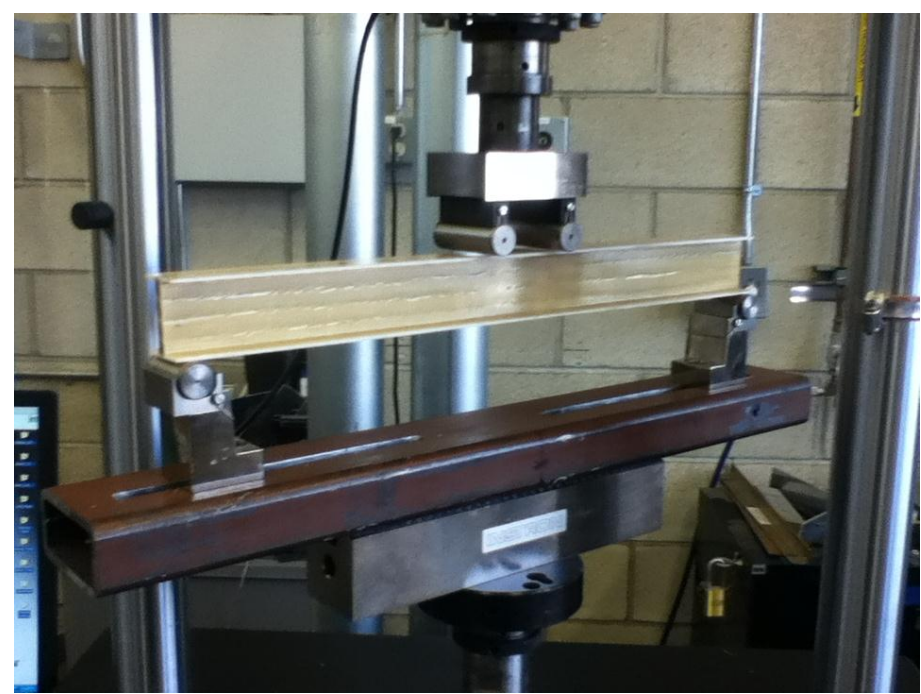

Figure 7-47. Test set-up for bamboo-hemp I-beam

centerline of the support span. Once positioned correctly, the loading fixture is lowered until contact is made with the top flange of the I-beam-a $1-\mathrm{Ib}_{\mathrm{f}}$ reading from the loading cell output in the Bluehill 2 software assures contact. Subsequently, the loading rate is set in the Bluehill 2 software to be 0.10 -in./min. and the test may begin.

\subsubsection{Results of the I-beam Test}

The purpose of this test is to observe the practicality of utilizing veneer bamboo fibers for structures such as an I-beam under flexural loading. Also, the flexural behavior of this biocomposite I-beam can lend insight on its use in real applications. The results of each beam's test are shown in Figure 7-48 below. As seen from the figure, these I-beams are capable of enduring notable loads with little deflection. For the first design, the maximum load endured is $1596.9-\mathrm{Ib}_{\mathrm{f}}$ with a corresponding deflection of 0.962 -in. In the second design, the maximum load endured is $2057.4-\mathrm{lb}_{\mathrm{f}}$ with a corresponding deflection of 0.717-in. The third design demonstrates a maximum load endured of $2742.8-\mathrm{Ib}_{\mathrm{f}}$ with a corresponding deflection of 0.759 -in. As observed from the figure, the stiffest and strongest design is number three. The first design performs the least well with larger flexural displacement than other I-beams, and the second design has moderate 
performance in relation to the others. The strength-to-weight ratio of each I-beam is supplied in Table 7-4 to properly compare the performance of each design. Again, the third design performs the best in terms of strength.

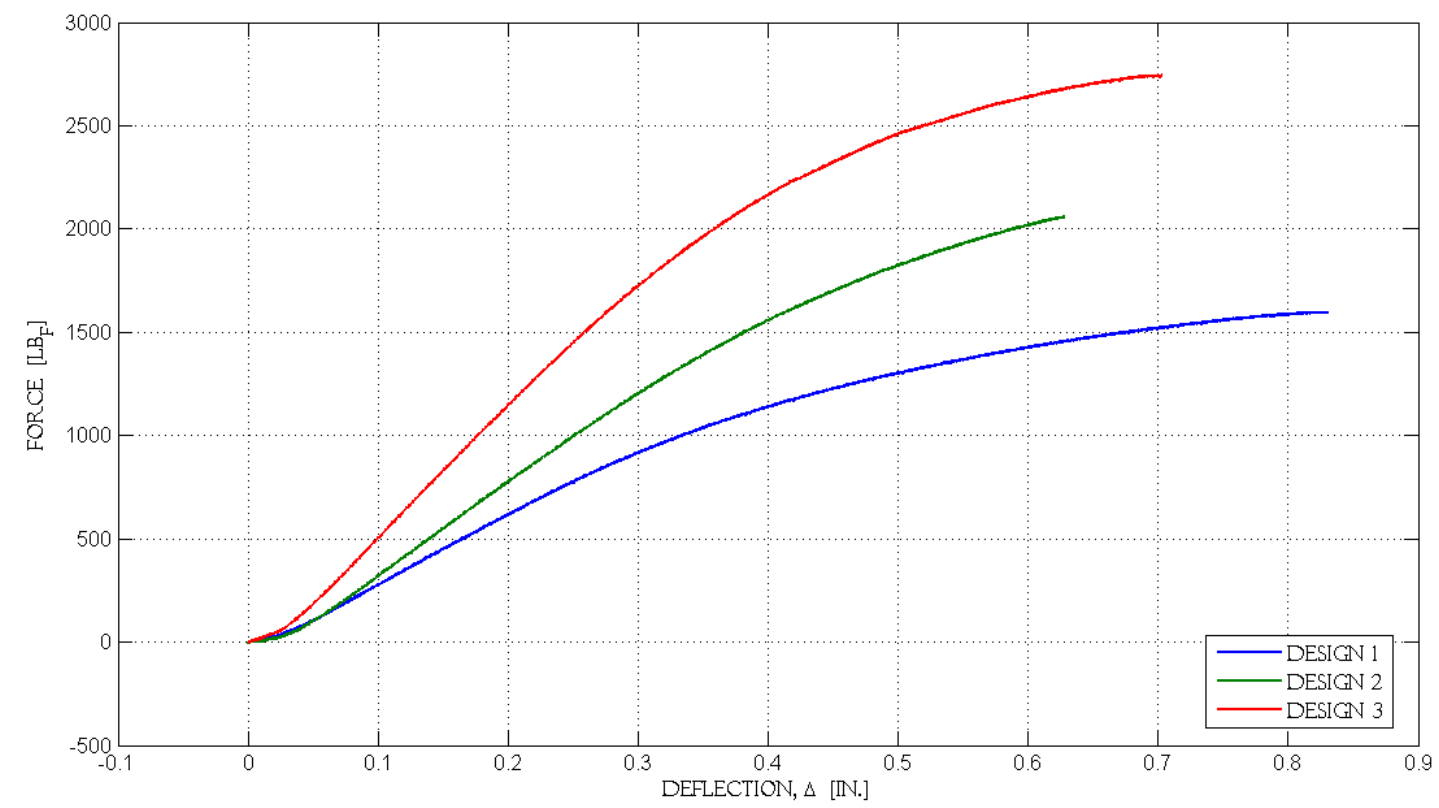

Figure 7-48. Load-deflection curves of I-beam designs

To illustrate the failure of the first design, Figure $7-49$ is shown to indicate the critical zones on the structure. As seen from the figure, little plastic deformation developed on the beam although this I-beam had the most flexural displacement among the three. Most of the deformation is seen in the top flange at the load point locations. Also, the beam developed an irreversible bend in the center, but this deformation is quite small. Most importantly, it is observed at the end of the test that the bamboo plies remained intact—suggesting the beam can withstand higher loads. 

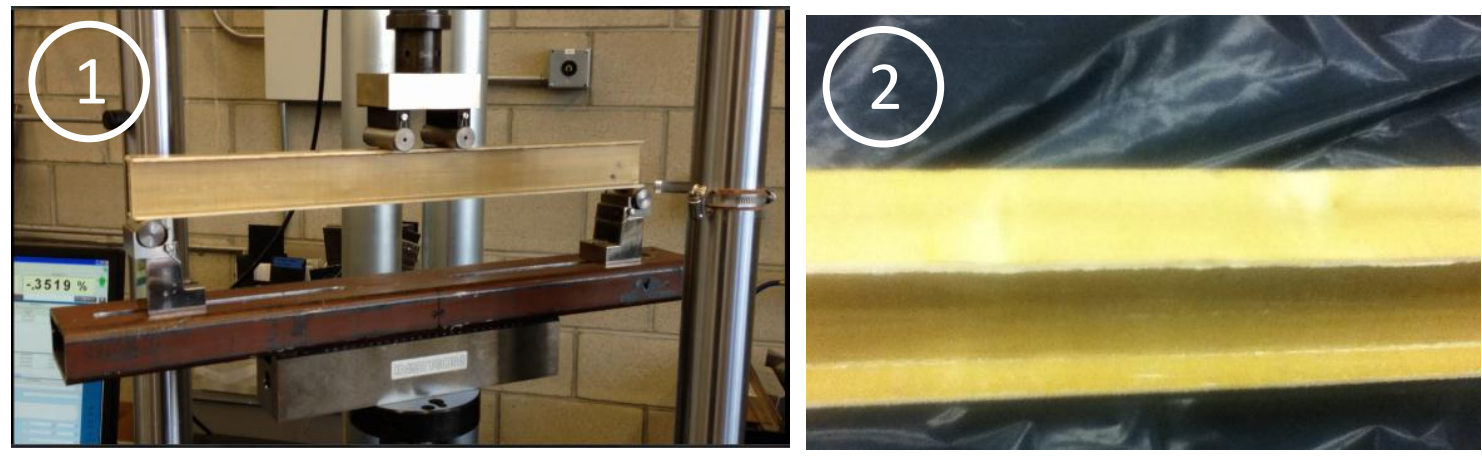

Figure 7-49. Instants up until failure for design 1: (1. left) pre-failure state with minor deflection (2. right) conclusion of test reveals little plastic deformation in the central region of the I-beam

The critical failure location of the second design is shown in Figure 7-50. The first image on the upper left corner of the figure illustrates the state of the beam moments prior to initial hemp ply delamination and crack propagation. Once the beam neared its ultimate load, hemp ply delamination occurred and is seen by the small white circle developing at the intersection of the bottom flange and web. Within milliseconds of hemp delamination, a crack formed in the bottom portion of the I-beam as seen from the lower left corner of the figure. Crack propagation ensued within milliseconds and ended in the center region
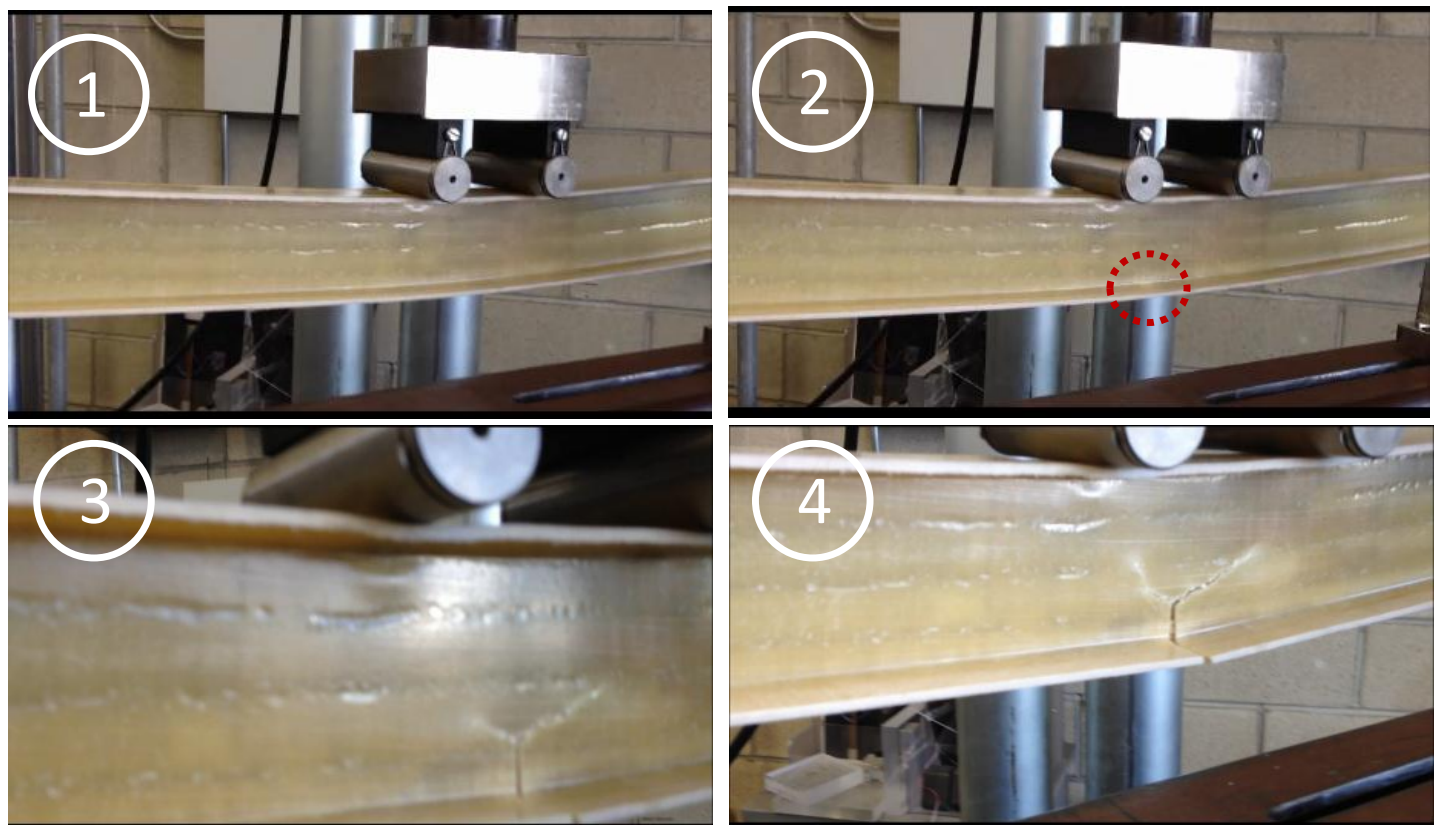

Figure 7-50. Instants up until failure for design 2: (1. upper left) pre-failure state without crack initiation (2. upper right) minor ply delamination from the core begins (3. lower left) crack propagation (4. lower right) final failure state 
of the web. It is important to note, that the bamboo plies in the web also displayed no plastic deformation at the conclusion of the test. Again, this observation suggests that the failure of the beam is largely due to the hemp fabric plies and not the bamboo core.

The resulting plastic deformation of the third design is shown in Figure 7-51. As mentioned earlier, this I-beam performed with the most stiffness, so the upper left portion of the figure displays the minor deflection of the beam. The first signs of plastic deformation are shown in the upper right portion the curve. At the onset of plastic deformation, the hemp fabric slightly delaminates from the core at the top flange where the two load points exist. As the test continued and the load increased, the bottom flange finally failed by a delamination between the U-channels and the bamboo ply in the flange. The primary reason for delamination is that the waxy outer wall of the bamboo strips makes it difficult for other constituent materials to adhere to the bamboo ply. After the ply delaminated from the core, the U-channel plies failed under tension as seen from
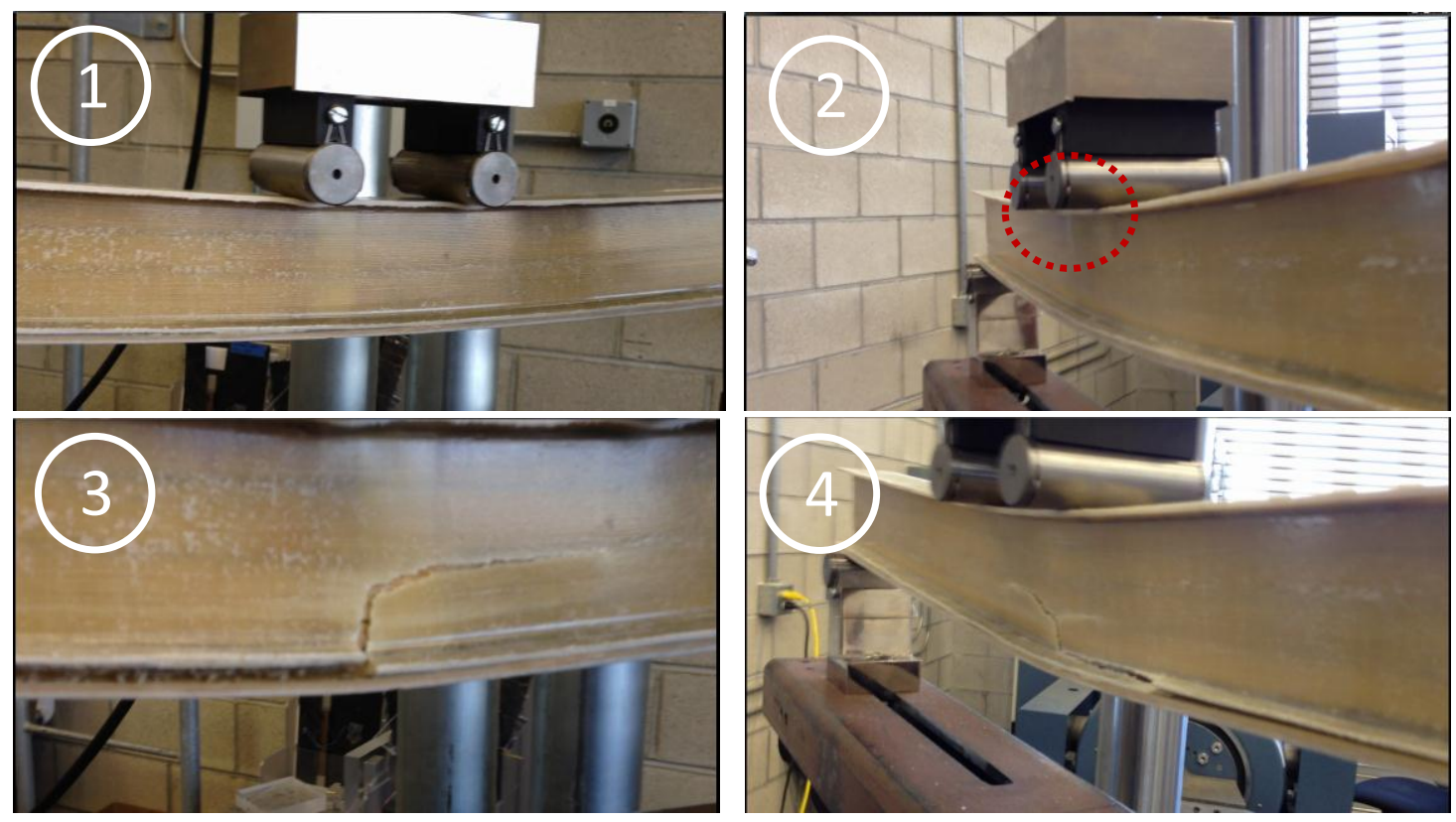

Figure 7-51. Instants up until failure for design 3: (1. upper left) pre-failure state without crack initiation (2. upper right) ply delamination near load points (3. lower left) bamboo ply delamination from web and crack propagation on one side (4. lower right) bamboo ply delamination and crack propagation on other side 
the crack formation on the bottom flange. The two photographs in the lower portion of the figure display the crack propagation toward the center of the web on both sides of the I-beam. Further inspection also showed that the bamboo ply did not fail after delaminating from the core-indicating the structure can withstand more load with better preparation. It is interesting to note that the crack geometry differs on both sides of the beam. As shown in the lower right region of the figure, the crack propagates along the corner of the flange and web for a considerable distance before moving toward the center of the beam. In contrast, the lower right portion of the figure illustrates the crack propagating immediately through the flange and up toward the center of the web. These two observations indicate that a better bond existed on one side between the bamboo ply in the flange and the U-channel.

Table 7-4. Strength-to-weight ratio of I-beam designs

\begin{tabular}{|c||c||c|c|}
\hline Design Number & Characteristic & Ultimate Load $\left(\mathrm{lb}_{\mathrm{f}}\right)$ & $\begin{array}{c}\text { Strength-to-Weight } \\
\text { Ratio (-) }\end{array}$ \\
\hline 1 & $\begin{array}{c}\text { 2-Ply Bamboo Web } \\
\text { Core }\end{array}$ & $1,596.9$ & 1412.6 \\
\hline 2 & $\begin{array}{c}\text { 3-Ply Bamboo Web } \\
\text { Core }\end{array}$ & $2,057.4$ & 1700.4 \\
\hline 3 & $\begin{array}{c}\text { 4-Ply Bamboo Web } \\
\text { Core, Bamboo } \\
\text { Flange, and Hemp } \\
\text { Twine }\end{array}$ & $2,742.8$ & 1729.4 \\
\hline
\end{tabular}

Comprehensive analysis of the testing results indicates utilizing the third design provides the best performance among the three in terms of stiffness and strength. The ultimate load applied to these I-beams indicates that this design has an exemplary strength-toweight ratio under this type of loading condition; their values are shown in Table 7-4. 
Among the three, the second design improves upon the first by $20.4 \%$ while the third demonstrates an improvement of $22.4 \%$. Previous tests for similar I-beams also demonstrate exceptional performance-winners of the last competition reached ultimate loads of $2570-\mathrm{Ib}_{\mathrm{f}}[63]$. In this respect, the third design improves on past I-beams by surpassing this ultimate load. Further improvement can be achieved by adding more hemp plies to the U-channel section since this region consistently showed the most failure-prone areas.

\subsubsection{Finite Element Method to Validate I-beam Performance Results from}

\section{Experiment}

A numerical method is once again employed through Abaqus/CAE to validate the experimental results obtained from the test performed on the bamboo biocomposite Ibeam. Again, the implementation of finite element analysis on this structure is utilized to demonstrate a scheme to adequately model this structure and to efficiently design similar structures for the future. For the conclusion of this study, a comparison is provided between the numerical analysis results and experimental results. In addition, the finite element analysis will also highlight any unexpected discrepancies that occurred during the test of the actual I-beam. Displacement and stress field plots are provided to illustrate the local behavior on the structure. As outlined previously, the procedure of the analysis consists of: identifying the model's geometry, defining the material properties, specifying the interaction between any individual parts in the model, assigning the boundary/load conditions, and finally generating the mesh to represent the model. A quadratic implicit solver is utilized to analyze the model under a four-point bend loading condition at the center of the beam and two simple roller supports at the ends. 


\subsubsection{Numerical Analysis Methodology for Bamboo I-beam}

Before beginning the analysis, a CAD model is created of the actual I-beam structure to properly simulate the test. The dimensions of the hemp plies, bamboo plies, and overall dimensions of the beam are defined in the model to the tolerance measured during the manufacturing process. The CAD model developed for this analysis is created in the CAE pre-processor of Abaqus. This analysis is a demonstration for replicating the test conditions for either design under the same loading conditions, so only Design 2 is analyzed in this study. Although the other designs incorporate different features into their structure, these additions can easily be defined in the model properties with the example shown in this analysis.

First, the geometry of the truss model must be in accordance with the actual structure. The general dimensions are modeled in the Abaqus pre-processor software along with the mechanical properties of the bamboo and hemp. Figure 7-52 illustrates the transformation of the real I-beam to the model created in Abaqus. It can be noted that the model is constructed using planar surfaces for the flanges and web. For a composite

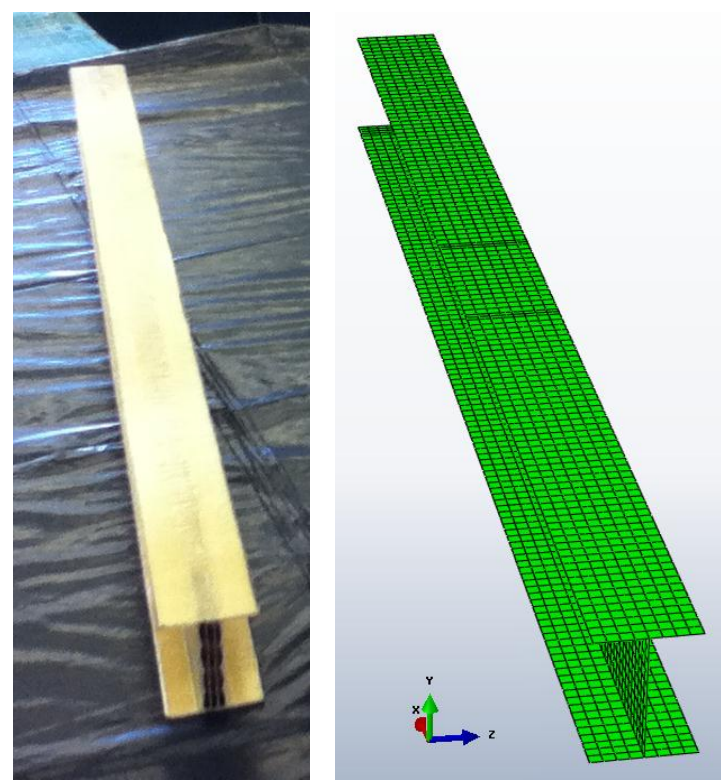

Figure 7-52. Abaqus/CAE model based on I-beam design 2

structure, this type of geometric model is appropriate for the analysis sought in this study. Certain features, however, such as the node length and thickness in each strip of bamboo are not modeled. These assumptions do not negatively impact the results of the analysis. 
The model's material properties are defined by the values measured and calculated from the experimental results of the optimum bamboo treatment. Table 7-5 and Table 7-6 are provided to supply the respective material properties for the bamboo strips utilized in the web core and the hemp fabric plies used throughout the structure. The hemp material properties are obtained from Melendez and Muy's study in working with the hemp-based composites [16]. The assumption is made again for the analysis that the bamboo is orthotropic for improved accuracy. The hemp fabric is modeled as a bi-directional weave of cloth with similar material properties in the principal and transverse directions. Consequently, an orthotropic material property model is defined for the analysis. The proper material orientations for the model are defined based on the longitudinal direction of the bamboo fibers. A rectangular coordinate system is utilized in the model to accommodate these material orientations. The principal direction is defined to be parallel to the longitudinal direction of the bamboo fibers.

Table 7-5. Material properties of bamboo strips in web core

\begin{tabular}{|c|c|}
\hline Material Property & Value \\
\hline \hline$E_{11}$ & $1,467,000-p s i$ \\
\hline$E_{22}$ & $8,829-p s i$ \\
\hline$E_{33}$ & $8,829-p s i$ \\
\hline$v_{12}$ & 0.260 \\
\hline$v_{13}$ & 0.052 \\
\hline$v_{23}$ & 0.052 \\
\hline$G_{12}$ & $50,976-p s i$ \\
\hline$G_{13}$ & $50,976-p s i$ \\
\hline$G_{23}$ & $4,196-p s i$ \\
\hline
\end{tabular}

The material orientations of the beam are defined through the composite material property manager, so each ply thickness and orientation is referenced in relation to the principal axes established previously. For the web, a total of nine plies are defined in the lay-up manager where three of the plies consist of bamboo with 0.1 -in. thickness and six 
of hemp with 0.025 -in. thickness. All the plies in this material lay-up are oriented in the principal direction, so each ply is oriented zero degrees. For the flanges, four hemp plies make up the composite in the lay-up manager with the same thickness and orientations mentioned previously. The plies of this material model are also assumed to have perfect bonding at the fiber-matrix interaction level and at the contact area between each face sheet. Once the material model is properly defined, the step size for a general static loading condition is applied in the model. A maximum of 100 increments with a size of $5.0 \times 10^{-5}$ are designated for the step size.

Table 7-6. Material properties of hemp fabric plies

\begin{tabular}{|c|c|}
\hline \multicolumn{1}{|c||}{ Material Property } & Value \\
\hline $\mathrm{E}_{11}$ & $888,465-\mathrm{psi}$ \\
\hline $\mathrm{E}_{22}$ & $888,465-\mathrm{psi}$ \\
\hline $\mathrm{v}_{12}$ & 0.12 \\
\hline $\mathrm{G}_{12}$ & $396,636-\mathrm{psi}$ \\
\hline $\mathrm{G}_{13}$ & $312,011-\mathrm{psi}$ \\
\hline $\mathrm{G}_{23}$ & $396,636-\mathrm{psi}$ \\
\hline
\end{tabular}

The following step in the analysis process is defining the correct boundary and load conditions. First, the experiment is validated in the linear-elastic region of the test by applying a 1000- $\mathrm{Ib}_{f}$ load in the central area of the beam. The total load is divided into half-loads spaced 3-in. apart in the center span of the model. This loading

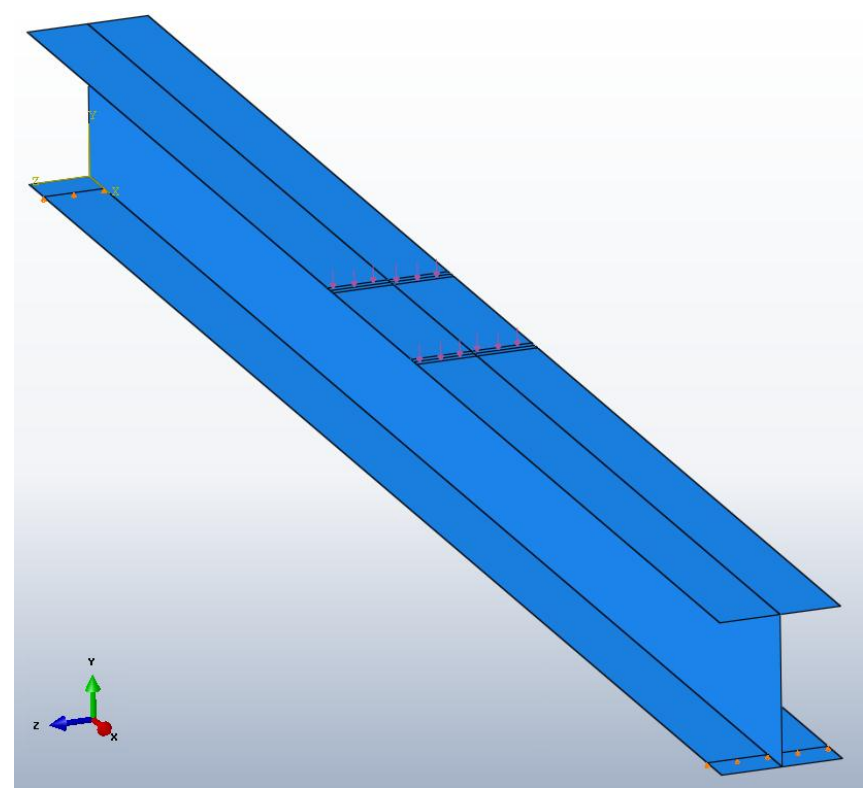

Figure 7-53. Loads and boundary conditions imposed on I-beam model 
condition is then simulated by partitioning the model three inches from the center and selecting this resultant region for the location of the load. Boundary conditions from the experiment are simulated in the model by partitioning a line on the bottom flange 0.5 -in. toward the center from each end edge. The model is then restrained to have zero displacement in the vertical y-direction. Figure 7-53 illustrates the boundary conditions imposed on the beam colored in orange triangles while the loads applied are illustrated in pink arrows. Consequently, the model is free to rotate about the roller support boundary condition during the loading time step—similarly to the actual experiment.

The mesh is subsequently defined after establishing the appropriate boundary conditions. Figure 7-55 shows the mesh generated for the model in Abaqus. The

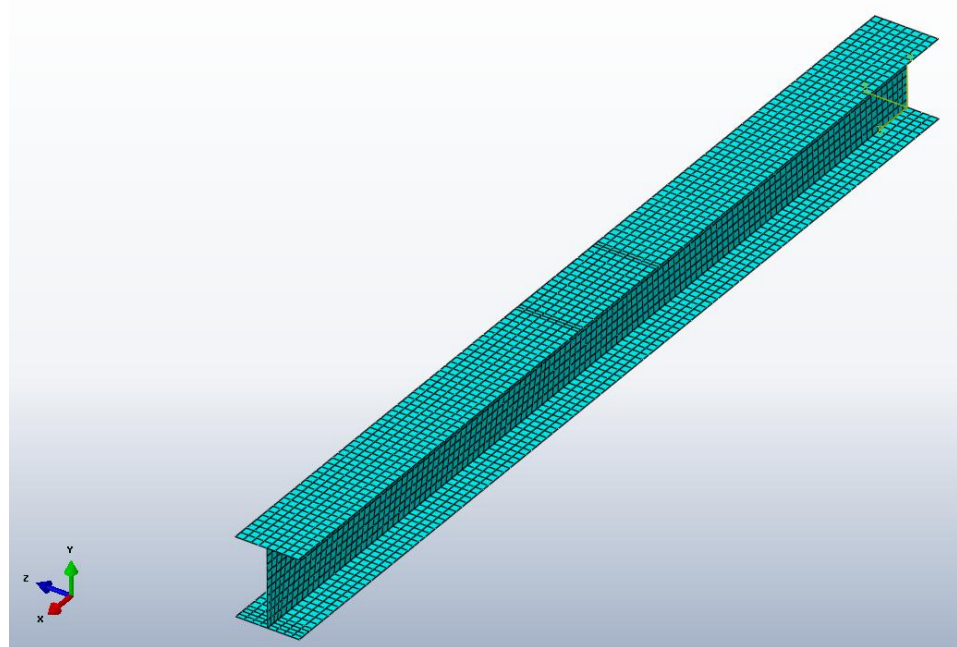

Figure 7-55. Generated mesh of I-beam model

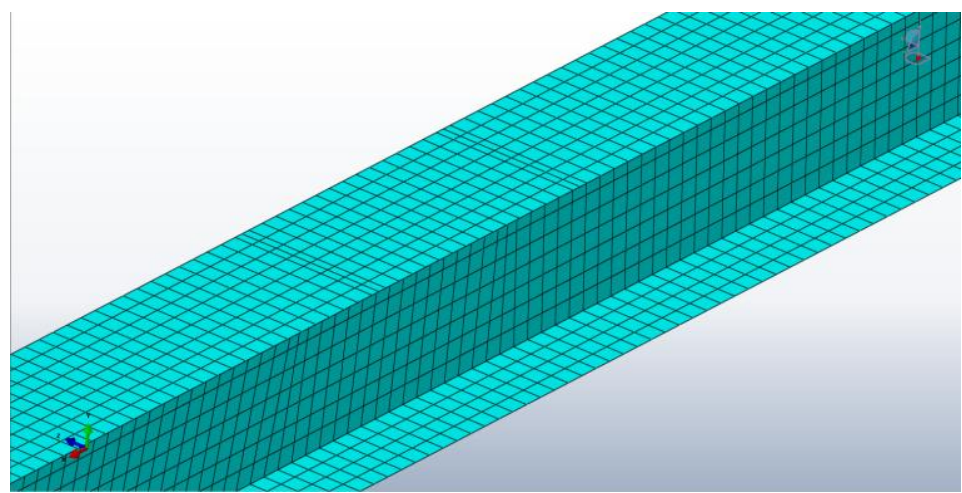

Figure 7-54. Visually enhanced views of critical area in the meshed Ibeam model model's mesh is created with a Structured meshing algorithm technique that minimizes mesh transitions at the intersection points of the web and flanges. As seen from the figure, quadratic shell elements are utilized in the mesh. Figure 7-54 displays a close-up view of the central region of the I-beam where the critical area is expected to be located during loading. The 
simple geometry of the I-beam easily accommodates the use of these elements. The specific element type used is $S 8 R$ which accounts for an 8-node doubly curved thick shell with reduced integration for the analysis—as defined by Abaqus.

\subsubsection{Comparison of Finite Element Analysis and Experimental Results for I-beam}

For the finite element analysis, a series of deformation, stress, and displacement models are generated from the conclusive numerical results. Figure 7-56 illustrates the deformation of the model for the analysis, and the shown deformation scale is set $5: 1$ for visual clarity. As seen from the figure, the deformation in the flange and web are exaggerated greatly to enhance the bending characteristic determined by the analysis. Overall deformations developed in the analysis comply with those observed in the experiment. Note must also be taken in the flexural behavior that the numerical results yield in the analysis. Higher deformations are seen in the flange regions of the structure.
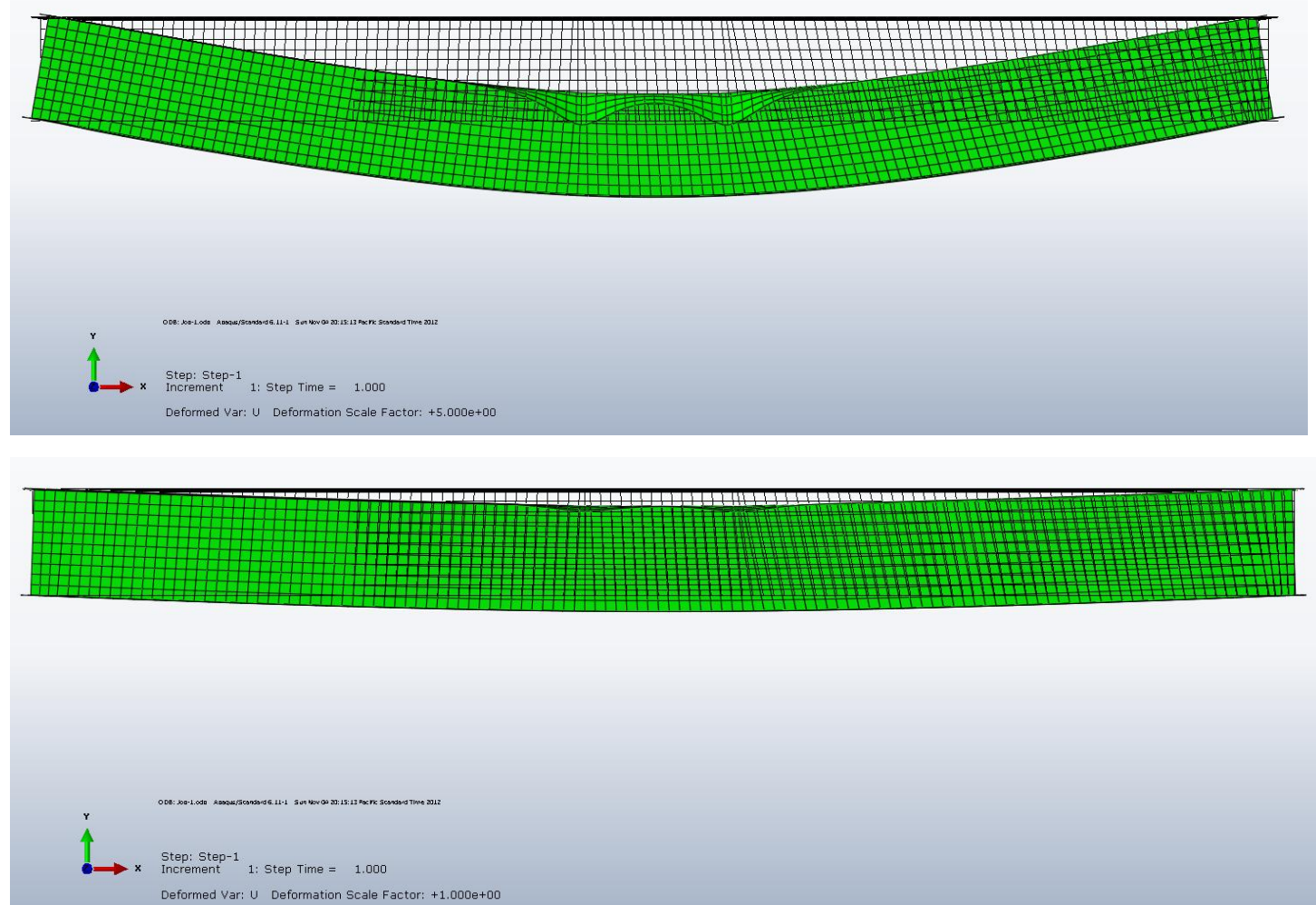

Figure 7-56. Deformation models of I-beam: deformation scale 5:1 (top) and deformation scale 1:1 (bottom) 
The top flange of the structure is observed to have the highest deformation since this encompasses the location of the load points. A close-up view of this critical deformation region is also provided in Figure 7-57-the deformation scale is 1:1. The beam deforms vertically and slightly out of the y-plane. This observation was also noted in the actual experiment, so the resulting deformation reassures the test data. It can be seen in Figure 7-57 that the out-of-plane deformation occurs near the center of the beam where the loads are applied.

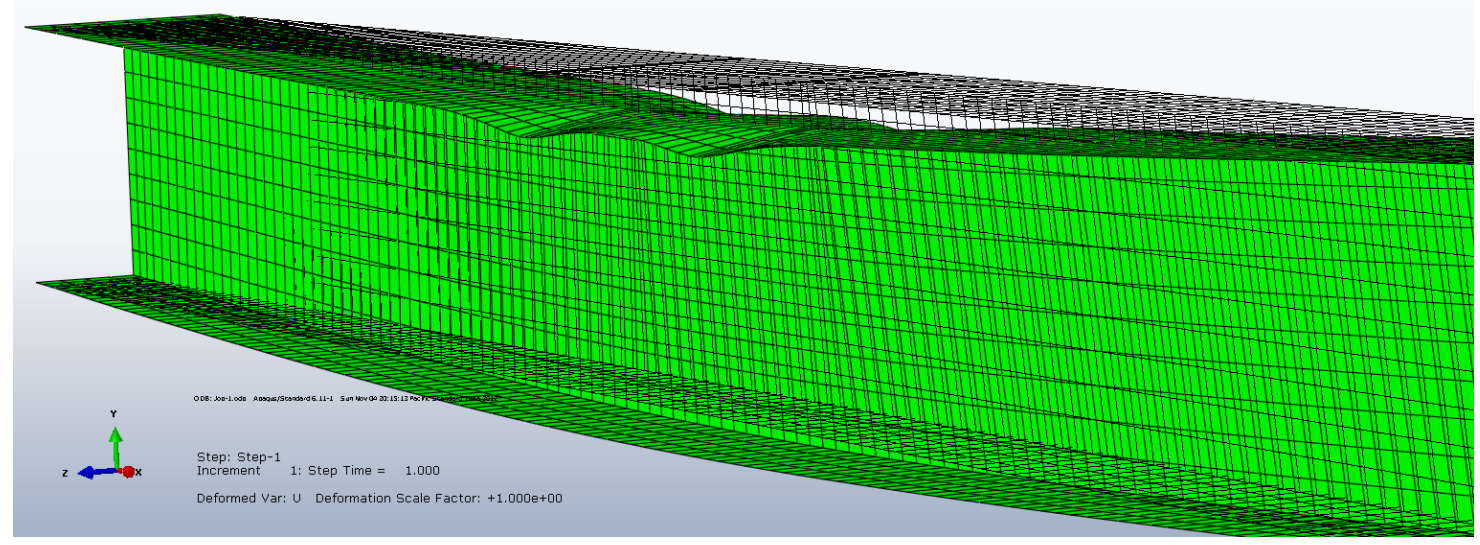

Figure 7-57. Enhanced view of I-beam deformation at critical area with deformation scale of 1:1

Figure 7-58 shows the stress model of the I-beam from the analysis. The provided figures illustrate the estimated stresses throughout the structure of the I-beam under flexural loading. As seen from the Von Mises stress figure, the highest stresses are located in the top flange region of the bamboo-reinforced biocomposite I-beam. It can also be seen from the figure that the highly stressed zones of the top flange occur in the center where the bamboo core lies below the load points. The analysis reveals that the core withstands much of the load, and the flange disperses the stress throughout the surrounding region. Moderately low stresses are also evident in the bottom central portion of the beam. Specifically, the intersection point of the web and flanges are prone 
to greater stresses. The stress field on the bottom flange in the center indicates a uniform region of stress that extends beyond the three inch span of the half loads. The areas near the boundary conditions, as shown in Figure 7-59, also displays fairly low stress levels as the region near the roller support is incredibly small. Such a stress field suggests that the ends of the I-beam can be tapered in order to optimize the structure's weight.

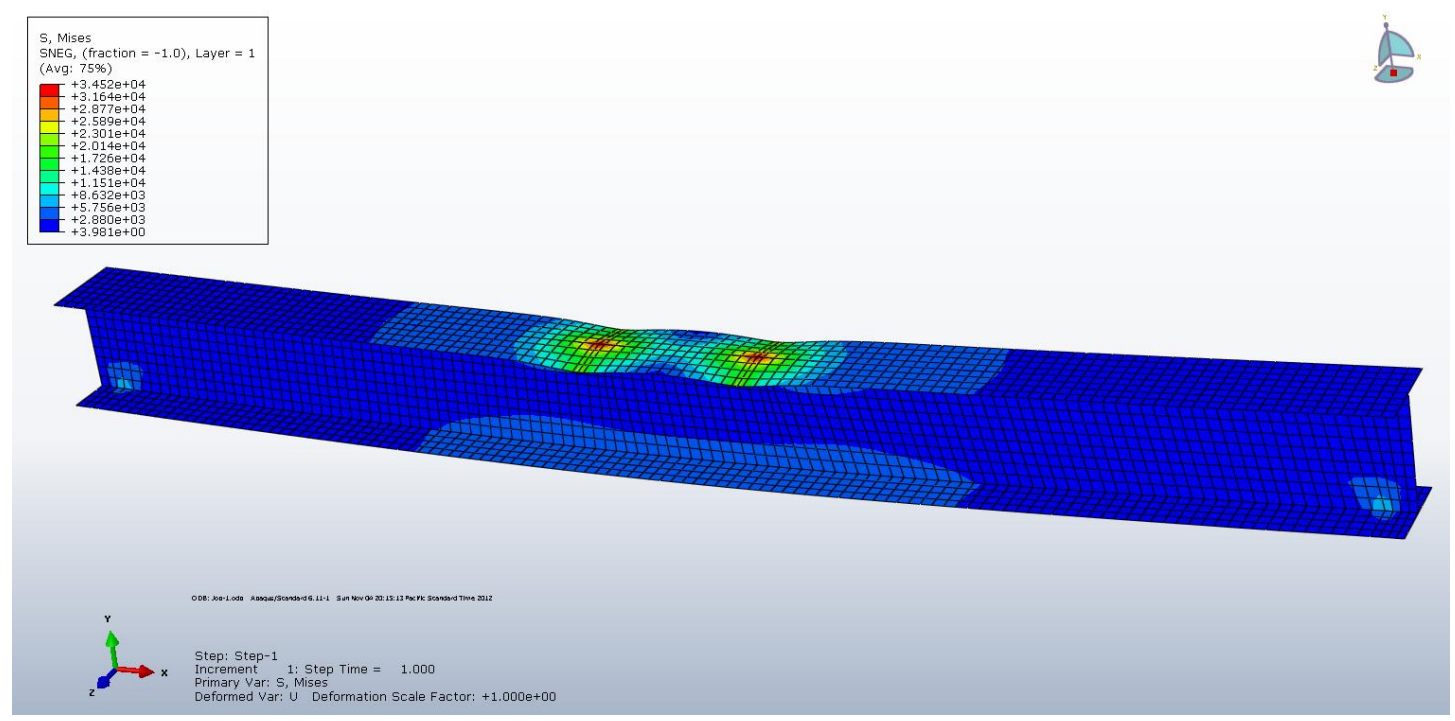

Figure 7-58. Overall stress field from numerical analysis of bamboo-reinforced biocomposite I-beam

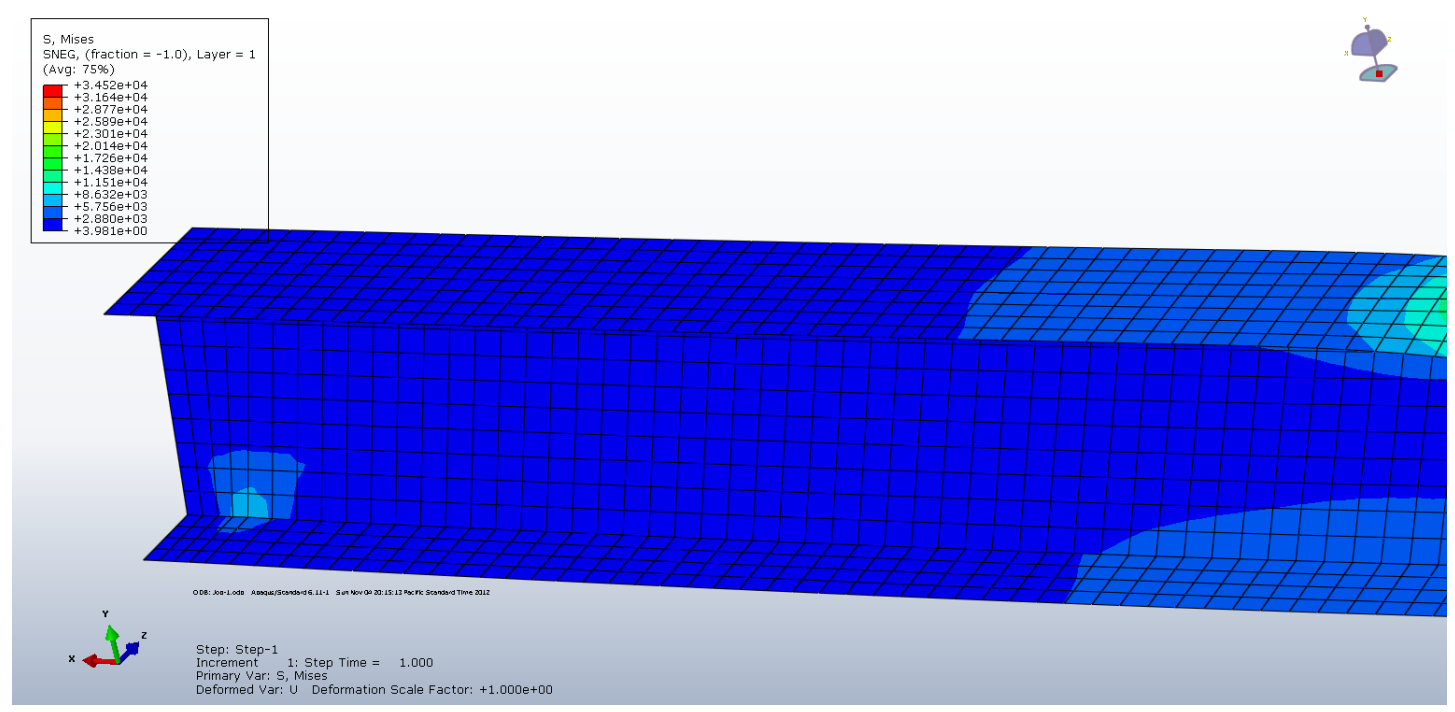

Figure 7-59. Enhanced views of the stress field in the region near boundary condition (right) 
The displacement of the I-beam model caused by the applied load is shown in Figure 7-60. The deflection in the vertical direction is shown in order to compare the results of the analysis to the test. The result of the displacement model agrees well with the deformation observed in the lab experiment. As seen from the figure, the greatest displacement is found in the central area of the flange where the load points are located. It must be noted that the region in between the load points deflects the most over the rest of the structure. Regions marked in dark blue have deformations also apparent in the actual structure. The deflection in the remaining areas follows a uniform path across the length of the beam. This trend is also observed in the actual I-beam of the experiment.

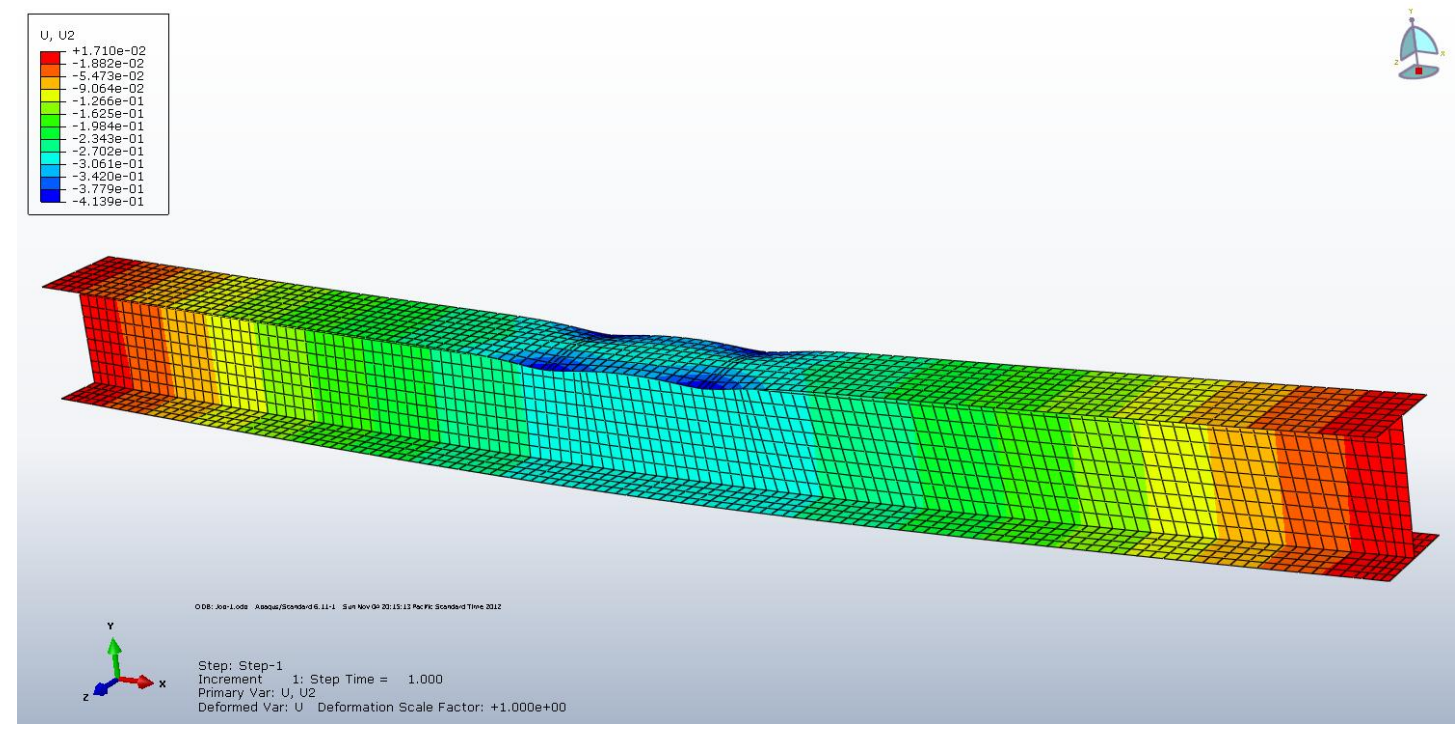

Figure 7-60. Vertical deflection field of bamboo-reinforced biocomposite I-beam

A comparison of the numerical and experimental results is shown in Table 7-7. It is important to note that the Instron machine measures the displacement change referenced from the starting position of the fixture prior to load application. Once again, only the displacement in relation to the vertical axis is shown for comparing both results. The Instron testing machine measured the vertical deflection in the experiment, so it is 
appropriate to simulate this type of displacement in the model. The table displays the maximum displacement of the area directly below the load points, so the nodes lying within the region of the load points are probed in the numerical analysis to illustrate the displacement in the given table. The error obtained from the numerical analysis in relation to the experimental results is calculated to be $13.7 \%$.

Table 7-7. Comparison of experimental and numerical results of I-beam

\begin{tabular}{|c|c|}
\hline \multirow{2}{*}{$\begin{array}{c}\text { Bamboo-Hemp l-beam } \\
\text { Analysis }\end{array}$} & $\begin{array}{c}\text { Static } 1000-\mathrm{lb}_{\mathrm{f}} \text { vertical force on top central region of } \\
\text { simply supported I-beam }\end{array}$ \\
\hline & Displacement in center (in.) \\
\hline Experimental & 0.2552 \\
\hline Numerical & 0.2901 \\
\hline Error (\%) & 13.7 \\
\hline
\end{tabular}

It is important to state that this analysis does not take into account any biological defects that may be present in the selection of bamboo utilized for the I-beam structures. Also, this analysis assumes perfectly rectangular plies in the core without voids. Cutting bamboo strips into perfect segments that fit together is an unrealistic notion considering the natural curvature of the outer wall in the plant material, so it is possible that voids filled with resin could have developed in the cross section of the I-beam. Consequently, the material model would differ from the actual model due to these resin-filled voids. In addition, the material properties of the hemp fabric may also be erroneous as further detail is not available on the manufacturing and test processes of the study. In spite of these shortcomings, the finite element analysis method employed is a suitable manner to predict the flexural behavior of the bamboo-reinforced biocomposite I-beams. 


\subsubsection{Mesh Convergence Study for Bamboo I-beam}

Efficiency is then optimized by conducting a mesh convergence study for the biocomposite I-beam analysis. The purpose of this study is to determine the optimum mesh model for solution convergence on the deflection value obtained previously. The methodology for conducting this study follows the same procedure as that from the truss mesh convergence study. As a reminder, the process consists of starting from a small mesh element density for the model to run the analysis and subsequently increasing the mesh density until the obtained solution reaches convergence. A node in the central top portion of the I-beam is probed for the deflection value at the conclusion of the current analysis in the iteration, and the result is recorded as data point for the convergence study. To begin the next iteration, the global element size is reduced to increase the mesh density of the model and the analysis is run again. This process is continued until a set tolerance of 0.001 -in. is reached in the analysis. Mesh convergence is then reached once this iterative scheme yields a value within the defined tolerance of the
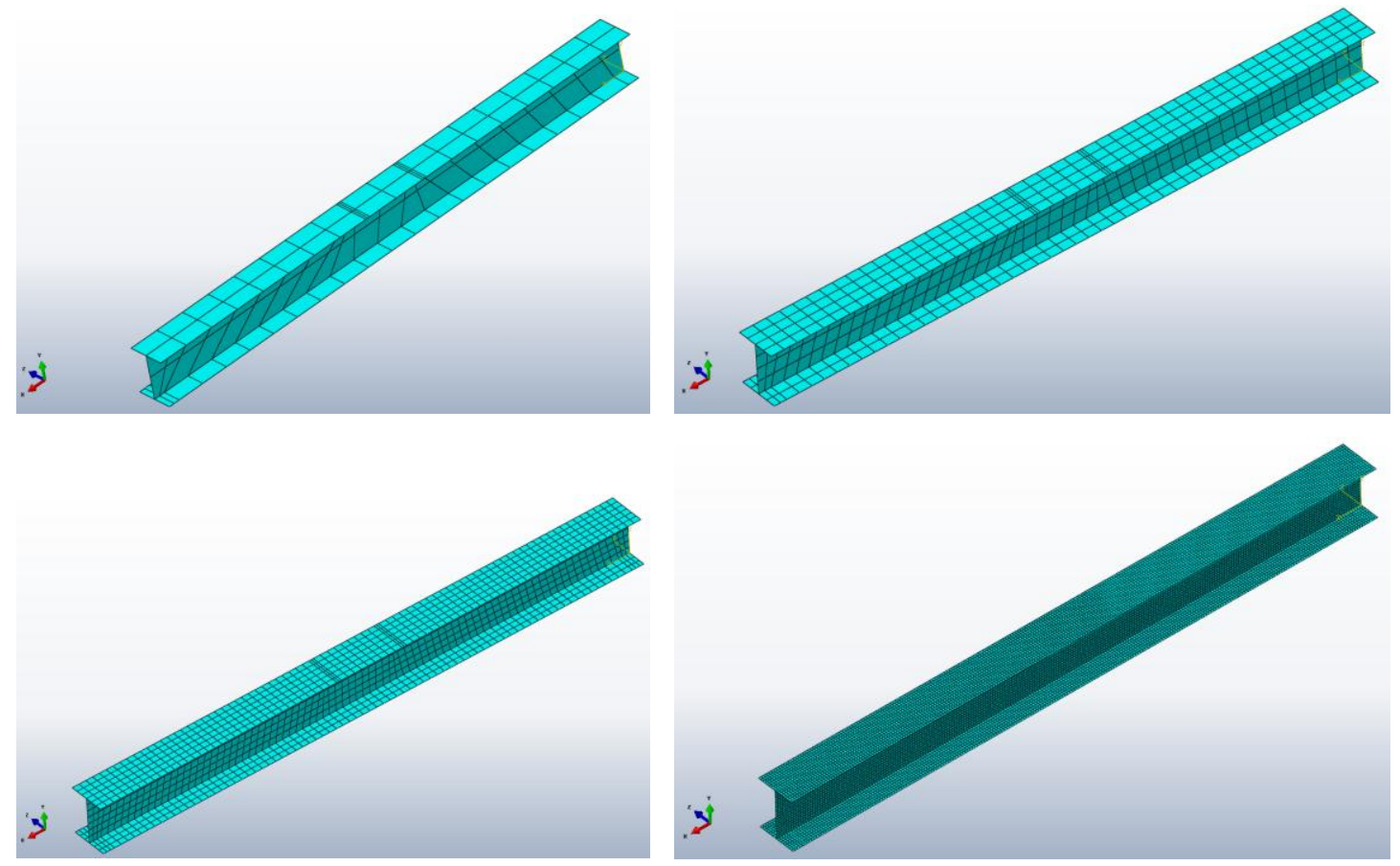

Figure 7-61. Mesh models from the I-beam convergence study: 132 elements (top left), 588 elements (top right), 1577 elements (bottom left), and 49920 (bottom right) 
previous iteration. A few of the meshed models are shown in Figure 7-61. As seen from Figure 7-62, the study indicates that mesh convergence is reached with the model containing 3172 elements. Therefore, the use of 3172 elements is required to analyze the biocomposite I-beam model under a four-point bend loading configuration.

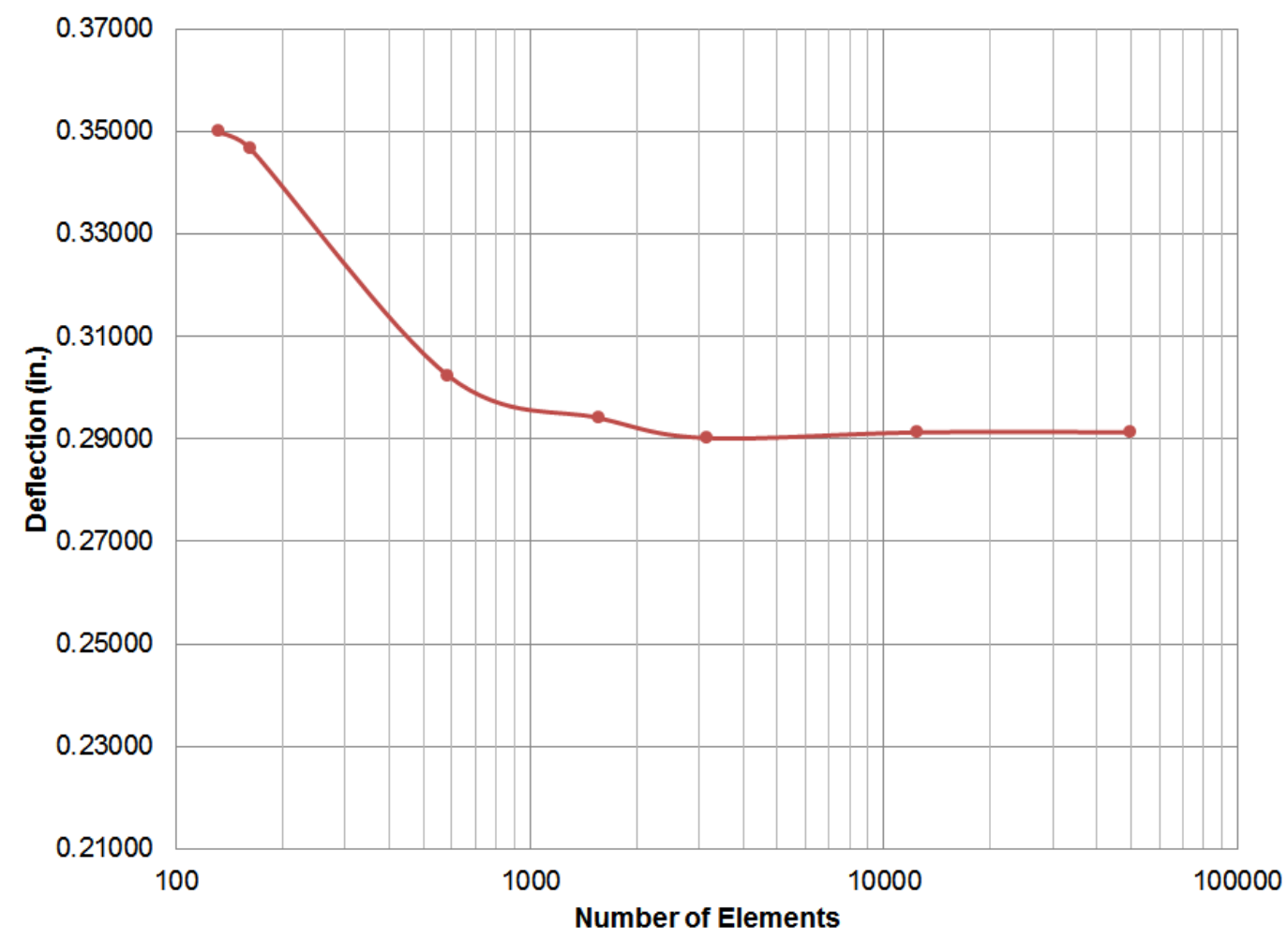

Figure 7-62. Mesh convergence study of bamboo-reinforced biocomposite I-beam

\subsubsection{Conclusion of I-beam Performance and Future Notes}

In summary of the bamboo biocomposite I-beam testing results, the ultimate load and strength-to-weight ratio of all the designs indicate favorable performance under flexural loading conditions, and the numerical scheme employed adequately predicts the behavior observed in the mechanical test. The best performing I-beam is the third design followed by the second and first designs. The load-carrying capabilities of these designs are an improvement over previous iterations. As noted from a previous winner of the 
SAMPE contest, the highest performing I-beam demonstrated an ultimate load of 2570$\mid b_{f}[6]$. The third design surpasses this result by $173-\mathrm{lb}_{\vdash}$-an improvement of $7 \%$. At Cal Poly SLO, previous iterations yield an average strength-to-weight ratio of 1290. These current designs improved upon the previous ones by $25 \%$; comparing the best among the current and previous designs indicates an improvement of $17 \%$.

Although the third design indicates an improvement over the winner of the previous contest, there is still room to improve performance by making changes in the design. A few noted considerations in the design are made by inspecting the deformation of the structure at the end of the test and analyzing the numerical model. Strength and stiffness can be added to the system by introducing more hemp layers in the center portion of the beam. The resultant damage found in the central region of the tested structure and the high stresses observed in this region of the simulated model warrant the implementation of this design change in a future iteration.

Secondly, another ply of bamboo may be added to the bottom flange to minimize stress and deflection. This note is derived from the separation of the bamboo ply from the hemp plies in the flange and web of the tested structure. Thirdly, the weight of the structure may also be optimized by tapering the flanges near the ends of the beam. The results of the numerical analysis indicate that low stresses are found in a small region near the boundary conditions, so this design change sprouts from this observation in the model. Lastly, bonding between each ply face can be improved by determining a proper manner to sand the bamboo strips into rectangular cross sections without ruining the strength of the bamboo. The delamination of the bamboo ply in the flange as inspected in the tested structure attributes to this suggested note. Overall, these structures give insight into the benefits of utilizing bamboo veneers in a biocomposite structure, and future design iterations may be improved by implementing the suggested notes. 


\section{Chapter 8. Conclusion}

In summary of this research, utilizing optimally treated bamboo for structural applications exhibiting flexural behavior demonstrates great promise and has room to improve with further research. As emphasized in the body of this work, innovative solutions are explored in the field of biocomposites for structural applications to address the issue of preserving the world's ecology in a polluting society. The transition from eco-unfriendly manufacturing processes and materials to greener practices and solutions is an eminent movement that will only become exceedingly critical with time. This study indicated that bamboos can be a successful solution for many structural applications-whether construction, automotive, or even aeronautic.

The ultimate goal of this research was to improve the mechanical properties of bamboo for use in biocomposite structural applications under bending loading conditions. A total of seven different treatments were investigated to optimize the material properties of this bamboo. Each treatment was followed by standardized manufacturing and testing procedures recommended by ISO standard 22157. An optimization analysis was performed on the mechanical properties of the bamboo to determine the optimum treatment. The optimum bamboo was then studied under four-point flexural loading to determine the characteristic bending behavior for two different configurations: in its natural cylindrical form and in a prepared veneer form. Once the bending characteristics were determined for the bamboo, two exemplary structural applications were investigated for their flexural performance in a bending test. Finite element methods are then employed to validate the results obtained from the structures' experiments. Finally, the results from the tests were assessed to determine the benefits of utilizing bamboo in biocomposite structures under flexural loading conditions. 
Primary objectives of this research included: obtain the base and resultant mechanical properties after treating the bamboo, conduct an optimization analysis to determine the ideal treatment, establish design guidelines for using the optimum bamboo under flexural loading conditions in the different geometric and node configurations, apply the optimally treated bamboo to two structural applications that exhibit bending to evaluate its usage for potential biocomposite structures, and validate the performance results of the experiments with numerical methods.

The mechanical properties of untreated and treated bamboo were determined for compression and tension loading conditions, so more bamboo biocomposites structures can be designed with this information. The following summarizes the results of these tests.

\section{Compression testing results show:}

- The specific strength was observed to increase by over $100 \%$ across all treatments, although the treatments with $220^{\circ} \mathrm{F}$ thermal treatment were observed to yield the highest average increase. Therefore, utilizing the natural chemical treatmentsparticularly the salt solutions-in conjunction with the $220^{\circ} \mathrm{F}$ thermal cure can benefit from their protective treatments without degrading the compressive strength of bamboo.

- Specific compressive stiffness was observed to increase by over $50 \%$ in relation to untreated bamboo with the inclusion of chemical and thermal treatments. In addition, employing any of the chemical treatments showed absent deteriorating effects to the stiffness of bamboos. 
- Similar to the specific strength, the ultimate and yield stresses were seen to improve with chemical treatment. Thermal treatments, however, above $200^{\circ} \mathrm{F}$ demonstrated increased yield and ultimate strains.

\section{Tension testing results show:}

- Tensile specific strength was observed to increase for all thermal and chemical treatments by an average of $60 \%$. The $3 \%$ salt treatment was noted to have a higher average improvement of approximately $80 \%$ relative to the untreated bamboo. Therefore, utilizing a $220 \mathrm{~F}$ thermal treatment with a $3 \%$ salt solution improves the tensile strength of bamboos in addition to increasing its longevity with the salt treatment.

- An investigation into the specific tensile modulus indicates that stiffness improves by an average of $150 \%$ with thermal and chemical treatments-particularly for the $220^{\circ} \mathrm{F}, 3 \%$ salt, and lime treatments. Consequently, these chemical treatments can provide their protective characteristics without negatively impacting the tensile stiffness of bamboos.

- All treatments demonstrate improved yield stress in comparison to the green untreated bamboo; ultimate stress only increases for a few of the chemical treatments. Lastly, thermal and chemical treatments did not significantly affect the yield strain of the green bamboo but it does considerably lower the ultimate strain.

Analysis on selecting an optimum treatment for the bamboo concludes that the $3 \%$ salt treatment provides the ideal mechanical properties for structural application. This optimization analysis favored bamboo in flexure, but bamboos can be treated differently for other specific design requirements. The properties regarded in the study include the strength and specific modulus in compression and tension. All of these parameters are valued equally to retain an objective understanding of the most well-balanced bamboo 
treatment. Initial data analysis indicates a close match between the $3 \%$ salt and $220^{\circ} \mathrm{F}$ treatments. Further analysis reveals that the optimum treatment is designated to be the $3 \%$ salt treatment due to its resulting effect on the mechanical characteristics and additional benefits of preserving the bamboo for longer life.

A cylindrical and rectangular configuration were investigated under three node locations, and a series of observations from the four-point flexural experiments form the basis for the following design guidelines. By applying these engineering guidelines, the designer can maximize the flexural performance of their bamboo biocomposite design.

\section{Established engineering guidelines for flexural loading conditions:}

- Strength is observed to be higher for both geometric configurations utilizing the internode; the effect, however, on the material's strength is minimal. Therefore, it is recommended for designs utilizing bamboo to place nodes away from the immediate vicinity of an applied flexural load, when permissible, to maximize strength capabilities.

- Stiffness appears to improve when a node is located in the proximity of a load point by $30 \%$ for the cylindrical configuration whereas the veneer strip configuration indicates minimal difference in stiffness. Consequently, it is advised that designs aimed at maximizing stiffness and implementing the natural form of the bamboo have a node located directly underneath an applied flexural load or sufficiently far from it while designs utilizing the veneer strip can disregard the location of the node.

- Yielding strains can be expected to be similar for either configuration, but the veneer strip configuration with a node located near the point load can minimize the ultimate strain. Similarly, the yield and ultimate stresses of this bamboo material under any configuration produces similar performance. 
Optimally treated bamboos in conjunction with hemp fabric and an organic resin system demonstrated feasible structural applications with favorable performance. To exhibit the benefits of utilizing bamboo in structural applications, a biocomposite truss and I-beam were fabricated. The following summary outlines the manufacturing processes of these structures as well as the suggestions proposed for future designs. Although the processes developed for both applications are deemed adequate, better efficiency can be achieved by using better tools to minimize manufacturing times.

\section{Biocomposite truss:}

- The manufacturing process entailed cutting cylindrical bamboo internodes to size and mitering the ends in an appropriate manner. Joint lashings fabricated out of hemp fabric and resin were subsequently wrapped around the truss members with a tight fit. The entire structure was then finally cured under a vacuum bagging process at room temperature.

- Testing consisted of applying a vertical load at the top joint while vertically supporting the truss on both of the bottom joints. Results yielded an ultimate load of $15761-\mathrm{lb}_{\mathrm{f}}$ with a corresponding deflection of 0.4696 -in. The strength-to-weight ratio of this structure is determined to be 7067 .

- Overall, the design shows great promise for biocomposite structures in high performance applications. The design may be improved to withstand greater loads by adding hemp plies to the top joint and consequently reducing the hemp plies around the lower central joint.

\section{Biocomposite I-beam:}


- The manufacturing process encompassed joining organic resin, bamboo veneer strips, and hemp fabric with the use of tightly clamped molds for a room-temperature cure.

- Testing consisted of applying two vertical half loads at the center of the beam under a four-point bending configuration. The ultimate loads of the first, second, and third designs yielded values of $1570-\mathrm{lb}_{\mathrm{f}}, 2057-\mathrm{lb}_{\mathrm{f}}$, and $2743-\mathrm{l}_{\mathrm{f}}$, respectively. The corresponding deflections were measured to be 0.962 -in., 0.717 -in., and 0.759 -in., respectively. The measured strength-to-weight ratios of the three designs are 1412.6, 1700.4, and 1729.4. An improvement of $25 \%$ is observed over previous iterations at Cal Poly SLO, and the design surpasses the winners of the last SAMPE competition by $173-\mathrm{Ib}_{\mathrm{f}}$.

- Overall, the design shows great promise for biocomposite structures in high performance applications. The design may be improved by introducing more hemp plies in the center region of the beam, adding another ply of bamboo in the flange, and devising a way in which to improve the bonding between plies without ruining the strength of the bamboo strip's outer wall.

A finite element analysis was employed to validate the experimental results of the two bamboo biocomposite structures-demonstrating the effectiveness of FEA for design. Both of the numerical analyses demonstrate agreement with results obtained from the tests. The truss application yielded an error of $8 \%$ between the numerical analysis and experimental results. Similarly, the I-beam application yielded an error of $14 \%$ between the numerical analysis and experimental results. Better agreement may be obtained in the analyses by conducting further tests to validate the material properties of the models and constructing the geometric models more closely to the actual structures. 


\section{Chapter 9. Future Work}

The entirety of this work demonstrates that bamboo can be an appealing material in the use of structural biocomposites for certain applications. However, this research only embodies a fragment of the total spectrum of uses for bamboos. In regards to this research, a few things may be additionally investigated for further insight.

First, higher thermal treatment temperatures and dwell times should be investigated to determine other optimum treatments for bamboos. Due to technical problems associated with the autoclave, temperatures above $220^{\circ} \mathrm{F}$ and dwell times longer than 4 hours could not be explored. To determine the true optimum treatment, the cure temperature would be increased until the bamboo reaches thermal degradation at which experimental tests would reveal significantly lower strength. Also longer dwell times at constant temperature can determine whether this practice may improve the strength and stiffness of the bamboo.

Second, further analysis on nodes should be studied with longer specimen spans to additionally validate the findings of this research. In this research, the effects of nodes were studied on the localized level of an applied load. Further investigation can continue on a more global level where much longer specimens with multiple nodes endure a fourpoint bending test. In this manner, additional insight can be gained for the flexural behavior of bamboo in larger structures. The dwindling supply of bamboo available in this research prevented conducting this study, so it is left for another study to explore this issue.

A truss was manufactured in this study for a smaller-scale application, but it would also be beneficial to create a large-scale version to investigate its performance in grander 
structures. Under this setup, more pertinent data can be attained for a life-size model. In addition, an optimization analysis should be performed on the joint methods investigated in this research. Due to time constraints and limited hemp fabric resources, additional joints could not be investigated. Joint design can ultimately determine the effectiveness of this structure; bad joints can prevent the structure from reaching its true performance potential during testing and operation.

Although bamboo is explicitly used in some of its most basic form throughout this study, bamboo laminates should also be studied for biocomposite design. It is known that bamboo culms and strips provide good performance characteristics, but the outermost layer of the bamboo culm is the strongest section. If bamboo unidirectional and bidirectional laminates can be created, biocomposite sandwiches can have reduced weight and higher strengths without the bamboo's extra weak inner layers in the laminate. As a result, this particular study was not investigated since the required machine and time to create these laminates was not available.

Lastly, this research investigated the compression, tension, and bending of this bamboo species, but a thorough investigation on the buckling, impact, and dynamic characteristics is also necessary for other applications. Preliminary research suggests that bamboos generally have good damping and buckling characteristics, but a study on the treatments' effects on these variables is pertinent. Also, considering other species of bamboo would also provide further understanding on the effect of these treatments. Although all bamboos have similar biological composition, it is valuable to confirm the obtained results with other bamboo species. 


\section{References}

[1] "0677-2809P-MTDC: Preservative-Treated Wood and Alternative Products in the Forest Service." 0677-2809P-MTDC: Preservative-Treated Wood and Alternative Products in the Forest Service. United States Forest Service, n.d. Web. 01 Oct. 2012. <http://www.fs.fed.us/t-d/pubs/htmlpubs/htm06772809/page04.htm>.

[2] "100 Bamboo Applications." 100 Bamboo Applications. Bamboo House India, 27 Aug. 2009. Web. 17 Sept. 2012. <http://www.slideshare.net/greenlivelihoods/100bamboo-applications>.

[3] "About Jungle Supply Company." About Jungle Supply Company. Jungle Supply Company, 2008. Web. 17 Sept. 2012.

<http://www.junglesupplyco.com/aboutus.html>.

[4] "Aerospace Metal Distributor." ASM Aerospace Specification Metals Inc. ASM Aerospace Specification Metals Inc., n.d. Web. 01 Oct. 2012.

$<$ http://www.aerospacemetals.com/index.html>.

[5] "Bamboo Grower's Guide." Growing Bamboo. Complete Bamboo, 2012. Web. 16 Sept. 2012. <http://www.completebamboo.com/grow_bamboo.html>.

[6] "Bamboo Growth Rates - The Life Cycle of This Fascinating Grass." Bamboo Growth Rates. Bamboo KI, 30 Aug. 2011. Web. 16 Sept. 2012.

<http://www.bambooki.com/blog/bamboo-growth-rates/>.

[7] "Bamboo Report." Bamboo Village Hawaii. Bamboo Village Hawaii, Feb. 2004. Web. 17 Sept. 2012.

[8] "Ecogreen4us." Bio Composite: Strength, Lightweight and Cheaper Car. Ecogreen4us, 4 Apr. 2012. Web. 15 Sept. 2012. $<$ http://www.ecogreen4us.com/stories/green-technology-stories/compositelightweight-cheaper-car/>.

[9] "Motive Bio-Composite Car." Motive Bio-Composite Car. Motive, n.d. Web. 15 Sept. 2012. <http://www.biocompositecar.com/>.

[10] "Our Environment." The White House. The White House, 2012. Web. 15 Sept. 2012. <http://www.whitehouse.gov/energy/our-environment>.

[11] "Shaku Bamboo Water." Shaku Bamboo Water. Shaku Design, 28 Apr. 2008. Web. 16 Sept. 2012. <http://www.navaching.com/shaku/water.html>.

[12] "Tata Steel Swears CO2 Emission Cuts by 2012." The Economic Times. ET Bureau, 21 Dec. 2009. Web. 14 Sept. 2012. <http://articles.economictimes.indiatimes.com/2009-12-21/news/28434568_1_h-mnerurkar-tata-steel-mr-nerurkar>. 
[13] "What Is My Hardiness Zone?" The Arbor Day Foundation. The Arbor Day Foundation, n.d. Web. 17 Sept. 2012.

$<$ http://www.arborday.org/treeinfo/Zonelnfo.cfm?ZipCode=95658>.

[14] ASTM. "Standard Test Method for Poisson's Ratio at Room Temperature." ASTM (n.d.): n. pag. Print.

[15] Airframe. N.d. Photograph. Airframes, Engines, and Systems -- Part 1. Langley Flying School, 2012. Web. 27 Oct. 2012.

$<$ http://www.langleyflyingschool.com/Pages/Airframes,\%20Engines\%20and\%20Sys tems--Part\%201.html>.

[16] Alma, Melendez, and Muy Terry. A Study on Organic Epoxy and Hemp Composite Plates with an Emphasis on Mechanical and Finite Element Analysis. Tech. San Luis Obispo: California Polytechnic State University, 2012. Print.

[17] Amada, Shigeyasu. Fiber Texture and Mechanical Graded Structure of Bamboo. Tech. N.p.: n.p., n.d. Print.

[18] Amini, Ahmad J. "Static and Fatigue Failure Response of Woven Carbon Fiber Specimens with Double-Edge Notches." Thesis. California Polytechnic State University, San Luis Obispo, 2010. Print.

[19] An F-22 Raptor Shoots out a Flare during an Air Combat Training Mission. N.d. Photograph. An F-22 Raptor Shoots out a Flare during an Air Combat Training Mission. Air-Attack, 12 Jan. 2012. Web. 2 Oct. 2012. <http://www.airattack.com/images/single/842/An-F-22-Raptor-shoots-out-a-flare-during-an-aircombat-training-mission.html>.

[20] Anatomical Features of Bamboo Internode. N.d. Photograph. Bamboo Structure. Luit Nirman. Web. 2 Oct. 2012. <http://www.assambambooworld.com/bamboostructural-material.htm>.

[21] Autoclave. N.d. Photograph. Autoclave. Upper Saddle River: Pearson Education, 2004. N. pag. Print.

[22] Bamboo Anatomy. N.d. Photograph. Bamboo Stem Anatomy. Guadua Bamboo, 2012. Web. 2 Oct. 2012. <http://www.guaduabamboo.com/bamboo-stemanatomy.html>.

[23] Bamboo Node. N.d. Photograph. Flickr. Flickr. Web. 2 Oct. 2012. <http://www.flickr.com/photos/27632962@N04/3493764206>.

[24] Biocomposite Samples Made from Natural Oils, Fibers, and Agricultural Coproducts. N.d. Photograph. Biopolymers and Biocomposites Research Team. lowa State University, 2012. Web. 2 Oct. 2012. <http://www.biocom.iastate.edu/research/biocomposites.html>. 
[25] Biomass Research and Development Act of 2000, United States Code (2000). Print.

[26] Bonide Bonide Hydrated Lime. N.d. Photograph. Sears. Sears, 2012. Web. 3 Oct. 2012. <http://www.sears.com/bonide-hydrated-lime-5-pound-bag/pSPM6446858301P>.

[27] California. California Invasive Plant Council. Cal-IPC: Invasive Plants. N.p., 2012. Web. 26 Oct. 2012. <http://www.calipc.org/landscaping/dpp/planttypes.php?region=state>.

[28] Canola Oil. N.d. Photograph. Canola Oil -- A Well Crafted Scheme. Preparedness Pro, 2012. Web. 2 Oct. 2012. <http://preparednesspro.com/canola-oil-a-wellcrafted-scheme/>.

[29] Compression Characteristics. N.d. IBTR. IBTR, 2012. Web. 3 Oct. 2012. <http://ibtr.jp/Curves-stress-strain-Can-Ime-(4730ml)-p1989.html>.

[30] Carbon Swatch. N.d. Photograph. Composite Boats 101, Part 2: Materials. Pocock Racing Shells, 14 Sept. 2010. Web. 2 Oct. 2012. <http://www.pocock.com/rowingboat-information/composite-racing-shells2/>.

[31] Chalet \& Bamboo. "Treatment of Bamboo - Chalet \& Bamboo." Treatment of Bamboo. Chalet \& Bamboo, 2011. Web. 07 Nov. 2012. <http://www.chaletbamboo.com/treatment.html>.

[32] Composite Beam Bridge. N.d. Photograph. Composite Beam Bridge. Reid Steel, 2012. Web. 27 Oct. 2012. <http://www.steel-bridges.com/highway-bridgecomposite-beam.htm>.

[33] Crawford, Ronald L. Lignin Biodegradation and Transformation. New York: Wiley, 1981. Print.

[34] Diagram Showing Interactions between These Elements in a Plant Cell Wall. N.d. Photograph. Processes and Texture. Eusol. Web. 2 Oct. 2012. <http://www.eusol.net/public/better-food/tomato-texture/behind-the-scenes>.

[35] Farrelly, David. The Book of Bamboo. San Francisco: Sierra Club, 1984. Print.

[36] Formidable Kiln Drying Systems. Perf. Phillipe Dawson. Kiln Drying Wood. Camille Foster and Jacob Wiler, n.d. Web. 02 Oct. 2012. <http://www.finewoodworking.com/Materials/MaterialsArticle.aspx?id=29500>.

[37] Fine Woodworking Video. Prod. Matt Berger and Michael Dobsevage. Perf. Mario Rodriguez, Dave Spacht. Fine Woodworking Video. FineWoodworking.com, 2012. Web. 07 Oct. 2012. <http://www.finewoodworking.com/Materials/MaterialsArticle.aspx?id=29500>. 
[38] Fowler, Paul, Dr. Clean, Green Technology Makes Sustainable Resins from Vegetable Oil. Rep. Aylesbury: Oakdene Hollins, n.d. Print.

[39] Graphic Breakdown of Water Salinity, Defining Freshwater, Brackish Water, Saltwater, and Brine Water. N.d. Photograph. Salinity. Peter Summerlin, 9 Feb. 2011. Web. 1 Oct. 2012. <http://en.wikipedia.org/wiki/File:Water_salinity_diagram.png>.

[40] Green Lime. N.d. Photograph. Green Lime Wallpaper. Zastavki, 2012. Web. 2 Oct. 2012. <http://www.zastavki.com/eng/Widescreen/wallpaper-4694.htm>.

[41] Guadua Bamboo. "When and How to Harvest Bamboo?" Guadua Bamboo. Guadua Bamboo, 2012. Web. 07 Nov. 2012. <http://www.guaduabamboo.com/harvestbamboo.html>.

[42] Harper, Mark. "Steel Industry: Carbon Fiber an Environmental Culprit." SmartPlanet. N.p., 13 Oct. 2011. Web. 14 Sept. 2012. $<$ http://www.smartplanet.com/blog/intelligent-energy/steel-industry-carbon-fiber-anenvironmental-culprit/9584>.

[43] Hemicellulose. N.d. Photograph. What Is Lignocellulose? LignoFuel, 15 Sept. 2010. Web. 2 Oct. 2012. <http://lignofuel.wordpress.com/tag/hemicellulose/>.

[44] ISO. "Bamboo—Determination of Physical and Mechanical Properties." ISO 221571 (2004): n. pag. Print.

[45] International FCBA. Water absorption data. 2012. Photograph. Materials Engineerig Team. FCBA, 2012. 6 Nov. 2012. Print.

[46] Janssen, Jules J.A. Building with Bamboo. Rep. no. 20. Eindhoven: Technical University of Eindhoven, 2000. Print.

[47] Jones, Robert M. Mechanics of Composite Materials. Washington: Scripta Book, 1975. Print.

[48] King Post Truss. N.d. Photograph. 10 Meter King Post Truss. Altham Oak, 2012. Web. 27 Oct. 2012. <http://www.oak-beams.co.uk/products/oakframes/attachment/img_0242/>.

[49] Kopeliovich, Dimitri. "Materials Engineering." Carbon Fiber Reinforced Polymer Composites [SubsTech]. SubsTech: Substances and Technology, n.d. Web. 14 Sept. 2012.

$<$ http://www.substech.com/dokuwiki/doku.php?id=carbon_fiber_reinforced_polymer _composites>.

[50] La Vardera, Gregory. N.d. Photograph. Modern House Floor Beam Day. Gregory La Vardera, 3 Mar. 2006. Web. 27 Oct. 2012.

<http://blog.lamidesign.com/2006/03/6030-house-floor-beam-day.html>. 
[51] Lignocellulose. 2012. Photograph. What Is Lignocellulose. Ligno Fuel, 2012. Web. 6 Nov. 2012. <http://lignofuel.wordpress.com/tag/hemicellulose/>.

[52] McGraw-Hill, ed. "Composites." McGraw-Hill Encyclopedia of Science \& Technology. 8th ed. Vol. 1. Columbus: McGraw-Hill, 1997. 375. Print.

[53] Mercedes-Benz A-Class. N.d. Photograph. Mercedes-Benz A-Class Concept. Motor Trend, 7 Apr. 2011. Web. 2 Oct. 2012. <http://www.motortrend.com/future/concept_vehicles/1104_2012_mercedes_benz_ a_class_concept/s.

[54] Merchant, Brian. "How Fast Does Bamboo Grow?" Planet Green. Planet Green, 23 Apr. 2009. Web. 16 Sept. 2012. <http://planetgreen.discovery.com/tv/go-for-thegreen/green-brain-bamboo-grow.html>.

[55] Microfibrils. N.d. Photograph. Carbohydrate Polymers. A Level Notes. Web. 2 Oct. 2012. <http://alevelnotes.com/Carbohydrate-polymers/65>.

[56] Monerai Wing Joint. N.d. Photograph. Sailplane Spar Joining Methods. HomeBuiltAirplanes.com, 28 May 2009. Web. 27 Oct. 2012. <http://www.homebuiltairplanes.com/forums/aircraft-design-aerodynamics-newtechnology/5536-sailplane-spar-joining-methods.html>.

[57] Instant Ocean Sea Salt. N.d. Photograph. Bulk Reef Supply. Bulk Reef Supply, 2012. Web. 2 Oct. 2012. <http://www.bulkreefsupply.com/instant-ocean-sea-saltmix-160-gallon.html>.

[58] NASA. "Salinity - NASA Science." Salinity - NASA Science. NASA, 2012. Web. 07 Nov. 2012. <http://science.nasa.gov/earth-science/oceanography/physicalocean/salinity/>.

[59] NY 74 Crossing over a Truss Bridge in Essex County. N.d. Photograph. Gribble Nation. Web. <http://www.gribblenation.net/nypics/nthway/nthway.html>.

[60] Pretreatments of Natural Fibers and Their Application as Reinforcing Material in Polymer Composites--a Review. Rep. The Free Library, n.d. Web. 17 Sept. 2012. $<$ http://www.thefreelibrary.com/Pretreatments+of+natural+fibers+and+their+applicati on+as+reinforcing...-a0203766365>.

[61] Rosenberg, Tina. "In Africa's Vanishing Forests, the Benefits of Bamboo." The New York Times 13 Mar. 2012: n. pag. Print.

[62] Rush, Susan. "Carbon Fiber: Life Beyond the Landfill : CompositesWorld." Carbon Fiber: Life Beyond the Landfill : CompositesWorld. High-Performance Composites, 5 May 2007. Web. 14 Sept. 2012. <http://www.compositesworld.com/articles/carbon-fiber-life-beyond-the-landfill>.

[63] SAMPE. 2012 SAMPE Student Bridge Contest Rules. Covina: SAMPE, 2012. PDF. 
[64] Sea Salt. N.d. Photograph. Sea Salt Ice Cream. French Blue, 8 July 2008. Web. 2 Oct. 2012. <http://frenchblue-frenchblue.blogspot.com/2011/07/sea-salticecream.html>.

[65] Twin I-beam Suspsension. N.d. Photograph. The Suspension Bible. Car Bibles, 2012. Web. 27 Oct. 2012. <http://www.carbibles.com/suspension_bible.html>.

[66] Two Spar Wing Construction. N.d. Photograph. Wings. Integrated, 2012. Web. 27 Oct. 2012. <http://navyaviation.tpub.com/14014/css/14014_80.htm>.

[67] Underlying Structure of the Wall of a Wood Cell, Showing the Substructure of Loadbearing Cellulose Microfibrils. N.d. Photograph. Wood Pulp Extract Stronger than Carbon Fiber or Kevlar. Gizmag, 3 Sept. 2012. Web. 6 Nov. 2012. $<$ http://www.gizmag.com/cellulose-nanocrystals-stronger-carbon-fiberkevlar/23959/pictures\#9>.

[68] Vishay Measurements Group. Student Strain Gage Application Manual. 1992. Instruction Manual.

[69] Wilson, Carl L., and Walter E. Loomis. Botany. New York: Holt, Rinehart and Winston, 1967. Print.

[70] Wood Moisture Calculations. Tech. Forestry \& Natural Resources, n.d. Web. $<$ http://www.extension.purdue.edu/extmedia/FNR/FNR-156.pdf>.

[71] Young, Gary. "Introduction to "Bamboo Theory"' Introduction to "Bamboo Theory" Bamboo Surfboards, n.d. Web. 17 Sept. 2012. $<$ http://www.bamboosurfboardshawaii.com/theory.html>.

[72] Schott, Wolfram. Bamboo in the Laboratory. Tech. N.p.: Schott, 2006. Print.

[73] Graymont. "High Calcium Quicklime | Products | Graymont." High Calcium Quicklime | Products | Graymont. Graymont, 2012. Web. 08 Nov. 2012. $<$ http://www.graymont.com/prod_high_calcium_quicklime.shtml>.

[74] MATE253. "Elastic Properties." Oct. 2012. Lecture. 


\section{Appendix}

\section{A1. Validation of ISO standard for Node Effect on Compressive Properties}

Table A - 0-1. Bamboo Specimens with Nodes under Compression (Tonkin bamboo data)

\begin{tabular}{|c|c|c|c|c|}
\hline Specimen Label & Modulus (ksi) & $\begin{array}{c}\text { Max } \\
\text { Compressive } \\
\text { Load (lbf) }\end{array}$ & $\begin{array}{c}\text { Compressive } \\
\text { Strain @ Max } \\
\text { Compressive } \\
\text { Load (\%) }\end{array}$ & $\begin{array}{c}\text { Compressive } \\
\text { Stress @ Max } \\
\text { Compressive } \\
\text { Load (ksi) }\end{array}$ \\
\hline 1 & 2620.81814 & 6895.36521 & 0.51166 & 10.92964 \\
\hline 2 & 2288.69452 & 4248.99052 & 0.51348 & 8.14401 \\
\hline 3 & 2000.43816 & 7721.75799 & 0.67080 & 9.92489 \\
\hline 4 & 2471.42148 & 7291.46975 & 0.50161 & 9.14239 \\
\hline 5 & 2166.28050 & 10857.19614 & 0.66843 & 10.77400 \\
\hline Average & 2309.5306 & 7402.956 & 0.573196 & 9.782986 \\
\hline $\begin{array}{c}\text { Standard } \\
\text { Deviation }\end{array}$ & 218.90704 & 2109.549 & 0.078833 & 1.040545 \\
\hline SD \% & 9.478421 & 28.49604 & 13.75324 & 10.63627 \\
\hline
\end{tabular}

Table A - 0-2. Bamboo Specimens without Nodes under Compression (Tonkin bamboo data)

\begin{tabular}{|c|c|c|c|c|}
\hline Specimen Label & Modulus (ksi) & $\begin{array}{c}\text { Max } \\
\text { Compressive } \\
\text { Load (lbf) }\end{array}$ & $\begin{array}{c}\text { Compressive } \\
\text { Strain @ Max } \\
\text { Compressive } \\
\text { Load (\%) }\end{array}$ & $\begin{array}{c}\text { Compressive } \\
\text { Stress @ Max } \\
\text { Compressive } \\
\text { Load (ksi) }\end{array}$ \\
\hline 1 & 2538.07621 & 11247.17816 & 0.90368 & 13.43877 \\
\hline 2 & 2248.78983 & 5780.84731 & 0.57045 & 8.16059 \\
\hline 3 & 2440.30815 & 4864.99288 & 0.45782 & 8.22411 \\
\hline 4 & 1868.28235 & 5595.19510 & 0.75444 & 10.09435 \\
\hline 5 & 1761.09777 & 9482.15236 & 0.94528 & 10.93621 \\
\hline Average & 2171.311 & 7394.073 & 0.726334 & 10.17081 \\
\hline $\begin{array}{c}\text { Standard } \\
\text { Deviation }\end{array}$ & 307.5648 & 2507.636 & 0.187925 & 1.954578 \\
\hline SD \% & 14.16494 & 33.91414 & 25.87308 & 19.21753 \\
\hline
\end{tabular}

The pair of compression tests for the mechanical behavior of bamboos began with the group having nodes. It is important to note the data shown is for a different species of bamboo, but the difference in the mechanical properties of bamboos with/without nodes remains the same between species. As seen from the table, the average Young's Modulus in compression for the group is over $2300 \mathrm{ksi}$. This value is significantly important because it is higher than most wood. For a natural material, this Young's Modulus is quite impressive. Also, the average strain at the maximum compressive load of less than $1 \%$ is another impressive characteristic of the bamboo. The second group for analysis comprised of the bamboos 
without nodes. As seen from the table, the average Young's Modulus in compression for the group is over $2100 \mathrm{ksi}$. The average strain at the maximum compressive load is also less than $1 \%$.

Experienced intuition leads one to believe that incorporating the nodes for structural design should significantly improve performance. However, the data yielded results that contradict one's initial predictions on the bamboo's mechanical characteristics. Although the test group including nodes had a greater average Modulus and maximum compressive load value than the group without nodes, the difference is minimal. The percent difference between the Modulus' is only $6 \%$, and the difference between the strains at maximum compressive load is $0.1 \%$-basically illustrating the same mechanical properties for both groups.

The precision of the data seems to be fairly good for most of the characteristics across the board; a standard deviation of about $15 \%$ for all the average mechanical characteristics is observed. With the exception of the maximum compressive load average, most of the mechanical characteristics can be trusted with confidence. The high standard deviation for the characteristic in question may be attributed to the bamboo's naturally thick base culms. As bamboo grows, the thickest culms are the first to form and the subsequent culms grow slightly thinner in diameter. The higher Modulus for this particular stronger specimen may be the reason why the data is swayed towards a larger deviation among the group. Lastly, any errors committed during the test would later be seen in the data or in the aftermath of each specimen's structure. Each specimen was consequently inspected after the test, and no mysterious failure modes were found to lead one to believe that any major errors had occurred during the test. 


\section{A2. Compression Testing Data}
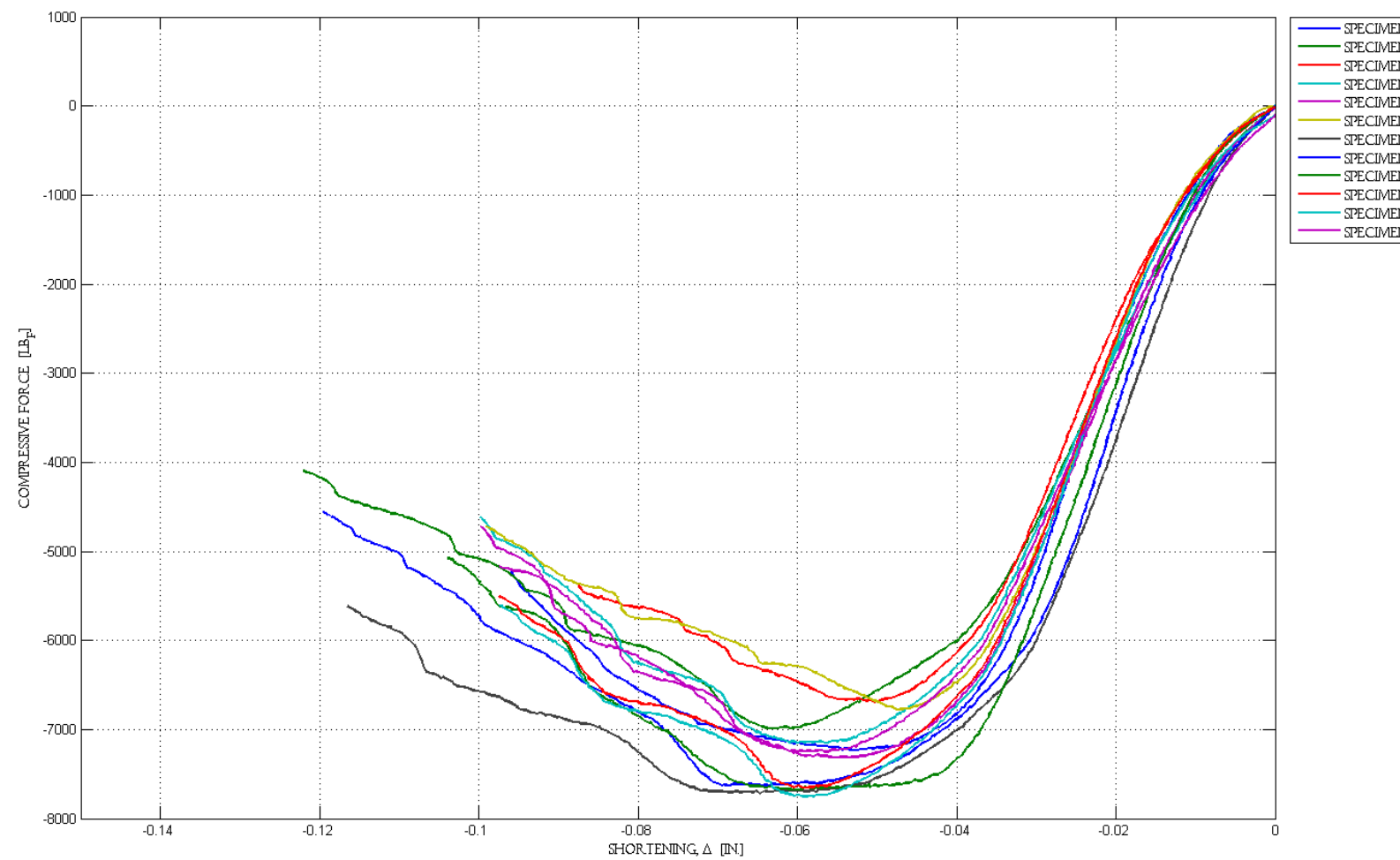

Figure A - 1. Force-Displacement Curves of untreated bamboo

Table A - 0-3. Compressive properties of untreated bamboo

\begin{tabular}{|c|c|c|c|c|c|c|c|}
\hline Specimen & $\begin{array}{l}\text { Modulus } \\
\quad(\mathrm{ksi})\end{array}$ & $\begin{array}{c}\text { Specific } \\
\text { Modulus } \\
\text { (kips-in.//lbm) } \\
\times 100\end{array}$ & $\begin{array}{c}\text { Yield } \\
\text { Strain } \\
(\%)\end{array}$ & $\begin{array}{c}\text { Yield } \\
\text { Stress } \\
\text { (ksi) }\end{array}$ & $\begin{array}{c}\text { Ultimate } \\
\text { Strain } \\
(\%)\end{array}$ & $\begin{array}{c}\text { Ultimate } \\
\text { Stress } \\
\text { (ksi) }\end{array}$ & $\begin{array}{c}\text { Specific } \\
\text { Strength } \\
\text { (kips-in./lbm) }\end{array}$ \\
\hline 1 & 488.742 & 123.732 & 1.962 & 6.684 & 3.318 & 8.677 & 219.679 \\
\hline 2 & 375.386 & 88.954 & 2.110 & 6.546 & 4.177 & 9.018 & 213.686 \\
\hline 3 & 422.998 & 101.682 & 2.205 & 6.919 & 3.289 & 8.688 & 208.857 \\
\hline 4 & 394.307 & 95.938 & 2.174 & 6.914 & 3.703 & 9.243 & 224.895 \\
\hline 5 & 429.096 & 102.410 & 1.912 & 6.066 & 3.611 & 9.440 & 225.300 \\
\hline 6 & 439.746 & 102.505 & 1.949 & 5.839 & 3.276 & 8.607 & 200.635 \\
\hline 7 & 493.865 & 113.013 & 1.985 & 7.625 & 4.410 & 10.022 & 229.336 \\
\hline 8 & 483.770 & 113.828 & 1.907 & 6.973 & 4.246 & 9.633 & 226.658 \\
\hline 9 & 472.701 & 110.187 & 2.157 & 7.695 & 4.143 & 9.900 & 230.769 \\
\hline 10 & 450.969 & 106.865 & 2.079 & 6.476 & 4.001 & 9.682 & 229.430 \\
\hline 11 & 398.687 & 94.926 & 2.136 & 7.548 & 3.995 & 9.389 & 223.558 \\
\hline 12 & 453.413 & 111.131 & 2.016 & 6.924 & 4.047 & 9.743 & 238.789 \\
\hline Mean & 441.973 & 105.431 & 2.049 & 6.851 & 3.851 & 9.337 & 222.633 \\
\hline $\begin{array}{l}\text { Standard } \\
\text { Deviation }\end{array}$ & 39.189 & 9.647 & 0.107 & 0.582 & 0.399 & 0.493 & 10.489 \\
\hline Maximum & 493.865 & 123.732 & 2.205 & 7.695 & 4.410 & 10.022 & 238.789 \\
\hline
\end{tabular}




\section{\begin{tabular}{|l||l|l|l|l|l|l|l|}
\hline Minimum & 375.386 & 88.954 & 1.907 & 5.839 & 3.276 & 8.607 & 200.635 \\
\hline
\end{tabular}}

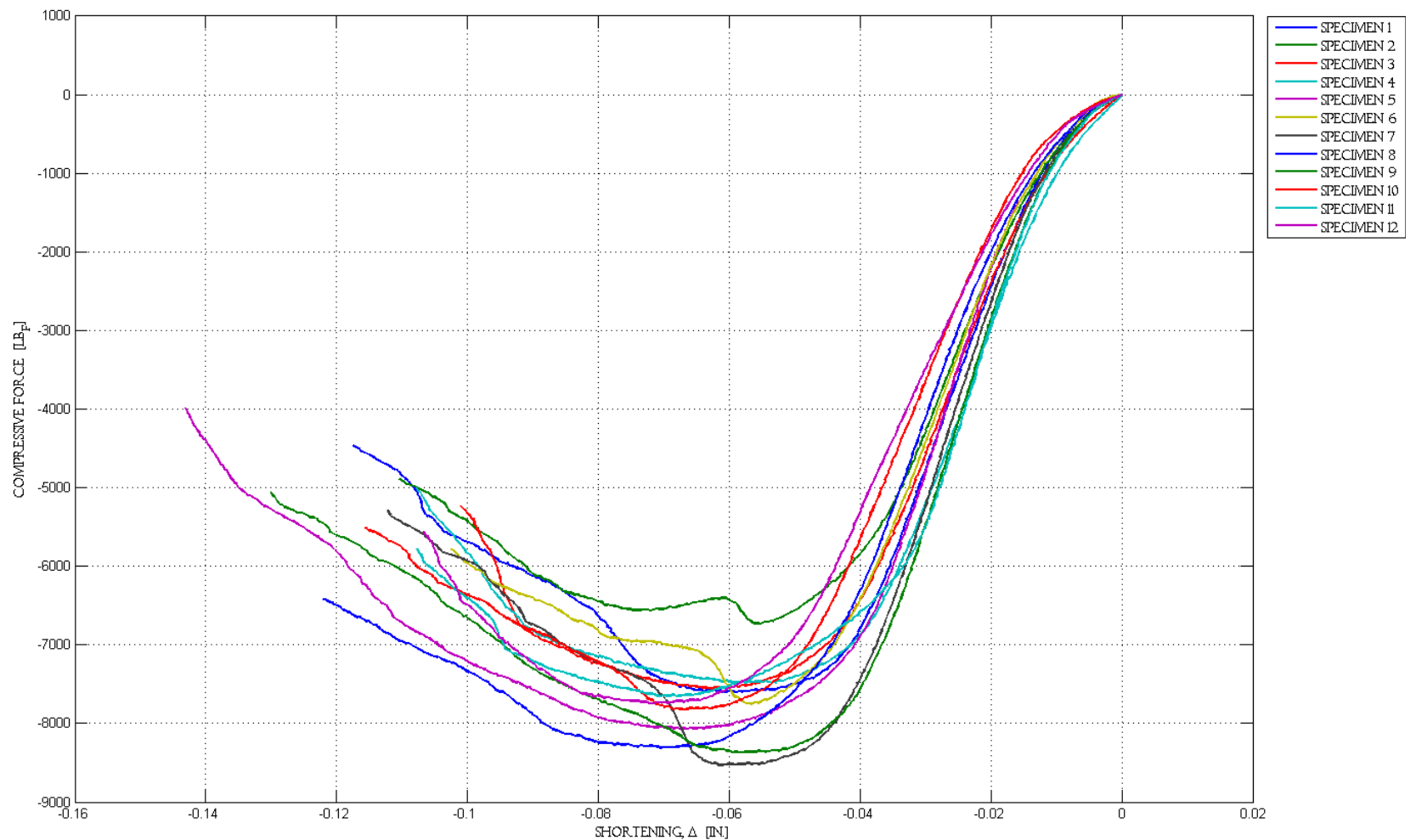

Figure A - 2. Compressive Force-Displacement curves for $150^{\circ} \mathrm{F}$ at $5^{\circ} \mathrm{F} / \mathrm{min}$. treatment

Table A - 0-4. Compressive properties of bamboo thermally treated at $150^{\circ} \mathrm{F}$ with $5^{\circ} \mathrm{F} / \mathrm{min}$. rate

\begin{tabular}{|c|c|c|c|c|c|c|c|}
\hline Specimen & $\begin{array}{c}\text { Modulus } \\
(\mathrm{ksi})\end{array}$ & $\begin{array}{c}\text { Specific } \\
\text { Modulus } \\
(\mathrm{kips}-\mathrm{in} / \mathrm{/l} \mathrm{b}) \\
\times 100\end{array}$ & $\begin{array}{c}\text { Yield } \\
\text { Strain } \\
(\%)\end{array}$ & $\begin{array}{c}\text { Yield } \\
\text { Stress } \\
(\mathrm{ksi})\end{array}$ & $\begin{array}{c}\text { Ultimate } \\
\text { Strain } \\
(\%)\end{array}$ & $\begin{array}{c}\text { Ultimate } \\
\text { Stress } \\
(\mathrm{ksi})\end{array}$ & $\begin{array}{c}\text { Specific } \\
\text { Strength } \\
\left(\mathrm{kips}-\text {-in./l }_{\mathrm{m}}\right)\end{array}$ \\
\hline 1 & 484.529 & 133.112 & 2.004 & 6.985 & 3.391 & 9.073 & 249.252 \\
\hline 2 & 435.726 & 117.446 & 1.861 & 5.698 & 3.130 & 7.927 & 213.661 \\
\hline 3 & 439.078 & 124.738 & 1.758 & 5.380 & 3.472 & 8.504 & 241.594 \\
\hline 4 & 481.900 & 128.850 & 1.663 & 5.953 & 3.057 & 8.889 & 237.678 \\
\hline 5 & 408.891 & 111.719 & 2.192 & 7.342 & 3.814 & 8.883 & 242.693 \\
\hline 6 & 519.083 & 136.961 & 1.919 & 5.370 & 3.894 & 8.889 & 234.534 \\
\hline 7 & 366.151 & 96.356 & 2.052 & 6.318 & 4.114 & 9.040 & 237.897 \\
\hline 8 & 487.846 & 127.708 & 2.521 & 6.665 & 4.378 & 9.180 & 240.306 \\
\hline 9 & 420.456 & 111.527 & 2.186 & 7.448 & 3.345 & 8.421 & 223.372 \\
\hline 10 & 388.839 & 108.011 & 2.441 & 7.008 & 4.518 & 8.957 & 248.807 \\
\hline 11 & 470.202 & 124.392 & 1.663 & 6.145 & 4.192 & 9.470 & 250.535 \\
\hline 12 & 451.874 & 123.126 & 2.710 & 7.684 & 3.878 & 9.207 & 250.878 \\
\hline Mean & 446.214 & 120.329 & 2.081 & 6.500 & 3.765 & 8.870 & 239.267 \\
\hline
\end{tabular}




\begin{tabular}{||c||c|c|c|c|c|c|c||}
\hline $\begin{array}{c}\text { Standard } \\
\text { Deviation }\end{array}$ & 45.037 & 11.667 & 0.341 & 0.807 & 0.485 & 0.413 & 11.305 \\
\hline Maximum & 519.083 & 136.961 & 2.710 & 7.684 & 4.518 & 9.470 & 250.878 \\
\hline Minimum & 366.151 & 96.356 & 1.663 & 5.370 & 3.057 & 7.927 & 213.661 \\
\hline
\end{tabular}

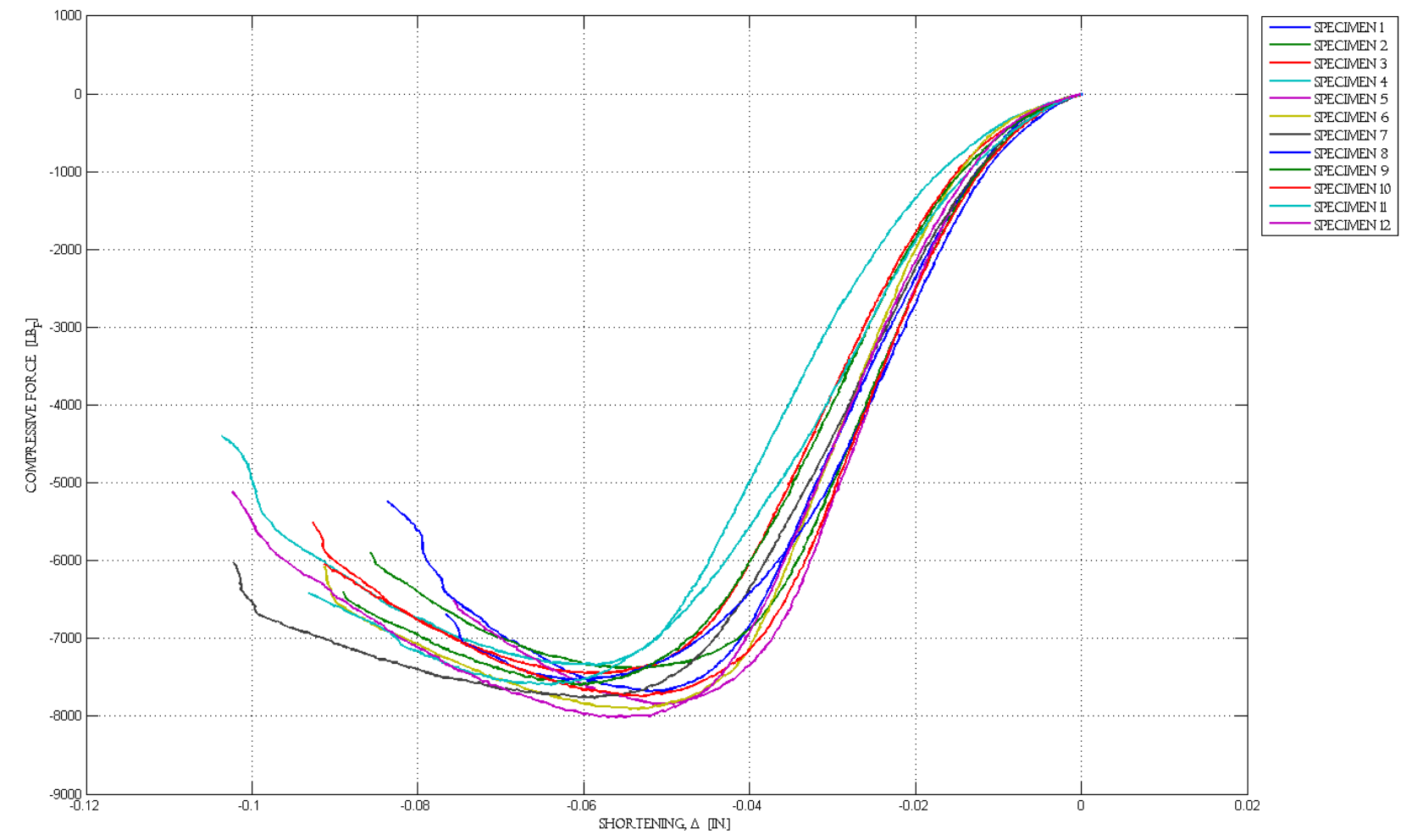

Figure A - 3. Compressive Force-Displacement curves for $180^{\circ} \mathrm{F}$ at $5^{\circ} \mathrm{F} / \mathrm{min}$. treatment

Table A - 0-5. Compressive properties of bamboo thermally treated at $180^{\circ} \mathrm{F}$ with $5^{\circ} \mathrm{F} / \mathrm{min}$. rate

\begin{tabular}{|c|c|c|c|c|c|c|c|}
\hline Specimen & $\begin{array}{c}\text { Modulus } \\
(\mathrm{ksi})\end{array}$ & $\begin{array}{c}\text { Specific } \\
\text { Modulus } \\
(\mathrm{kips-in.// \textrm {b }} \mathrm{m}) \\
\times 100\end{array}$ & $\begin{array}{c}\text { Yield } \\
\text { Strain } \\
(\%)\end{array}$ & $\begin{array}{c}\text { Yield } \\
\text { Stress } \\
(\mathrm{ksi})\end{array}$ & $\begin{array}{c}\text { Ultimate } \\
\text { Strain } \\
(\%)\end{array}$ & $\begin{array}{c}\text { Ultimate } \\
\text { Stress } \\
(\mathrm{ksi})\end{array}$ & $\begin{array}{c}\text { Specific } \\
\text { Strength } \\
(\mathrm{kips}-\mathrm{i} . / \mathrm{l} \mathrm{b})\end{array}$ \\
\hline 1 & 541.595 & 158.361 & 2.318 & 9.121 & 3.007 & 10.168 & 297.407 \\
\hline 2 & 620.298 & 164.973 & 1.996 & 8.504 & 3.162 & 10.525 & 279.926 \\
\hline 3 & 521.013 & 144.325 & 2.257 & 8.022 & 3.406 & 10.292 & 285.516 \\
\hline 4 & 490.757 & 134.087 & 2.730 & 8.629 & 3.433 & 10.047 & 274.503 \\
\hline 5 & 637.423 & 180.573 & 2.083 & 8.952 & 3.012 & 10.686 & 302.720 \\
\hline 6 & 659.103 & 176.231 & 2.135 & 9.028 & 3.084 & 11.134 & 297.688 \\
\hline 7 & 477.903 & 135.768 & 1.816 & 5.931 & 3.398 & 9.823 & 279.072 \\
\hline 8 & 444.637 & 125.250 & 1.668 & 5.431 & 3.710 & 9.426 & 266.135 \\
\hline 9 & 532.461 & 147.906 & 2.122 & 7.458 & 3.388 & 10.071 & 279.742 \\
\hline 10 & 604.242 & 170.690 & 1.858 & 7.553 & 3.078 & 10.291 & 290.694 \\
\hline
\end{tabular}




\begin{tabular}{|c|c|c|c|c|c|c|c||}
\hline 11 & 474.845 & 129.034 & 1.938 & 6.247 & 3.725 & 10.353 & 282.040 \\
\hline 12 & 524.333 & 148.958 & 2.301 & 8.290 & 3.414 & 10.268 & 291.707 \\
\hline \hline Mean & 544.051 & 151.346 & 2.102 & 7.764 & 3.318 & 10.257 & 285.596 \\
\hline $\begin{array}{c}\text { Standard } \\
\text { Deviation }\end{array}$ & 70.285 & 18.754 & 0.282 & 1.270 & 0.250 & 0.427 & 10.727 \\
\hline Maximum & 659.103 & 180.573 & 2.730 & 9.121 & 3.725 & 11.134 & 302.720 \\
\hline Minimum & 444.637 & 125.250 & 1.668 & 5.431 & 3.007 & 9.426 & 266.135 \\
\hline
\end{tabular}

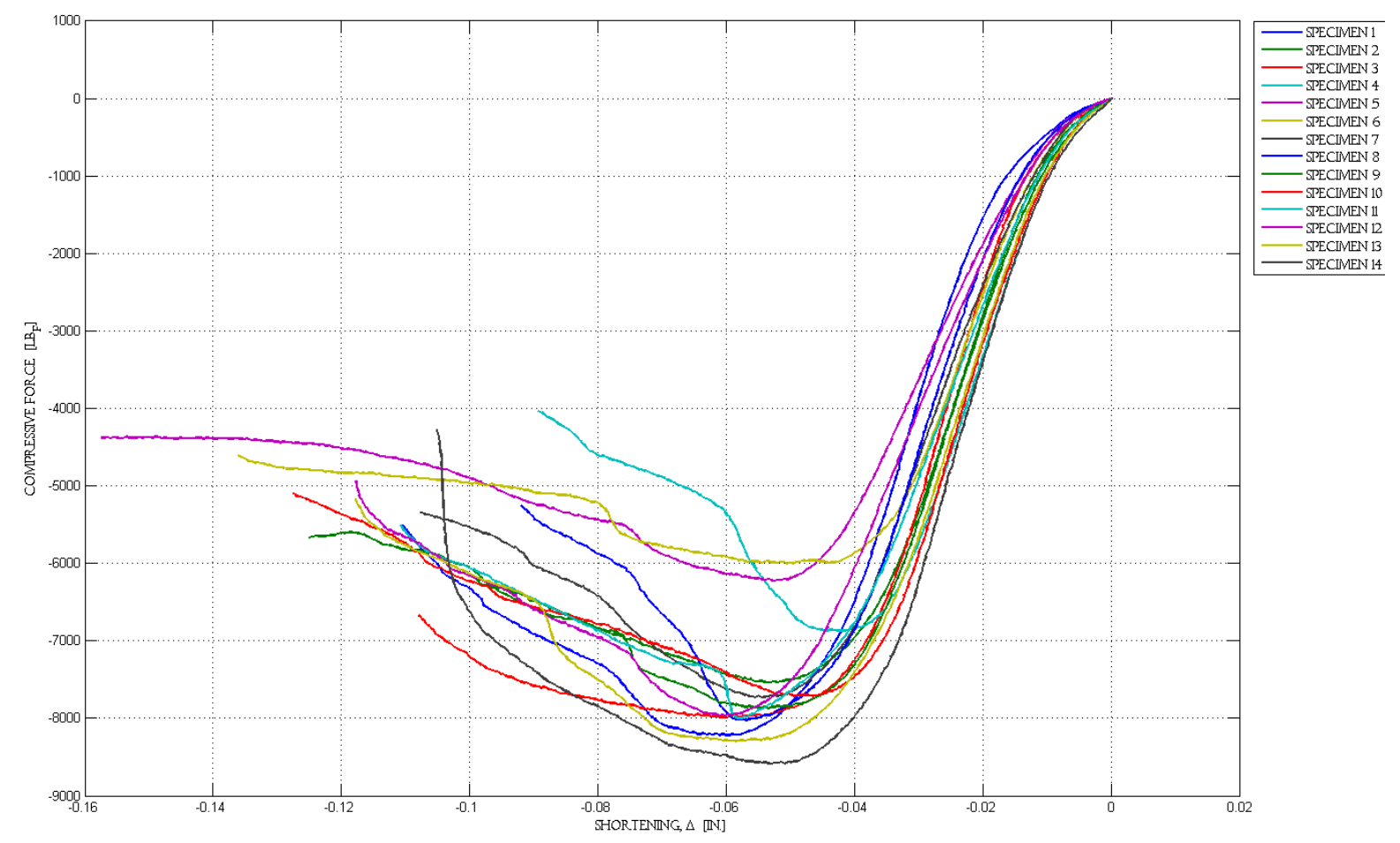

Figure A - 4. Compressive Force-Displacement curves for $180^{\circ} \mathrm{F}$ at $1^{\circ} \mathrm{F} / \mathrm{min}$. treatment

Table A - 0-6. Compressive properties of bamboo thermally treated at $180^{\circ} \mathrm{F}$ with $1^{\circ} \mathrm{F} / \mathrm{min}$. rate

\begin{tabular}{|c|c|c|c|c|c|c|c|}
\hline Specimen & $\begin{array}{c}\text { Modulus } \\
(\mathrm{ksi})\end{array}$ & $\begin{array}{c}\text { Specific } \\
\text { Modulus } \\
(\mathrm{kips}-\mathrm{in} . / \mathrm{l} \mathrm{b}) \\
\mathrm{x} 100\end{array}$ & $\begin{array}{c}\text { Yield } \\
\text { Strain } \\
(\%)\end{array}$ & $\begin{array}{c}\text { Yield } \\
\text { Stress } \\
(\mathrm{ksi})\end{array}$ & $\begin{array}{c}\text { Ultimate } \\
\text { Strain } \\
(\%)\end{array}$ & $\begin{array}{c}\text { Ultimate } \\
\text { Stress } \\
(\mathrm{ksi})\end{array}$ & $\begin{array}{c}\text { Specific } \\
\text { Strength } \\
(\mathrm{kips}-\mathrm{in} . / \mathrm{l} \mathrm{m})\end{array}$ \\
\hline 1 & 499.952 & 152.425 & 2.552 & 7.748 & 3.992 & 10.399 & 317.057 \\
\hline 2 & 558.410 & 143.550 & 1.963 & 7.831 & 3.565 & 11.283 & 290.040 \\
\hline 3 & 547.759 & 161.581 & 1.954 & 6.518 & 3.997 & 10.282 & 303.299 \\
\hline 4 & 488.795 & 150.398 & 1.958 & 7.216 & 3.153 & 9.612 & 295.751 \\
\hline 5 & 382.622 & 106.580 & 2.726 & 7.612 & 4.697 & 9.353 & 260.521 \\
\hline 6 & 486.002 & 133.151 & 2.144 & 7.265 & 4.601 & 9.096 & 249.207 \\
\hline 7 & 477.509 & 147.379 & 2.351 & 8.265 & 3.497 & 10.053 & 310.270 \\
\hline
\end{tabular}




\begin{tabular}{|c|c|c|c|c|c|c|c|}
\hline 8 & 497.074 & 142.837 & 2.555 & 8.345 & 4.124 & 11.328 & 325.514 \\
\hline 9 & 548.552 & 163.259 & 2.025 & 7.540 & 4.342 & 10.990 & 327.068 \\
\hline 10 & 556.442 & 157.187 & 2.047 & 8.210 & 4.340 & 11.136 & 314.568 \\
\hline 11 & 356.544 & 107.393 & 2.738 & 7.435 & 4.764 & 10.158 & 305.974 \\
\hline 12 & 375.262 & 103.951 & 3.124 & 8.773 & 4.926 & 10.798 & 299.104 \\
\hline 13 & 465.889 & 129.774 & 2.313 & 7.928 & 4.676 & 11.125 & 309.891 \\
\hline 14 & 474.767 & 145.634 & 1.816 & 5.952 & 4.505 & 10.698 & 328.160 \\
\hline \hline Mean & 479.684 & 138.936 & 2.305 & 7.617 & 4.227 & 10.451 & 302.602 \\
\hline $\begin{array}{c}\text { Standard } \\
\text { Deviation }\end{array}$ & 66.801 & 20.166 & 0.386 & 0.739 & 0.533 & 0.727 & 23.356 \\
\hline Maximum & 558.410 & 163.259 & 3.124 & 8.773 & 4.926 & 11.328 & 328.160 \\
\hline Minimum & 356.544 & 20.166 & 1.816 & 5.952 & 3.153 & 9.096 & 249.207 \\
\hline
\end{tabular}

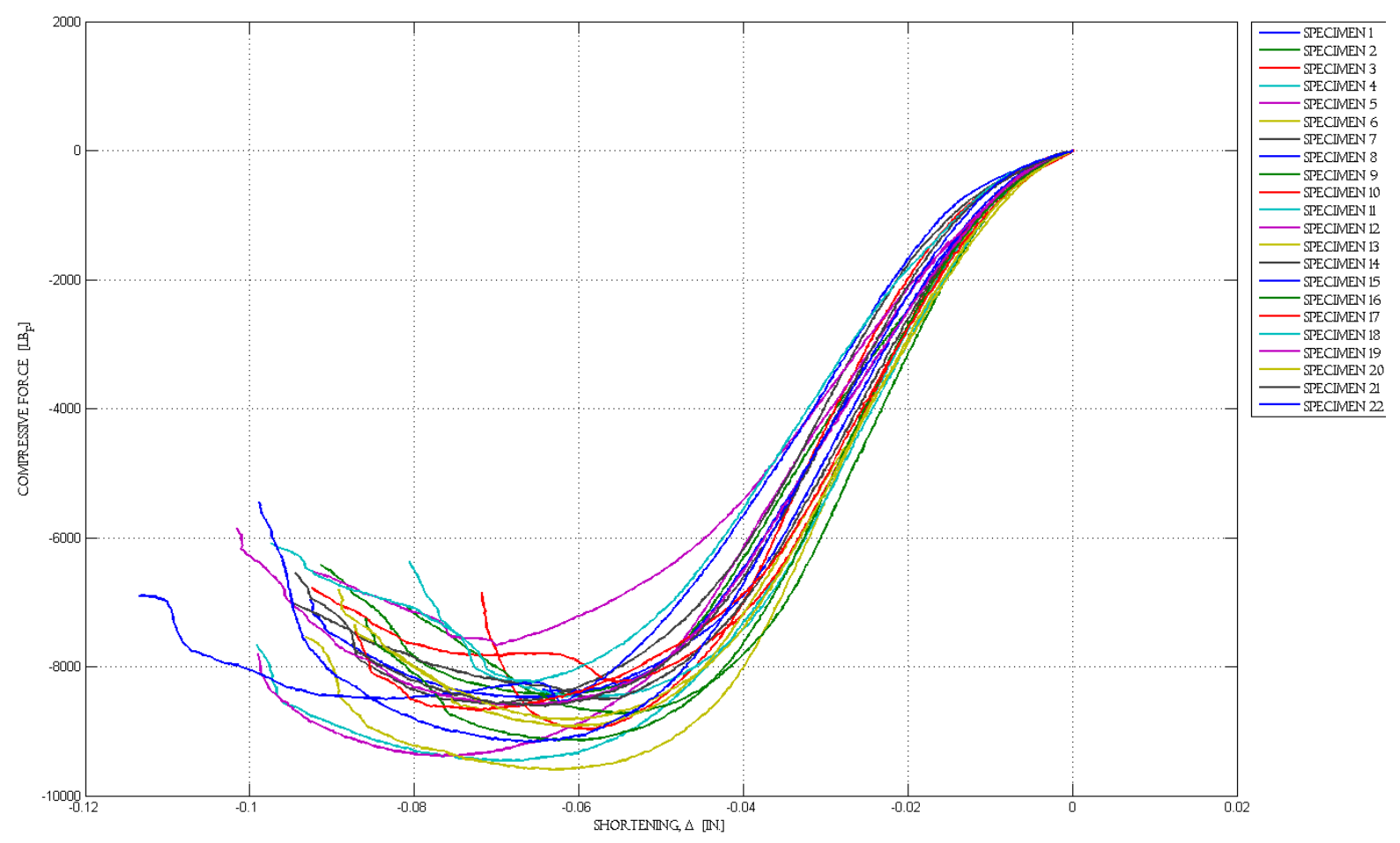

Figure A - 5. Comnressive Force-Disnlacement curves for $150^{\circ} \mathrm{F}$ at $5^{\circ} \mathrm{F} / \mathrm{min}$. treatment

Table A - 0-7. Compressive properties of bamboo thermally treated at $200^{\circ} \mathrm{F}$ with $5^{\circ} \mathrm{F} / \mathrm{min}$. rate

\begin{tabular}{|c|c|c|c|c|c|c|c|}
\hline Specimen & $\begin{array}{c}\text { Modulus } \\
(\mathrm{ksi})\end{array}$ & $\begin{array}{c}\text { Specific } \\
\text { Modulus } \\
\left(\mathrm{kips}-\mathrm{in} . / \mathrm{l} \mathrm{b}_{\mathrm{m}}\right) \\
\times 100\end{array}$ & $\begin{array}{c}\text { Yield } \\
\text { Strain } \\
(\%)\end{array}$ & $\begin{array}{c}\text { Yield } \\
\text { Stress } \\
(\mathrm{ksi})\end{array}$ & $\begin{array}{c}\text { Ultimate } \\
\text { Strain } \\
(\%)\end{array}$ & $\begin{array}{c}\text { Ultimate } \\
\text { Stress } \\
(\mathrm{ksi})\end{array}$ & $\begin{array}{c}\text { Specific } \\
\text { Strength } \\
\left(\mathrm{kips}-\mathrm{in} . / \mathrm{l} \mathrm{b}_{\mathrm{m}}\right)\end{array}$ \\
\hline 1 & 462.903 & 138.594 & 2.509 & 9.461 & 3.624 & 11.377 & 340.619 \\
\hline 2 & 495.921 & 152.591 & 2.686 & 10.160 & 3.625 & 11.727 & 360.824 \\
\hline 3 & 593.332 & 194.535 & 2.560 & 9.807 & 3.626 & 12.100 & 396.706 \\
\hline
\end{tabular}




\begin{tabular}{|c|c|c|c|c|c|c|c|}
\hline 4 & 442.375 & 140.437 & 1.800 & 5.966 & 3.918 & 10.142 & 321.963 \\
\hline 5 & 377.803 & 118.063 & 2.844 & 8.460 & 4.490 & 10.232 & 319.761 \\
\hline 6 & 522.911 & 157.979 & 2.264 & 8.356 & 3.875 & 11.003 & 332.404 \\
\hline 7 & 468.143 & 157.624 & 2.251 & 6.832 & 4.046 & 9.733 & 327.715 \\
\hline 8 & 538.497 & 166.717 & 2.138 & 8.381 & 3.906 & 11.367 & 351.933 \\
\hline 9 & 532.672 & 175.221 & 2.155 & 8.592 & 3.501 & 10.921 & 359.247 \\
\hline 10 & 614.436 & 185.071 & 1.999 & 8.798 & 3.223 & 11.601 & 349.426 \\
\hline 11 & 568.756 & 174.465 & 2.076 & 8.999 & 3.586 & 11.714 & 359.332 \\
\hline 12 & 391.366 & 118.956 & 2.435 & 7.639 & 4.218 & 10.739 & 326.425 \\
\hline 13 & 449.584 & 136.238 & 2.307 & 8.791 & 3.495 & 10.970 & 332.429 \\
\hline 14 & 470.127 & 143.331 & 2.197 & 7.746 & 3.448 & 10.460 & 318.905 \\
\hline 15 & 393.286 & 126.866 & 2.292 & 6.033 & 3.686 & 9.508 & 306.702 \\
\hline 16 & 569.305 & 167.937 & 2.094 & 8.465 & 3.468 & 11.411 & 336.619 \\
\hline 17 & 447.146 & 134.683 & 2.053 & 7.387 & 4.245 & 10.449 & 314.727 \\
\hline 18 & 421.922 & 128.244 & 2.534 & 7.866 & 3.751 & 10.277 & 312.362 \\
\hline 19 & 446.339 & 135.665 & 2.348 & 8.358 & 3.843 & 10.745 & 326.588 \\
\hline 20 & 547.670 & 170.084 & 1.964 & 8.282 & 3.317 & 10.860 & 337.273 \\
\hline 21 & 537.842 & 162.983 & 1.985 & 7.084 & 3.609 & 10.831 & 328.200 \\
\hline 22 & 429.792 & 142.315 & 2.473 & 8.112 & 3.627 & 9.864 & 326.617 \\
\hline Mean & 487.369 & 151.300 & 2.271 & 8.163 & 3.733 & 10.820 & 335.762 \\
\hline Standard & 68.518 & 21.447 & 0.259 & 1.065 & 0.312 & 0.693 & 20.573 \\
\hline Deviation & & & & & & \\
\hline Maximum & 614.436 & 194.535 & 2.844 & 10.160 & 4.490 & 12.100 & 396.706 \\
\hline Minimum & 377.803 & 118.063 & 1.800 & 5.966 & 3.223 & 9.508 & 306.702 \\
\hline
\end{tabular}




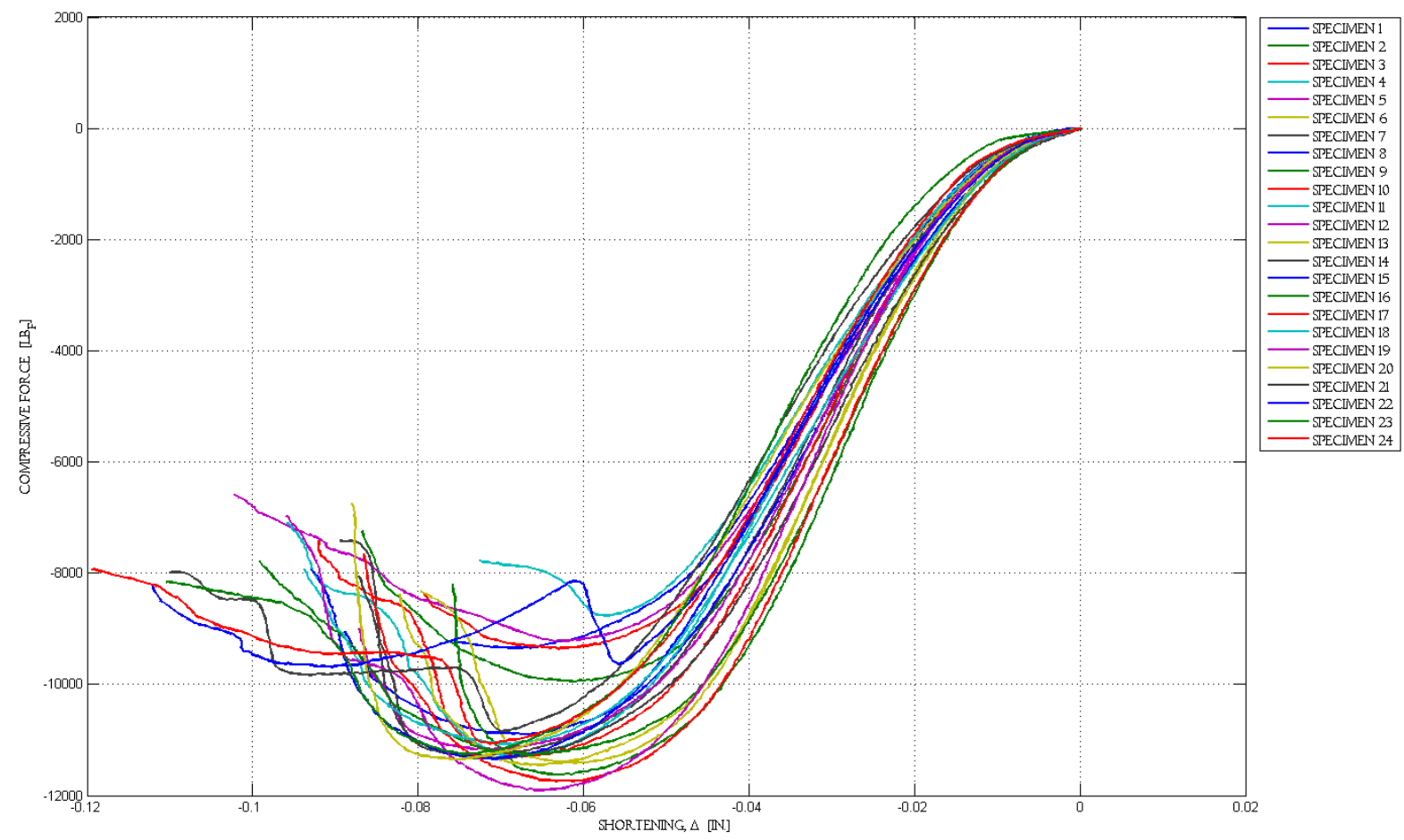

Figure A - 6. Combressive Force-Disblacement curves for $150^{\circ} \mathrm{F}$ at $5^{\circ} \mathrm{F} / \mathrm{min}$. treatment

Table A - 0-8. Compressive properties of bamboo thermally treated at $200^{\circ} \mathrm{F}$ with $1^{\circ} \mathrm{F} / \mathrm{min}$. rate

\begin{tabular}{|c|c|c|c|c|c|c|c|}
\hline Specimen & $\begin{array}{l}\text { Modulus } \\
(\mathrm{ksi})\end{array}$ & $\begin{array}{c}\text { Specific } \\
\text { Modulus } \\
\left.\text { (kips-in.//l } \mathrm{b}_{\mathrm{m}}\right) \\
\text { x } 100\end{array}$ & $\begin{array}{c}\text { Yield } \\
\text { Strain } \\
(\%)\end{array}$ & $\begin{array}{c}\text { Yield } \\
\text { Stress } \\
\text { (ksi) }\end{array}$ & $\begin{array}{l}\text { Ultimate } \\
\text { Strain } \\
(\%)\end{array}$ & $\begin{array}{l}\text { Ultimate } \\
\text { Stress } \\
\text { (ksi) }\end{array}$ & $\begin{array}{l}\text { Specific } \\
\text { Strength } \\
\text { (kips-in.//l } \mathrm{b}_{\mathrm{m}} \text { ) }\end{array}$ \\
\hline 1 & 582.755 & 179.309 & 2.399 & 9.366 & 3.831 & 12.149 & 373.808 \\
\hline 2 & 567.607 & 188.574 & 2.227 & 8.365 & 3.601 & 11.533 & 383.142 \\
\hline 3 & 636.651 & 191.186 & 2.470 & 10.427 & 3.658 & 12.753 & 382.972 \\
\hline 4 & 545.365 & 167.805 & 2.635 & 9.927 & 3.355 & 11.624 & 357.662 \\
\hline 5 & 512.870 & 164.381 & 2.323 & 8.506 & 3.743 & 11.623 & 372.521 \\
\hline 6 & 667.493 & 181.384 & 2.295 & 10.377 & 3.525 & 13.581 & 369.042 \\
\hline 7 & 613.401 & 168.517 & 2.335 & 9.309 & 4.272 & 14.273 & 392.127 \\
\hline 8 & 605.460 & 158.913 & 2.572 & 10.761 & 4.019 & 14.078 & 369.495 \\
\hline 9 & 757.181 & 200.844 & 2.135 & 10.564 & 3.709 & 14.844 & 393.734 \\
\hline 10 & 652.143 & 174.370 & 2.293 & 9.480 & 4.116 & 14.491 & 387.464 \\
\hline 11 & 607.228 & 159.797 & 2.770 & 11.518 & 4.153 & 14.825 & 390.122 \\
\hline 12 & 654.002 & 169.871 & 2.413 & 10.454 & 4.245 & 14.834 & 385.297 \\
\hline 13 & 747.915 & 190.795 & 2.352 & 11.665 & 3.768 & 15.026 & 383.326 \\
\hline 14 & 693.005 & 173.251 & 2.192 & 10.391 & 4.017 & 15.056 & 376.408 \\
\hline 15 & 605.103 & 156.357 & 2.676 & 11.191 & 4.076 & 14.550 & 375.975 \\
\hline 16 & 707.541 & 181.887 & 2.174 & 10.769 & 3.794 & 14.477 & 372.167 \\
\hline 17 & 708.385 & 197.873 & 2.073 & 10.032 & 3.378 & 13.333 & 372.442 \\
\hline 18 & 549.804 & 141.338 & 2.609 & 10.771 & 3.969 & 14.039 & 360.908 \\
\hline
\end{tabular}




\begin{tabular}{|c|c|c|c|c|c|c|c|}
\hline 19 & 719.002 & 206.018 & 2.166 & 9.800 & 3.548 & 13.554 & 388.353 \\
\hline 20 & 570.062 & 142.515 & 2.838 & 11.752 & 4.227 & 14.602 & 365.062 \\
\hline 21 & 512.200 & 138.808 & 2.731 & 9.606 & 4.078 & 13.035 & 353.247 \\
\hline 22 & 528.863 & 150.673 & 2.480 & 8.935 & 3.221 & 11.169 & 318.193 \\
\hline 23 & 623.963 & 172.365 & 2.634 & 2.634 & 4.148 & 13.298 & 367.343 \\
\hline 24 & 593.332 & 155.730 & 2.496 & 2.496 & 4.014 & 13.398 & 351.642 \\
\hline \hline Mean & 623.389 & 171.357 & 2.429 & 9.546 & 3.853 & 13.589 & 372.602 \\
\hline $\begin{array}{l}\text { Standard } \\
\text { Deviation }\end{array}$ & 72.270 & 18.858 & 0.218 & 2.334 & 0.304 & 1.223 & 16.638 \\
\hline Maximum & 757.181 & 206.018 & 2.838 & 11.752 & 4.272 & 15.056 & 393.734 \\
\hline Minimum & 512.200 & 138.808 & 2.073 & 2.496 & 3.221 & 11.169 & 318.193 \\
\hline
\end{tabular}

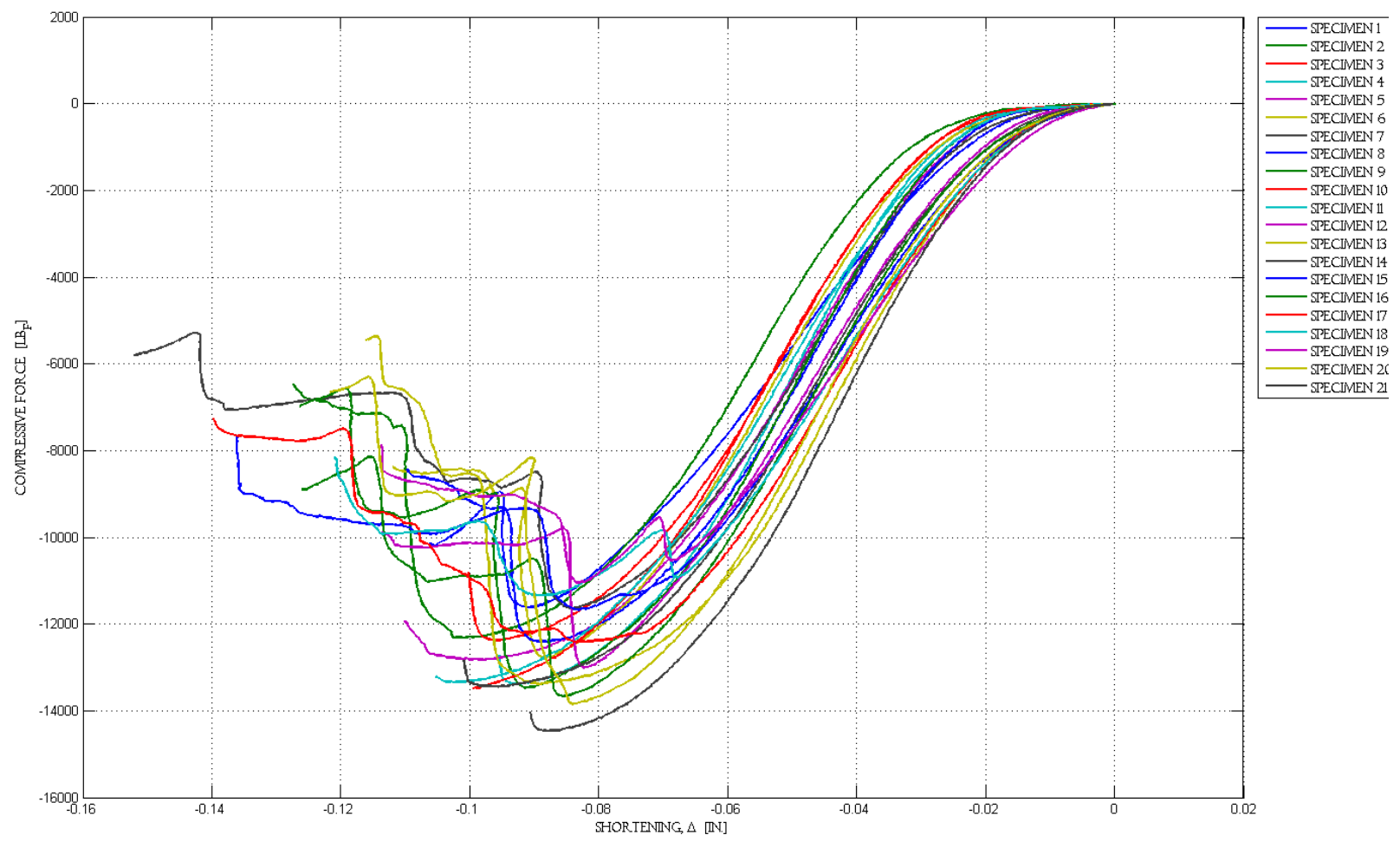

Figure A - 7. Compressive Force-Displacement curves for $220^{\circ} \mathrm{F}$ at $1^{\circ} \mathrm{F} / \mathrm{min}$. treatment

Table A - 0-9. Compressive properties of bamboo thermally treated at $220^{\circ} \mathrm{F}$ with $1^{\circ} \mathrm{F} / \mathrm{min}$. rate

\begin{tabular}{|c|c|c|c|c|c|c|c|}
\hline Specimen & $\begin{array}{c}\text { Modulus } \\
(\mathrm{ksi})\end{array}$ & $\begin{array}{c}\text { Specific } \\
\text { Modulus } \\
\left(\mathrm{kips}-\mathrm{in} / / \mathrm{l} \mathrm{b}_{\mathrm{m}}\right) \\
\times 100\end{array}$ & $\begin{array}{c}\text { Yield } \\
\text { Strain } \\
(\%)\end{array}$ & $\begin{array}{c}\text { Yield } \\
\text { Stress } \\
(\mathrm{ksi})\end{array}$ & $\begin{array}{c}\text { Ultimate } \\
\text { Strain } \\
(\%)\end{array}$ & $\begin{array}{c}\text { Ultimate } \\
\text { Stress } \\
(\mathrm{ksi})\end{array}$ & $\begin{array}{c}\text { Specific } \\
\text { Strength } \\
(\mathrm{kips}-\mathrm{in} . / \mathrm{l} \mathrm{m})\end{array}$ \\
\hline 1 & 476.427 & 137.696 & 3.763 & 11.654 & 5.395 & 16.480 & 476.299 \\
\hline 2 & 618.909 & 172.880 & 4.085 & 12.771 & 6.211 & 18.112 & 505.915 \\
\hline 3 & 607.661 & 165.125 & 4.343 & 14.220 & 6.121 & 18.247 & 495.846 \\
\hline
\end{tabular}




\begin{tabular}{|c|c|c|c|c|c|c|c|}
\hline 4 & 640.673 & 186.785 & 4.030 & 14.306 & 5.719 & 18.486 & 538.955 \\
\hline 5 & 574.982 & 157.099 & 3.979 & 13.234 & 6.267 & 18.503 & 505.537 \\
\hline 6 & 592.302 & 169.229 & 3.761 & 12.817 & 4.940 & 16.369 & 467.672 \\
\hline 7 & 635.210 & 175.958 & 3.212 & 11.461 & 4.837 & 17.163 & 475.432 \\
\hline 8 & 640.669 & 181.493 & 3.472 & 12.473 & 5.313 & 18.100 & 512.750 \\
\hline 9 & 631.052 & 194.170 & 3.260 & 11.111 & 5.120 & 16.807 & 517.131 \\
\hline 10 & 624.202 & 180.928 & 4.051 & 13.921 & 5.887 & 17.974 & 520.998 \\
\hline 11 & 591.557 & 172.970 & 3.716 & 12.546 & 5.901 & 17.819 & 521.009 \\
\hline 12 & 506.514 & 151.651 & 3.623 & 12.093 & 4.896 & 16.246 & 486.405 \\
\hline 13 & 647.653 & 189.928 & 3.131 & 12.886 & 5.180 & 17.824 & 522.685 \\
\hline 14 & 599.429 & 128.633 & 4.148 & 15.784 & 6.416 & 20.931 & 449.162 \\
\hline 15 & 538.520 & 158.388 & 3.260 & 12.016 & 4.724 & 15.482 & 455.338 \\
\hline 16 & 506.629 & 159.820 & 3.539 & 11.952 & 4.989 & 15.953 & 503.234 \\
\hline 17 & 540.022 & 156.528 & 3.250 & 11.740 & 4.938 & 15.698 & 455.003 \\
\hline 18 & 519.700 & 157.963 & 3.222 & 11.268 & 5.213 & 14.371 & 436.818 \\
\hline 19 & 452.966 & 134.811 & 3.528 & 11.730 & 4.155 & 13.470 & 400.884 \\
\hline 20 & 629.068 & 182.339 & 3.145 & 12.635 & 4.714 & 17.708 & 513.287 \\
\hline 21 & 585.023 & 165.729 & 3.599 & 13.644 & 5.576 & 18.270 & 517.566 \\
\hline \hline Mean & 579.008 & 165.720 & 3.625 & 12.679 & 5.358 & 17.143 & 489.425 \\
\hline \multirow{2}{*}{$\begin{array}{c}\text { Standard } \\
\text { Deviation }\end{array}$} & 58.962 & 17.942 & 0.372 & 1.176 & 0.607 & 1.638 & 34.940 \\
\cline { 2 - 8 } Maximum & 647.653 & 194.170 & 4.343 & 15.784 & 6.416 & 20.931 & 538.955 \\
\hline Minimum & 452.966 & 128.633 & 3.131 & 11.111 & 4.155 & 13.470 & 400.884 \\
\hline
\end{tabular}

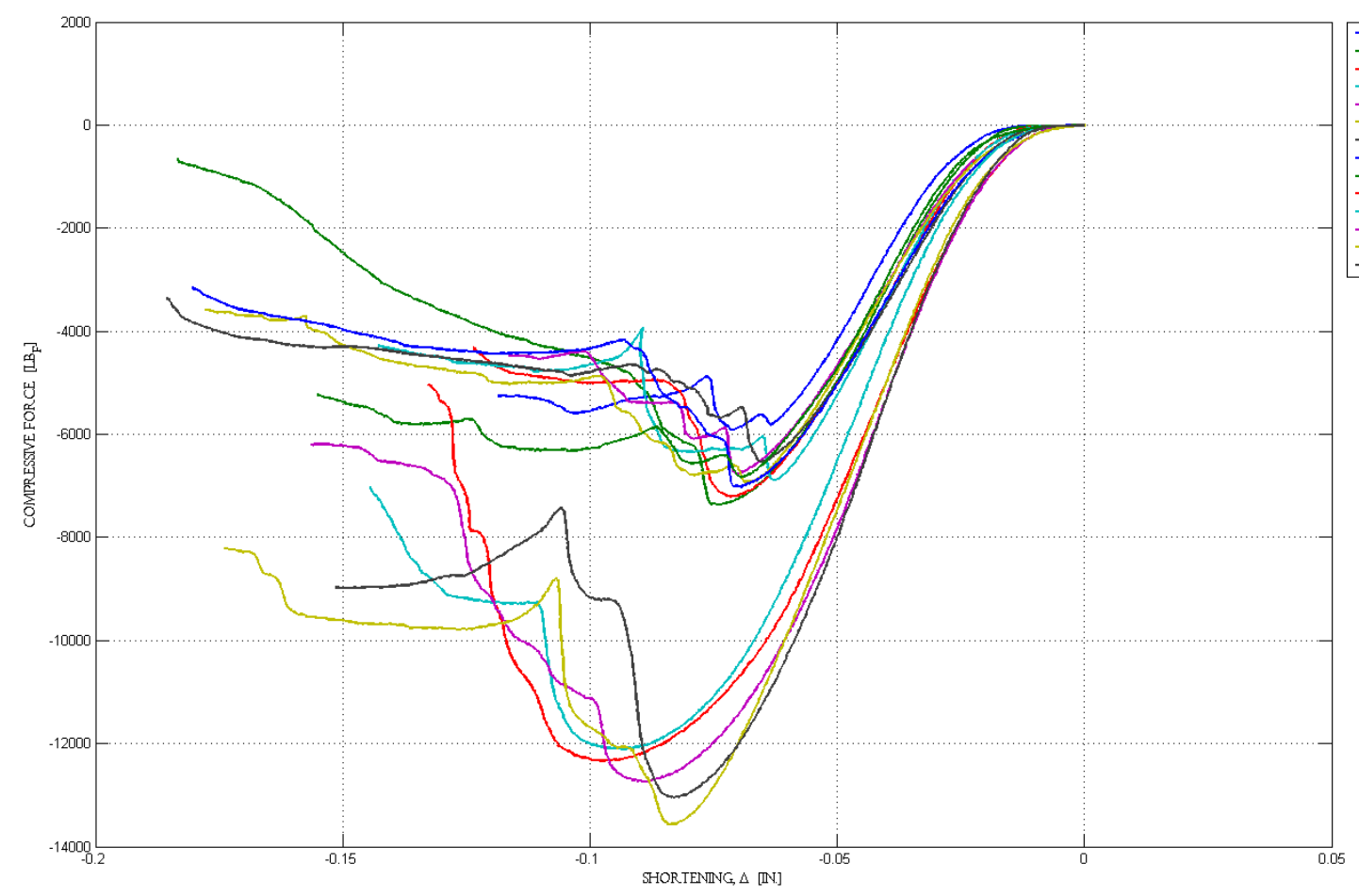

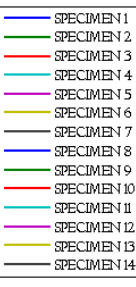

Figure A - 8. Compressive force-displacement curves of bamboo chemically treated with $3 \%$ salt solution 
Table A - 0-10. Compressive properties of bamboo chemically treated with $3 \%$ salt solution

\begin{tabular}{|c|c|c|c|c|c|c|c|}
\hline Specimen & $\begin{array}{l}\text { Modulus } \\
\text { (ksi) }\end{array}$ & $\begin{array}{c}\text { Specific } \\
\text { Modulus } \\
\text { (kips-in.//lbm) } \\
\text { x } 100\end{array}$ & $\begin{array}{c}\text { Yield } \\
\text { Strain } \\
(\%)\end{array}$ & $\begin{array}{c}\text { Yield } \\
\text { Stress } \\
\text { (ksi) }\end{array}$ & $\begin{array}{l}\text { Ultimate } \\
\text { Strain } \\
(\%)\end{array}$ & $\begin{array}{c}\text { Ultimate } \\
\text { Stress } \\
\text { (ksi) }\end{array}$ & $\begin{array}{c}\text { Specific } \\
\text { Strength } \\
\text { (kips-in.// } \mathrm{b}_{\mathrm{m}} \text { ) }\end{array}$ \\
\hline 1 & 538.377 & 177.098 & 3.961 & 10.742 & 5.254 & 13.404 & 422.835 \\
\hline 2 & 579.165 & 192.414 & 4.246 & 4.246 & 5.472 & 16.512 & 520.878 \\
\hline 3 & 511.024 & 169.213 & 3.835 & 11.362 & 5.476 & 16.343 & 522.147 \\
\hline 4 & 538.340 & 177.085 & 3.736 & 11.339 & 4.688 & 14.914 & 471.963 \\
\hline 5 & 496.678 & 163.381 & 3.799 & 10.989 & 5.207 & 15.354 & 481.307 \\
\hline 6 & 509.215 & 172.615 & 4.078 & 12.566 & 5.796 & 15.733 & 502.657 \\
\hline 7 & 502.290 & 169.121 & 3.845 & 11.943 & 5.084 & 14.734 & 478.390 \\
\hline 8 & 529.509 & 183.857 & 3.869 & 12.758 & 5.187 & 16.067 & 525.061 \\
\hline 9 & 498.479 & 156.263 & 3.619 & 9.446 & 5.092 & 13.973 & 490.281 \\
\hline 10 & 466.242 & 147.545 & 3.436 & 10.902 & 5.954 & 15.525 & 471.881 \\
\hline 11 & 506.028 & 158.134 & 3.357 & 10.085 & 5.494 & 14.967 & 473.630 \\
\hline 12 & 521.825 & 163.070 & 3.057 & 9.844 & 5.193 & 15.275 & 489.577 \\
\hline 13 & 471.694 & 151.184 & 3.821 & 12.085 & 5.433 & 15.585 & 514.361 \\
\hline 14 & 520.639 & 166.871 & 3.177 & 9.785 & 5.182 & 15.537 & 497.979 \\
\hline Mean & 513.536 & 167.704 & 3.703 & 10.578 & 5.322 & 15.280 & 490.210 \\
\hline $\begin{array}{l}\text { Standard } \\
\text { Deviation }\end{array}$ & 28.582 & 12.462 & 0.338 & 2.096 & 0.316 & 0.853 & 27.282 \\
\hline Maximum & 579.165 & 192.414 & 4.246 & 12.758 & 5.954 & 16.512 & 525.061 \\
\hline Minimum & \begin{tabular}{|l|}
466.242 \\
\end{tabular} & 147.545 & 3.057 & 4.246 & 4.688 & 13.404 & 422.835 \\
\hline
\end{tabular}




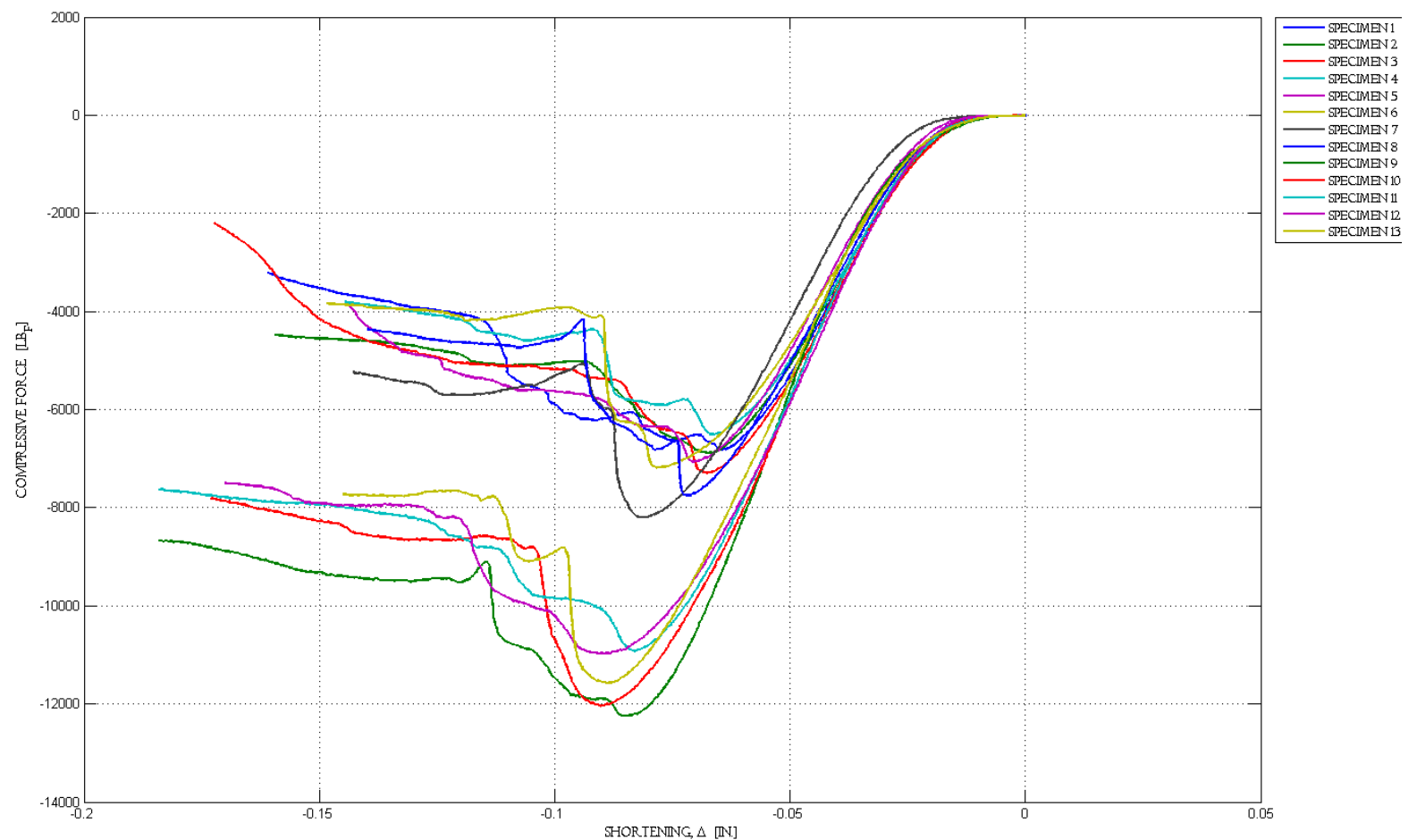

Figure A - 9. Compressive force-displacement curves of bamboo chemically treated with $6 \%$ salt solution

Table A - 0-11. Compressive properties of bamboo chemically treated with $6 \%$ salt solution

\begin{tabular}{|c|c|c|c|c|c|c|c|}
\hline Specimen & $\begin{array}{c}\text { Modulus } \\
(\mathrm{ksi})\end{array}$ & $\begin{array}{c}\text { Specific } \\
\text { Modulus } \\
\left(\mathrm{kips}-\mathrm{in} . / \mathrm{l} \mathrm{b}_{\mathrm{m}}\right) \\
\times 100\end{array}$ & $\begin{array}{c}\text { Yield } \\
\text { Strain } \\
(\%)\end{array}$ & $\begin{array}{c}\text { Yield } \\
\text { Stress } \\
(\mathrm{ksi})\end{array}$ & $\begin{array}{c}\text { Ultimate } \\
\text { Strain } \\
(\%)\end{array}$ & $\begin{array}{c}\text { Ultimate } \\
\text { Stress } \\
(\mathrm{ksi})\end{array}$ & $\begin{array}{c}\text { Specific } \\
\text { Strength } \\
(\mathrm{kips}-\mathrm{in} . / \mathrm{lb} \mathrm{m})\end{array}$ \\
\hline 1 & 453.712 & 175.857 & 3.371 & 8.960 & 5.203 & 11.564 & 448.228 \\
\hline 2 & 526.611 & 168.246 & 3.366 & 10.654 & 5.068 & 14.310 & 457.197 \\
\hline 3 & 573.965 & 185.150 & 3.344 & 11.299 & 4.835 & 15.088 & 486.712 \\
\hline 4 & 526.136 & 163.396 & 3.195 & 9.879 & 4.902 & 13.680 & 424.838 \\
\hline 5 & 558.700 & 179.070 & 3.448 & 10.640 & 5.125 & 14.716 & 471.653 \\
\hline 6 & 491.264 & 157.963 & 3.667 & 11.029 & 5.082 & 14.815 & 476.377 \\
\hline 7 & 520.393 & 181.322 & 3.522 & 9.217 & 5.196 & 14.735 & 513.421 \\
\hline 8 & 524.151 & 168.537 & 3.911 & 12.671 & 4.911 & 15.726 & 505.667 \\
\hline 9 & 558.419 & 186.140 & 3.900 & 12.402 & 4.872 & 15.160 & 505.331 \\
\hline 10 & 520.200 & 160.062 & 3.594 & 10.780 & 5.664 & 16.136 & 496.507 \\
\hline 11 & 503.446 & 150.732 & 3.706 & 11.416 & 5.345 & 14.876 & 445.379 \\
\hline 12 & 485.500 & 142.375 & 3.255 & 9.291 & 5.721 & 15.098 & 442.747 \\
\hline 13 & 494.415 & 146.711 & 3.354 & 8.756 & 5.344 & 15.413 & 457.371 \\
\hline \hline Mean & 518.224 & 166.582 & 3.510 & 10.534 & 5.174 & 14.717 & 471.648 \\
\hline $\begin{array}{c}\text { Standard } \\
\text { Deviation }\end{array}$ & 33.270 & 14.583 & 0.232 & 1.267 & 0.284 & 1.126 & 28.257 \\
\hline Maximum & 573.965 & 186.140 & 3.911 & 12.671 & 5.721 & 16.136 & 513.421 \\
\hline
\end{tabular}




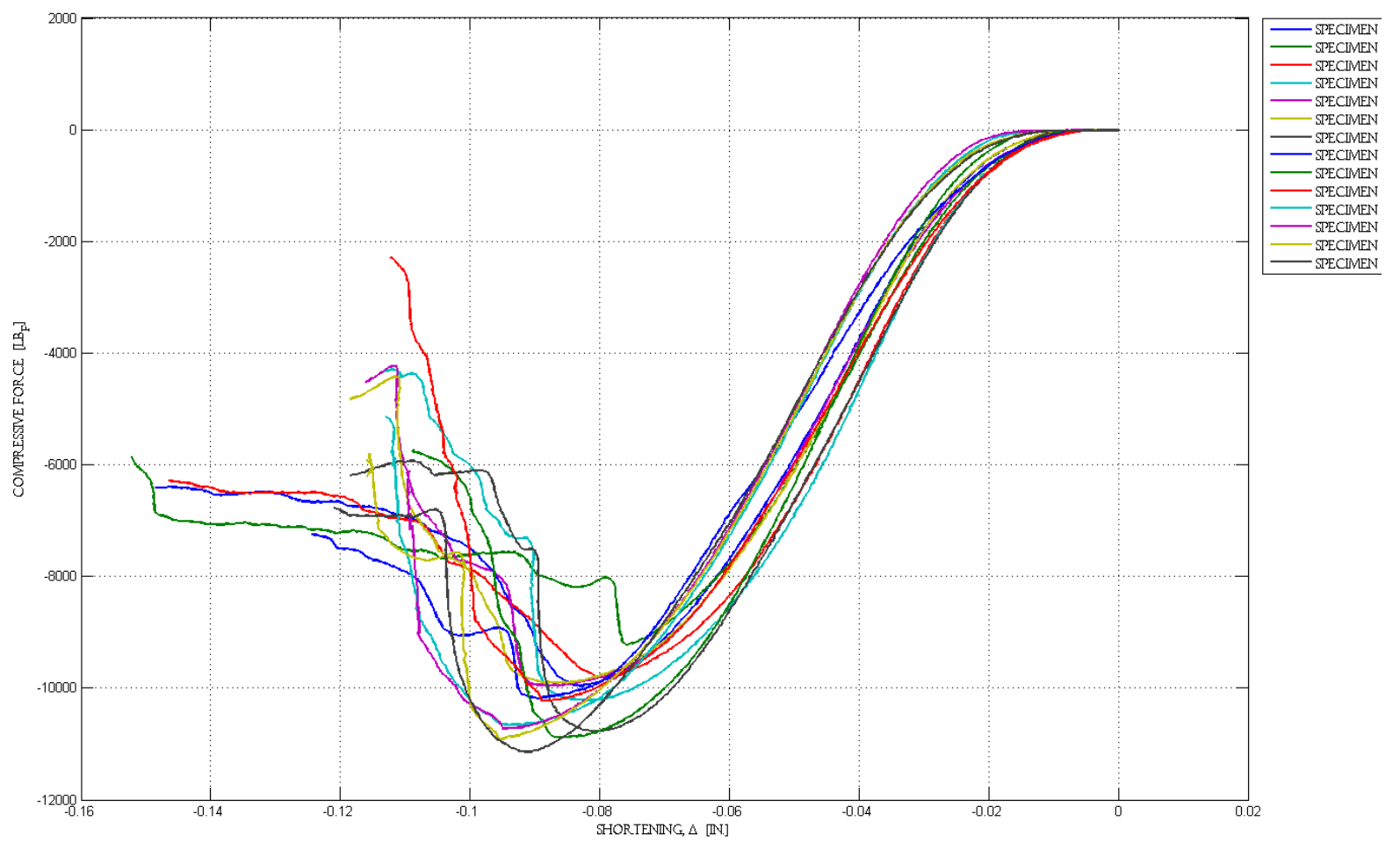

Figure A - 10. Compressive force-displacement curves of bamboo chemicallv treated with $9 \%$ salt solution

Table A - 0-12. Compressive properties of bamboo chemically treated with $9 \%$ salt solution

\begin{tabular}{|c|c|c|c|c|c|c|c|}
\hline Specimen & $\begin{array}{c}\text { Modulus } \\
(\mathrm{ksi})\end{array}$ & $\begin{array}{c}\text { Specific } \\
\text { Modulus } \\
(\mathrm{kips}-\mathrm{in} . / \mathrm{l} \mathrm{b}) \\
\times 100\end{array}$ & $\begin{array}{c}\text { Yield } \\
\text { Strain } \\
(\%)\end{array}$ & $\begin{array}{c}\text { Yield } \\
\text { Stress } \\
(\mathrm{ksi})\end{array}$ & $\begin{array}{c}\text { Ultimate } \\
\text { Strain } \\
(\%)\end{array}$ & $\begin{array}{c}\text { Ultimate } \\
\text { Stress } \\
(\mathrm{ksi})\end{array}$ & $\begin{array}{c}\text { Specific } \\
\text { Strength } \\
(\mathrm{kips}-\mathrm{in} . / \mathrm{lb} \text { ) }\end{array}$ \\
\hline 1 & 563.267 & 165.181 & 3.394 & 10.656 & 5.322 & 16.698 & 489.685 \\
\hline 2 & 554.409 & 154.863 & 3.540 & 11.590 & 5.309 & 15.973 & 446.163 \\
\hline 3 & 591.564 & 176.586 & 3.107 & 10.265 & 5.340 & 16.259 & 485.343 \\
\hline 4 & 588.162 & 174.529 & 3.051 & 10.060 & 5.346 & 16.475 & 488.881 \\
\hline 5 & 566.665 & 168.650 & 3.493 & 11.345 & 5.680 & 16.726 & 497.790 \\
\hline 6 & 572.761 & 165.538 & 3.263 & 10.343 & 5.799 & 16.866 & 487.465 \\
\hline 7 & 499.769 & 151.445 & 3.595 & 11.305 & 5.445 & 16.542 & 501.272 \\
\hline 8 & 524.192 & 142.443 & 3.131 & 9.422 & 5.172 & 16.081 & 436.980 \\
\hline 9 & 711.905 & 180.229 & 3.298 & 12.826 & 5.172 & 18.801 & 475.966 \\
\hline 10 & 577.917 & 144.119 & 3.222 & 11.326 & 5.351 & 17.446 & 435.051 \\
\hline 11 & 614.890 & 156.063 & 3.615 & 11.847 & 5.536 & 17.671 & 448.504 \\
\hline 12 & 565.035 & 145.628 & 3.827 & 12.014 & 5.673 & 17.217 & 443.746 \\
\hline 13 & 631.063 & 158.559 & 3.508 & 11.812 & 5.576 & 18.780 & 471.861 \\
\hline 14 & 628.845 & 159.605 & 3.668 & 12.570 & 5.470 & 19.439 & 493.381 \\
\hline
\end{tabular}




\begin{tabular}{|c||c|c|c|c|c|c|c||}
\hline Mean & 585.032 & 160.246 & 3.408 & 11.241 & 5.442 & 17.212 & 471.578 \\
\cline { 2 - 8 } & 51.441 & 12.125 & 0.235 & 0.981 & 0.190 & 1.093 & 24.206 \\
\hline $\begin{array}{c}\text { Standard } \\
\text { Deviation }\end{array}$ & 711.905 & 180.229 & 3.827 & 12.826 & 5.799 & 19.439 & 501.272 \\
\hline Maximum & 71.49 & 14.423 & 3.051 & 9.422 & 5.172 & 15.973 & 435.051 \\
\hline Minimum & 499.769 & 142.443 \\
\hline
\end{tabular}

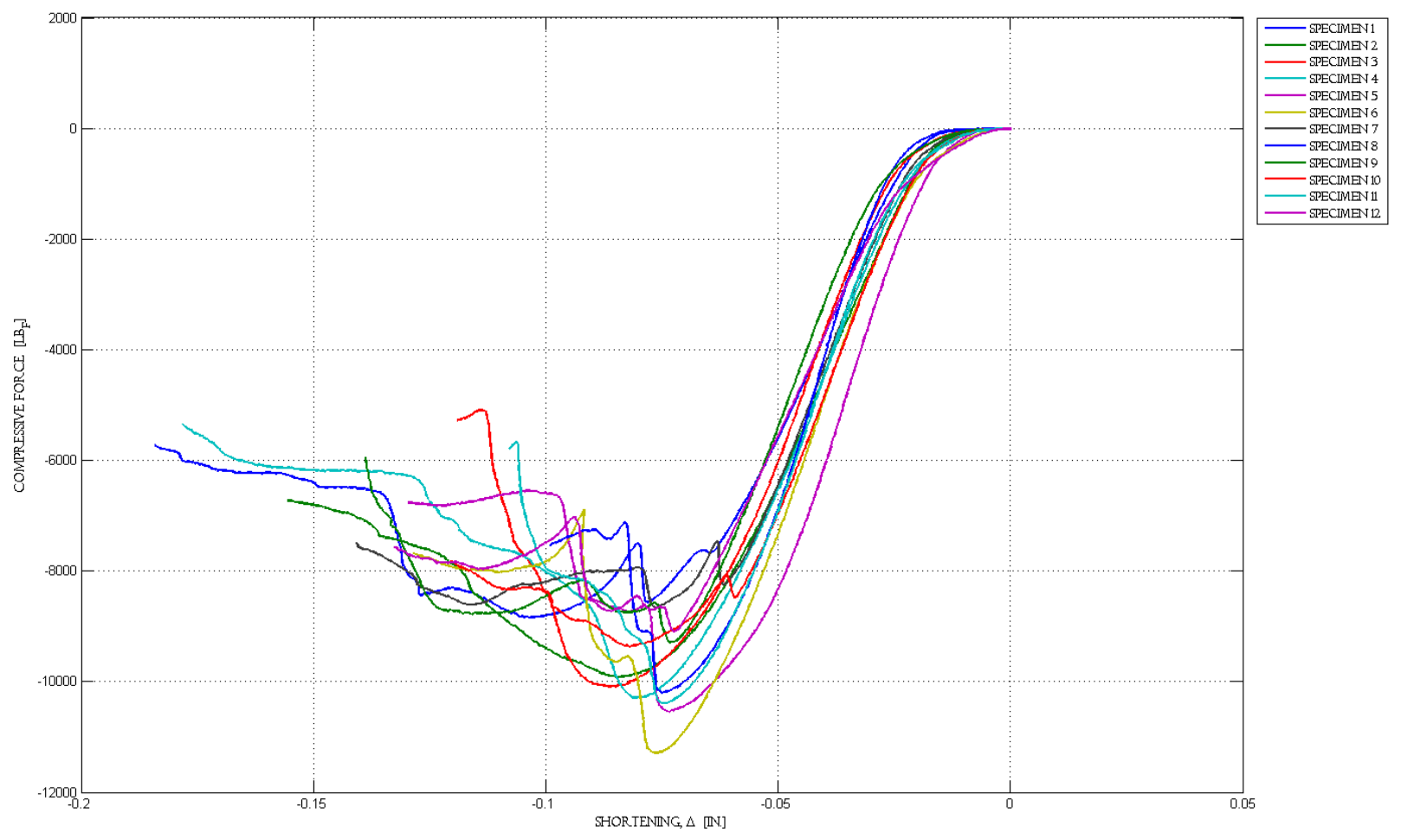

Figure A - 11. Compressive force-displacement curves of bamboo chemically treated with lime

Table A - 0-13. Compressive properties of bamboo chemically treated with lime

\begin{tabular}{|c|c|c|c|c|c|c|c|}
\hline Specimen & $\begin{array}{c}\text { Modulus } \\
(\mathrm{ksi})\end{array}$ & $\begin{array}{c}\text { Specific } \\
\text { Modulus } \\
\left(\mathrm{kips}-\mathrm{in} . / \mathrm{l} \mathrm{b}_{\mathrm{m}}\right) \\
\mathrm{x} 100\end{array}$ & $\begin{array}{c}\text { Yield } \\
\text { Strain } \\
(\%)\end{array}$ & $\begin{array}{c}\text { Yield } \\
\text { Stress } \\
(\mathrm{ksi})\end{array}$ & $\begin{array}{c}\text { Ultimate } \\
\text { Strain } \\
(\%)\end{array}$ & $\begin{array}{c}\text { Ultimate } \\
\text { Stress } \\
(\mathrm{ksi})\end{array}$ & $\begin{array}{c}\text { Specific } \\
\begin{array}{c}\text { Strength } \\
\left(\mathrm{kips} \text {-in.// } \mathrm{b}_{\mathrm{m}}\right)\end{array}\end{array}$ \\
\hline 1 & 418.049 & 116.125 & 3.961 & 10.171 & 6.805 & 13.663 & 379.530 \\
\hline 2 & 523.310 & 133.839 & 3.265 & 10.577 & 5.914 & 16.649 & 425.815 \\
\hline 3 & 585.574 & 156.153 & 3.333 & 9.983 & 5.785 & 16.853 & 449.401 \\
\hline 4 & 463.591 & 124.957 & 3.973 & 11.344 & 5.936 & 15.724 & 423.825 \\
\hline 5 & 619.667 & 182.255 & 2.841 & 10.276 & 4.923 & 16.179 & 475.853 \\
\hline 6 & 590.844 & 181.798 & 3.132 & 11.151 & 4.714 & 16.772 & 516.062 \\
\hline 7 & 532.517 & 154.353 & 3.456 & 11.106 & 6.685 & 13.264 & 384.457 \\
\hline 8 & 754.240 & 215.497 & 3.106 & 10.929 & 4.557 & 16.660 & 475.987 \\
\hline 9 & 568.970 & 162.100 & 3.489 & 10.398 & 4.702 & 15.192 & 432.809 \\
\hline 10 & 597.894 & 164.257 & 3.051 & 10.950 & 5.225 & 15.715 & 431.721 \\
\hline
\end{tabular}




\begin{tabular}{|c|c|c|c|c|c|c|c|}
\hline 11 & 581.503 & 165.670 & 3.417 & 11.110 & 5.090 & 16.530 & 470.952 \\
\hline 12 & 430.317 & 125.457 & 3.638 & 10.146 & 4.708 & 13.904 & 405.350 \\
\hline Mean & 555.540 & 156.872 & 3.388 & 10.678 & 5.420 & 15.592 & 439.314 \\
\hline $\begin{array}{l}\text { Standard } \\
\text { Deviation }\end{array}$ & 91.948 & 28.707 & 0.347 & 0.472 & 0.787 & 1.302 & 40.376 \\
\hline Maximum & 754.240 & 215.497 & 3.973 & 11.344 & 6.805 & 16.853 & 516.062 \\
\hline Minimum & 418.049 & 116.125 & 2.841 & 9.983 & 4.557 & 13.264 & 379.530 \\
\hline
\end{tabular}

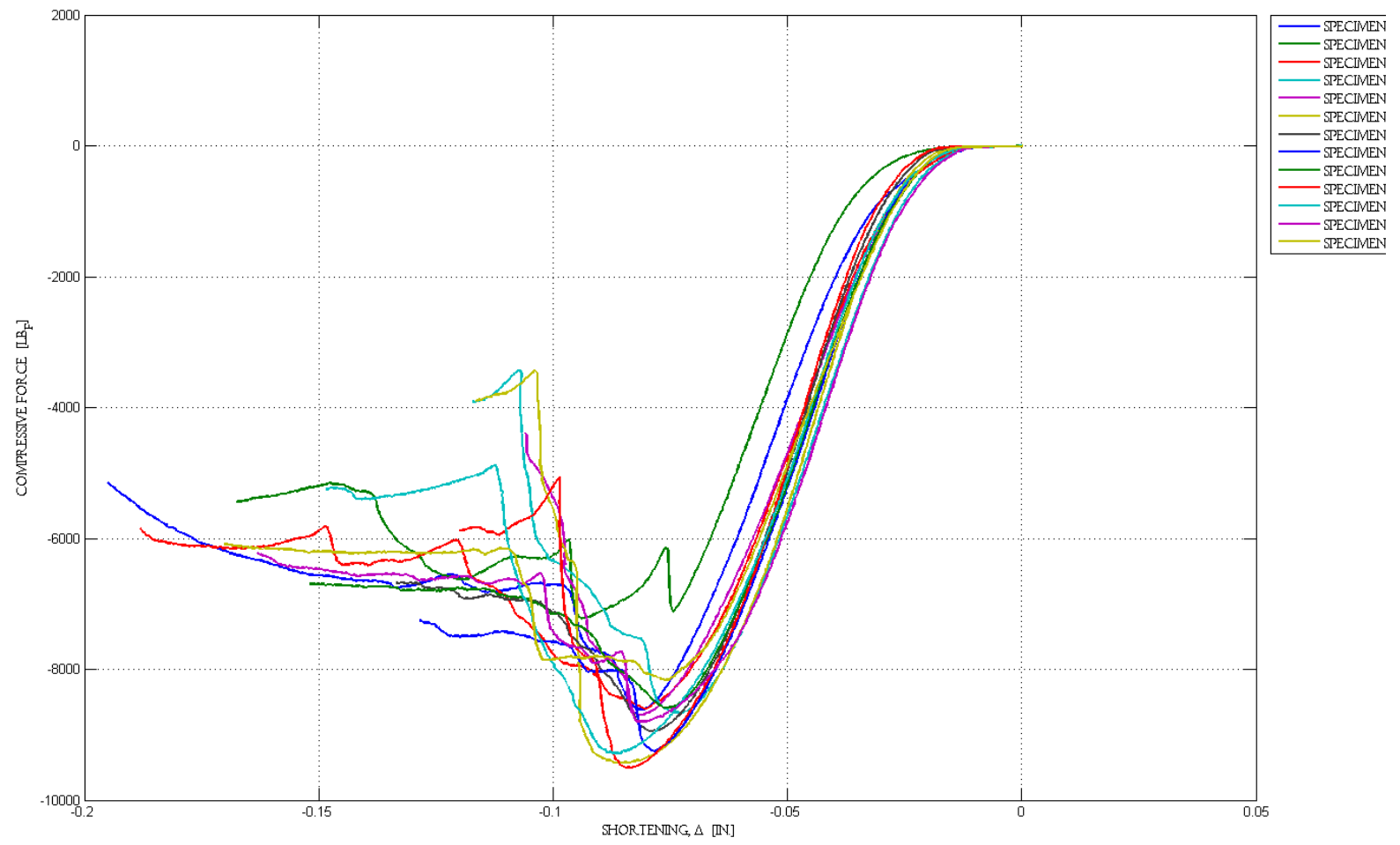

Figure A - 12. Compressive force-displacement curves of bamboo chemically treated with oil

Table A - 0-14. Compressive properties of bamboo chemically treated with oil

\begin{tabular}{|c|c|c|c|c|c|c|c|}
\hline Specimen & $\begin{array}{c}\text { Modulus } \\
(\mathrm{ksi})\end{array}$ & $\begin{array}{c}\text { Specific } \\
\text { Modulus } \\
(\mathrm{kips}- \\
\text { in.//bm) } \\
\times 100\end{array}$ & $\begin{array}{c}\text { Yield } \\
\text { Strain } \\
(\%)\end{array}$ & $\begin{array}{c}\text { Yield } \\
\text { Stress } \\
(\mathrm{ksi})\end{array}$ & $\begin{array}{c}\text { Ultimate } \\
\text { Strain } \\
(\%)\end{array}$ & $\begin{array}{c}\text { Ultimate } \\
\text { Stress } \\
(\mathrm{ksi})\end{array}$ & $\begin{array}{c}\text { Specific } \\
\text { Strength } \\
\left(\mathrm{kips}-\mathrm{in}_{\mathrm{m}} / \mathrm{lb}_{\mathrm{m}}\right)\end{array}$ \\
\hline 1 & 538.928 & 116.125 & 4.05094 & 10.52852 & 6.10653 & 15.64575 & 453.50000 \\
\hline 2 & 536.4895 & 133.839 & 4.5988 & 11.46892 & 6.5627 & 13.64993 & 382.35098 \\
\hline 3 & 510.237 & 156.153 & 4.07799 & 11.50041 & 5.97735 & 14.86475 & 446.38889 \\
\hline 4 & 551.7009 & 124.957 & 3.71268 & 11.11794 & 5.37045 & 16.00398 & 457.25657 \\
\hline 5 & 505.8645 & 182.255 & 3.93512 & 10.92906 & 5.63611 & 15.96006 & 459.94409 \\
\hline 6 & 612.94 & 181.798 & 3.72729 & 11.72446 & 5.90703 & 17.3885 & 487.07283 \\
\hline 7 & 588.8467 & 154.353 & 3.77577 & 10.453 & 5.48389 & 15.67257 & 462.31770 \\
\hline 8 & 501.5958 & 215.497 & 3.97351 & 10.83503 & 5.25395 & 14.26256 & 483.47661 \\
\hline
\end{tabular}




\begin{tabular}{|c|c|c|c|c|c|c|c|}
\hline 9 & 539.9849 & 162.100 & 3.61599 & 9.93212 & 5.88012 & 15.15181 & 450.94673 \\
\hline 10 & 585.3551 & 164.257 & 4.05937 & 11.03371 & 5.94414 & 16.22204 & 484.24000 \\
\hline 11 & 512.908 & 165.670 & 3.79531 & 9.49189 & 6.19961 & 16.14377 & 479.04362 \\
\hline 12 & 532.7865 & 125.457 & 3.4803 & 9.72854 & 5.82252 & 14.77157 & 453.11564 \\
\hline 13 & 437.896 & 125.457 & 4.24374 & 11.08502 & 6.05636 & 13.21563 & 419.54381 \\
\hline \hline Mean & 535.041 & 156.872 & 3.926678 & 10.75605 & 5.861597 & 15.30407 & 455.3229 \\
\hline $\begin{array}{c}\text { Standard } \\
\text { Deviation }\end{array}$ & 45.02889 & 28.707 & 0.293236 & 0.69695 & 0.357286 & 1.145556 & 28.84699 \\
\hline Maximum & 612.94 & 215.497 & 4.5988 & 11.72446 & 6.5627 & 17.3885 & 487.0728 \\
\hline Minimum & 437.896 & 116.125 & 3.4803 & 9.49189 & 5.25395 & 13.21563 & 382.351 \\
\hline
\end{tabular}




\section{A3. Tension Testing Data}

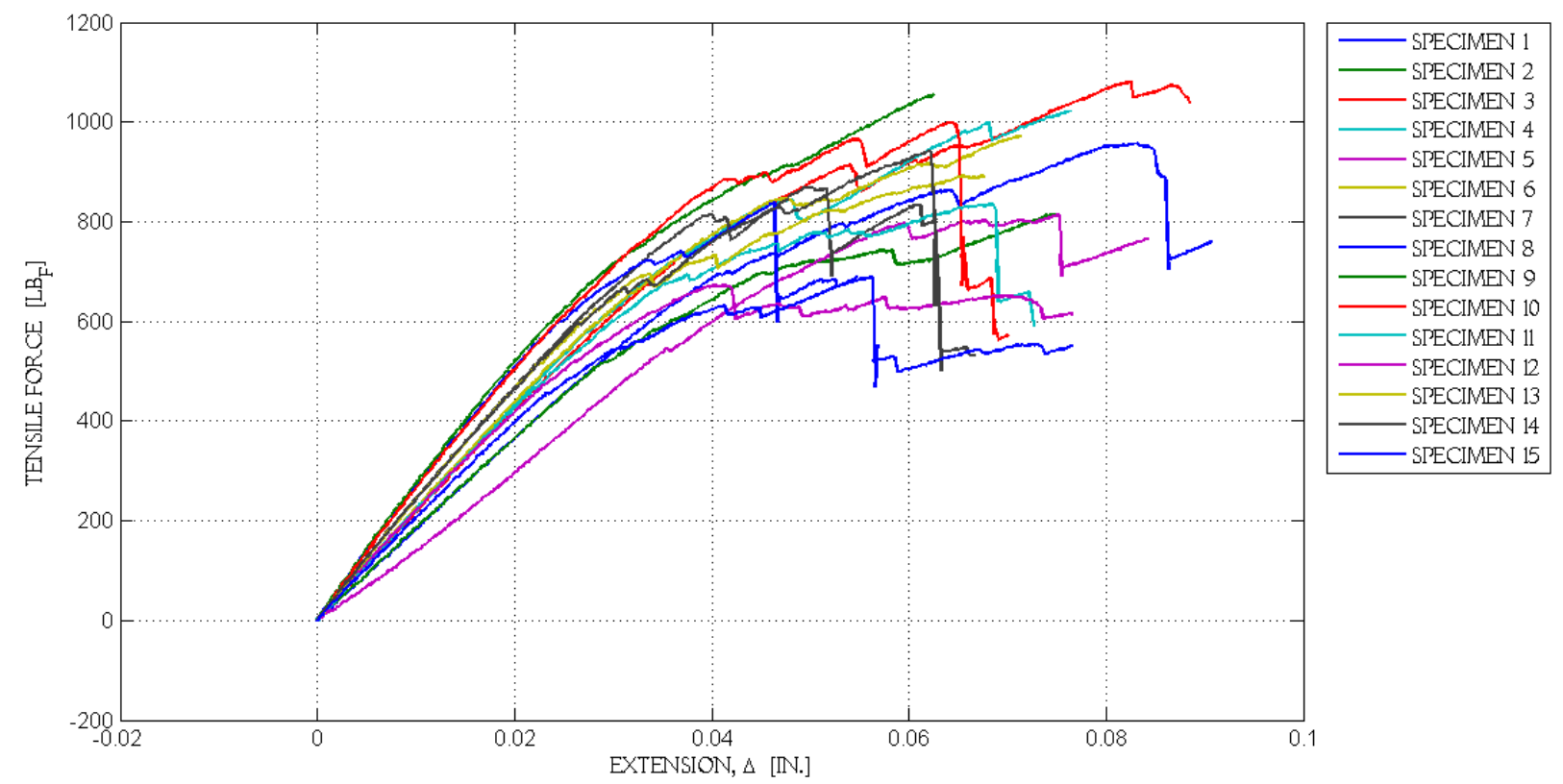

Figure A - 13. Tensile force-displacement curves of untreated bamboo

Table A - 0-15. Tensile properties of untreated bamboo

\begin{tabular}{|c|c|c|c|c|c|c|c|}
\hline Specimen & $\begin{array}{l}\text { Modulus } \\
(\mathrm{ksi})\end{array}$ & $\begin{array}{c}\text { Specific } \\
\text { Modulus } \\
\text { (kips-in.//lb }) \\
\times 100\end{array}$ & $\begin{array}{c}\text { Yield } \\
\text { Strain } \\
(\%)\end{array}$ & $\begin{array}{c}\text { Yield } \\
\text { Stress } \\
\text { (ksi) }\end{array}$ & $\begin{array}{l}\text { Ultimate } \\
\text { Strain } \\
(\%)\end{array}$ & $\begin{array}{c}\text { Ultimate } \\
\text { Stress } \\
\text { (ksi) }\end{array}$ & $\begin{array}{r}\text { Specific } \\
\text { Strength } \\
\text { (kips-in./lb } \mathrm{b}_{\mathrm{m}}\end{array}$ \\
\hline 1 & 565.762 & 175.394 & 2.604 & 14.705 & 6.910 & 25.111 & 778.479 \\
\hline 2 & 769.977 & 235.673 & 1.756 & 13.693 & 4.559 & 25.737 & 787.737 \\
\hline 3 & 697.187 & 202.755 & 1.884 & 13.384 & 6.761 & 30.003 & 872.546 \\
\hline 4 & 675.700 & 199.082 & 1.616 & 11.086 & 6.025 & 26.066 & 767.975 \\
\hline 5 & 592.12 & 182.439 & 2.734 & 15.875 & 6.700 & 23.121 & 712.387 \\
\hline 6 & 753.78 & 215.806 & 1.613 & 12.352 & 5.961 & 28.163 & 806.296 \\
\hline 7 & 674.018 & 218.972 & 1.324 & 9.335 & 3.869 & 20.397 & 662.638 \\
\hline 8 & 876.401 & 264.314 & 1.188 & 10.920 & 3.536 & 23.297 & 702.624 \\
\hline 9 & 896.622 & 274.768 & 1.327 & 12.416 & 5.251 & 30.633 & 938.731 \\
\hline 10 & 796.391 & 242.005 & 1.497 & 12.287 & 5.033 & 25.805 & 784.141 \\
\hline 11 & 842.395 & 281.017 & 1.605 & 13.738 & 5.191 & 26.581 & 886.731 \\
\hline 12 & 786.676 & 246.995 & 1.581 & 12.729 & 3.326 & 21.401 & 671.935 \\
\hline 13 & 894.751 & 260.314 & 1.559 & 14.247 & 5.751 & 29.367 & 854.380 \\
\hline 14 & 850.207 & 267.610 & 1.678 & 14.786 & 4.709 & 28.440 & 895.184 \\
\hline 15 & 926.549 & 286.383 & 1.662 & 15.564 & 4.596 & 26.575 & 821.408 \\
\hline Mean & |773.236 & 236.902 & 1.709 & 13.141 & 5.212 & 26.046 & 796.213 \\
\hline $\begin{array}{l}\text { Standard } \\
\text { Deviation }\end{array}$ & $\mid 112.421$ & 34.941 & 0.428 & 1.815 & 1.142 & 3.037 & 83.943 \\
\hline
\end{tabular}




\begin{tabular}{||l||c|c|c|c|c|c|c||}
\hline Maximum & 926.549 & 286.383 & 2.734 & 15.875 & 6.910 & 30.633 & 938.731 \\
\hline Minimum & 565.762 & 175.394 & 1.188 & 9.335 & 3.326 & 20.397 & 662.638 \\
\hline
\end{tabular}

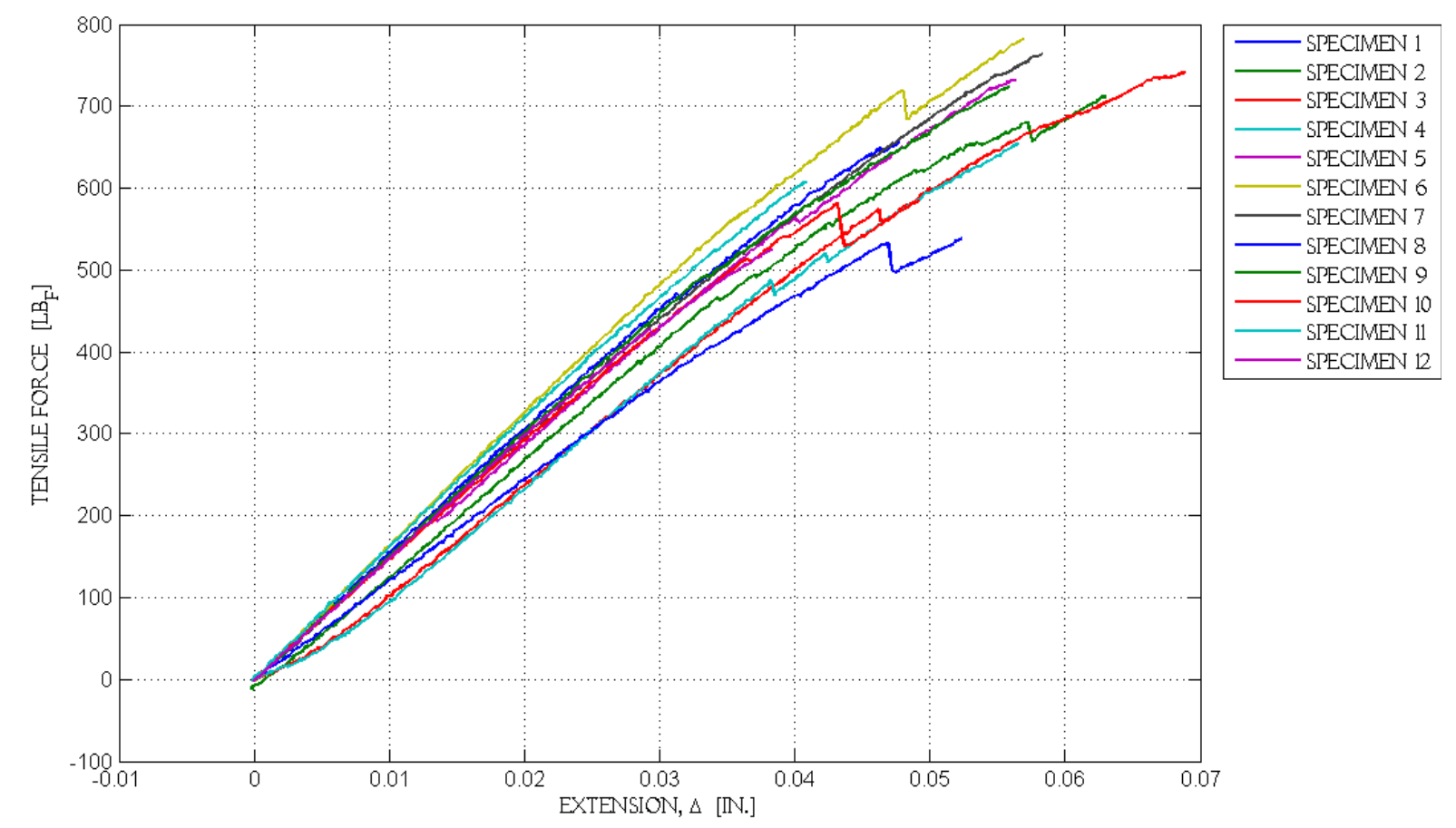

Figure A - 14. Tensile force-displacement curves of bamboo thermally treated at $180^{\circ} \mathrm{F}$ with $1^{\circ} \mathrm{F} / \mathrm{min}$. rate

Table A - 0-16. Tensile properties of bamboo thermally treated at $180^{\circ} \mathrm{F}$ with $1^{\circ} \mathrm{F} / \mathrm{min}$. rate

\begin{tabular}{|c|c|c|c|c|c|c|c|}
\hline Specimen & $\begin{array}{c}\text { Modulus } \\
(\mathrm{ksi})\end{array}$ & $\begin{array}{c}\text { Specific } \\
\text { Modulus } \\
\left(\mathrm{kips}-\mathrm{in} . / \mathrm{l} \mathrm{b}_{\mathrm{m}}\right) \\
\mathrm{\times} 100\end{array}$ & $\begin{array}{c}\text { Yield } \\
\text { Strain } \\
(\%)\end{array}$ & $\begin{array}{c}\text { Yield } \\
\text { Stress } \\
(\mathrm{ksi})\end{array}$ & $\begin{array}{c}\text { Ultimate } \\
\text { Strain } \\
(\%)\end{array}$ & $\begin{array}{c}\text { Ultimate } \\
\text { Stress } \\
(\mathrm{ksi})\end{array}$ & $\begin{array}{c}\text { Specific } \\
\text { Strength } \\
(\mathrm{kips}-\mathrm{in} . / \mathrm{l} \mathrm{b})\end{array}$ \\
\hline 1 & 1692.52 & 729.277 & 1.57 & 25.67 & 2.40 & 35.70 & 1538.424 \\
\hline 2 & 1211.92 & 514.318 & 2.86 & 28.80 & 3.15 & 30.09 & 1277.176 \\
\hline 3 & 1468.03 & 572.825 & 2.32 & 30.60 & 3.46 & 38.86 & 1516.145 \\
\hline 4 & 1261.16 & 520.523 & 1.91 & 21.69 & 2.82 & 29.12 & 1201.902 \\
\hline 5 & 1295.81 & 581.060 & 2.68 & 24.92 & 3.50 & 32.43 & 1454.276 \\
\hline 6 & 1458.42 & 610.378 & 2.40 & 31.83 & 2.85 & 34.65 & 1450.306 \\
\hline 7 & 1396.18 & 638.250 & 2.93 & 35.65 & 2.93 & 35.65 & 1629.484 \\
\hline 8 & 1183.94 & 548.529 & 2.63 & 25.60 & 2.65 & 25.86 & 1197.934 \\
\hline 9 & 1285.04 & 567.919 & 2.79 & 30.57 & 2.79 & 30.57 & 1351.068 \\
\hline 10 & 1066.97 & 464.530 & 2.15 & 21.07 & 2.45 & 21.19 & 922.6538 \\
\hline 11 & 1530.77 & 600.730 & 2.06 & 29.09 & 2.06 & 29.09 & 1141.516 \\
\hline 12 & 1423.57 & 641.967 & 1.47 & 20.48 & 1.92 & 25.07 & 1130.483 \\
\hline \hline Mean & 1350.069 & 582.526 & 2.39 & 27.77 & 2.82 & 31.20 & 1334.626 \\
\hline Standard & 171.773 & 69.522 & 0.49 & 4.72 & 0.49 & 5.10 & 207.629 \\
\hline
\end{tabular}




\begin{tabular}{||c||c|c|c|c|c|c|c||}
\hline Deviation & & & & & & & \\
\hline Maximum & 1692.52 & 729.277 & 2.93 & 35.65 & 3.5 & 38.86 & 1629.484 \\
\hline Minimum & 1066.97 & 464.530 & 1.47 & 20.48 & 1.92 & 21.19 & 922.6538 \\
\hline
\end{tabular}

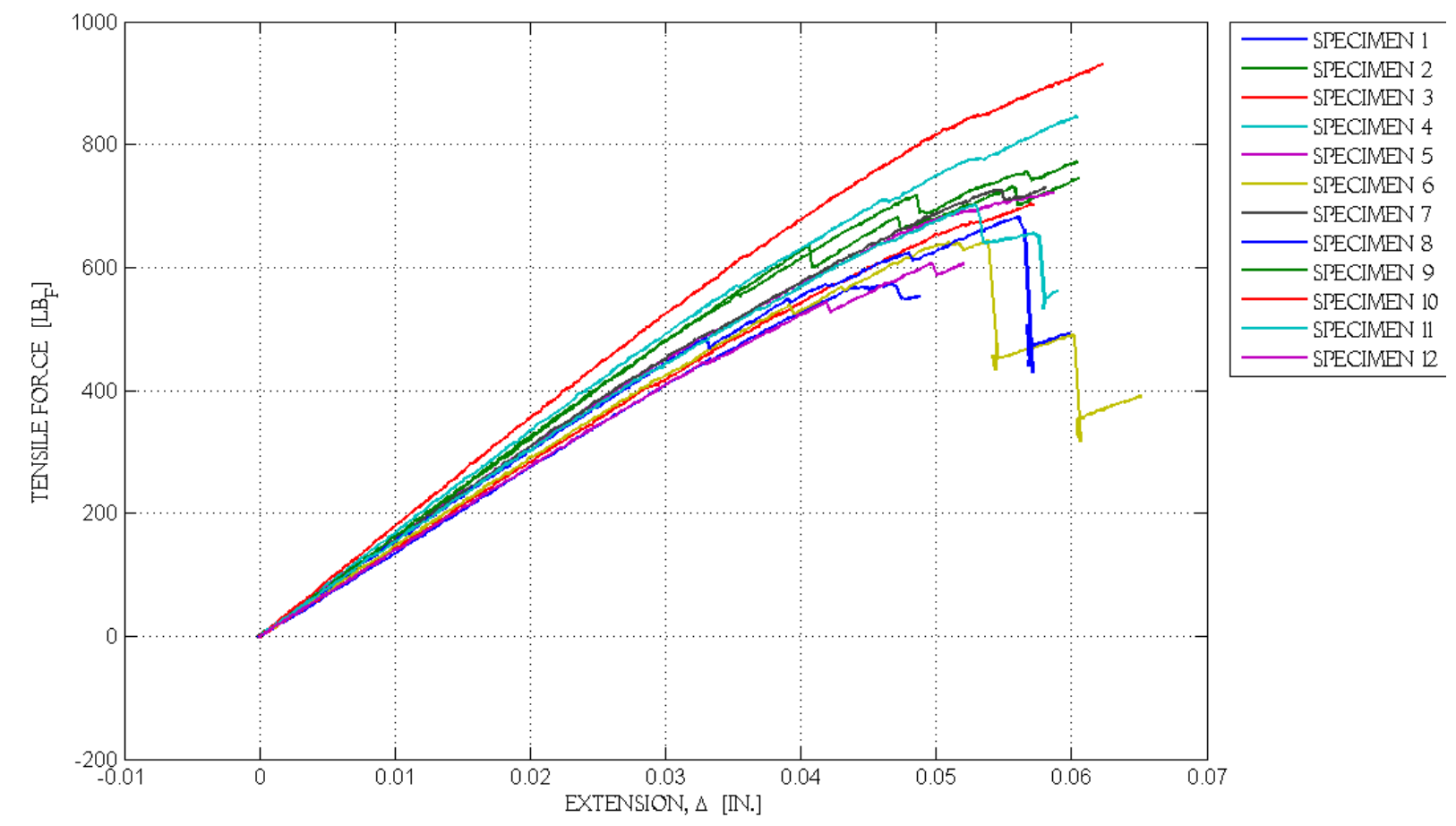

Figure A - 15. Tensile force-displacement curves of bamboo thermallv treated at $200^{\circ} \mathrm{F}$ with $1^{\circ} \mathrm{F} / \mathrm{min}$. rate

Table A - 0-17. Tensile properties of bamboo thermally treated at $200^{\circ} \mathrm{F}$ with $1^{\circ} \mathrm{F} / \mathrm{min}$. rate

\begin{tabular}{|c|c|c|c|c|c|c|c|}
\hline Specimen & $\begin{array}{c}\text { Modulus } \\
(\mathrm{ksi})\end{array}$ & $\begin{array}{c}\text { Specific } \\
\text { Modulus } \\
(\mathrm{kips-in.// \textrm {b } m}) \\
\times 100\end{array}$ & $\begin{array}{c}\text { Yield } \\
\text { Strain } \\
(\%)\end{array}$ & $\begin{array}{c}\text { Yield } \\
\text { Stress } \\
(\mathrm{ksi})\end{array}$ & $\begin{array}{c}\text { Ultimate } \\
\text { Strain } \\
(\%)\end{array}$ & $\begin{array}{c}\text { Ultimate } \\
\text { Stress } \\
(\mathrm{ksi})\end{array}$ & $\begin{array}{c}\text { Specific } \\
\text { Strength } \\
\left(\mathrm{kips}-\mathrm{in} . / \mathrm{l} \mathrm{b}_{\mathrm{m}}\right)\end{array}$ \\
\hline 1 & 1158.72 & 523.422 & 2.35 & 24.00 & 2.79 & 24.91 & 1125.308 \\
\hline 2 & 1480.46 & 586.502 & 2.04 & 28.96 & 3.09 & 34.08 & 1350.155 \\
\hline 3 & 1371.65 & 531.005 & 2.96 & 34.37 & 2.96 & 34.37 & 1330.747 \\
\hline 4 & 1512.29 & 589.891 & 3.10 & 38.69 & 3.10 & 38.69 & 1509.351 \\
\hline 5 & 1195.57 & 512.267 & 3.01 & 28.19 & 3.01 & 28.19 & 1207.831 \\
\hline 6 & 1129.80 & 504.920 & 1.98 & 21.05 & 2.72 & 25.15 & 1123.874 \\
\hline 7 & 1319.11 & 544.502 & 2.73 & 30.61 & 3.00 & 31.48 & 1299.237 \\
\hline 8 & 1703.20 & 698.397 & 1.65 & 26.75 & 2.79 & 37.48 & 1536.917 \\
\hline 9 & 1462.66 & 552.002 & 2.43 & 32.29 & 3.02 & 34.72 & 1310.415 \\
\hline 10 & 1707.44 & 625.877 & 3.16 & 44.39 & 3.16 & 44.39 & 1627.186 \\
\hline 11 & 1336.70 & 523.574 & 2.67 & 30.67 & 2.67 & 30.67 & 1201.272 \\
\hline 12 & 1305.00 & 615.614 & 2.11 & 25.58 & 2.71 & 29.30 & 1382.065 \\
\hline \hline Mean & 1390.217 & 567.331 & 2.51 & 30.46 & 2.91 & 32.78 & 1333.696 \\
\hline
\end{tabular}




\begin{tabular}{||c||c|c|c|c|c|c|c||}
\hline $\begin{array}{c}\text { Standard } \\
\text { Deviation }\end{array}$ & 191.0984 & 57.729 & 0.50 & 6.43 & 0.17 & 5.71 & 160.5297 \\
\hline Maximum & 1707.44 & 698.397 & 3.16 & 44.39 & 3.16 & 44.39 & 1627.186 \\
\hline Minimum & 1129.8 & 504.920 & 1.65 & 21.05 & 2.67 & 24.91 & 1123.874 \\
\hline
\end{tabular}

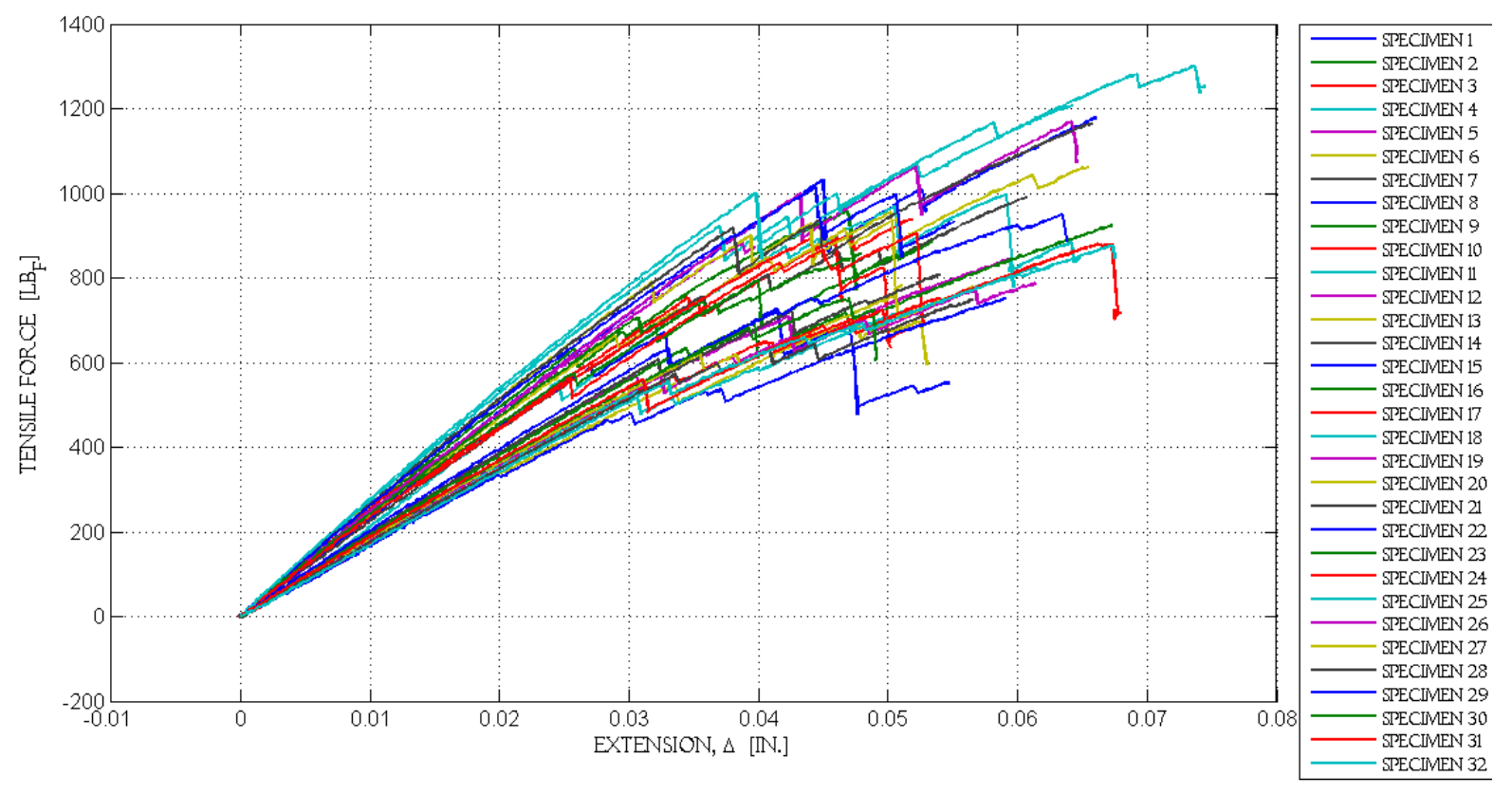

Figure A - 16. Tensile force-displacement curves of bamboo thermallv treated at $220^{\circ} \mathrm{F}$ with $1^{\circ} \mathrm{F} / \mathrm{min}$. rate

Table A - 0-18. Tensile properties of bamboo thermally treated at $220^{\circ} \mathrm{F}$ with $1^{\circ} \mathrm{F} / \mathrm{min}$. rate

\begin{tabular}{|c|c|c|c|c|c|c|c|}
\hline Specimen & $\begin{array}{c}\text { Modulus } \\
(\mathrm{ksi})\end{array}$ & $\begin{array}{c}\text { Specific } \\
\text { Modulus } \\
(\text { (kips-in./lb } \\
\times 100\end{array}$ & $\begin{array}{c}\text { Yield } \\
\text { Strain } \\
(\%)\end{array}$ & $\begin{array}{c}\text { Yield } \\
\text { Stress } \\
(\mathrm{ksi})\end{array}$ & $\begin{array}{c}\text { Ultimate } \\
\text { Strain } \\
(\%)\end{array}$ & $\begin{array}{c}\text { Ultimate } \\
\text { Stress } \\
(\mathrm{ksi})\end{array}$ & $\begin{array}{c}\text { Specific } \\
\text { Strength } \\
(\mathrm{kips}-\mathrm{in} . / \mathrm{l} \mathrm{b})\end{array}$ \\
\hline 1 & 1708.04 & 552.481 & 2.27 & 36.63 & 3.40 & 42.32 & 1369.02 \\
\hline 2 & 1866.62 & 530.842 & 1.39 & 24.80 & 2.53 & 37.57 & 1068.374 \\
\hline 3 & 2079.09 & 668.351 & 1.73 & 32.41 & 1.69 & 41.89 & 1346.496 \\
\hline 4 & 1819.84 & 543.295 & 1.27 & 22.05 & 3.07 & 39.60 & 1182.086 \\
\hline 5 & 1729.60 & 528.217 & 2.17 & 35.34 & 3.22 & 41.35 & 1262.795 \\
\hline 6 & 1740.89 & 644.652 & 1.49 & 25.22 & 3.38 & 40.29 & 1491.853 \\
\hline 7 & 2131.90 & 716.882 & 1.86 & 37.57 & 3.22 & 50.27 & 1690.426 \\
\hline 8 & 1803.15 & 669.739 & 1.69 & 26.02 & 2.14 & 28.30 & 1051.135 \\
\hline 9 & 1935.50 & 717.509 & 1.59 & 29.12 & 2.87 & 37.26 & 1381.337 \\
\hline 10 & 1903.37 & 686.238 & 0.72 & 12.61 & 2.35 & 34.15 & 1231.253 \\
\hline 11 & 1947.36 & 547.212 & 1.86 & 33.68 & 3.71 & 47.51 & 1334.94 \\
\hline 12 & 1779.06 & 557.546 & 1.96 & 31.54 & 1.96 & 31.54 & 988.5779 \\
\hline 13 & 1900.00 & 525.731 & 1.60 & 28.76 & 2.53 & 34.74 & 961.3642 \\
\hline 14 & 1905.16 & 521.043 & 1.94 & 34.14 & 3.38 & 43.44 & 1188.089 \\
\hline
\end{tabular}




\begin{tabular}{|c|c|c|c|c|c|c|c|}
\hline 15 & 1864.30 & 584.259 & 2.25 & 36.22 & 2.25 & 36.22 & 1135.134 \\
\hline 16 & 1890.05 & 652.234 & 1.31 & 22.56 & 2.45 & 33.79 & 1166.101 \\
\hline 17 & 1793.35 & 599.033 & 1.30 & 21.96 & 2.29 & 35.27 & 1178.238 \\
\hline 18 & 2009.85 & 587.551 & 1.99 & 37.01 & 3.22 & 44.73 & 1307.665 \\
\hline 19 & 1995.98 & 634.285 & 2.12 & 36.21 & 3.02 & 43.67 & 1387.702 \\
\hline 20 & 1831.38 & 759.708 & 1.57 & 26.85 & 2.42 & 37.45 & 1553.512 \\
\hline 21 & 1760.32 & 672.744 & 1.65 & 27.83 & 2.89 & 37.86 & 1446.955 \\
\hline 22 & 1626.07 & 701.745 & 1.52 & 23.09 & 3.10 & 36.95 & 1594.745 \\
\hline 23 & 1850.05 & 797.796 & 2.02 & 33.78 & 3.52 & 45.94 & 1981.15 \\
\hline 24 & 1827.64 & 603.020 & 2.07 & 33.61 & 2.86 & 40.59 & 1339.103 \\
\hline 25 & 1860.60 & 701.227 & 1.54 & 27.88 & 3.27 & 43.97 & 1657.167 \\
\hline 26 & 1905.54 & 685.945 & 1.64 & 30.11 & 3.22 & 42.90 & 1544.215 \\
\hline 27 & 1930.63 & 718.909 & 1.80 & 32.96 & 3.00 & 42.45 & 1580.791 \\
\hline 28 & 1776.87 & 780.254 & 2.01 & 29.36 & 3.01 & 39.01 & 1713.175 \\
\hline 29 & 1912.62 & 661.916 & 1.67 & 30.32 & 3.21 & 45.59 & 1577.622 \\
\hline 30 & 1878.27 & 724.394 & 1.98 & 30.77 & 2.35 & 37.18 & 1433.911 \\
\hline 31 & 1799.11 & 707.316 & 1.58 & 27.28 & 2.25 & 42.94 & 1688.105 \\
\hline 32 & 1952.68 & 688.875 & 1.66 & 31.24 & 3.38 & 49.20 & 1735.664 \\
\hline \hline Mean & 1866.09 & 645.967 & 1.73 & 29.65 & 2.84 & 40.19 & 1392.772 \\
\hline $\begin{array}{c}\text { Standard } \\
\text { Deviation }\end{array}$ & 105.894 & 79.949 & 0.33 & 5.55 & 0.51 & 5.07 & 245.3761 \\
\hline Maximum & 2131.9 & 797.796 & 2.27 & 37.57 & 3.71 & 50.27 & 1981.15 \\
\hline Minimum & 1626.07 & 521.043 & 0.72 & 12.61 & 1.69 & 28.3 & 961.3642 \\
\hline
\end{tabular}

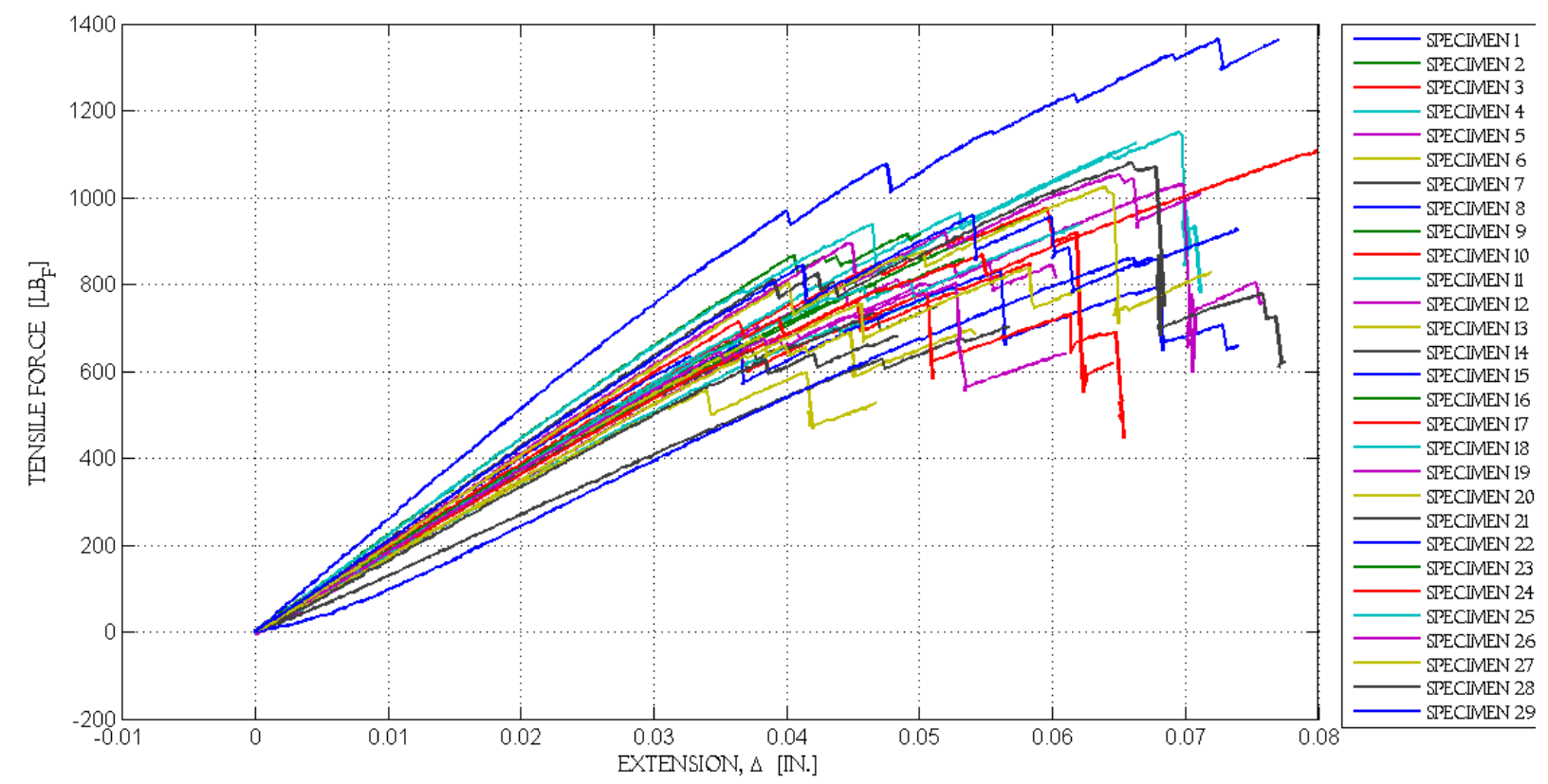

Figure A - 17. Tensile force-displacement curves of bamboo chemically treated with $3 \%$ salt solution 
Table A - 0-19. Tensile properties of bamboo chemically treated with $3 \%$ salt solution

\begin{tabular}{|c|c|c|c|c|c|c|c|}
\hline Specimen & $\begin{array}{l}\text { Modulus } \\
(\mathrm{ksi})\end{array}$ & $\begin{array}{c}\text { Specific } \\
\text { Modulus } \\
\text { (kips-in.//b }) \\
\text { x } 100\end{array}$ & $\begin{array}{c}\text { Yield } \\
\text { Strain } \\
(\%)\end{array}$ & $\begin{array}{c}\text { Yield } \\
\text { Stress } \\
\text { (ksi) }\end{array}$ & $\begin{array}{l}\text { Ultimate } \\
\text { Strain } \\
(\%)\end{array}$ & $\begin{array}{c}\text { Ultimate } \\
\text { Stress } \\
\text { (ksi) }\end{array}$ & $\begin{array}{r}\text { Specific } \\
\text { Strength } \\
\left.\text { (kips-in./lb } \mathrm{b}_{\mathrm{m}}\right)\end{array}$ \\
\hline 1 & 1639.97 & 648.314 & 1.64 & 25.50 & 2.31 & 31.63 & 1250.372 \\
\hline 2 & 1345.02 & 641.982 & 3.02 & 35.20 & 3.02 & 35.20 & 1680.152 \\
\hline 3 & 1690.82 & 725.785 & 1.79 & 28.75 & 1.79 & 28.75 & 1658.15 \\
\hline 4 & 1507.39 & 609.724 & 2.19 & 30.54 & 2.68 & 34.94 & 1413.146 \\
\hline 5 & 1527.42 & 654.547 & 1.96 & 28.66 & 2.74 & 35.51 & 1521.658 \\
\hline 6 & 1627.67 & 611.600 & 1.70 & 25.75 & 2.07 & 27.76 & 1043.238 \\
\hline 7 & 1595.58 & 656.653 & 1.87 & 27.92 & 2.67 & 32.74 & 1347.369 \\
\hline 8 & 1666.50 & 572.976 & 1.83 & 29.41 & 2.80 & 36.77 & 1264.194 \\
\hline 9 & 1436.99 & 590.417 & 2.30 & 29.21 & 2.63 & 31.44 & 1291.856 \\
\hline 10 & 1382.61 & 540.314 & 2.37 & 30.34 & 3.01 & 35.02 & 1368.613 \\
\hline 11 & 1472.09 & 559.368 & 2.63 & 35.37 & 3.45 & 42.17 & 1602.3 \\
\hline 12 & 1552.22 & 668.847 & 2.21 & 31.90 & 3.49 & 41.27 & 1778.342 \\
\hline 13 & 1665.95 & 662.848 & 1.91 & 29.85 & 2.82 & 31.30 & 1245.235 \\
\hline 14 & 1440.12 & 871.942 & 2.36 & 32.04 & 2.91 & 36.24 & 2194.017 \\
\hline 15 & 1378.37 & 663.219 & 3.30 & 39.18 & 3.85 & 43.40 & 2088.153 \\
\hline 16 & 1698.36 & 612.634 & 1.80 & 29.63 & 2.39 & 37.46 & 1351.402 \\
\hline 17 & 1672.87 & 660.195 & 2.27 & 35.26 & 2.53 & 35.94 & 1418.425 \\
\hline 18 & 1732.90 & 635.161 & 2.46 & 36.33 & 3.14 & 42.50 & 1557.632 \\
\hline 19 & 1706.09 & 648.800 & 1.76 & 29.99 & 2.64 & 36.17 & 1375.66 \\
\hline 20 & 1732.76 & 641.688 & 2.28 & 36.82 & 2.92 & 40.91 & 1515.135 \\
\hline 21 & 1832.64 & 646.710 & 1.93 & 33.46 & 2.53 & 37.68 & 1329.766 \\
\hline 22 & 1724.67 & 581.911 & 2.35 & 35.48 & 3.84 & 45.17 & 1524.106 \\
\hline 23 & 1629.14 & 551.614 & 2.01 & 31.13 & 2.01 & 31.13 & 1157.027 \\
\hline 24 & 1719.51 & 585.735 & 2.63 & 34.62 & 2.63 & 34.62 & 1634.971 \\
\hline 25 & 1823.02 & 575.269 & 2.33 & 38.20 & 3.33 & 45.81 & 1445.701 \\
\hline 26 & 1548.02 & 573.160 & 2.24 & 32.66 & 3.24 & 38.51 & 1426.029 \\
\hline 27 & 1616.00 & 621.315 & 1.97 & 30.57 & 3.15 & 38.94 & 1497.101 \\
\hline 28 & 1691.71 & 638.144 & 1.96 & 32.07 & 2.13 & 32.55 & 1113.131 \\
\hline 29 & 1711.39 & 653.103 & 2.68 & 37.91 & 2.68 & 37.91 & 1446.585 \\
\hline Mean & 1612.68 & 631.171 & 2.20 & 32.20 & 2.81 & 36.53 & 1466.878 \\
\hline $\begin{array}{l}\text { Standard } \\
\text { Deviation }\end{array}$ & 131.01 & 62.986 & 0.39 & 3.66 & 0.51 & 4.69 & 253.4497 \\
\hline Maximum & $\mid 1832.64$ & 871.942 & 3.30 & 39.18 & 3.85 & 45.81 & 2194.017 \\
\hline Minimum & $\mid 1345.02$ & 540.314 & 1.64 & 25.5 & 1.79 & 27.76 & 1043.238 \\
\hline
\end{tabular}




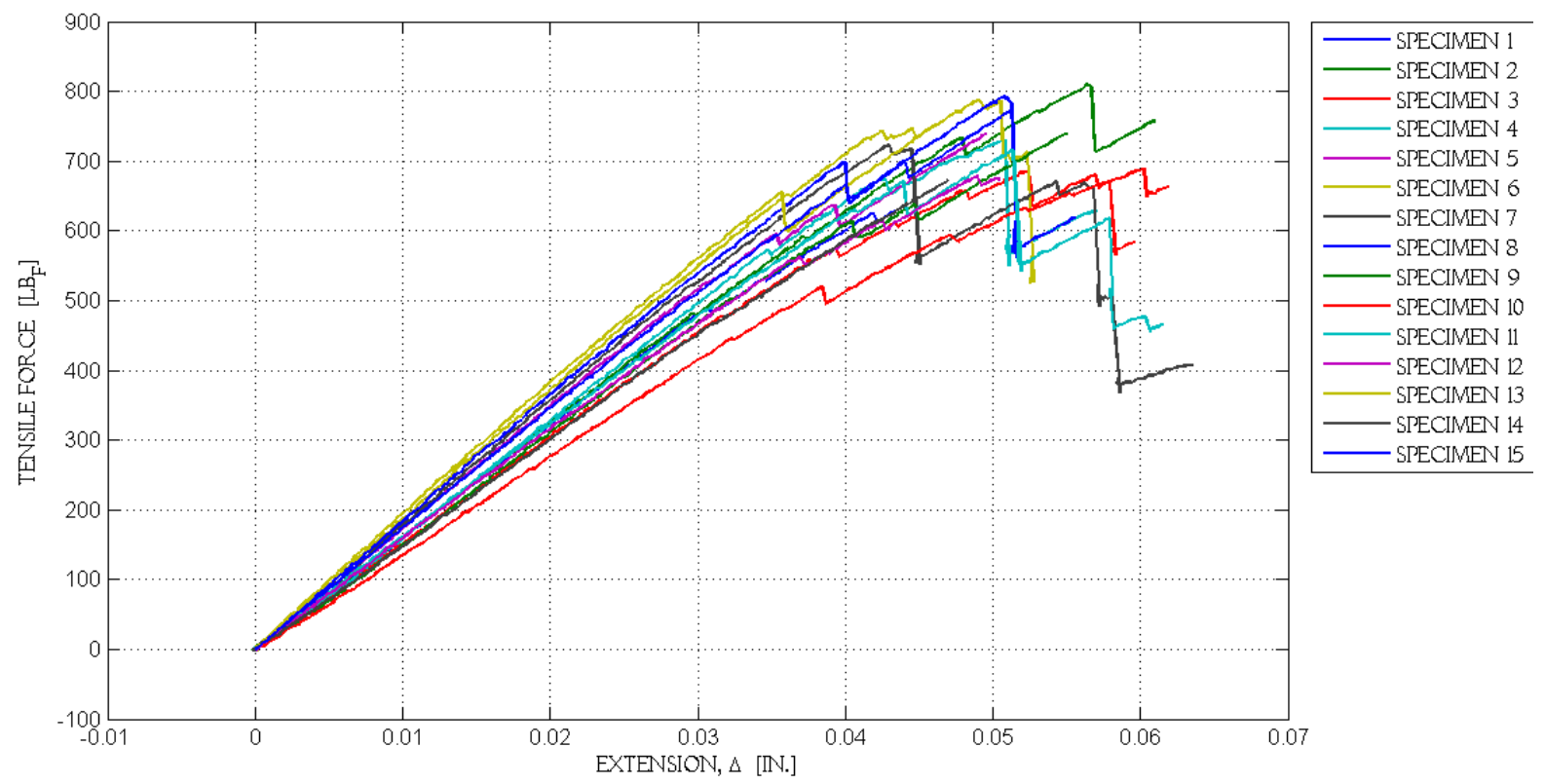

Figure A - 18. Tensile force-displacement curves of bamboo chemically treated with $6 \%$ salt solution

Table A - 0-20. Tensile properties of bamboo chemically treated with $6 \%$ salt solution

\begin{tabular}{|c|c|c|c|c|c|c|c|}
\hline Specimen & $\begin{array}{l}\text { Modulus } \\
\text { (ksi) }\end{array}$ & $\begin{array}{l}\text { Specific } \\
\text { Modulus } \\
\text { (kips-in.//lbm) } \\
\text { x } 100\end{array}$ & $\begin{array}{c}\text { Yield } \\
\text { Strain } \\
(\%)\end{array}$ & $\begin{array}{c}\text { Yield } \\
\text { Stress } \\
\text { (ksi) }\end{array}$ & $\begin{array}{c}\text { Ultimate } \\
\text { Strain } \\
(\%)\end{array}$ & $\begin{array}{l}\text { Ultimate } \\
\text { Stress } \\
\text { (ksi) }\end{array}$ & $\begin{array}{c}\text { Specific } \\
\text { Strength } \\
\left.\text { (kips-in.// } \mathrm{b}_{\mathrm{m}}\right)\end{array}$ \\
\hline 1 & 1399.24 & 636.246 & 2.08 & 30.49 & 2.50 & 26.64 & 1386.251 \\
\hline 2 & 1441.17 & 603.685 & 2.42 & 35.73 & 2.85 & 32.37 & 1496.757 \\
\hline 3 & 1495.09 & 593.959 & 1.97 & 33.52 & 2.62 & 28.14 & 1331.702 \\
\hline 4 & 1367.72 & 605.519 & 2.13 & 29.26 & 2.53 & 26.98 & 1295.181 \\
\hline 5 & 1415.53 & 628.545 & 1.90 & 28.76 & 2.43 & 24.73 & 1276.867 \\
\hline 6 & 1412.30 & 648.403 & 2.15 & 29.69 & 2.47 & 28.00 & 1363.047 \\
\hline 7 & 1544.39 & 692.826 & 2.19 & 31.23 & 2.19 & 31.23 & 1401.207 \\
\hline 8 & 1353.80 & 559.197 & 2.00 & 29.37 & 2.54 & 25.88 & 1213.234 \\
\hline 9 & 1331.82 & 592.266 & 2.23 & 31.43 & 2.87 & 26.11 & 1397.843 \\
\hline 10 & 1234.26 & 553.748 & 1.94 & 30.11 & 3.03 & 22.67 & 1351.039 \\
\hline 11 & 1221.82 & 487.189 & 2.25 & 27.64 & 2.63 & 25.91 & 1102.079 \\
\hline 12 & 1298.95 & 566.840 & 1.84 & 27.61 & 2.58 & 22.72 & 1204.714 \\
\hline 13 & 1306.72 & 532.029 & 1.80 & 25.82 & 2.46 & 22.31 & 1051.283 \\
\hline 14 & 1308.25 & 555.690 & 2.38 & 28.83 & 2.38 & 28.83 & 1224.612 \\
\hline 15 & 1200.43 & 525.319 & 2.00 & 27.25 & 2.65 & 23.04 & 1192.399 \\
\hline Mean & 1355.43 & 585.431 & 2.08 & 26.37 & 2.582 & 29.78 & 1285.881 \\
\hline $\begin{array}{l}\text { Standard } \\
\text { Deviation }\end{array}$ & 98.70 & 53.342 & 0.19 & 3.04 & 0.210 & 2.52 & 121.309 \\
\hline Maximum & 1544.39 & 692.826 & 2.42 & 32.37 & 3.03 & 35.73 & 1496.757 \\
\hline
\end{tabular}




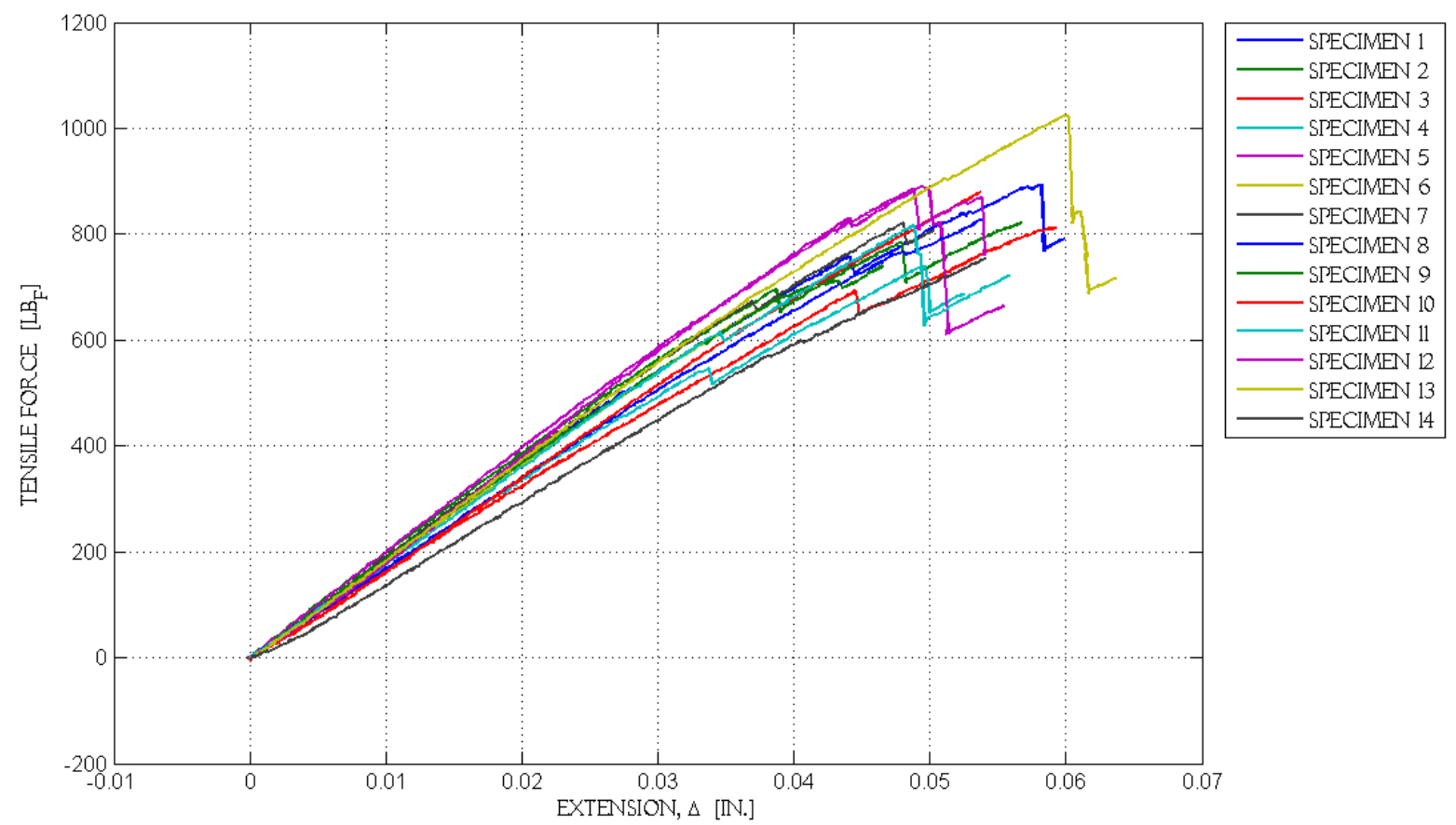

Figure A - 19. Tensile force-disblacement curves of bamboo chemicallv treated with $9 \%$ salt solution

Table A - 0-21. Tensile properties of bamboo chemically treated with $9 \%$ salt solution

\begin{tabular}{|c|c|c|c|c|c|c|c|}
\hline Specimen & $\begin{array}{c}\text { Modulus } \\
(\mathrm{ksi})\end{array}$ & $\begin{array}{c}\text { Specific } \\
\text { Modulus } \\
\left(\mathrm{kips}-\mathrm{in} . / \mathrm{l} \mathrm{b}_{\mathrm{m}}\right) \\
\mathrm{x} 100\end{array}$ & $\begin{array}{c}\text { Yield } \\
\text { Strain } \\
(\%)\end{array}$ & $\begin{array}{c}\text { Yield } \\
\text { Stress } \\
(\mathrm{ksi})\end{array}$ & $\begin{array}{c}\text { Ultimate } \\
\text { Strain } \\
(\%)\end{array}$ & $\begin{array}{c}\text { Ultimate } \\
\text { Stress } \\
(\mathrm{ksi})\end{array}$ & $\begin{array}{c}\text { Specific } \\
\text { Strength } \\
(\mathrm{kips}-\mathrm{in} . / \mathrm{lb} \mathrm{m})\end{array}$ \\
\hline 1 & 1418.29 & 515.323 & 2.20 & 26.64 & 2.90 & 33.42 & 1214.253 \\
\hline 2 & 1290.11 & 490.026 & 1.94 & 27.62 & 2.93 & 32.37 & 1049.182 \\
\hline 3 & 1461.82 & 529.307 & 2.34 & 28.14 & 3.23 & 38.47 & 1392.923 \\
\hline 4 & 1468.23 & 606.707 & 1.69 & 26.98 & 2.48 & 32.43 & 1339.916 \\
\hline 5 & 1466.83 & 567.455 & 2.46 & 24.73 & 2.46 & 32.81 & 1269.356 \\
\hline 6 & 1441.58 & 593.863 & 1.99 & 26.99 & 1.99 & 28.00 & 1111.869 \\
\hline 7 & 1521.63 & 611.489 & 2.41 & 31.23 & 2.60 & 32.75 & 1316.263 \\
\hline 8 & 1454.68 & 613.612 & 2.41 & 25.88 & 2.74 & 35.44 & 1494.781 \\
\hline 9 & 1565.37 & 582.238 & 2.18 & 26.11 & 2.43 & 31.82 & 1183.602 \\
\hline 10 & 1572.57 & 553.011 & 2.73 & 22.67 & 2.73 & 39.02 & 1372.102 \\
\hline 11 & 1306.97 & 499.443 & 2.42 & 25.91 & 2.42 & 28.40 & 1085.282 \\
\hline 12 & 1388.56 & 526.122 & 2.20 & 22.72 & 2.44 & 30.01 & 1137.229 \\
\hline 13 & 1435.25 & 570.015 & 3.00 & 22.31 & 3.00 & 38.54 & 1530.755 \\
\hline 14 & 1620.23 & 670.413 & 2.02 & 23.04 & 2.79 & 39.79 & 1646.314 \\
\hline \hline Mean & 1458.001 & 566.359 & 2.29 & 25.78 & 2.65 & 33.81 & 1295.988 \\
\hline
\end{tabular}




\begin{tabular}{|c||c|c|c|c|c|c|c||}
\hline $\begin{array}{c}\text { Standard } \\
\text { Deviation }\end{array}$ & 93.403 & 51.011 & 0.33 & 2.51 & 0.31 & 3.90 & 179.5995 \\
\cline { 2 - 5 } & 1620.23 & 670.413 & 3 & 31.23 & 3.23 & 39.79 & 1646.314 \\
\hline Maximimum & 1290.11 & 490.026 & 1.69 & 22.31 & 1.99 & 28 & 1049.182 \\
\hline
\end{tabular}

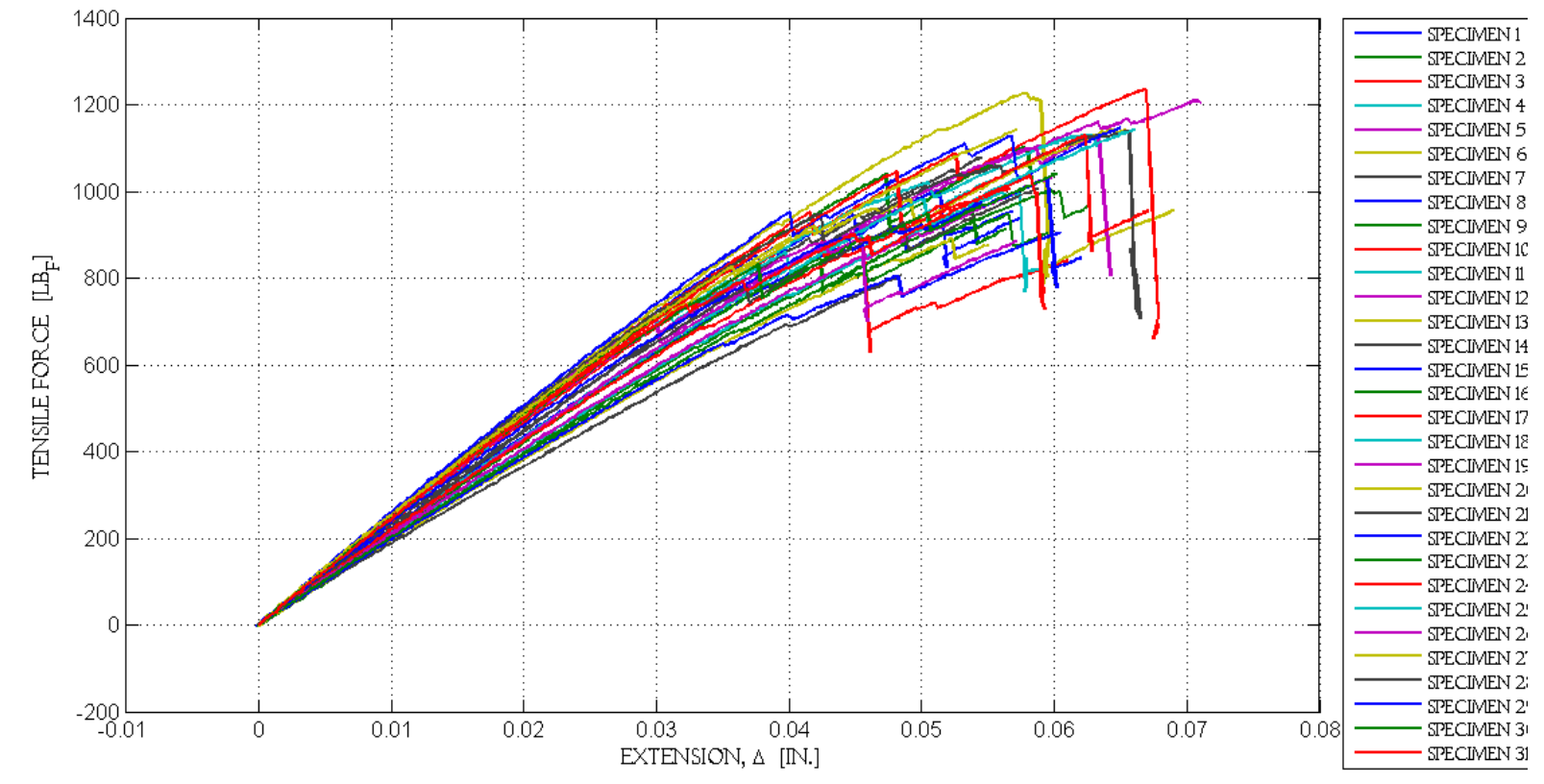

Figure A - 20. Tensile force-displacement curves of bamboo chemically treated with lime solution

Table A - 0-22. Tensile properties of bamboo chemically treated with lime solution

\begin{tabular}{|c|c|c|c|c|c|c|c|}
\hline Specimen & $\begin{array}{c}\text { Modulus } \\
(\mathrm{ksi})\end{array}$ & $\begin{array}{c}\text { Specific } \\
\text { Modulus } \\
(\mathrm{kips}-\mathrm{in} . / \mathrm{l} \mathrm{b}) \\
\mathrm{x} \text { ) }\end{array}$ & $\begin{array}{c}\text { Yield } \\
\text { Strain } \\
(\%)\end{array}$ & $\begin{array}{c}\text { Yield } \\
\text { Stress } \\
(\mathrm{ksi})\end{array}$ & $\begin{array}{c}\text { Ultimate } \\
\text { Strain } \\
(\%)\end{array}$ & $\begin{array}{c}\text { Ultimate } \\
\text { Stress } \\
(\mathrm{ksi})\end{array}$ & $\begin{array}{c}\text { Specific } \\
\text { Strength } \\
\left(\mathrm{kips}-\mathrm{i} . / \mathrm{l} \mathrm{b}_{\mathrm{m}}\right)\end{array}$ \\
\hline 1 & 1577.20 & 587.169 & 1.849 & 26.966 & 2.960 & 34.900 & 981.920 \\
\hline 2 & 1567.56 & 602.100 & 1.949 & 29.026 & 2.720 & 35.550 & 1000.340 \\
\hline 3 & 1652.20 & 590.308 & 0.900 & 14.622 & 3.130 & 31.950 & 898.870 \\
\hline 4 & 1667.51 & 678.775 & 2.187 & 33.300 & 2.970 & 40.940 & 1151.900 \\
\hline 5 & 1434.96 & 553.469 & 2.143 & 29.283 & 3.510 & 41.590 & 1170.370 \\
\hline 6 & 1580.04 & 547.110 & 1.981 & 29.054 & 2.900 & 38.680 & 1088.430 \\
\hline 7 & 1514.37 & 497.464 & 2.090 & 29.625 & 2.860 & 37.380 & 1051.820 \\
\hline 8 & 1898.69 & 761.082 & 1.234 & 22.763 & 2.570 & 37.740 & 1061.840 \\
\hline 9 & 1686.23 & 619.953 & 1.996 & 30.839 & 2.900 & 37.490 & 1054.800 \\
\hline 10 & 1684.01 & 663.649 & 2.049 & 31.570 & 3.350 & 41.700 & 1173.240 \\
\hline 11 & 1933.64 & 678.870 & 2.062 & 35.819 & 3.150 & 45.070 & 1268.130 \\
\hline 12 & 1850.51 & 626.517 & 1.541 & 24.257 & 3.190 & 41.660 & 1172.090 \\
\hline 13 & 1878.56 & 693.277 & 2.024 & 34.165 & 3.380 & 43.750 & 1230.900 \\
\hline 14 & 1714.20 & 660.494 & 1.538 & 24.826 & 3.330 & 38.980 & 1096.870 \\
\hline
\end{tabular}




\begin{tabular}{|c|c|c|c|c|c|c|c|}
\hline 15 & 1849.05 & 668.540 & 2.003 & 32.033 & 5.340 & 41.620 & 1171.160 \\
\hline 16 & 1784.04 & 567.741 & 2.233 & 36.328 & 2.830 & 42.360 & 1191.810 \\
\hline 17 & 1826.50 & 580.192 & 1.710 & 29.110 & 2.960 & 43.270 & 1217.600 \\
\hline 18 & 1742.17 & 572.162 & 1.665 & 27.513 & 2.820 & 42.150 & 1186.080 \\
\hline 19 & 1794.21 & 559.869 & 2.070 & 34.094 & 3.010 & 45.040 & 1267.260 \\
\hline 20 & 1744.40 & 643.708 & 2.110 & 33.916 & 2.890 & 40.790 & 1147.840 \\
\hline 21 & 1767.95 & 576.853 & 2.052 & 33.411 & 2.550 & 39.950 & 1124.140 \\
\hline 22 & 1795.53 & 587.990 & 1.728 & 29.284 & 3.120 & 42.200 & 1187.320 \\
\hline 23 & 1867.67 & 658.125 & 1.815 & 31.871 & 3.150 & 47.190 & 1327.800 \\
\hline 24 & 1901.05 & 692.348 & 2.033 & 35.784 & 3.140 & 44.220 & 1244.180 \\
\hline 25 & 1956.33 & 668.656 & 1.632 & 30.678 & 3.650 & 50.890 & 1431.880 \\
\hline 26 & 1925.40 & 653.297 & 1.498 & 27.475 & 2.980 & 36.300 & 1021.500 \\
\hline 27 & 1885.73 & 670.341 & 1.826 & 30.693 & 2.860 & 42.970 & 1209.100 \\
\hline 28 & 1874.39 & 675.823 & 1.849 & 30.377 & 3.070 & 42.410 & 1193.320 \\
\hline 29 & 2082.51 & 769.090 & 1.612 & 31.356 & 2.970 & 45.560 & 1282.070 \\
\hline 30 & 1904.64 & 703.639 & 1.629 & 29.210 & 3.100 & 41.870 & 1178.000 \\
\hline 31 & 1939.55 & 659.679 & 1.652 & 29.107 & 2.250 & 41.150 & 1157.820 \\
\hline \hline Mean & 1783.25 & 634.461 & 1.828 & 29.947 & 3.08 & 41.20 & 1159.370 \\
\hline $\begin{array}{c}\text { Standard } \\
\text { Deviation }\end{array}$ & 147.94 & 62.973 & 0.296 & 4.353 & 0.50 & 3.84 & 108.020 \\
\hline Maximum & 2082.51 & 769.090 & 2.233 & 36.328 & 5.34 & 50.89 & 1431.880 \\
\hline Minimum & 1434.96 & 497.464 & 0.900 & 14.622 & 2.25 & 31.95 & 898.870 \\
\hline
\end{tabular}

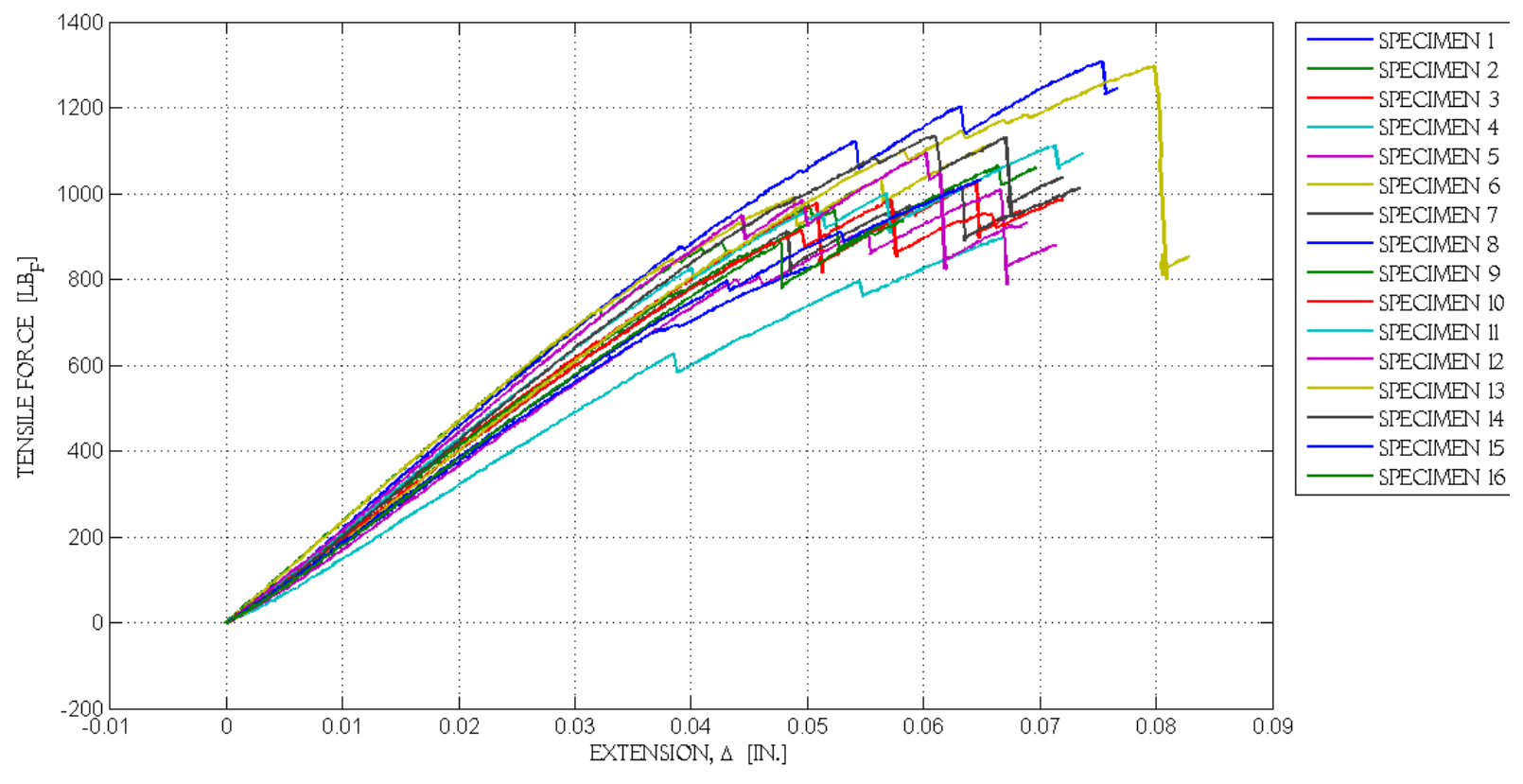

Figure A - 21. Tensile force-displacement curves of bamboo chemically treated with oil 
Table A - 0-23. Tensile properties of bamboo chemically treated with oil

\begin{tabular}{|c|c|c|c|c|c|c|c|}
\hline Specimen & $\begin{array}{l}\text { Modulus } \\
\quad(\mathrm{ksi})\end{array}$ & $\begin{array}{c}\text { Specific } \\
\text { Modulus } \\
\text { (kips-in./lb }) \\
\text { x } 100\end{array}$ & $\begin{array}{l}\text { Yield } \\
\text { Strain } \\
(\%)\end{array}$ & $\begin{array}{l}\text { Yield } \\
\text { Stress } \\
\text { (ksi) }\end{array}$ & $\begin{array}{l}\text { Ultimate } \\
\text { Strain } \\
(\%)\end{array}$ & $\begin{array}{c}\text { Ultimate } \\
\text { Stress } \\
\text { (ksi) }\end{array}$ & $\begin{array}{l}\text { Specific } \\
\text { Strength } \\
\text { (kips-in.//lbm) }\end{array}$ \\
\hline 1 & 1334.38 & 505.251 & 1.953 & 25.057 & 3.726 & 37.3 & 1109.186 \\
\hline 2 & 1530.27 & 559.054 & 1.594 & 23.745 & 2.894 & 34.48 & 1025.403 \\
\hline 3 & 1586.70 & 594.597 & 2.435 & 35.710 & 3.271 & 39.12 & 1163.155 \\
\hline 4 & 1605.40 & 603.448 & 1.957 & 27.263 & 3.366 & 41.84 & 1244 \\
\hline 5 & 1455.55 & 526.661 & 2.193 & 28.913 & 3.304 & 37.18 & 1105.441 \\
\hline 6 & 1453.83 & 476.540 & 1.948 & 25.942 & 3.395 & 34.94 & 1039.012 \\
\hline 7 & 1447.54 & 554.087 & 2.043 & 28.057 & 3.847 & 37.34 & 1110.188 \\
\hline 8 & 1712.46 & 614.263 & 1.889 & 30.205 & 2.591 & 36.93 & 1098.128 \\
\hline 9 & 1605.93 & 551.724 & 1.995 & 30.232 & 3.584 & 42.02 & 1249.618 \\
\hline 10 & 1494.72 & 521.057 & 2.076 & 29.797 & 3.749 & 37.68 & 1120.518 \\
\hline 11 & 1475.47 & 540.202 & 2.047 & 27.195 & 3.876 & 37.97 & 1128.959 \\
\hline 12 & 1294.61 & 482.495 & 1.984 & 24.347 & 3.047 & 31.34 & 932.0342 \\
\hline 13 & 1341.48 & 479.047 & 2.175 & 27.410 & 3.801 & 40.04 & 1190.569 \\
\hline 14 & 1400.98 & 469.527 & 2.352 & 30.032 & 3.033 & 35.22 & 1047.216 \\
\hline 15 & 1479.18 & 541.459 & 2.178 & 30.311 & 3.338 & 39.64 & 1178.679 \\
\hline 16 & 1561.05 & 523.155 & 2.379 & 34.210 & 3.031 & 37.22 & 1106.69 \\
\hline Mean & 1486.22 & 533.910 & 2.075 & 28.652 & 3.366 & 37.52 & 1115.55 \\
\hline $\begin{array}{l}\text { Standard } \\
\text { Deviation }\end{array}$ & 112.27 & 45.220 & 0.210 & 3.271 & 0.380 & 2.74 & 81.42 \\
\hline Maximum & 1712.46 & 614.263 & 2.435 & 35.710 & 3.876 & 42.02 & 1249.62 \\
\hline Minimum & \begin{tabular}{|l|}
1294.61 \\
\end{tabular} & 469.527 & 1.594 & 23.745 & 2.591 & 31.34 & 932.03 \\
\hline
\end{tabular}




\section{A4. Flexural Testing Data}

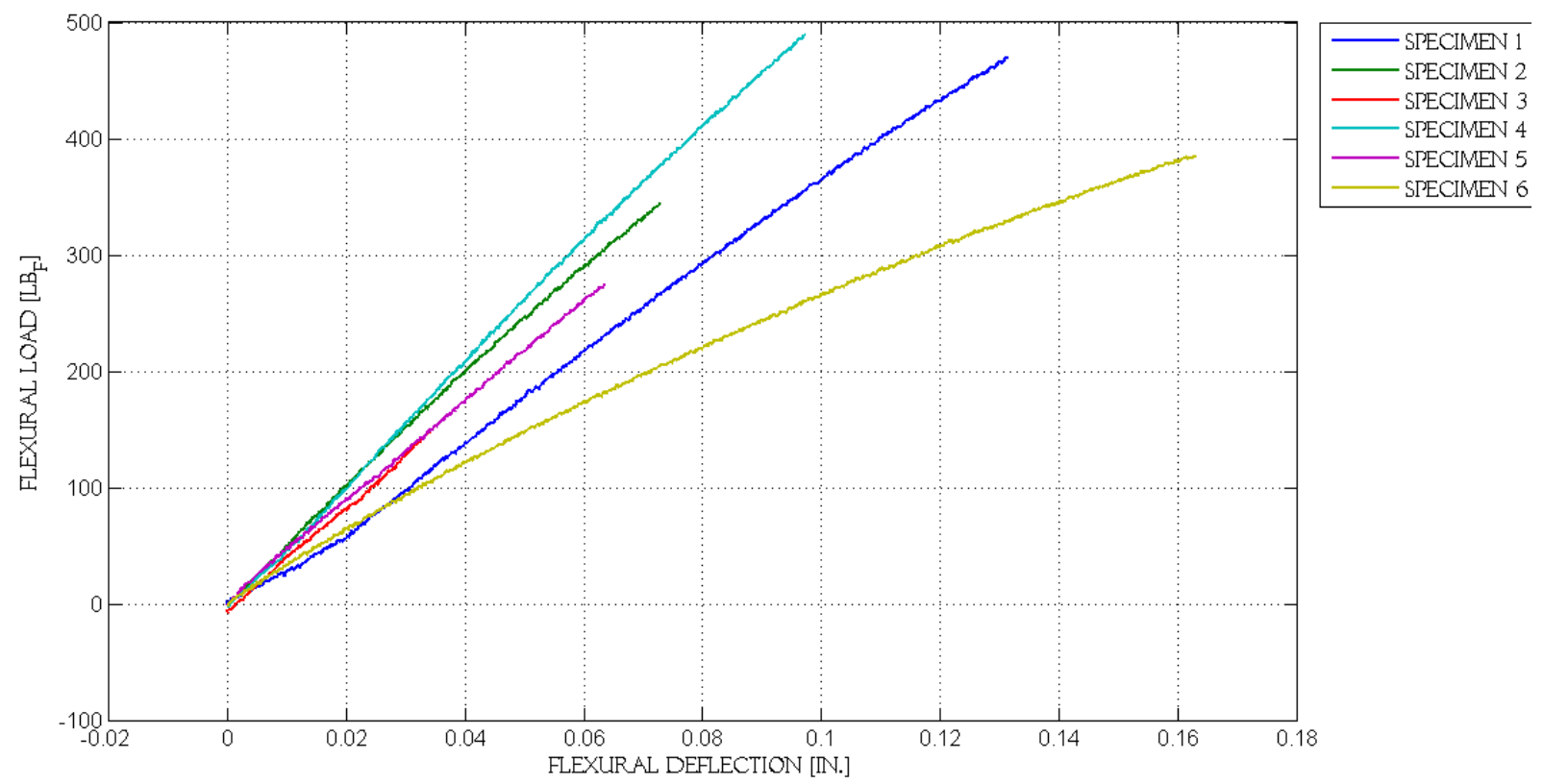

Figure A - 22. Tensile force-displacement curves of internode bamboo for cylindrical configuration

Table A - 0-24. Flexural properties of internode bamboo for cylindrical configuration

\begin{tabular}{|c|c|c|c|c|c|c|c|}
\hline Specimen & $\begin{array}{l}\text { Modulus } \\
\text { (ksi) }\end{array}$ & $\begin{array}{c}\text { Specific } \\
\text { Modulus } \\
\left.\text { (kips-in.//b } \mathrm{b}_{\mathrm{m}}\right) \\
\text { x } 100\end{array}$ & $\begin{array}{c}\text { Yield } \\
\text { Strain } \\
(\%)\end{array}$ & $\begin{array}{c}\text { Yield } \\
\text { Stress } \\
\text { (ksi) }\end{array}$ & $\begin{array}{l}\text { Ultimate } \\
\text { Strain } \\
(\%)\end{array}$ & $\begin{array}{c}\text { Ultimate } \\
\text { Stress } \\
\text { (ksi) }\end{array}$ & $\begin{array}{c}\text { Specific } \\
\text { Strength } \\
\text { (kips-in.// } \mathrm{b}_{\mathrm{m}} \text { ) }\end{array}$ \\
\hline 1 & 504.935 & 192.723 & 1.20 & 5.798 & 1.20 & 5.806 & 143.5 \\
\hline 2 & 594.601 & 229.576 & 0.68 & 4.015 & 0.68 & 4.015 & 171.2 \\
\hline 3 & 605.402 & 219.349 & 0.31 & 1.813 & 0.31 & 1.813 & 168.2 \\
\hline 4 & 595.437 & 225.544 & 0.91 & 5.321 & 0.92 & 5.330 & 163.7 \\
\hline 5 & 494.712 & 178.596 & 0.59 & 2.953 & 0.59 & 2.953 & 178.4 \\
\hline 6 & 381.618 & 153.260 & 0.96 & 4.051 & 1.42 & 5.394 & 143.8 \\
\hline Mean & 529.451 & 199.841 & 0.78 & 3.992 & 0.85 & 4.219 & 161.7 \\
\hline $\begin{array}{l}\text { Standard } \\
\text { Deviation }\end{array}$ & 87.212 & 30.322 & 0.32 & 1.474 & 0.41 & 1.586 & 14.3 \\
\hline Maximum & 605.402 & 229.576 & 1.20 & 5.798 & 1.42 & 5.806 & 178.4 \\
\hline Minimum & 381.618 & 153.260 & 0.31 & 1.813 & 0.31 & 1.813 & 143.5 \\
\hline
\end{tabular}




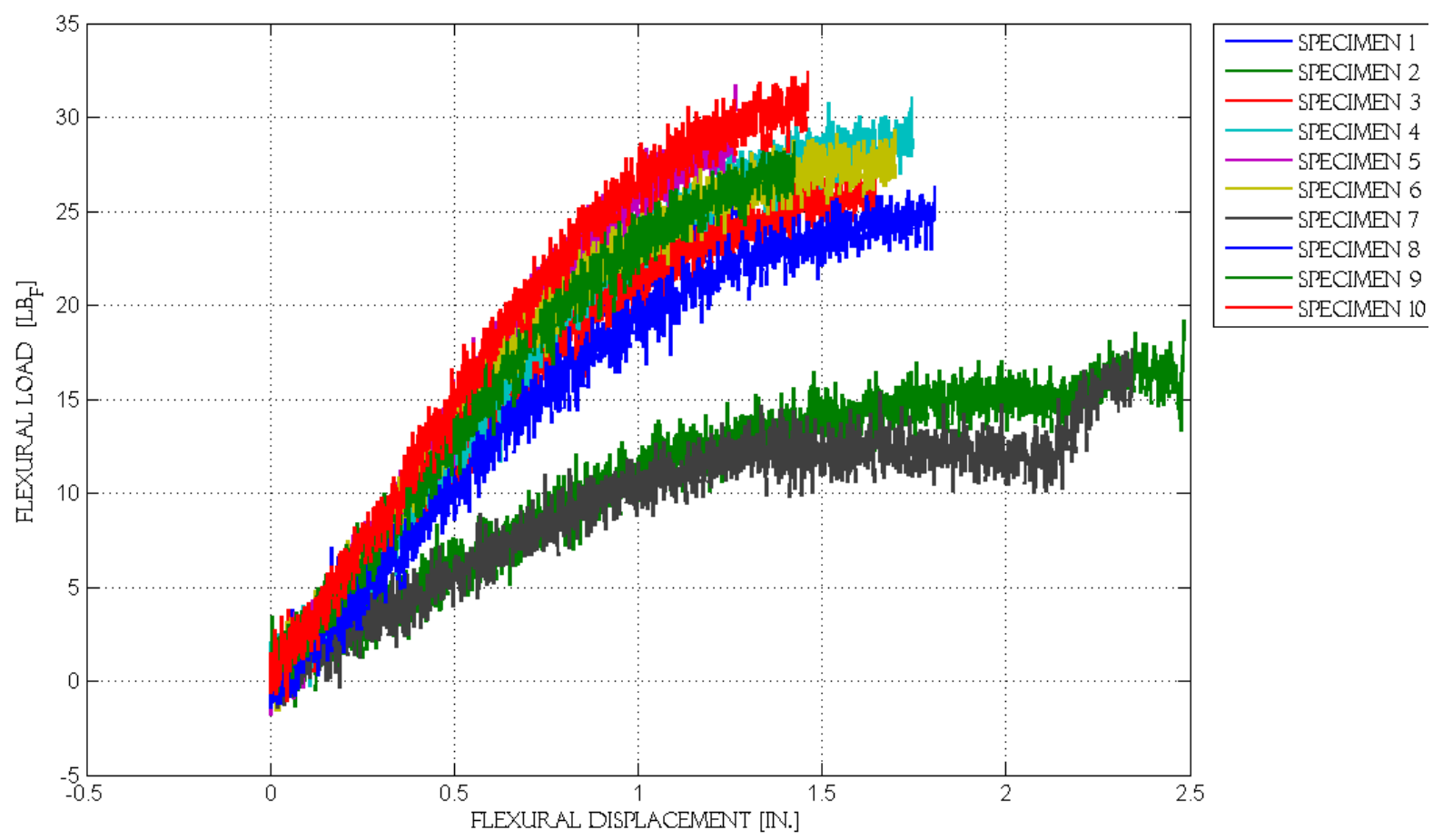

Figure A - 23. Tensile force-displacement curves of internode bamboo for rectangular configuration

Table A - 0-25. Flexural properties of internode bamboo for rectangular configuration

\begin{tabular}{|c|c|c|c|c|c|c|c|}
\hline Specimen & $\begin{array}{c}\text { Modulus } \\
(\mathrm{ksi})\end{array}$ & $\begin{array}{c}\text { Specific } \\
\text { Modulus } \\
\left(\mathrm{kips}-\mathrm{in} . / \mathrm{l} \mathrm{b}_{\mathrm{m}}\right) \\
\times 100\end{array}$ & $\begin{array}{c}\text { Yield } \\
\text { Strain } \\
(\%)\end{array}$ & $\begin{array}{c}\text { Yield } \\
\text { Stress } \\
(\mathrm{ksi})\end{array}$ & $\begin{array}{c}\text { Ultimate } \\
\text { Strain } \\
(\%)\end{array}$ & $\begin{array}{c}\text { Ultimate } \\
\text { Stress } \\
(\mathrm{ksi})\end{array}$ & $\begin{array}{c}\text { Specific } \\
\text { Strength } \\
\left(\mathrm{kips}-\text {-n.// }_{\mathrm{m}}\right)\end{array}$ \\
\hline 1 & 1839.357 & 690.786 & 0.823 & 13.885 & 1.151 & 18.969 & 712.403 \\
\hline 2 & 2071.718 & 707.675 & 0.734 & 15.841 & 1.666 & 24.630 & 841.336 \\
\hline 3 & 1879.348 & 759.547 & 0.761 & 13.536 & 1.412 & 19.533 & 789.431 \\
\hline 4 & 1952.632 & 734.901 & 0.742 & 14.645 & 1.535 & 21.002 & 790.426 \\
\hline 5 & 1944.042 & 739.742 & 0.670 & 12.748 & 1.166 & 19.298 & 734.320 \\
\hline 6 & 2044.601 & 776.500 & 0.717 & 14.100 & 1.497 & 19.612 & 744.829 \\
\hline 7 & 2083.469 & 722.173 & 0.745 & 14.529 & 1.585 & 22.899 & 793.739 \\
\hline 8 & 1806.236 & 677.406 & 0.795 & 12.530 & 1.560 & 19.110 & 716.686 \\
\hline 9 & 1784.871 & 690.900 & 0.772 & 13.247 & 1.313 & 18.878 & 730.751 \\
\hline 10 & 1858.406 & 686.544 & 1.080 & 16.346 & 1.408 & 19.482 & 719.729 \\
\hline Mean & 1926.468 & 718.617 & 0.784 & 14.141 & 1.429 & 20.341 & 757.365 \\
\hline $\begin{array}{c}\text { Standard } \\
\text { Deviation }\end{array}$ & 110.374 & 33.565 & 0.112 & 1.244 & 0.174 & 1.942 & 43.442 \\
\hline Maximum & 2083.469 & 776.500 & 1.080 & 16.346 & 1.666 & 24.630 & 841.336 \\
\hline Minimum & 1784.871 & 677.406 & 0.670 & 12.530 & 1.151 & 18.878 & 712.403 \\
\hline
\end{tabular}




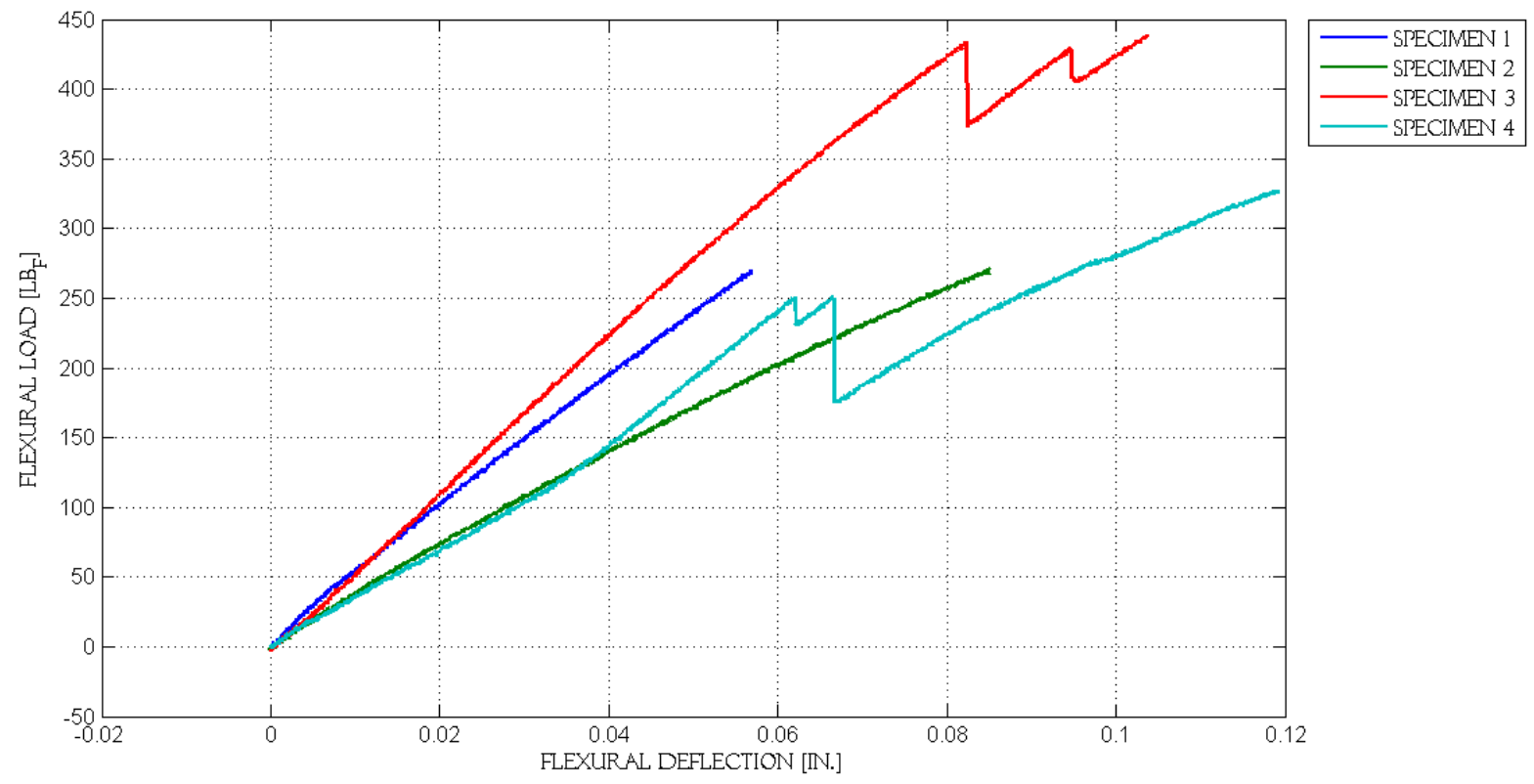

Figure A - 24. Tensile force-displacement curves of mid-node bamboo for cylindrical configuration

Table A - 0-26. Flexural properties of mid-node bamboo for cylindrical configuration

\begin{tabular}{|c|c|c|c|c|c|c|c|}
\hline Specimen & $\begin{array}{c}\text { Modulus } \\
(\mathrm{ksi})\end{array}$ & $\begin{array}{c}\text { Specific } \\
\text { Modulus } \\
\left(\mathrm{kips}-\mathrm{in} . / \mathrm{l} \mathrm{b}_{\mathrm{m}}\right) \\
\mathrm{x} 100\end{array}$ & $\begin{array}{c}\text { Yield } \\
\text { Strain } \\
(\%)\end{array}$ & $\begin{array}{c}\text { Yield } \\
\text { Stress } \\
(\mathrm{ksi})\end{array}$ & $\begin{array}{c}\text { Ultimate } \\
\text { Strain } \\
(\%)\end{array}$ & $\begin{array}{c}\text { Ultimate } \\
\text { Stress } \\
(\mathrm{ksi})\end{array}$ & $\begin{array}{c}\text { Specific } \\
\text { Strength } \\
\left(\mathrm{kips}-\mathrm{in} . / \mathrm{lb}_{\mathrm{m}}\right)\end{array}$ \\
\hline 1 & 842.694 & 260.091 & 0.46 & 3.928 & 0.47 & 4.005 & 136.8 \\
\hline 2 & 649.302 & 146.569 & 0.69 & 4.494 & 0.69 & 4.494 & 105.4 \\
\hline 3 & 780.245 & 215.537 & 0.74 & 4.679 & 0.90 & 5.310 & 136.7 \\
\hline 4 & 740.313 & 221.651 & 0.48 & 4.225 & 0.93 & 5.585 & 170.5 \\
\hline Mean & 753.139 & 210.962 & 0.59 & 4.331 & 0.75 & 4.848 & 137.4 \\
\hline $\begin{array}{c}\text { Standard } \\
\text { Deviation }\end{array}$ & 81.038 & 47.241 & 0.14 & 0.328 & 0.22 & 0.729 & 26.6 \\
\hline Maximum & 842.694 & 260.091 & 0.74 & 4.679 & 0.93 & 5.585 & 170.5 \\
\hline Minimum & 649.302 & 146.569 & 0.46 & 3.928 & 0.47 & 4.005 & 105.4 \\
\hline
\end{tabular}



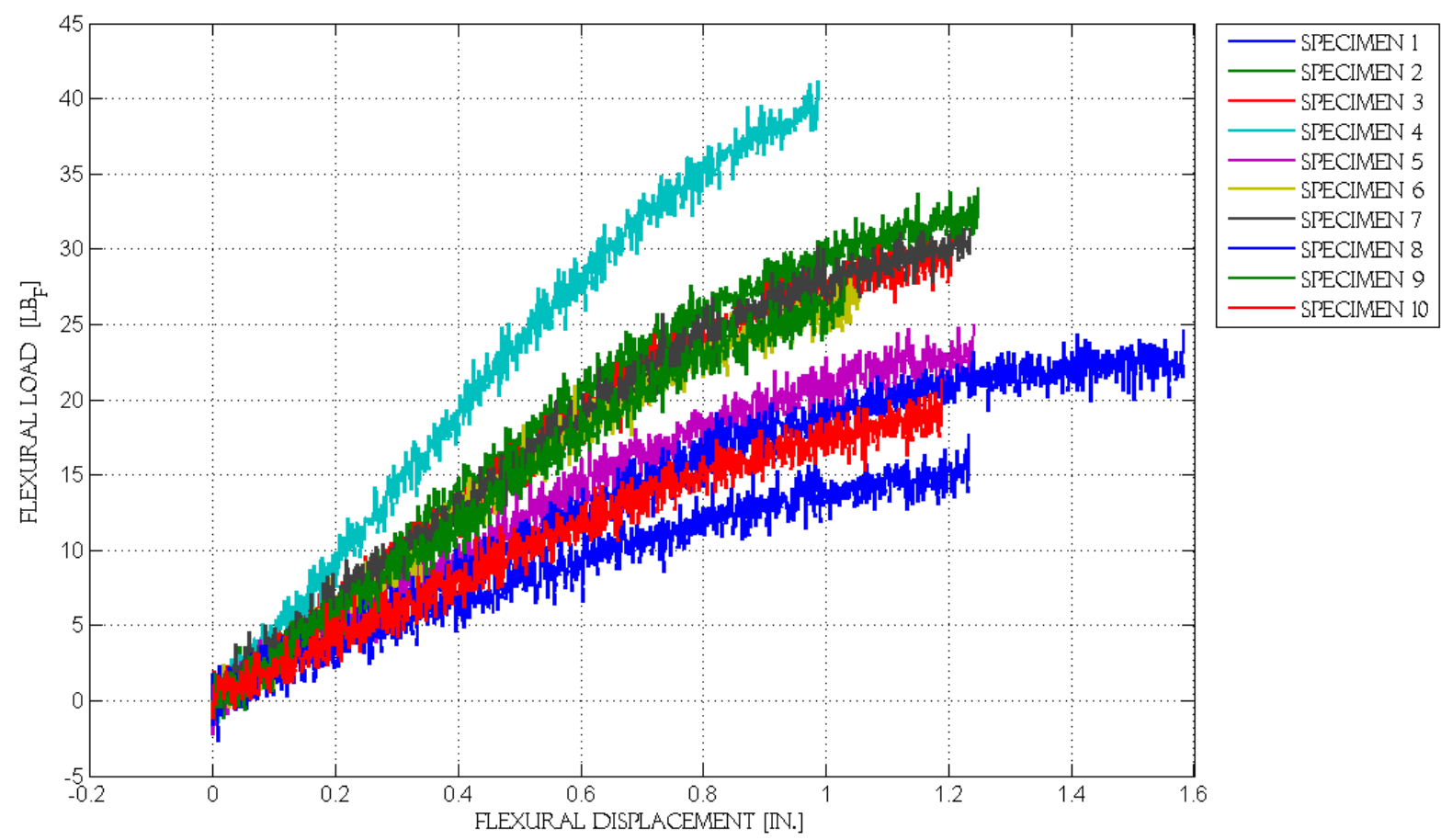

Figure A - 25. Tensile force-displacement curves of mid-node bamboo for rectangular configuration

Table A - 0-27. Flexural properties of mid-node bamboo for rectangular configuration

\begin{tabular}{|c|c|c|c|c|c|c|c|}
\hline Specimen & $\begin{array}{c}\text { Modulus } \\
(\mathrm{ksi})\end{array}$ & $\begin{array}{c}\text { Specific } \\
\text { Modulus } \\
(\mathrm{kips}-\mathrm{in} . / \mathrm{lb}) \\
\times 100\end{array}$ & $\begin{array}{c}\text { Yield } \\
\text { Strain } \\
(\%)\end{array}$ & $\begin{array}{c}\text { Yield } \\
\text { Stress } \\
(\mathrm{ksi})\end{array}$ & $\begin{array}{c}\text { Ultimate } \\
\text { Strain } \\
(\%)\end{array}$ & $\begin{array}{c}\text { Ultimate } \\
\text { Stress } \\
(\mathrm{ksi})\end{array}$ & $\begin{array}{c}\text { Specific } \\
\text { Strength } \\
(\mathrm{kips}-\mathrm{in} . / \mathrm{l} \mathrm{b})\end{array}$ \\
\hline 1 & 1476.757 & 642.618 & 0.726 & 10.676 & 1.048 & 15.461 & 672.796 \\
\hline 2 & 2175.876 & 770.245 & 0.714 & 15.124 & 1.227 & 20.878 & 739.052 \\
\hline 3 & 2175.652 & 734.820 & 0.914 & 16.308 & 1.101 & 19.840 & 670.103 \\
\hline 4 & 1994.368 & 641.336 & 0.753 & 15.082 & 1.041 & 19.659 & 632.181 \\
\hline 5 & 1895.841 & 733.584 & 0.696 & 11.639 & 1.072 & 17.982 & 695.789 \\
\hline 6 & 2220.686 & 765.500 & 0.926 & 17.891 & 1.015 & 19.738 & 680.407 \\
\hline 7 & 2273.037 & 818.386 & 0.835 & 17.731 & 1.112 & 20.673 & 744.330 \\
\hline 8 & 2081.200 & 780.541 & 0.806 & 16.219 & 1.346 & 21.353 & 800.821 \\
\hline 9 & 1996.656 & 731.343 & 0.793 & 15.115 & 1.019 & 18.675 & 684.019 \\
\hline 10 & 2183.846 & 603.741 & 0.859 & 16.791 & 0.983 & 19.944 & 551.376 \\
\hline Mean & 2047.392 & 722.211 & 0.802 & 15.258 & 1.096 & 19.420 & 687.087 \\
\hline $\begin{array}{c}\text { Standard } \\
\text { Deviation }\end{array}$ & 232.7721 & 70.013 & 0.081 & 2.394 & 0.111 & 1.711 & 67.468 \\
\hline Maximum & 2273.037 & 818.386 & 0.926 & 17.891 & 1.346 & 21.353 & 800.821 \\
\hline Minimum & 1476.757 & 603.741 & 0.696 & 10.676 & 0.983 & 15.461 & 551.376 \\
\hline
\end{tabular}




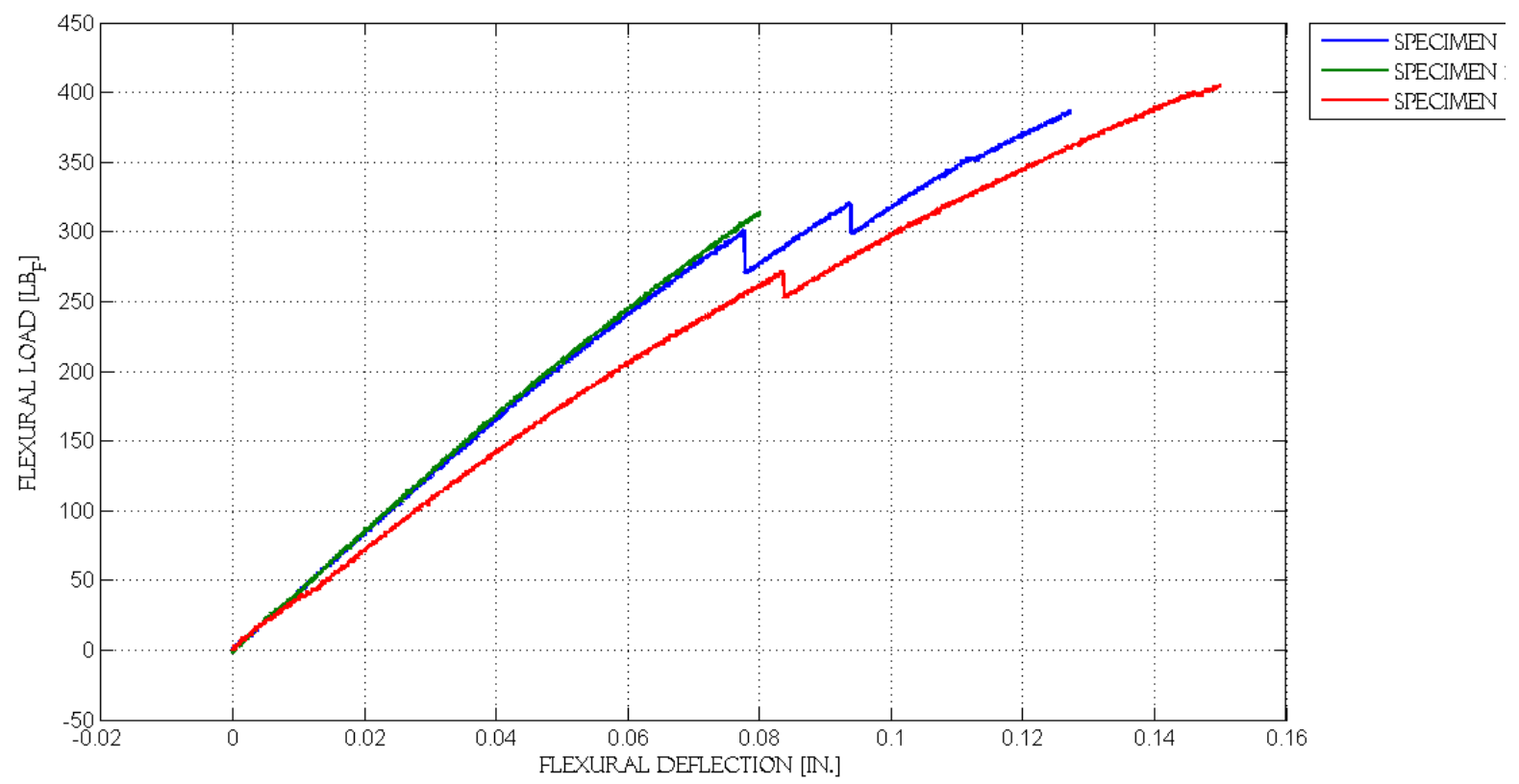

Figure A - 26. Tensile force-displacement curves of quarternode bamboo for cylindrical configuration

Table A - 0-28. Flexural properties of quarter-node bamboo for cylindrical configuration

\begin{tabular}{|c|c|c|c|c|c|c|c|}
\hline Specimen & $\begin{array}{c}\text { Modulus } \\
(\mathrm{ksi})\end{array}$ & $\begin{array}{c}\text { Specific } \\
\text { Modulus } \\
(\mathrm{kips}-\mathrm{in} / \mathrm{ll} \mathrm{b}) \\
\times 100\end{array}$ & $\begin{array}{c}\text { Yield } \\
\text { Strain } \\
(\%)\end{array}$ & $\begin{array}{c}\text { Yield } \\
\text { Stress } \\
(\mathrm{ksi})\end{array}$ & $\begin{array}{c}\text { Ultimate } \\
\text { Strain } \\
(\%)\end{array}$ & $\begin{array}{c}\text { Ultimate } \\
\text { Stress } \\
(\mathrm{ksi})\end{array}$ & $\begin{array}{c}\text { Specific } \\
\text { Strength } \\
(\mathrm{kips}-\mathrm{in} . / \mathrm{l} \mathrm{b})\end{array}$ \\
\hline 1 & 469.939 & 128.750 & 0.73 & 3.294 & 1.20 & 4.271 & 113.4 \\
\hline 2 & 494.151 & 152.988 & 0.74 & 3.505 & 0.74 & 3.505 & 125.2 \\
\hline \hline 3 & 426.978 & 114.471 & 1.36 & 4.762 & 1.40 & 4.842 & 118.9 \\
\hline Mean & 463.690 & 132.070 & 0.94 & 3.854 & 1.11 & 4.206 & 120.2 \\
\hline $\begin{array}{c}\text { Standard } \\
\text { Deviation }\end{array}$ & 34.020 & 19.472 & 0.36 & 0.794 & 0.34 & 0.671 & 15.6 \\
\hline Maximum & 494.151 & 152.988 & 1.36 & 4.762 & 1.40 & 4.842 & 125.2 \\
\hline Minimum & 426.978 & 114.471 & 0.73 & 3.294 & 0.74 & 3.505 & 113.4 \\
\hline
\end{tabular}




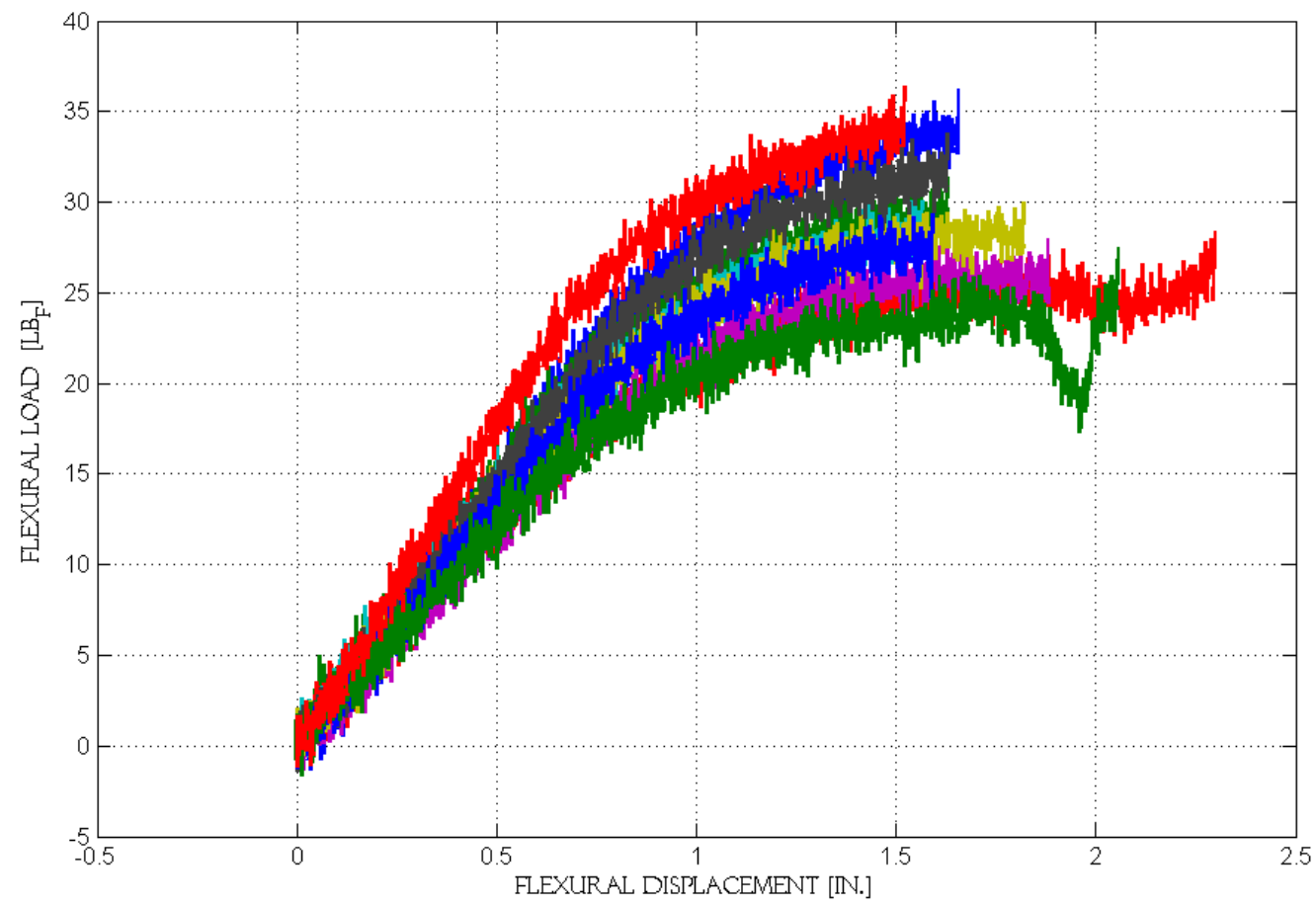

- SPECIMEN 1 SPECIMEN 2 - SPECIMEN 3 SPECIMEN 4

SPECIMEN 5 SPECIMEN 6 - SPECIMEN 7 SPECIMEN 8 SPECIMEN 9 SPECIMEN 10

Figure A - 27. Tensile force-displacement curves of quarternode bamboo for cylindrical configuration

Table A - 0-29. Flexural properties of quarter-node bamboo for rectangular configuration

\begin{tabular}{|c|c|c|c|c|c|c|c|}
\hline Specimen & $\begin{array}{c}\text { Modulus } \\
(\mathrm{ksi})\end{array}$ & $\begin{array}{c}\text { Specific } \\
\text { Modulus } \\
(\mathrm{kips}-\mathrm{in} . / \mathrm{lb}) \\
\times 100\end{array}$ & $\begin{array}{c}\text { Yield } \\
\text { Strain } \\
(\%)\end{array}$ & $\begin{array}{c}\text { Yield } \\
\text { Stress } \\
(\mathrm{ksi})\end{array}$ & $\begin{array}{c}\text { Ultimate } \\
\text { Strain } \\
(\%)\end{array}$ & $\begin{array}{c}\text { Ultimate } \\
\text { Stress } \\
(\mathrm{ksi})\end{array}$ & $\begin{array}{c}\text { Specific } \\
\text { Strength } \\
(\mathrm{kips}-\mathrm{in} . / \mathrm{lb} \mathrm{m})\end{array}$ \\
\hline 1 & 2150.276 & 693.836 & 0.971 & 17.653 & 1.472 & 21.982 & 709.309 \\
\hline 2 & 2134.371 & 659.951 & 1.091 & 19.118 & 1.442 & 21.968 & 679.269 \\
\hline 3 & 2038.801 & 615.256 & 1.334 & 20.030 & 1.923 & 22.922 & 691.716 \\
\hline 4 & 2340.215 & 723.972 & 1.119 & 20.962 & 1.401 & 23.215 & 718.177 \\
\hline 5 & 2170.595 & 753.392 & 0.976 & 18.397 & 1.527 & 21.221 & 736.573 \\
\hline 6 & 2629.806 & 787.875 & 0.842 & 21.090 & 1.488 & 24.989 & 748.649 \\
\hline 7 & 2747.548 & 804.689 & 1.129 & 24.035 & 1.325 & 26.099 & 764.374 \\
\hline 8 & 2310.381 & 680.511 & 0.950 & 18.241 & 1.323 & 21.639 & 637.375 \\
\hline 9 & 2355.841 & 694.596 & 0.962 & 19.560 & 1.645 & 23.781 & 701.149 \\
\hline 10 & 2457.851 & 744.581 & 0.666 & 15.278 & 1.402 & 23.106 & 699.986 \\
\hline \hline Mean & 2333.568 & 715.866 & 1.004 & 19.436 & 1.495 & 23.092 & 708.658 \\
\hline $\begin{array}{c}\text { Standard } \\
\text { Deviation }\end{array}$ & 226.663 & 58.509 & 0.180 & 2.349 & 0.178 & 1.537 & 36.419 \\
\hline Maximum & 2747.548 & 804.689 & 1.334 & 24.035 & 1.923 & 26.099 & 764.374 \\
\hline Minimum & 2038.801 & 615.256 & 0.666 & 15.278 & 1.323 & 21.221 & 637.375 \\
\hline
\end{tabular}




\section{A5. Manufacturing Hemp Mold Joints for Bamboo Truss Application}

The entire construction of the truss structure is done in the Cal Poly Structures/Composites lab, and all the materials used in the truss fabrication are green certified. Also, it is decided to fabricate a small-scale prototype version of this application in order to evaluate the effectiveness of bamboo in the structure. A full-scale prototype is left for future work. The mentioned materials include bamboo internodes cured with the $3 \%$ salt treatment, joints made of the same bidirectional hemp fabric, and biodegradable putty from Ecopoxy. The joints are fabricated through a wet lay-up process using organic resin and hardener, and the entire truss is vacuum bagged to properly cure the part for a desirable final product.
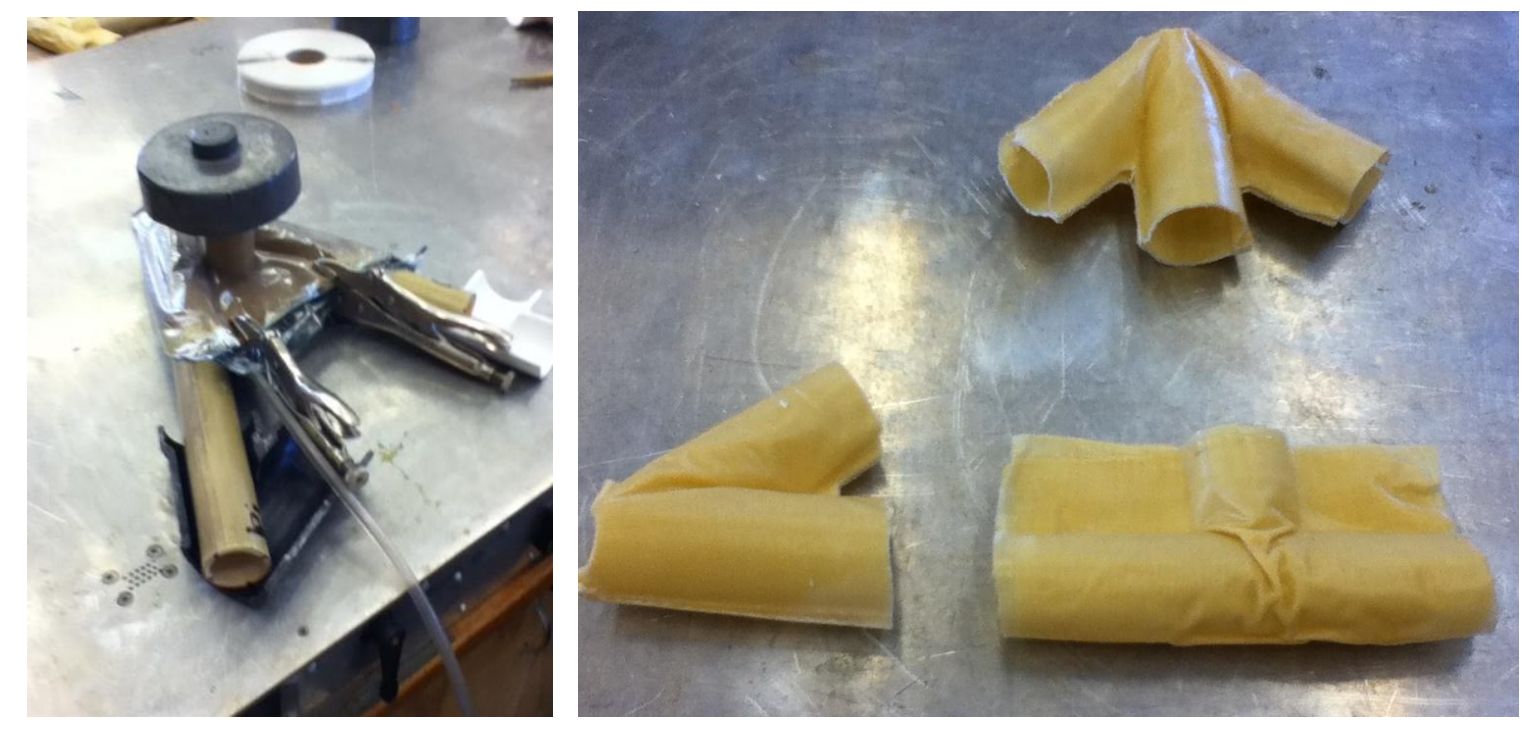

Figure A-28. Attempt at fabricating molded joints for bamboo truss

The manufacturing of the mold design is discussed for potential improvement on the fabrication technique of the joint—as it would greatly simplify manufacturing on a larger scale. First, the properly cured bamboo poles are cut to the appropriate length. Afterward, biocomposite laminates are prepared for each molded fitting, so two 5 -in. $x$-in. rectangular hemp fabric cutsouts are prepared for each of the four joints. The fabric is then coated with Ecopoxy resin ER500 and EH725 medium hardener with a 4:1 resin-hardener ratio. Plastic spreaders are 
utilized to ensure even coats of the resin over the fabric. Once soaked, the bi-directional plies are laid-up with special care taken in aligning the fibers of each ply. Details of the lay-up process are discussed shortly in the next segment. Then the laminate is folded around the connection point at each joint, and the entire joint is encased in a vacuum bag following contemporary composite bagging techniques. Details of this portion of the composite molding process are also further discussed in the subsequent section. Once the lay-up has cured, the molded joints are inspected for quality. At this point, the prepared joints did not pass the quality check and were consequently discarded for the other design. Figure A- depicts some of the attempts made to fabricate these molded joints. 


\section{A6. Flexural Strain Derivation}

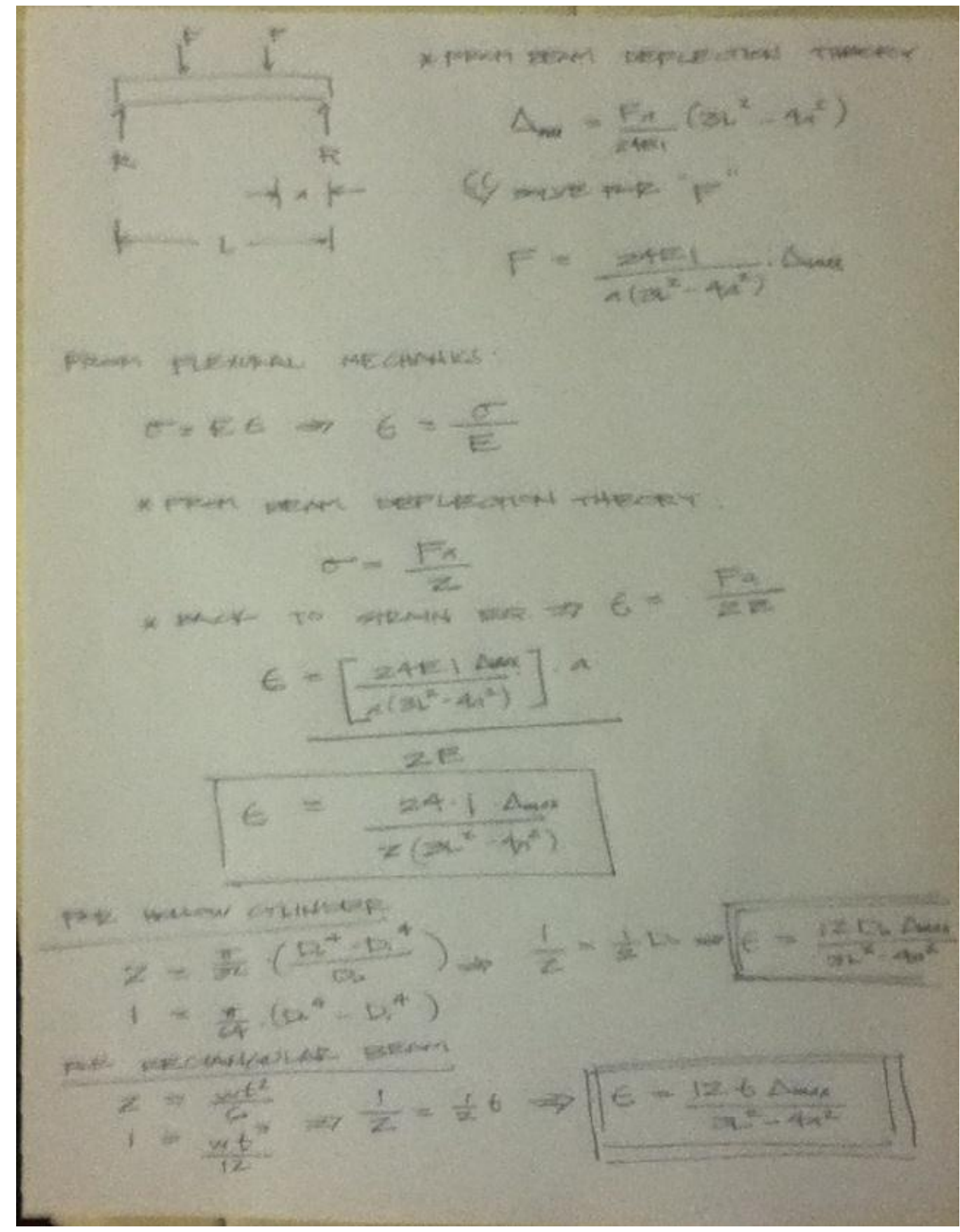

\section{A7. MATLAB Codes}

\section{Elastic Modulus}

function[Ymodulus, rsq] = modulus (stress, strain, reg) $\circ\{$

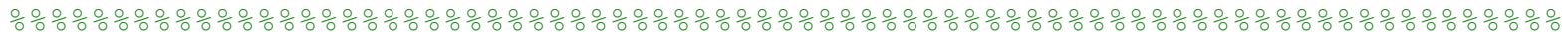

THIS CODE DETERMINES THE YOUNG'S MODULUS OF A MATERIAL SPECIMEN

FROM ITS STRESS-STRAIN CURVE. THE USER SUPPLIES THE LINEAR REGION OF

THE CURVE, AND THE MODULUS IS COMPUTED BY FITTING A LINEAR REGRESSION 
LINE. THE ACCURACY OF THE MODULUS IS ANALYZED BY USING A R-SQUARED VALUE DETERMINE FROM THE FITTED REGRESSION LINE.

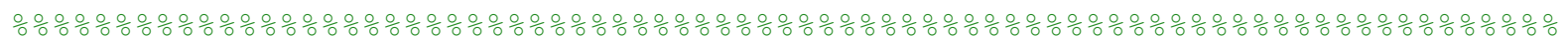
INPUTS :

stress = array of stress data points [ksi]

strain = array of strain data points [\%]

reg = region of the curve believed to be linear on the stress-strain curve

[1x2] vector where the first element is the lower percentile and

the second is the higher percentile of the original curve.

\section{OUTPUTS :}

modulus = Young's Modulus [*same units as 'stress']

rsq $=$ R-squared value of fitted modulus equation [-]

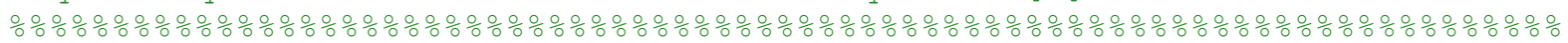

응

응 Setting lower/upper limit of the linear region of curve

lower_lim = reg(1);

higher_lim $=\operatorname{reg}(2)$;

$\mathrm{b}=\operatorname{round}($ length (strain)*lower_lim);

if $\mathrm{b}==0$

$\mathrm{b}=1$;

end

$c=$ round(length (strain)*higher_lim);

lin_strain $=$ strain $(b: c) / 100 ; \%$ convert strain from [\%] to [in/in]

lin_stress $=$ stress $(\mathrm{b}: \mathrm{c})$;

응 Fitting a regression line to stress-strain curve

$\mathrm{x}=$ lin_strain;

$y=$ lin_stress;

$\mathrm{p}=\operatorname{polyfit}(\mathrm{x}, \mathrm{y}, 1)$;

Ymodulus $=\mathrm{p}(1)$;

yfit $=$ polyval $(\mathrm{p}, \mathrm{x})$;

yresid = y - yfit;

SSresid = sum(yresid.^2);

SStotal $=($ length $(y)-1) * \operatorname{var}(y)$;

rsq $=1$ - SSresid/SStotal;

plot (strain, stress)

hold all

plot (x.*100, yfit, '--r', 'linewidth', 1.5)

legend('Stress-Strain Curve', 'Fitted Line', 'location', 'best')

xlabel ('Strain [\%]')

ylabel ('Stress [ksi]')

hold off

grid on

end

\section{YIELDING CONDITIONS}

function[yield strain, yield stress, eval, rl] = thresh_yield (sťress, strain, regions, slope, modulus) 응

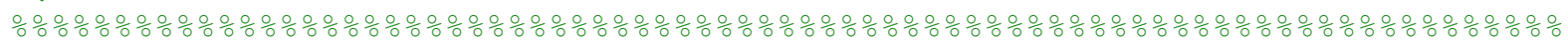
THIS CODE DETERMINES THE YIELD STRESS OF A MATERIAL SPECIMEN 
FROM ITS STRESS-STRAIN CURVE THROUGH THE SLOPE THRESHOLD METHOD. THE STRESSSTRAIN CURVE IS DIVIDED INTO THE SPECIFIED NUMBER OF REGIONS FROM THE $1 / 4$ LENGTH ON THE CURVE TO THE MAX STRESS LOCATION. THE SLOPE OF EACH REGION IS CALCULATED AND THE FIRST REGION'S SLOPE TO DROP TO/BELOW THE GIVEN SLOPE THRESHOLD PERCENTAGE OF THE MODULUS IS THE INTERVAL THAT CONTAINS THE SOLUTION. THE CENTER OF THAT INTERVAL IS DETERMINED TO BE THE YIELD STRESS/STRAIN POINT.

TIPS: INCREASING THE NUMBER OF REGIONS NORMALLY INCREASES ACCURACY. FOR DUCTILE MATERIALS, A THRESHOLD SLOPE PERCENTAGE VALUE OF 50\% IS ADVISED. FOR BRITTLE MATERIALS, A THRESHOLD SLOPE PERCENTAGE VALUE OF 10\% IS RECOMMENDED.

REQ. CODES: find_max

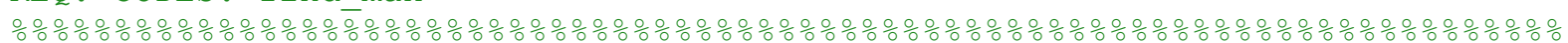

INPUTS :

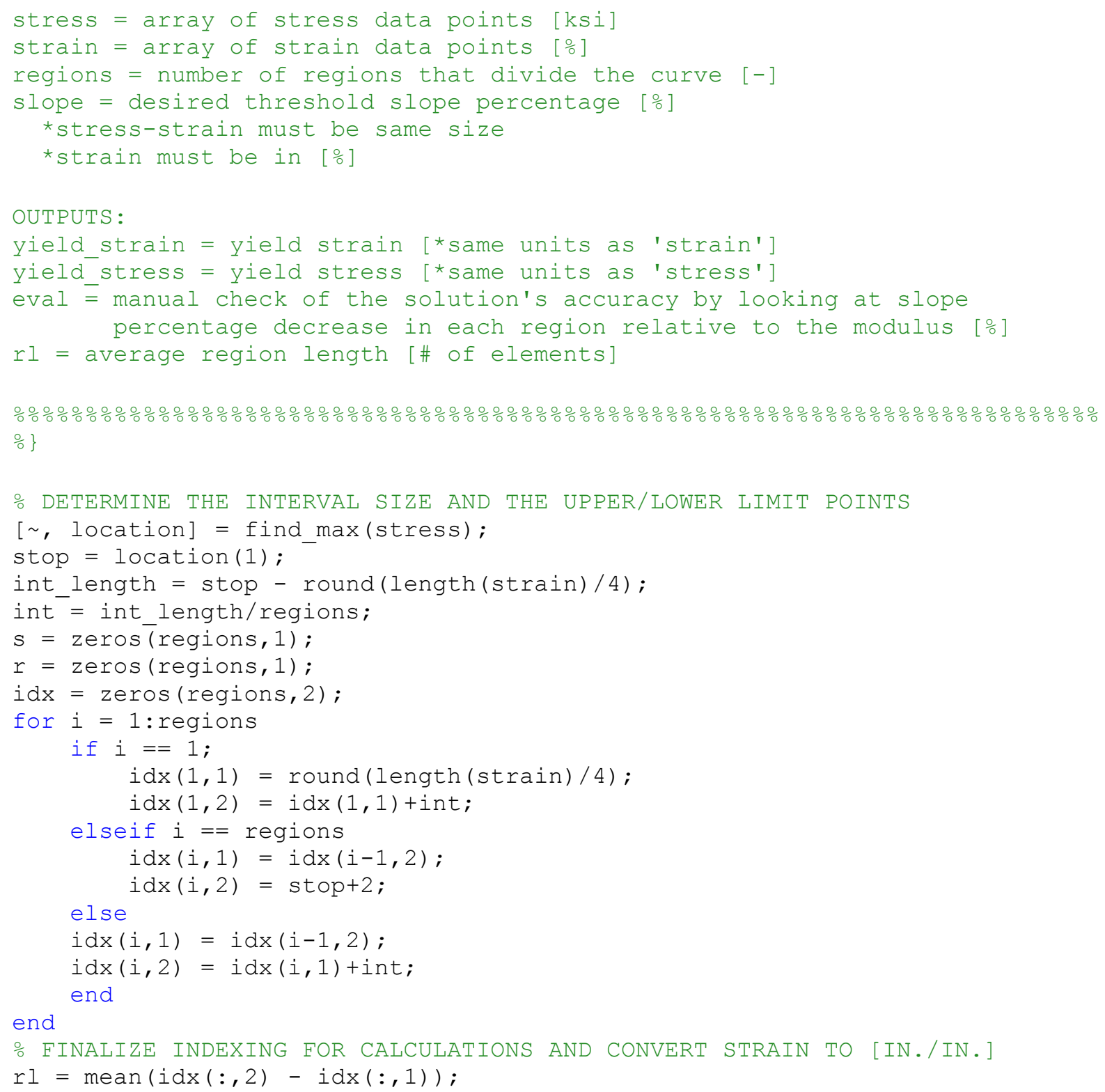




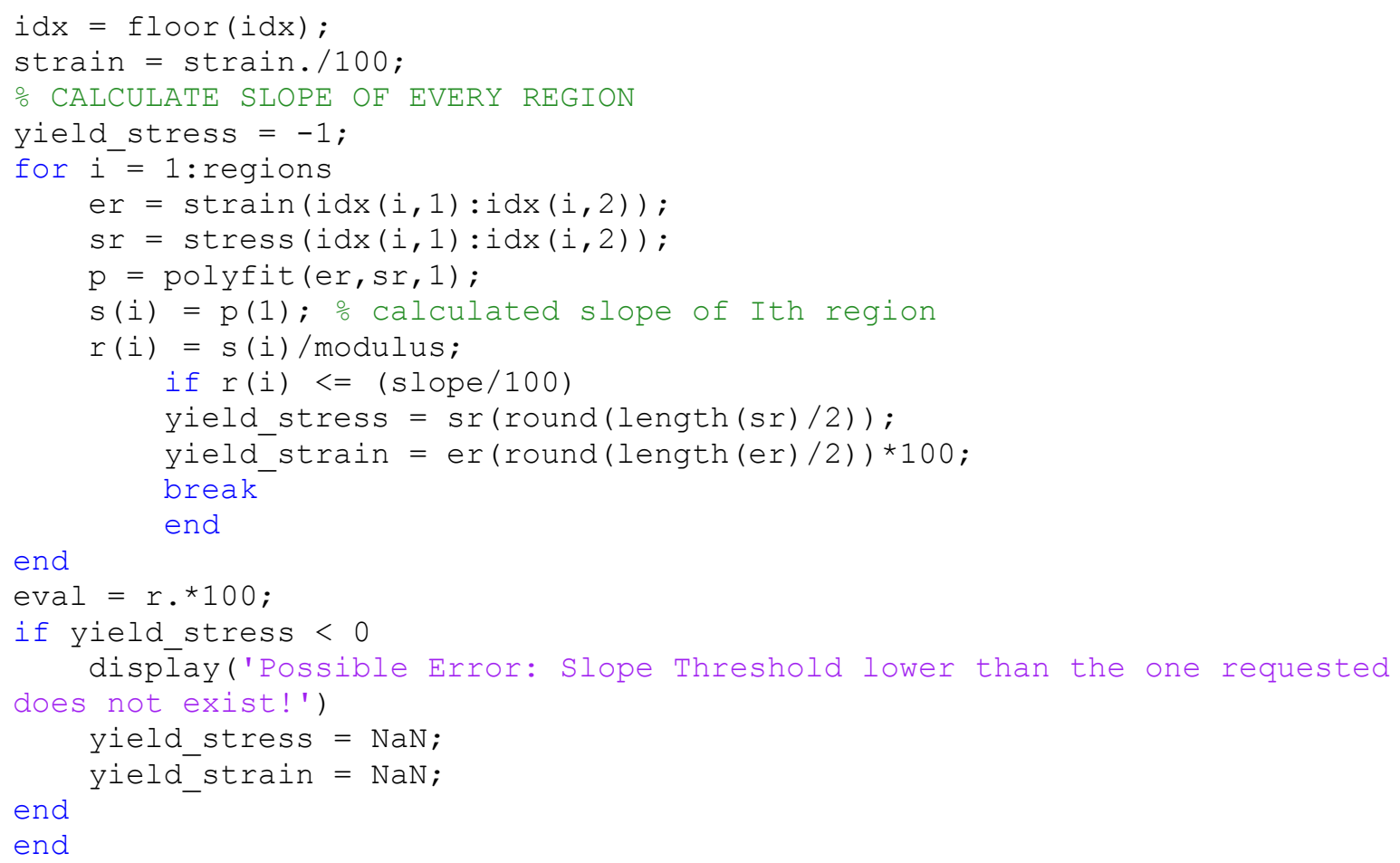

\section{MAXIMUM}

function [maximum, location] = find $\max (\mathrm{A})$

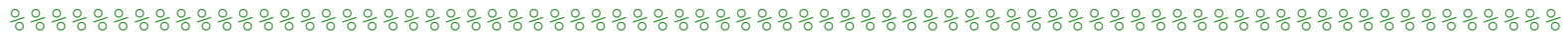
응응 THIS CODE FINDS THE MAXIMUM OF A VECTOR AND SPITS OUT ITS LOCATION IN 응 THE VECTOR.

\section{POISSON'S RATIO}

function[poisson, rsq_t,rsq_l] = poisson(load,strain_t,strain_l,reg) $\circ\{$

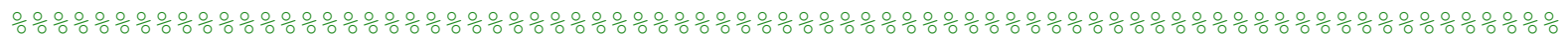
THIS CODE DETERMINES THE POISSON'S OF A MATERIAL SPECIMEN FROM ITS LOAD-STRAIN CURVE. THE USER SUPPLIES THE LINEAR REGION OF THE CURVE, AND THE POISSON RATIO IS COMPUTED BY TAKING THE RATIO OF THE FITTED LINEAR REGRESSION LINES' SLOPES TO EACH STRESS-STRAIN CURVE IN THE LONGITUDINAL AND TRANSVERSE DIRECTION. THE ACCURACY OF EACH SLOPE IS ANALYZED BY USING AN R-SQUARED VALUE DETERMINED FROM THE FITTED REGRESSION LINES. A 
PLOT IS FINALLY GENERATED OF THE ABSOLUTE VALUE OF EACH RESPECTIVE STRAIN ON THE Y-AXIS AND LOAD ON THE X-AXIS.

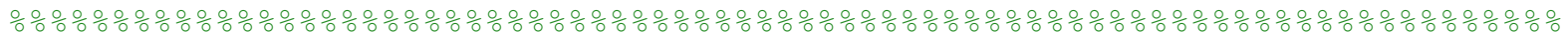
INPUTS :

load = array of load data points

strain_t = array of strain data points in the transverse direction [\%]

strain_l = array of strain data points in the longitudinal direction [\%] reg $=$ region of the curve believed to be linear on the stress-strain curve

[1x2] vector where the first element is the lower percentile and the second is the higher percentile of the original curve.

OUTPUTS :

poisson = Poisson's Ratio [-]

rsq $t=R$-squared value of fitted transverse equation [-]

rsq_l = R-squared value of fitted longitudinal equation [-]

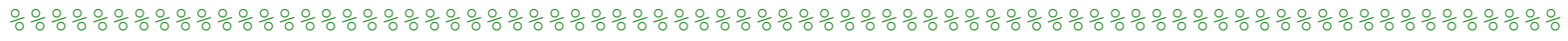

의

응 Setting lower/upper limit of the linear region of curve

lower_lim = reg(1);

higher_lim $=\operatorname{reg}(2)$;

$\mathrm{b}=\operatorname{round}($ length $($ strain_l $) *$ lower_lim);

if $\mathrm{b}==0$

$\mathrm{b}=1$;

end

$\mathrm{c}=\operatorname{round}($ length (strain_l) *higher_lim);

\% adjust strain from $\%$ to decimal

lin_strain $t=$ strain $t(\mathrm{~b}: \mathrm{c}) / 100$;

lin strain_l = strain ${ }^{-}$- $(\mathrm{b}: \mathrm{c}) / 100$;

lin load $=$ load (b:c);

$\%$ Fitting a regression line to stress-strain curve

$\mathrm{x}=$ lin_load;

$y_{-} t=1 \bar{i}{ }_{-}$strain_t;

$\mathrm{y}_{-} 1=$ lin_strain_1; $_{-}^{-}$

p_t $=$ polyfit $\left(x, y_{-} t, 1\right)$

$\mathrm{P}_{-}{ }^{-}=$polyfit $\left(\mathrm{x}, \mathrm{y}_{-}{ }_{1}, 1\right)$;

nu_t $=p_{-} t(1) ;$

nu_l $=p_{-}^{-1}(1)$;

poisson $=-n u_{-}$t/nu_l;

yfit $t=$ polyval $\left(p \_t, x\right)$;

yfit_l $=$ polyval $\left(p_{-}{ }_{1}, \mathrm{x}\right)$;

yresid_t $=y_{-} t-$ yfit_t $_{-}$

yresid_l $=y_{-}^{-}{ }^{-}$yfit_l; $^{-}$

SSresidt $=\operatorname{sum}(y r e s i d t . \wedge 2)$;

SSresid_l = $\operatorname{sum}($ yresid_l.^2);

SStotal $t=\left(\right.$ length $\left.\left(y \_t\right)-1\right) * \operatorname{var}\left(y \_t\right)$;

SStotal_l ${ }_{-}^{-}=\left(\right.$length $\left.\left(y_{-}{ }^{-}\right)-1\right) * \operatorname{var}\left(y_{-}{ }^{-}\right)$; 


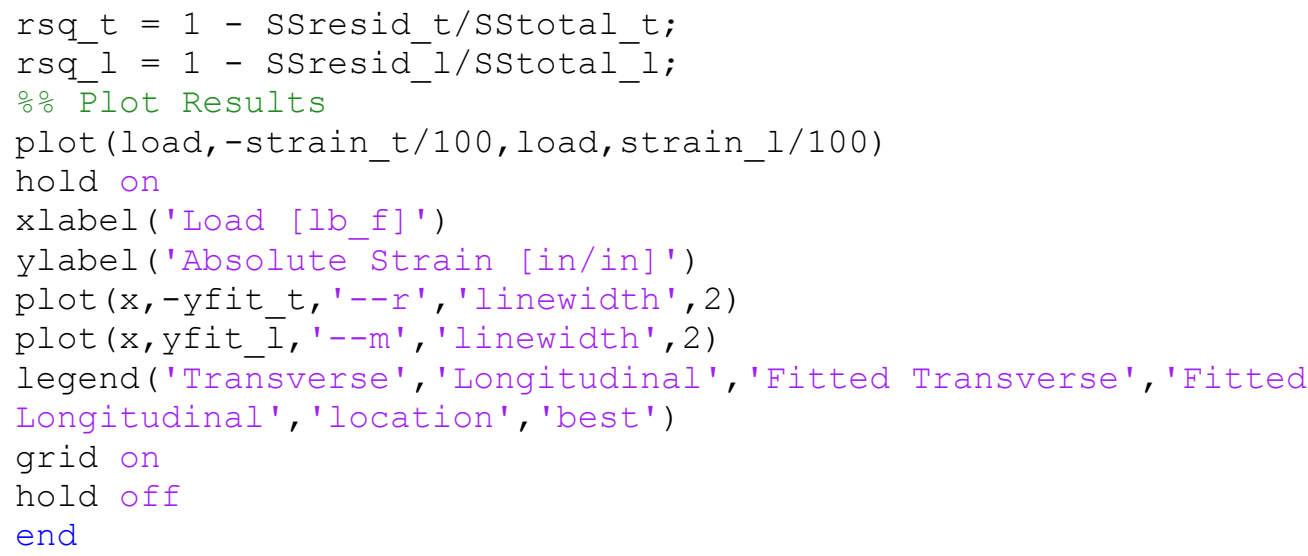

